WHOI-76-I0I
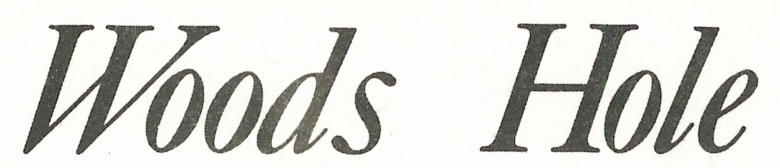

Oceanographic

Institution
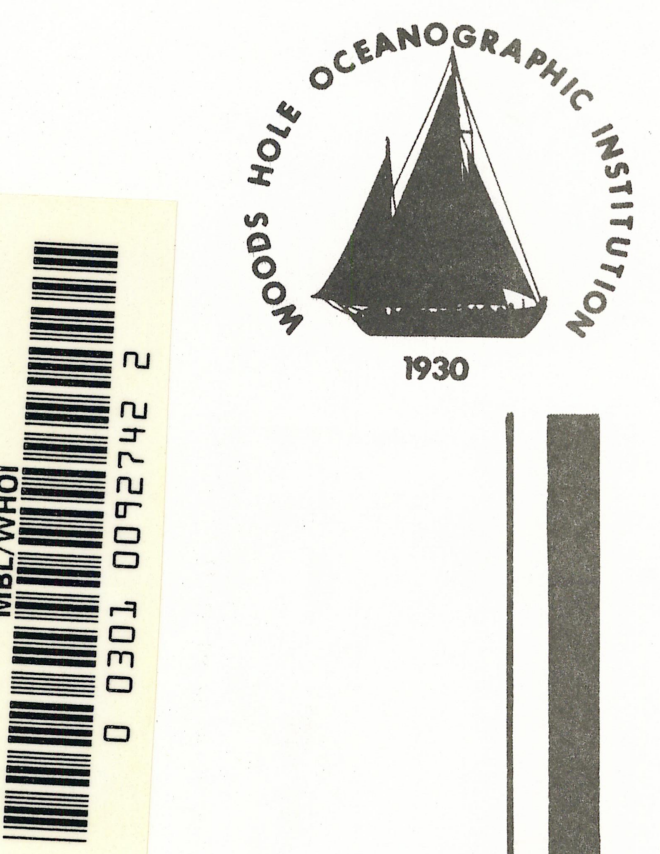

\title{
A COMPILATION OF MOORED CURRENT DATA AND ASSOCIATED OCEANOGRAPHIC OBSERVATIONS, VOLUME XII (1973 MID-OCEAN DYNAMICS EXPERIMENT (MODE))
}

\section{By}

D. Chausse and S. Tarbe11

November 1976

TECHNICAL REPORT

Prepared for the Office of Naval Research under Contract N00014-66-C-0241; NR 083-004 and N00014-76-C-0197; NR 083-400.

Approved for public release; distribution unlimited.

WOODS HOLE, MASSACHUSETTS 02543

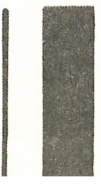





\section{WHOI-76-101}

\section{A COMPILATION OF MOORED CURRENT DATA AND ASSOCIATED OCEANOGRAPHIC OBSERVATIONS, VOLUME XII (1973 MID-OCEAN DYNAMICS EXPERIMENT (MODE))}

by
D. Chausse and S. Tarbell

WOODS HOLE OCEANOGRAPHIC INSTITUTION

Woods Hole, Massachusetts 02543

November 1976

\section{TECHNICAL REPORT}

Prepared for the Office of Naval Research under Contracts N00014-66-C-0241; NR 083-004 and N00014-76-C-0197; NR 083-400.

Reproduction in whole or in part is permitted for any purpose of the United States Government. In citing this manuscript in a bibliography, the reference should be followed by the phrase: UNPUBLISHED MANUSCRIPT.

Approved for public release; distribution uniimited.

Approved for Distribution

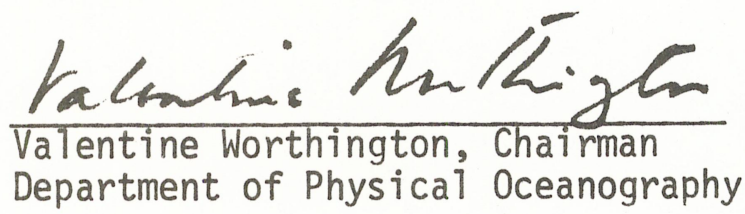





\begin{abstract}
Summaries are presented of basic current, temperature and pressure measurements which were made from moored instruments as a part of the Mid-Ocean Dynamics Experiment (MODE) which took place March to July 1973. Current data are presented as Basic Statistics, Spectral Diagrams, Progressive Vector Diagrams, East vs. North Plots, and Variables vs. Time Plots.
\end{abstract}


Table of Contents

Abstract

Page

Table of Contents

Preface

Acknowledgments

Introduction

Hydrography

Moorings

Temperature/Pressure

Depth

Time

Current Meters

Current Meter Types

Current Meter Problems

Data Processing

Data Identifiers

Data Presentation

Computer Programs

References

iii

$\mathrm{v}$

vi

vii

viii

viii

xii

xii

xiv

$\mathrm{xv}$

$\mathrm{xV}$

xvi

xviij

xviii

xix

xxii

xxvi

Data Section 1

Mooring 480

481

482

483

484

485

486

488

489

493

494

495

497

498

499

500

501

110

128

134

144

158

168

178

200

Data Section 2

Composites

223

Appendix

281 

This volume is the twelfth of a series of Data Reports presenting data collected by the W.H.O.I. Buoy Group.

Volume I W.H.O.I. Ref. 65-44 (unpublished manuscript) Webster, F., and N. P. Fofonoff, 1965

"A compilation of moored current meter observations, Volume I".

Volume II W.H.O.I. Ref. 66-60 (unpublished manuscript)

Webster, F., and N. P. Fofonoff, 1966

"A compilation of moored current meter observations, Volume II".

Volume III W.H.O.I. Ref. 67-66 (unpublished manuscript)

Webster, F., and N. P. Fofonoff

"A compilation of moored current meter observations, Volume III".

Volume IV W.H.O.I. Ref. 70-40 (unpublished manuscript)

Pollard, R. T., 1970

"A compilation of moored wind and current meter observations, Volume IV".

Volume V W.H.O.I. Ref. 71-50 (unpublished manuscript)

Tarbell, S., and F. Webster

"A compilation of moored current meter and wind observations, Volume V (1966 measurements)".

Volume VI $\frac{\text { W.H.O.I. Ref. } 74-7}{\text { Tarbell, S., 1974 (unpublished manuscript) }}$

"A compilation of moored wind and current observations taken in 1967, Volume VI".

Volume VII W.H.O.I. Ref. 74-52 (unpublished manuscript)

Chausse, D., and S. Tarbell, 1974

"A compilation of moored current meter and wind observations, Volume VII (1968 measurements)" .

Volume VIII W.H.O.I. Ref. 75-7 (unpublished manuscript)

Pollard, R. T., and S. Tarbell, 1975

"A compilation of moored current meter and wind observations, Volume VIII (1970 array experiment)".

Volume IX W.H.O.I. Ref. 75-68 (unpublished manuscript)

Tarbell, S., M. G. Briscoe, and D. Chausse, 1976

"A compilation of moored current data and associated oceanographic observations, Volume IX (1973 Internal Wave Experiment (IWEX))".

Volume X W.H.O.I. Ref. 76-40 (unpublished manuscript) Tarbell, S., 1976

"A compilation of moored current data and associated oceanographic observations, Volume X (early 1969 measurements)" .

Volume XI W.H.O.I. Ref.76-4I (unpublished manuscript)

Tarbell, S., 1976

"A compilation of moored current data and associated oceanographic observations, Volume XI (late 1969 measurements)".

Volume XII presents data from moored instruments collected as a part

of the Mid-Ocean Dynamics Experiment (MODE). 


\section{ACKNOWIEDGMENTS}

MODE constituted a major effort for all sections of the Buoy Group. Over 50 miles of mooring line with over $700 \mathrm{glass}$ spheres and almost 200 instruments, including releases, radios and lights were set from the Research Vessel Chain in 12 days and recovered in 9 days. This was made possible by much hard work and good humor from everyone involved in the planning and execution of the MODE W.H.O.I. buoy moorings, including the R. V. Chain and her crew who were, as usual, dependable and helpful. This report was prepared with the help of all members of the Data Processing section of the Buoy Group and in particular by Audrey Williams. 


\section{INTRODUCTION}

The Mid-Ocean Dynamics Experiment (MODE) was a successful effort to measure large scale, slow moving physical ocean features with a wide variety of instruments. Additional MODE objectives include determining the effects of bottom topography on the mesoscale motions and comparing the interpretation of data gathered from the different types of instruments. MODE was planned as a series of concurrent experiments where each instrument type would comprise an experiment of its own as well as be an addition to the MODE data array.

The W.H.O.I. moorings were only one part of the MODE program. SOFAR floats, airdropsondes, vertical profilers, STDs and tow fish all had experiments of their own. The Institute of Oceanographic Sciences in England and the University of Rhode Island set additional moored current meter stations. The bottom experiment added pressure gauges, electromagnetic transport meters, inverted echo sounders, magnetic field detectors and detectors which measure the vertical component of the electric field to the instrument array.

The active field work involved the crews and scientists aboard six ships and three airplanes which covered the MODE area (Bermuda Triangle) for four months gathering the data from these assorted instruments. An uncounted number of people worked before, during and after MODE towards obtaining high quality data.

For information on other aspects of the MODE project see the following appendixes:

Appendix I List of the participating institutions and principal investigators by project.

Appendix II List of papers about MODE by MODE Contribution Number. Appendix III List of contributions to the MODE Hot-Line News.

This report presents the data gathered on the W.H.O.I. buoy moorings by current meters and temperature/pressure recorders plus mean CTD temperature and salinity data taken during the mooring recovery phase of the operation. 
Hydrography

Ten CTD stations were made on the buoy recovery cruise of the Research Vessel Chain, cruise 112, Leg 6. The CTD was designed by Neil Brown (Brown, 1975; Fofonoff, Hayes and Millard, 1974) and measures conductivity, temperature and pressure. Temperature and salinity profiles, spatially averaged over ten CTD stations, are shown in Fig. I (Millard and Bryden, 1973).

\section{Moorings}

There were 16 subsurface moorings set and retrieved by the Woods Hole Oceanographic Buoy Group for the MODE array. A seventeenth mooring, the only surface mooring, was set as a reference marker to indicate the center of the MODE area at $28^{\circ} 00^{\prime} \mathrm{N}, 69^{\circ} 10^{\prime} \mathrm{W}$. This mooring was considered lost after May 23.

The sixteen subsurface moorings had the same basic design with current meters and temperature/pressure recorders shackled into the mooring line at designated depths. Jacketed 3/16" wire was used for the top of the mooring line which was in the fishbite zone. Below the fishbite zone 3/8" dacron was used. The flotation was provided by 16" and 17" glass spheres in hard hat clusters which were attached at intervals along the mooring line. Details on moorings, components and procedures may be found in Heinmiller (1973a), Heinmiller (1973b) and Heinmiller (1975).

Table 1 lists the MODE mooring number, the W.H.O.I. mooring number and the times and location of launch and retrieval.

Figure 2 is a map showing mooring positions and bottom topography. 

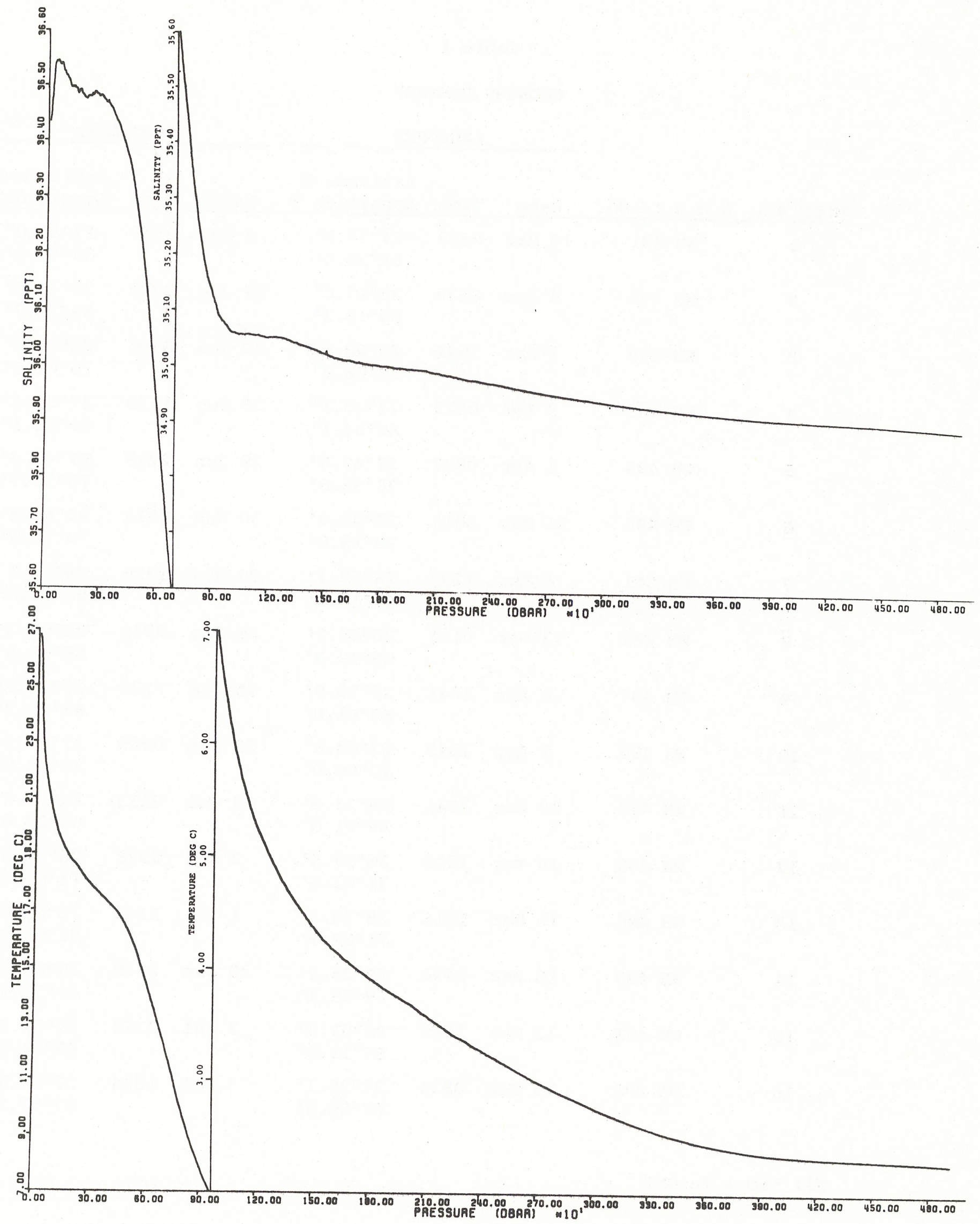

Figure 1. Plots of salinity and temperature from 10 CTD stations 
Table 1

MOORING SUMMARY

I.AUNCHED

RECOVERED

\begin{tabular}{|c|c|c|c|c|c|c|c|c|}
\hline DDE & No. & W.H.O.I. NO. & Date & Time & $\begin{array}{l}\text { Latitude } \mathrm{N} \\
\text { Longitude } \mathrm{W}\end{array}$ & Date & Time & $\begin{array}{l}\text { Latitude N } \\
\text { Longitude W }\end{array}$ \\
\hline & 1 & WH 481 & 10 Mar & 2358 & $\begin{array}{l}27^{\circ} 59.8^{\prime} \\
69^{\circ} 39.0^{\prime}\end{array}$ & $4 \mathrm{JuI}$ & 1552 & $\begin{array}{l}27^{\circ} 38.0^{\prime} \\
69^{\circ} .11 .6^{\prime}\end{array}$ \\
\hline & 2 & WH 500 & 4 Apr & 0436 & $\begin{array}{l}28^{\circ} 17.0^{\circ} \\
69^{\circ} 16.3^{\circ}\end{array}$ & 27 Jun & 0829 & $\begin{array}{l}28^{\circ} 16.5^{\prime} \\
69^{\circ} 16.7^{\prime}\end{array}$ \\
\hline & 3 & WH 499 & 3 Apr & 1641 & $\begin{array}{l}28^{\circ} 08.98^{\prime} \\
70^{\circ} 08.1^{\prime}\end{array}$ & 28 Jun & 0305 & $\begin{array}{l}28^{\circ} 09.0^{\circ} \\
70^{\circ} 08.1^{\prime \prime}\end{array}$ \\
\hline & 4 & WH 498 & 3 Apr & 0623 & $\begin{array}{l}27^{\circ} 33.1^{\prime} \\
69^{\circ} 34.1^{\prime \prime}\end{array}$ & 28 Jun & 1138 & $\begin{array}{l}27^{\circ} .33 .1^{\prime} \\
69^{\circ} 34.1^{\prime \prime}\end{array}$ \\
\hline & 5 & WH 494 & $I$ Apr & 0807 & $\begin{array}{l}27^{\circ} 49.8^{\prime} \\
70^{\circ} 39.8^{\prime}\end{array}$ & 29 Jun & 2219 & $\begin{array}{l}27^{\circ} 49.3^{\prime \prime} \\
70^{\circ} 39.9^{\prime \prime}\end{array}$ \\
\hline & 6 & WH 493 & 31 Mar & 1815 & $\begin{array}{l}28^{\circ} 42.0^{\circ} \\
70^{\circ} 15.8^{\prime}\end{array}$ & 30 Jun & 0514 & $\begin{array}{l}28^{\circ} 4.1 .8^{\circ} \\
70^{\circ}=6.2^{\circ}\end{array}$ \\
\hline & 7 & WH 501 & 4 Apr & 1200 & $\begin{array}{l}28^{\circ} 50.1^{\prime} \\
69^{\circ} 18.0^{\prime}\end{array}$ & 30 Jun & 1730 & $\begin{array}{l}28^{\circ} 50.5^{\prime \prime} \\
\left.69^{\circ}\right] .9 .0^{\prime \prime}\end{array}$ \\
\hline & 8 & WH 482 & $12 \operatorname{Mar}$ & 0131 & $\begin{array}{l}28^{\circ} 09.3^{\circ} \\
68^{\circ} 39.3^{\prime}\end{array}$ & 26 Jun & 0940 & $\begin{array}{l}28^{\circ} 09.3^{\prime \prime} \\
68^{\circ} 38.5^{\prime \prime}\end{array}$ \\
\hline & 9 & WH 497 & 2 Apr & 1641 & $\begin{array}{l}27^{\circ} 18.0^{\prime \prime} \\
69^{\circ} 01.0^{\circ}\end{array}$ & 28 Jun & 2336 & $\begin{array}{l}\left.28^{\circ}\right] .8 .4^{\prime} \\
69^{\circ} 01.2^{\prime}\end{array}$ \\
\hline & 10 & WH 495 & 1 Apr & 1645 & $\begin{array}{l}27^{\circ} 08.8^{\prime} \\
70^{\circ} 00.0^{\prime}\end{array}$ & 29 Jun & 0955 & $\begin{array}{l}27^{\circ}\left(18.5^{\prime}\right. \\
70^{\circ}\left(11.0^{\prime}\right.\end{array}$ \\
\hline & 11 & WH 485 & 13 Mar & 2241 & $\begin{array}{l}26^{\circ} 23.8^{\prime} \\
69^{\circ} 21.0^{\prime}\end{array}$ & $2 \mathrm{Jul}$ & 2113 & $\begin{array}{l}26^{\circ} 2: 3.8^{\circ} \\
69^{\circ} 20.6^{\prime}\end{array}$ \\
\hline & 12 & WH 486 & 14 Mar & 1513 & $\begin{array}{l}26^{\circ} 57.5^{\prime} \\
71^{\circ} 02.6^{\prime}\end{array}$ & 2 JuI & 0523 & $\begin{array}{l}26^{\circ} 55.5^{\circ} \\
71^{\circ} \mathrm{C} 6.5^{\circ}\end{array}$ \\
\hline & 13 & WH 488 & 15 Mar & 1516 & $\begin{array}{l}28^{\circ} 33.1^{\prime} \\
71^{\circ} 22.9^{\prime \prime}\end{array}$ & $1 \mathrm{JuI}$ & 1348 & $\begin{array}{l}28^{\circ}=9.3^{\circ} \\
71^{\circ}<3.9^{\circ}\end{array}$ \\
\hline & 14 & WH 489 & 16 Mar & 0434 & $\begin{array}{l}29^{\circ} 35.0^{\prime \prime} \\
69^{\circ} 59.1^{\prime}\end{array}$ & 30 Jun & 2325 & $\begin{array}{l}29^{\circ} 36.4^{\prime} \\
69^{\circ}: 59.4^{\prime}\end{array}$ \\
\hline & 15 & WH 483 & 12 Mar & 1519 & $\begin{array}{l}29^{\circ} 02.3^{\prime \prime} \\
68^{\circ} 13.8^{\circ}\end{array}$ & $3 \mathrm{JuI}$ & 2322 & $\begin{array}{l}29^{\circ} \mathrm{C}=.6^{\prime} \\
68^{\circ} 14.0^{\prime \prime}\end{array}$ \\
\hline & 16 & WH 484 & $13 \operatorname{Mar}$ & 0834 & $\begin{array}{l}27^{\circ} 25.1^{\prime \prime} \\
67^{\circ} 59.5^{\circ}\end{array}$ & $3 \mathrm{Jul}$ & 1004 & $\begin{array}{l}27^{\circ} 22.7^{\prime} \\
67^{\circ} 57.9^{\circ}\end{array}$ \\
\hline
\end{tabular}

All times in GMT 


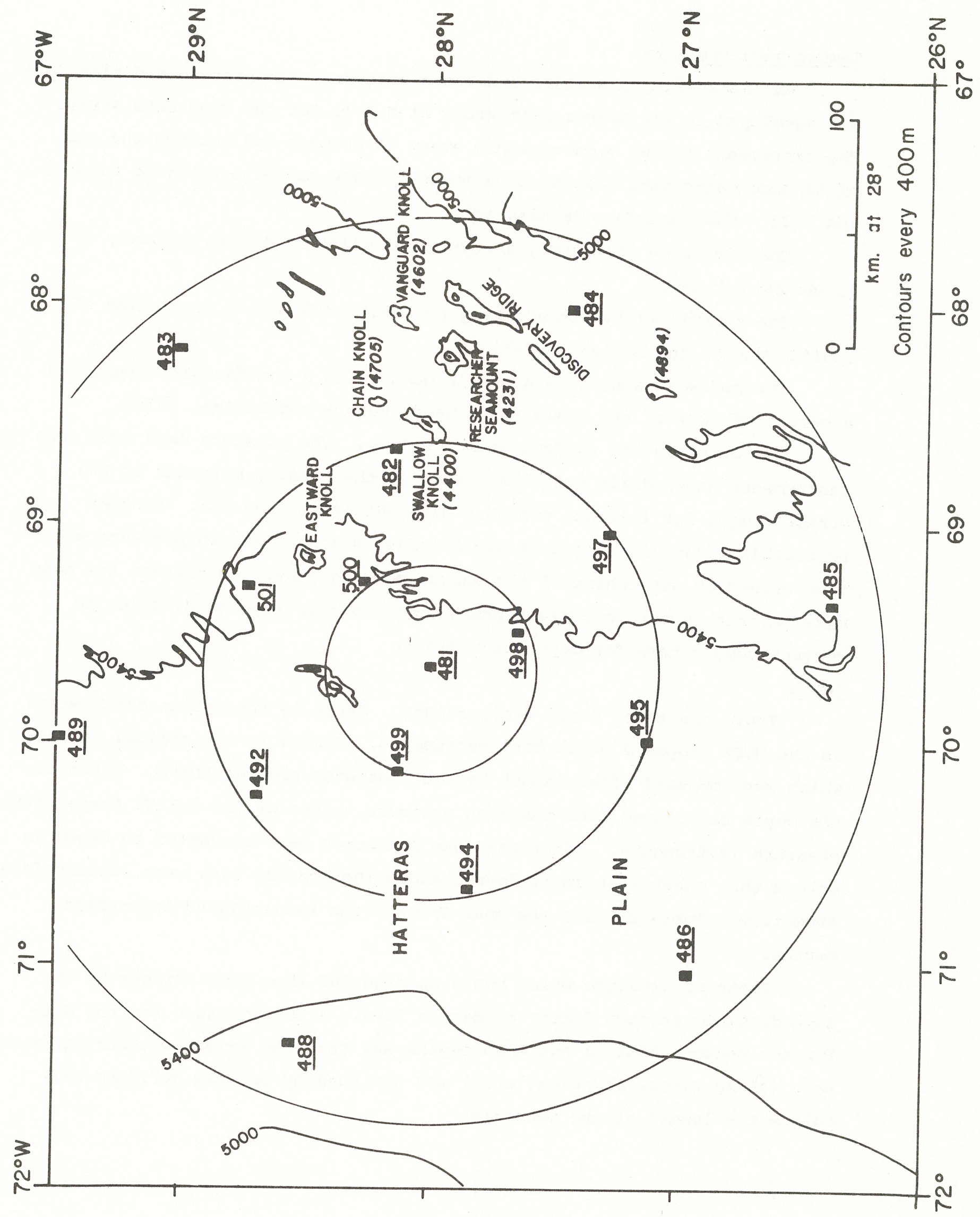

Figure 2. Mooring Location 


\section{Temperature/Pressure}

An instrument to measure and store pressure, temperature and time data was developed in the Draper Laboratory at M.I.T. for the MODE Data Array. The instrument stores a data sample every 15 seconds and records the sum of 64 successive data samples on a magnetic tape cassette every 16 minutes $(64 \times 15=960$ seconds $=16$ minutes $)$.

The time base generator, a crystal oscillator, has an accuracy of \pm 1 second per day.

The thermistor has an accuracy of about $.01^{\circ} \mathrm{C}$ and a resolution of $.001^{\circ} \mathrm{C}$ (Wunsch and Dahlen, 1974).

The pressure sensor is a strain gauge with a manufacturer specified accuracy of .03\% of the scale range used (Wunsch and Dahlen, 1974).

Two problems are evident with $T / P$ data. The pressure data from some instruments shows drift due to a change in the bonding material of the strain gauge, for instance pressure data 485,11 on page 235. Another noticeable effect in many pressure records is a decrease in pressure with time caused by stretching of the dacron mooring line. No attempt has been made in this report to correct pressure for either type of drift or to correct temperature for depth excursions.

Depth

There are three depth designations. First is the design depth which is the MODE proposed depth for instruments. Second is the nominal depth which was computed after launch by buoy computer program NOYFB. Third is the depth calculated from the mean pressure values of the M.I.T. temperature/ pressure instruments. After the mean pressures were converted to depth in meters then other instrument depths along the mooring line were interpolated from them. Table 2 lists the mean $T / P$ and the recalculated instrument depths.

Plots of pressure which begin on page 240 show some effects of the unpredictable stretch factor of dacron line. A second cause for the difference between nominal and mean depths was that the length of mooring hardware (sling rings, shackles, etc.) was not used by program NOYFB to calculate the length of the moorings. 


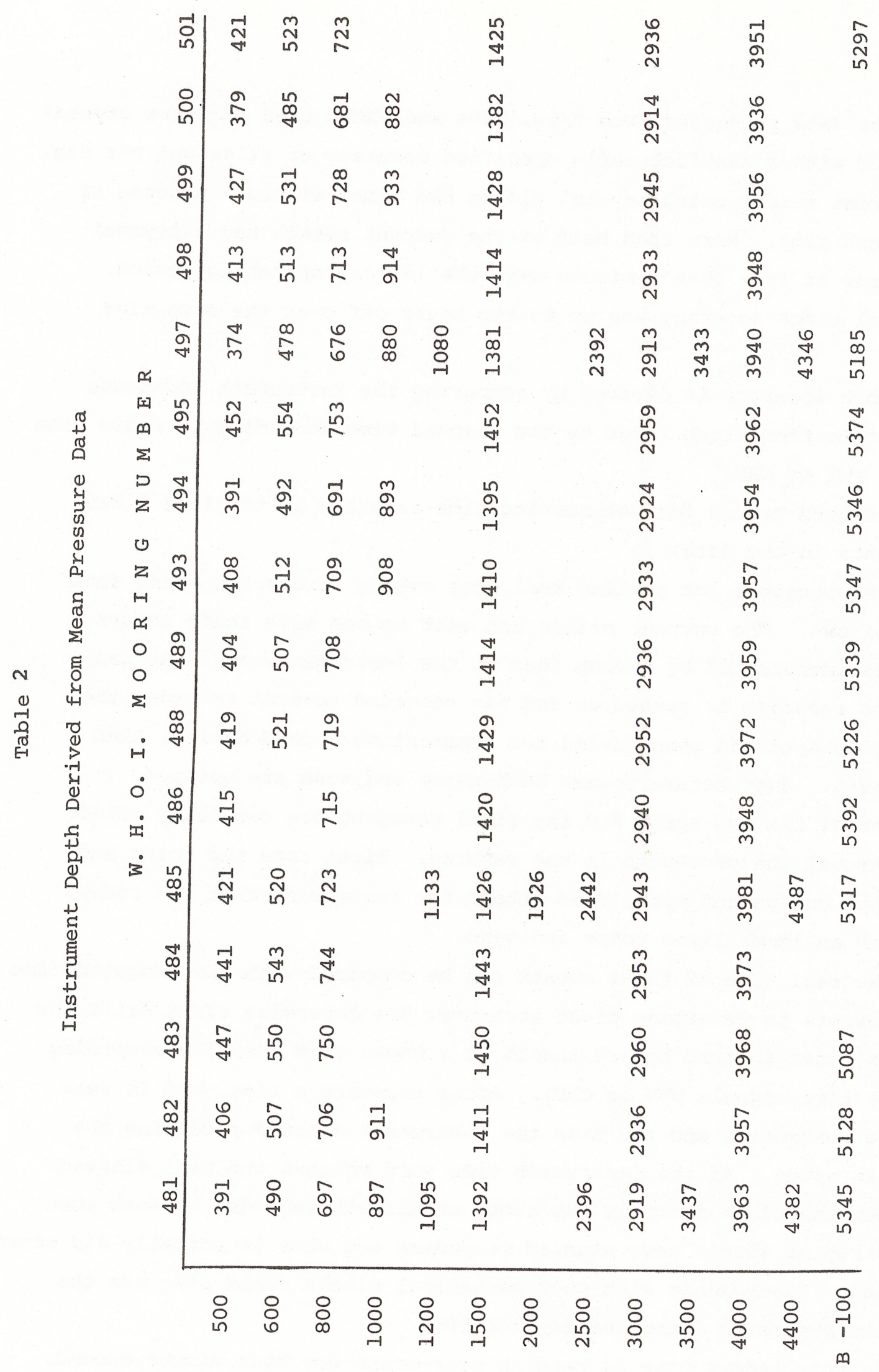

(ux) $\mathrm{H}$ 山 व 
Time

Most data presented from T/Ps, $850 \mathrm{~s}$ and VACMs used a quartz crystal oscillator with a manufacturer's specified accuracy of \pm 1 second per day. MODE current meters using crystal clocks had times within 4 minutes of the correct time. More than half of the current meters had a crystal clock error of less than 1 minute over the 130 day operating period. Mechanical clock accuracy was up to two hours off over the same time period.

Clock accuracy is derived by comparing the instrument indicated elapsed time from clock reset to the elapsed time according to radio time stations WWV or $\mathrm{CHU}$.

A second method for determining time accuracy is to place timed real events in the data.

The procedure for putting real time events in current meter data is as follows. The current meters are sent to sea with their rotors and vanes immobilized by taping them to the instrument case. At sea, after the recorder is turned on and has recorded several records, the rotor is untaped and spun during two consecutive record cycles, then taped again. Just before launch both rotor and vane are untaped. The times of the two spins and the final untaping are carefully noted. For retireval the procedure is the reverse. First tape the rotor and vane, then untape and spin, then retape the sensors so that the record ends with an immobilized rotor and vane.

The real time of these events can be compared with the computed time of the events to determine clock accuracy. To determine clock drift the clock is reset to zero before launch at a known time (usually according to radio time signals WWV or $\mathrm{CHU}$ ). After recovery a time word is read from the instrument and the time the instrument started recording the record is noted. If the instrument time word matches the real elapsed time then the clock drift is the time, usually in seconds, between when the instrument should have started recording and when it actually did start recording. Instruments with good mechanical clocks could also use the real time events to determine clock drift.

In this report time is read as year-month-day hour.minute. second. 


\section{Current Meters}

Eighty-four current meters were set and retrieved by the W.H.O.I. Buoy Group during MODE. One instrument, the only wind recorder, is presumed stolen as it was on our missing MODE Center surface mooring. Sixty-two of the instruments were vector Averaging Current Meters (VACMS) built by American Machine and Foundry (AMF). Three instruments were prototype Vector Averaging Current Meters built by Geodyne now a part of Edgerton, Germeshausen and Grier (EG\&G). Thirteen Model 850 current meters were also built by Geodyne as were 4 film recording current meters. An additional film recording current meter was loaned by the U. S. Navy. The following institutions contributed current meters: W.H.O.I. (63), I.O.S. (7), U.R.I. (9), Nova University (4) and U. S. Navy (1). Current Meter Types

The VACM (Vector Averaging Current Meter) gathers compass and vane information and computes $\mathrm{E}$ and $\mathrm{N}$ components each time a pair of rotor magnets passes the sensing diode and sums these components through the entire recording interval. There are 16 magnets on the rotor so one complete rotor revolution would cause eight compute cycles. The pulses out of the $\mathrm{V} / \mathrm{F}$ converter, whose output frequency is related to the thermistor resistance at its input, are summed over the recording interval. In the decoding these numbers are converted to mean temperatures. The variables are recorded on a cassette tape at the end of each recording interval.

The Model 850 current meter stores burst sampled data on magnetic tape cartridges. For MODE, each Model 850 that had a crystal clock collected and stored 13 current samples at a 5.27 second sampling rate then turned off for the remainder of the 30 minute cycle. Those Model 850 s that were modified to measure temperature store the output count from the temperature circuit for one 5.19 second period at the beginning of each 30 minute interval. Model 850 instruments that used mechanical clocks to measure time collected current information only. Their 13 compass, vane and rotor samples were of 5 second duration each in the 30 minute interval.

Data from the five instruments that recorded on film and also used mechanical clocks are not presented in this report. 


\section{Current Meter Problems}

The VACMs had two types of instrument failure during MODE. Figure 3 is a current record from MODE with both types of failure. One problem was a chemical deposition in the rotor and vane bearings which hampered the movement of the sensor. The other problem was a diode drifting in and out of its sensing range causing varying and unknown quantities of rotor occurrences to be ignored.

The chemical deposition problem (Dexter, Milliman and Schmitz, 1975) was solved by isolating the bearings so they would no longer be cathodic crevices.

The rotor drop out problem was an electrical one. The semiconductor magneto-diodes sense rotation as magnet pairs attached to the rotor pass by them. The voltage drop across these diodes varies with the magnetic field. As the rotor turns an ac signal is produced. This signal, low in amplitude (about 40 millivolts peak-to-peak), is superimposed on a $61 / 2$ volts $d c$ level and balanced by an offset contrcl. in the amplifier. This design proved to be unstable; changes in temperature, pressure, and time caused the dc signal to drift. When the signal drifted out of sensing range the turning rotor went undetected. Adding an ac coupled amplifier to the instrument after MODE eliminated the drift sensitivity and the resultant loss of rotor data. It also made the rotor axial adjustment less critical.

The five Model 850 current meter failures were caused by unrelated problems.

All five film instruments had related problems. First the mechanical readout of the film was not very accurate in either the data reading or the time domain. One film was reread at W.H.O.I. by eye. When compared to the mechanical reading it proved that some data records had not been read and that a few sections of data had been moved out of sequence and reinserted later in the record. A second problem was the accuracy of the time base which relates partly to the first problem and partly to the inaccuracies of mechanical clocks. 

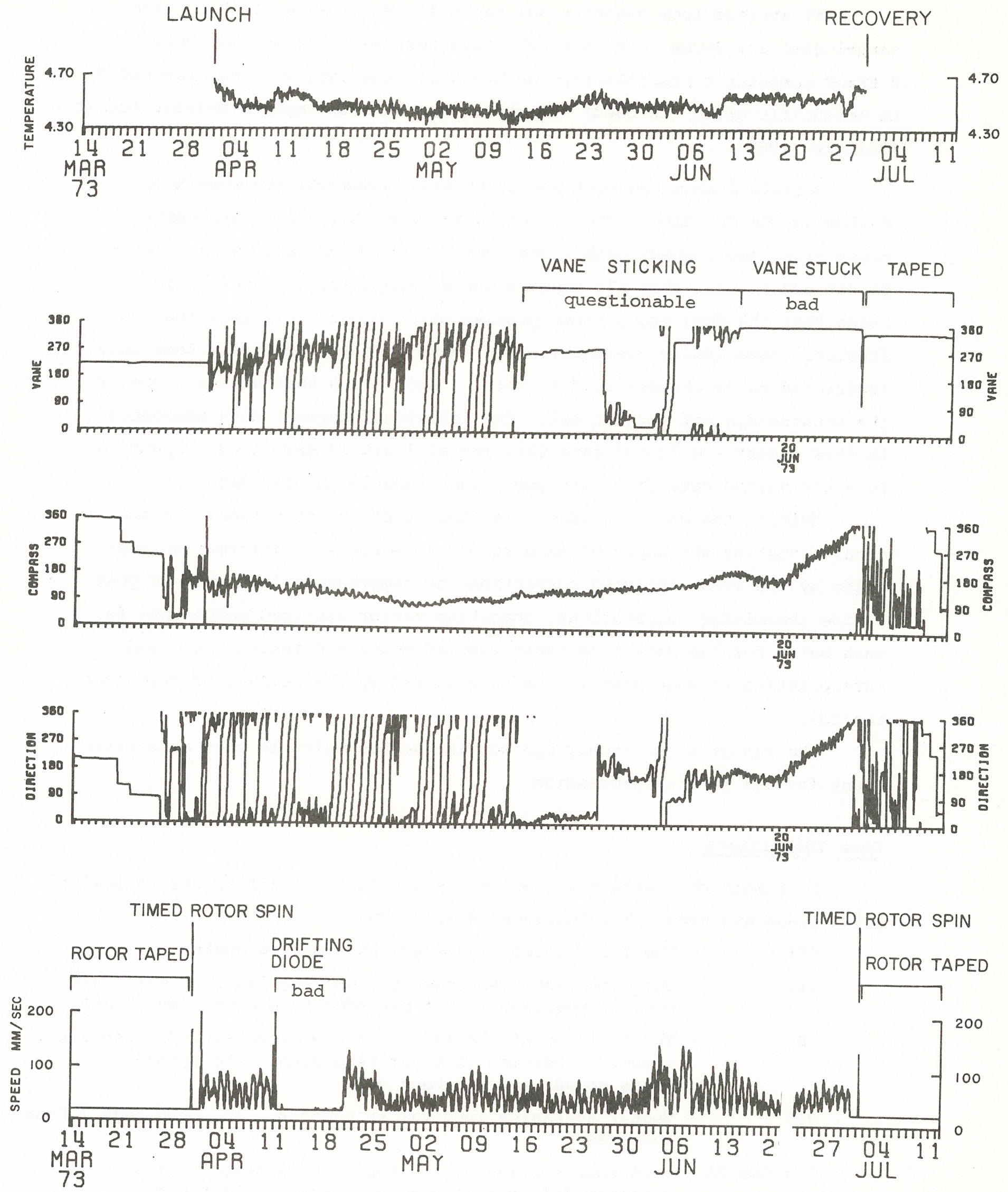

Figure 3. VACM data with instrument problems 


\section{Data Processing}

Bit strings from magnetic sea tapes for Model 850 s (1/4" 2 track cartridges) and VACMS ( $1 / 8 " 4$ track cassettes) were transcribed onto 9 track computer compatible tape at W.H.O.I. The data was then converted to scientific units (decoded) and stored on magnetic tape in Maltais format (Maltais, 1969).

Decisions about current and temperature data quality were made following the decoding. The current data were classified into three categories (good, questionable and bad) for the MODE data users. "Good" is defined to mean that all sensors worked properly. 'Questionable' means that the data had a known problem which might not affect the low frequency data (daily averages). One example is a sticky vane that only registered major changes in direction. 'Bad' means that at least one of the sensors did not work at all. The individual current data presented in this report are 'good' data that are at least 13 days long. Figure 3 is a pre edited data file with good, questionable and bad data.

Editing the data included selecting start and stop times for the data, adjusting the depth of each record to agree with information supplied by the T/Ps, applying corrections to temperature indicated by post cruise thermistor calibrations, computing vector averaged components for each burst for the data from burst sampled Model 850 instruments, and interpolating through gaps in the data caused by the removal of erroneous records.

The result is an evenly spaced time series which is used as a basic input for all further processing.

\section{Data Identifiers}

To insure that each data series has a unique identifier the following guide lines are used. For instance, 481,12B900:

481 - The first three digits are the mooring number.

.12 - The 12th instrument down the mooring line. A one digit mooring position is not preceded by a comma (4812D900).

B - The position of the letter in the alphabet indicates the amount of editing that has been done. The symbol \$ means no editing has been done.

900

- Vector averaging interval in seconds. IH would mean 1 hour averages.

I d Gau 24 - A 24 hour average over a Gaussian filtered series, Gaussian filter having a half width of 24 hours. 
The data are presented three ways: data from individual current meters are arranged by data number; current meter and temperature/pressure data are displayed as composites by mooring; current vectors are displayed as arrays by depth.

The first section presents current meter data ordered by mooring and instrument position numbers. Each mooring has two pages on which are rresented a description and a diagram of the mooring.

The following abbreviations and symbols are used:

* Current meter data presented

\# T/P data, presented in Data Section 2

+ Temperature only, presented in Data Section 2

$850 t$ Model 850 with temperature modification

FCM Film recording current meter

Film Film recording current meter (Navy)

I.O.S. Institute of Oceanographic Sciences, formerly N.I.O.

U.R.I. University of Rhode Island

M.I.T. Massachusetts Institute of Technology

T/P M.I.T. temperature/pressure recorder

VACM Vector Averaging Current Meter

Following the mooring information are sets of four pages that display data for each current meter that had 'good' current data (Figure 4). Each set of four pages includes data sampling information, the quality of the various variables, a list of general statistical parameters, and four plots: a scatter plot of $U$ vs. $V$, a spectral plot, a progressive vector plot and a plot of temperature, $U, V$, speed and direction vs. time. Temperature is plotted for the time period of good current data.

In Section 2 the current meter and $T / P$ data are presented as a series of composite plots. Both $\mathrm{CM}$ and T/P temperature data (Table 3) are sub-sampled and plotted by mooring number and increasing depth. Pressure data are also subsampled and plotted by mooring number and increasing depth. The temperature and pressure data for each mooring are displayed on facing pages to facilitate comparisons. Stick diagrams of $U$ and $V$ are plotted from 1 day Gaussian filtered data and presented by mooring and increasing depth.

Geographic displays of 4-day averaged current vectors are presente:d by depth and time. 


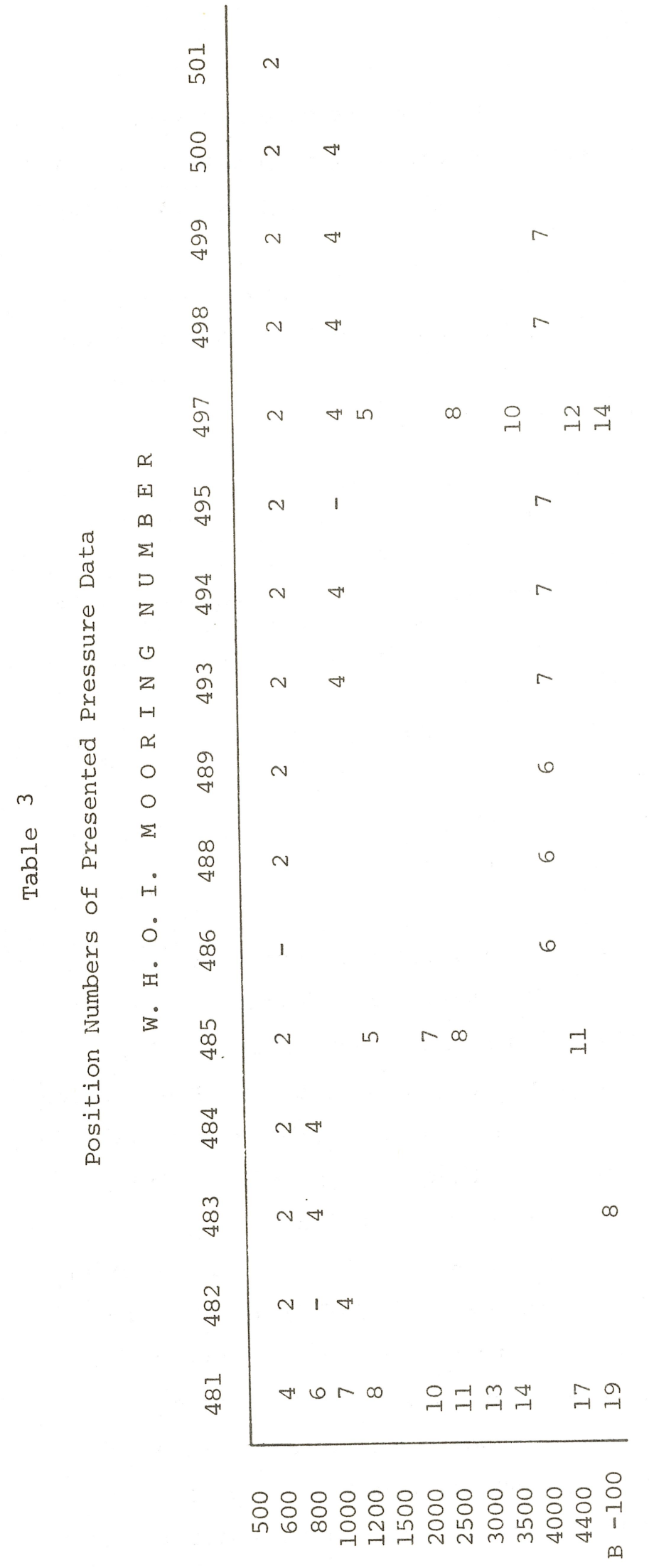

(u) บ7dəव u6țsəa

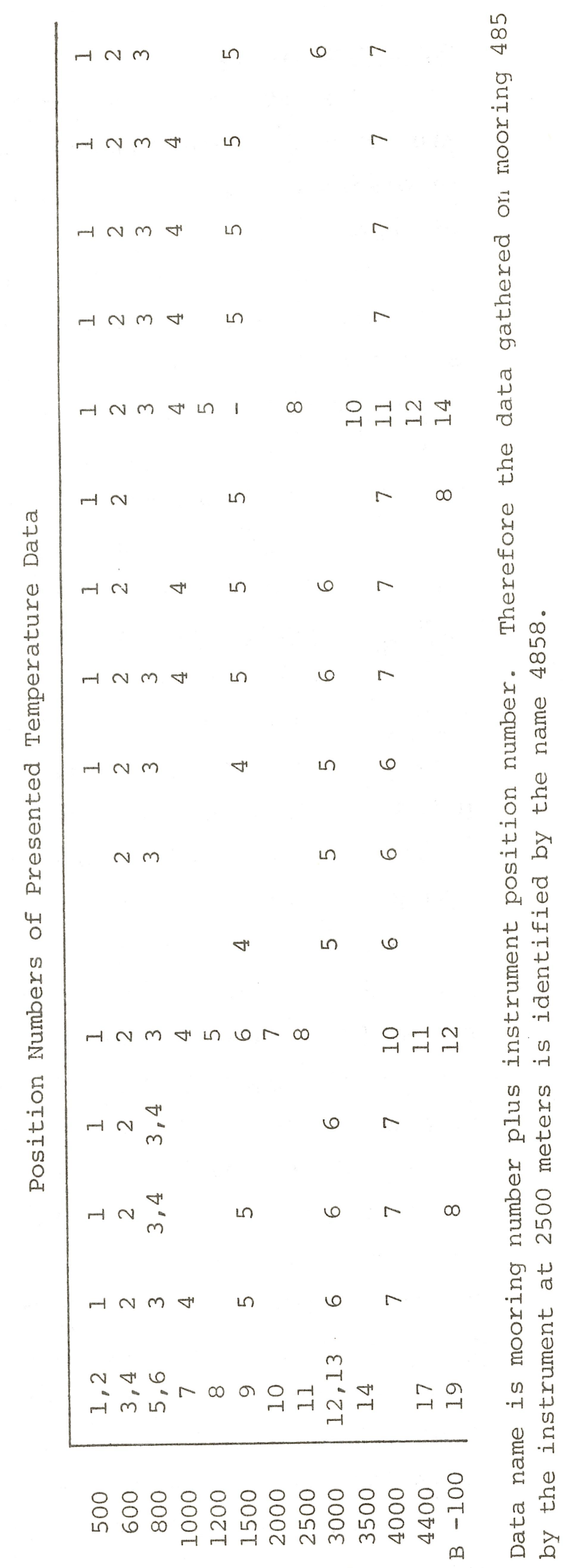

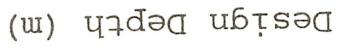



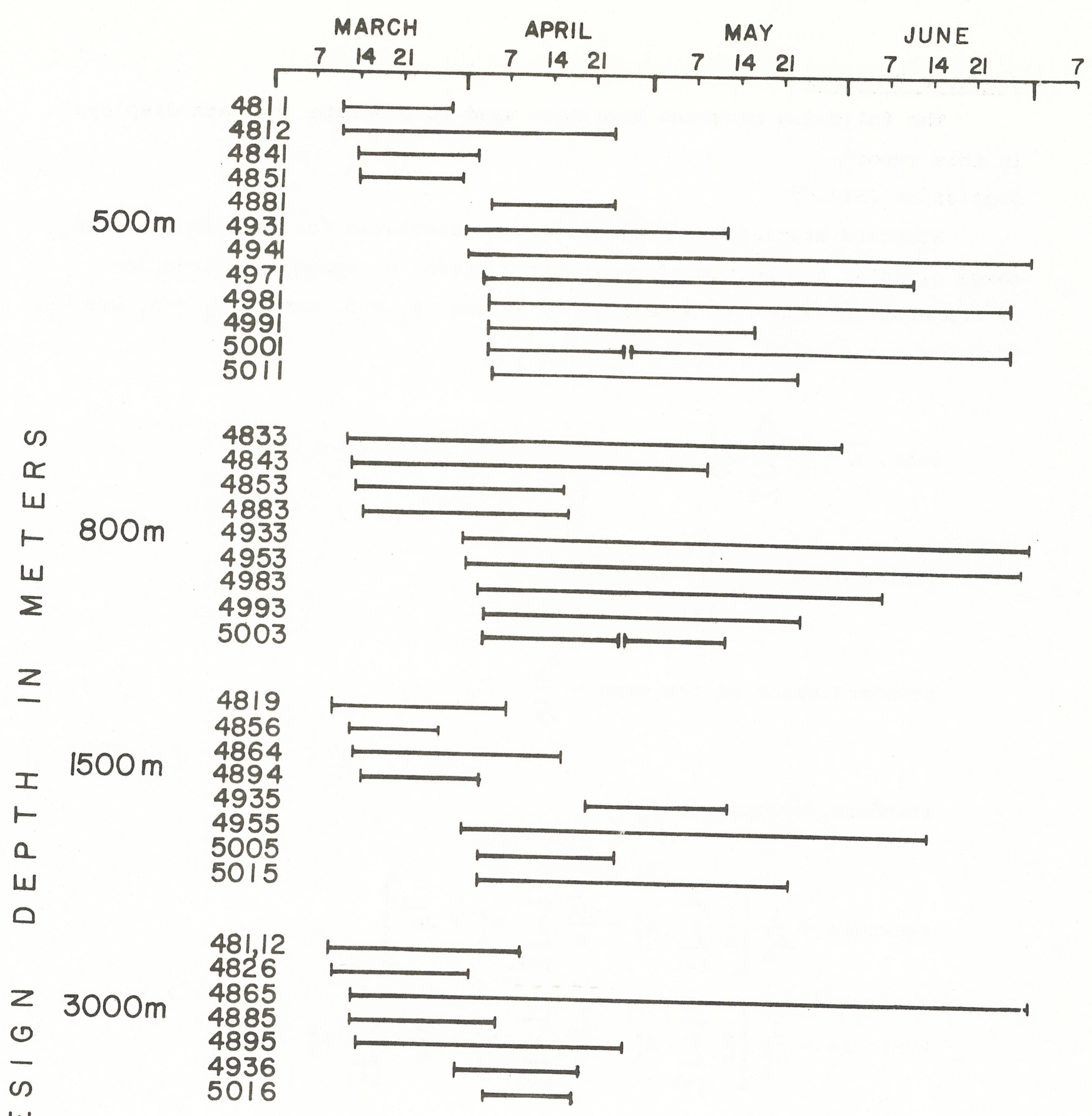

w

○
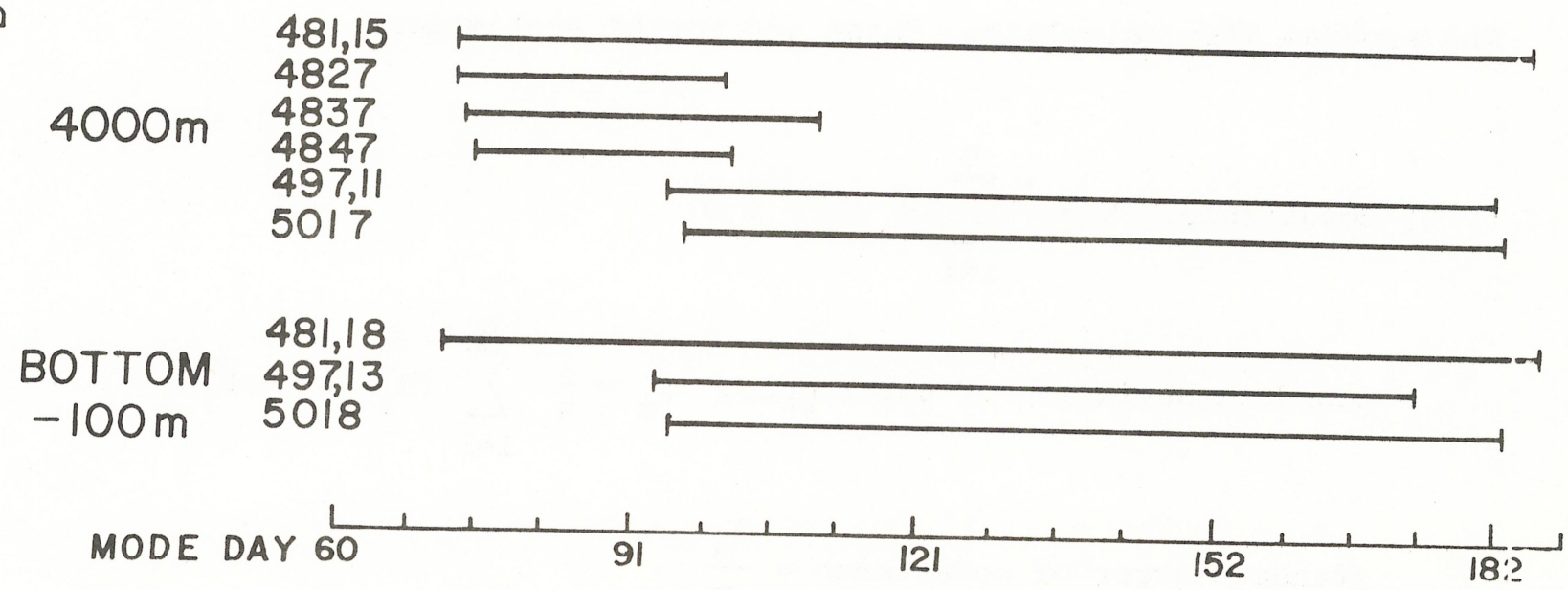

Figure 4. Duration of current data 
The following programs have been used to generate the data displays in this report:

Statistics (STATS)

Standard statistical parameters are calculated for data in the time range given at the bottom of the table. Given $n$ speed and direction or temperature values in a sample, we define $E_{i}=S_{i} \sin \Theta_{i}, N_{i}=S_{i} \cos \theta_{i}$ ' then for $A=E, N, S$, and $T$,

$$
\begin{aligned}
& \text { mean, } \bar{A}=\frac{1}{n} \sum_{i=1}^{n} A_{i} \\
& \text { variance, } \sigma_{A}^{2}=\frac{1}{n} \sum_{i=1}^{n} A_{i}^{2}-\bar{A}^{2} \\
& \text { standard error of the mean }=\frac{\sigma_{A}}{\sqrt{n}} \\
& \text { standard deviation }=\sigma_{\mathrm{A}} \\
& \begin{aligned}
\text { skewness } & =\frac{1}{\sigma_{A}^{3}}\left[\frac{1}{n} \sum_{i=1}^{n} A_{i}^{3}-\frac{3 \bar{A}}{n} \sum_{i=1}^{n} A_{i}^{2}+2 \bar{A}^{3}\right] \\
\text { kurtosis } & =\frac{1}{\sigma_{A}^{4}}\left[\frac{1}{n} \sum_{i=1}^{n} A_{i}^{4}-\frac{4 A}{n} \sum_{i=1}^{n} A_{i}^{3}+\frac{\overline{6 A}^{2}}{n} \sum_{i=1}^{n} A_{i}^{2}-3 \bar{A}^{4}\right]
\end{aligned}
\end{aligned}
$$

The program also calculates "East and North" statistics,

$$
\begin{aligned}
& \text { covariance, } \mathrm{M}=\frac{1}{\mathrm{n}} \sum_{i=1}^{\mathrm{n}} \mathrm{E}_{i} \mathrm{~N}_{i}-\overline{\mathrm{E}} \overline{\mathrm{N}} \\
& \text { standard deviation of covariance, } \sigma_{m}=\frac{1}{\mathrm{n}} \sum_{i=1}^{\mathrm{n}}\left(\mathrm{E}_{i} \mathrm{~N}_{i}\right)^{2}-\overline{\mathrm{E}}_{i} \mathrm{~N}^{2} \\
& \text { standard error of covariance }=\frac{\sigma_{\mathrm{m}}}{\sqrt{\mathrm{n}}}
\end{aligned}
$$




$$
\text { correlation coefficient, } M^{\prime}=\frac{M}{\sigma_{E} \sigma_{N}} \text {. }
$$

The program also calculates parameters related to vector quantities: the scalar amplitude of the vector mean, $V_{m}=\bar{E}^{2}+\bar{N}^{2}$; vector variance, $\mathrm{V}_{\mathrm{V}}^{2}=\frac{1}{2}\left(\sigma_{\mathrm{E}}^{2}+\sigma_{\mathrm{N}}^{2}\right)$; standard deviation $=\mathrm{V}_{\mathrm{V}}$.

\section{Scatterplot}

The vector components are plotted against each other to give a pictorial indication of the DIRECTION and SPEED of the velocity vectors. This type plot can be helpful in finding instrument malfunctions and characteristics not easily noticed elsewhere.

Progressive Vector Diagram (PROVEC)

The vector progressive displacements are plotted. The plot begins with an asterisk (*) on a day boundary. All following day boundaries are indicated with a (+). This type of plot accentuates very low frequency events at the expense of higher frequency oscillations which may be hidden by a large amplitude low-frequency current.

Variable vs. Time Plot

This is a diagram of any variable plotted as a function of TIME. The plot is generated from the 1 hour averaged series. This type of plot is complementary to the PROGRESSIVE VECTOR diagram since it accentuates higher frequency events such as inertial and tidal oscillations.

\section{Spectra}

The program TIMSAN (TIme Series ANalysis) uses the Fast Fourier Transform algorithm of Singleton (1969) and is restricted to data segments of length $\mathrm{N}$ points, where $\mathrm{N}$ must be an even number which has no prime factor larger than 5, and must be less than 8000 points; data series longer than this must be broken into two or more pieces.

The number of degrees of freedom for the first 40 plotted points is given by $v=a \mathrm{~m}$ where $\mathrm{m}$ is the number of adjacent frequency bands being averaged as stated in the label, $s$ is the number of independent data pieces being averaged, again as stated in the label, and a should be two for temperature spectra and for Horizontal Kinetic Energy [HKE] spectra for which the EAST and NORTH components seem statistically 
independent. In the absence of information regarding NORTH-EAST Correlation, one should use $a=2$ to be safe.

The log-log plot is further averaged during plotting so that more and more points are averaged together as frequency increases. This eliminates the bunching together of points at high frequencies, increases the degrees of freedom of the high frequency estimates, and still permits low-frequency resolution. The averaging algorithm is as follows: counting from the left of the plot, the first 40 plotted points represent data that has been averaged as stated in the label; the data for the next 15 plotted points has been averaged over twice as many frequency bands; the next 6 over five times as many, the next 40 over ten times as many, the next 15 over twenty times as many, the next 6 over fifty times as many, the next 40 over 100 times as many and so on. In this way, for example, 7900 data points with no averaging indicated in the label would be plotted as only 176 points, and the last 14 estimates would be averaged over 200 basic frequency bands. The $m$ in the formula $v=a \mathrm{~m}$ for degrees of freedom is, in this example, 200 times larger at the highest frequencies than at the lowest frequencies.

For $v>30$, the confidence limits for the spectral estimates are given approximately by $\left(1-2 / 9 \mathrm{~V} \pm \mathrm{z} \sqrt{2 / 9 \mathrm{v}}{ }^{1 / 3}\right.$ where $\mathrm{z}=1.28375$ for $80 \%$ confidence limits, $\mathrm{z}=1.645$ for $90 \%, \mathrm{z}=1.96$ for $97 \%$ and $z=2.5757$ for $99 \%$. In the example above, if the HKE spectral plot label had indicated 2 pieces and averaging over 8 adjacent frequency bands then $v=2 \times 2 \times 8=32$ for the lowest frequencies (assuming NORTH and EAST components are highly correlated) and $200 \times 32=6400$ for the highest frequencies. The 95\% confidence intervals (i.e., 95\% of the time one would expect the spectral estimates to vary no more than this much) would be $(0.57,1.55)$ at low frequencies, and $(0.97,1.03)$ at high frequencies.

For $v \leq 30$, one must obtain confidence intervals from Chi-Squared distribution tables in standard statistical references. 
Temperature spectra from 850 current meters show aliasing at high frequencies because of the sampling scheme. speed and direction are burst sampled 14 times per recording interval. These 14 samples are vector averaged to attenuate high frequency aliasing in the current measurement. There is only one 5.19 second temperature sample per recording interval, however. The temperature spectrum for data series 497,11 shows clearly the resulting high frequency aliasing. In contrast, the VACM averages all variables over the full recording interval, virtually eliminating the problem.

Stick Plot

The basic $U$ and $V$ time series is filtered using a symmetrical running Gaussian filter with a half-width of 24 hours followed by a simple running hat filter. The filtering is sequential and the resultant time series is 48 hours shorter than the input time series (The first and last 24 hours are lost.). Each consecutive plotted vector is the result of a 24 hour average running from midnight to midnight. Vector direction follows normal direction conventions, i.e., north is up. 


\section{References}

Brown, N., 1975, A Precision CTD Microprofiler. W.H.O.I. Ref. 75-18 (unpublished manuscript).

Dexter, S. C.., J. D. Milliman and W. J. Schmitz, Jr., 1975, Mineral deposition in current meter bearings. Deep-Sea Res., 22, 703-706.

Fofonoff, N. P., S. P. Hays, and R. C. Millard, Jr., 1974, W.H.O.I./Brown microprofiler: methods of calibration and data handling. W.H.O.I. Ref. 74-89 (unpublished manuscript).

Heinmiller, R. H., 1973a, Cruise Report CHAIN 112, Legs I and II, W.H.O.I. Ref. 73-5I (unpublished manuscript).

Heinmiller, R. H., 1973b, Cruise Report CHAIN 112, Leg VI, W.H.O.I. Ref. 73-50 (unpublished manuscript).

Heinmiller, R. H., 1975, Mooring Operations of the Woods Hole Buoy Group for the Mid-Ocean Dynamics Experiment, W.H.O.I. Ref. 75-42 (unpublished manuscript).

Maltais, J. A., 1969, A nine channel digital magnetic tape format for storing oceanographic data. W.H.O.I. Ref. 69-55 (unpublished manuscript).

Millard, R. C., Jr., and H. Bryden, 1973, Spatially-averaged MODE-I CTD stations. MODE Hot-Line News no. 43 (unpublished manuscript).

Singleton, R. C., 1969, An algorithm for computing the mixed radix Fast Fourier Transform. I.E.E.E. Trans. on Audio and Electroacoustics, $\mathrm{AU}-17$ (2) 93-103.

Wunsch, C., and J. Dahlen, 1974, A moored temperature and pressure recorder. Deep-Sea Res., 21, 145-154. 


\section{STATION 480}

$3 / 8 " 3 \times 19$ WIRE ROPE

$5 / 8 "$ NYLON

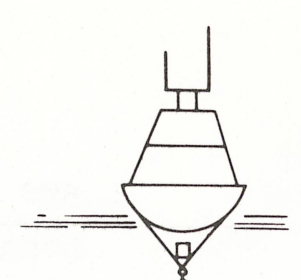

LIGHT

CB BEACON

HF BEACON

RADAR TRANSPONDER

NOVA TRANSPONDER

WIND RECORDER - 4801

TELEM. TENSION CELL

$10 \mathrm{~m} \mathrm{1/2" \text {CHAIN }}$

SWIVEL

$100 \mathrm{~m}$

$100 \mathrm{~m}$

$100 \mathrm{~m}$

$100 \mathrm{~m}$

$500 \mathrm{~m}$

$500 \mathrm{~m} \quad$ Surface mooring 480 was set as a reference marker in the center of the MODE area at $28^{\circ} 00^{\circ} \mathrm{N}$,

$500 \mathrm{~m} \quad 69^{\circ} 10^{\prime} \mathrm{W}$. Its instruments included four radios, a strobe light,

$22 \mathrm{~m}$ a tension cell and a wind recorder. Early in the morning on May $23 r d$

$515 \mathrm{~m}$ the bridge personnel of the Research Vessel Chain noticed a ship hove to at the mooring position with deck lights on.

$502 \mathrm{~m}$ As the Cahin got closer the unknown ship turned on her running lights and departed without responding to radio calls.

$529 \mathrm{~m}$ The missing buoy is assumed to have been stolen by that ship as it was never seen again.

$512 \mathrm{~m}$

$480 \mathrm{~m}$

$589 \mathrm{~m}$

$20 \mathrm{~m} \mathrm{3/4"} \mathrm{NYLON}$

3 m 1/2" CHAIN

STIMSON ANCHOR, 5850 LBS.

\section{MODE CENTRAL SURFACE MOORING ( LOST)}


Mooring No. 481

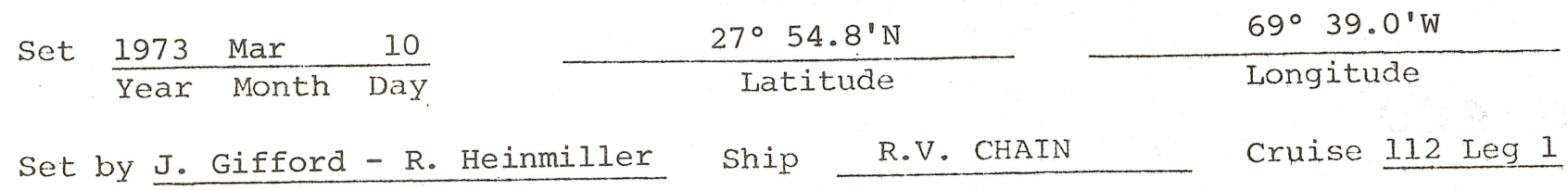

Retrieved $\frac{1973 \text { July }}{\text { Year Month Day }}$

Retrieved by G. Tupper - R. Heinmiller Ship R.V. CHAIN Cruise 112 Leg 6

Purpose of Mooring: Mooring \#1 of MODE 1 array

Mooring Type: Subsurface mooring

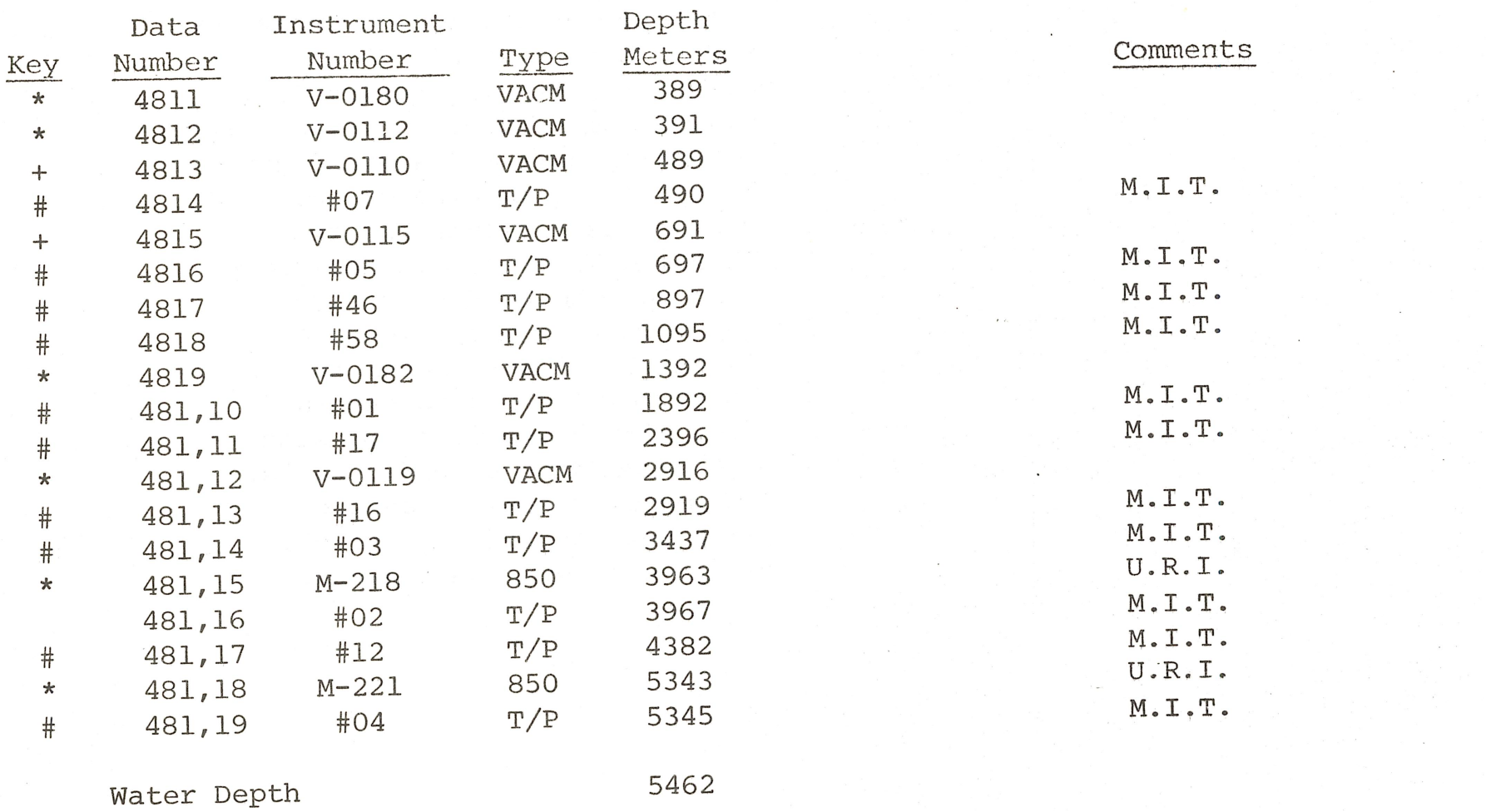

COMMENTS ON MOORING: 

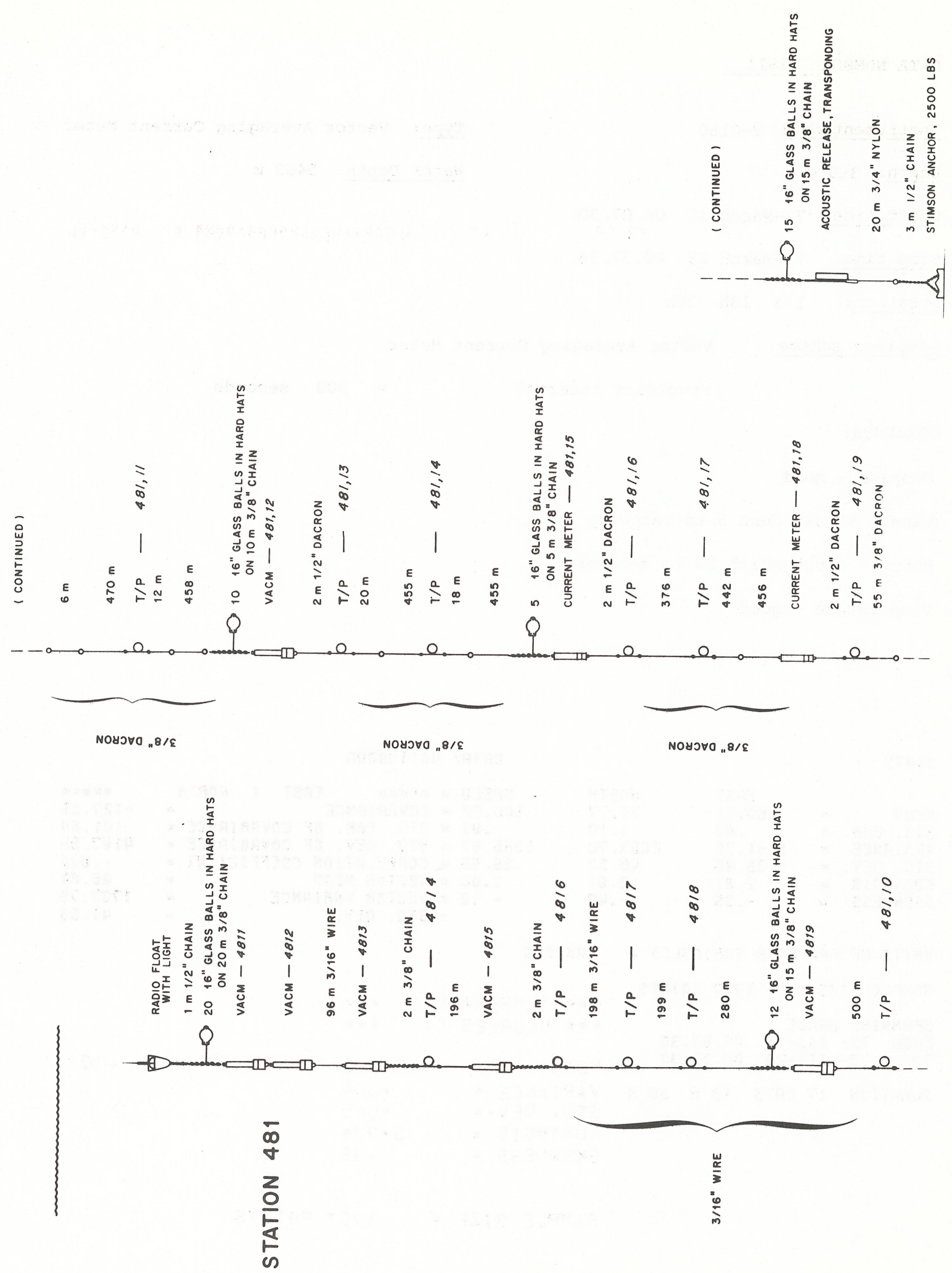
DATA NUMBER 4811

Instrument No.: $\mathrm{V}-0180$

Depth: $389 \mathrm{~m}$

Start time: 73-March 1106.07 .30$.

Stop time: 73-March 2900.37 .36$.

Duration: $\quad 17 \mathrm{~d}$ 18h $30 \mathrm{~m}$

Sampling scheme: Vector Averaging Current Meter

$$
\text { recording interval }=900 \text { seconds }
$$

COMMENTS :

Compass - good

Vane - sticky June 6 to recovery

Rotor - stuck March 29 to recovery

Temperature - good

STATS

$\begin{array}{lrr} & \text { EAST } & \text { NOATH } \\ \text { MEAN } & -60.31 & 75.77 \\ \text { STD. EAR. } & .89 & 1.10 \\ \text { VARIANCE }= & 1361.86 & 2053.70 \\ \text { STD DEV. } & 36.90 & 45.32 \\ \text { KURTESIS }= & 2.81 & 2.97 \\ \text { SKEWNESS }= & -.35 & -.43\end{array}$

UNITS OF RAW DATA VARJABLES =

MMPSEC

SAMPLE S1ZE - 1707 POINTS

SPANNING RANGE

FROM 73- I』I-12 06.07.30

TO 73- II-29 00.37 .30

DURATION 17 DAYS $18 \mathrm{H} 30 \mathrm{M}$
Type: Vector Averaging Current Meter Water Depth: $5462 \mathrm{~m}$ 

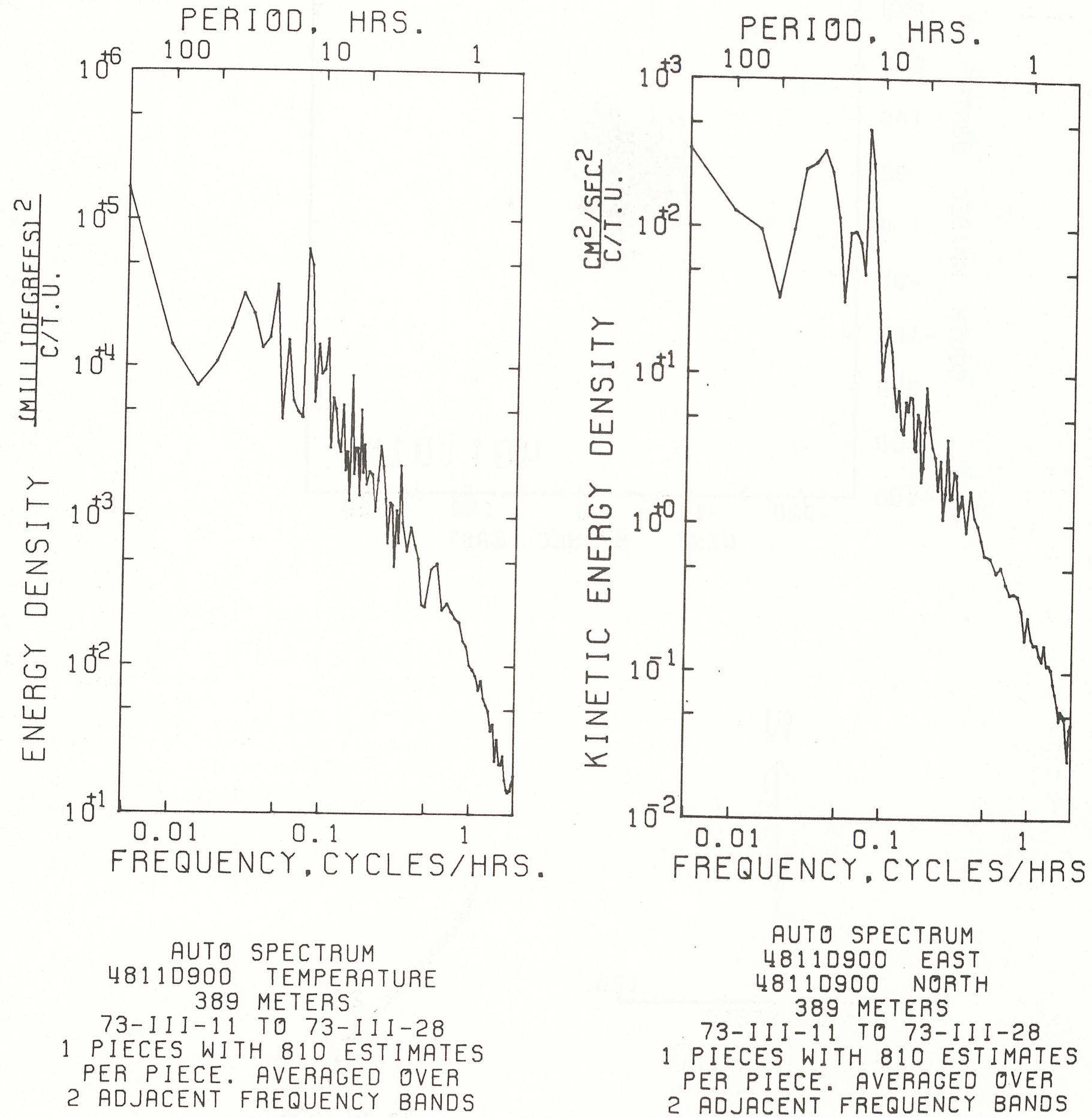

73-III-11 TO 73-III-28

1 PIECES WITH 810 ESTIMATES PER PIECE. AVERAGED OVER 2 ADJACENT FREQUENCY BANDS 

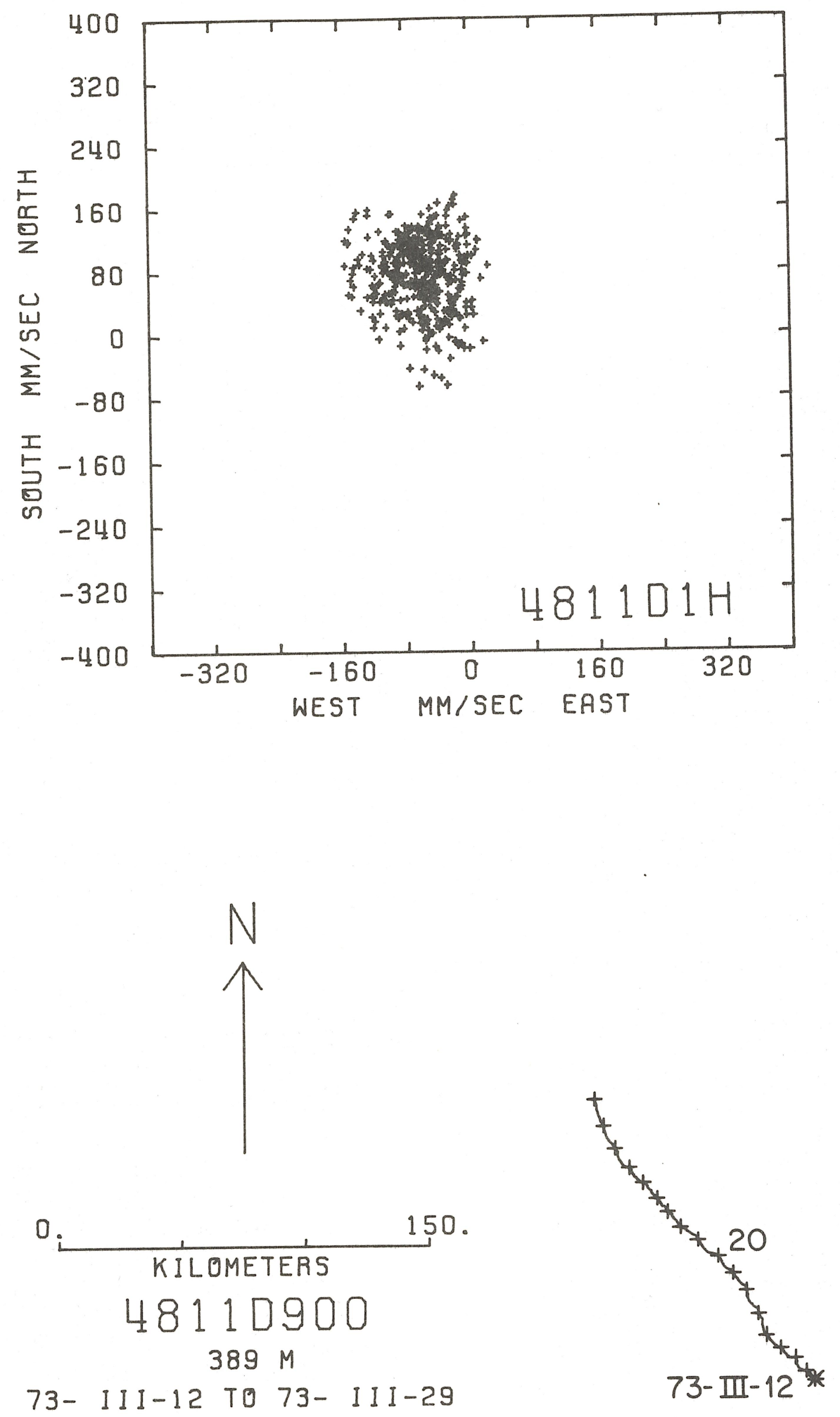

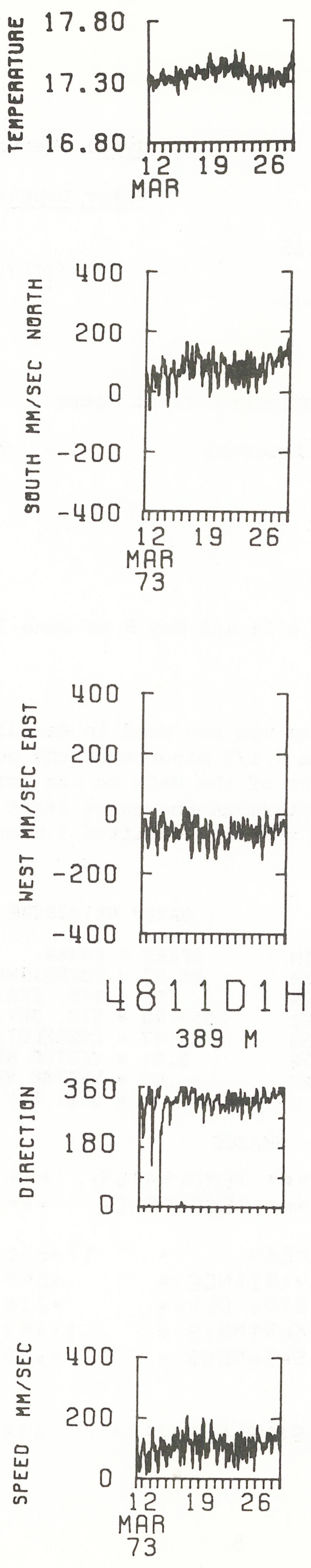
DATA NUMBER 4812

Instrument No.: $\mathrm{V}-0112$

Depth: $391 \mathrm{~m}$

Start time: 73-March-11 07.11.15.

Stop time: 73-April-23 20.56.15.

Duration: $43 \mathrm{~d}$ 13h $45 \mathrm{~m}$

Sampling scheme:

Vector Averaging Current Meter

recording interval $=900$ seconds
Type: Vector Averaging Current Meter Water Depth: $5462 \mathrm{~m}$

COMMENTS :

Compass - good

Vane stuck May 20 to end

Rotor below threshold April 23 \& 24 and May 8 to June 16

Temperature good

Clock had problem in counting so was not used in decoding. Last events show that the decoded data is within $71 / 2$ minutes of the correct time. Because of the recording characteristics of the VACM we can not position events within the 15 minute interval to determine the exact start time of the record. The clock check indicates that the clock drifted 1 minute 13 seconds from February 20 to July 17, 1973.

STATS

DATAP 48120900 OA

$\begin{array}{lrr} & \text { EAST } & \text { NOATH } \\ \text { MEAN } & -42.12 & 67.18 \\ \text { STD. ERT. } ~ & .78 & .79 \\ \text { YARIANCE }= & 2548.80 & 2634.61 \\ \text { STD. DEV. }= & 50.49 & 51.33 \\ \text { KURTOSIS }= & 3.17 & 3.72 \\ \text { SKENNESS }= & .12 & .29\end{array}$

SPEEO H EAST \& NOATH 96.97 COVARJANCE $=-64.21$ .70 5T0. ERR. OF COVARJANCE $=70.20$ 2057.40 STO. OEV. OF COVARJANCE $=4540.60$ 45.47 CORAELATION COEFF JCJENT = -.025 3.01 VECTOR MEAN $=79.29$ .45 VECTOA VAAIANCE $=2591.76$ * STO. OEV.

50.91

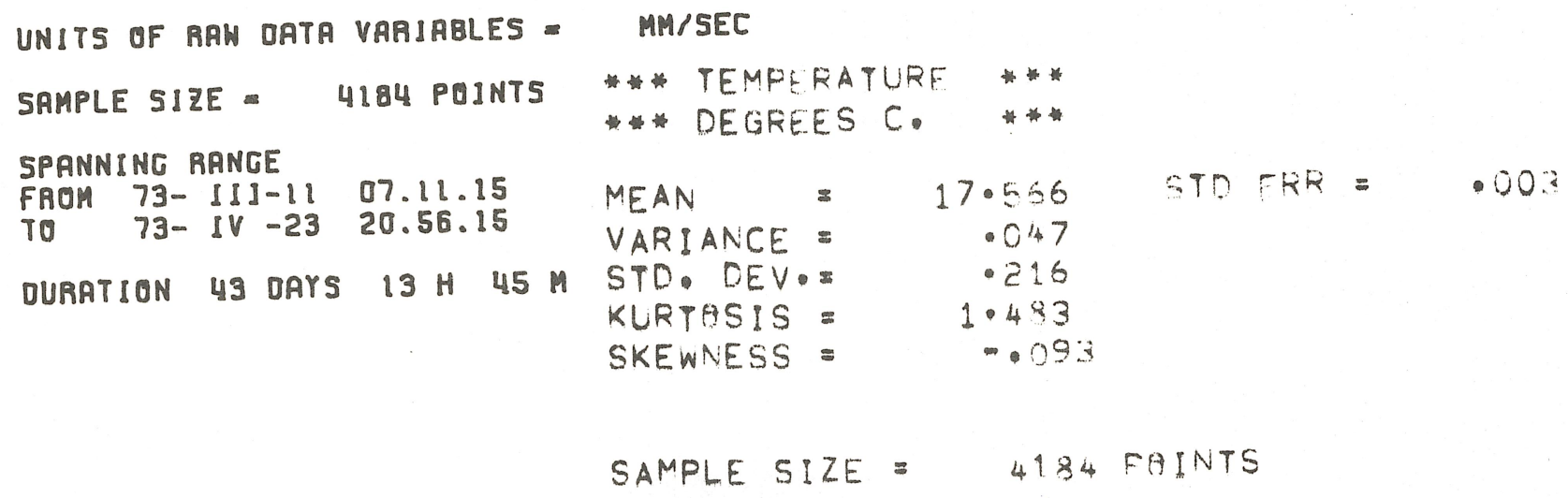



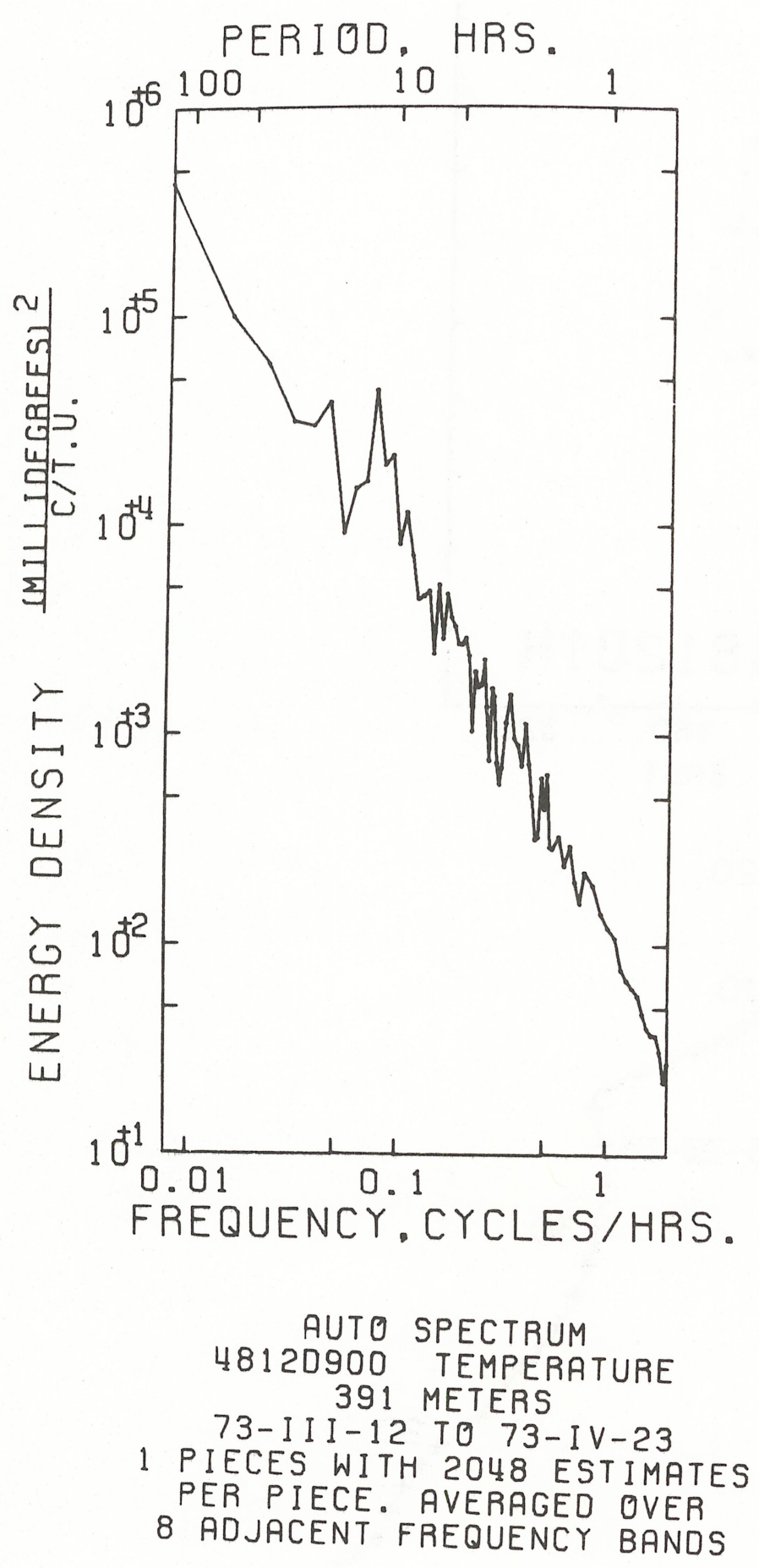

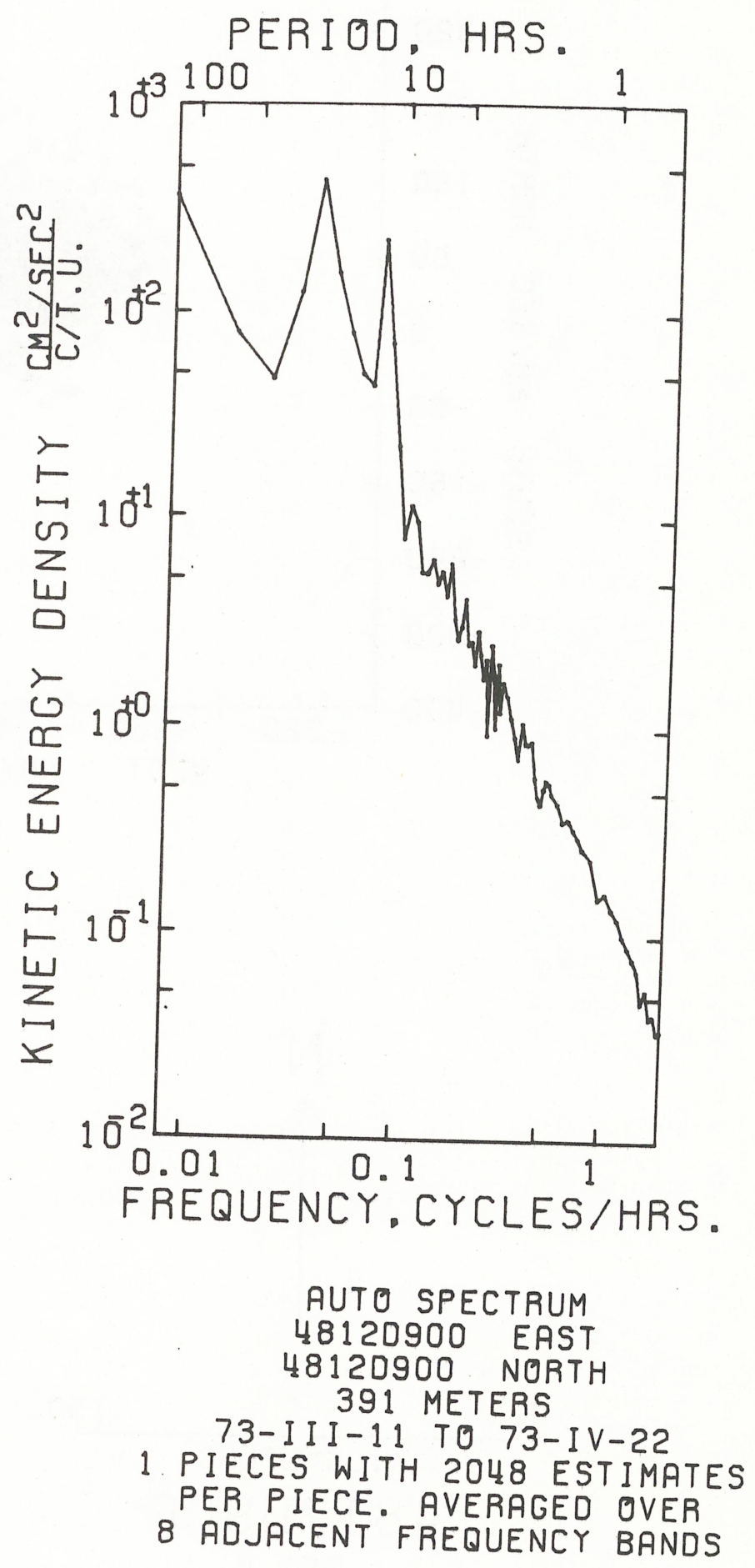



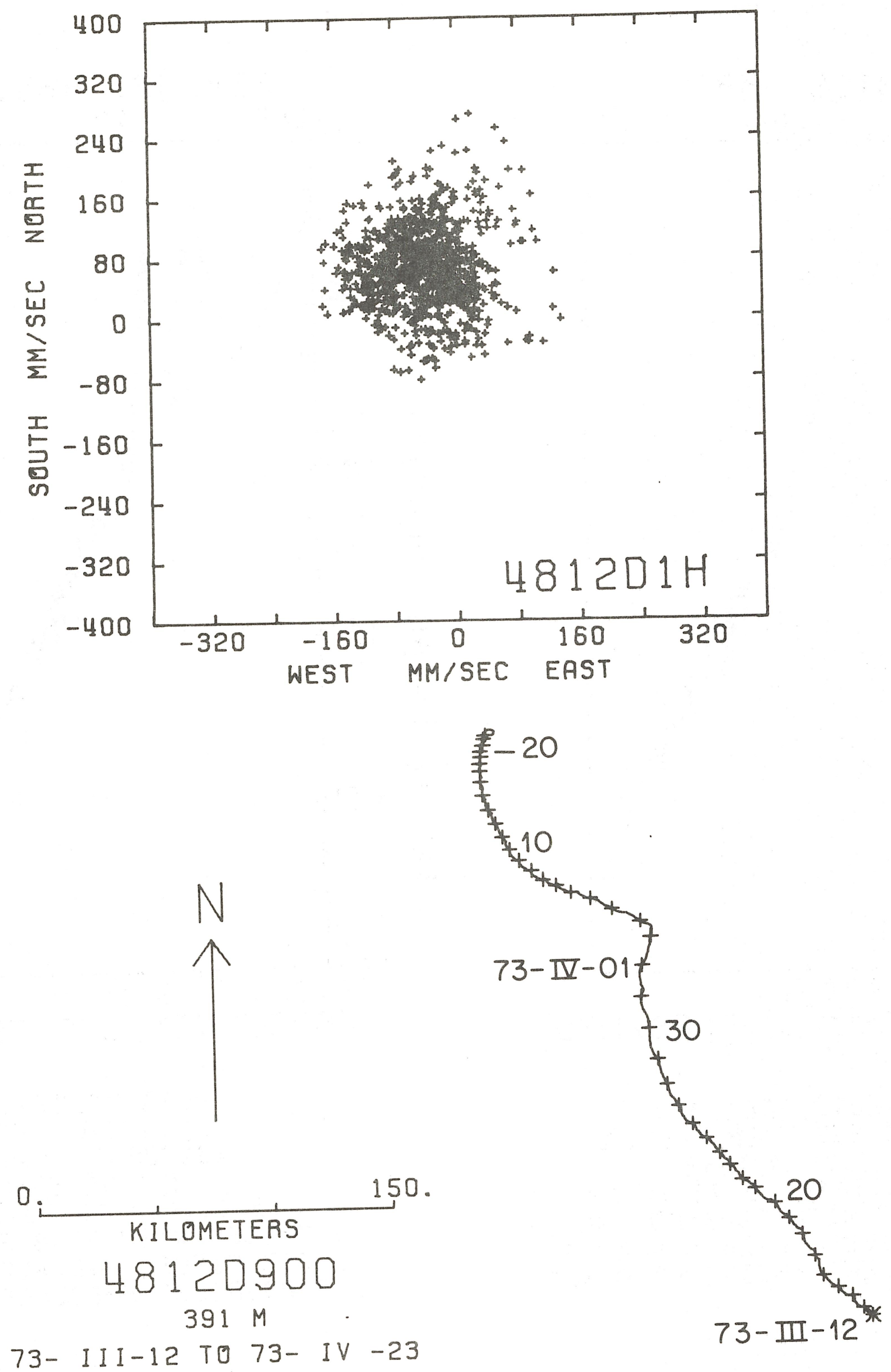

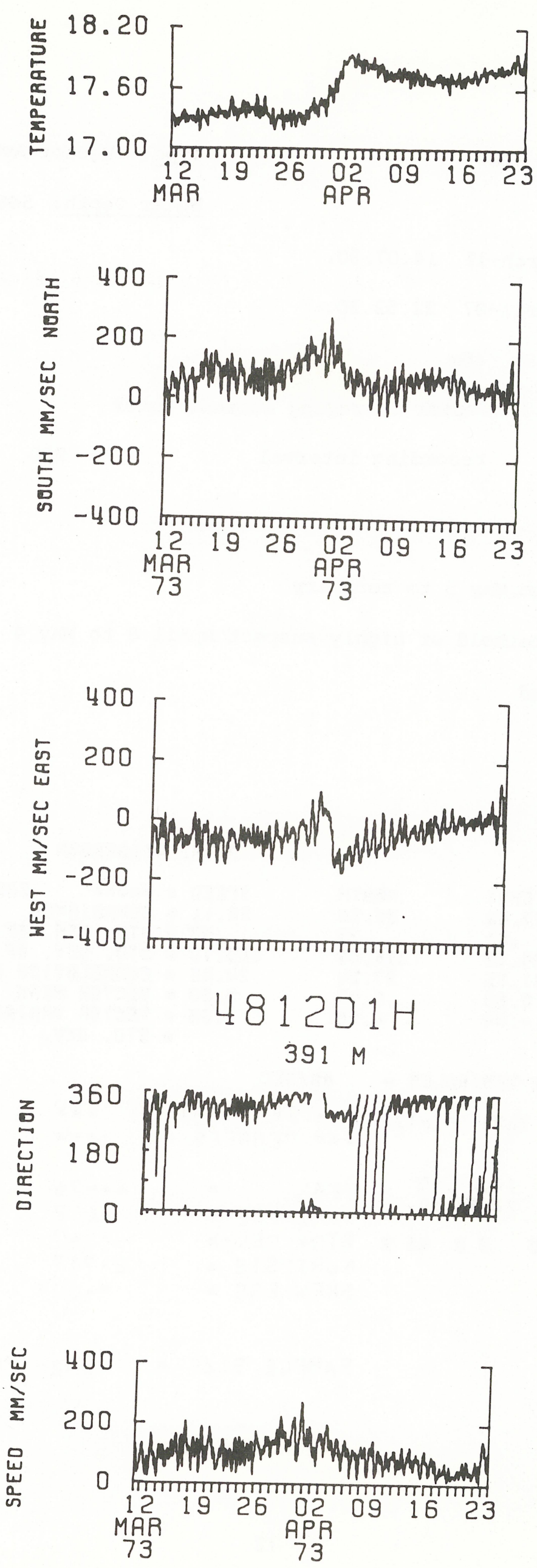
DATA NUMBER 4819

Instrument No: : V-0182

Depth: $1392 \mathrm{~m}$
Type: Vector Averaging Current Meter Water Depth: $5462 \mathrm{~m}$

Start time: 73-March-11 14.07.30.

Stop time: 73-Apri1-07 22.52.30.

Duration: $27 \mathrm{~d}$. $45 \mathrm{~m}$

Sampling scheme: Vector Averaging Current Meter

recording interval $=900$ seconds

COMMENTS :

Compass - good

Vane - sticky from May 3 to recovery

Rotor - below threshold or highly suspect April 8 to May 4

Temperature - good

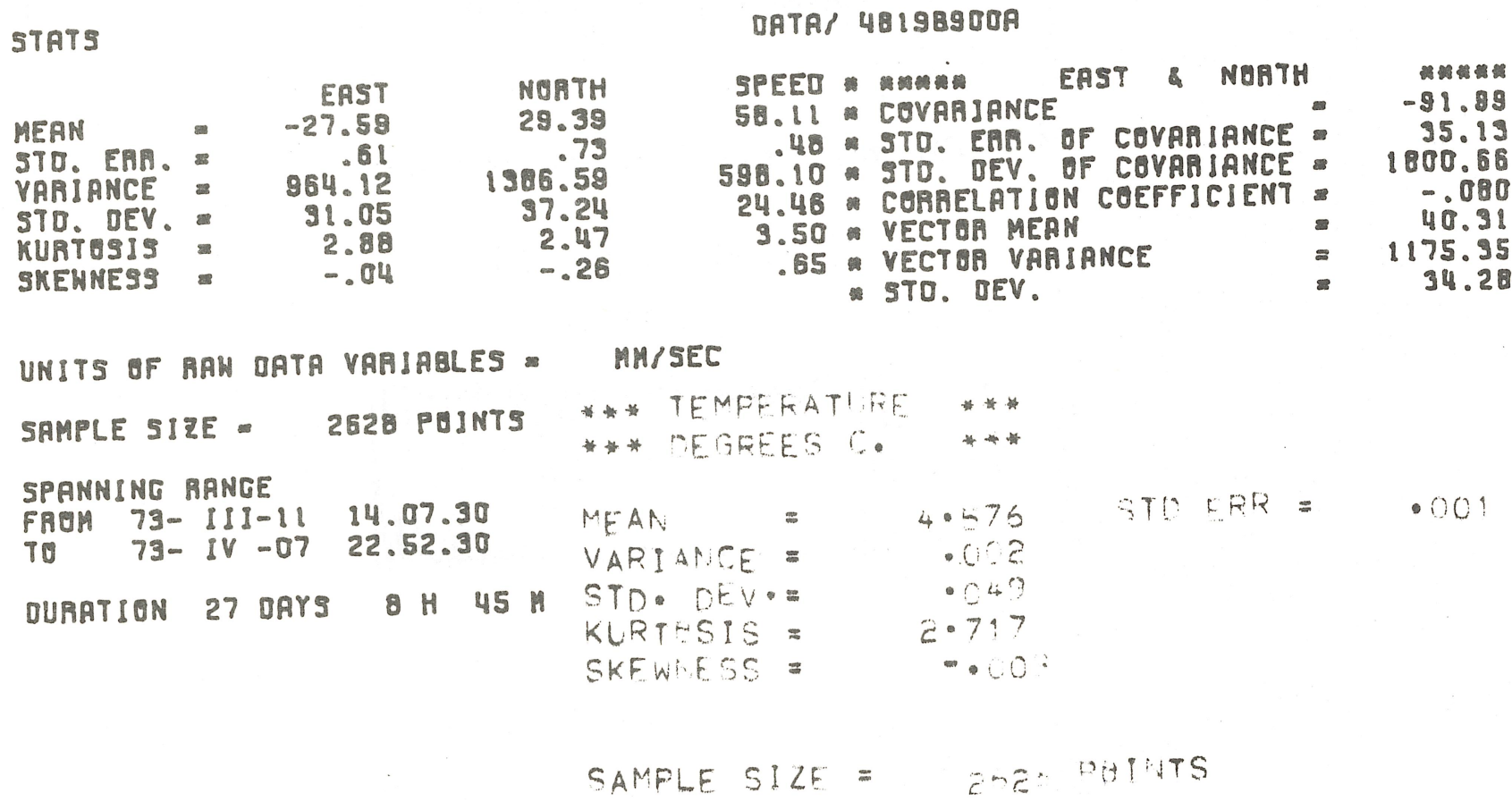



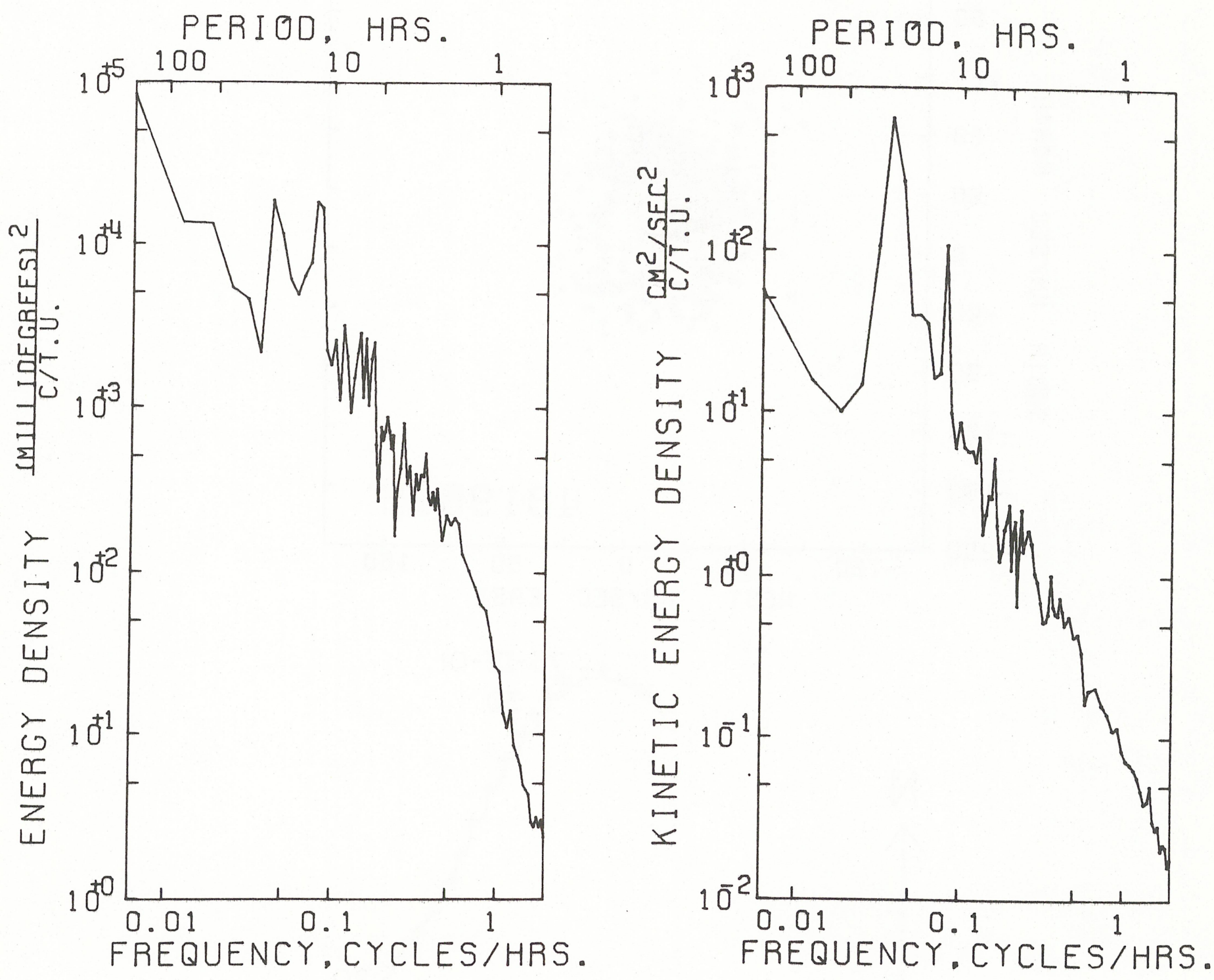

AUTO SPECTRUM

$4819 B 900$ TEMPERATURE 1392 METERS

73-III-11 TO 73-IV-06

1 PIECES WITH 1296 ESTIMATES

PER PIECE. AVERAGED OVER

4 ADJACENT FREQUENCY BANDS

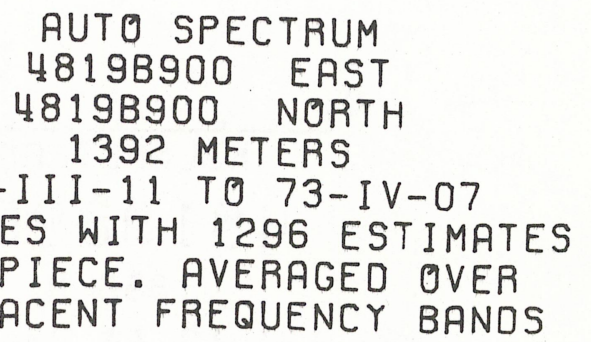



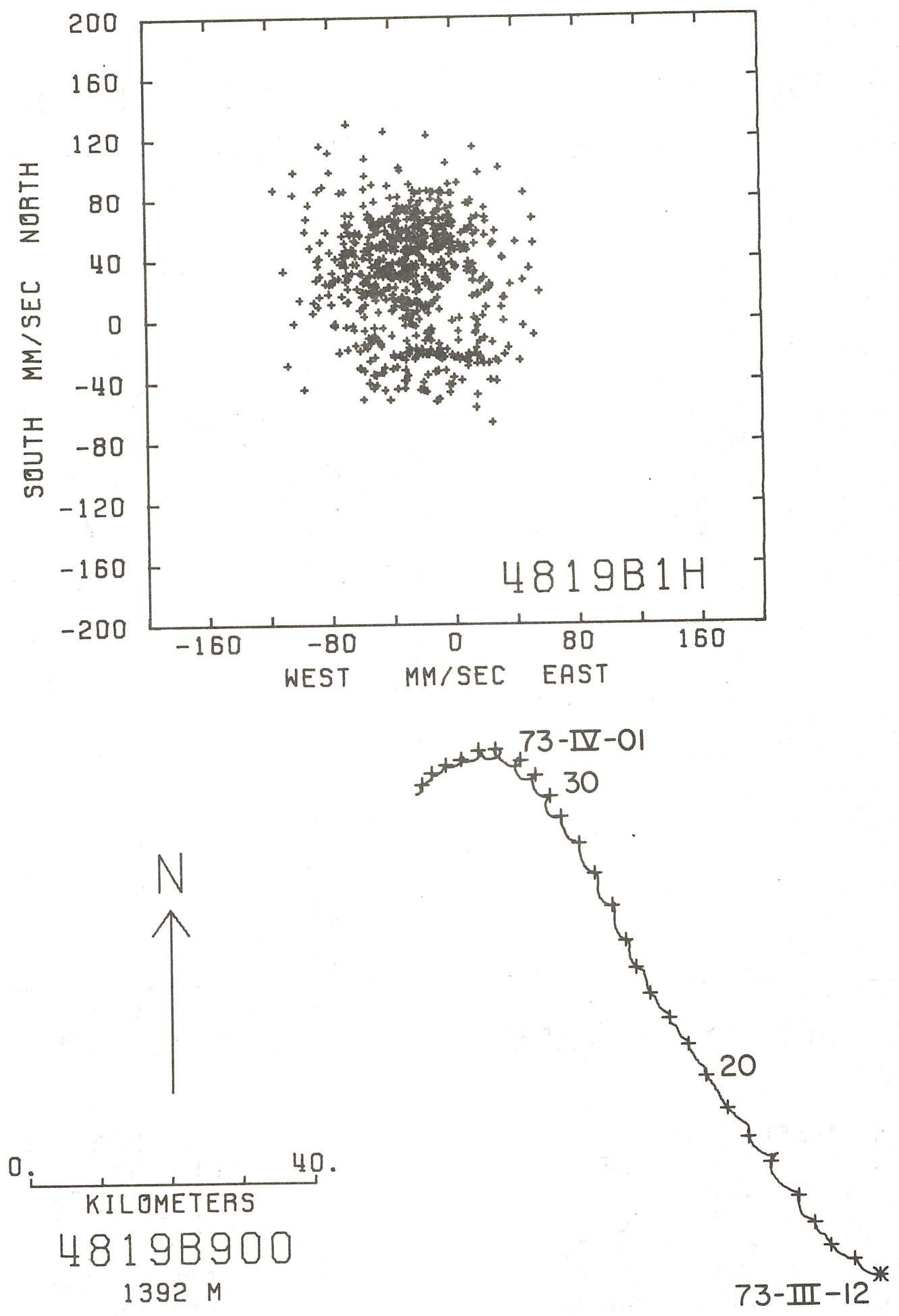

73- III-12 TO 73- IV -07 

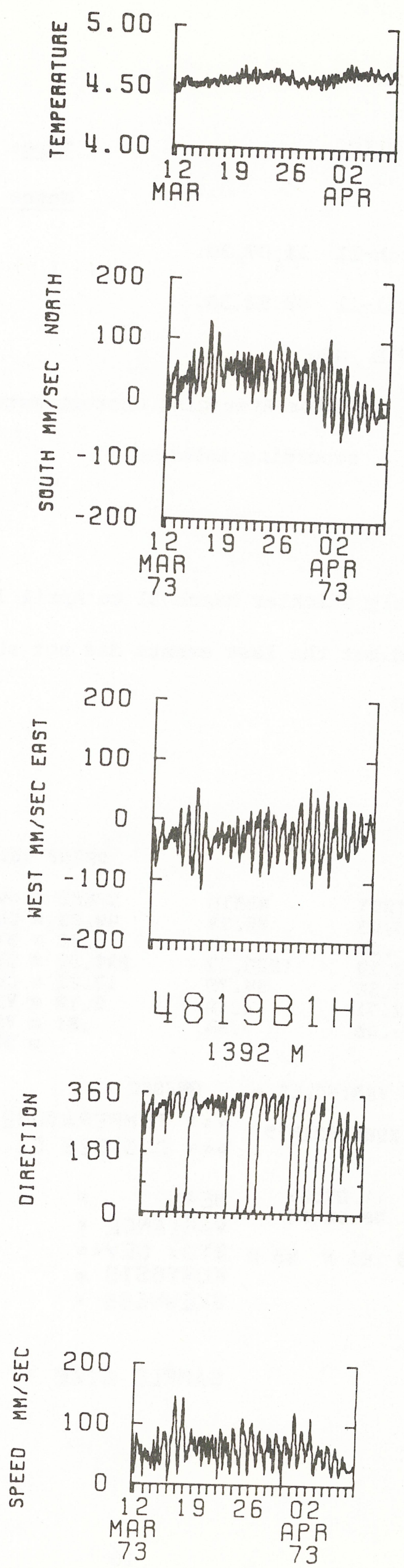
DATA NUMBER 481,12

Instrument No.: $\mathrm{V}-0119$

Depth: $2916 \mathrm{~m}$
Type: Vector Averaging Current Meter Water Depth: $5462 \mathrm{~m}$

Start time: 73-March-11 11.07.30.

Stop time: 73-April-11 08.52.30.

Duration: $30 \mathrm{~d} 21 \mathrm{~h} \quad 45 \mathrm{~m}$

Sampling scheme: Vector Averaging Current Meter

recording interval $=900$ seconds

COMMENTS:

Compass - good

Vane - progressively stickier March 31 to April 15, stuck April 29 to June 9

Rotor - looks good but the last events did not show up in the deck data

Temperature - good

STATS

\begin{tabular}{|c|c|c|c|}
\hline & & EAST & MONTH \\
\hline $\begin{array}{l}\text { EAN } \\
\text { TO. ENA. }\end{array}$ & & -23.55 & 25.79 \\
\hline $\begin{array}{l}\text { TO, ENA. } \\
\text { ARI ANCE }\end{array}$ & & 306.30 & 1206.37 \\
\hline $\begin{array}{l}\text { DEV. } \\
\text { TTESIS }\end{array}$ & $=$ & 17.50 & 34.79 \\
\hline 10315 & $=$ & 2.71 & 2.18 \\
\hline & 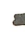 & -.00 & \\
\hline
\end{tabular}

DคTA8 481.128900 ค

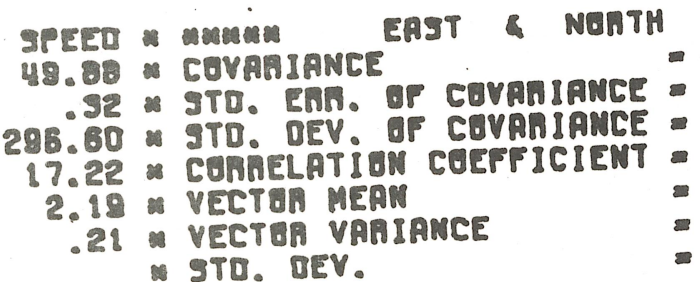

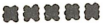

47.11

19.4ว

1058.48

.070

35.67

756.94

27.50

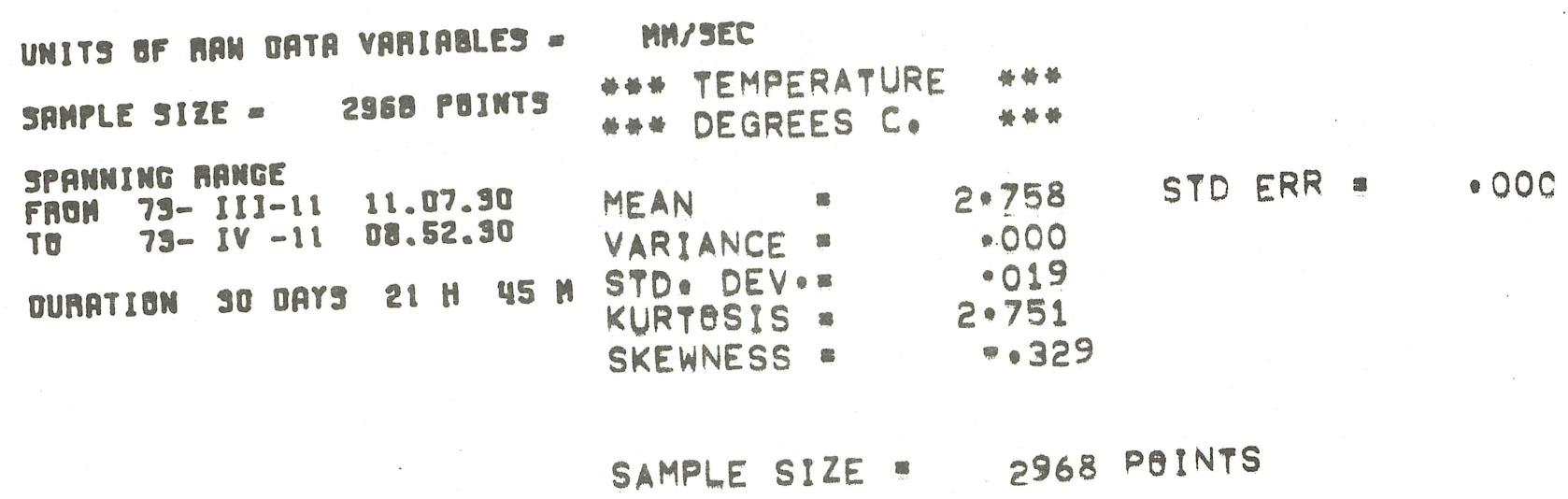



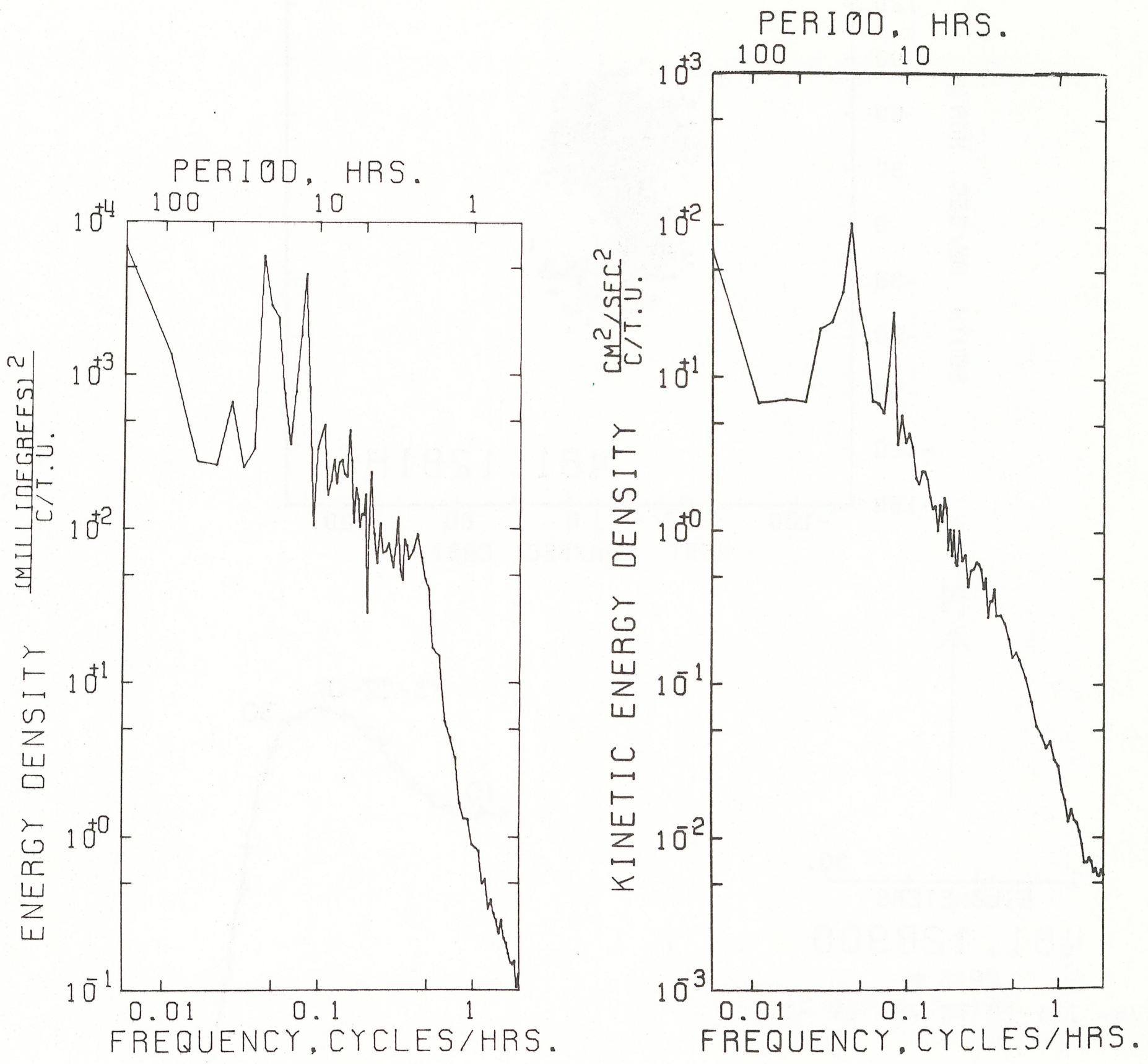

AUTO SPECTRUM

481.12B900 TEMPERATURE 2916 METERS

73-III-11 TO 73-IV-10

1 PIECES WITH 1458 ESTIMATES

PER PIECE. AVERAGED OVER

4 ADJACENT FREQUENCY BANDS

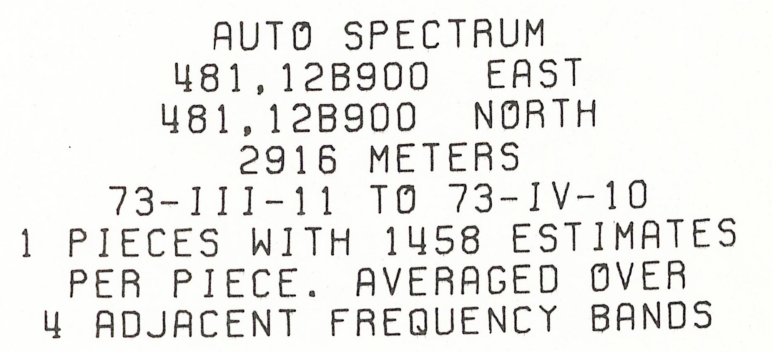



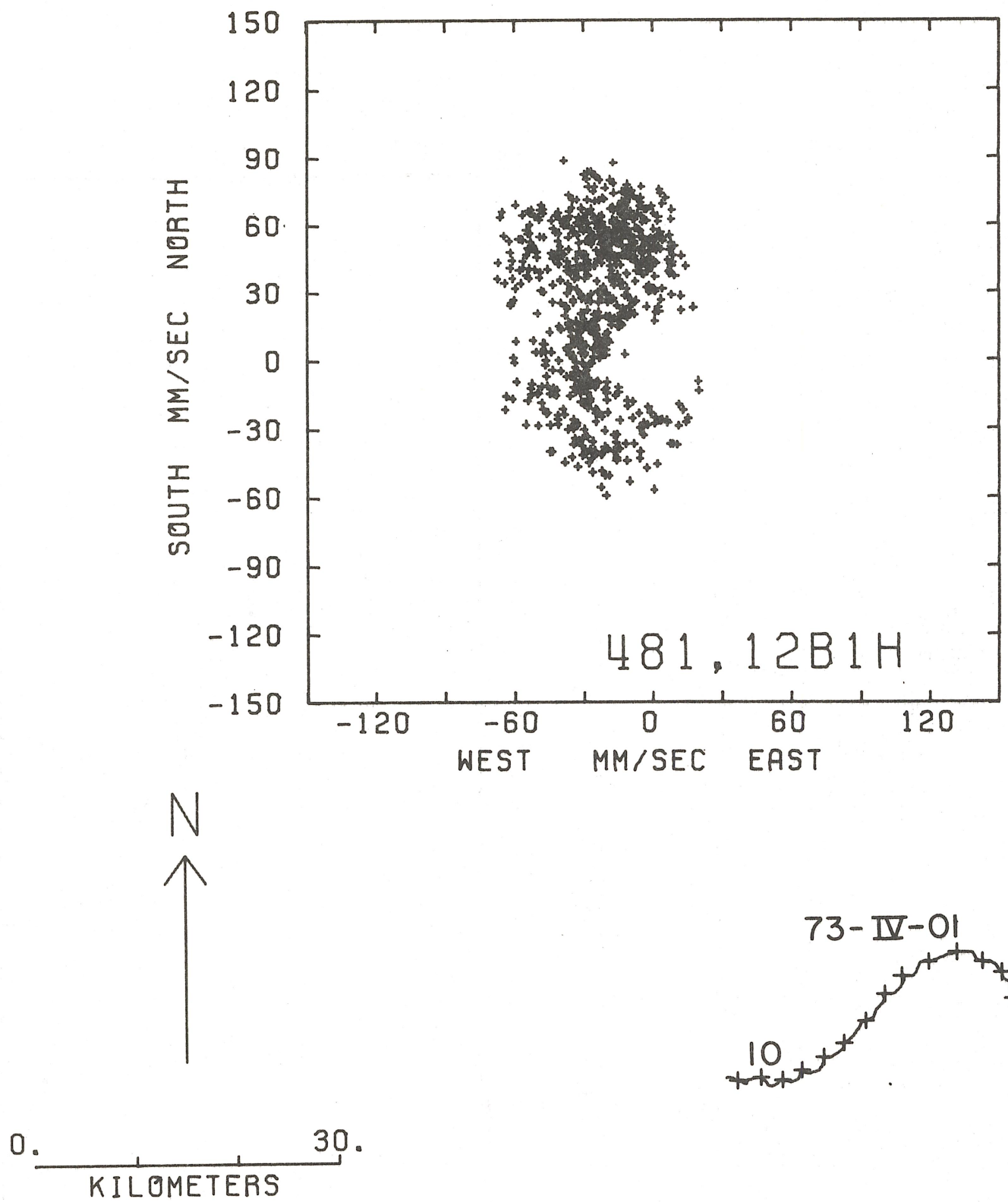

$481,12 \mathrm{~B} 900$ $2916 \mathrm{M}$

73- III-12 TO 73- IV - 11

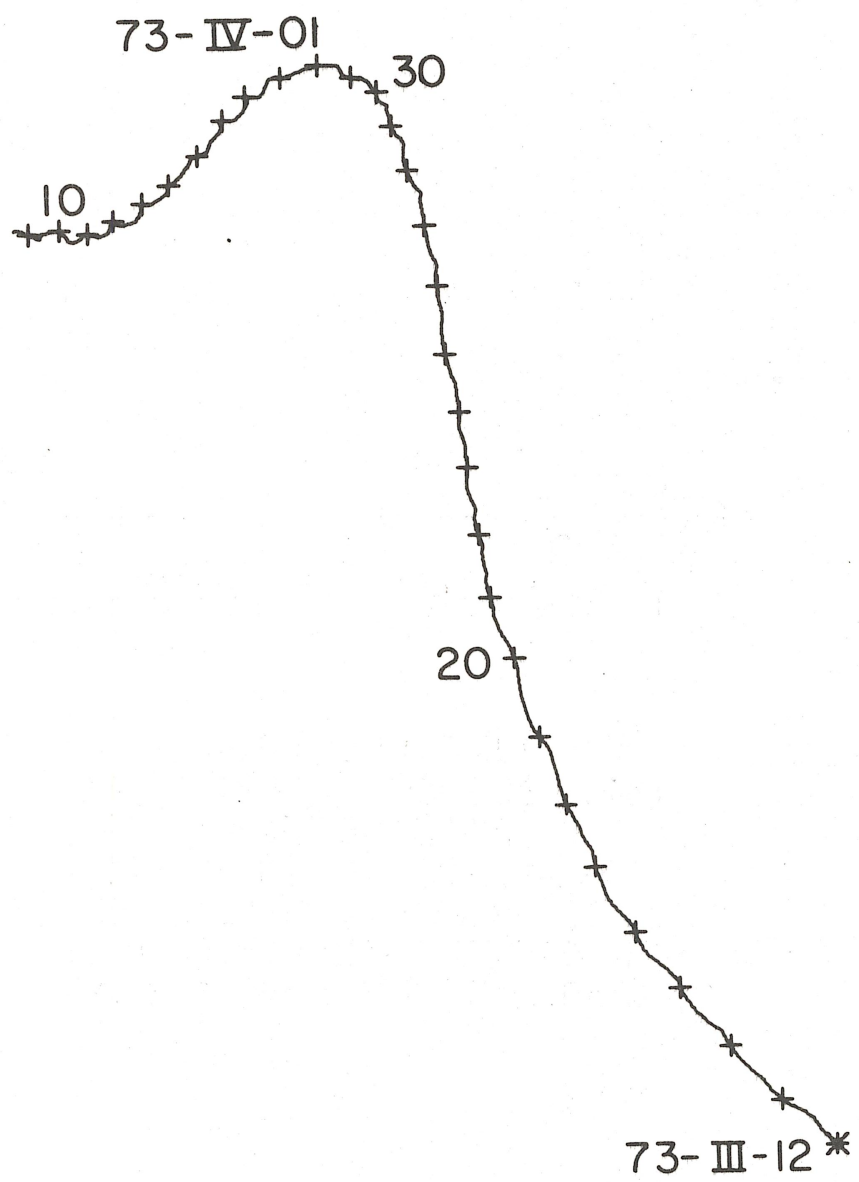



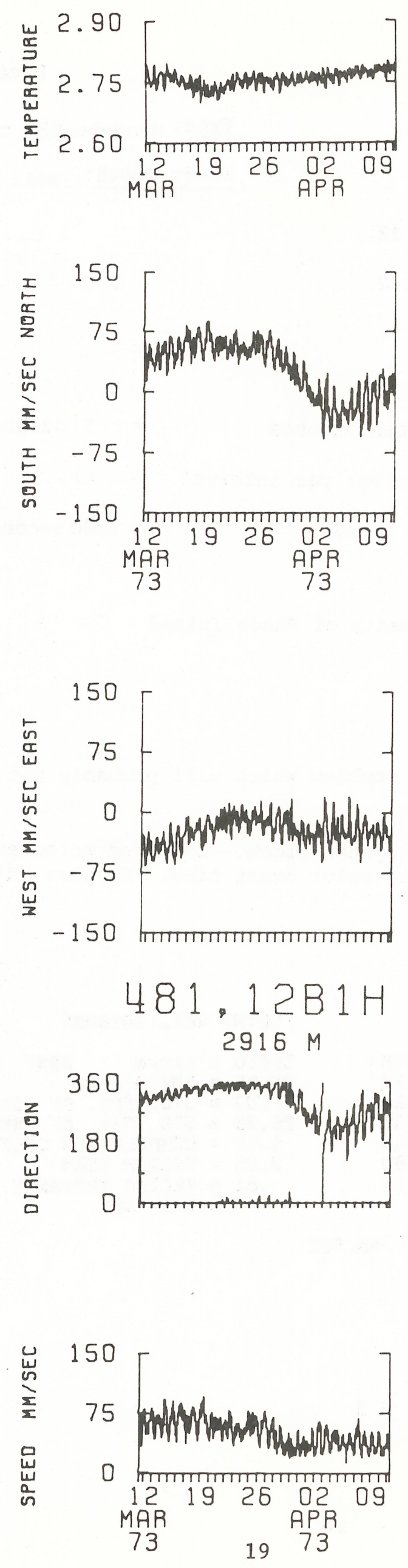
DATA NUMBER 481,15

Data number 481,15

Instrument No. : $\mathrm{M}-218$

Type: Geodyne 850 current meter

Depth: $3963 \mathrm{~m}$

Water depth: $5462 \mathrm{~m}$

Start time: 73-March-11 05.19.42.

Stop time: 73-July-04 09.49.42.

Duration: $115 \mathrm{~d}$ 4h $30 \mathrm{~m}$

Sampling scheme: Interval

$\begin{array}{ll}\text { time between strobes } & =5 \text { seconds } \\ \text { no. of strobes per interval } & =13 \\ \text { recording interval } & =1800 \text { seconds }\end{array}$

COMMENTS:

Instrument owned by the University of Rhode Island

Compass - good

Vane - good

Rotor count - minor order bit problem which will probably not affect the vector averages

Clock - mechanical clock not crystal clock. Computed rotor event time occurs $11 / 2$ hours sooner than the real rotor event time. No time adjustmant made.

STATS

\begin{tabular}{|c|c|c|c|}
\hline & & EAST & MOATH \\
\hline MEAN & - & 2.43 & 3.27 \\
\hline STD. EAh. & $=$ & .23 & \\
\hline VARI ANCE & $=$ & 281.36 & 379.56 \\
\hline STD. OEV. & 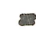 & 18.77 & 10.48 \\
\hline RURTOSIS & 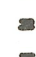 & 1.61 & 1.63 \\
\hline EMNES9 & $=$ & .08 & \\
\hline
\end{tabular}

UNITS OF คAM DATA VARIABLES - MMPSEC

SRMPLE SIEE - 5530 POIMTS

SPANMING AARGE

FHOM 79- III-11 05.18 .42

TO 79- VIIDO 00.49 .42

DURATION 115 DATS 4 H 30 M $O$
DATAS 481.1501800A

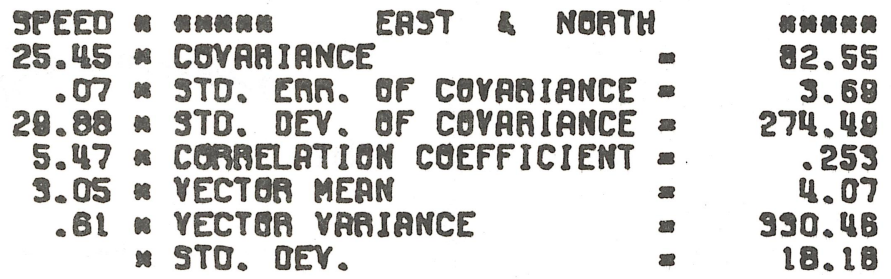




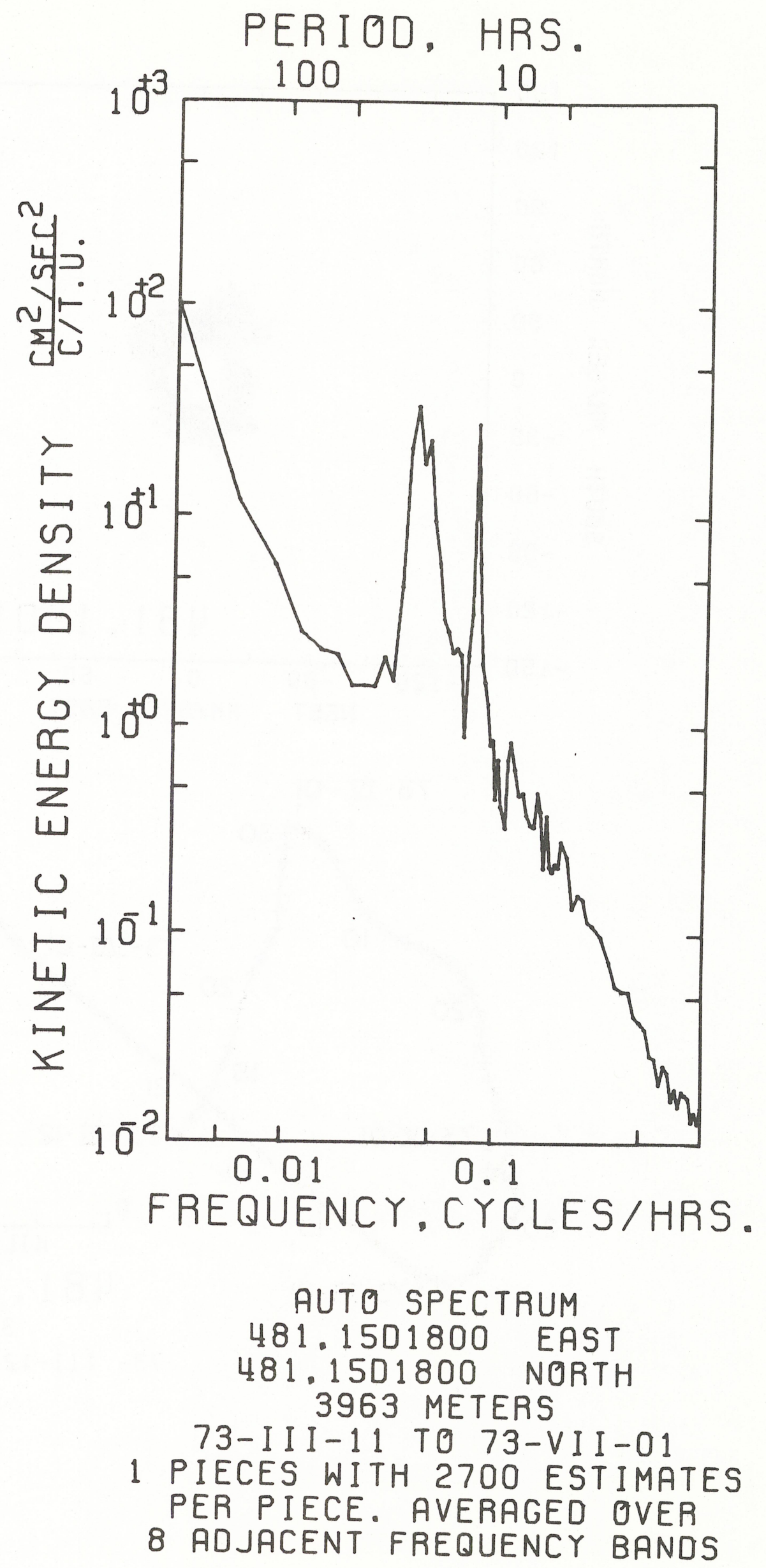



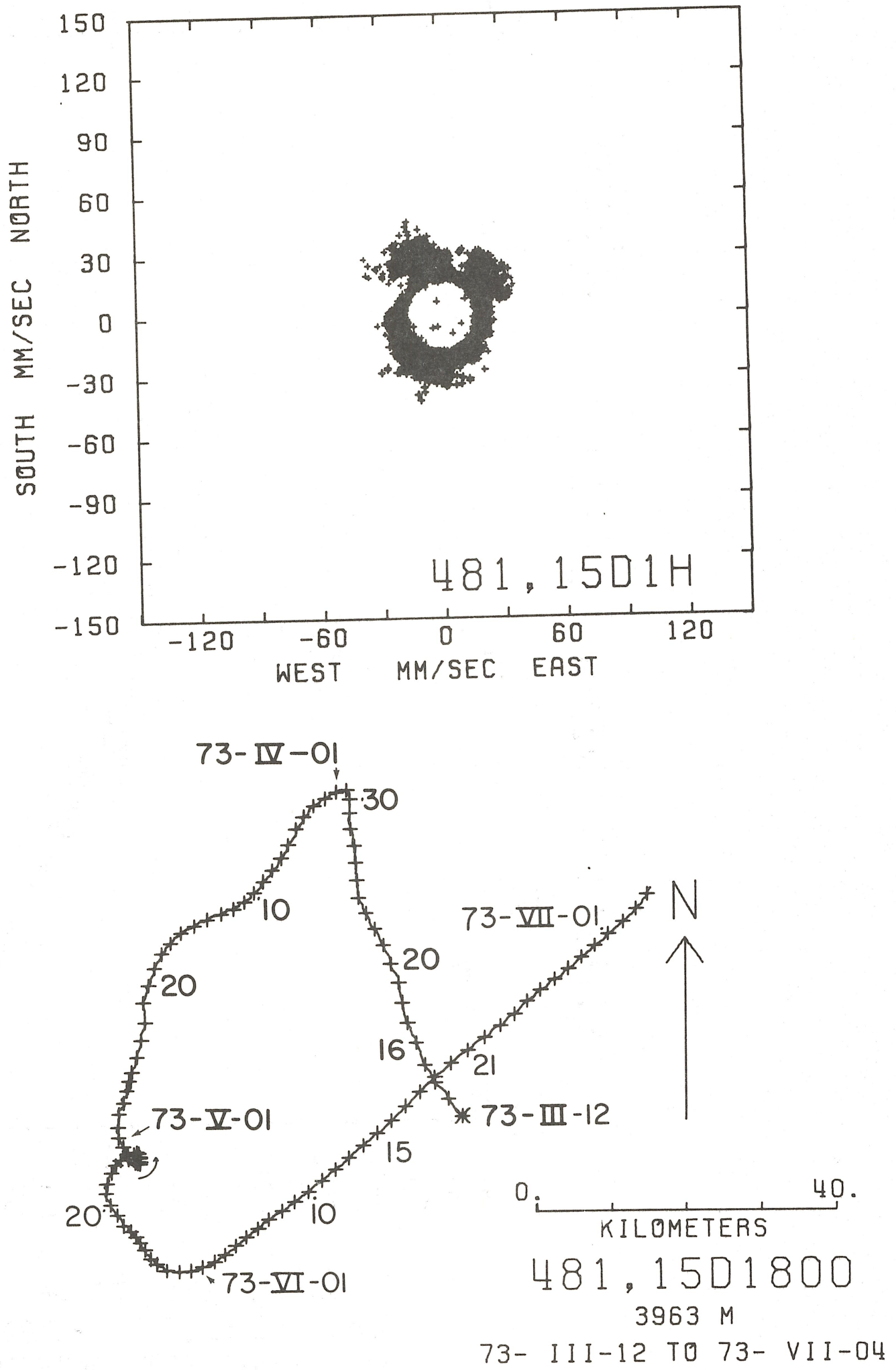

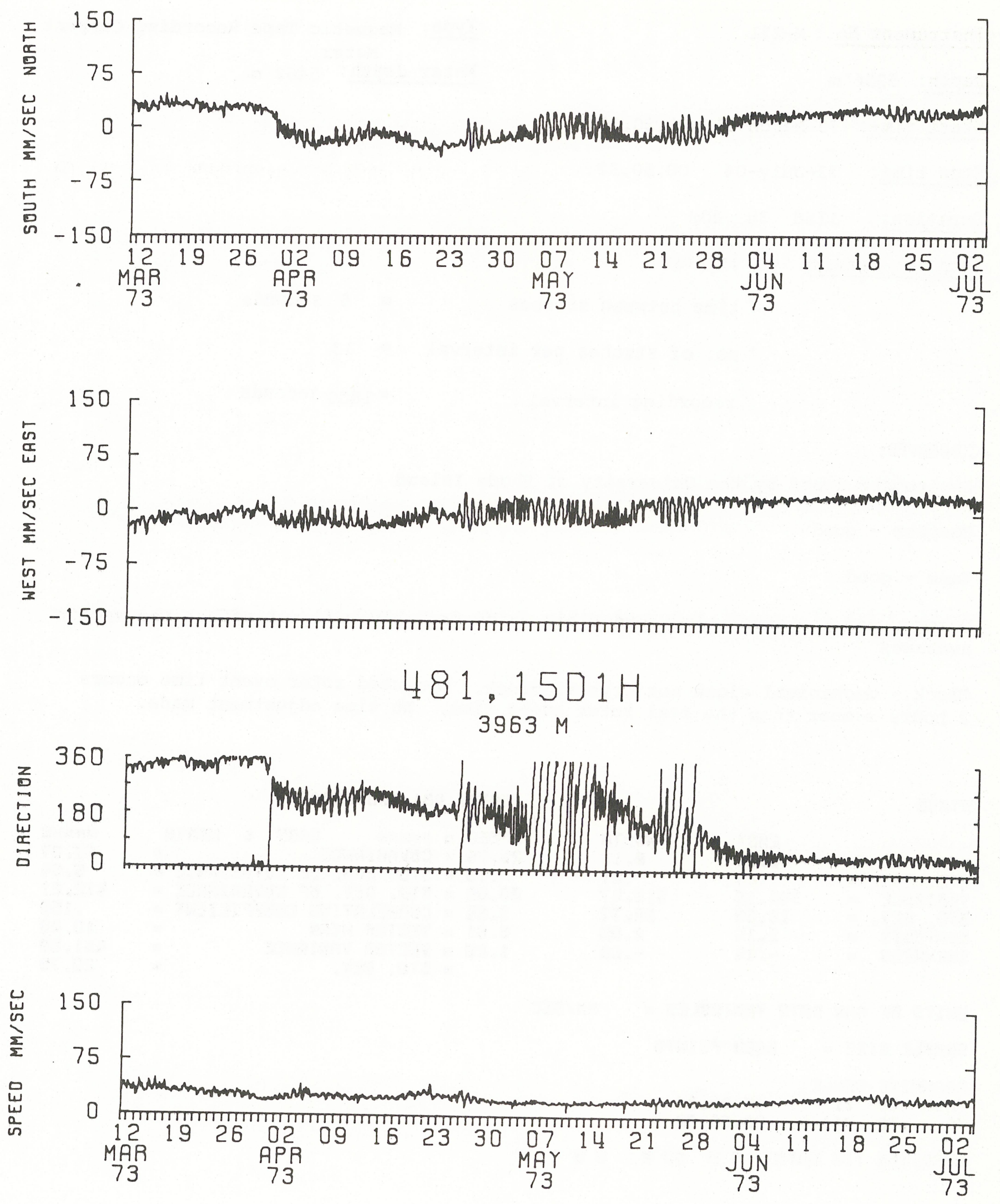
DATA NUMBER 481,18

Instrument No.: $\mathrm{M}-221$

Depth: $5356 \mathrm{~m}$

Start time: 73-March-11 05.20.32.

Stop time: $73-J u l y-04 \quad 08.50 .32$.

Duration: $115 \mathrm{~d}$ 3h 30m

Sampling scheme: Interval

$$
\begin{array}{ll}
\text { time between strobes } & =5 \text { seconds } \\
\text { no. of strobes per interval } & =13 \\
\text { recording interval } & =1800 \text { seconds }
\end{array}
$$

COMMENTS:

Instrument owned by the University of Rhode Island

Compass - good

Vane - good

Rotor count - a stuck low order bit which probably will not affect the vector averages

Clock - mechanical clock not crystal clock. Computed rotor event time occurs 2 hours sooner than the real rotor event time. No time adjustment made.

STATS

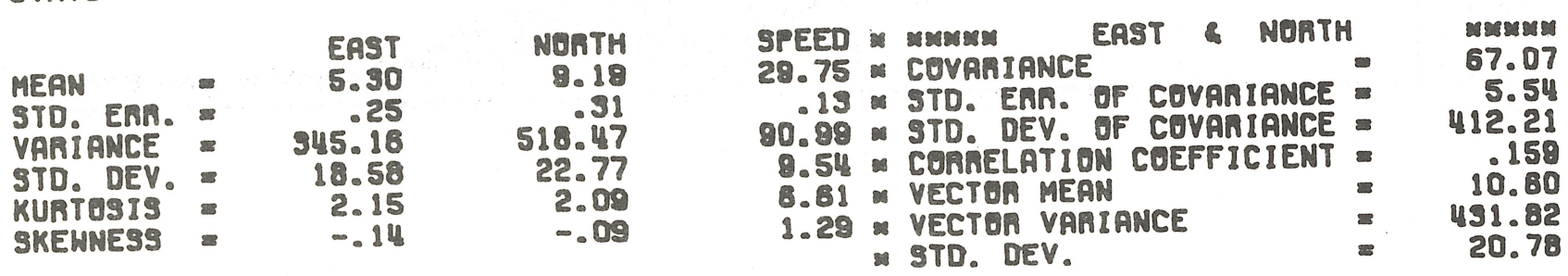

UNITS OF NAM DATA VAAIABLES = MM/SEC

SAMPLE SIZE = 5520 POINTS

SPANNING RANGE

FROM 73- III-11 05.20.32

TO 73-VII-O4 08.50.32

DURATION 115 DAYS 3 H. 30 M 0 S
DATA/ 481.18D1800A

Type: Magnetic Tape Recording Current Water depth: $5462 \mathrm{~m}$ 

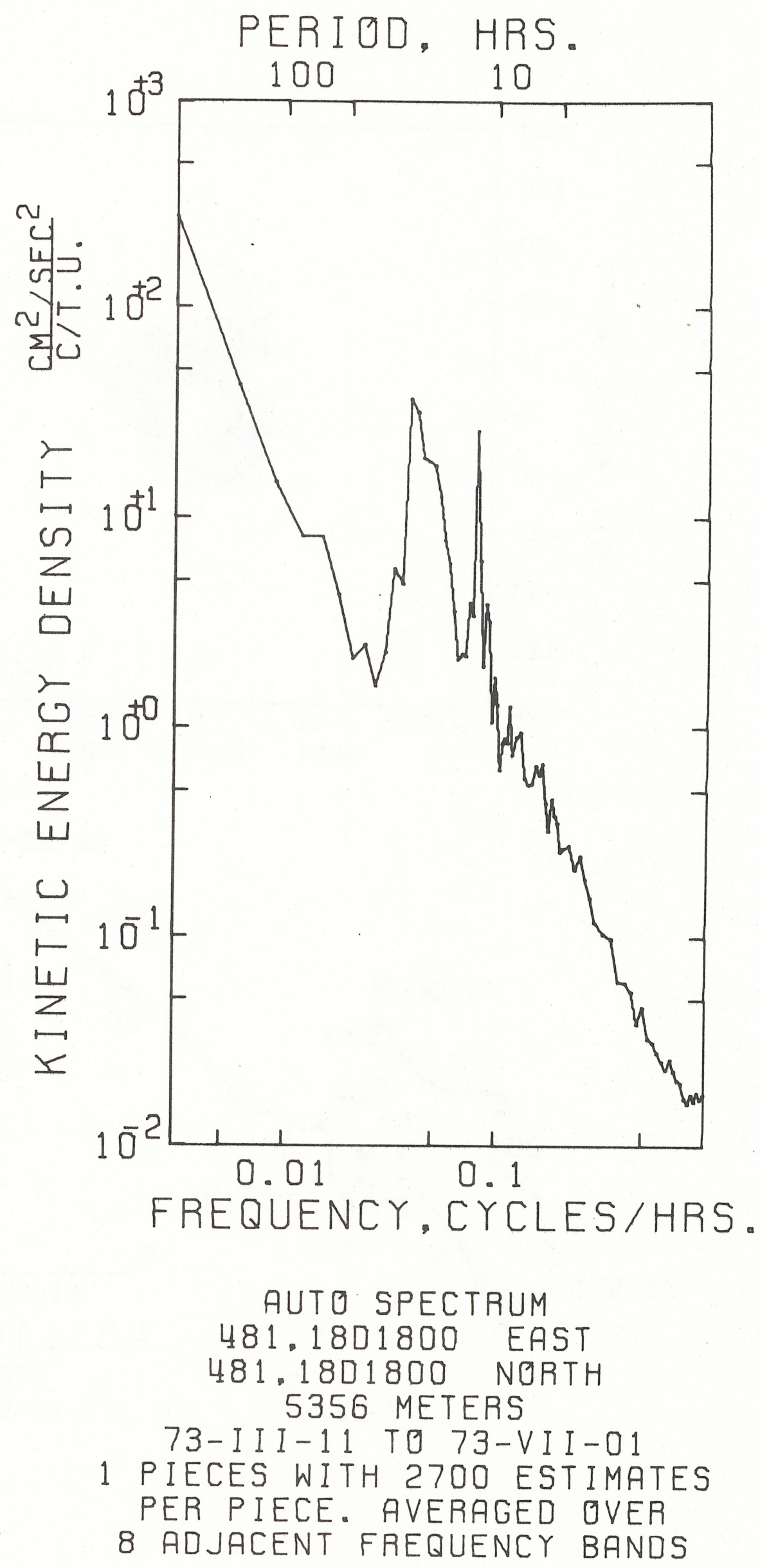

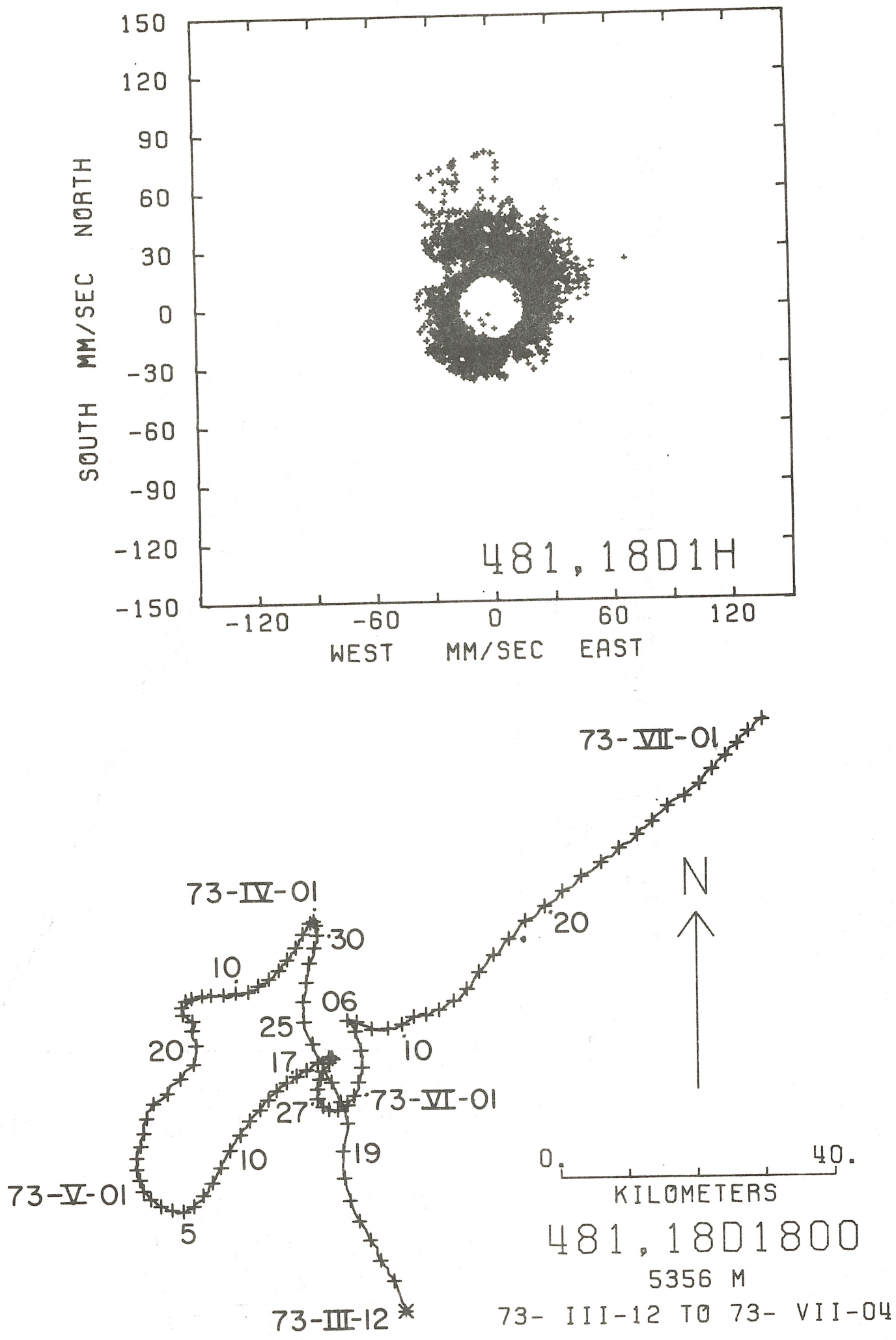

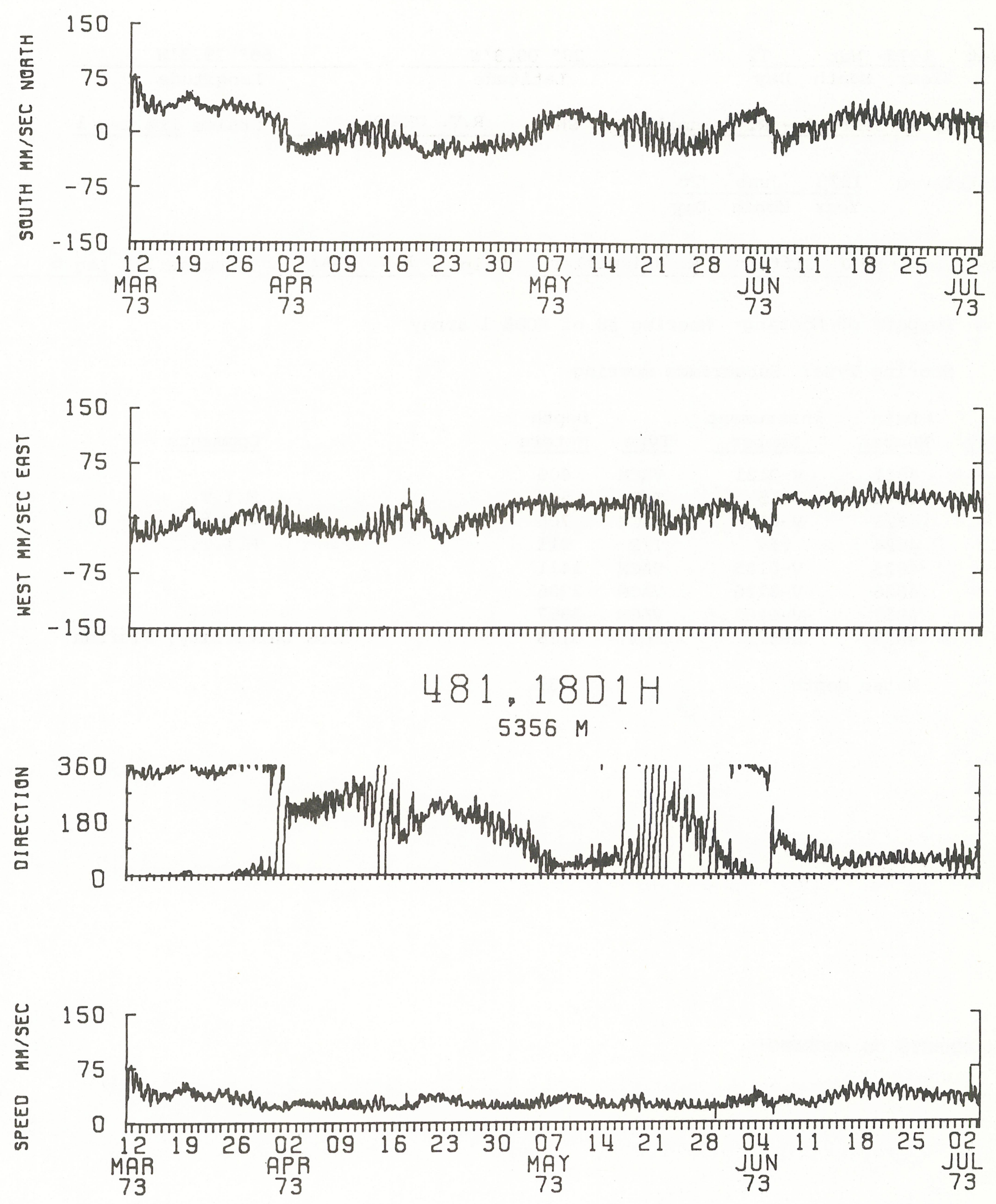
Mooring No. 482

Set $\frac{1973 \text { Mar } \quad 12}{\text { Year Month Day }}$

$28^{\circ} 09.3^{\prime} \mathrm{N}$

$68^{\circ} 39.3^{\prime} \mathrm{W}$

Latitude

Longitude

Set by J. Gifford - R. Heinmiller

Ship R.V. CHAIN

Cruise 112 Leg 1

Retrieved $\frac{1973 \text { June } 26}{\text { Year Month Day }}$

Retrieved by J. Gifford - R. Heinmiller Ship R.V. CHAIN Cruise 112 Leg 6

Purpose of Mooring: Mooring \#8 of MODE 1 array

Mooring Type: Subsurface mooring

\begin{tabular}{|c|c|c|c|c|}
\hline Key & $\begin{array}{c}\text { Data } \\
\text { Number } \\
\end{array}$ & $\begin{array}{c}\text { Instrument } \\
\text { Number }\end{array}$ & Type & $\begin{array}{l}\text { Depth } \\
\text { Meters }\end{array}$ \\
\hline+ & 4821 & $V-0121$ & VACM & 406 \\
\hline$\#$ & 4822 & \#15 & $\mathrm{T} / \mathrm{P}$ & 507 \\
\hline+ & 4823 & $V-0130$ & VACM & 706 \\
\hline 非 & 4824 & \#54 & $\mathrm{T} / \mathrm{P}$ & 911 \\
\hline+ & 4825 & $V-0135$ & VACM & 1411 \\
\hline * & 4826 & $V-0126$ & VACM & 2936 \\
\hline * & 4827 & $V-0165$ & VACM & 3957 \\
\hline & 4828 & $\mathrm{H}-275$ & FCM & 5128 \\
\hline \multicolumn{3}{|c|}{ Water depth } & & 5239 \\
\hline
\end{tabular}

Comments

$M . I . T$.

I.O.S.

M.I.T.

Nova University, Florida

COMMENTS ON MOORING: 

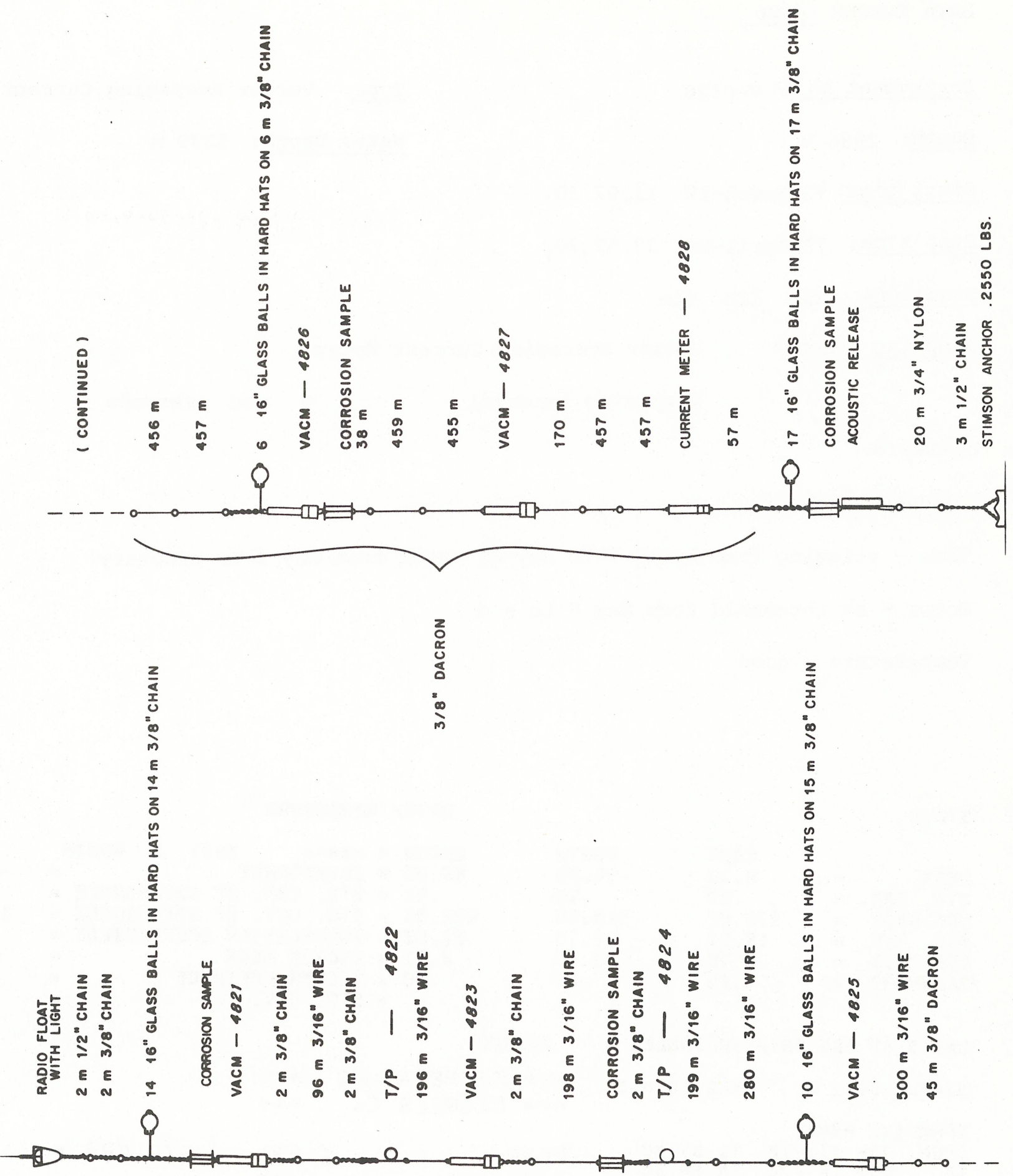

$\infty$
0
0
$Z$
0
$\frac{E}{E}$
0 
DATA NUMBER 4826

Instrument No.: V-0126

Depth: $2936 \mathrm{~m}$
Type: Vector Averaging Current Meter

Water Depth: $5239 \mathrm{~m}$

Start time: 73-March-12 11.07.30.

Stop time: 73-April-06 23.52,30,

Duration: $25 \mathrm{~d} \quad 12 \mathrm{~h} \quad 45 \mathrm{~m}$

Sampling scheme: Vector Averaging Current Meter

$$
\text { recording interval }=900 \text { seconds }
$$

COMMENTS :

Compass - good

Vane - sticking from April 7 to May 8, stuck from May 8 to recovery

Rotor - at threshold from May 8 to end

Temperature - good

STATS

$\begin{array}{lrr} & \text { EAST } & \text { MONTH } \\ \text { MERR } & 6.42 & -44.83 \\ \text { STD. ENA. } & .39 & .46 \\ \text { VARIANCE } & 372.92 & 516.00 \\ \text { STD. DEV. } & 19.91 & 22.76 \\ \text { KURTESIS } & 3.78 & 9.15 \\ \text { SKEHNES9 } & .65 & .07\end{array}$

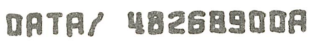

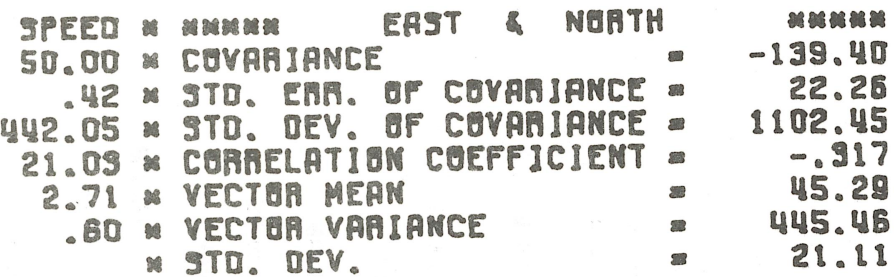

MMPSEC

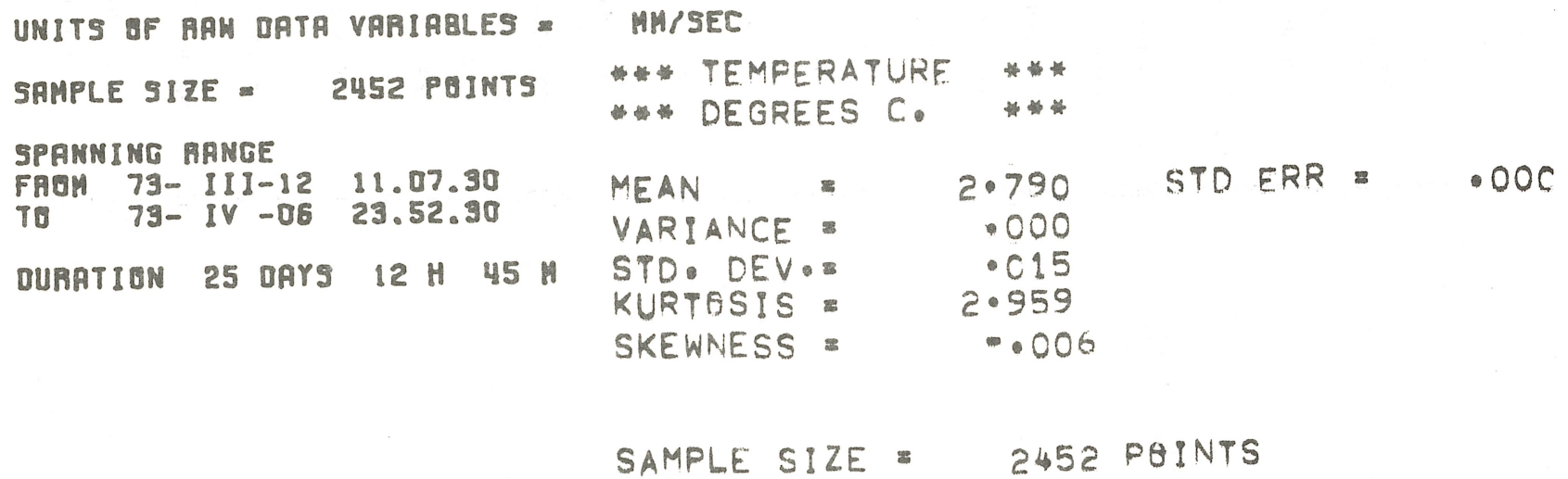




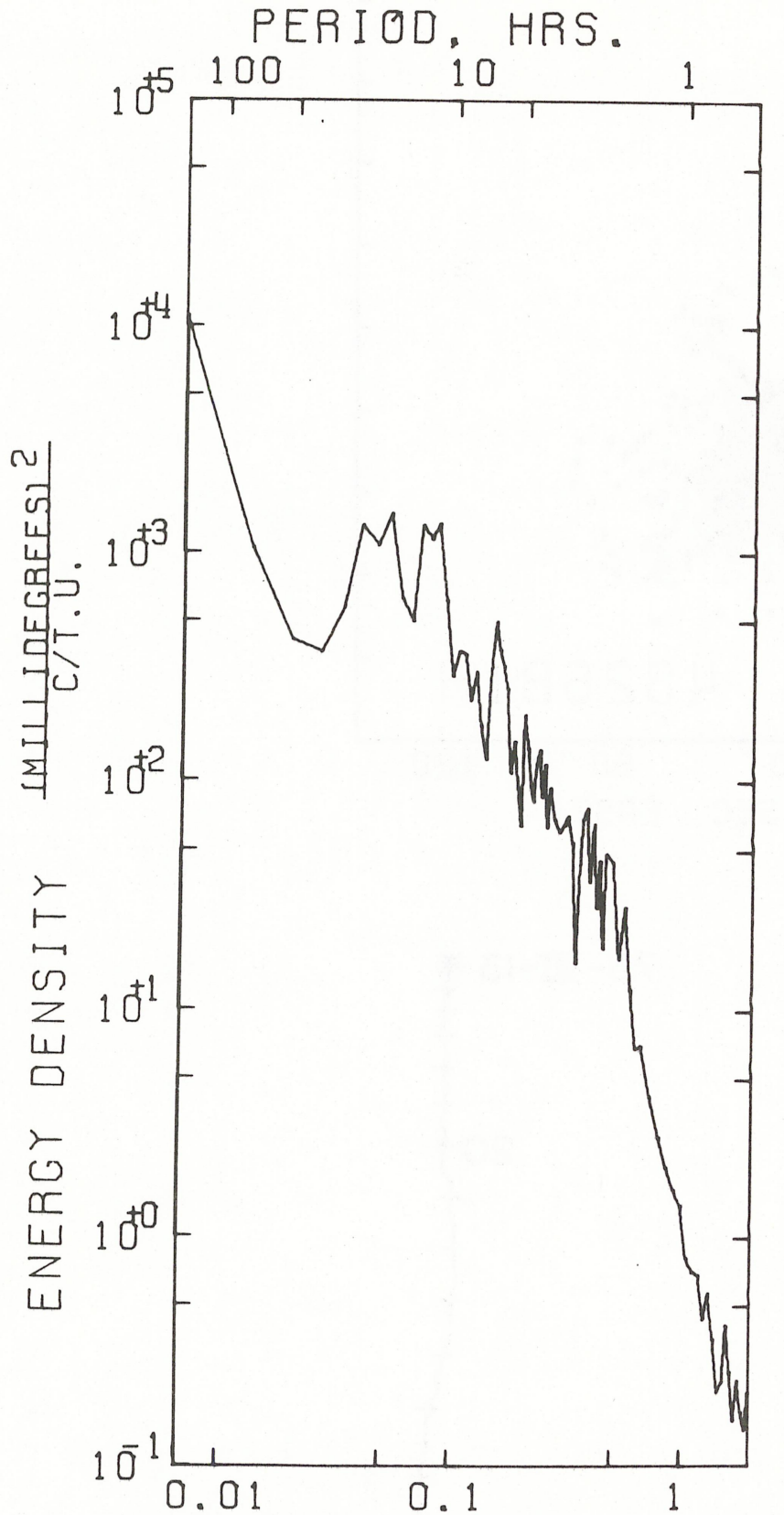

FREQUENCY, CYCLES/HRS.

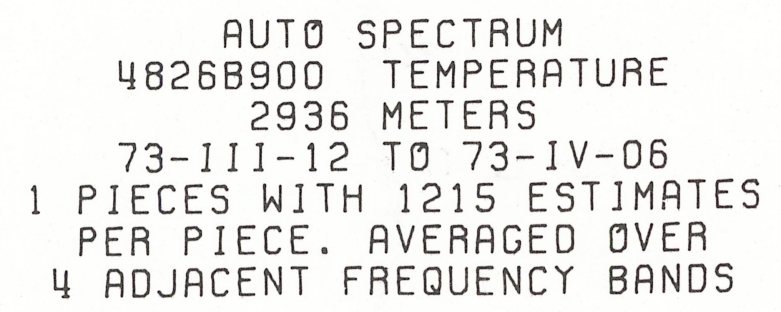

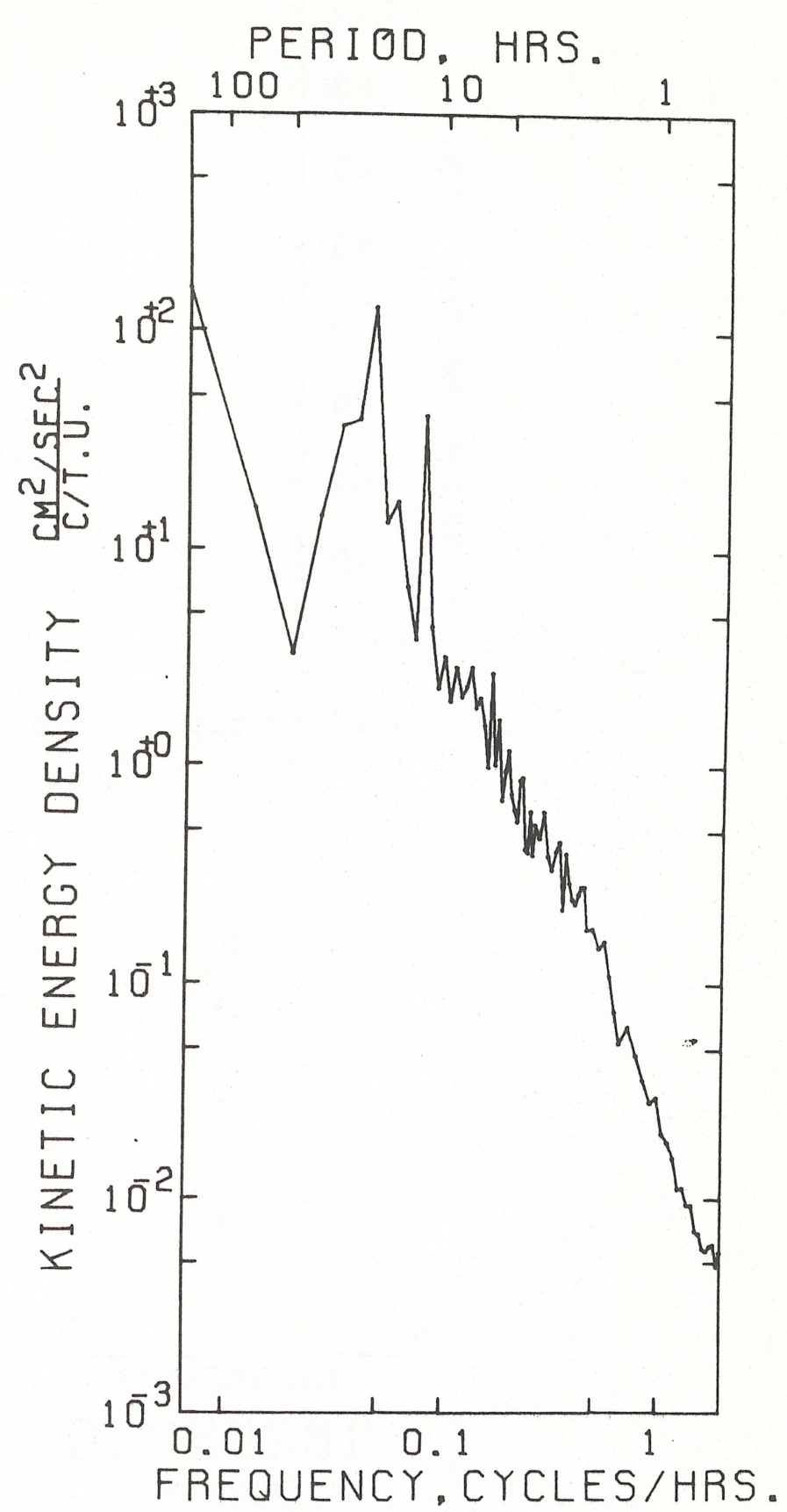

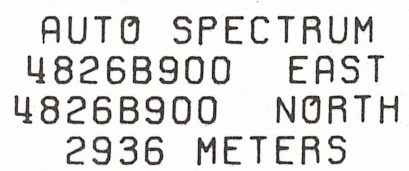

73-III-12 TO 73-IV-06

1 PIECES WITH 1215 ESTIMATES PER PIECE. AVERAGED OVER 4 ADJACENT FREQUENCY BANDS 

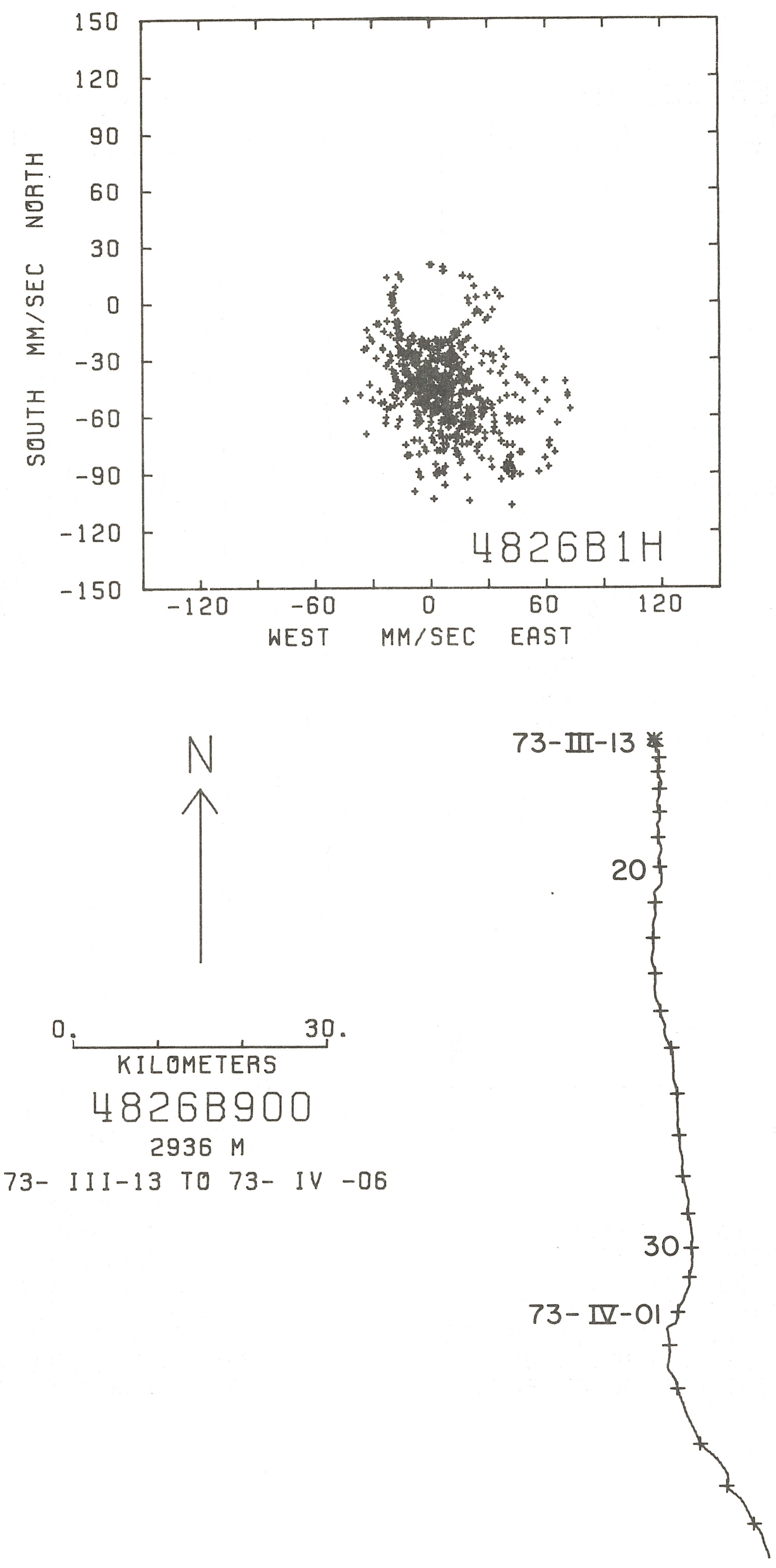

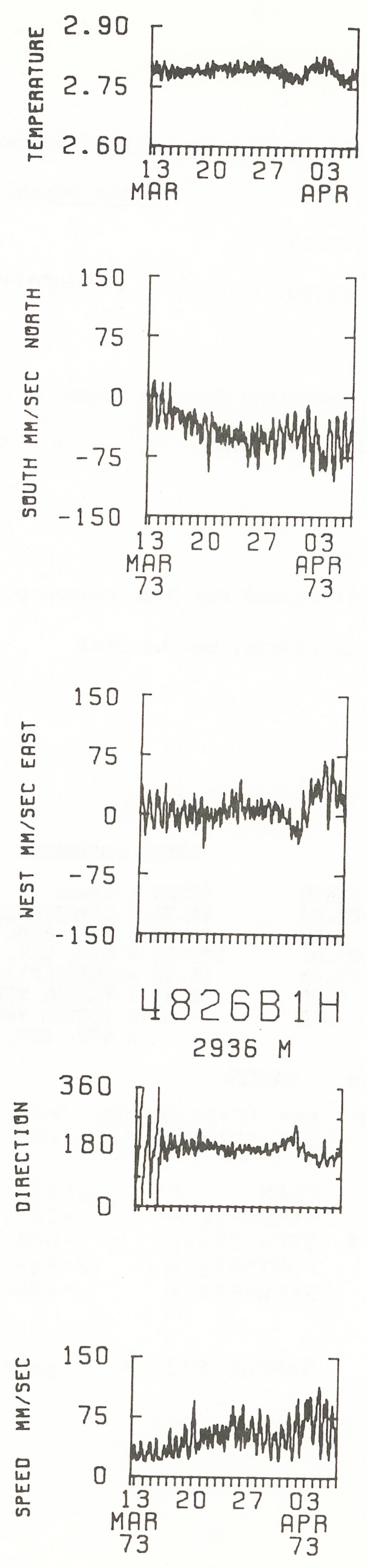
DATA NUMBER 4827

Instrument No.: $\mathrm{V}-0165$

Depth: $3957 \mathrm{~m}$
Type: Vector Averaging Current Meter Water Depth: $5239 \mathrm{~m}$

Start time: 73-March-12 10.07.30.

Stop time: 73-April-09 11.52.30.

Duration: $28 \mathrm{~d}$ ih $45 \mathrm{~m}$

Sampling scheme: Vector Averaging Current Meter

$$
\text { recording interval }=900 \text { seconds }
$$

COMMENTS :

Compass - good

Vane - stuck April 9 to April 24 and May 3 to recovery

Rotor -speeds are very low in places, may be real

Temperature - good

STคTS

\begin{tabular}{|c|c|c|c|}
\hline & & EดST & MURTH \\
\hline $\begin{array}{l}\text { MEAN } \\
\text { STD. EHA. }\end{array}$ & 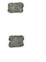 & $\begin{array}{r}-6.0 \\
.20\end{array}$ & -33.00 \\
\hline IARIANCE & $\approx$ & 218.33 & 298 \\
\hline STD. DEY. & $\$$ & 14.70 & 17.26 \\
\hline URTESIS & $=$ & 2.77 & 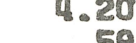 \\
\hline :HNESS & 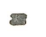 & 20 & \\
\hline
\end{tabular}

อคTคค แอ278900C

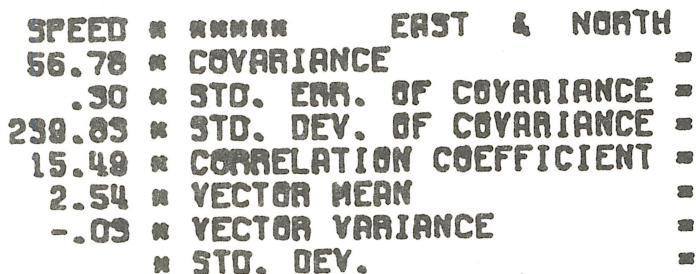

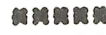
$-27.00$

16.20 845.05 $-.108$ 54.28 258.27

16.07

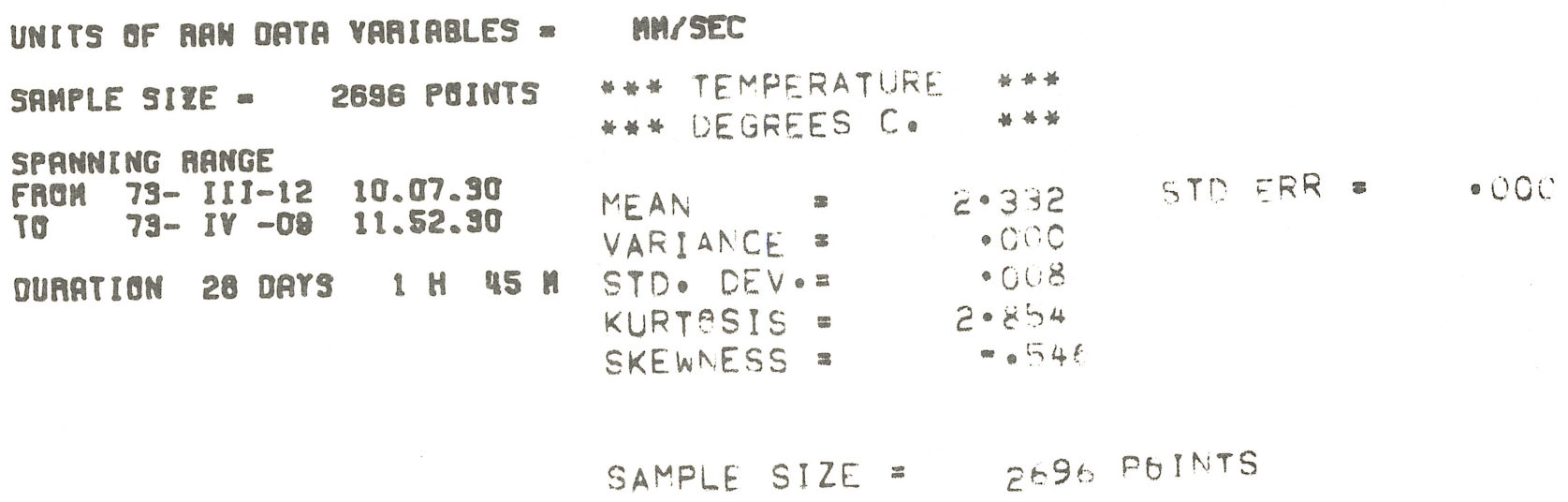



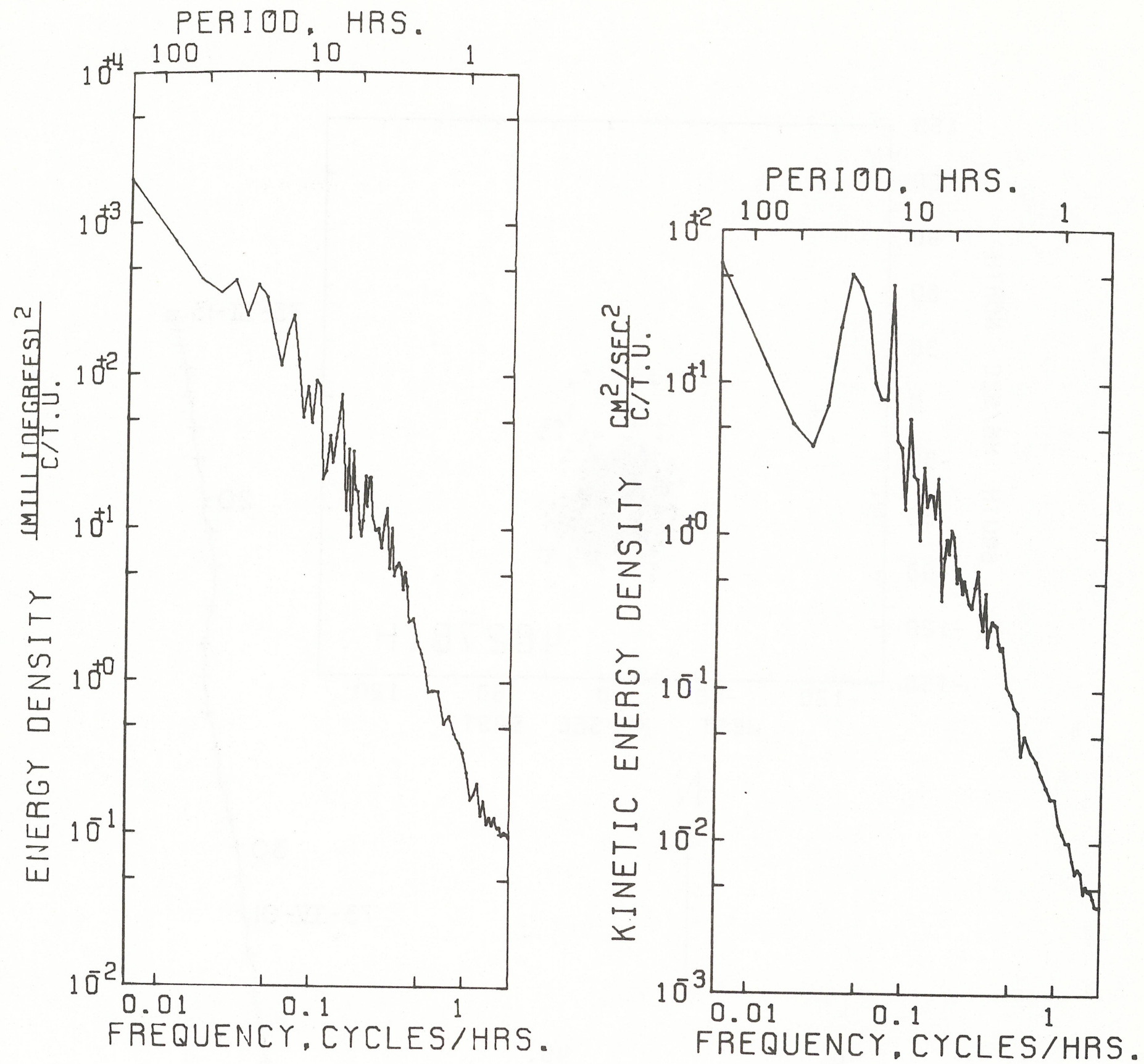

AUTO SPECTRUM

48278900 TEMPERATURE 3957 METERS

73-III-12 TO 73-IV-08

1 PIECES WITH 1296 ESTIMATES

PER PIECE. AVERAGED OVER

4 ADJACENT FREQUENCY BANDS

AUTO SPECTRUM

$4827 B 900$ EAST

$4827 B 900$ NORTH

3957 METERS

73-III-12 TO 73-IV-08

1 PIECES WITH 1296 ESTIMATES

PER PIECE. AVERAGED OVER

4 ADJACENT FREQUENCY BANDS 


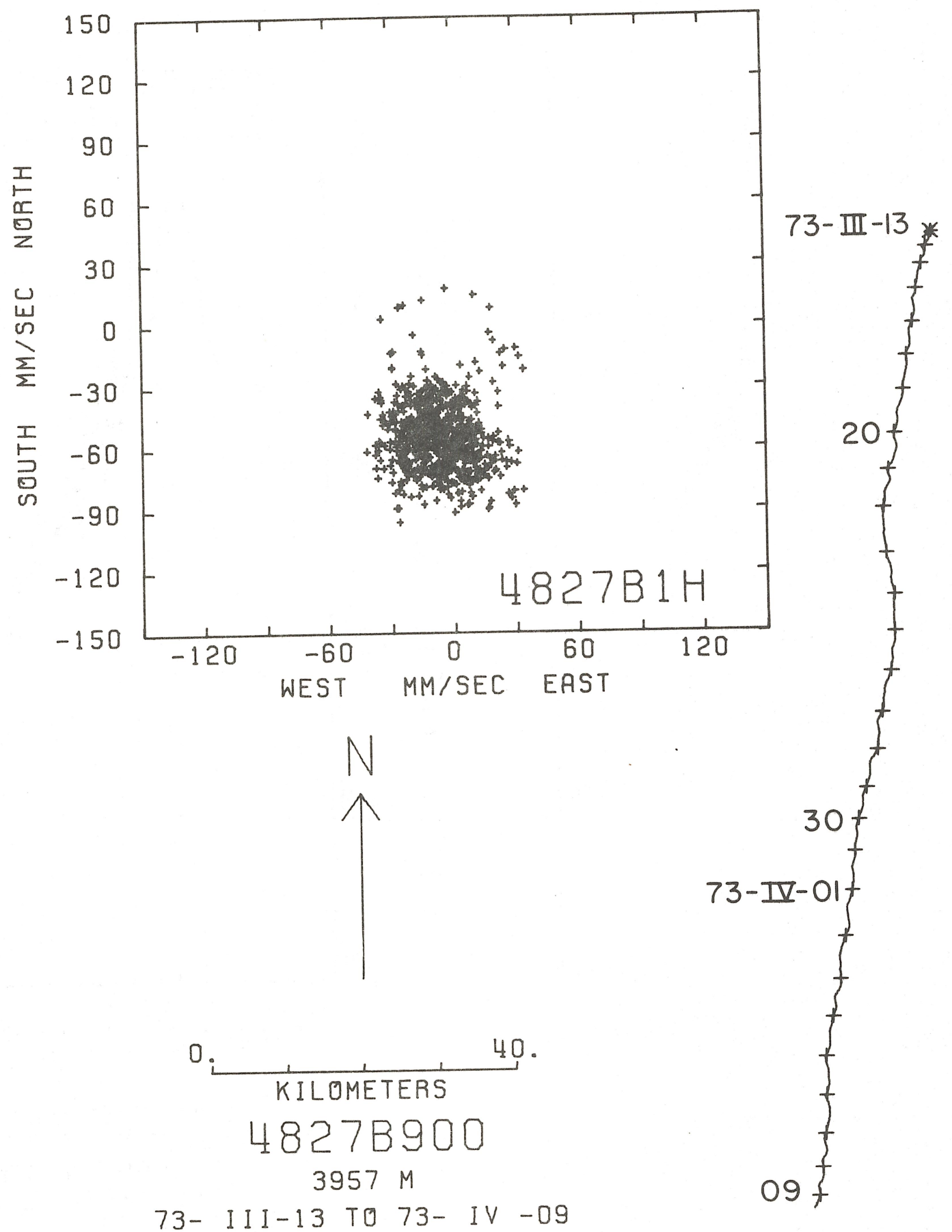



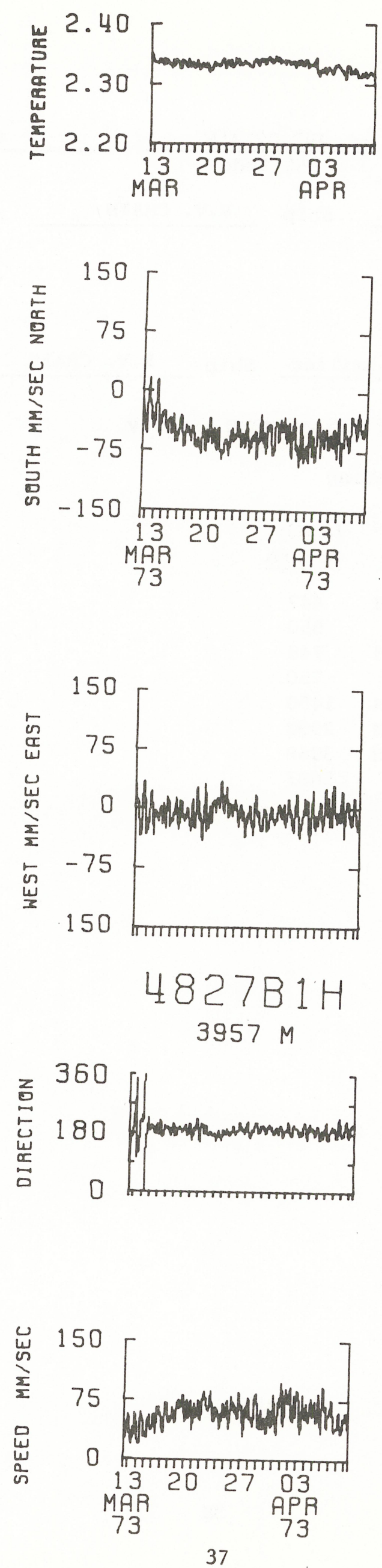
Mooring No. 483

Set $\frac{1973 \text { Mar } 12}{\text { Year Month Day }} \frac{29^{\circ} 02.3^{\prime} \mathrm{N}}{\text { Latitude }} \frac{68^{\circ} 13.8^{\prime} \mathrm{W}}{\text { Longitude }}$
Set by G. Tupper - R. Heinmiller Ship R.V. CHAIN $\quad$ Cruise 112 Leg 1

Retrieved $\frac{1973 \text { July } 3}{\text { Year Month Day }}$

Retrieved by J. Gifford - R. Heinmiller Ship R.V. CHAIN Cruise 112 Leg 6

Purpose of Mooring: Mooring \#15 of MODE 1 array

Mooring Type: Subsurface mooring

\begin{tabular}{|c|c|c|c|c|c|}
\hline Key & $\begin{array}{c}\text { Data } \\
\text { Number } \\
\end{array}$ & $\begin{array}{c}\text { Instrument } \\
\text { Number } \\
\end{array}$ & Type & $\begin{array}{l}\text { Depth } \\
\text { Meters }\end{array}$ & Comments \\
\hline+ & 4831 & $V-0113$ & VACM & 447 & \\
\hline \# & 4832 & \#43 & $\mathrm{T} / \mathrm{P}$ & 550 & M.I.T. \\
\hline * & 4833 & $V-0171$ & VACM & 748 & U.R.I. \\
\hline \# & 4834 & \#44 & $\mathrm{T} / \mathrm{P}$ & 750 & M.I.T. \\
\hline+ & 4835 & $V-0117$ & VACM & 1450 & \\
\hline+ & 4836 & V-0107 & VACM & 2998 & \\
\hline * & 4837 & V-0177 & VACM & 3968 & \\
\hline$\#$ & 4838 & \#11 & $\mathrm{T} / \mathrm{P}$ & 5087 & $M . I . T$. \\
\hline \multicolumn{3}{|c|}{ Water depth } & & 5192 & \\
\hline
\end{tabular}

COMMENTS ON MOORING: 


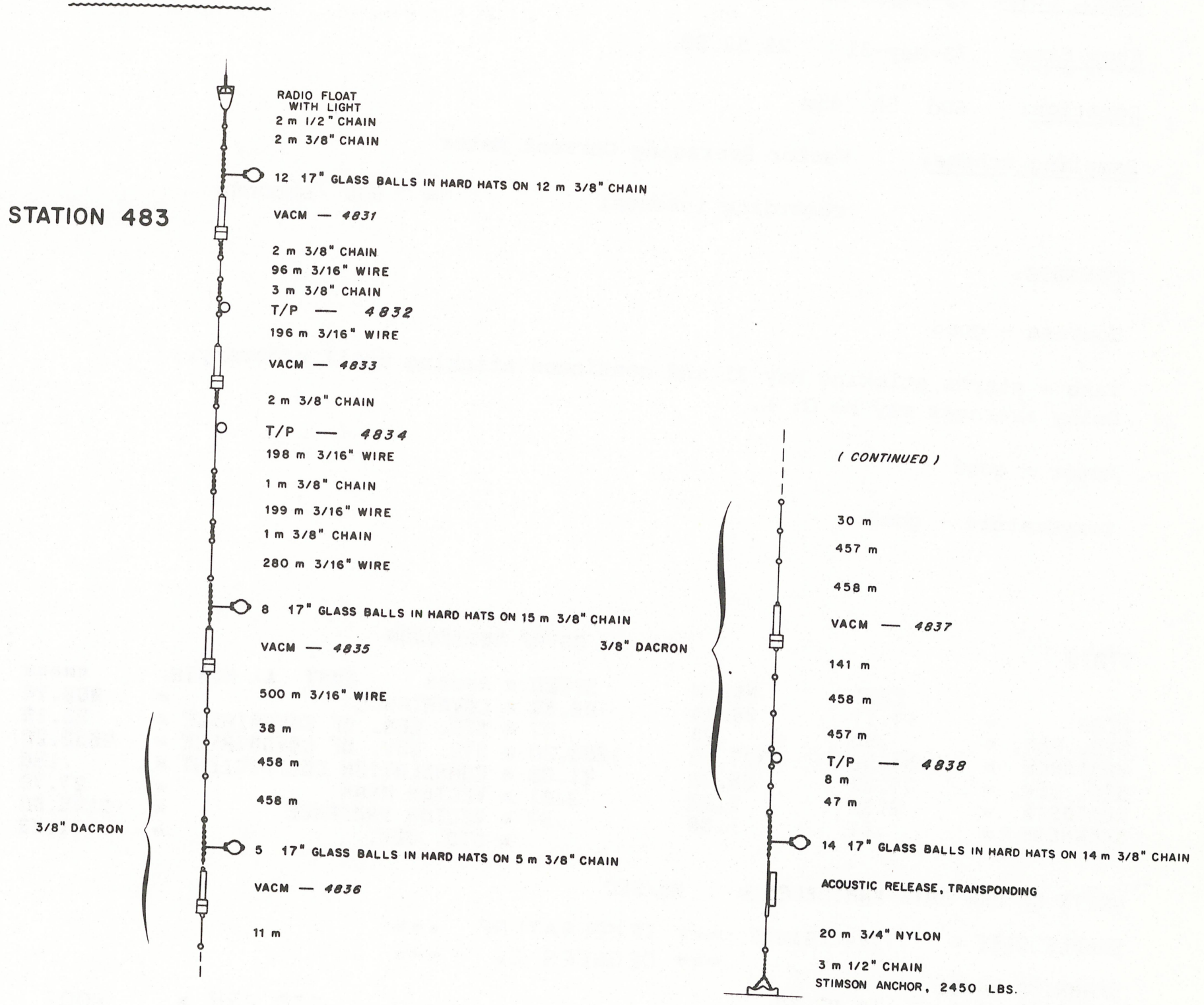


DATA NUMBER 4833

Instrument No.: V-0171

Depth: $748 \mathrm{~m}$

start time: 73-March-12 18.07.30.

Stop time: 73-May-31 23.52.30.

Duration: $60 \mathrm{~d}$ 5h $45 \mathrm{~m}$
Vector Averaging Current Meter recording interval

COMMENTS:

Compass - good Vane - starts sticking May 31 and continues sticking until recovery.
Daily averages may be $\mathrm{O} . \mathrm{K}$.

Rotor - good

Temperature - good

STATS

$\begin{array}{lrr} & \text { EAST } & \text { NOATH } \\ \text { MEAN } & -7.17 & 26.84 \\ \text { STD. EAR. } & .80 & .75 \\ \text { VARIANCE }= & 5964.32 & 4327.48 \\ \text { STD OEV. } & 77.23 & 65.78 \\ \text { KURTOSIS }= & 2.38 & 3.22 \\ \text { SKENNESS } & .11 & -.39\end{array}$

OATA/ $4833 E 9009$

$$
\begin{aligned}
& \text { UNITS OF RAW OATA VARIABLES - MAPSEC }
\end{aligned}
$$

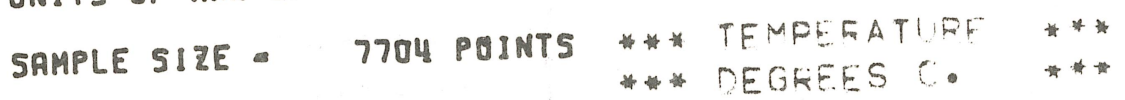

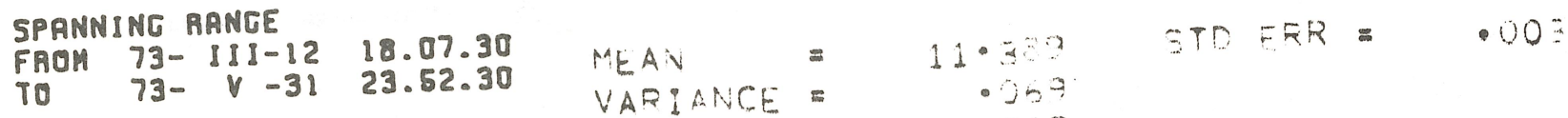

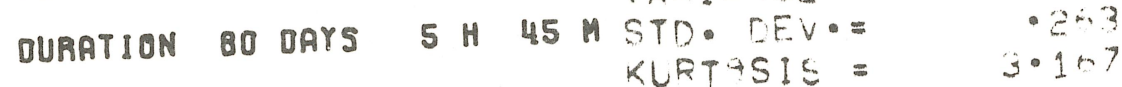

$$
\begin{aligned}
& \text { SKEWIESS }=46 \text { ? } \\
& \text { SAMPLE SILE = } 770: \text { OAIVTS }
\end{aligned}
$$




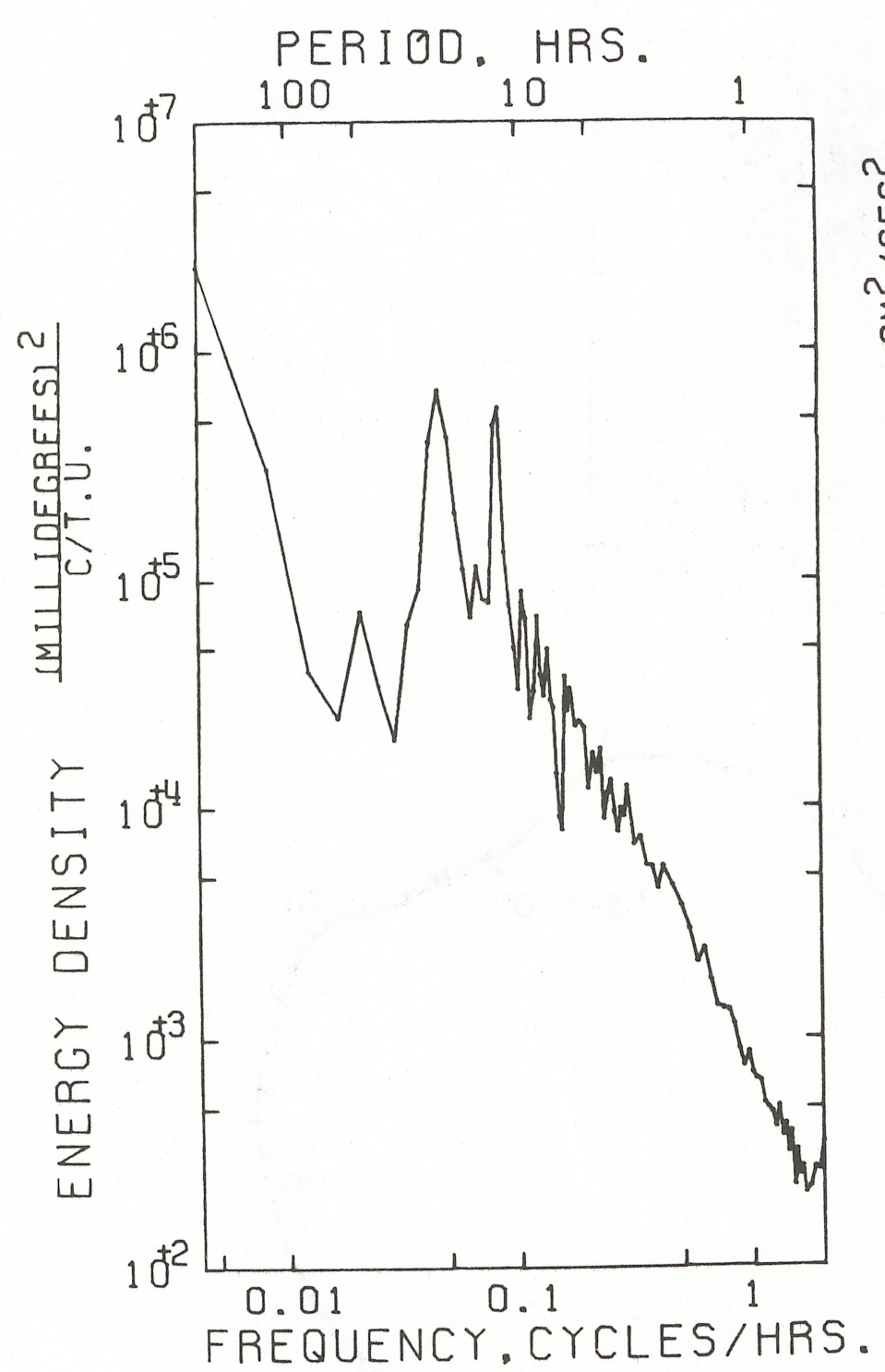

AUTO SPECTRUM

4833 E900 TEMPERATURE 748 METERS

73-III- 12 TO 73-V-31

1 PIECES WITH 3840 ESTIMATES

PER PIECE. AVERAGED OVER

8 ADJACENT FREQUENCY BANDS

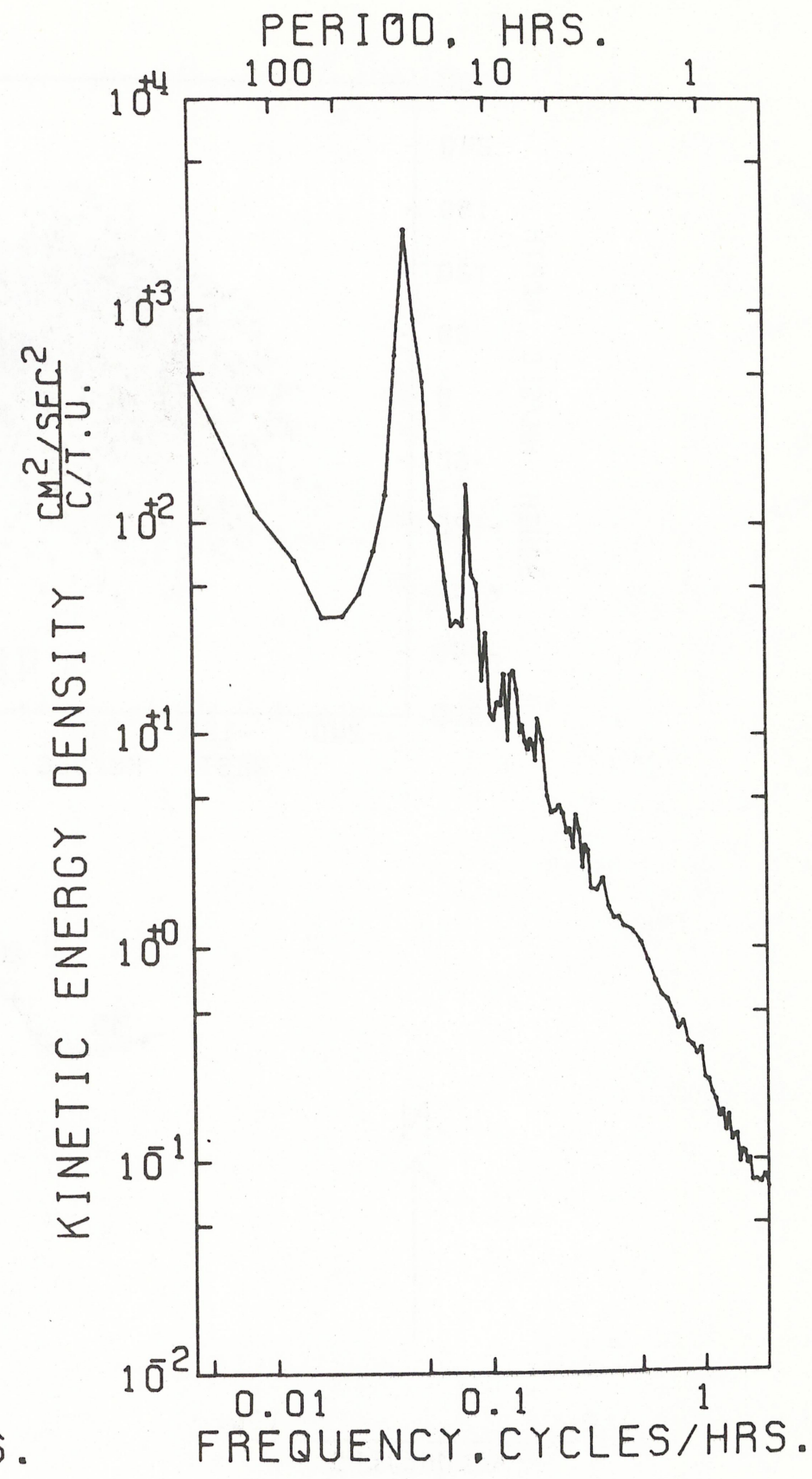

AUTO SPECTRUM

$4833 E 900$ EAST

$4833 E 900$ NORTH

748 METERS

73-I I I- 12 TO 73-V-31

1 PIECES WITH 3840 ESTIMATES

PER PIECE. AVERAGED OVER

8 ADJACENT FREQUENCY BANDS 

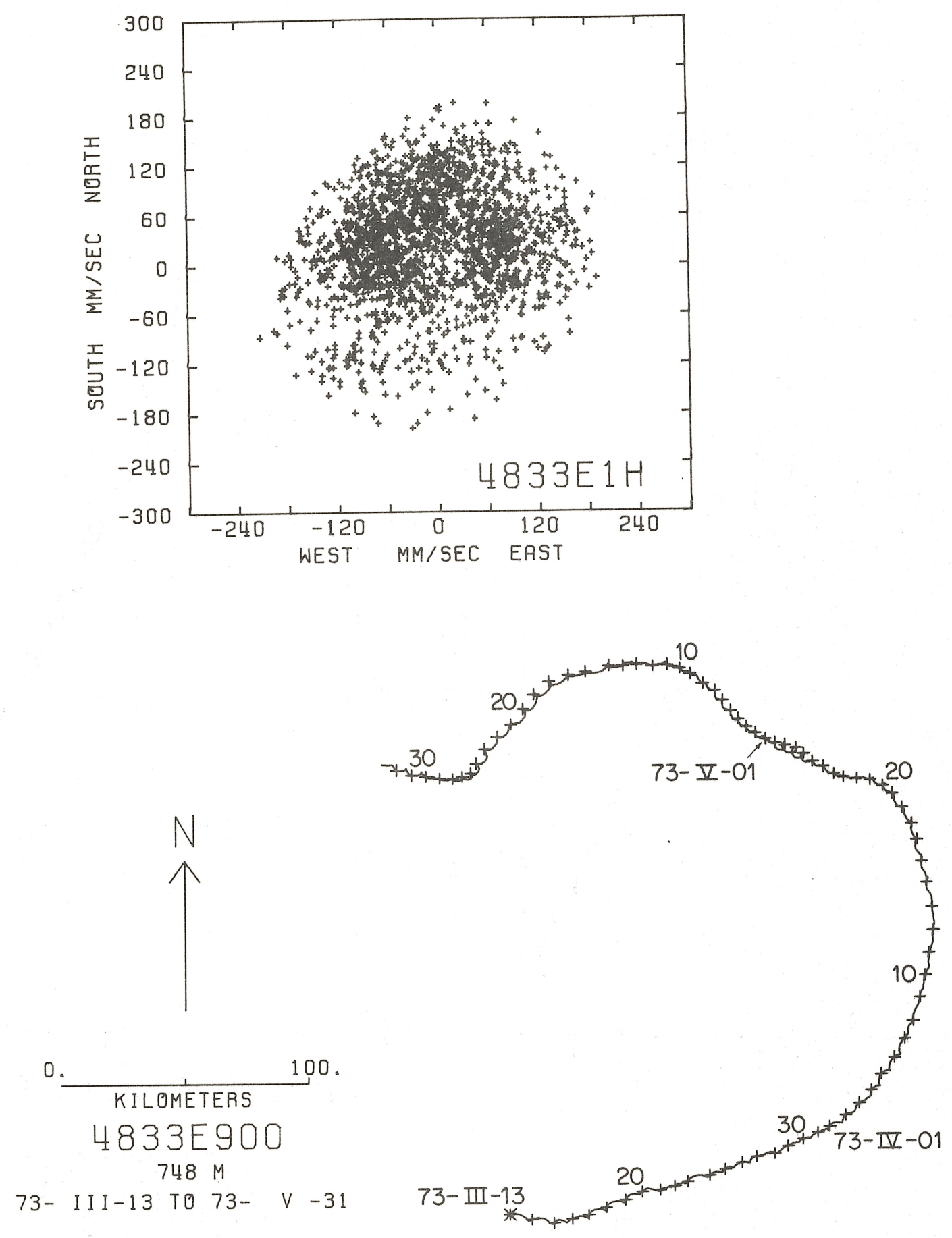

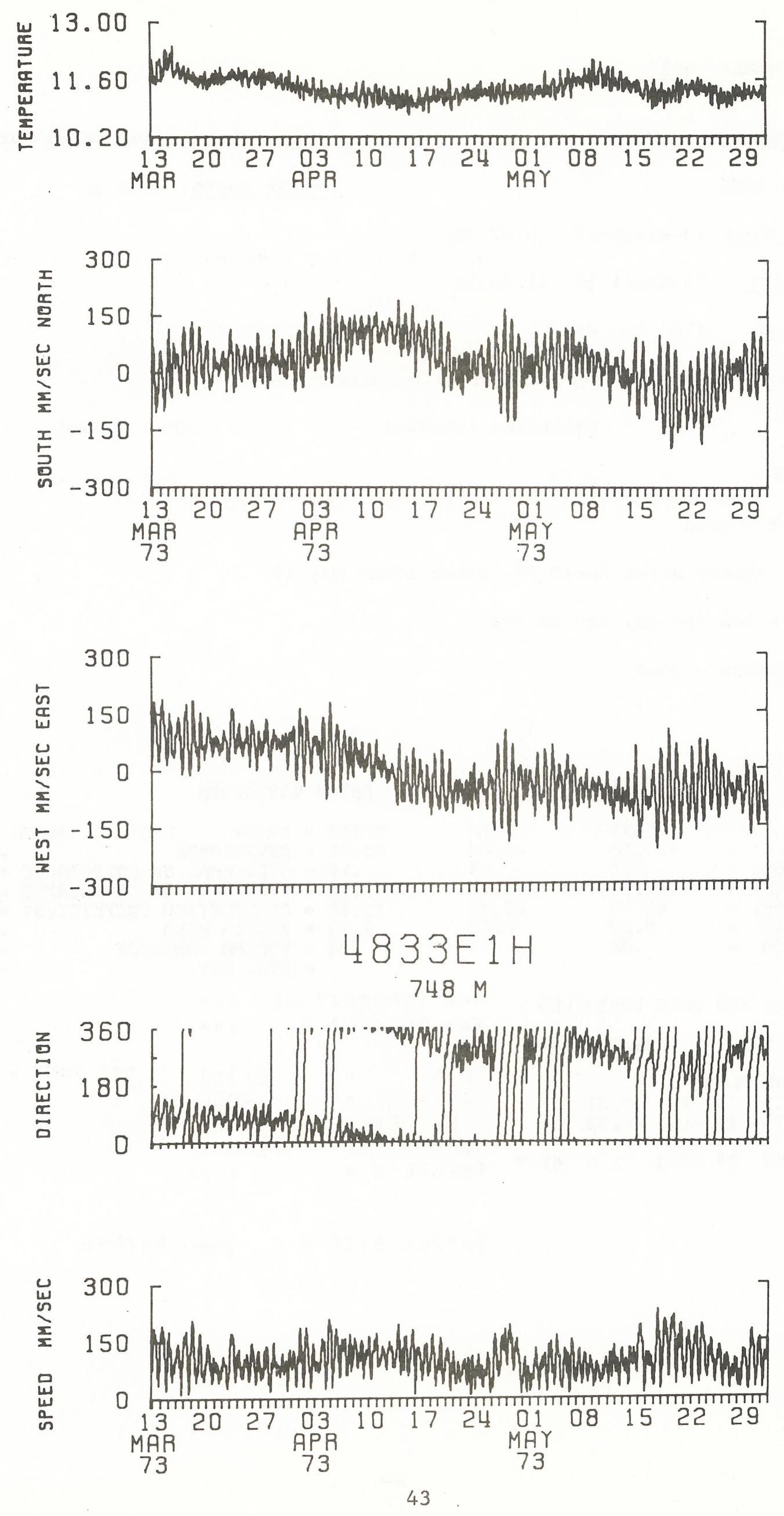
DATA NUMBER 4837

Instrument No.: $\mathrm{V}-0177$

Depth: 3968
Type: Vector Averaging Current Meter Water Depth: $5192 \mathrm{~m}$

Start time: 73-March-13 10.07.30.

Stop time: 73-April-19 11,52.30.

Duration: $\quad 37 \mathrm{~d}$ ih $45 \mathrm{~m}$

Sampling scheme: Vector Averaging Current Meter

$$
\text { recording interval }=900 \text { seconds }
$$

COMMENTS :

Compass - good

Vane - sticky after April 20, stuck after May 19

Rotor - low speeds, may be real

Temperature - good

รTคTร

DATPR 4Es7ag00A

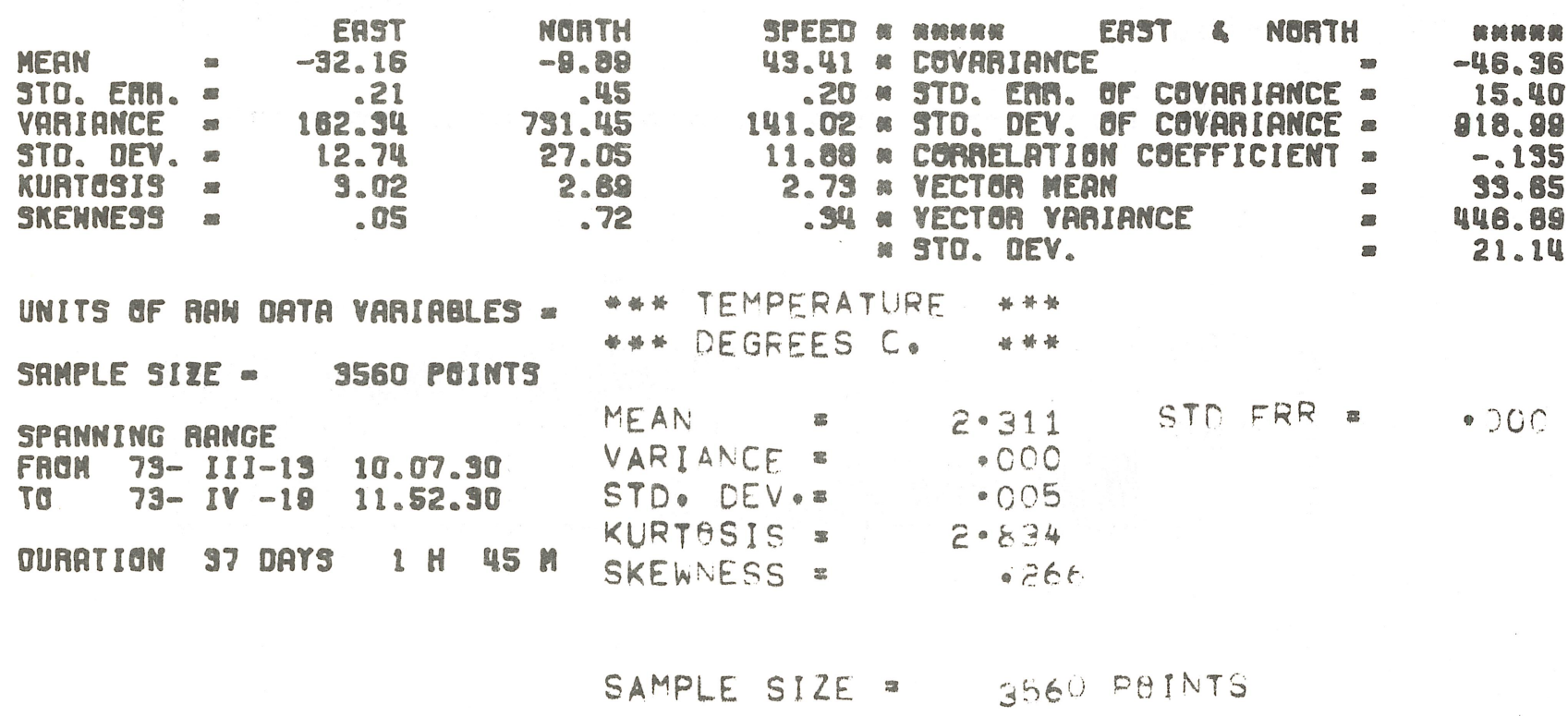



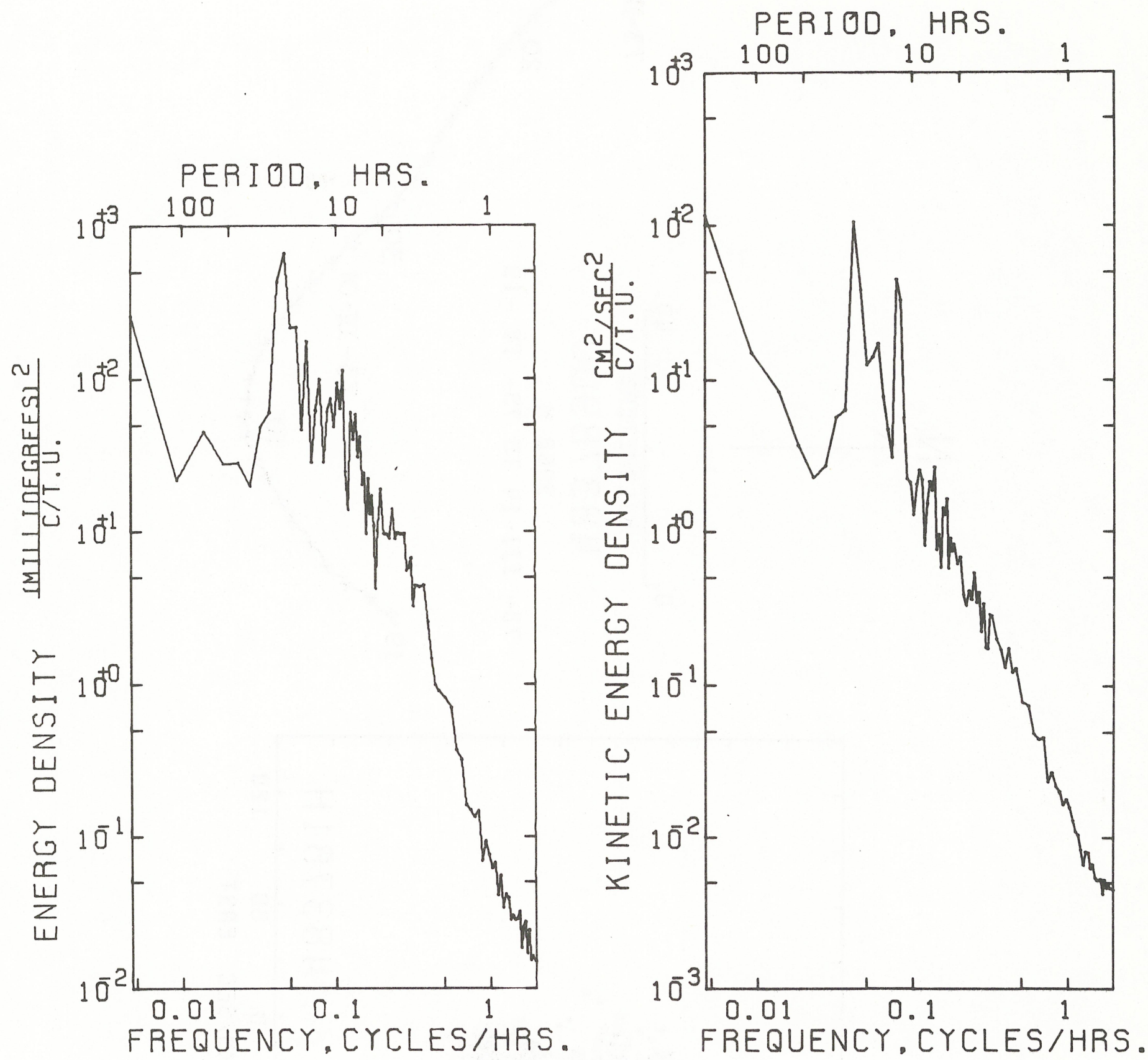

AUTO SPECTRUM

48378900 TEMPERATURE 3968 METERS

73-III-13 TO 73-IV-18

1 PIECES WITH 1728 ESTIMATES

PER PIECE. AVERAGED OVER

4 ADJACENT FREQUENCY BANDS

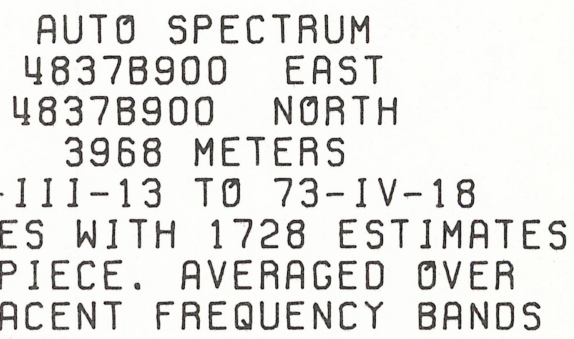



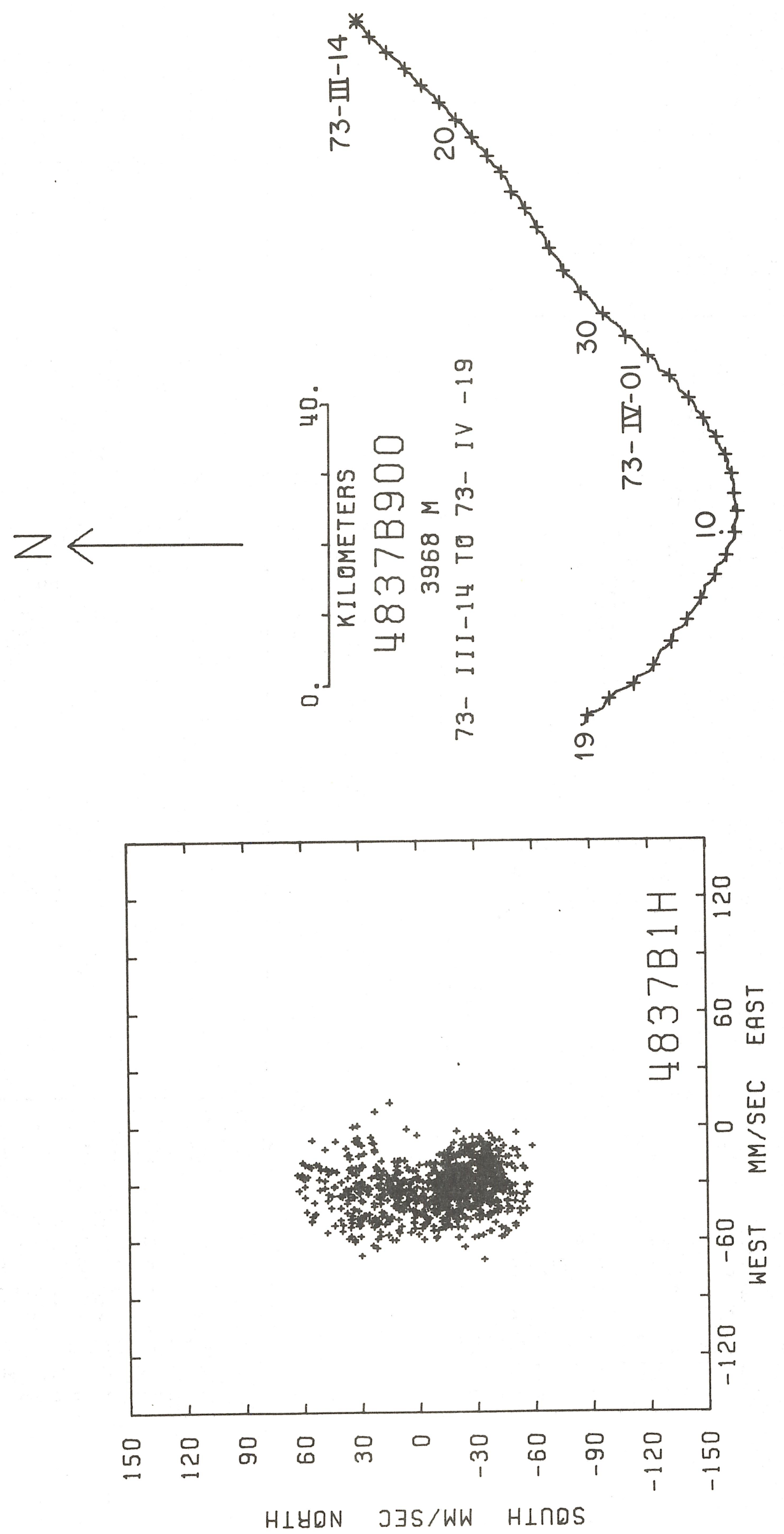

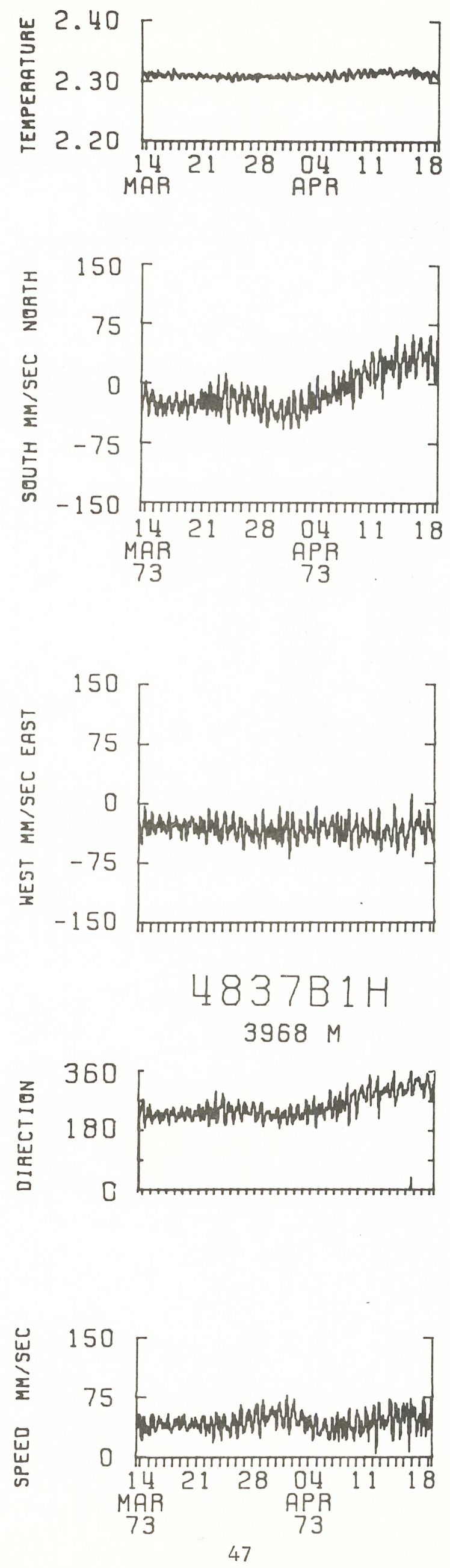
Mooring No. 484

Set $\frac{1973 \text { Mar } \quad 13}{\text { Year Month Day }}$

$27^{\circ} 25.1^{\prime \prime} \mathrm{N}$

$67^{\circ} 59.5^{\prime} \mathrm{W}$

Latitude

Longitude

Set by J. Gifford - R. Heinmiller

Ship R.V. CHAIN

Cruise 112 Leg 1

Retrieved $\frac{1973 \text { July }}{3}$

Retrieved by G. Tupper - R. Heinmiller

Ship

R.V. CHAIN

Cruise 112 Leg 6

Purpose of Mooring: Mooring \#16 of MODE 1 array

Mooring Type: Subsurface mooring

\begin{tabular}{|c|c|c|c|c|}
\hline Key & $\begin{array}{c}\text { Data } \\
\text { Number }\end{array}$ & $\begin{array}{c}\text { Instrument } \\
\text { Number }\end{array}$ & Type & $\begin{array}{l}\text { Depth } \\
\text { Meters }\end{array}$ \\
\hline * & 4841 & $V-0108$ & VACM & 441 \\
\hline$\#$ & 4842 & $\# 50$ & $\mathrm{~T} / \mathrm{P}$ & 543 \\
\hline * & 4843 & $V-0175$ & VACM & 741 \\
\hline$\#$ & 4844 & $\# 45$ & $\mathrm{~T} / \mathrm{P}$ & 744 \\
\hline & 4845 & $V-0114$ & VACM & 1443 \\
\hline+ & 4846 & $\mathrm{~V}-0181$ & VACM & 2953 \\
\hline * & 4847 & $V-0185$ & VACM & 3923 \\
\hline
\end{tabular}

Comments

$M . I . T$.

$U \cdot R \cdot I$.

M.I.T.

No recoverable data

COMMENTS ON MOORING: 


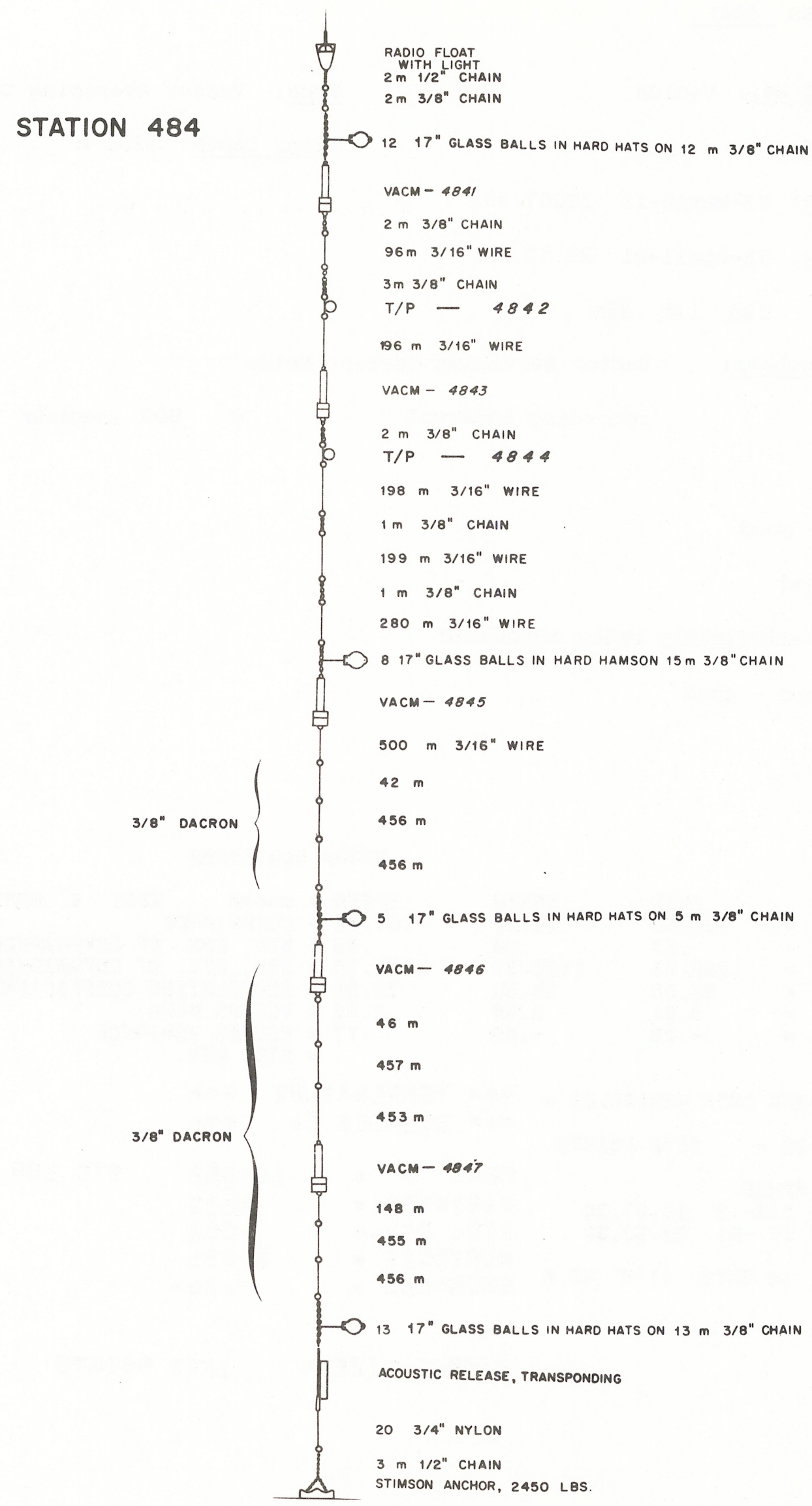


DATA NUMBER 4841

Instrument No.: V-0108

Depth: $441 \mathrm{~m}$
Type: Vector Averaging Current Meter Water Depth: $5151 \mathrm{~m}$

Start time: 73-March-13 12.07.30.

Stop time: 73-April-01 23.52.30.

Duration: $19 \mathrm{~d}$ 11h $45 \mathrm{~m}$

Sampling scheme: Vector Averaging Current Meter recording interval $=900$ seconds

COMMENTS :

Compass - good

Vane - good

Rotor - periodically below threshold

Temperature - good

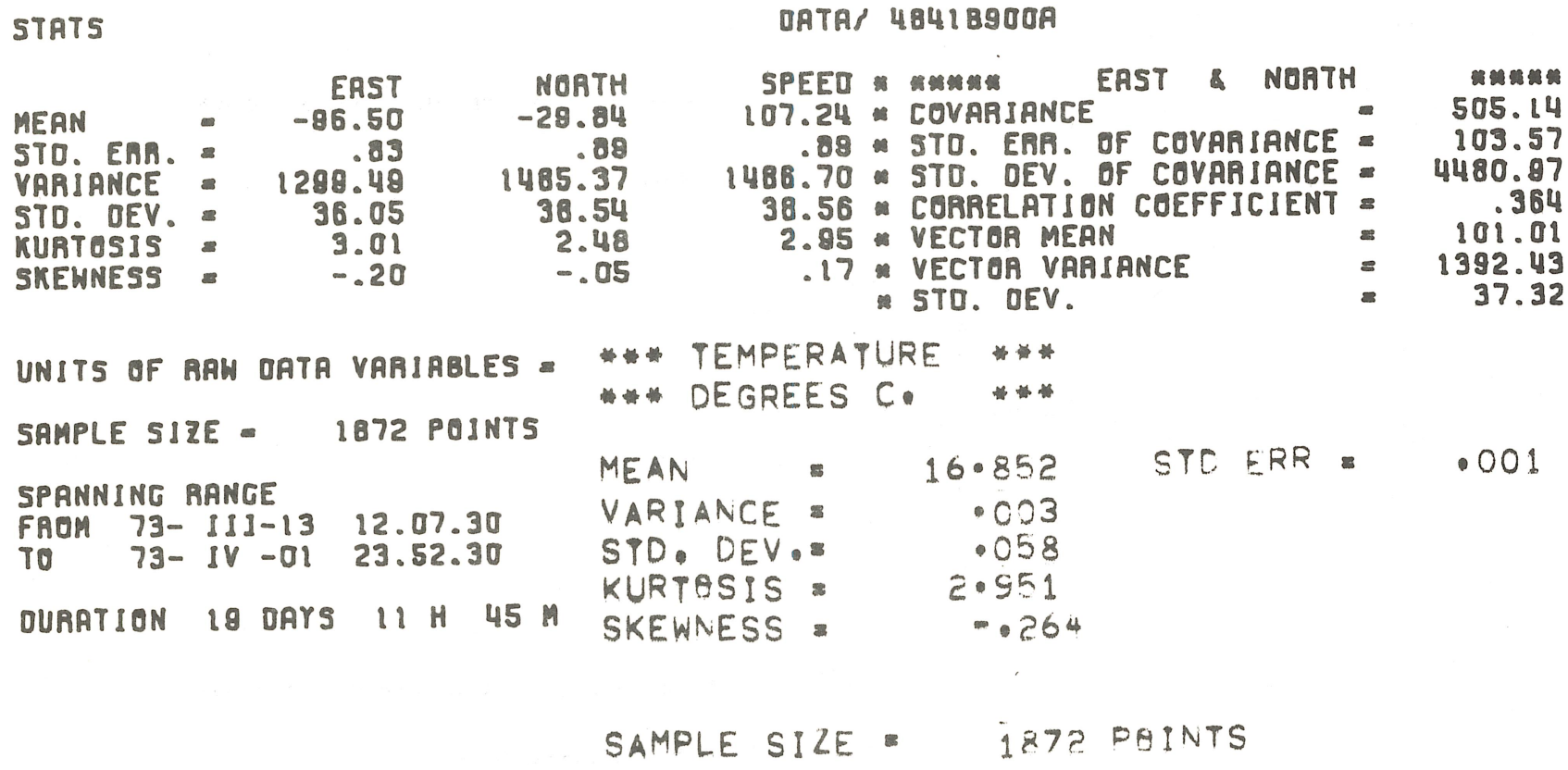



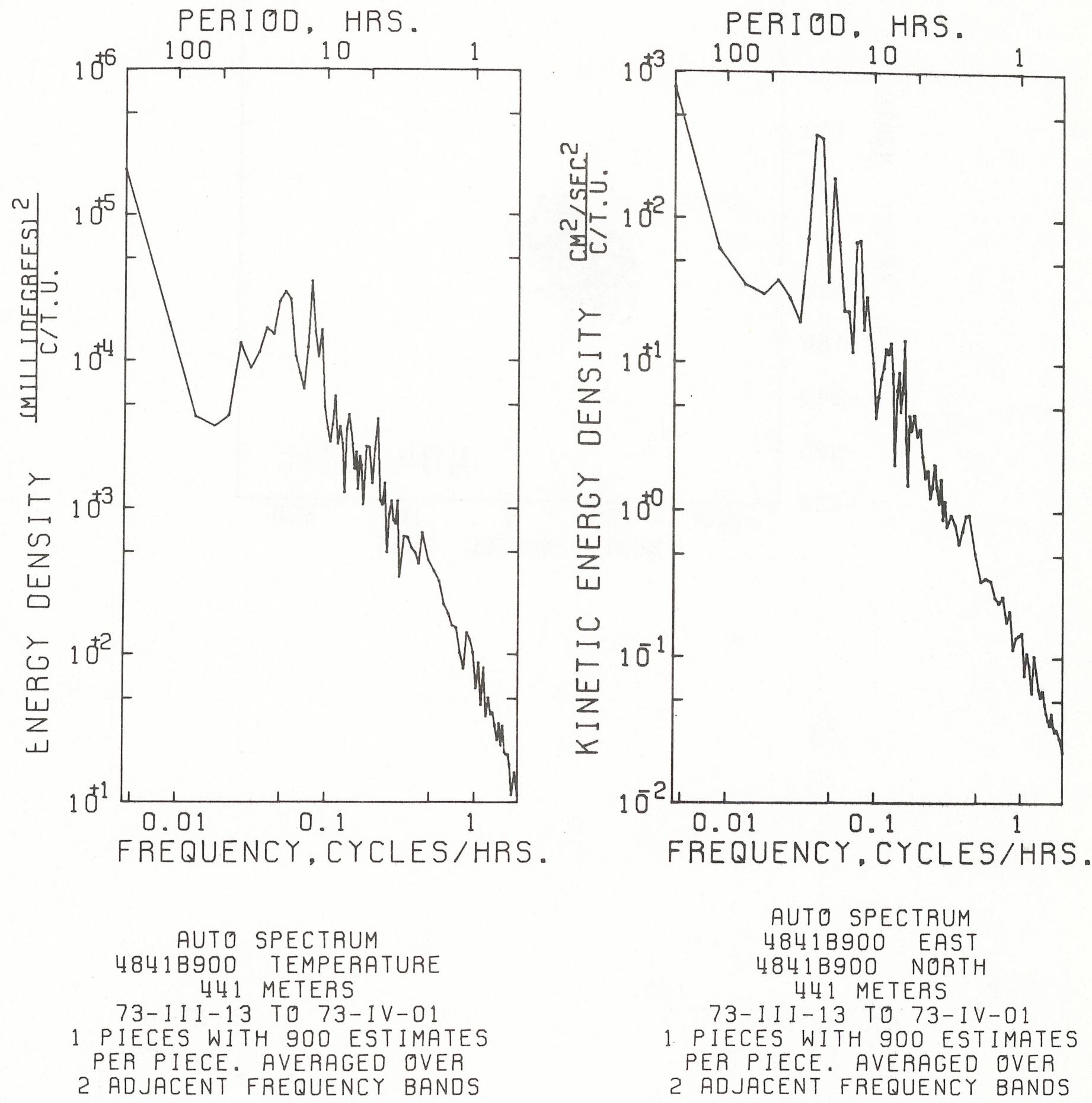

73-III-13 TO 73-IV-01

1. PIECES WITH 900 ESTIMATES

PER PIECE. AVERAGED OVER

2 ADJACENT FREQUENCY BANDS 

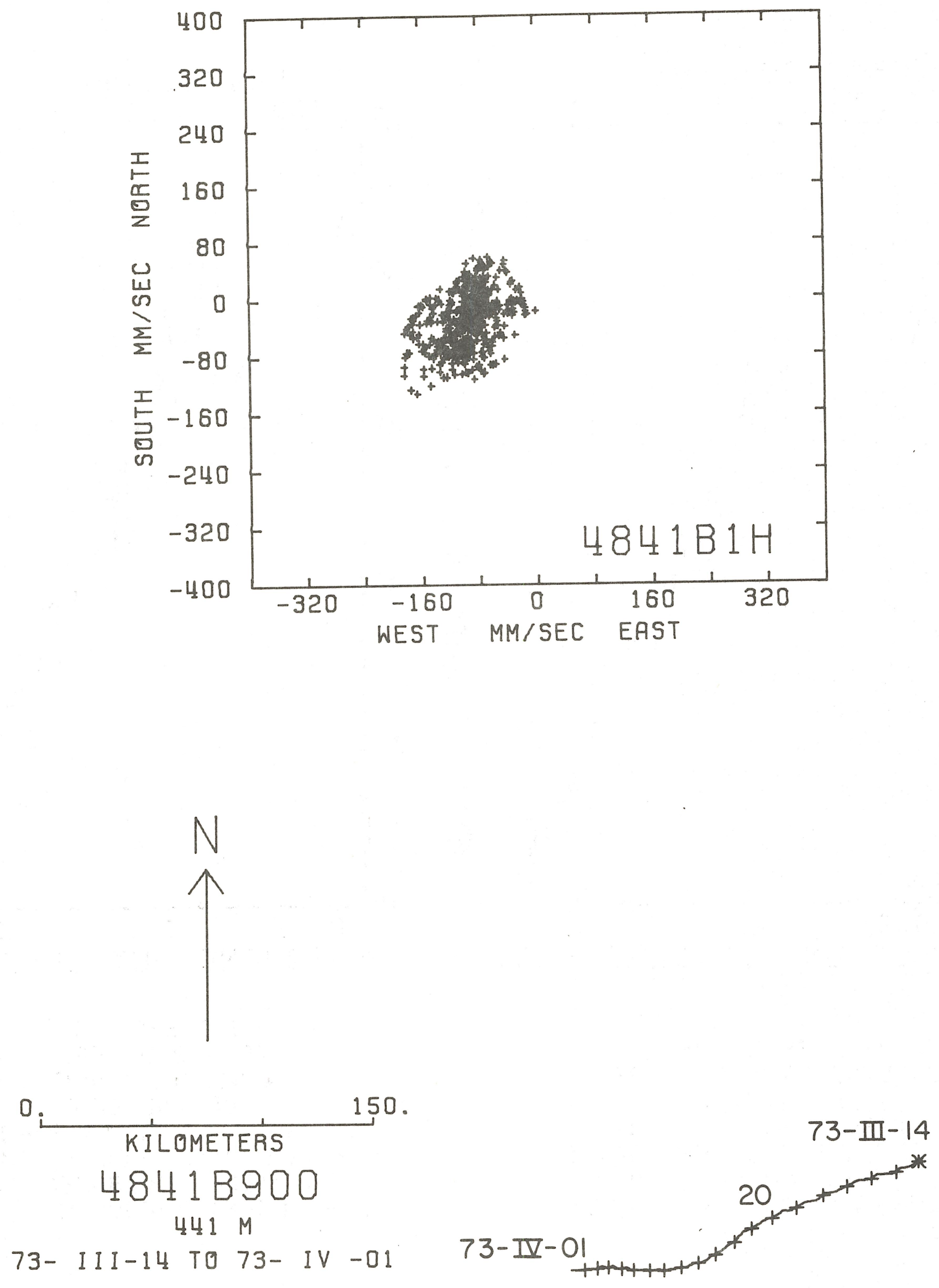

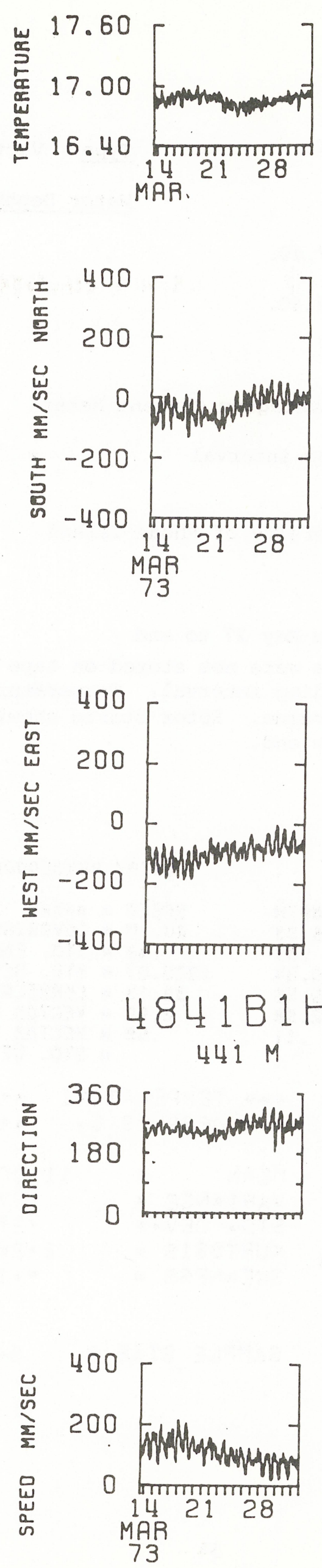
DATA NUMBER 4843

Instrument No.: V-0175

Depth: $741 \mathrm{~m}$
Type: Vector Averaging Current Meter Water Depth: $5151 \mathrm{~m}$

Start time: 73-March-13 16.07.40.

Stop time: 73-May-09 17.52.40.

Duration: $57 \mathrm{~d}$ ih $45 \mathrm{~m}$

Sampling scheme: Vector Averaging Current Meter

recording interval $=900$ seconds

COMMENTS:

Instrument owned by the University of Rhode Island

Compass - good

Vane - stuck or sticking from May 27 to end

Rotor - Compass and vane data were not stored on tape unless there was a rotor count during the recording interval. Temperature values were stored on tape regardless of rotor value. Rotor starts showing threshold values

(0 rotor count) from May 9 to end.

Temperature - good

STATS

OATA/ $4843 E 900$ A

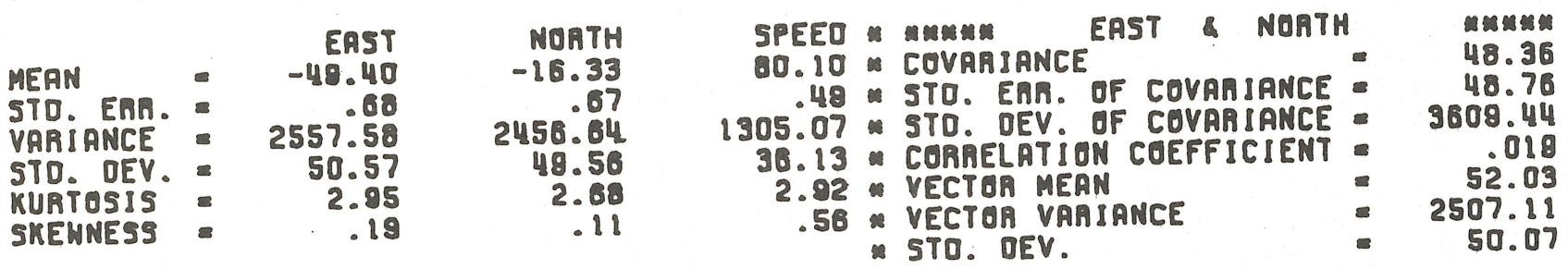

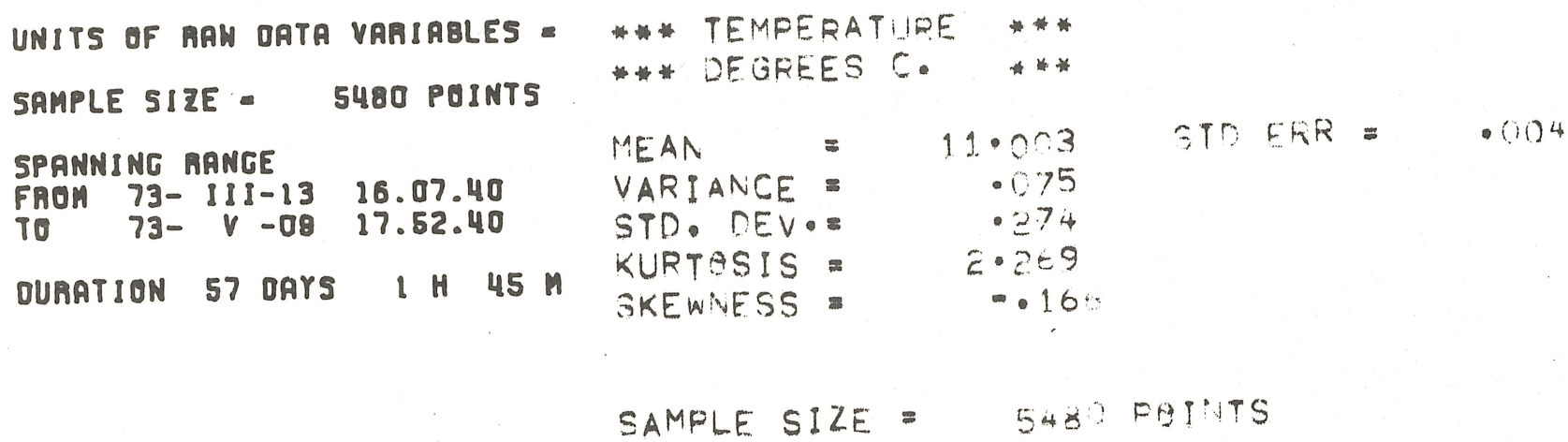




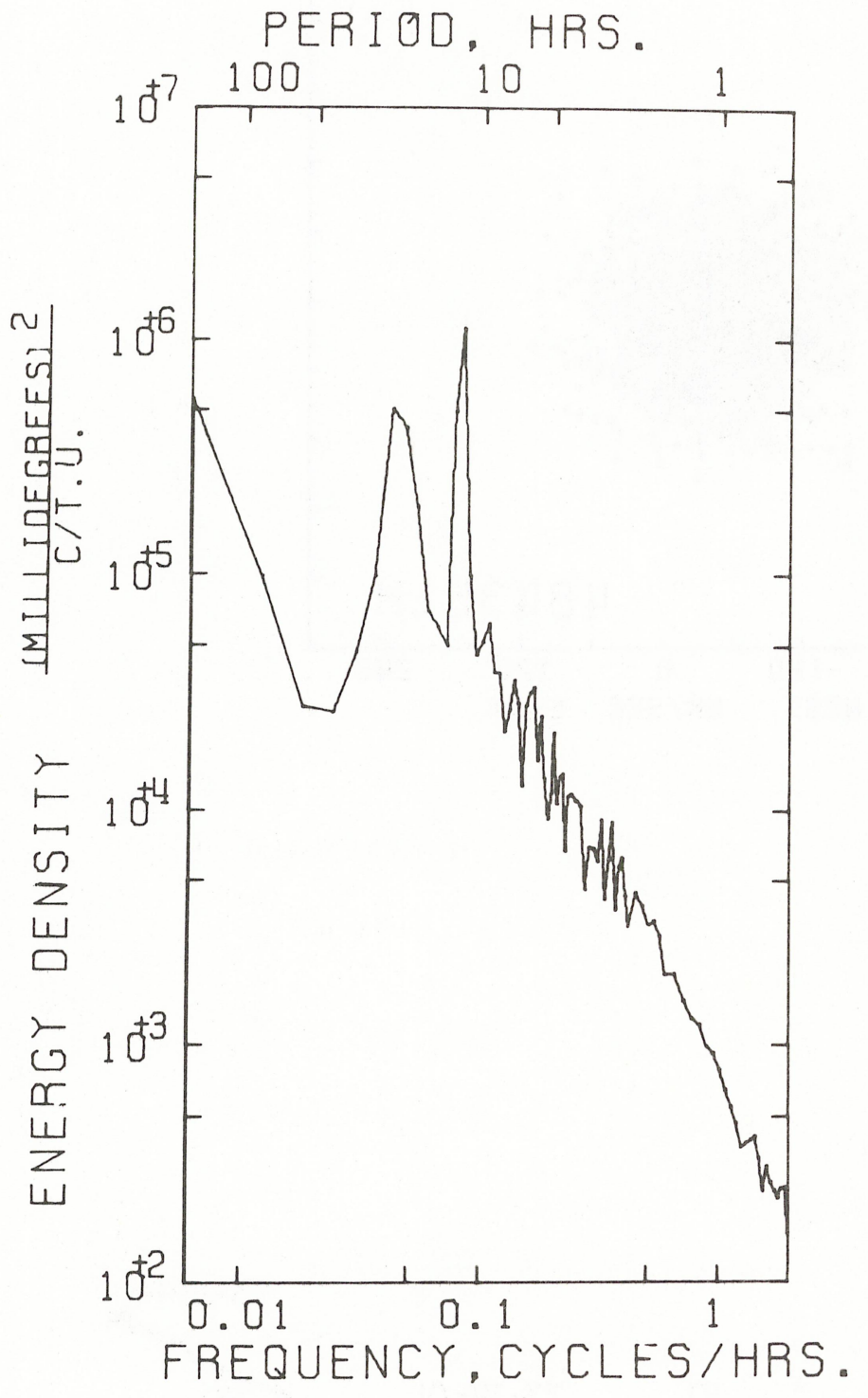

AUTO SPECTRUM

$4843 E 900$ TEMPERATURE

741 METERS

73-I I I-13 TO 73-V-08

1 PIECES WITH 2700 ESTIMATES

PER PIECE. AVERAGED OVER

8 ADJACENT FREQUENCY BANDS
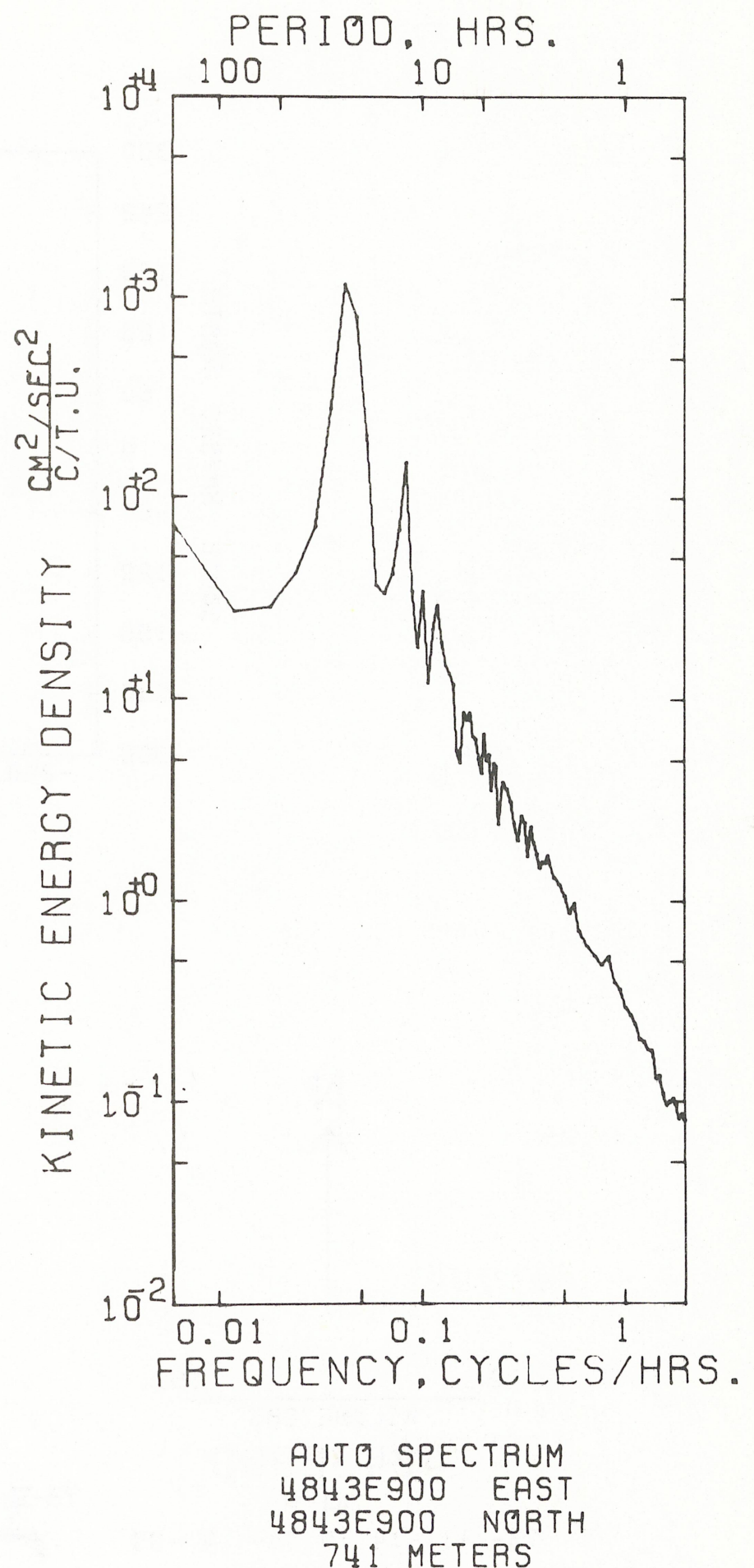

73-III-13 TO 73-V-08

1 PIECES WITH 2700 ESTIMATES

PER PIECE. AVERAGED OVER

8 ADJACENT FREQUENCY BANDS 

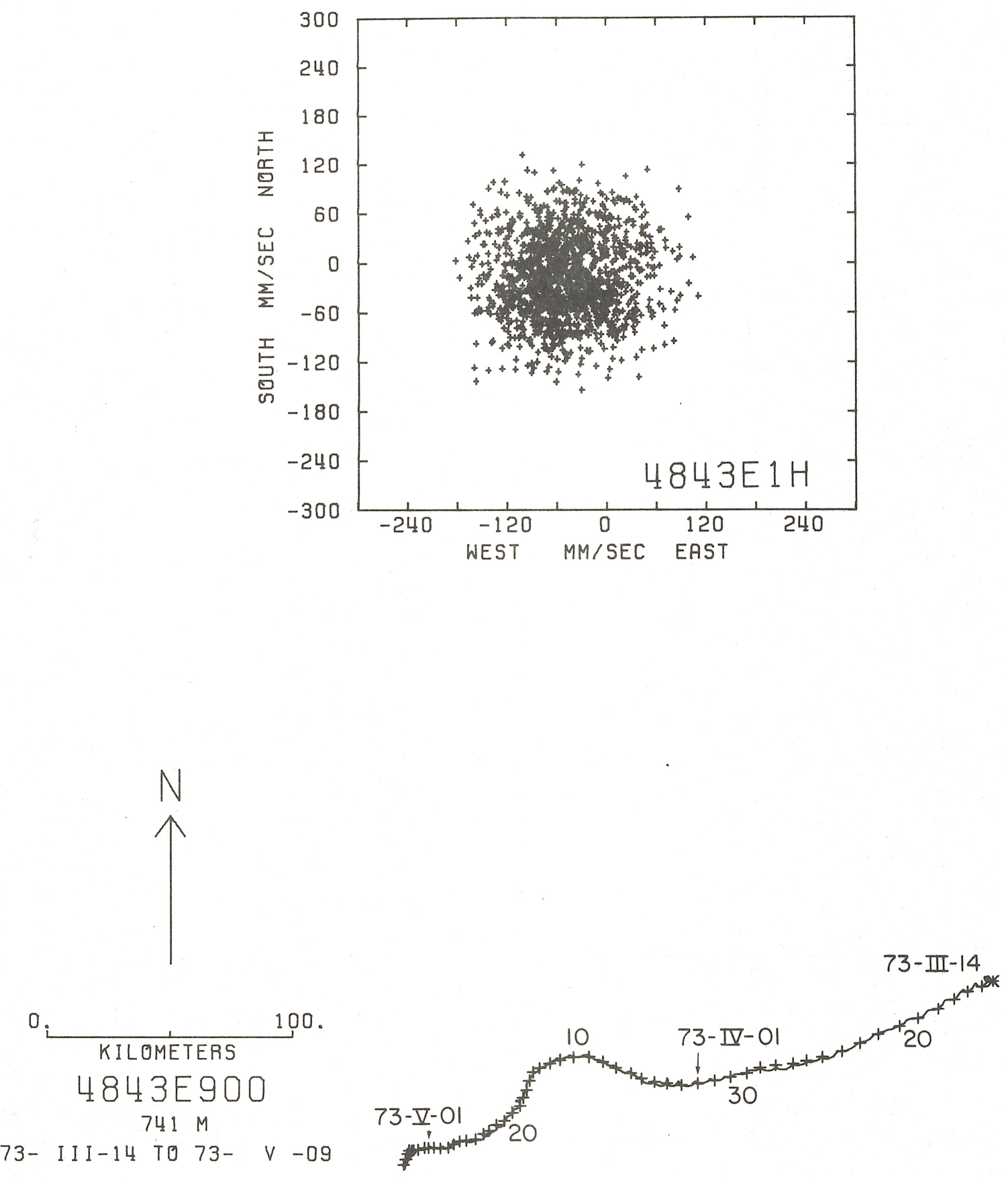

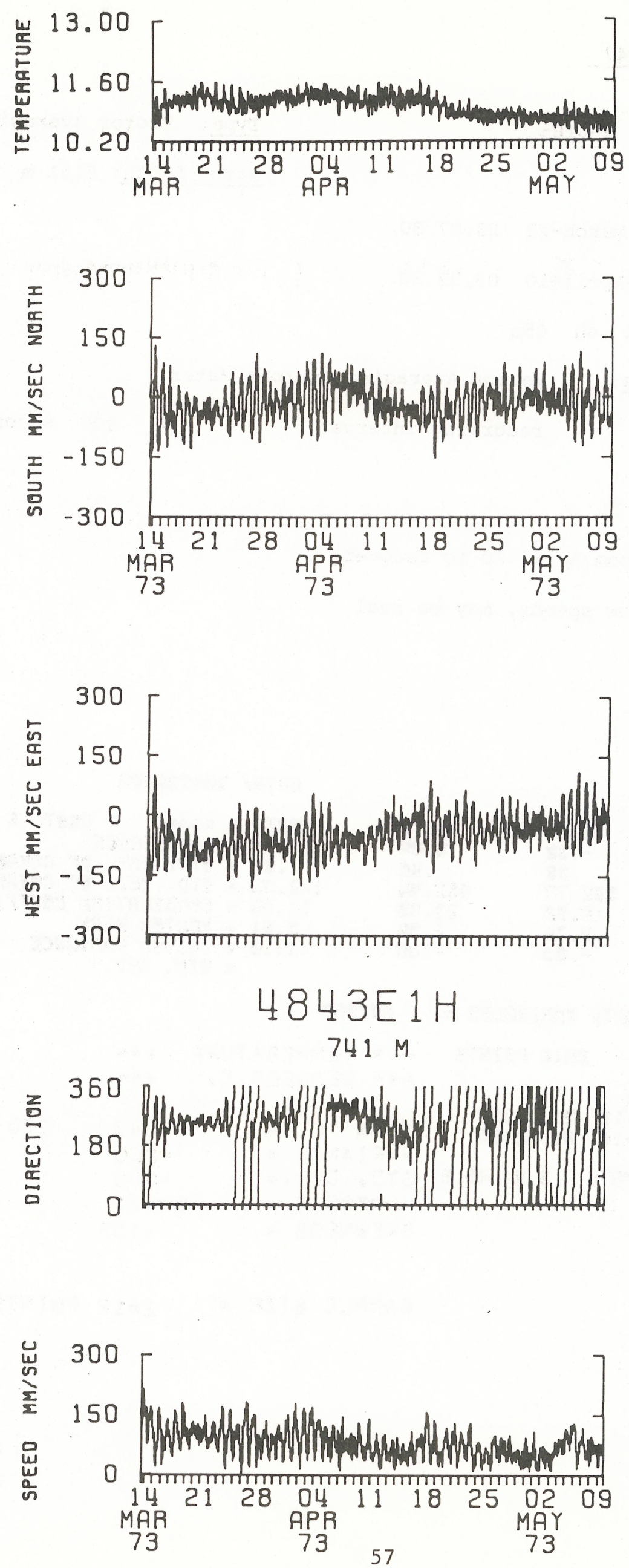
DATA NUMBER 4847

Instrument No.: V-0185

Depth: $3973 \mathrm{~m}$

Start time: 73-March-13 23,07.30.

Stop time: 73-April-10 03.52.30.

Duration: $27 \mathrm{~d}$ 4h $45 \mathrm{~m}$

Sampling scheme:

Vector Averaging Current Meter

recording interval

$=900$ seconds

COMMENTS:

Compass - good

Vane - stuck from April 10 to recovery

Rotor - very low speeds, may be real
Type: Vector Averaging Current Meter Water Depth: $5151 \mathrm{~m}$

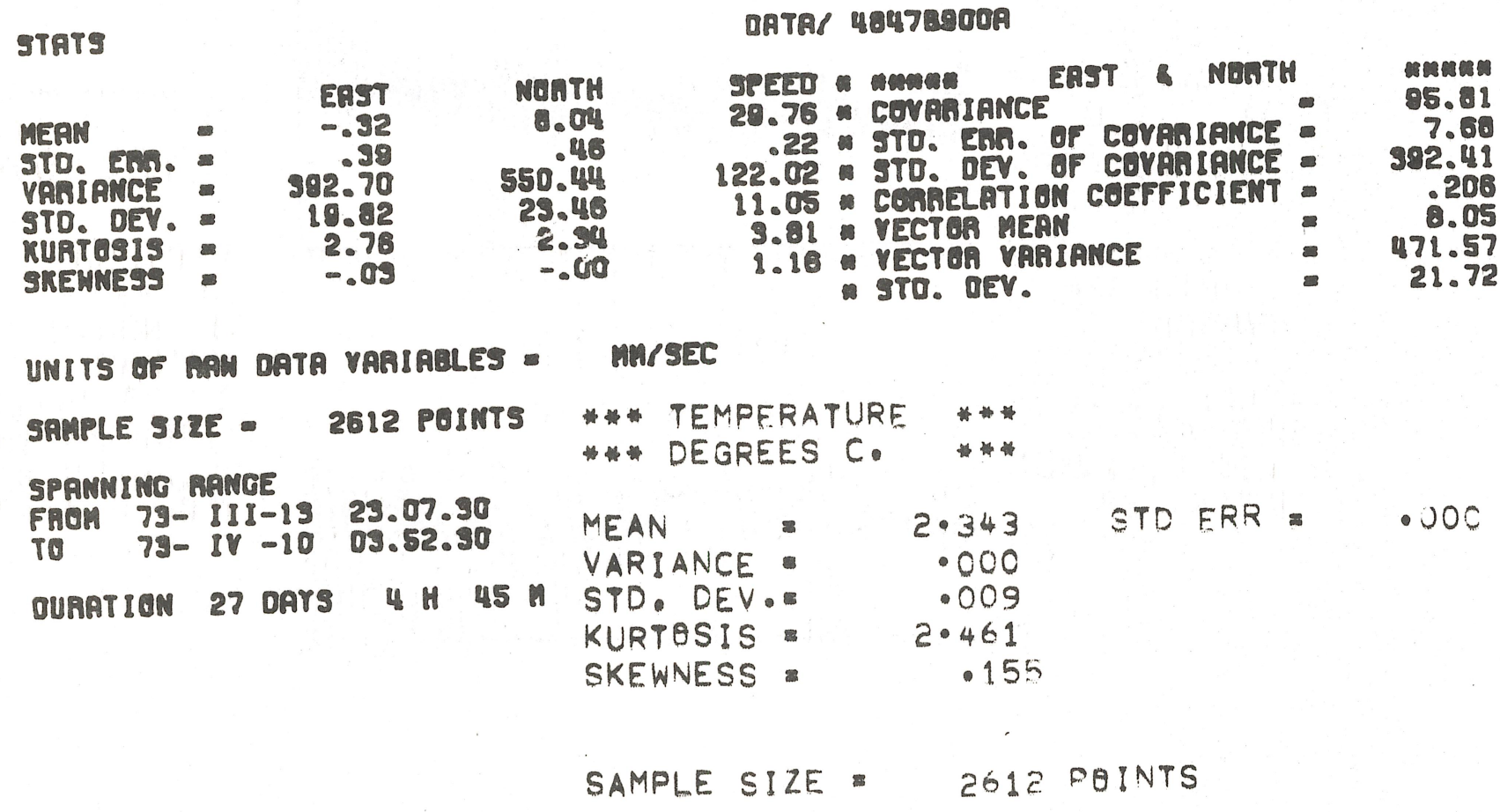



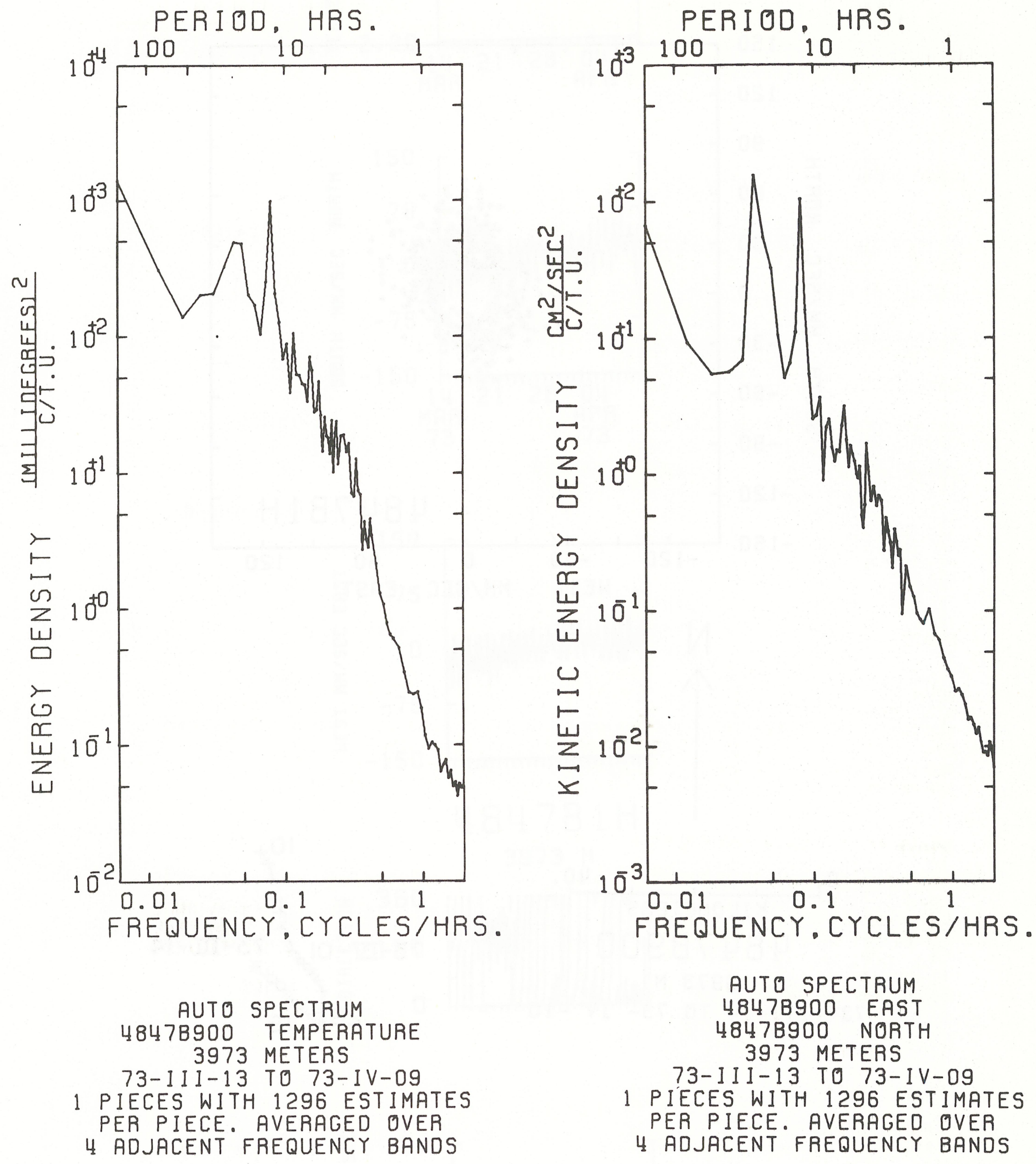

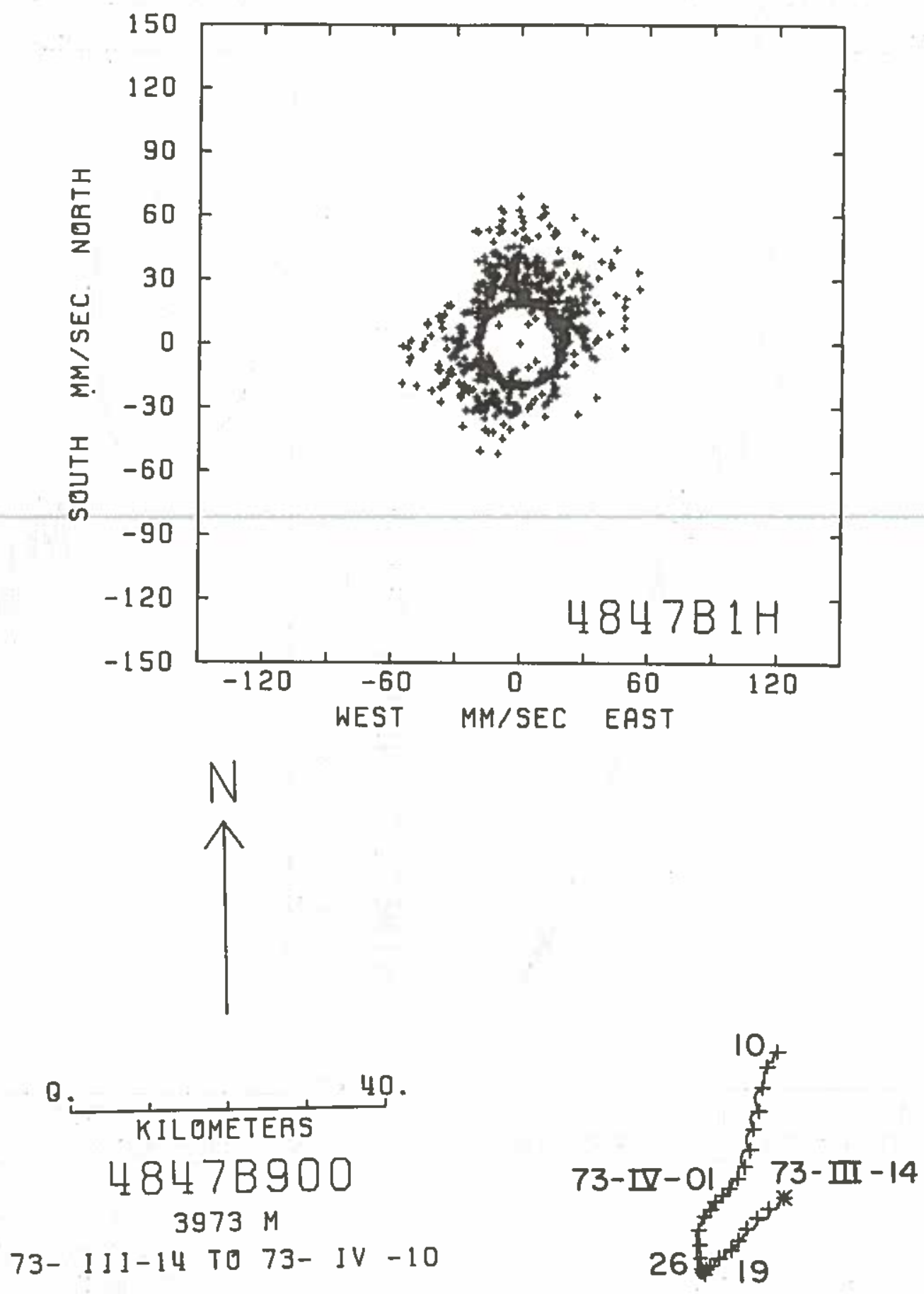

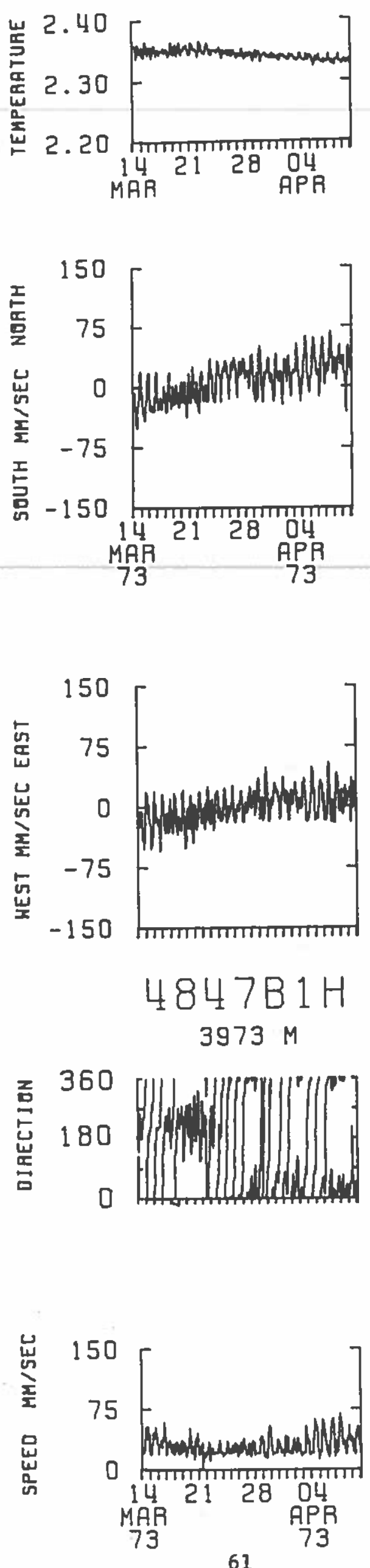
Mooring No. 485

Set $\frac{1973 \text { Mar } 13}{\text { Year Month Day }}$

$26^{\circ} 23.8^{\prime} \mathrm{N}$

$69^{\circ} 21.0^{\prime} \mathrm{W}$

Latitude

Longitude

Set by G. Tupper - R. Heinmiller

Ship R.V. CHAIN

Cruise 112 Leg 1

Retrieved $\frac{1973 \text { July }}{\text { Year Month Day }}$

Retrieved by J. Gifford - R. Heinmiller

Ship R.V. CHAIN

Cruise 112 Ieg 6

Purpose of Mooring: Mooring \#ll of MODE 1 array

Mooring Type: Subsurface mooring

\begin{tabular}{|c|c|c|c|c|c|}
\hline Key & $\begin{array}{l}\text { Data } \\
\text { Number }\end{array}$ & $\begin{array}{c}\text { Instrument } \\
\text { Number }\end{array}$ & Type & $\begin{array}{l}\text { Depth } \\
\text { Meters }\end{array}$ & Comments \\
\hline * & 4851 & $V-078$ & VACM & 421 & \\
\hline$\#$ & 4852 & \#39 & $\mathrm{T} / \mathrm{P}$ & 520 & M.I.T. \\
\hline \multirow[t]{2}{*}{ * } & 4853 & $V-0155$ & VACM & 723 & I.O.S. \\
\hline & 4854 & \#57 & $\mathrm{T} / \mathrm{P}$ & 928 & M.I.T., flooded \\
\hline \# & 4855 & \#60 & $\mathrm{T} / \mathrm{P}$ & 1133 & $M \cdot I . T$. \\
\hline * & 4856 & $V-0139$ & VACM & 1426 & \\
\hline \# & 4857 & \#20 & $\mathrm{T} / \mathrm{P}$ & 1926 & $M \cdot I . T$. \\
\hline \multirow[t]{2}{*}{ \# } & 4858 & \#19 & $\mathrm{T} / \mathrm{P}$ & 2442 & $M . I . T$. \\
\hline & 4859 & $V-0136$ & VACM & 2943 & Installation error, no data \\
\hline+ & 485,10 & \#27 & $\mathrm{T} / \mathrm{P}$ & 3981 & M.I.T. \\
\hline \# & 485,11 & \#32 & $\mathrm{T} / \mathrm{P}$ & 4387 & $M . I . T$. \\
\hline \multirow[t]{3}{*}{+} & 485,12 & \#10 & $\mathrm{T} / \mathrm{P}$ & 5305 & M.I.T. \\
\hline & 485,13 & $\mathrm{H}-871$ & Film & 5309 & Nova University \\
\hline & Water & th & & 5420 & \\
\hline
\end{tabular}

COMMENTS ON MOORING: 

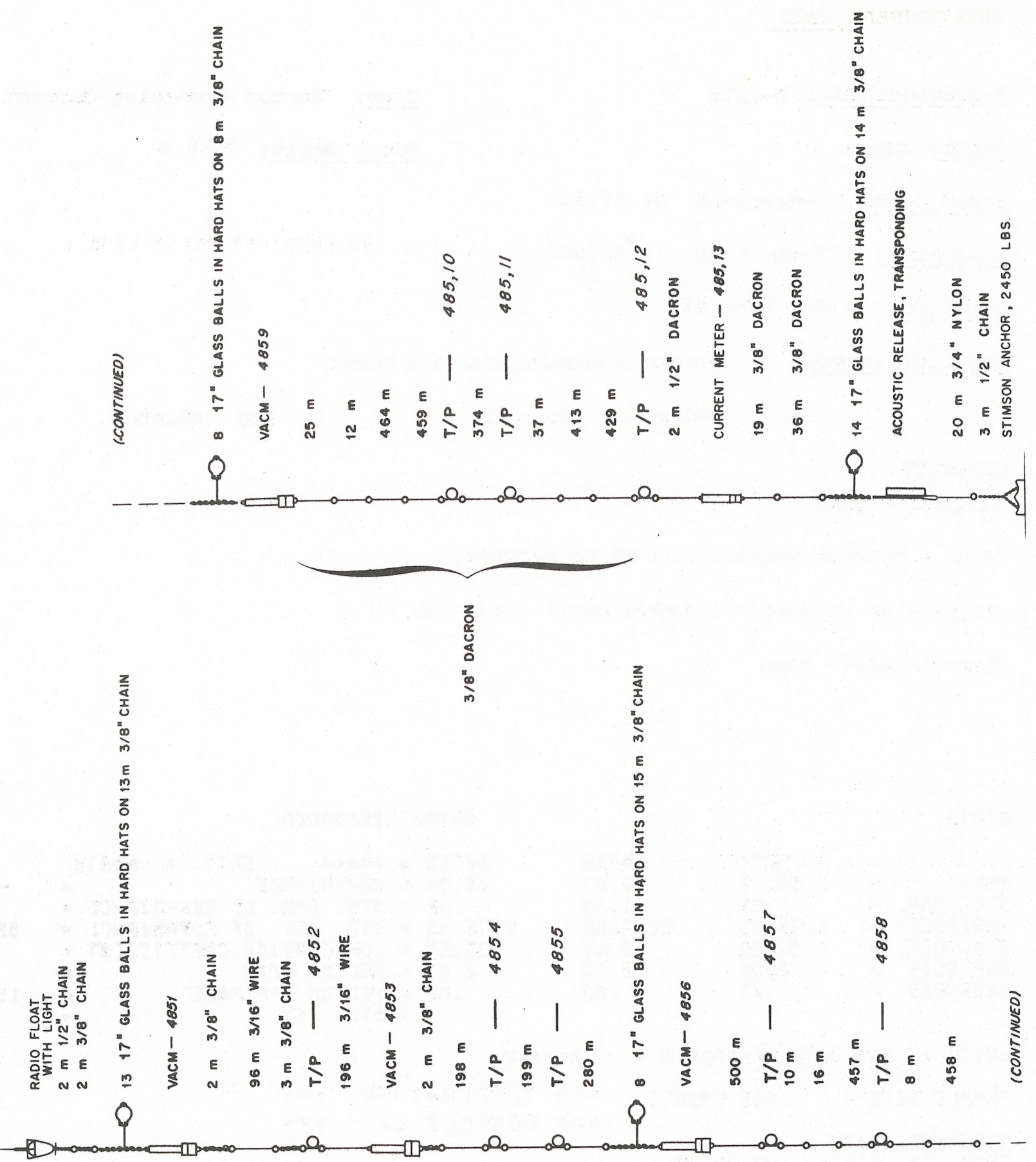

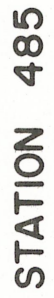


DATA NUMBER 4851

Instrument No.: $\mathrm{V}-0178$

Depth: $421 \mathrm{~m}$
Type: Vector Averaging Current Meter Water Depth: $5420 \mathrm{~m}$

Start time: 73-March-14 04.07.30.

Stop time: $73-\mathrm{March}-30 \quad 18.52 .30$.

Duration: $\quad 16 \mathrm{~d}$ 14h $45 \mathrm{~m}$

Sampling scheme: Vector Averaging Current Meter

$$
\text { recording interval }=900 \text { seconds }
$$

COMMENTS :

Compass - good

Vane - becomes stuck March 30 to recovery

Rotor - at threshold between April 1 and May 30

Temperature - good

5TATS

\section{OATAR 405109009}

$\begin{array}{lrr} & \text { EAST } & \text { NORTH } \\ \text { MEAN } & 83.10 & -2.67 \\ \text { STD. EAR. } & .06 & 1.14 \\ \text { VARIANCE }= & 1173.20 & 2062.02 \\ \text { STO. DEV. } & 34.25 & 45.41 \\ \text { KURTOSIS }= & 2.59 & 2.74 \\ \text { SKENNESS }= & .20 & .62\end{array}$

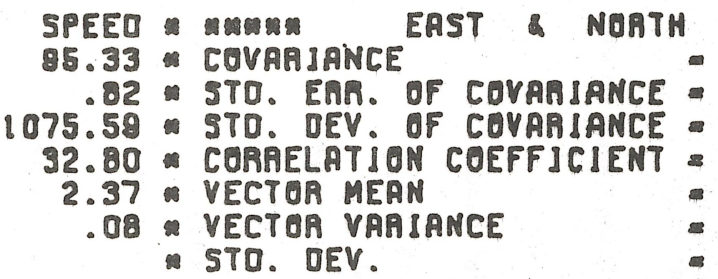

ตัตกต

$-10.58$

98. 47

3833.75

$-.007$

83.23

1617.61

40.22

UNITS OF RAN DATA VARIABLES \& MMSEC

SAMPLE SIZE - 1596 POJNTS * TEMPERATURE

SPANNING RANGE

FROM 73- \$I-14 04.07.30 MEAN $\quad$ 16.967 STOERR

T0 73- $\$ I-30 \quad 18.52 .30$

VARIANCE: $: 005$

DURATION 26 DAYS $24 \mathrm{H} 45 \mathrm{H}$ STD. DEV . 069

KURTOSIS: $\quad 2.547$

SKEWNESS : $\cdot 292$

SAMPLE SIZE $=1596$ POINTS 

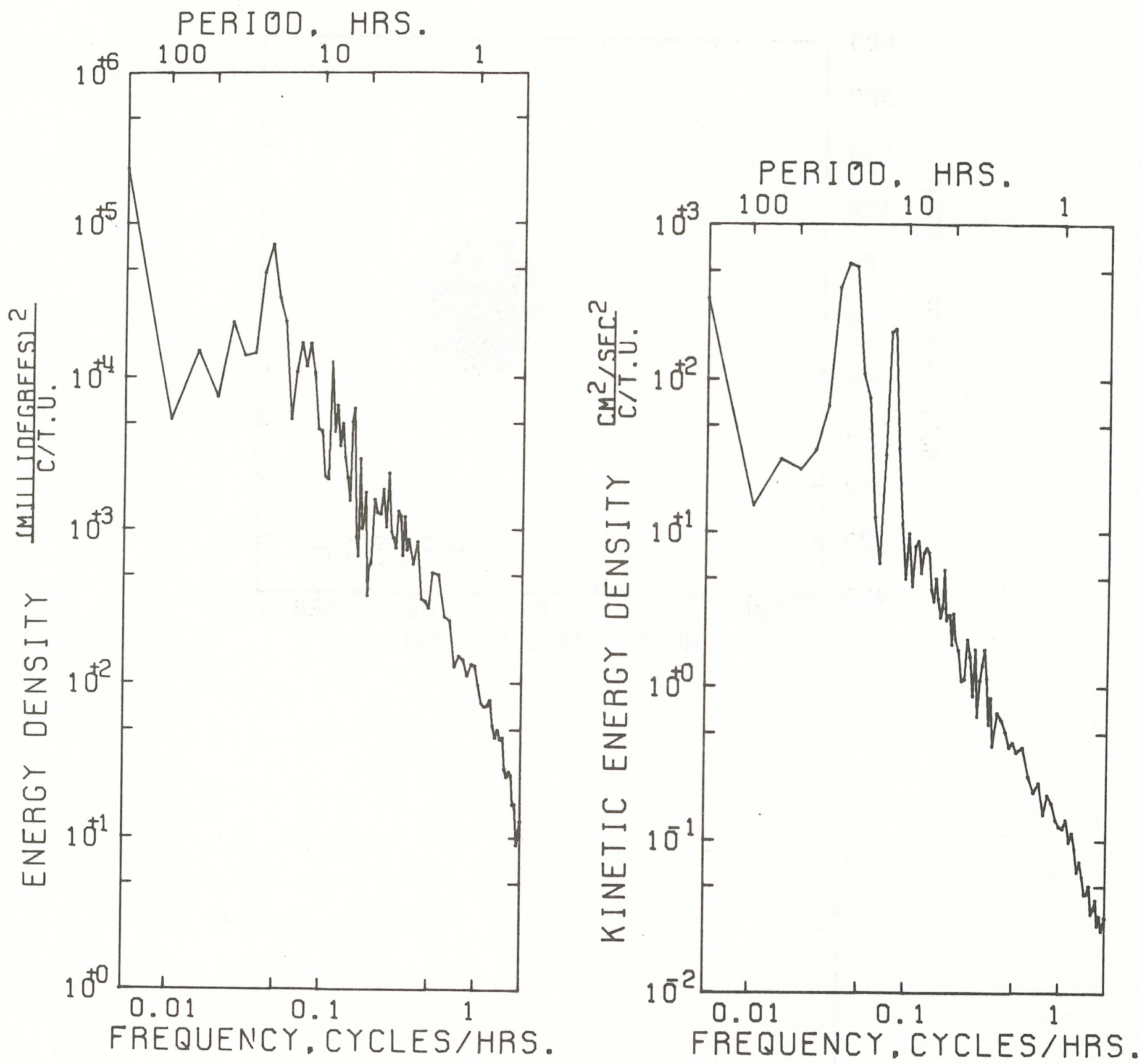

AUTO SPECTRUM

$4851 \mathrm{~B} 900$ TEMPERATURE

421 METERS

73-III-14 TO 73-III-30

1 PIECES WITH 768 ESTIMATES

PER PIECE. AVERAGED OVER

2 ADJACENT FREQUENCY BANDS
AUTO SPECTRUM

$4851 B 900$ EAST

4851 B900 NORTH

421 METERS

73-III-14 TO 73-III-30

1 PIECES WITH 768 ESTIMATES

PER PIECE. AVERAGED OVER

2 ADJACENT FREQUENCY BANDS 

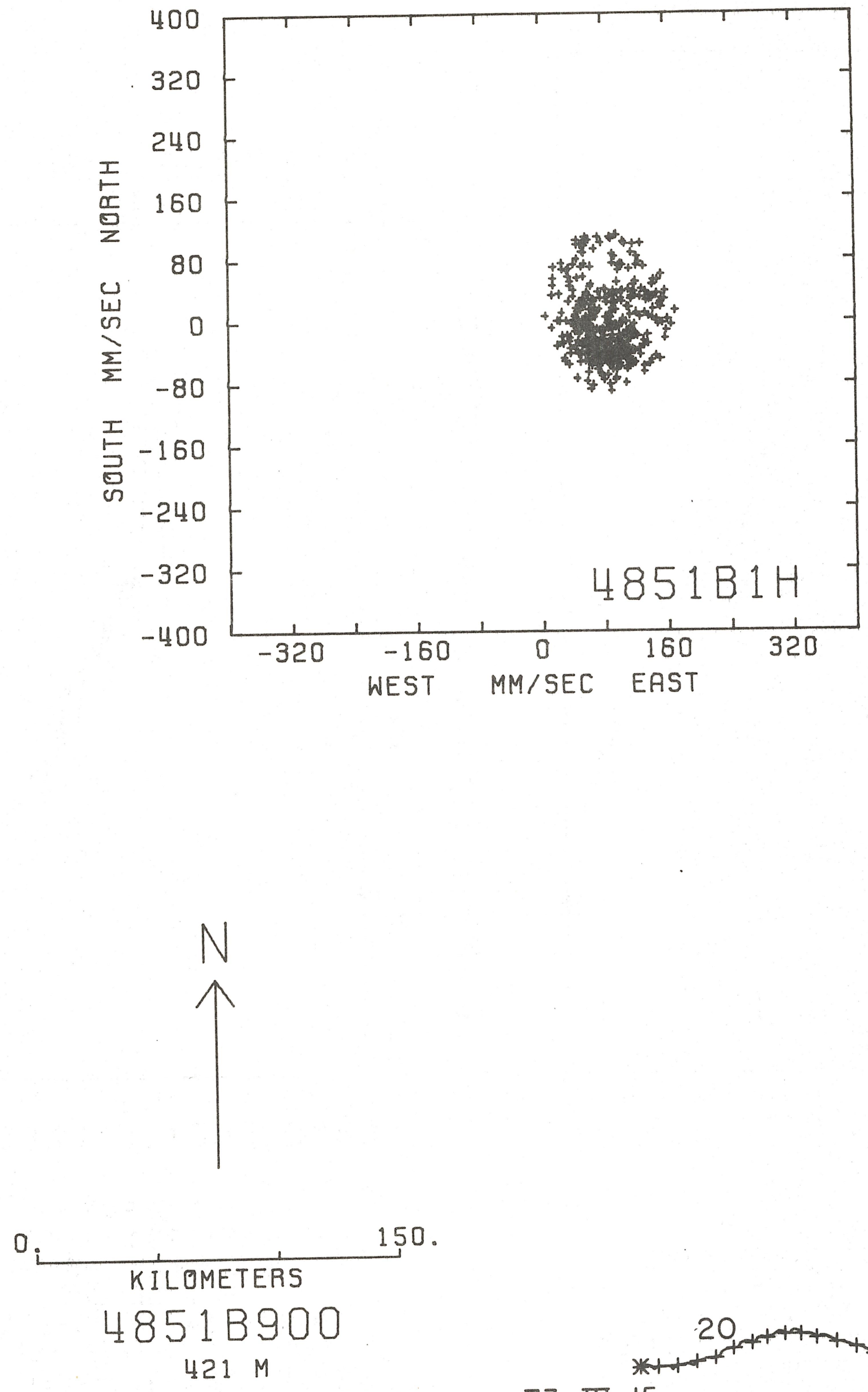

73- III-15 TO.73- III-30

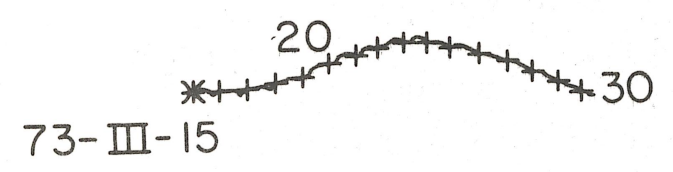



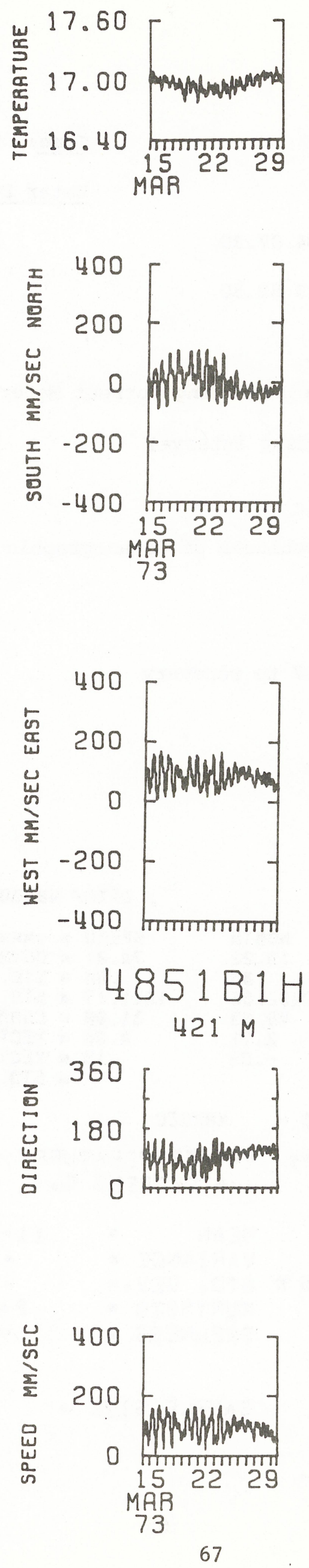
DATA NUMBER 4853

Instrument No.: V-0155

Depth: $723 \mathrm{~m}$
Type: Vector Averaging Current Meter Water Depth: $5420 \mathrm{~m}$

Start time: 73-March-14 04.07.30.

Stop time: 73-April-16 13.52.30.

Duration: $\quad 33 \mathrm{~d}$ 9h $45 \mathrm{~m}$

Sampling scheme: Vector Averaging Current Meter

recording interval $=900$ seconds

COMMENTS:

Instrument owned by the Institute of Oceanographic Sciences

Compass - good

Vane - sticky from April 17 to recovery

Rotor - good

Temperature - good

STATS

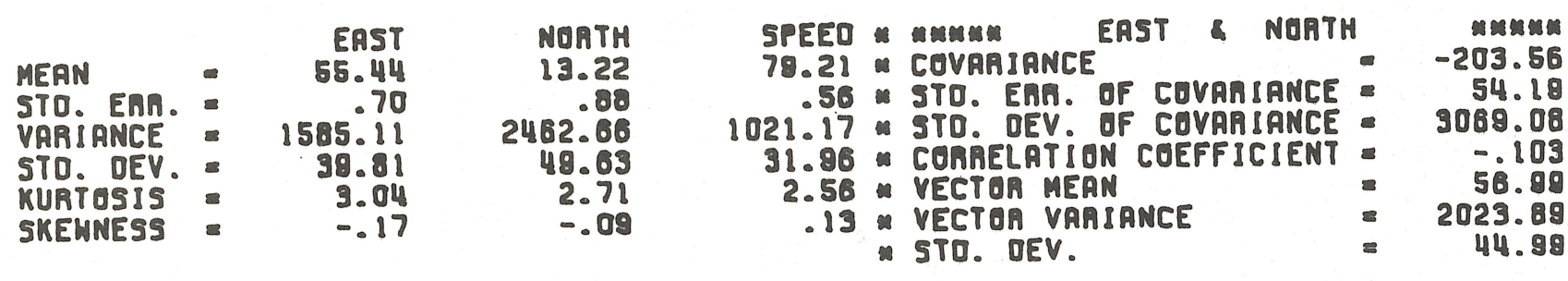

UNITS OF RAM DATA VARIABLES = MMISEC

SAMPLE SIZE = 3200 POINTS * TEMPERATURF * * * * * *REES C. * *

SPANNING AANGE

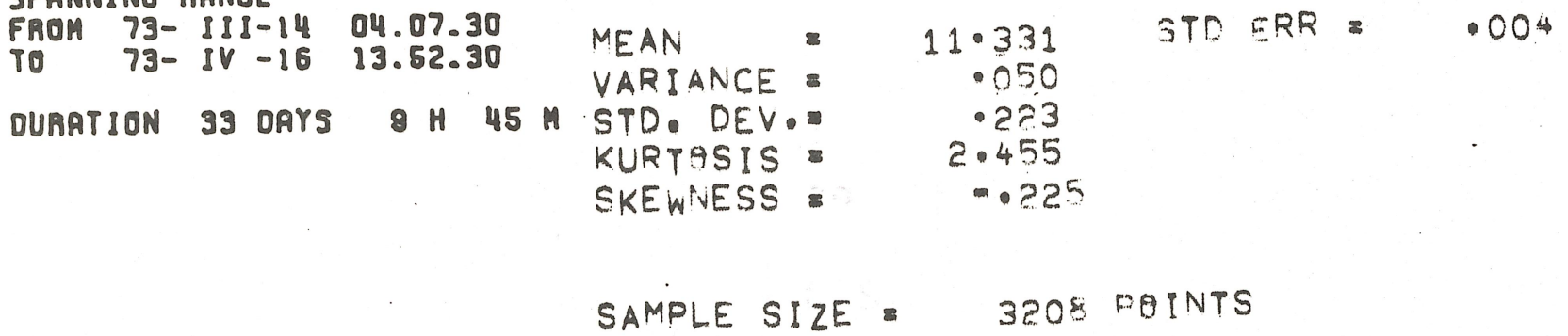



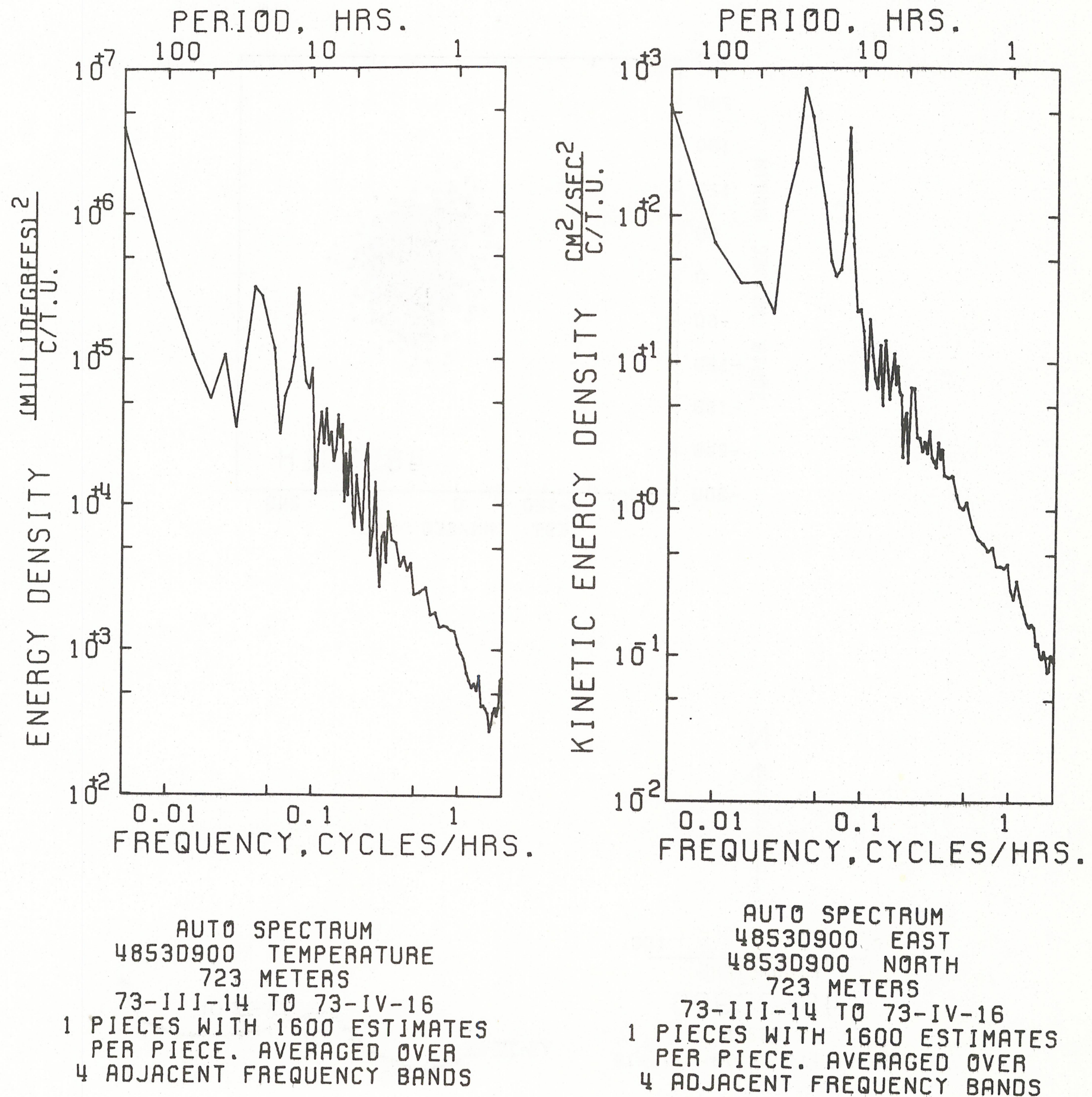

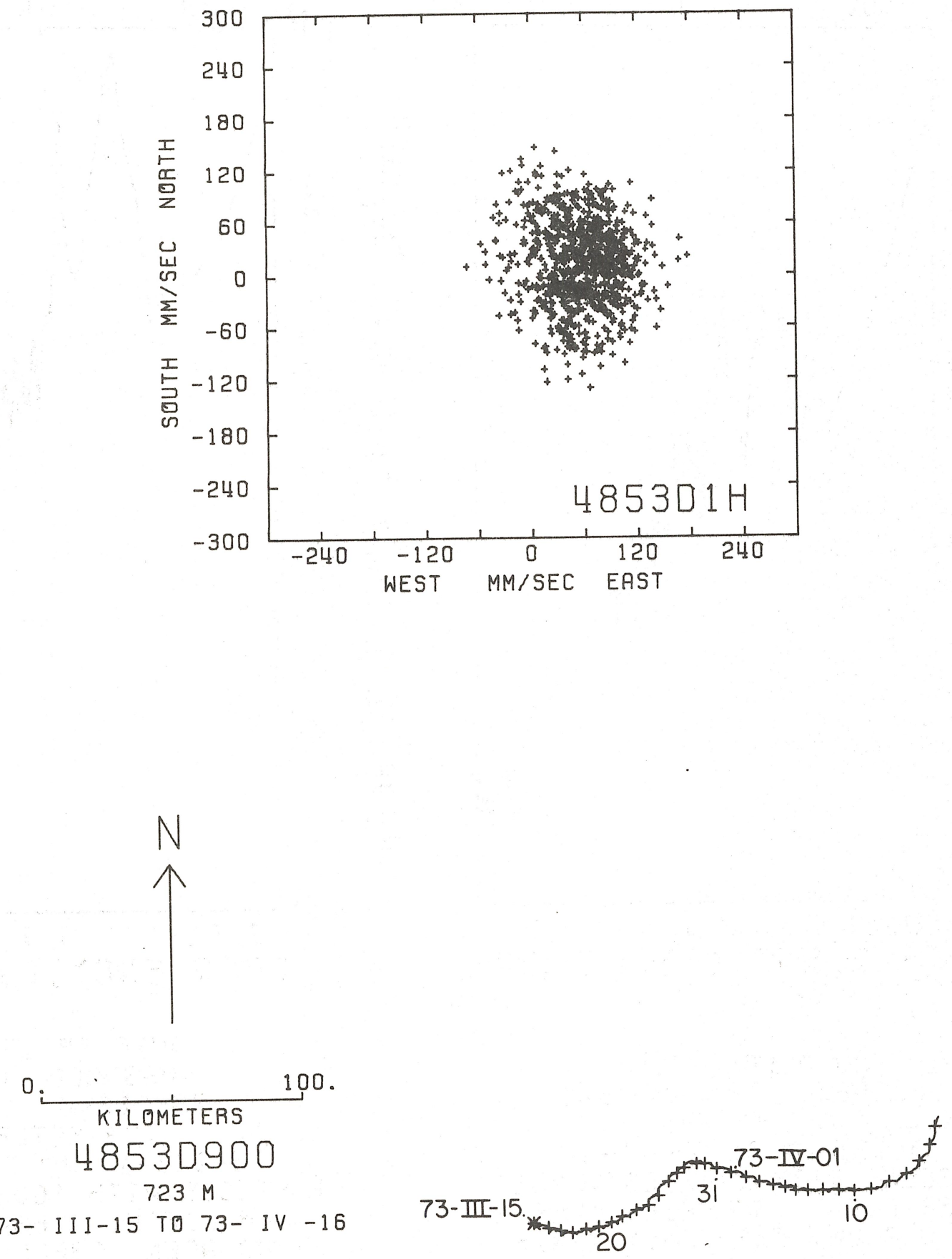

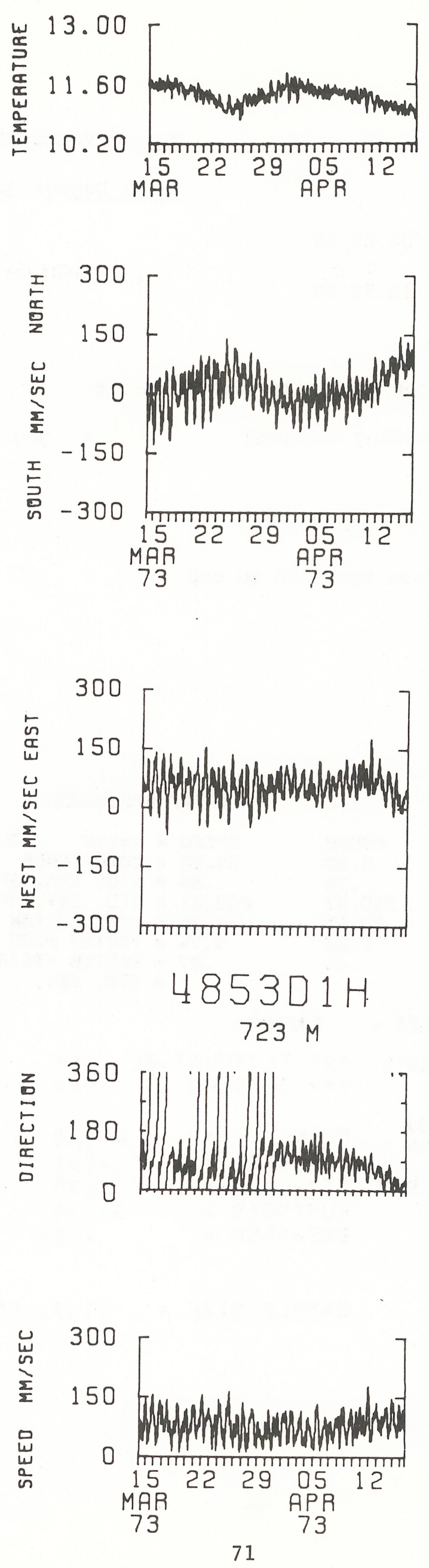
DATA NUMBER 4856

Instrument No:: V-0139

Depth: $1426 \mathrm{~m}$

start time: 73-March-14 04.07.30.

Stop time: 73-March-28 10.52.30.

Duration: $\quad 14 d$ h $45 \mathrm{~m}$

Sampling scheme: Vector Averaging Current Meter

$$
\text { recording interval }=900 \text { seconds }
$$

COMMENTS :

Compass - good

Vane - sticky or stuck from March 28 to end

Rotor - good

Temperature - good

STATS

\begin{tabular}{|c|c|c|c|}
\hline & & EAST & MORTH \\
\hline STD. ERH. & $=$ & $\begin{array}{r}.75 \\
.75\end{array}$ & \\
\hline VARIANCE & $=$ & 774.24 & 780.57 \\
\hline TO. DEV. & $=$ & 27.83 & 27.58 \\
\hline FUNESS & 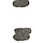 & 47 & 0 \\
\hline
\end{tabular}

DคTA

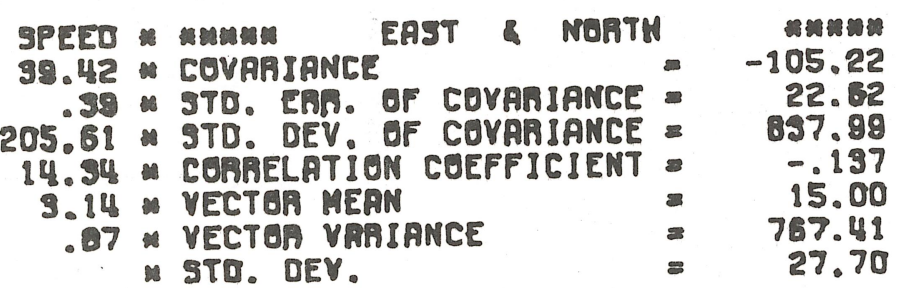

UNITS OF MPH DATA VARIABLES : MMPSC

SAMPLE SIEE * 1372 PEINTS * TEMPERATURE * *

SPANNING RANGE

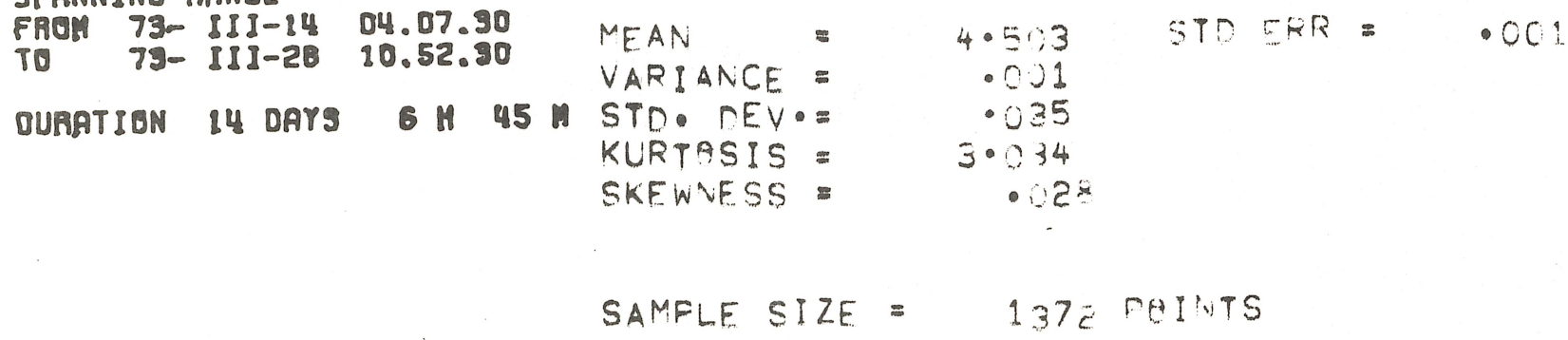



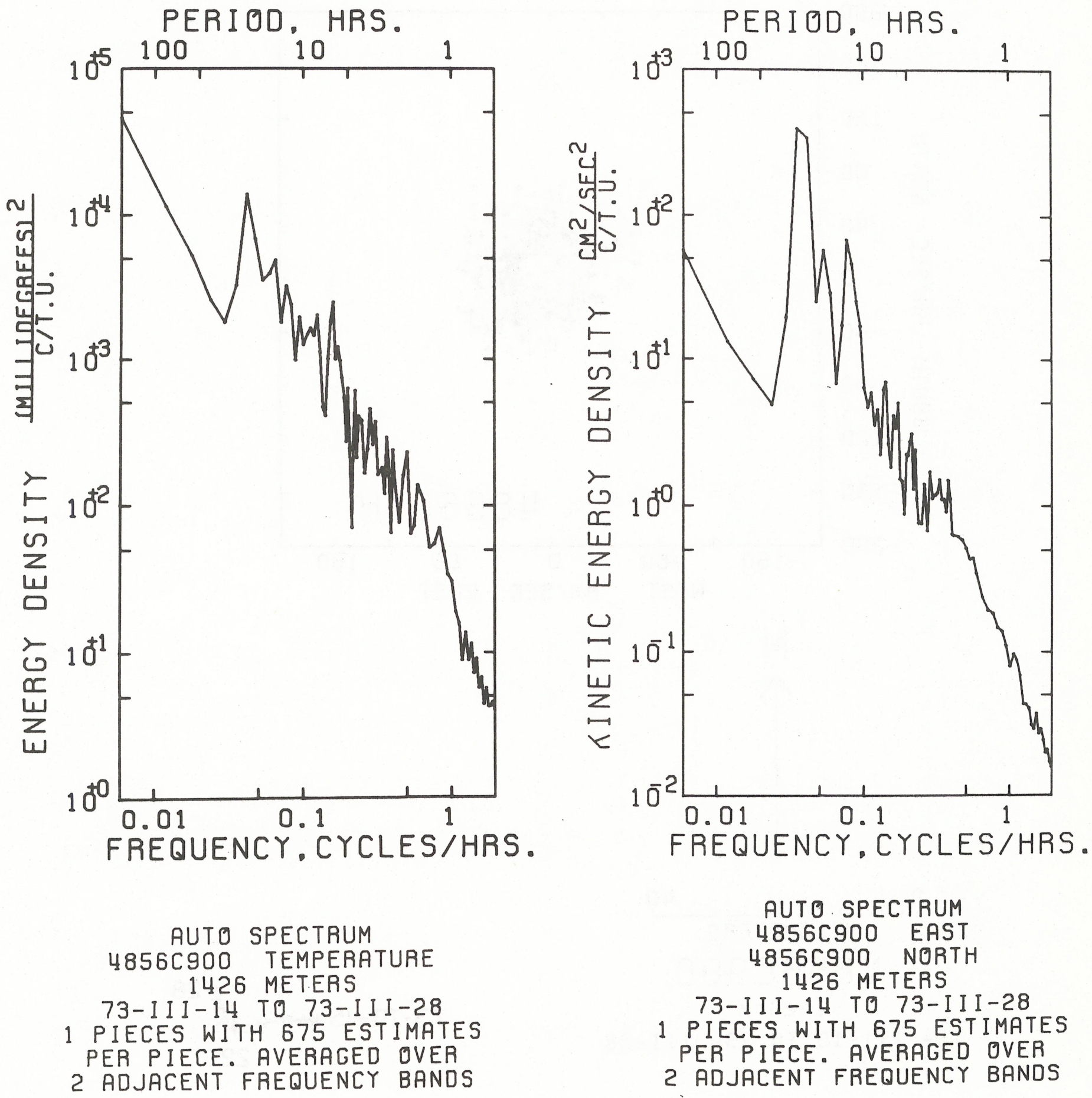

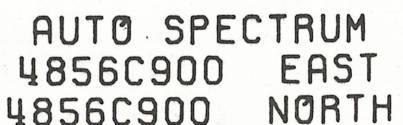$$
4856 \text { C900 NORTH }
$$$$
1426 \text { METERS }
$$$$
\text { 73-I I I-14 TO 73-I I I-28 }
$$

1 PIECES WITH 675 ESTIMATES

PER PIECE. AVERAGED OVER

2 ADJACENT FREQUENCY BANDS 

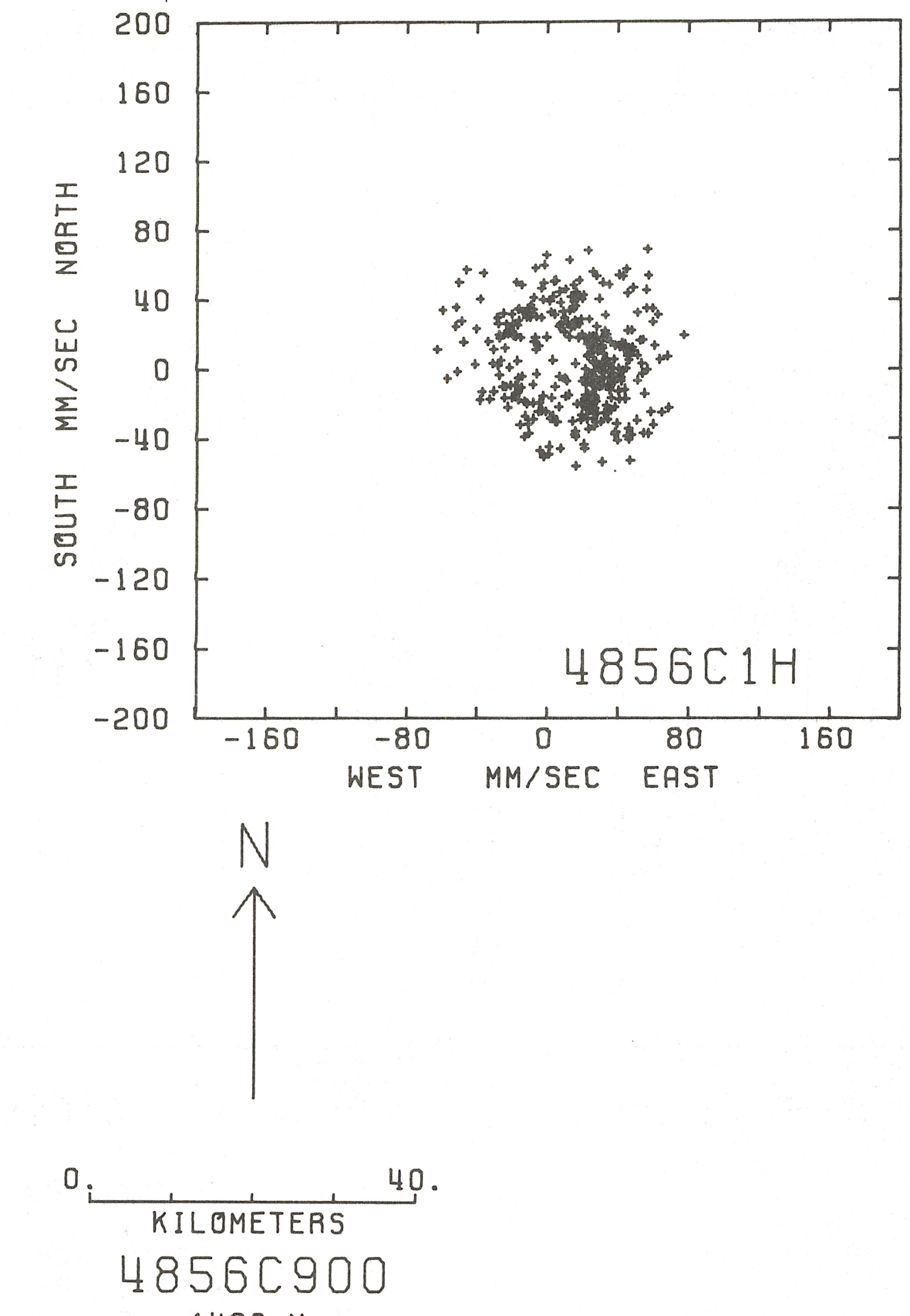

$1426 \mathrm{M}$

73- III-15 TO 73- III-28

$73-$ III- $15 *+15+t^{28}$
22 

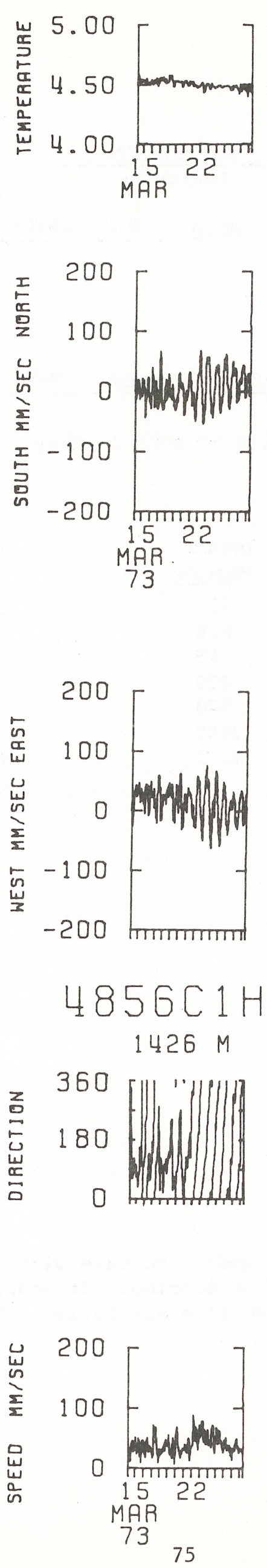
Mooring No. 486

Set $\frac{1973 \text { Mar } 14}{\text { Year Month Day }} \frac{26^{\circ} 57.5^{\prime} \mathrm{N}}{\text { Latitude }} \frac{71^{\circ} 02.6^{\prime} \mathrm{W}}{\text { Longitude }}$
Set by J. Gifford - R. Heinmiller Ship R.V. CHAIN $\quad$ Cruise 112 Leg 1

Retrieved $\frac{1973 \text { July } \quad 2}{\text { Year Month Day }}$

Retrieved by G. Tupper - R. Heinmiller Ship R.V. CHAIN Cruise 112 Leg 6

Purpose of Mooring: Mooring \#12 of MODE 1 array

Mooring Type: Subsurface

\begin{tabular}{|c|c|c|c|c|c|}
\hline Key & $\begin{array}{c}\text { Data } \\
\text { Number }\end{array}$ & $\begin{array}{c}\text { Instrument } \\
\text { Number } \\
\end{array}$ & Type & $\begin{array}{l}\text { Depth } \\
\text { Meters }\end{array}$ & Comments \\
\hline & 4861 & $V-0131$ & VACM & 415 & \\
\hline & 4862 & $\# 40$ & $\mathrm{~T} / \mathrm{P}$ & 519 & M.I.T., tape fouled \\
\hline & 4863 & $V-0172$ & VACM & 715 & U.R.I., tape unreadable \\
\hline * & 4864 & $V-0124$ & VACM & 1420 & \\
\hline * & 4865 & $V-0106$ & VACM & 2940 & \\
\hline$\#$ & 4866 & \#28 & $\mathrm{T} / \mathrm{P}$ & 3948 & $M . I . T$. \\
\hline & 4867 & $\mathrm{H}-877$ & Film & 5372 & Nova University, Florida \\
\hline \multicolumn{3}{|c|}{ Water depth } & & 5474 & \\
\hline
\end{tabular}

COMMENTS ON MOORING:

MODE cameraman overboard in Zodiac to take pictures of a launch. A tanker passed 2.3 miles astern of us during tow of mooring. It crossed the mooring line between item 20 (8 glass balls) and item 26 (5 glass balls). No apparent damage. 

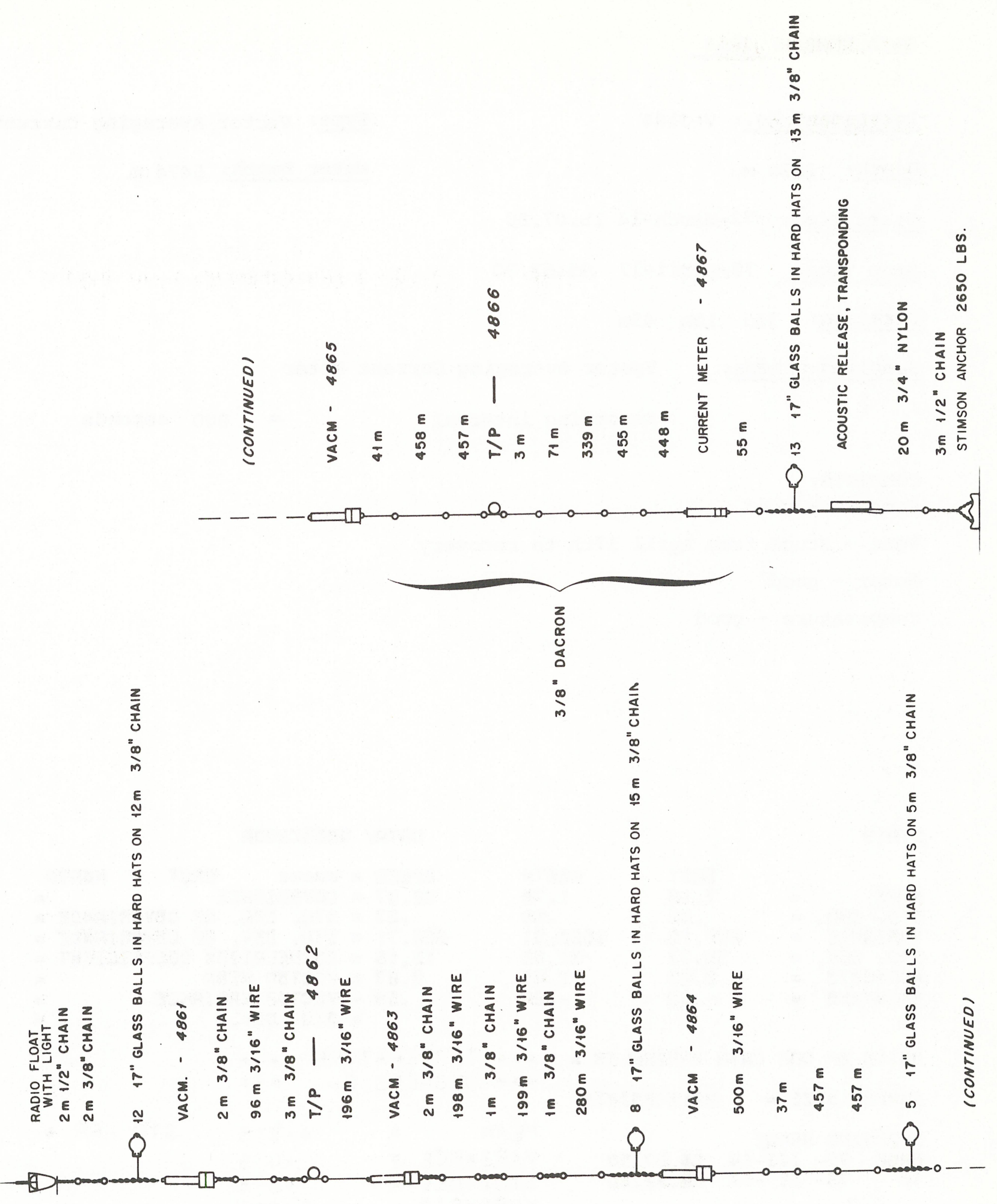

0
$\infty$
0
$z$
0
$E$
$\frac{1}{5}$
0 
DATA NUMBER 4864

Instrument No.: V-0184

Depth: $1420 \mathrm{~m}$
Type: Vector Averaging Current Meter Water Depth: $5474 \mathrm{~m}$

Start Time: 73-March-14 15.07.30

Stop Time: 73-April-17 05.52.30

Duration: $33 \mathrm{~d}$ 10h $45 \mathrm{~m}$

Sampling scheme: Vector Averaging Current Meter

recording interval $=900$ seconds

COMMENTS:

Compass - good

Vane - stuck from April 17th to recovery

Rotor - good

Temperature - good

sTATS

DATAR 4อEบESDOA

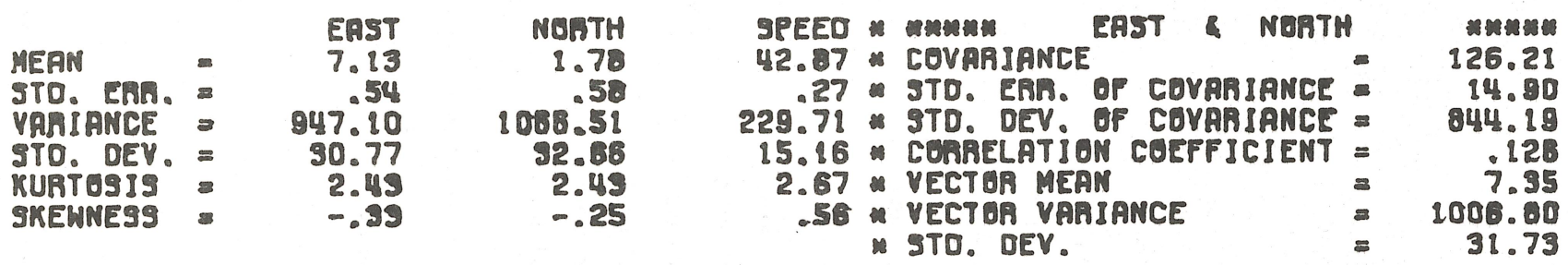

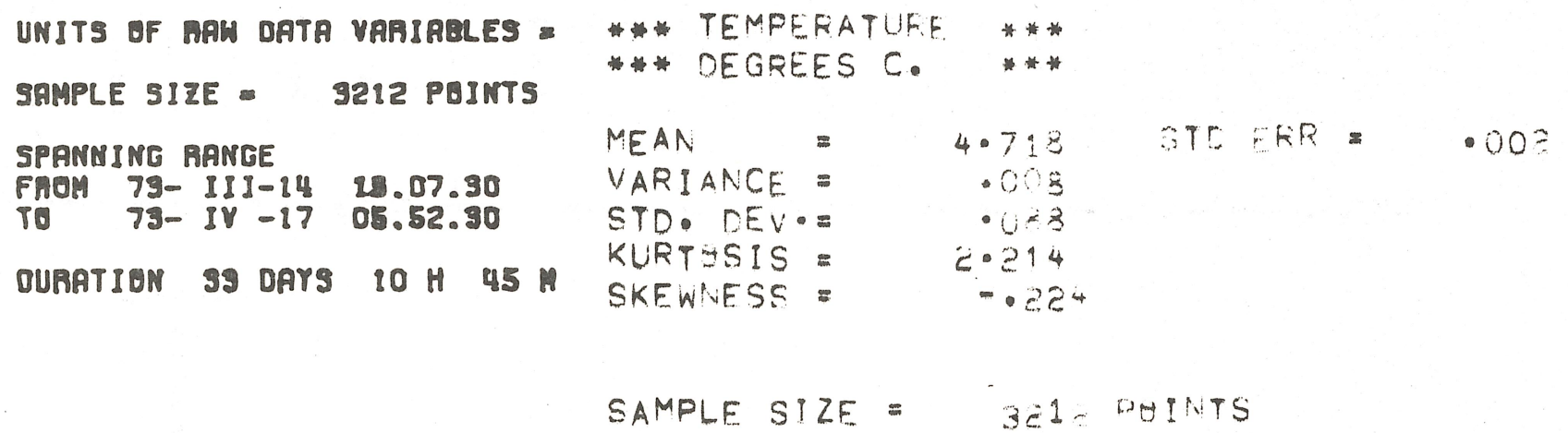



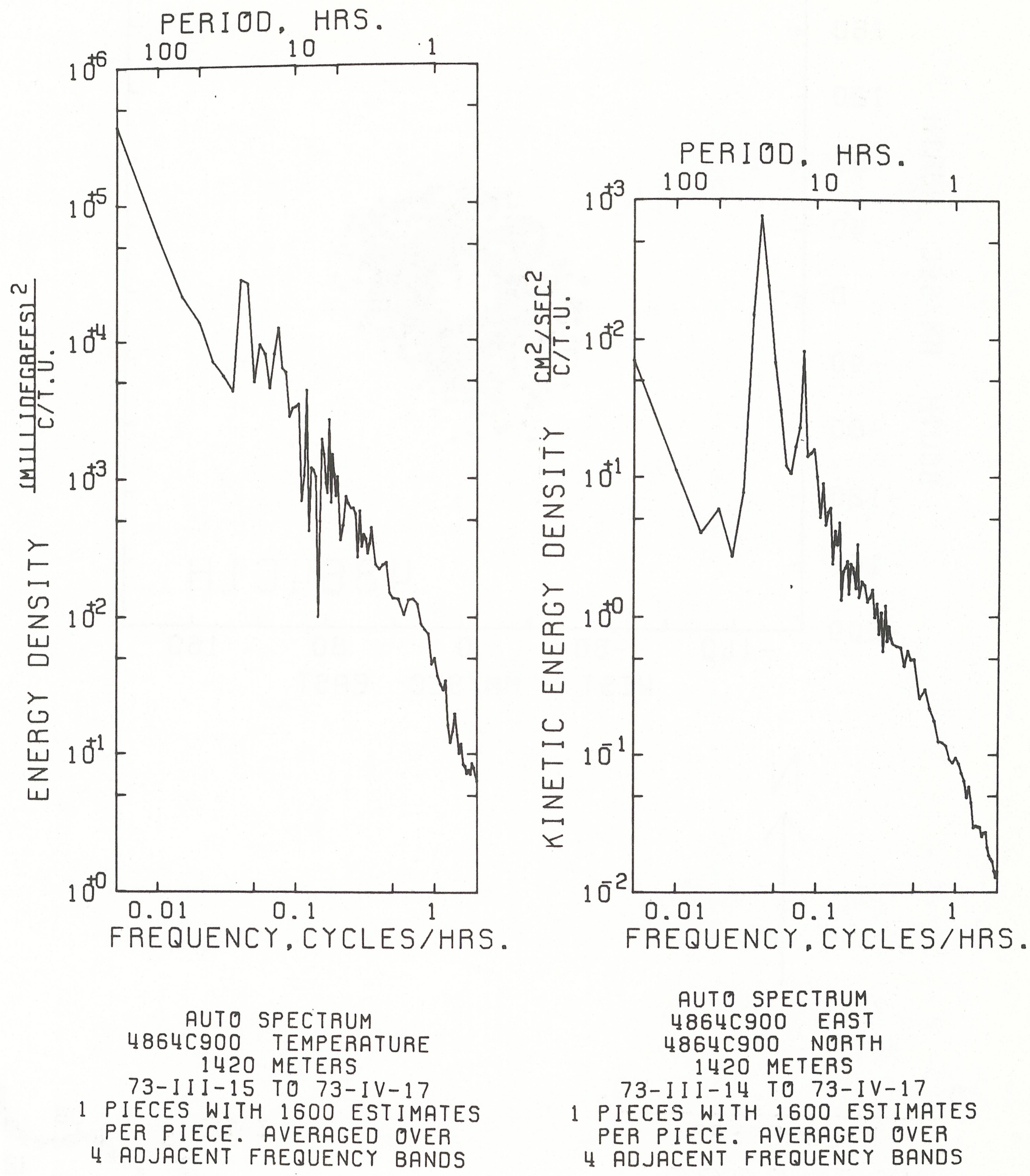

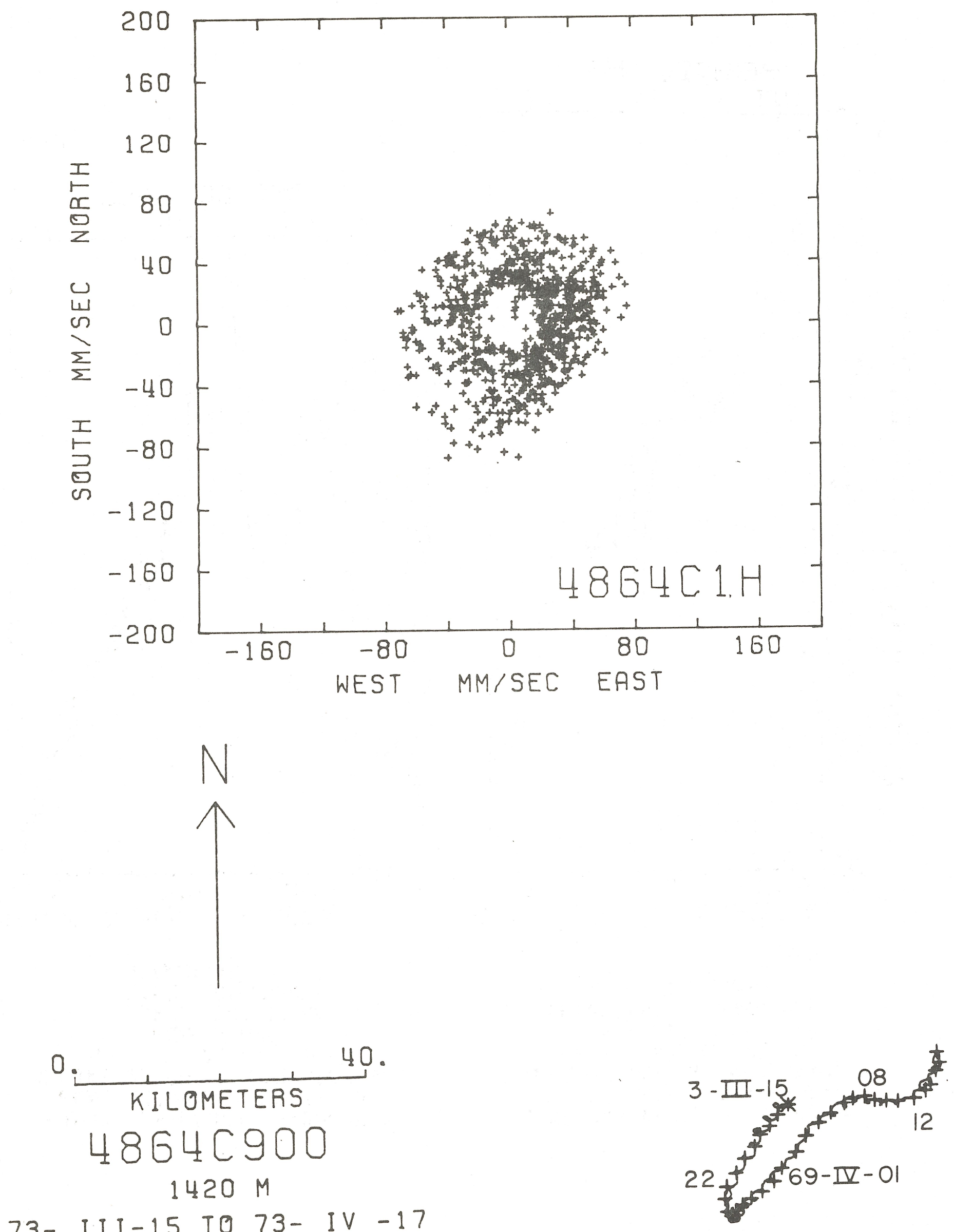

73- III-15 TO 73-IV - I7 

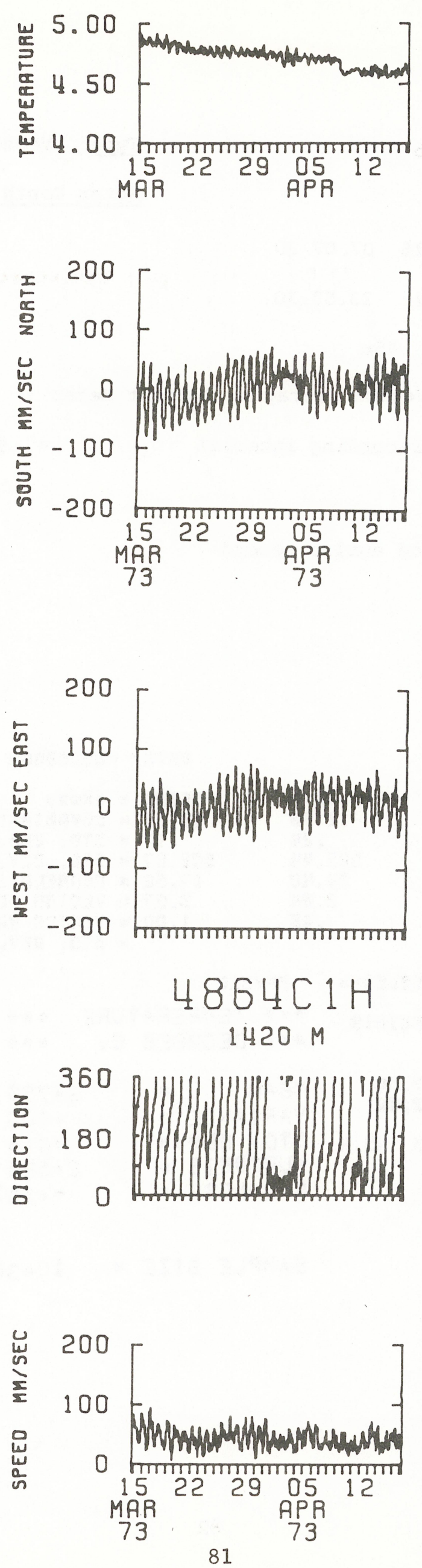
DATA NUMBER 4865

Instrument No.: V-0106

Depth: $2940 \mathrm{~m}$

Start time: 73-March-15 07.07.30.

Stop time: 73-July-01 23.52.30.

Duration: $108 \mathrm{~d}$ 16h $45 \mathrm{~m}$

Sampling scheme: Vector Averaging Current Meter

$$
\text { recording interval }=900 \text { seconds }
$$

COMMENTS :

All variables look good entire record

STคTร

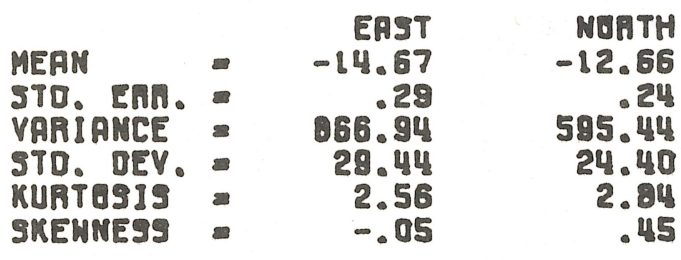

optar 4865cgoga

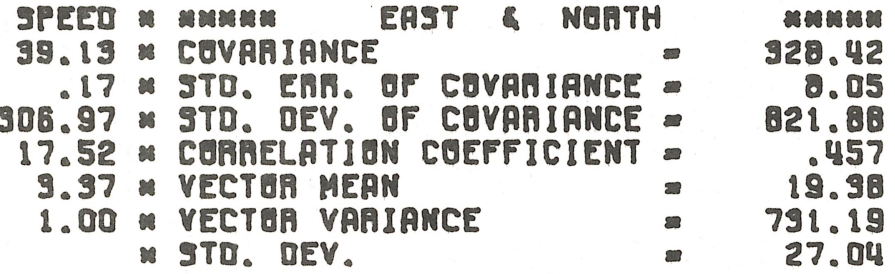

UMITS OF BAH DATA VARIABLES - MMPSE

SAMPLE 9IZE - 10436 PUINTS

SPANNING APNGE

FROH 73- III-15 07.07.30

TO 7\$-VII-0I 23.52.30

MEAN
DURATION 108 DAYS 16 H 45 M

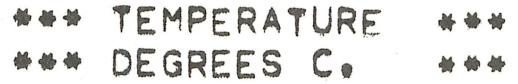

VARTANCE

.000

STD ERR : . .0OC

STD. DEV. 016

KURTOSIS $\quad 2.528$

SKEWNESS: 107

SAMPLE SIZE : 10436 POINTS 

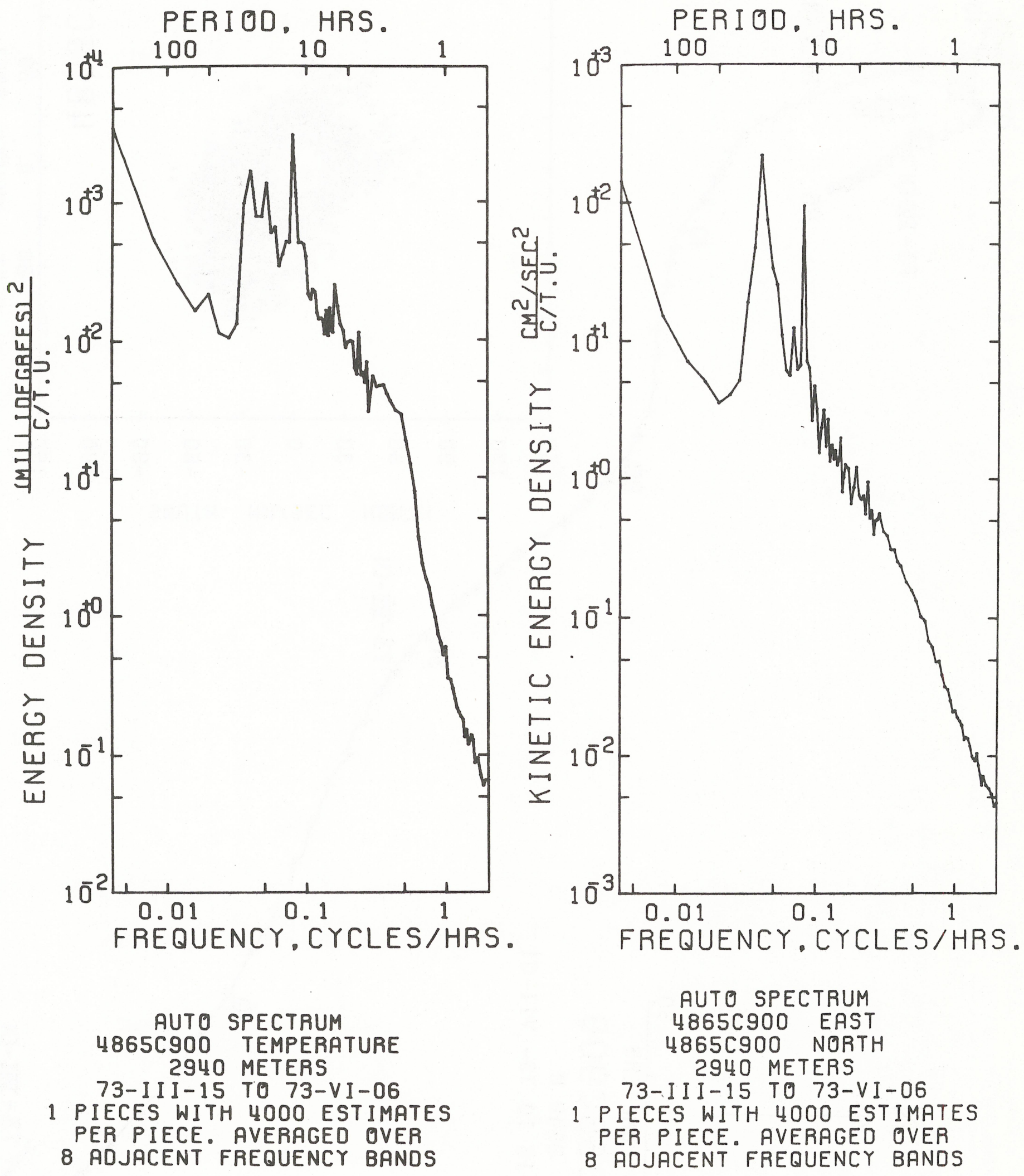

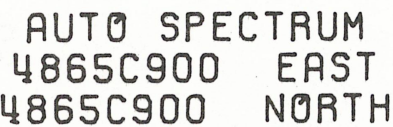$$
2940 \text { METERS }
$$$$
\text { 73-III-15 TO 73-VI-06 }
$$

1 PIECES WITH 4000 ESTIMATES

PER PIECE. AVERAGED OVER

8 ADJACENT FREQUENCY BANDS 


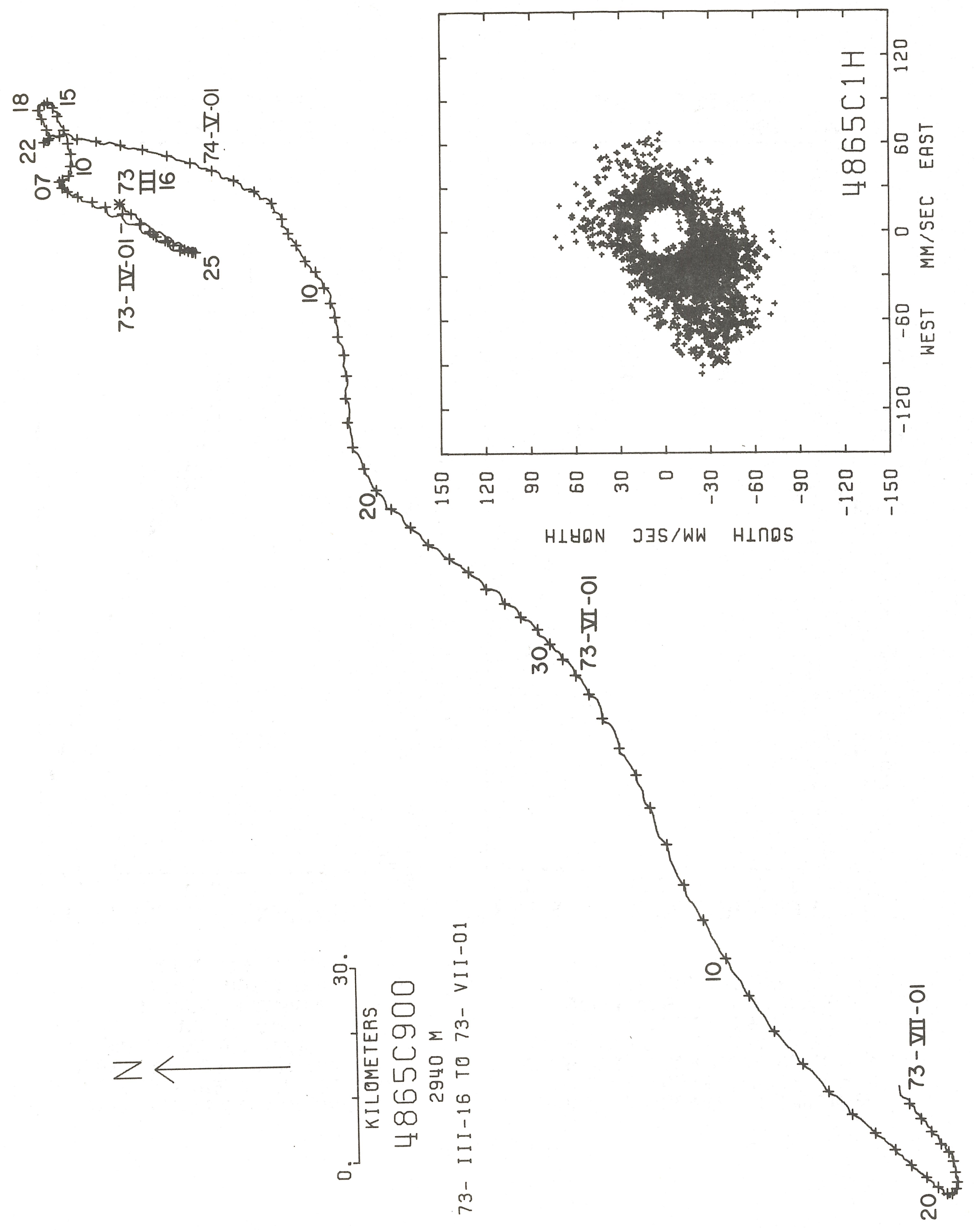



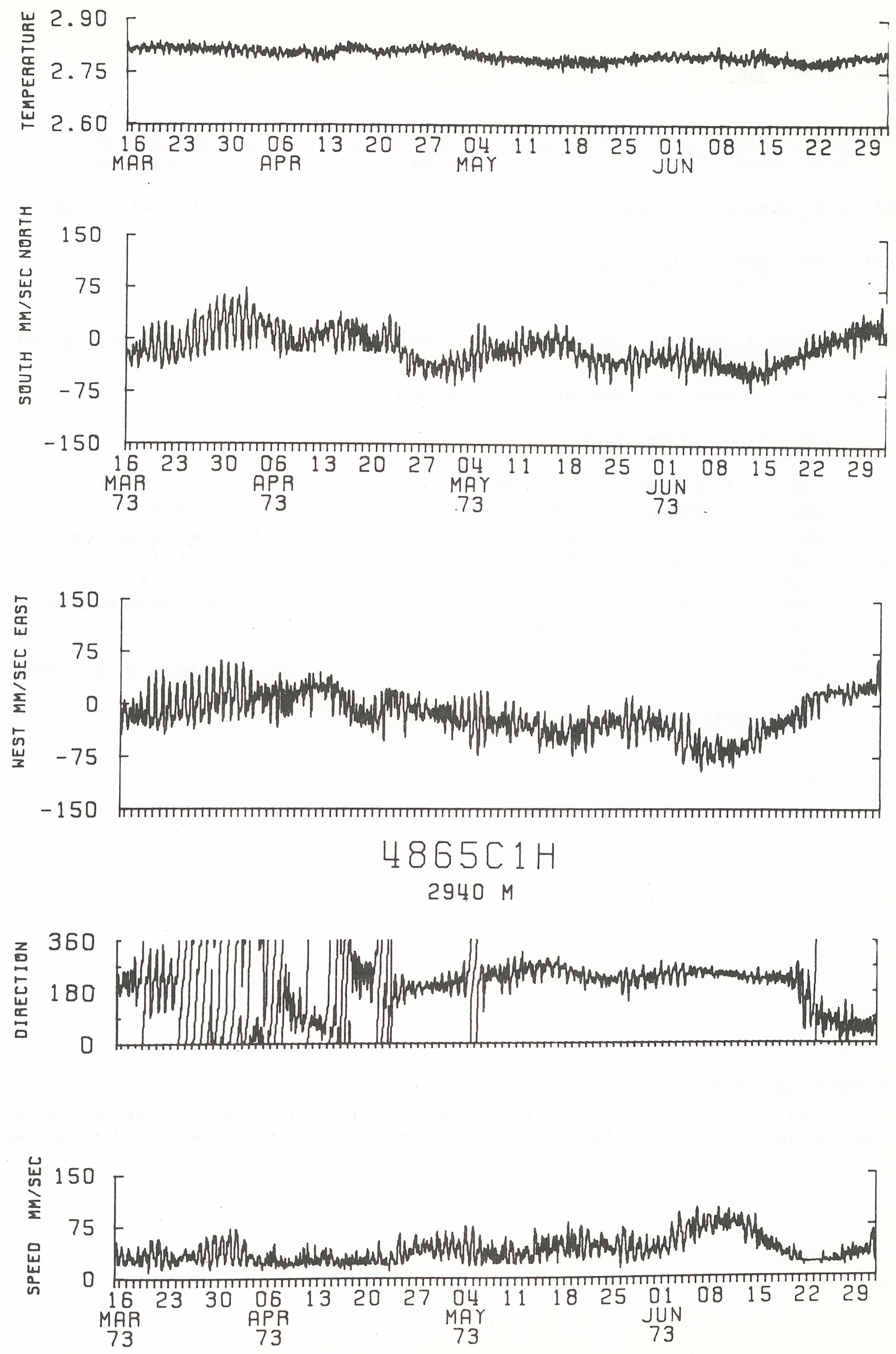
Mooring No. 488

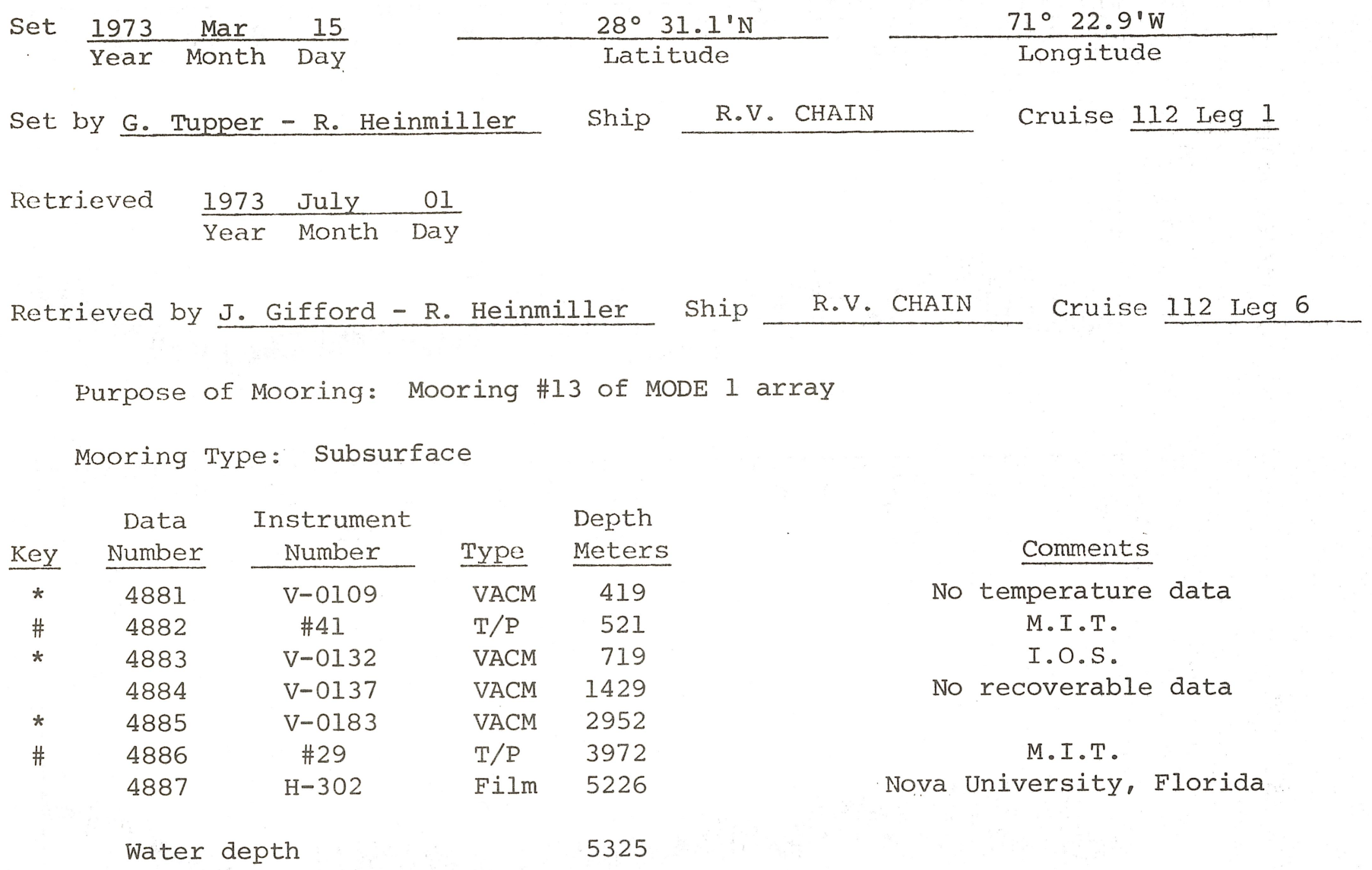

COMMENTS ON MOORING:

The 3/4" nylon just below the release on mooring 487 parted before the anchor reached the bottom. The mooring was retrieved and reset with a new mooring number (488). 

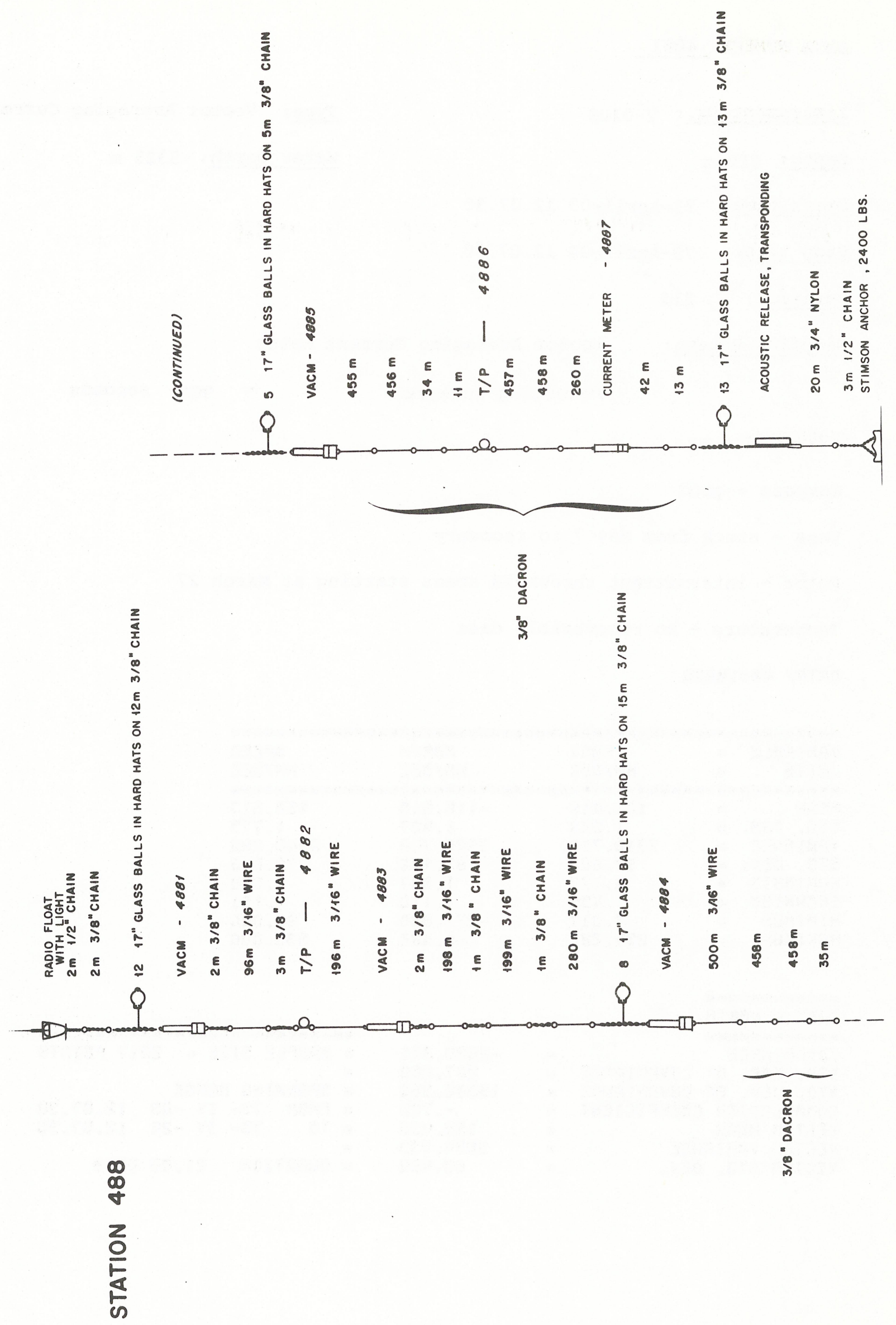
DATA NUMBER 4881

Instrument No.: V-0109

Depth: $419 \mathrm{~m}$
Type: Vector Averaging Current Meter

Water Depth: $5325 \mathrm{~m}$

Start time: 73-April-03 12.07.30

Stop time: 73-April-24 12.07.30

Duration: $21 d$

Sampling scheme: Vector Averaging Current Meter

recording interval $=900$ seconds

COMMENTS:

Compass - good

Vane - stuck from May 7 to recovery

Rotor - intermittent threshold areas starting at March 27

Temperature - no recoverable data

DATA/ 48818900

\begin{tabular}{|c|c|c|c|c|}
\hline $\begin{array}{l}\text { VARTI ABLE } \\
\text { UNITS }\end{array}$ & $\approx$ & $\begin{array}{r}\text { EAST } \\
\text { MM/SEC }\end{array}$ & $\begin{array}{l}\text { NORPH } \\
\text { MM/SEC }\end{array}$ & $\begin{array}{r}\text { SPEED } \\
\text { MM/SEC }\end{array}$ \\
\hline \multicolumn{5}{|c|}{ 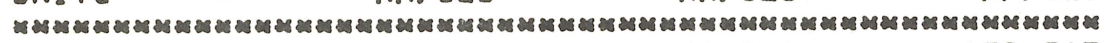 } \\
\hline $\begin{array}{l}\text { MEAN } \\
\text { STD. ERR. } \\
\text { VARIANCE } \\
\text { STO. DEV. } \\
\text { KURTESIS } \\
\text { SKEWNESS } \\
\text { MINIMUM } \\
\text { MAXIMUM }\end{array}$ & $\begin{array}{l}= \\
= \\
= \\
= \\
= \\
= \\
=\end{array}$ & $\begin{array}{r}102.419 \\
1.203 \\
3318.718 \\
57.608 \\
2.127 \\
.435 \\
7.017 \\
234.222\end{array}$ & $\begin{array}{r}-116.913 \\
1.407 \\
3990.748 \\
63.172 \\
1.997 \\
-.110 \\
-271.890 \\
-9.428\end{array}$ & $\begin{array}{r}158.517 \\
1.779 \\
6340.295 \\
79.626 \\
2.090 \\
.151 \\
20.000 \\
333.000\end{array}$ \\
\hline
\end{tabular}

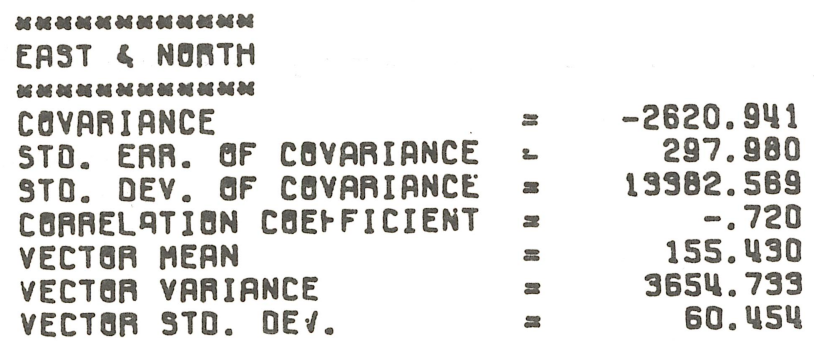

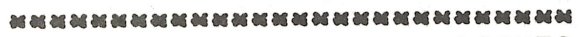

* SAMPLE SIZE - 2017 POINTS

$\varpi$

* SPANNING RANGE

* FRUM 73- IV -03 12.07.30

* TO 73- IV $-24 \quad 12.07 .30$

* DUAATION 21.00 DAYS 


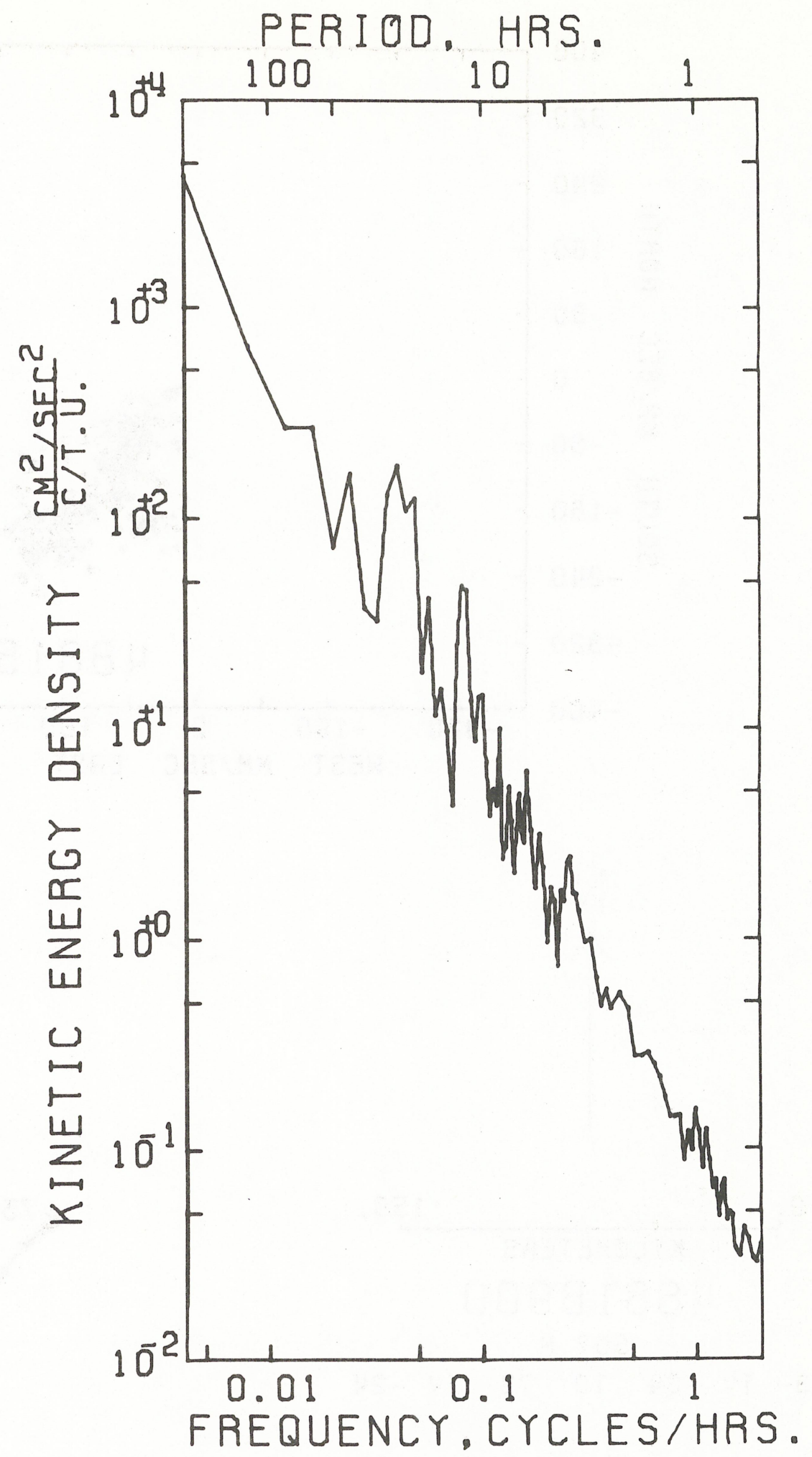

AUTO SPECTRUM $4881 B 900$ EAST 4881 B900 NORTH 507 METERS

73-IV-03 TO 73-IV-24

1 PIECES WITH 1000 ESTIMATES

PER PIECE. AVERAGED OVER

2 ADJACENT FREQUENCY BANDS 

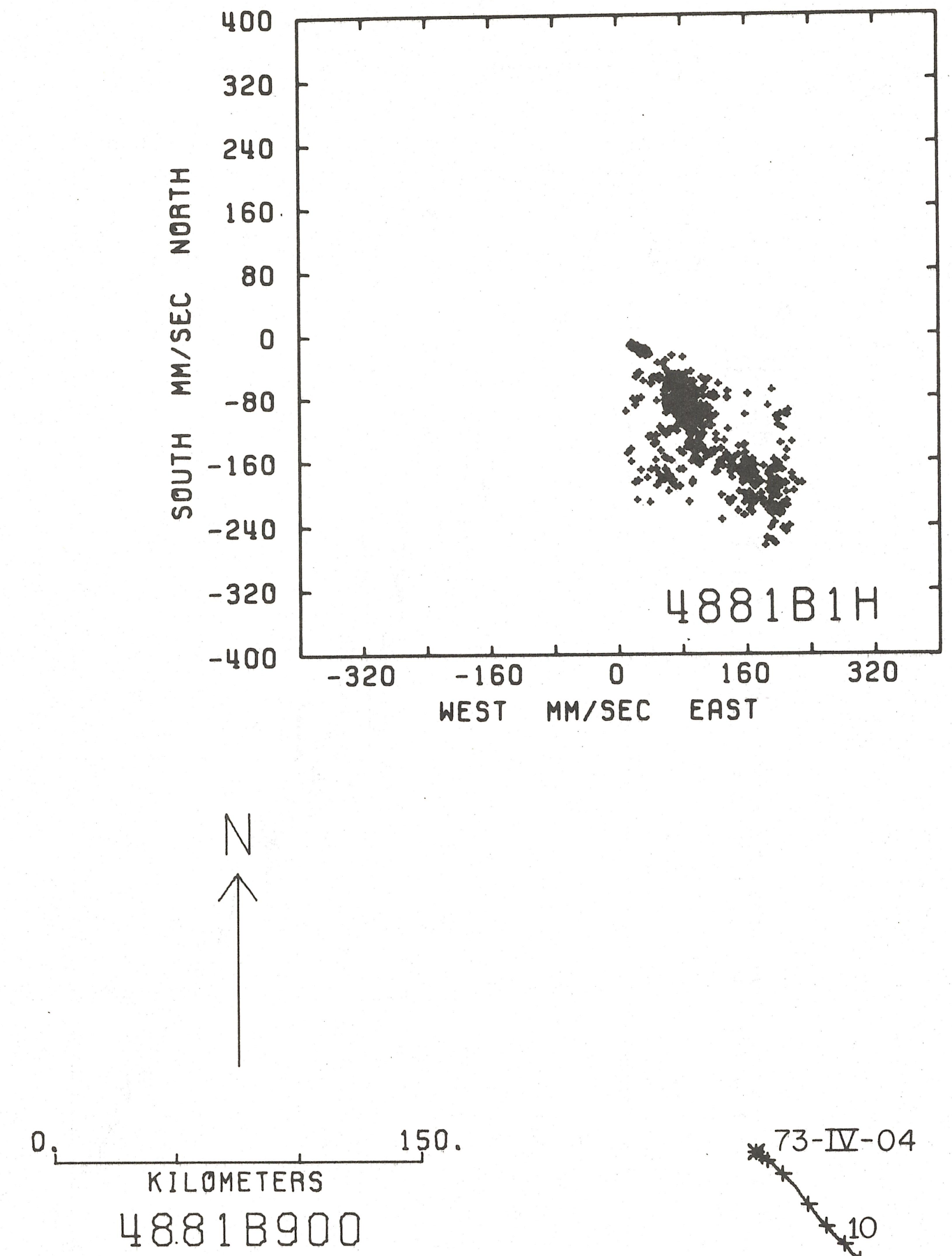

$507 \mathrm{M}$

73-IV-04 TO 73- IV - 24

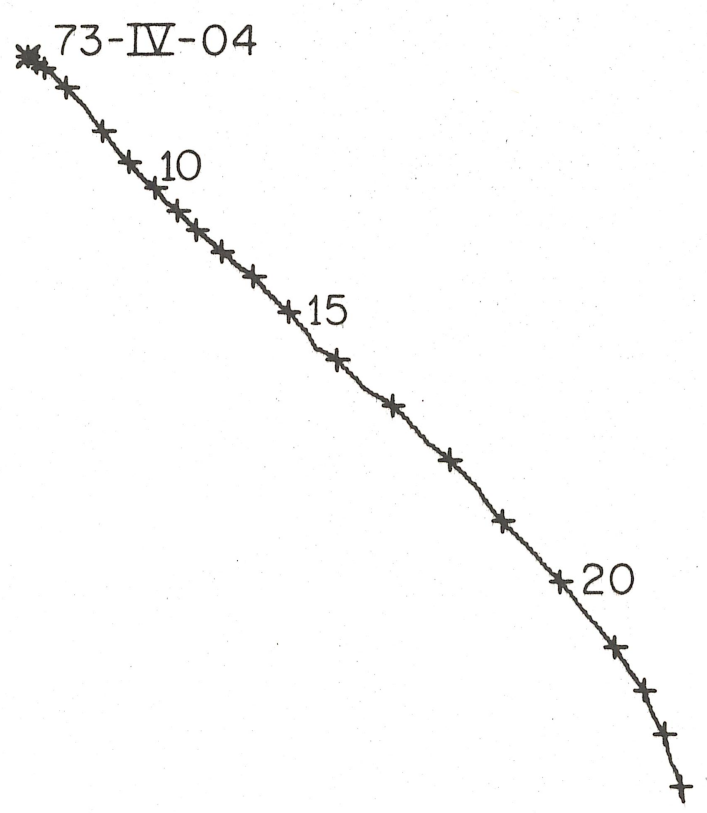



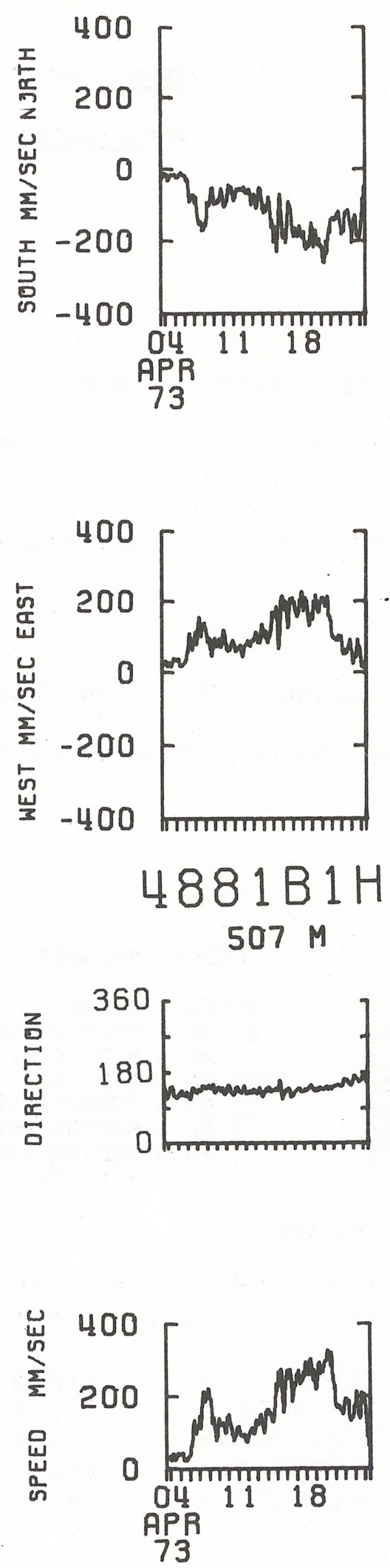
DATA NUMBER 4883

Instrument No.: V-0132

Depth: $719 \mathrm{~m}$
Type: Vector Averaging Current Meter

Water Depth: $5325 \mathrm{~m}$

Start time: 73-March-15 18.07.30.

Stop time: 73-April-17 11.52.30.

Duration: $\quad 32 \mathrm{~d} \quad 17 \mathrm{~h} 45 \mathrm{~m}$

Sampling scheme: Vector Averaging Current Meter

$$
\text { recording interval }=900 \text { seconds }
$$

COMMENTS :

Instrument owned by the Institute of Oceanographic Sciences

Compass - good

Vane - sticking from April 17 to April 22. Stuck from May 10 to recovery

Rotor - good until June 26 then drops to threshold values

Temperature - good

STATS

$\begin{array}{lrr} & \text { EAST } & \text { NOATH } \\ \text { MEAN } & 104.98 & -62.48 \\ \text { STD. EAR. } & .86 & 1.08 \\ \text { VARIANCE }= & 2323.07 & 3657.88 \\ \text { STO. OEV. } & 48.20 & 00.48 \\ \text { KUATOSIS }= & 2.54 & 2.55 \\ \text { SKEWNESS }= & -.13 & .32\end{array}$

OATA/ 4803 C900A

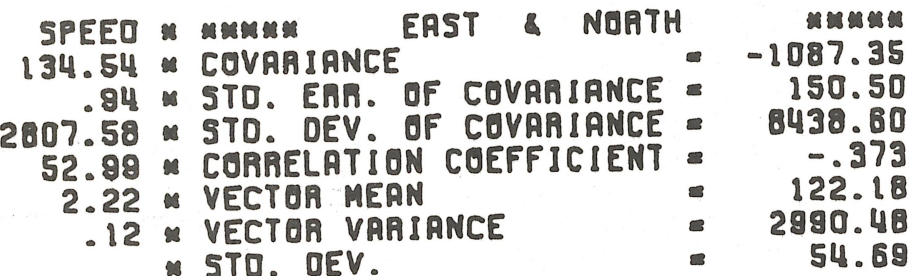

MMPSEC

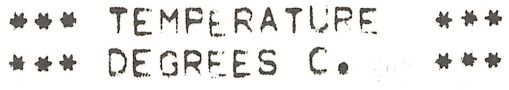

SPRNNING RANGE

$\begin{array}{lll}\text { FAOM } 73-111-15 & 18.07 .30 \\ \text { TO } & 73-1 V-17 & 11.52 .30\end{array}$

DURATION 32 DAYS 17 H $45 \mathrm{M}$
STO ERR = $\quad 00$

- 28

- 478

$2 \cdot 415$

$=.340$

SAMPLE SIZE $\quad 3144$ EINTS 

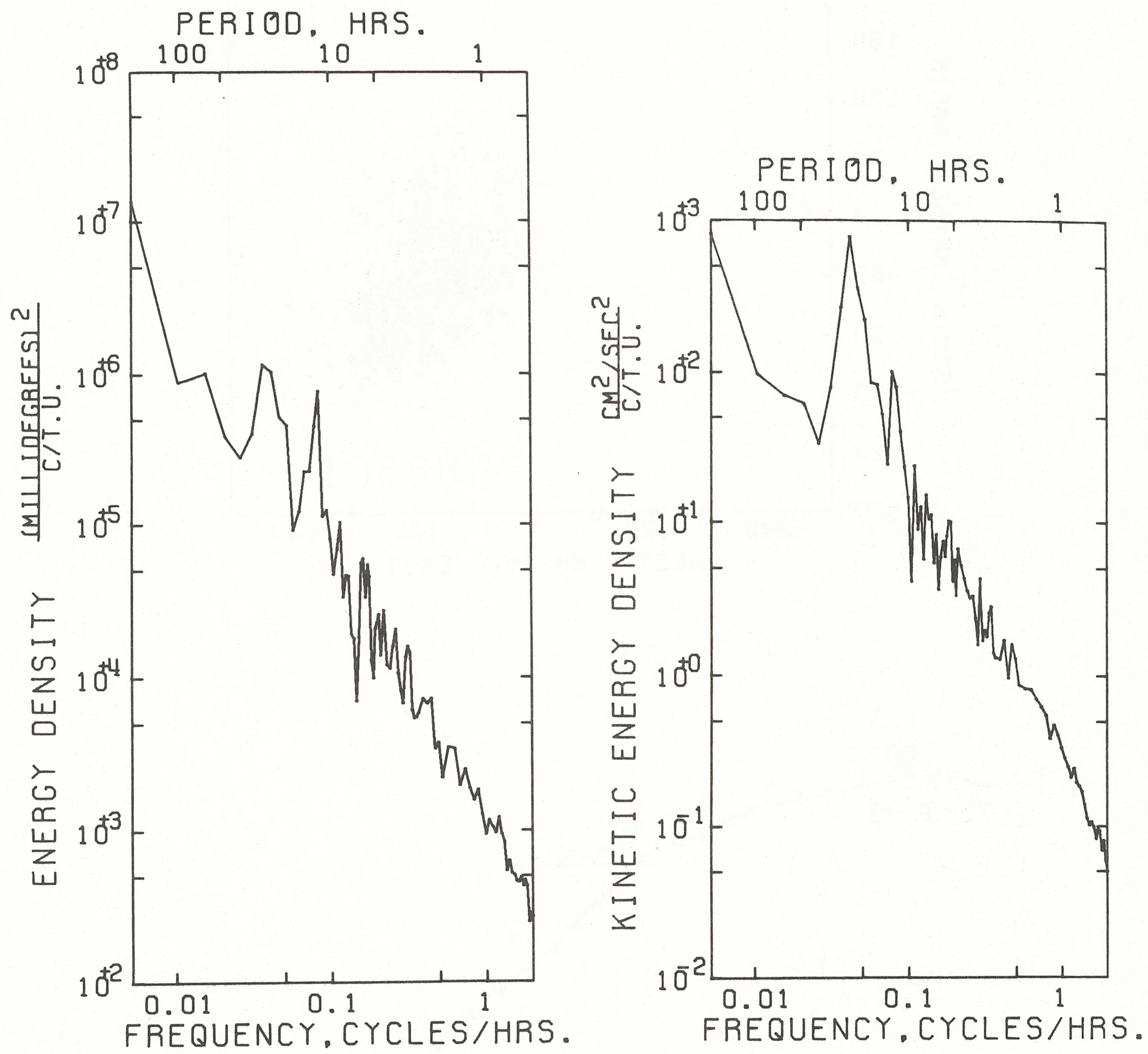

AUTO SPECTRUM

4883 C900 TEMPERATURE 719 METERS

73-III-15 TO 73-IV-16

1 PIECES WITH 1536 ESTIMATES

PER PIECE. AVERAGED OVER

4 ADJACENT FREQUENCY BANDS

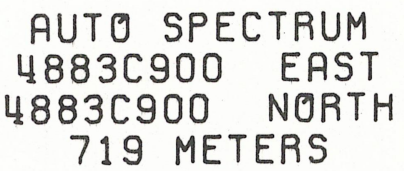

73-III-15 TO 73-IV-16

1 PIECES WITH 1536 ESTIMATES

PER PIECE. AVERAGED OVER

4 ADJACENT FREQUENCY BANDS 

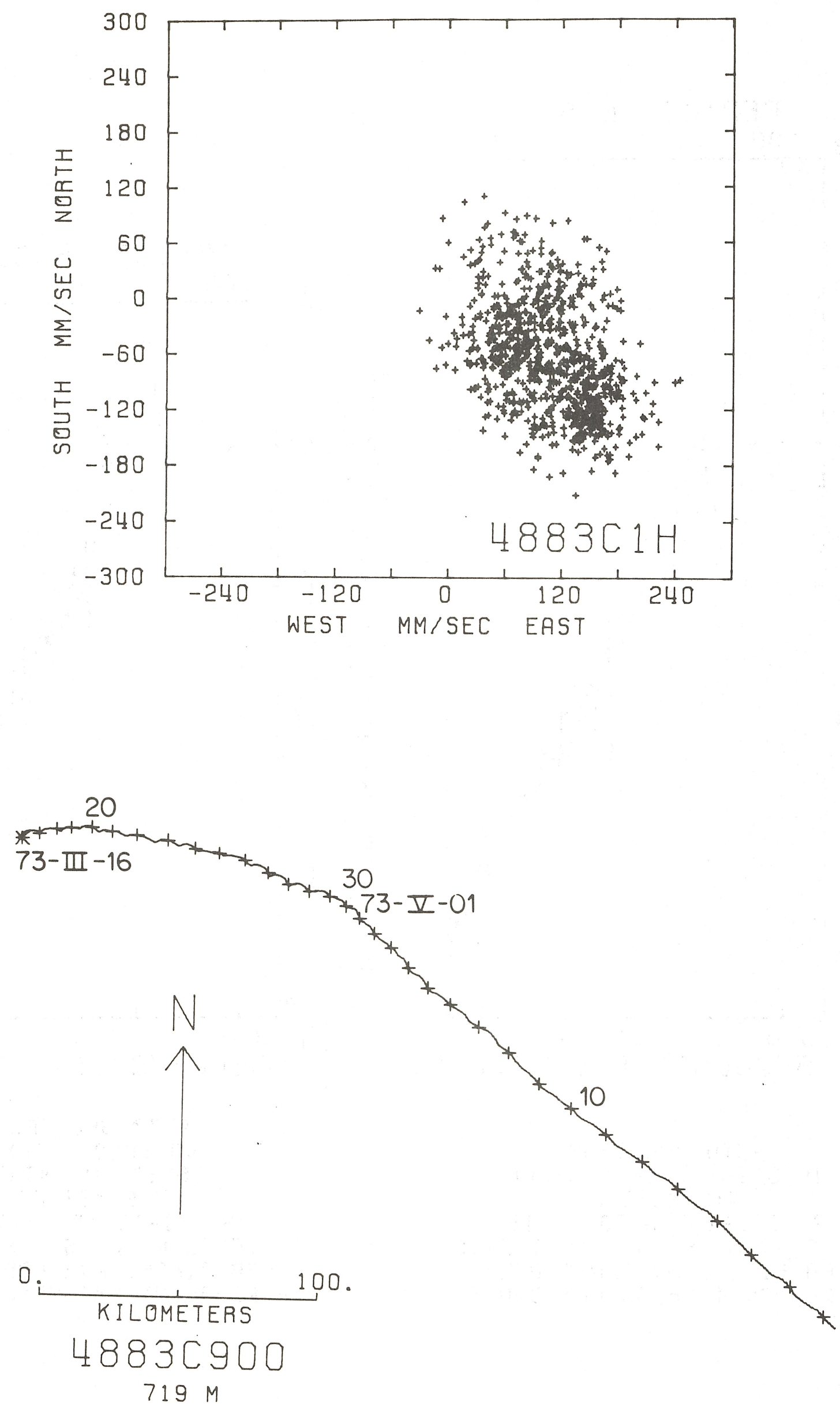

73- III-16 TO 73- IV - 17 

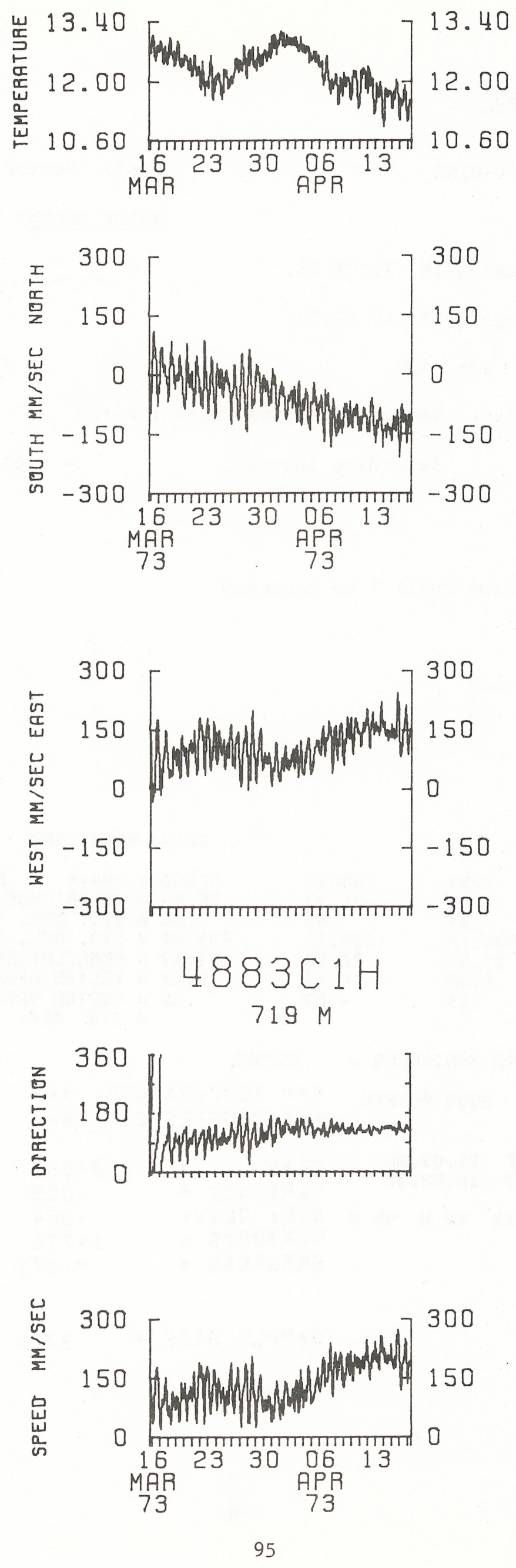
DATA NUMBER 4885

Instrument No.: $\mathrm{V}-0183$

Depth: $2952 \mathrm{~m}$

Start time: 73-March-15 21.07.36.

Stop time: 73-April-07 19.52.30.

Duration: $22 \mathrm{~d} \quad 22 \mathrm{~h} \quad 45 \mathrm{~m}$

Sampling scheme: Vector Averaging Current Meter

recording interval $=900$ seconds

COMMENTS :

Compass - good

Vane - sticky from April 7 to recovery

Rotor - good

Temperature - good

STคTร

\begin{tabular}{|c|c|c|c|}
\hline & & EAST & $\begin{array}{r}\text { MOFTH } \\
-45.77\end{array}$ \\
\hline DN Enn. & 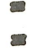 & $\begin{array}{r}.99 \\
.67\end{array}$ & .69 \\
\hline RIANCE & $\approx$ & 890.16 & 1056.21 \\
\hline STD. DEV. & $=$ & 91.47 & 2.50 \\
\hline $\begin{array}{l}\text { KURTOSIS } \\
\text { SREHNESS }\end{array}$ & 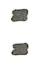 & $\begin{array}{l}.32 \\
.13\end{array}$ & - \\
\hline
\end{tabular}

Type: Vector Averaging Current Meter Water Depth: $5325 \mathrm{~m}$

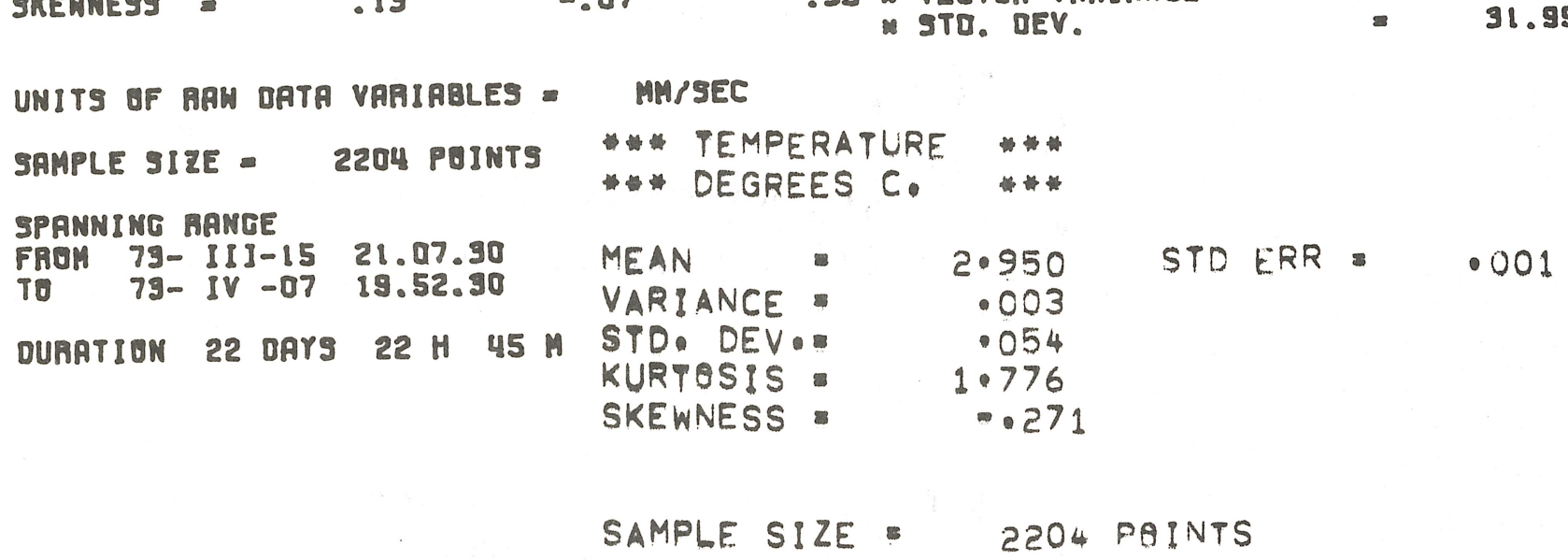



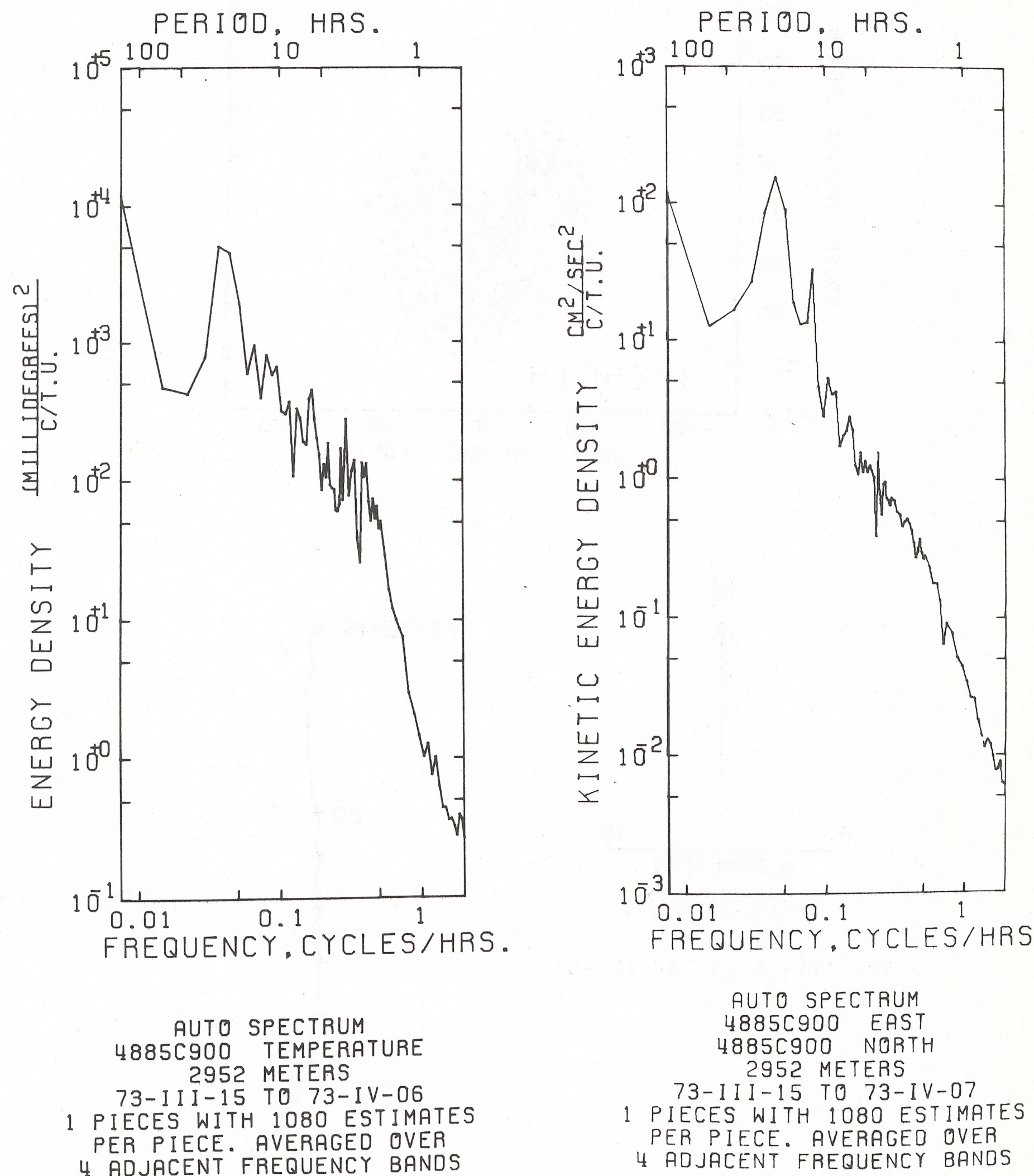

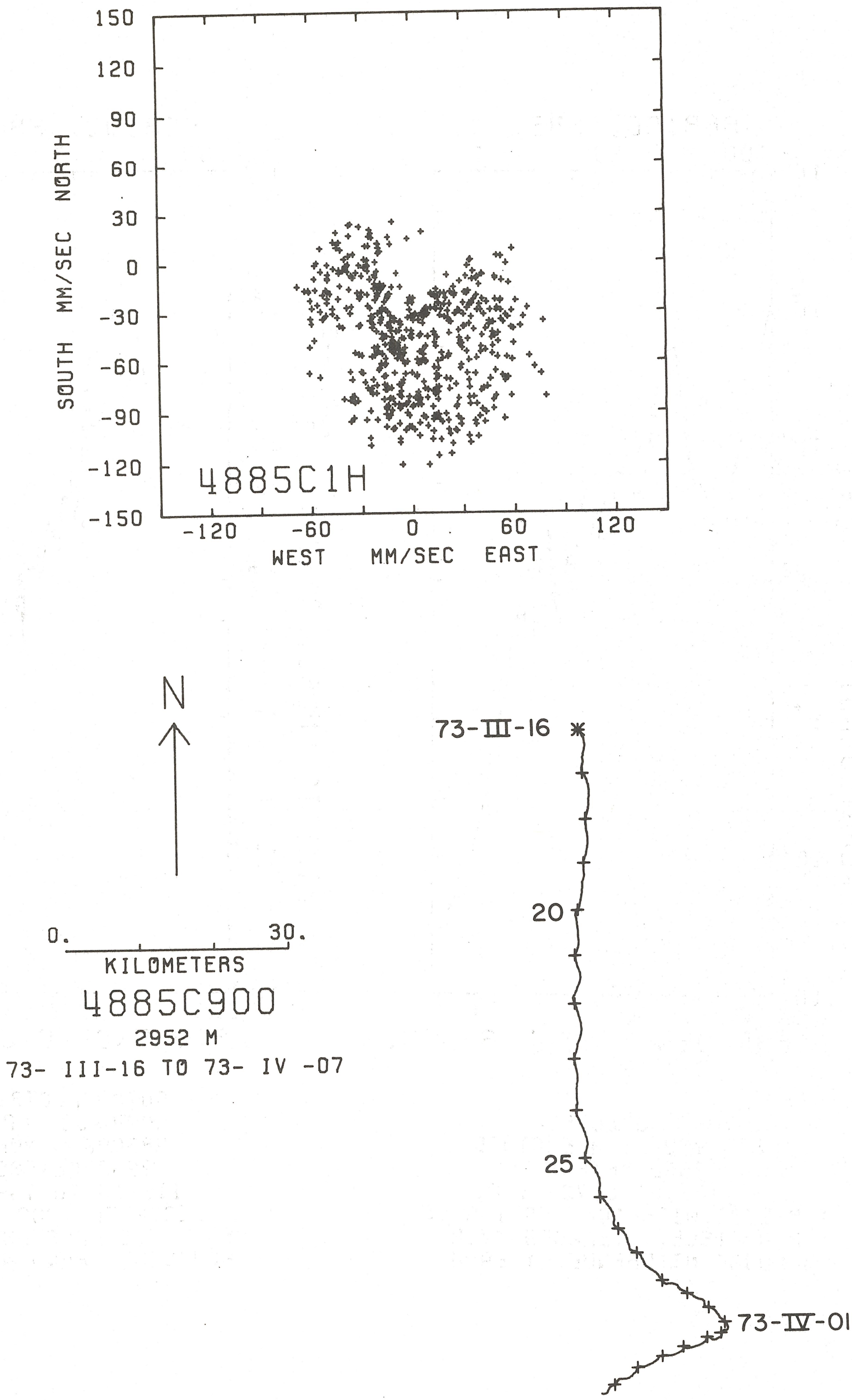

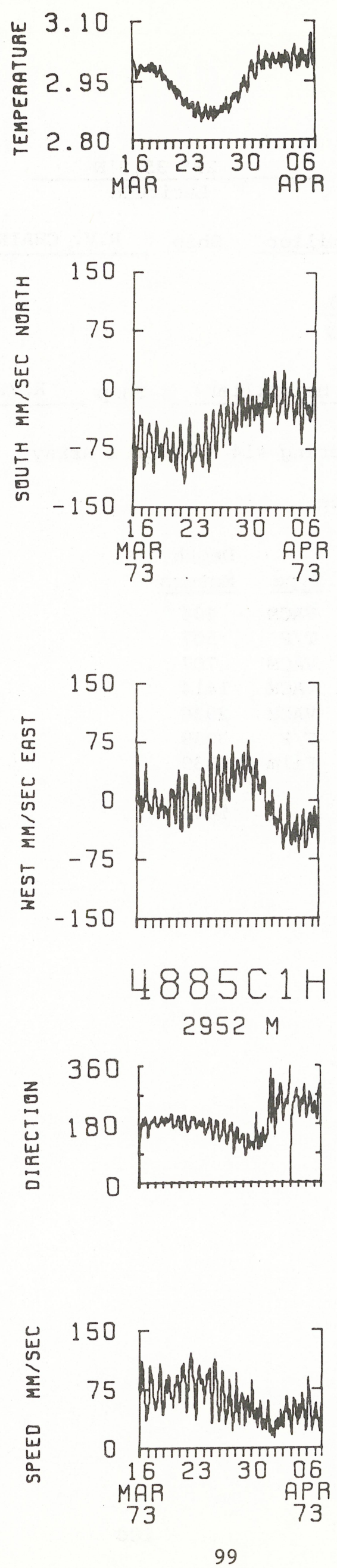
Mooring No. 489

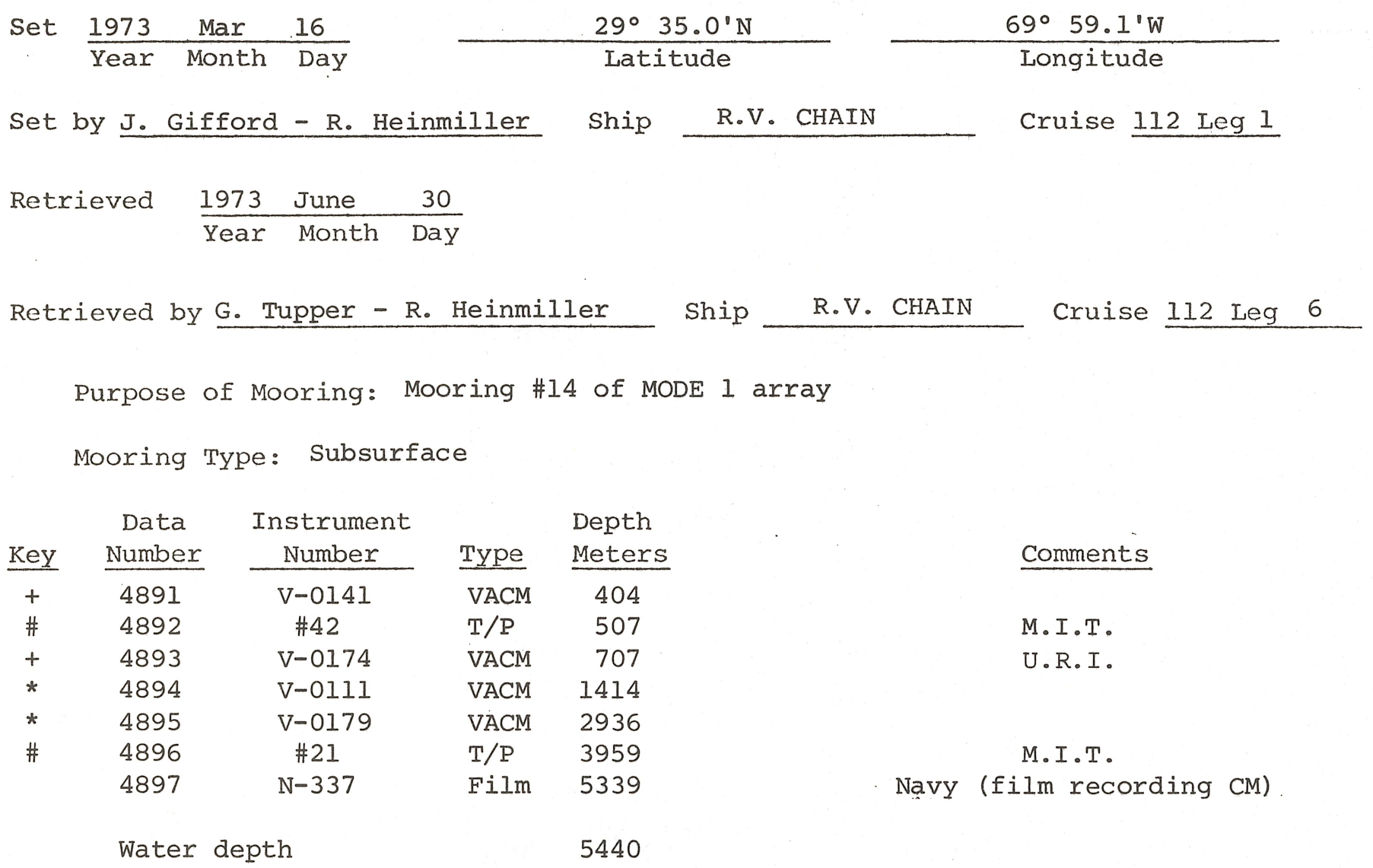

COMMENTS ON MOORING: 

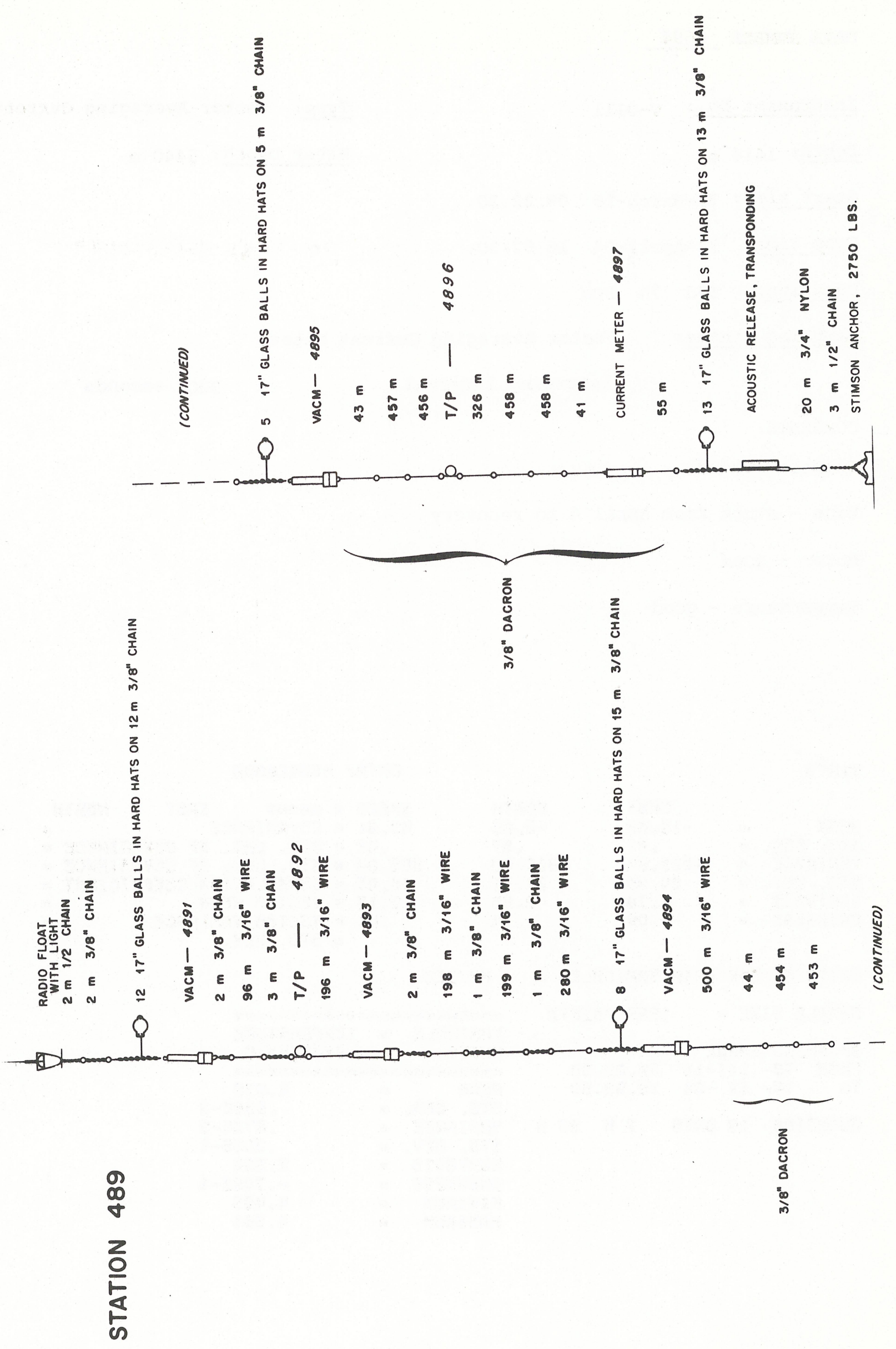
DATA NUMBER 4894

Instrument No.: $\quad \mathrm{V}-0111$

Depth: $1414 \mathrm{~m}$

Start time: 73-March-16 09.22.30.

Stop time: 73-April-04 16.52.30.

Duration: $\quad 19 \mathrm{~d}$ 7h $30 \mathrm{~m}$

Sampling scheme: Vector Averaging Current Meter

$$
\text { recording interval }=900 \text { seconds }
$$

COMMENTS :

Compass - good

Vane - stuck from April 4 to recovery

Rotor - good

Temperature - good

STATS

\section{DATAR 4OSHCSOOA}

\begin{tabular}{|c|c|c|c|}
\hline & & Е月ST & NOATH \\
\hline MEAN & 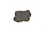 & -19.92 & -3.25 \\
\hline $\begin{array}{l}\text { STO. ERP. } \\
\text { VARIANCE }\end{array}$ & $\begin{array}{l}\nexists \\
\Rightarrow\end{array}$ & & \\
\hline STD. DEV. & $=$ & 34.98 & 24.51 \\
\hline KUATOSIS & $=$ & & \\
\hline WhES & 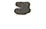 & & \\
\hline
\end{tabular}

UNITS OF RAW DATA VARJABLES :

SAMPLE SIZE = 2055 POINTS

SPANNING RANGE

FROM 73-III-16 09.22.30

TO $73-I V-04 \quad 16.52 .30$

OURATION 19 DAYS 7 H $30 \mathrm{M}$
Type: Vector Averaging Current Meter

Water Depth: $5440 \mathrm{~m}$ 

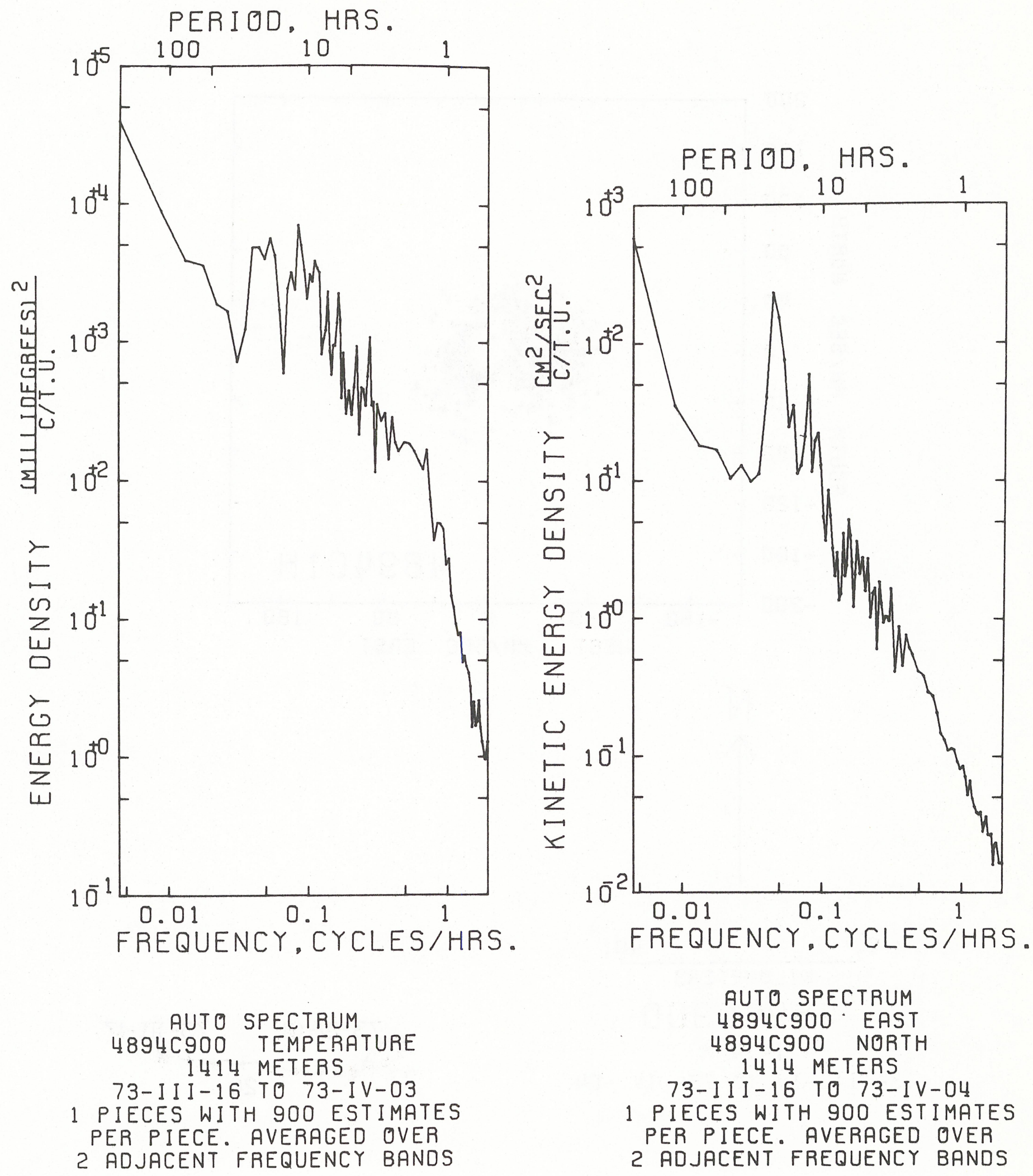

AUTO SPECTRUM 4894 C $9000^{\circ}$ EAST 4894 C900 NORTH 1414 METERS 73-III-16 TO 73-IV-04 1 PIECES WITH 900 ESTIMATES PER PIECE. AVERAGED OVER 2 ADJACENT FREQUENCY BANDS 

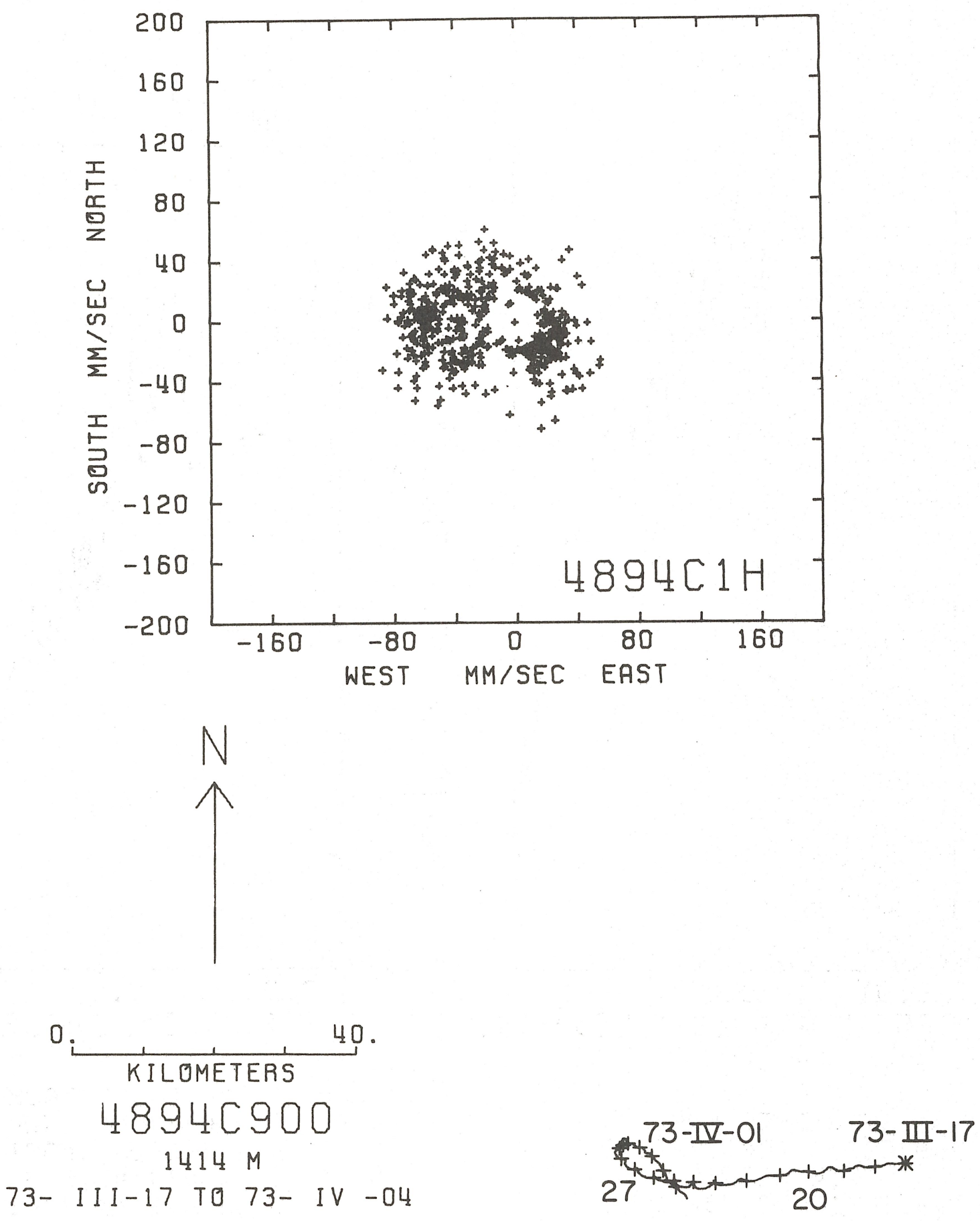

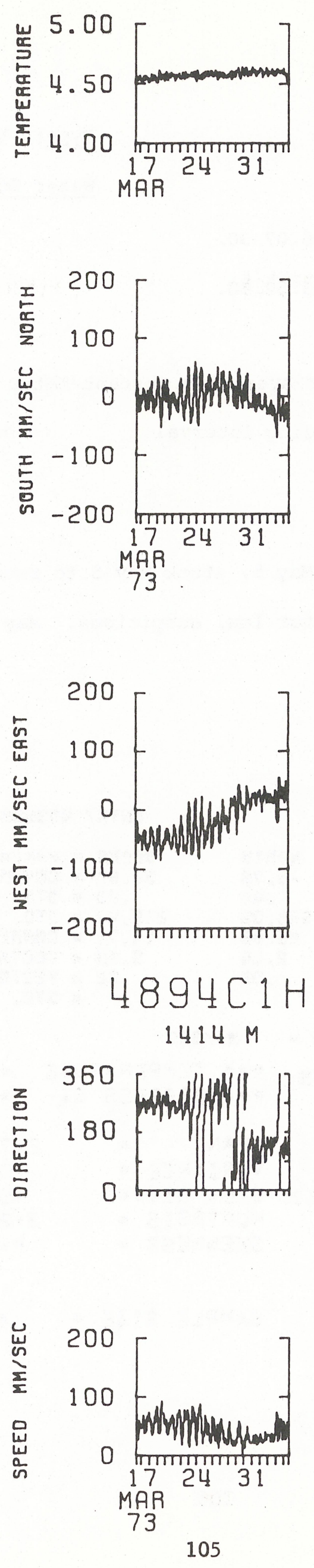
DATA NUMBER 4895

Instrument No.: $\mathrm{V}-0179$

Depth: $2936 \mathrm{~m}$
Type: Vector Averaging Current Meter Water Depth: $5440 \mathrm{~m}$

Start time: 73-March-16 16.07.30.

Stop time: 73-April-28 03.52.30.

Duration: $\quad 42 \mathrm{~d}$ 11h $45 \mathrm{~m}$

Sampling scheme: Vector Averaging Current Meter

$$
\text { recording interval } \quad=900 \text { seconds }
$$

COMMENTS :

Compass - good

Vane - sticky April 28 to May 5, stuck May 5 to recovery

Rotor - May 6 to May 19 rotor low, suspicious. May 19 to recovery rotor below threshold

Temperature - good

STATS

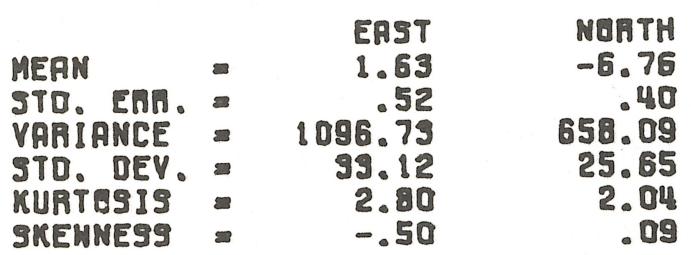

DคTคР 48958900ค

SPEED ะ $39.03 \times$ COVARIANCE $\cong-74.94$

$.23 \times$ 5TQ. ENR. OF COVARIANCE - 10.59

$216.47 \times$ STD. DEV. OF COVARIANCE $=676.50$

14.71 CORAELATION COEFFJCIENT $=-.088$

3.66 * VECTOA MEAN

.92 V VECTOA VARIANCE

M STQ. DEV.
6.95

077.41

29.62

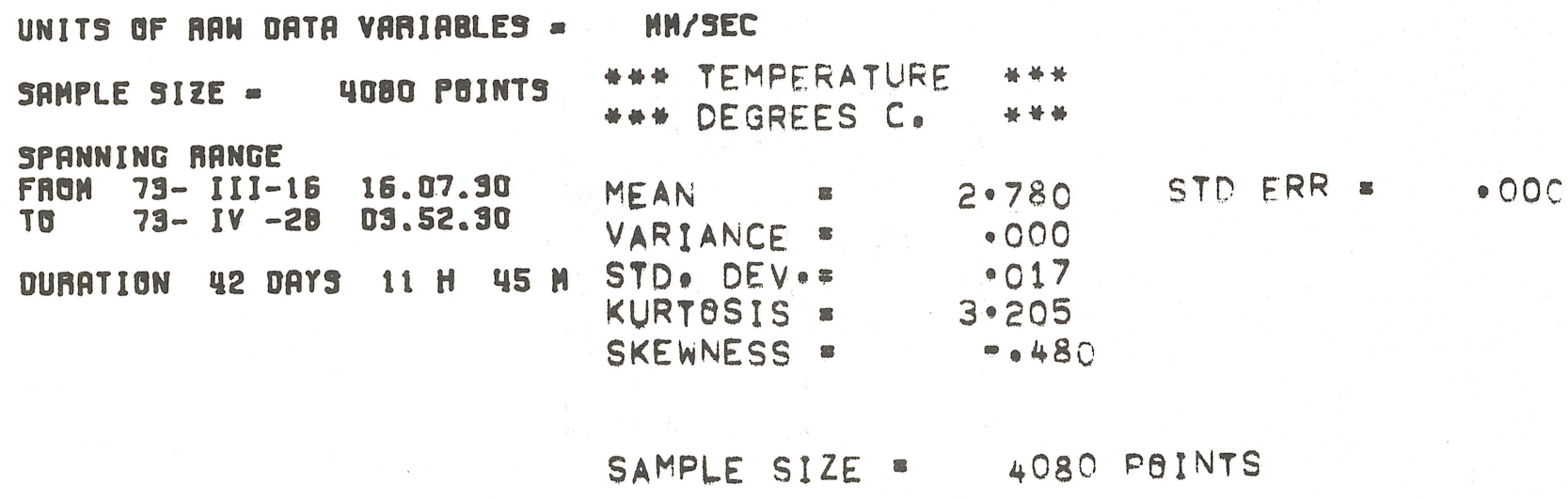



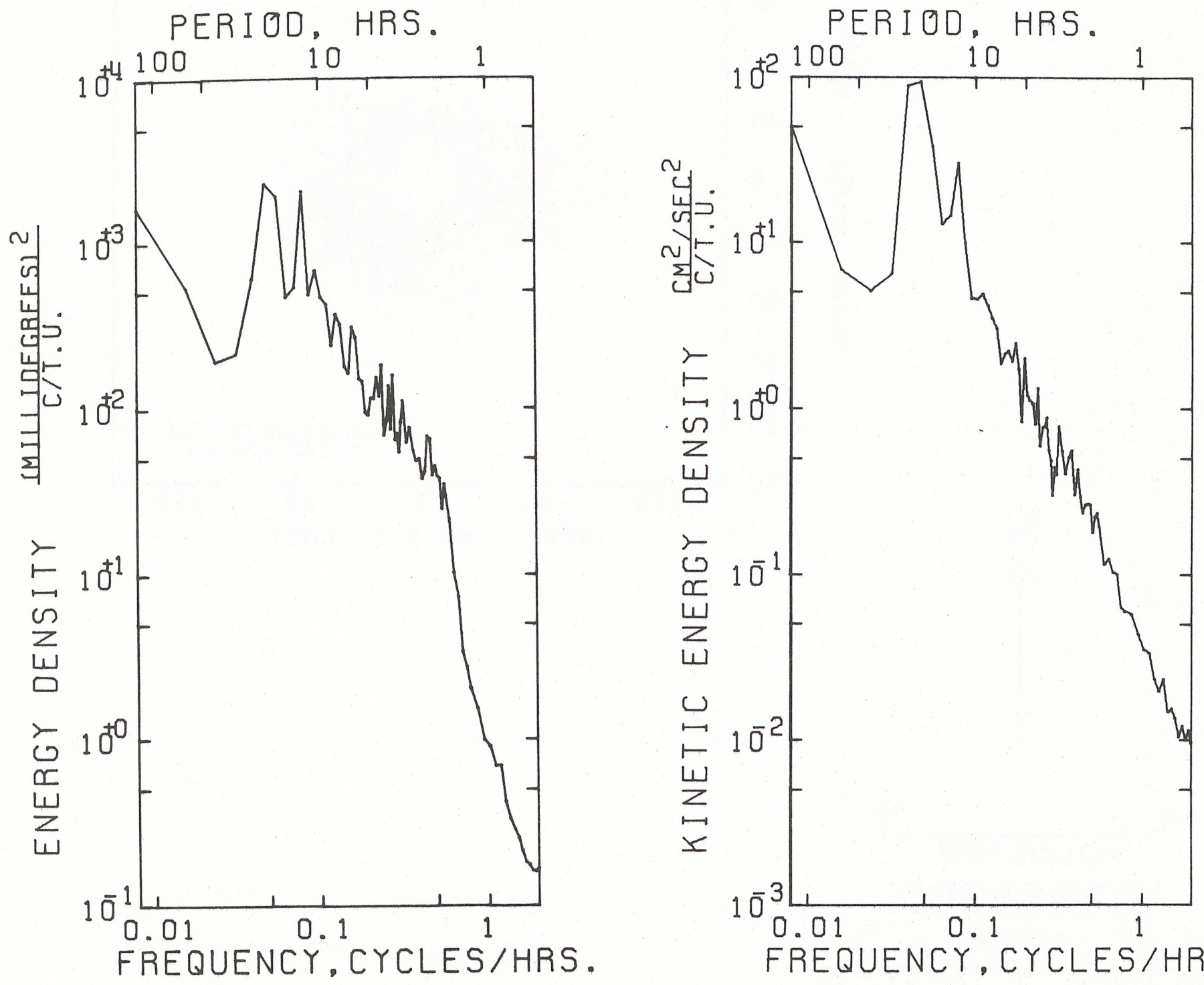

FREQUENCY, CYCLES/HRS.

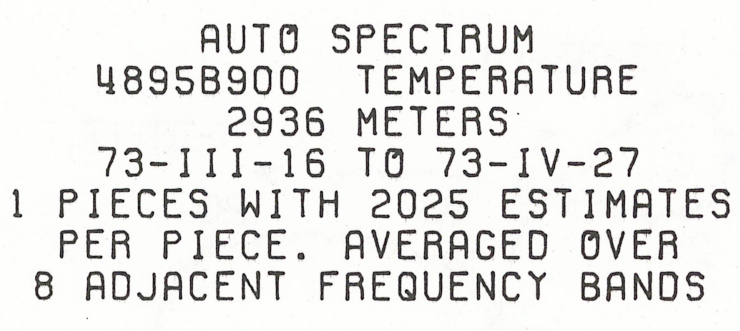

AUTO SPECTRUM

900 TEMPERATURE

I-16 TO 73-IV-27

PIECES WITH 2025 ESTIMATES

PER PIECE. AVERAGED OVER

ADJACENT FREQUENCY BANDS 

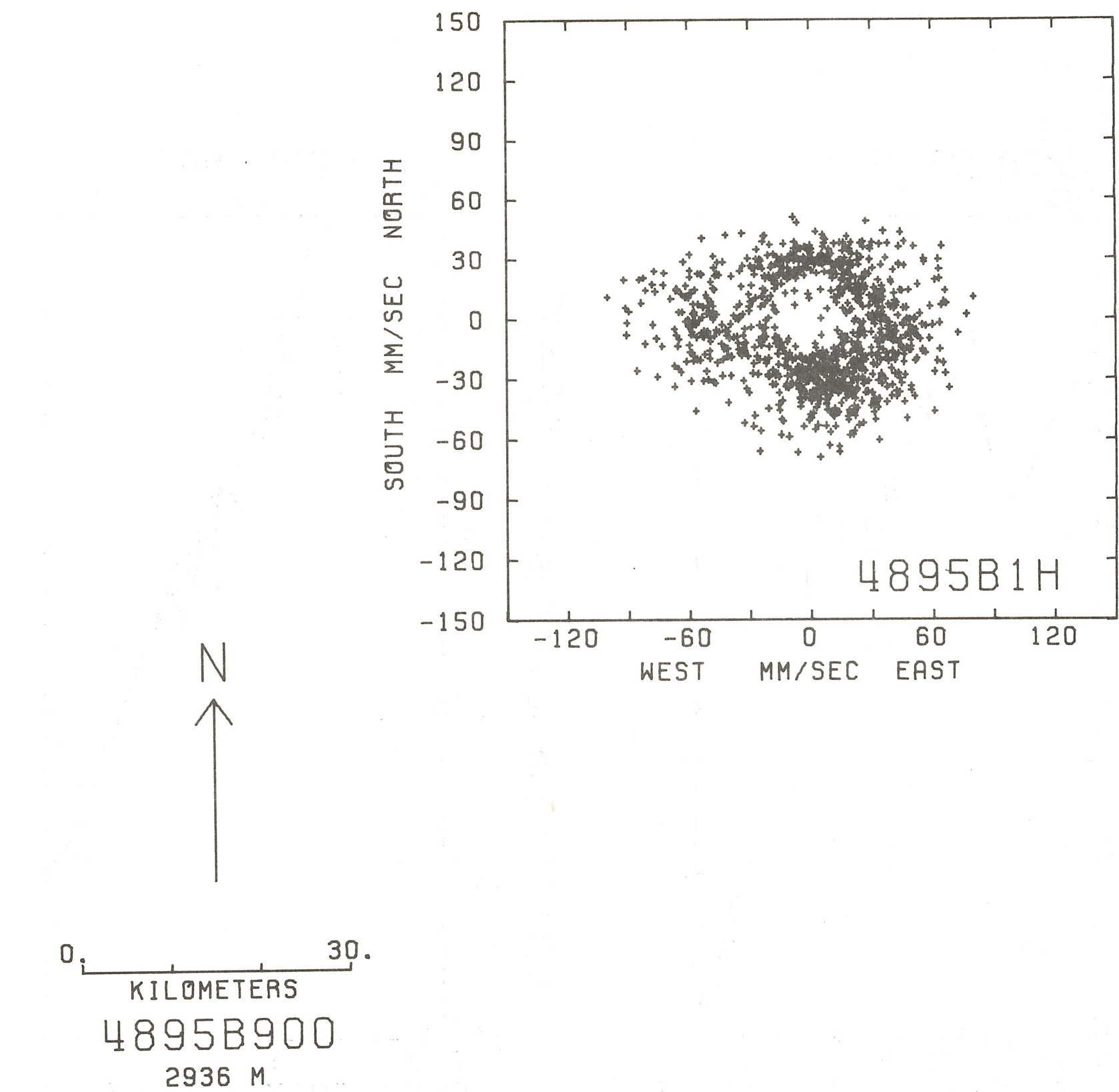

73- III-17 TO 73- IV - 28

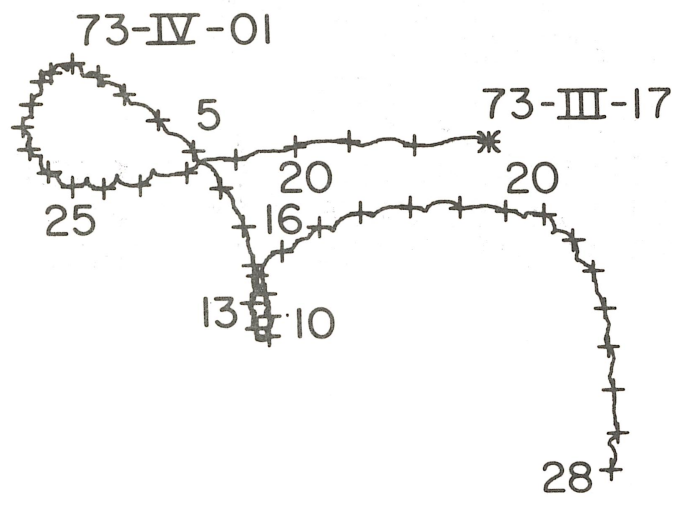



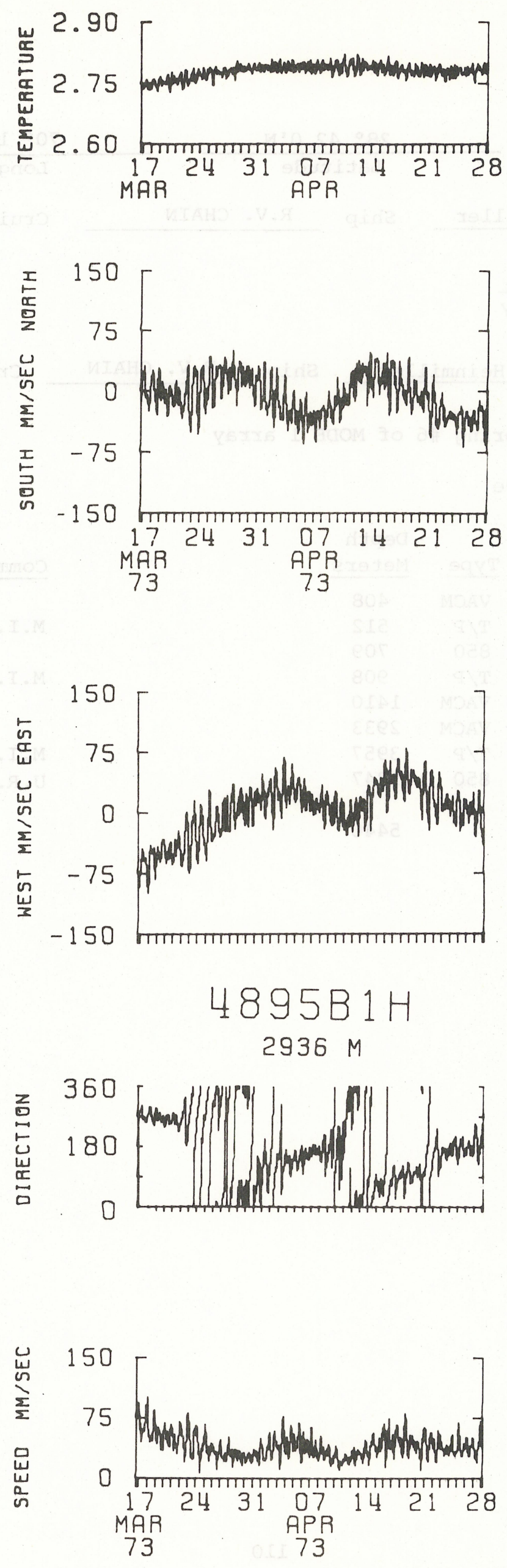
Mooring No. 493

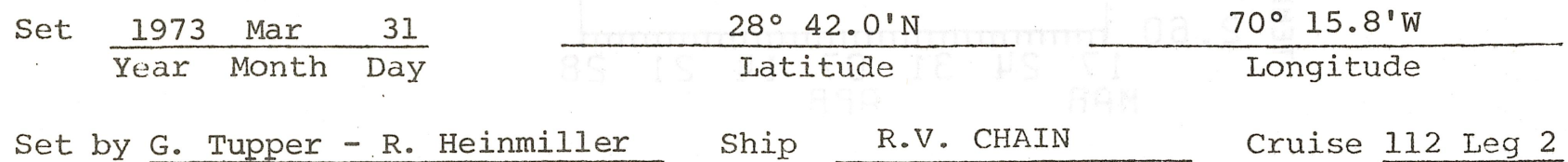

Set by G. Tupper - R. Heinmiller Ship R.V. CHAIN Cruise 112 Leg 2

Retrieved $\frac{1973 \text { June } \quad 30}{\text { Year Month Day }}$

Retrieved by G. Tupper - R. Heinmiller Ship R.V. CHAIN Cruise II2 Ieg 6

Purpose of Mooring: Mooring \#6 of MODE 1 array

Mooring Type: Subsurface

\begin{tabular}{|c|c|c|c|c|c|}
\hline Key & $\begin{array}{c}\text { Data } \\
\text { Number } \\
\end{array}$ & $\begin{array}{c}\text { Instrument } \\
\text { Number }\end{array}$ & Type & $\begin{array}{l}\text { Depth } \\
\text { Meters } \\
\end{array}$ & Comments \\
\hline * & 4931 & $V-0199$ & VAC.M & 408 & \\
\hline$\#$ & 4932 & \#34 & $\mathrm{T} / \mathrm{P}$ & 512 & M.I.T. \\
\hline * & 4933 & $M-142 t$ & 850 & 709 & \\
\hline$\#$ & 4934 & \#52 & $\mathrm{T} / \mathrm{P}$ & 908 & M.I.T. \\
\hline * & 4935 & $V-0195$ & $\mathrm{VACM}$ & 1410 & \\
\hline * & 4936 & $V-0138$ & VACM & 2933 & \\
\hline \multirow[t]{2}{*}{$\#$} & 4937 & \#25 & $\mathrm{T} / \mathrm{P}$ & 3957 & M.I.T. \\
\hline & 4938 & $M-179$ & 850 & 5347 & U.R.I. \\
\hline & Water & pth & & 5446 & \\
\hline
\end{tabular}

COMMENTS ON MOORING: 


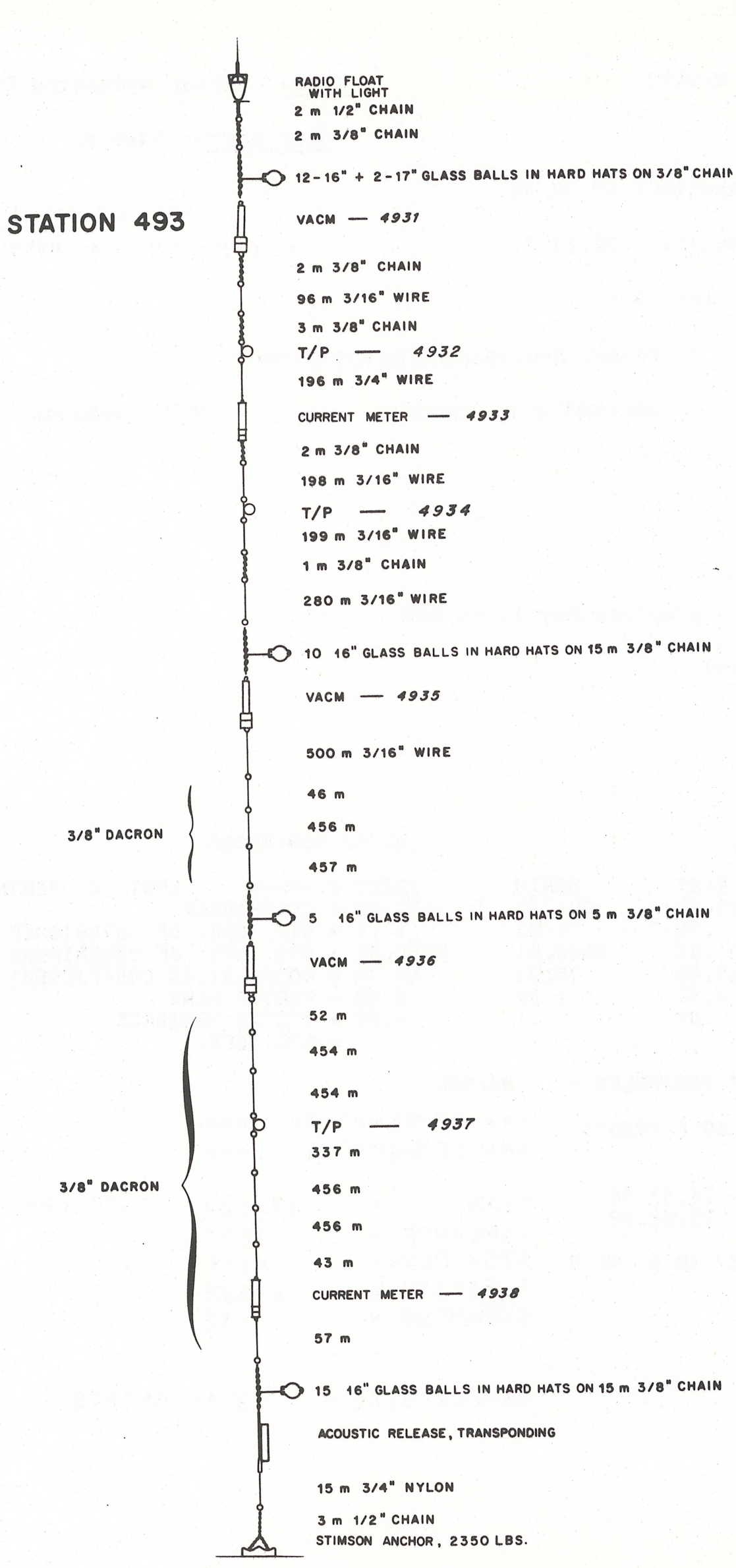


DATA NUMBER 4931

Instrument No.: V-0199

Depth: $408 \mathrm{~m}$

Start time: 73-April-01 09.07.30.

Stop time: 73-May-11 23.52.30.

Duration: $40 \mathrm{~d}$ 18h $45 \mathrm{~m}$

Sampling scheme: Vector Averaging Current Meter

$$
\text { recording interval = } 900 \text { seconds }
$$

COMMENTS :

Compass - good

Vane - good

Rotor - threshold problems May 12 to end

Temperature - good

STATS

$\begin{array}{lrrr} & & \text { EAST } & \text { NORTH } \\ \text { MEAN } & = & 84.24 & 241.85 \\ \text { STO. ERR. } & .76 & 1.23 \\ \text { VARIANCE }= & 2251.45 & 5886.81 \\ \text { STD. DEV. } & 47.45 & 76.73 \\ \text { KURTOSIS }= & 2.52 & 1.88 \\ \text { SKEHNESS }= & -.09 & .21\end{array}$

DATAR 4931B900A

\section{Type: Vector Averaging Current Meter} Water Depth: $5446 \mathrm{~m}$

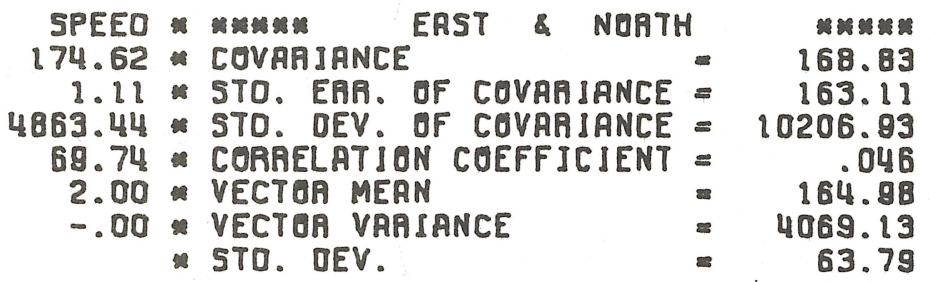

UN』TS OF RAW DATA VAR』ABLES = MMPSEC

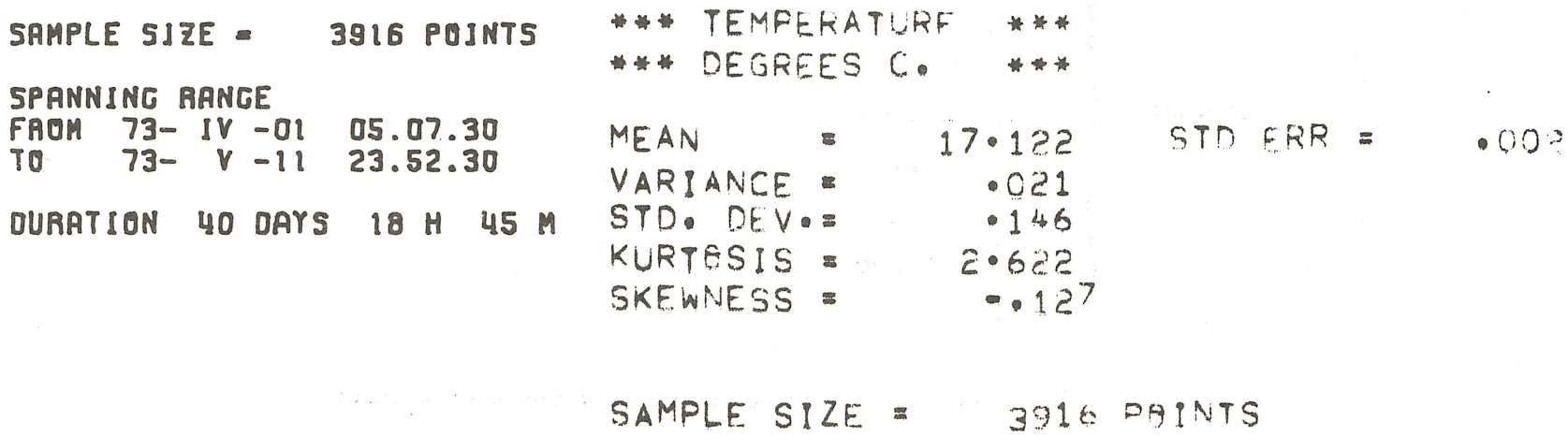



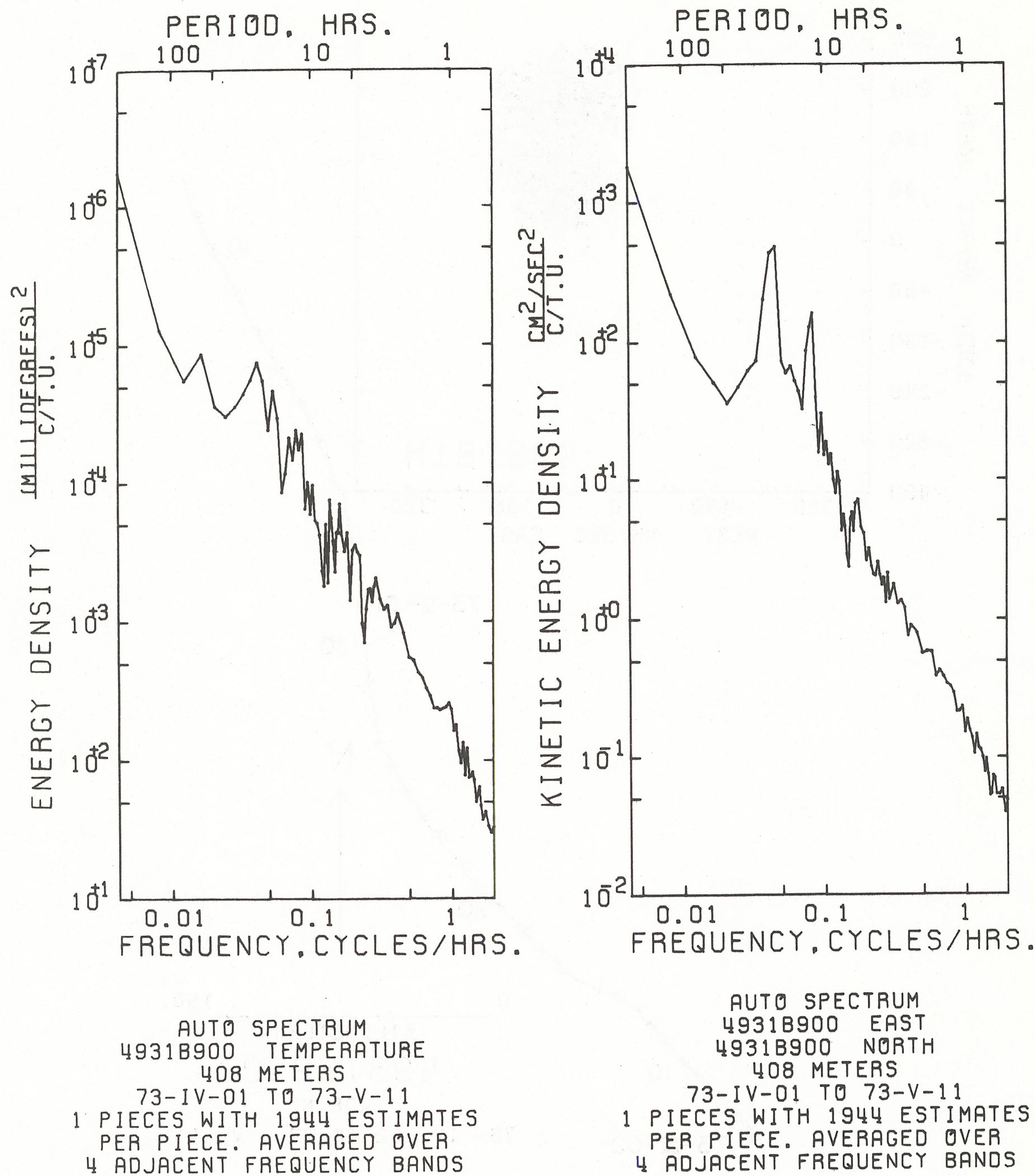


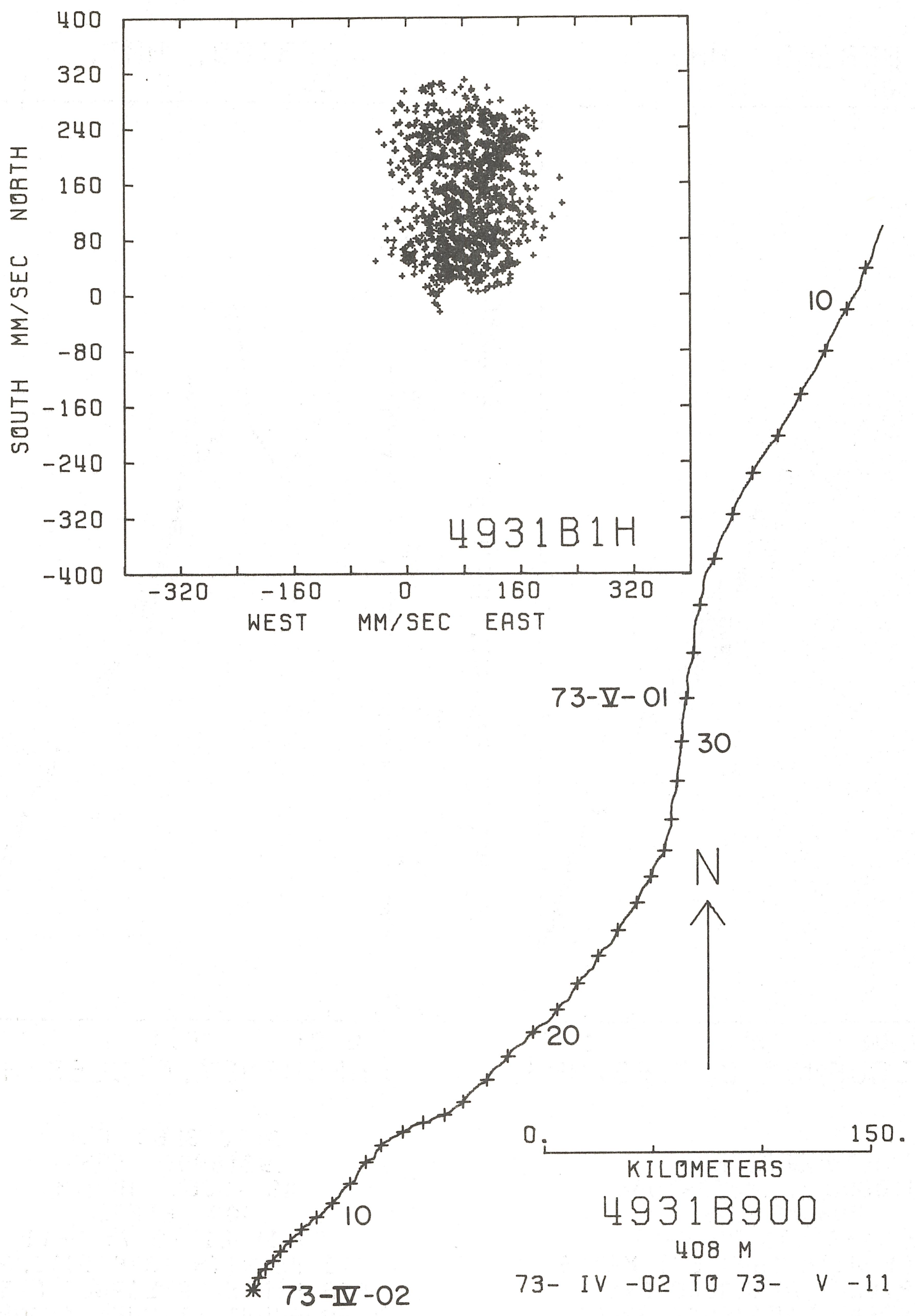



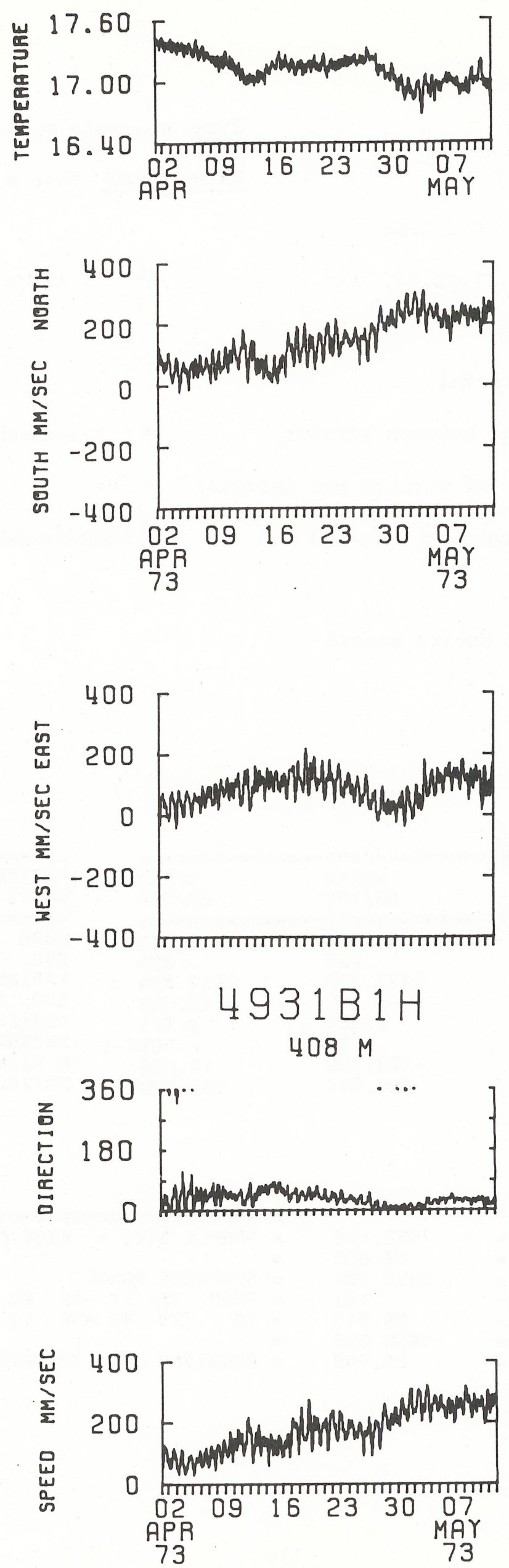
DATA NUMBER 4933

Instrument No.: $\mathrm{M}-142 \mathrm{t}$

Depth: $709 \mathrm{~m}$

Start time: 73-Apri1-02 00.03.34

Stop time: 73-June-30 17.33.34

Duration: $89 \mathrm{~d}$ 17h $30 \mathrm{~m}$

Sampling scheme: Interval
Type: Magnetic Tape Recording Current Meter Water depth: $5446 \mathrm{~m}$

$$
\begin{array}{ll}
\text { time between strobes } & =5.27 \text { seconds } \\
\text { no. of strobes per interval } & =13 \\
\text { recording interval } & =1800 \text { seconds }
\end{array}
$$

COMMENTS :

All variables look good entire record

DATA/ Y93301800

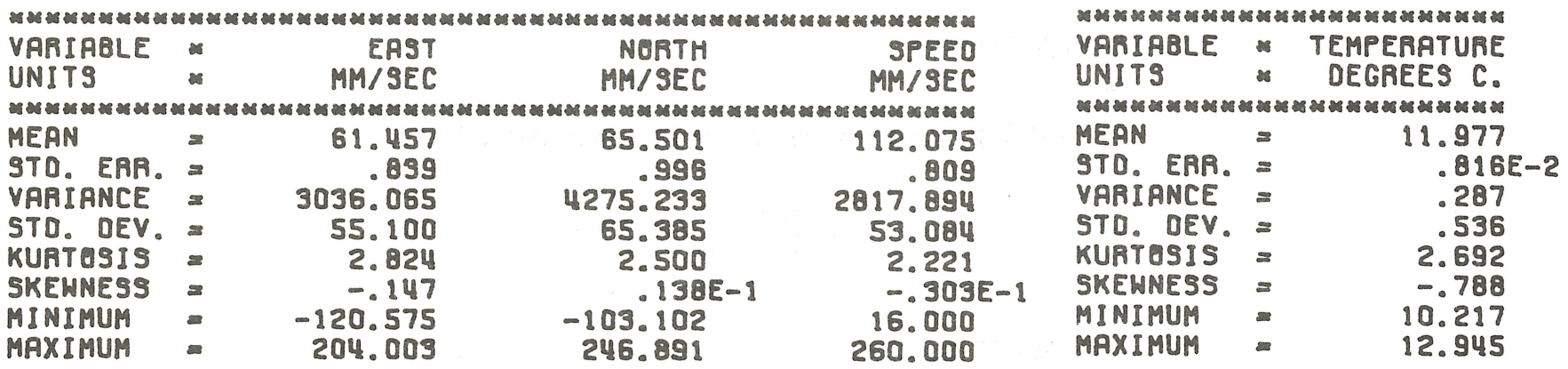

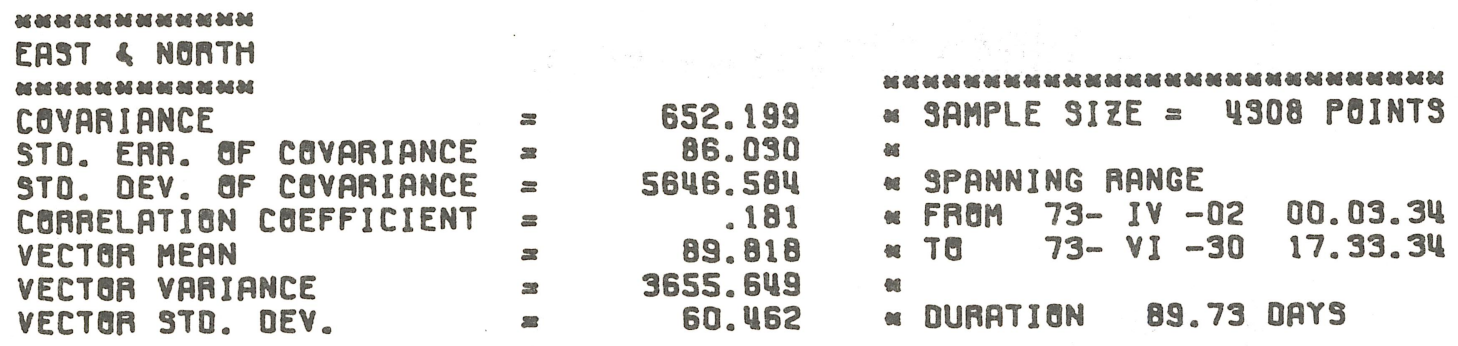



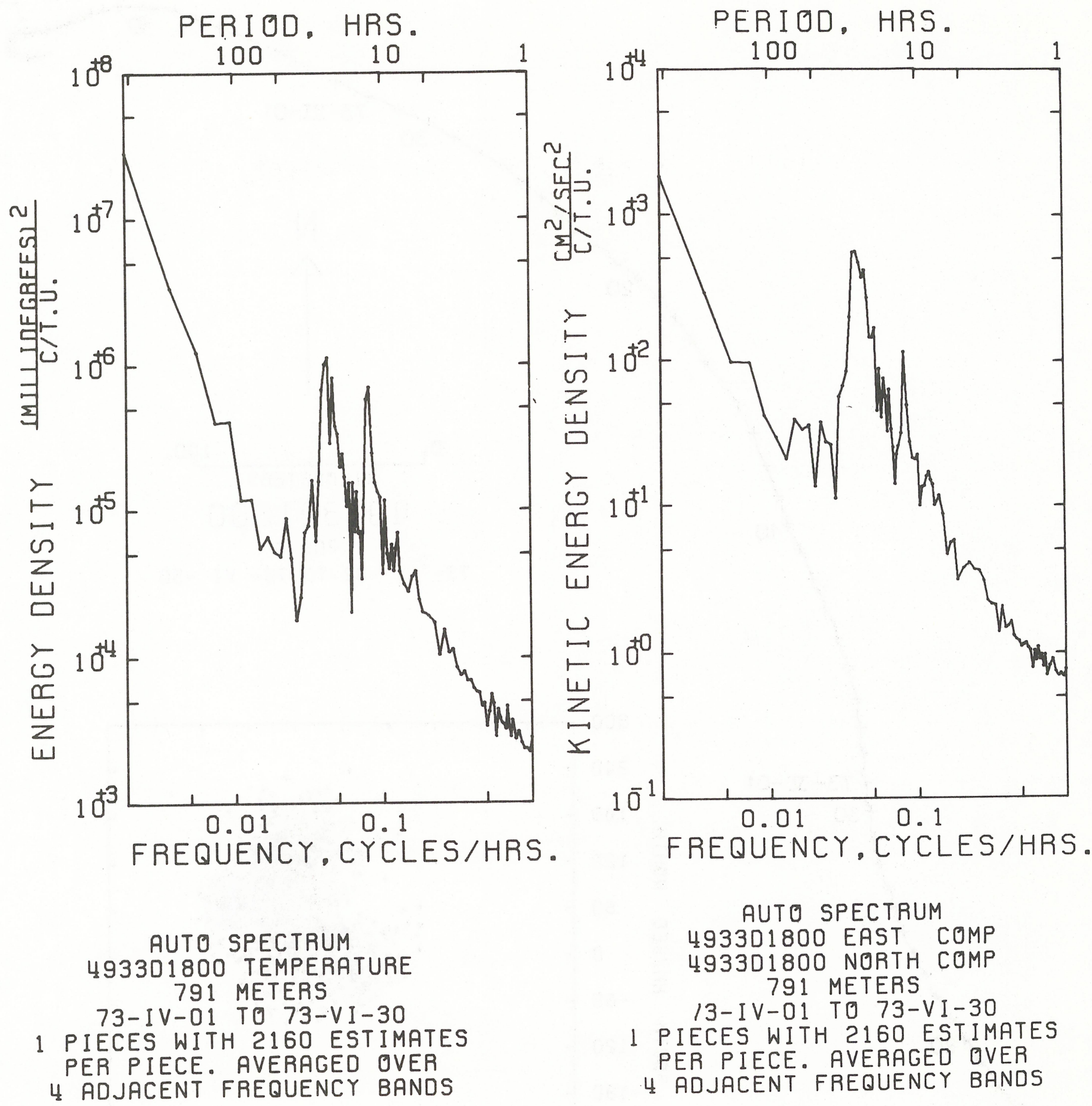

AUTO SPECTRUM 493301800 EAST COMP 493301800 NORTH COMP 791 METERS 13-IV-01 TO 73-VI-30

1 PIECES WITH 2160 ESTIMATES PER PIECE. AVERAGED OVER 4 ADJACENT FREQUENCY BANDS 


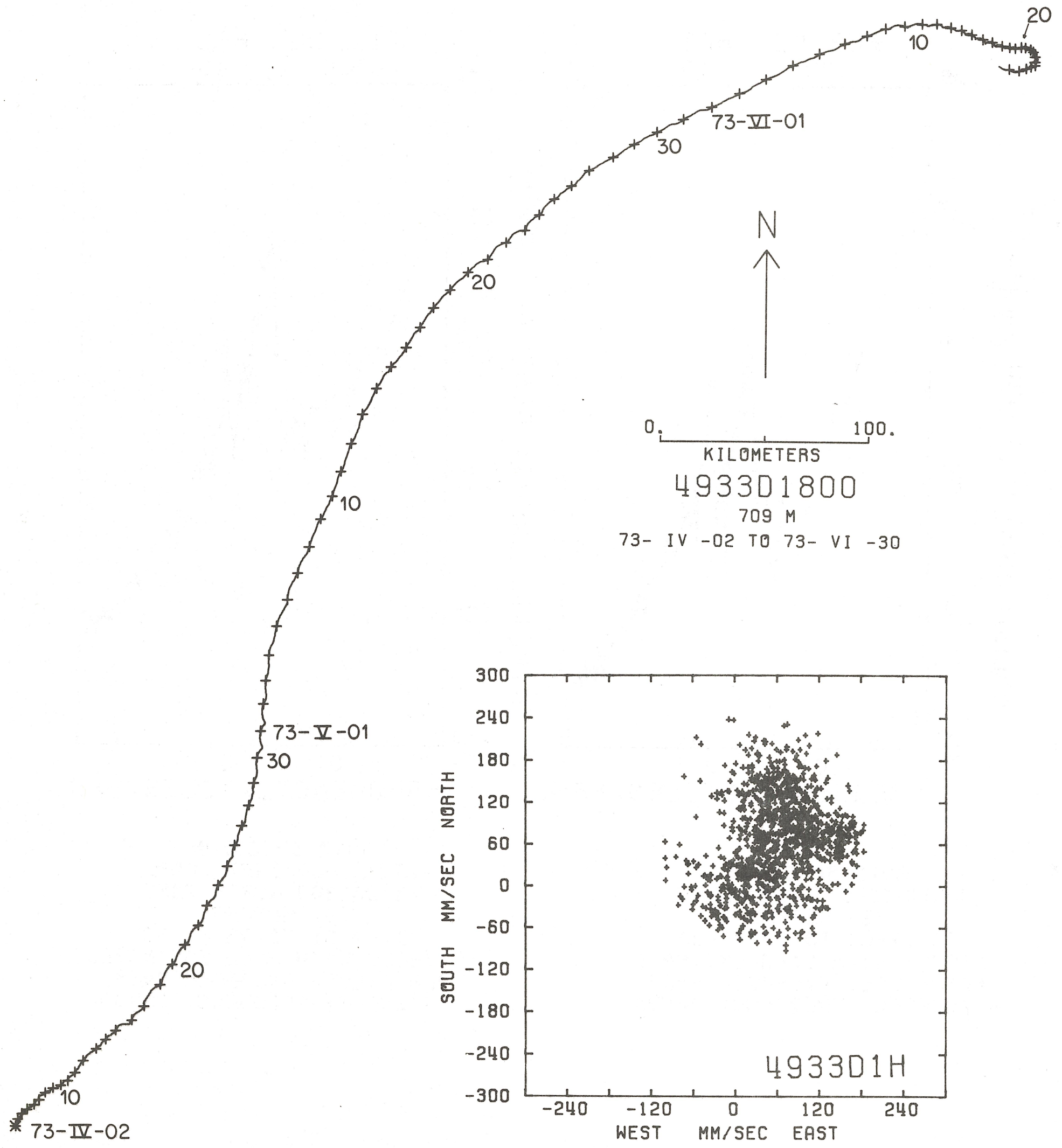



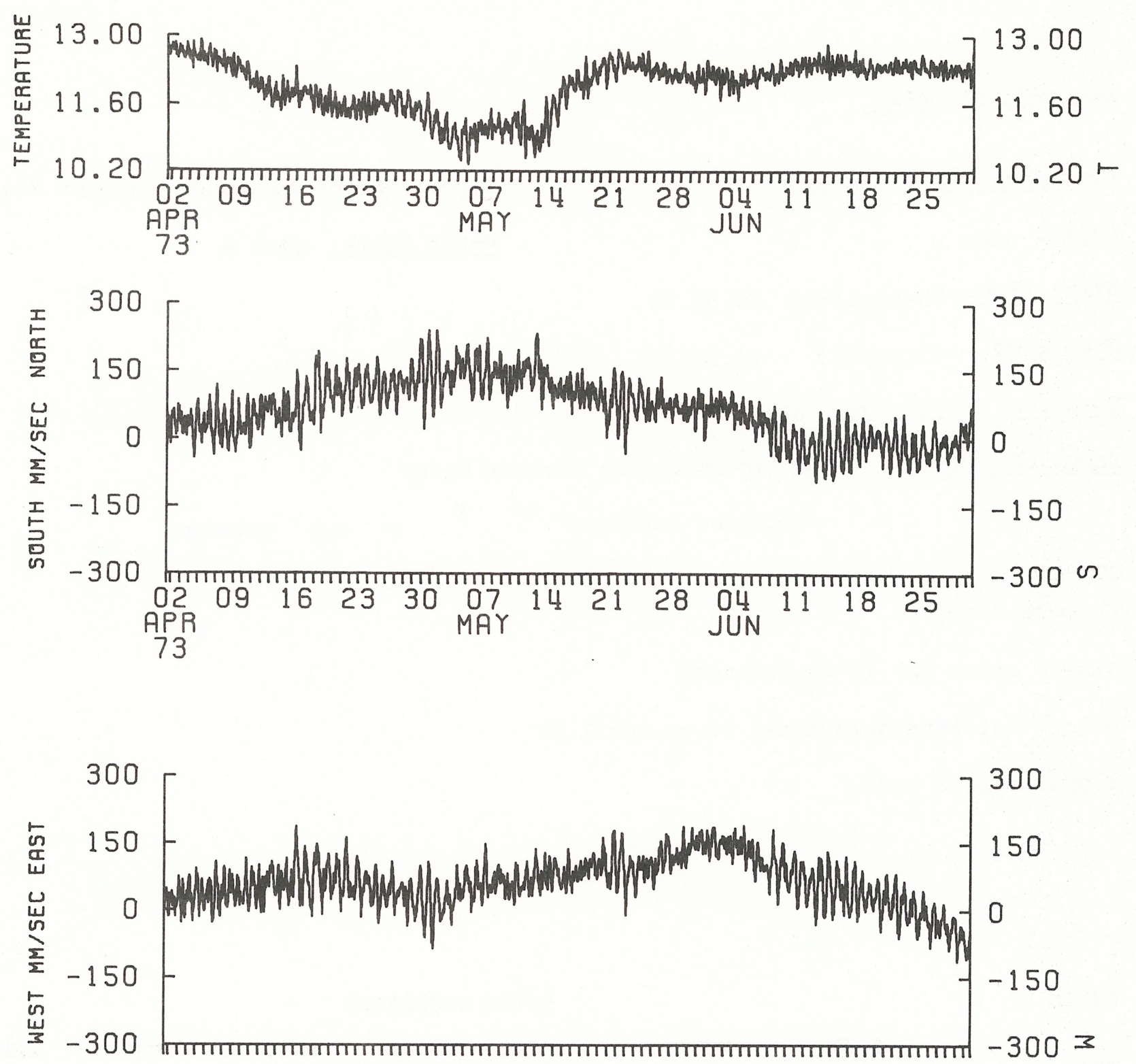
$493301 \mathrm{H}$
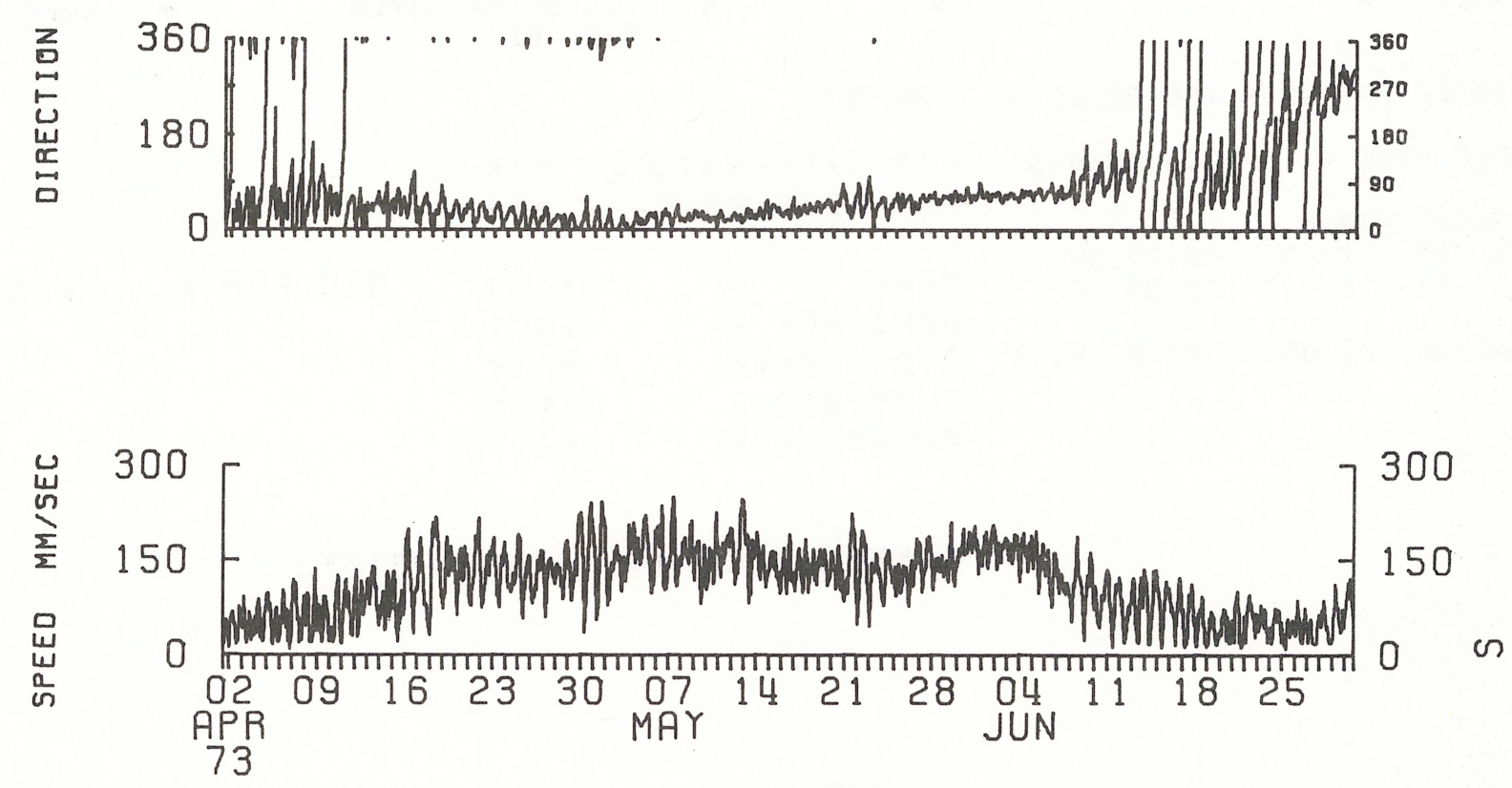
DATA NUMBER 4935

Instrument No.: $\mathrm{V}-0195$

Depth: $1410 \mathrm{~m}$

Start time: 73-April-21 00.07.30.

Stop time: 73-May-13 23.52.30.

Duration: $22 \mathrm{~d} \quad 23 \mathrm{~h} \quad 45 \mathrm{~m}$

Sampling scheme: Vector Averaging Current Meter

$$
\text { recording interval }=900 \text { seconds }
$$

COMMENTS :

Compass - good

Vane - stuck May 14 to recovery

Rotor - at threshold April 10 to April 20

Temperature - good

รTคTร

DATAR 49358900B

$\begin{array}{lrrr} & & \text { EAST } & \text { NORTH } \\ \text { MEAN } & 4.11 & 20.00 \\ \text { STD. ERR. } & 4 & .71 & .69 \\ \text { VARIANCE }= & 1116.11 & 1045.51 \\ \text { STD DEV. }= & 33.41 & 32.33 \\ \text { KURTESIS }= & 2.68 & 2.98 \\ \text { SKEWNESS } \Rightarrow & -.13 & -.00\end{array}$

UNITS OF RAW DATA VARIABLES $=$ MMSEC

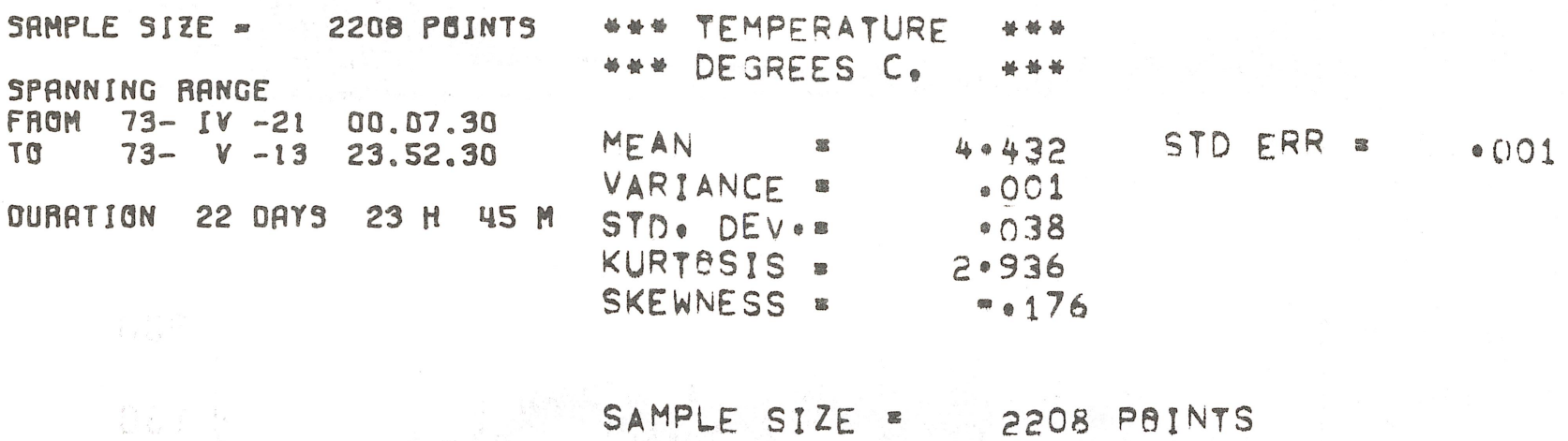



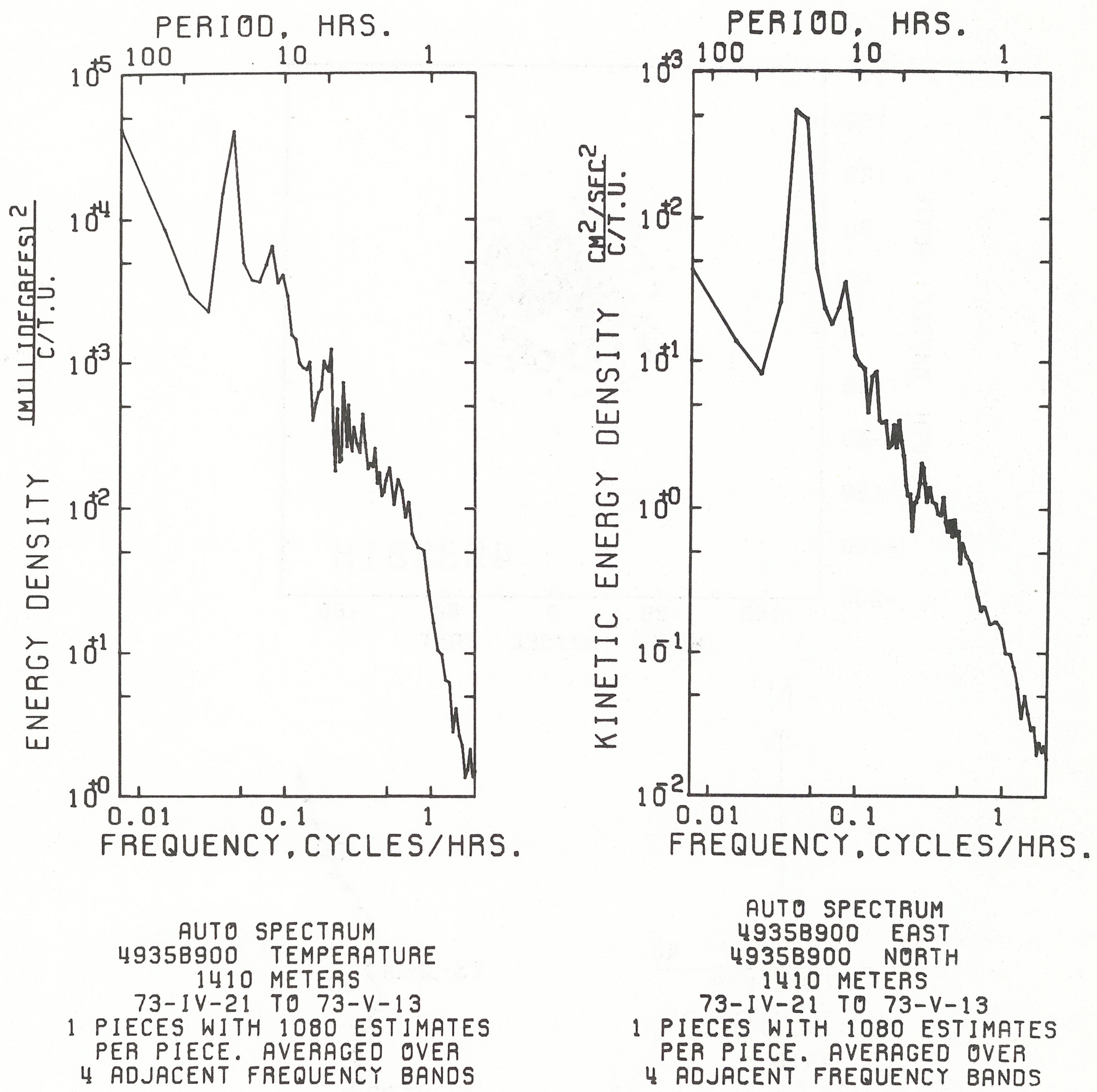

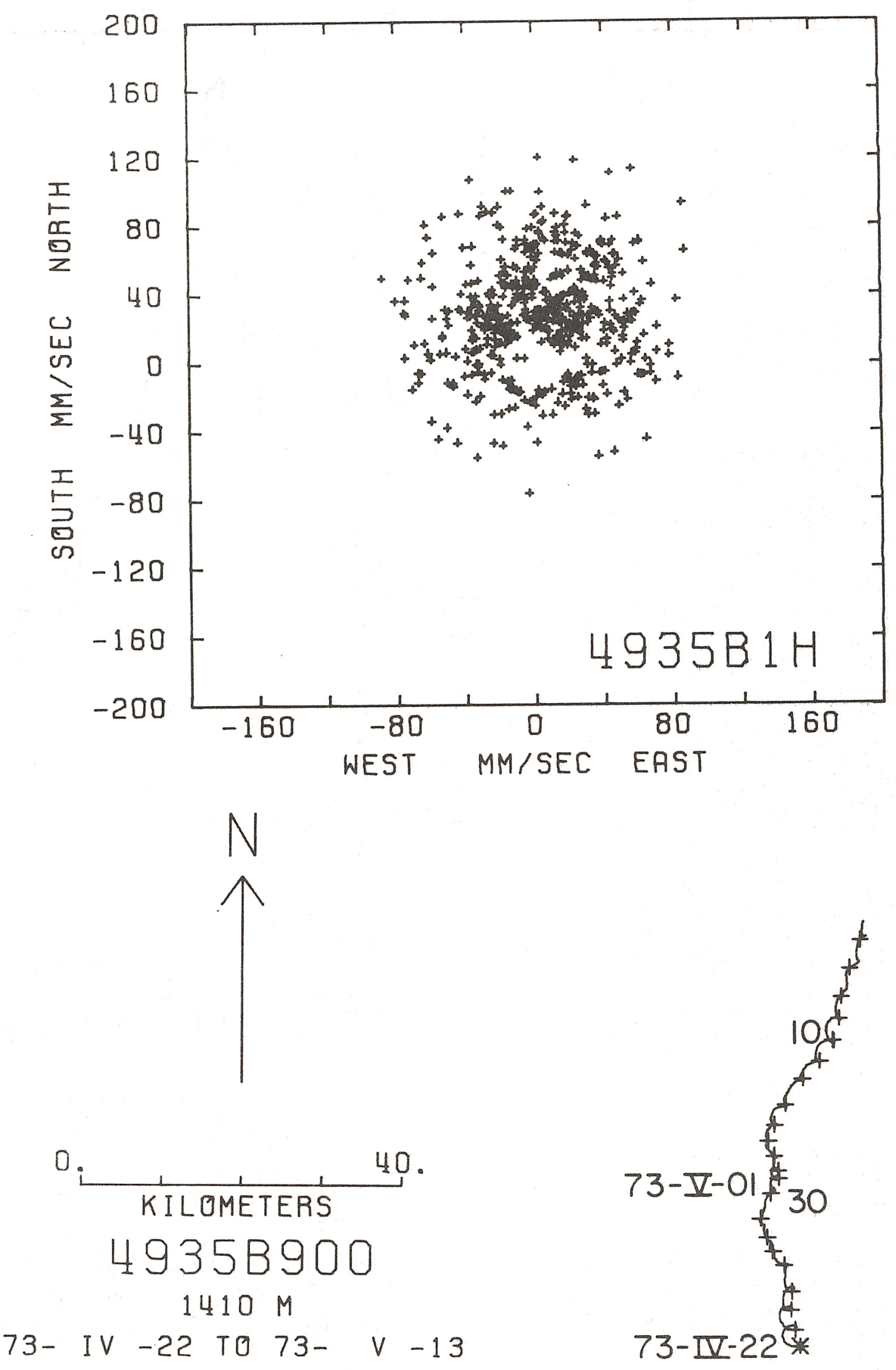

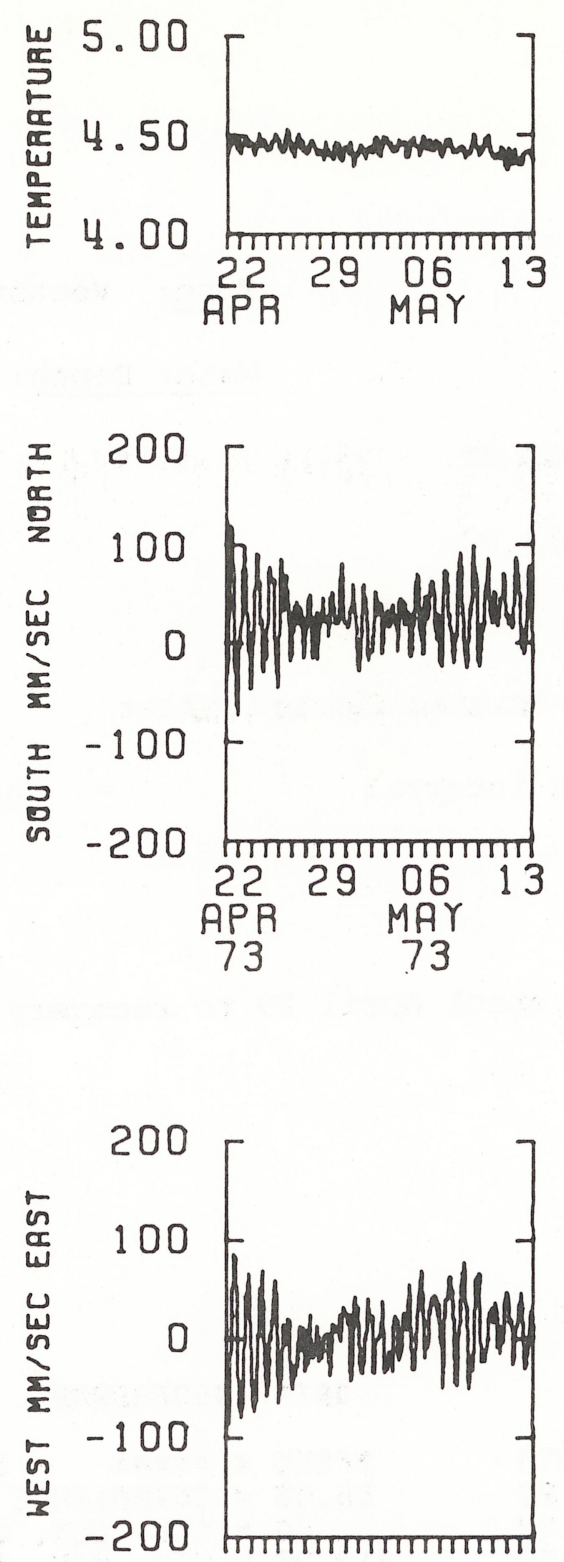

$4935 \mathrm{~B} 1 \mathrm{H}$

$1410 \mathrm{M}$
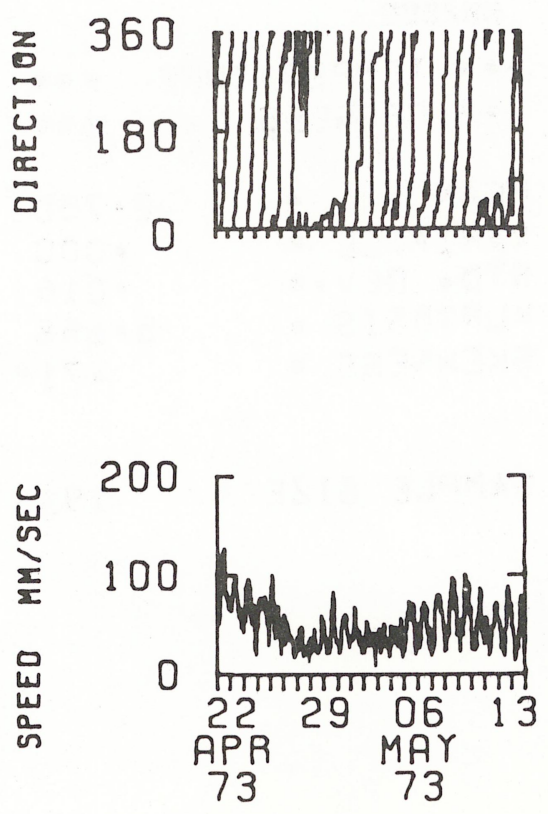
DATA NUMBER 4936

Instrument No.: $\mathrm{V}-0138$

Depth: $2933 \mathrm{~m}$

Start time: 73-Apri1-01 01.07.30.

Stop time: 73-April-21 04.52.30.

Duration: $\quad 20 d \quad 3 h \quad 45 m$

Sampling scheme: Vector Averaging Current Meter

$$
\text { recording interval }=900 \text { seconds }
$$

COMMENTS:

Compass - good

Vane - sticky April 21 to 29, stuck April 29 to recovery

Rotor - good

Temperature - good
Type: Vector Averaging Current Meter

Water Depth: $5446 \mathrm{~m}$
STATS

$\begin{array}{lrr} & \text { EAST } & \text { NOATH } \\ \text { MEAN } & -21.83 & 48.25 \\ \text { STD. ERA. } & .43 & .42 \\ \text { VARIANCE } \cong & 361.45 & 994.00 \\ \text { STD. DEV. } & 19.01 & 18.28 \\ \text { KURTESIS } \cong & 9.08 & 9.22 \\ \text { SKENNESS } & -.39 & -.20\end{array}$

DATAR $49360900 A$

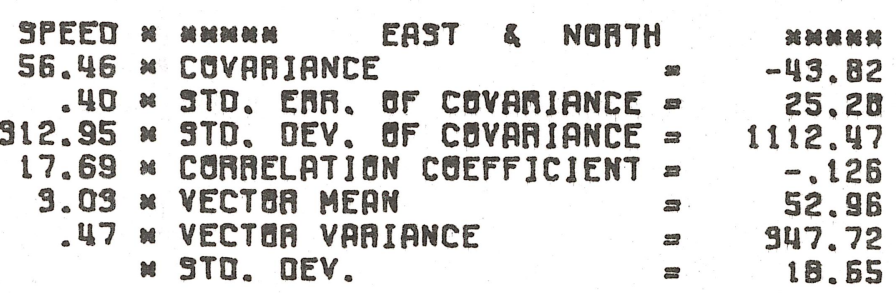

UNITS GF RAW DATA VARIABLES - MMPSEC

SAMPLE SIZE $=1936$ POINTS

* TEMPERATURE *\#

SPANNING RANGE

F月णM 73-IV -01 01.07.30

T0 73-IV -2104.52 .30$

DURATIEN 20 DAYS

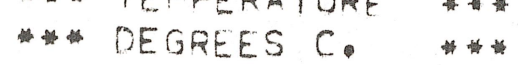

MEAN

VARIANCE

$2 \cdot 750$

.000

- 016

$5 \cdot 658$

$.71 x$

KURTESIS

SKEWNFSS$$
.71 \%
$$

SAMPLE SIZE $=1930$ PGINTS 

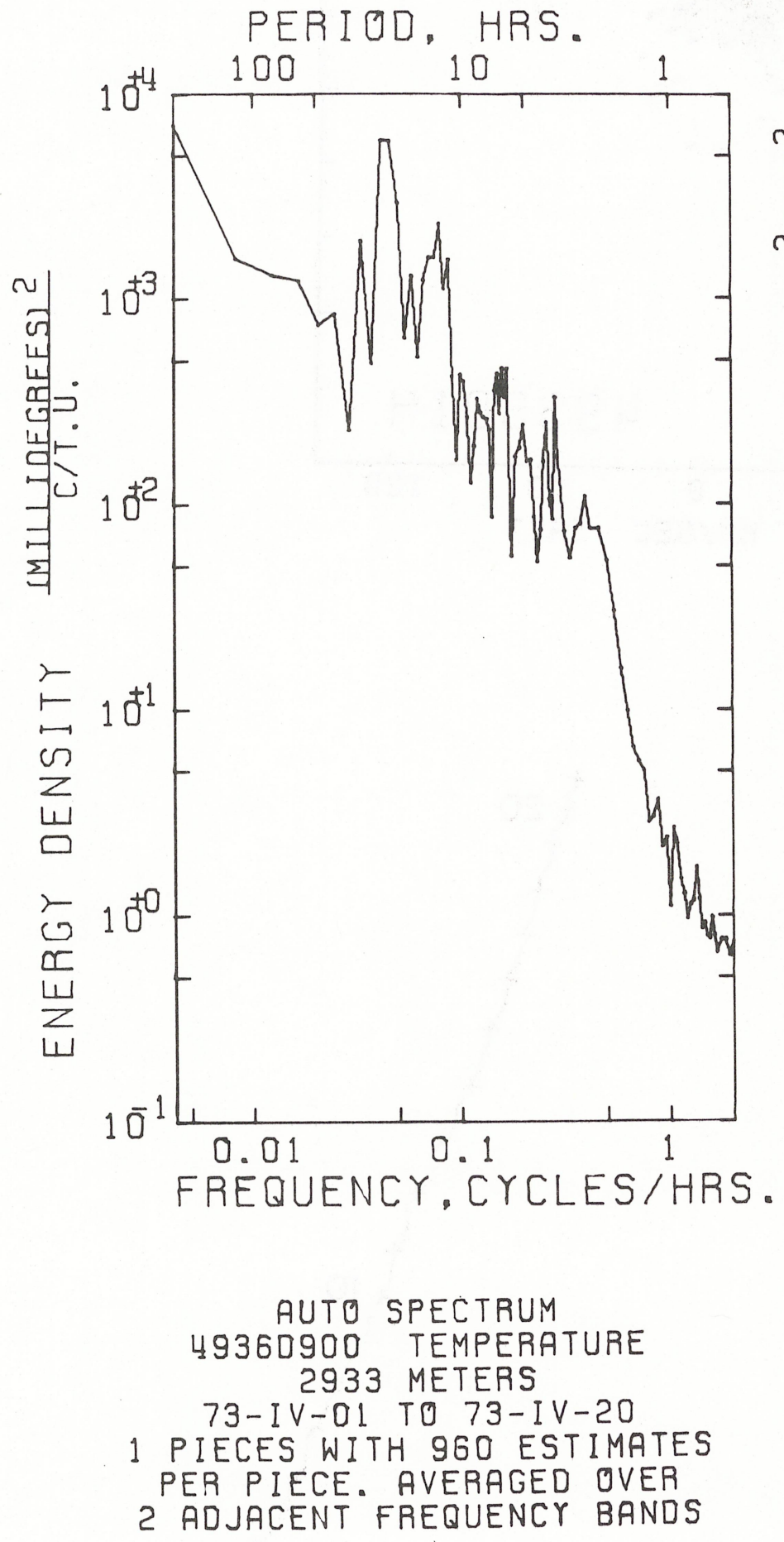
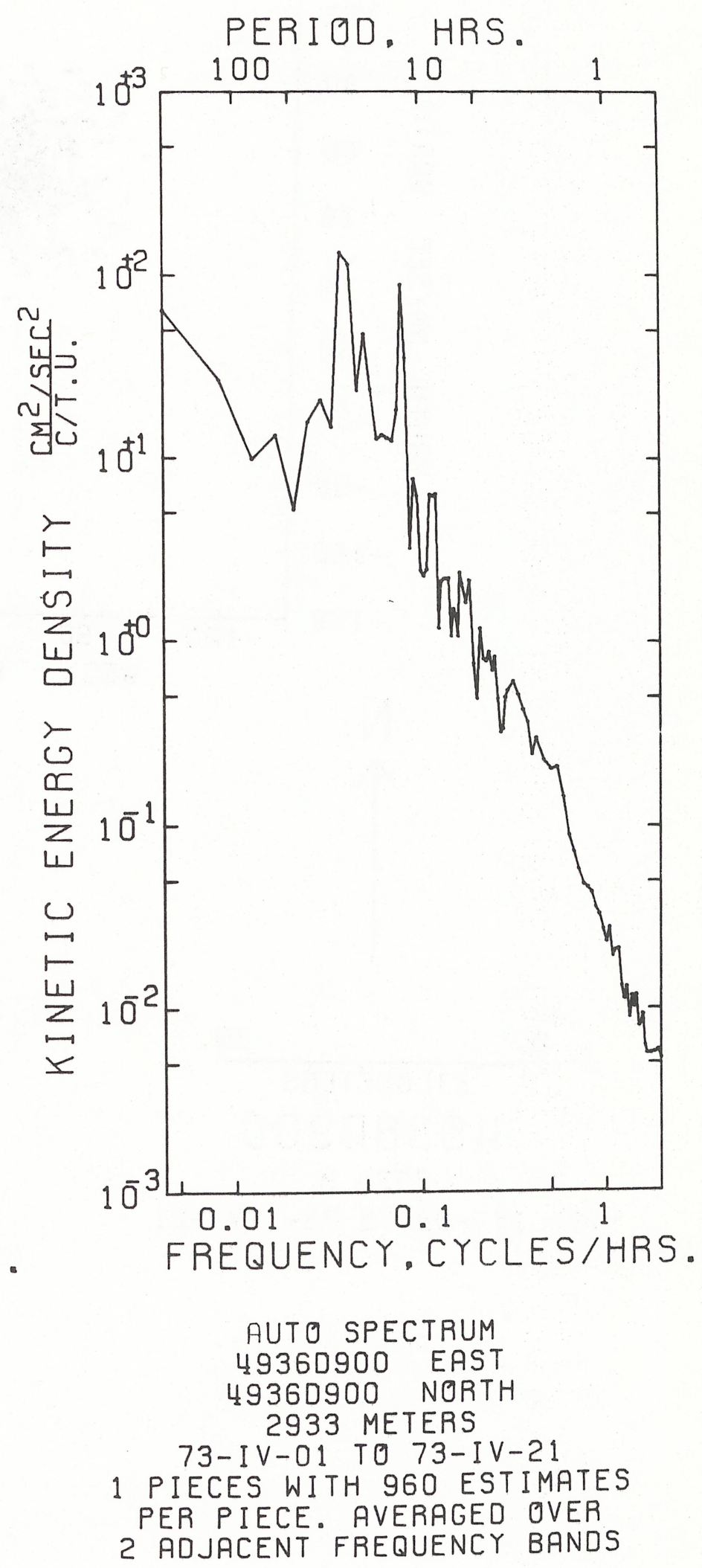


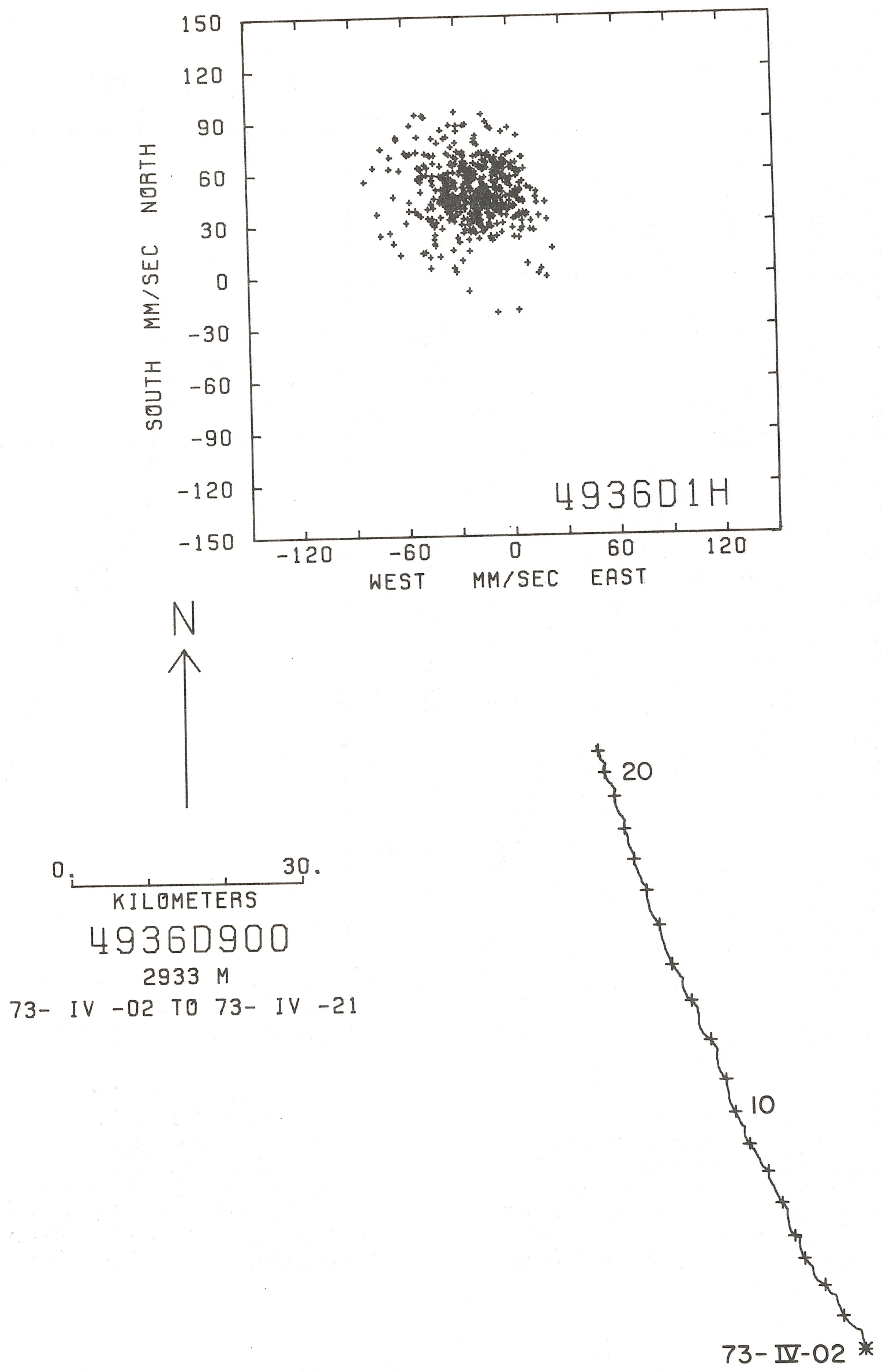



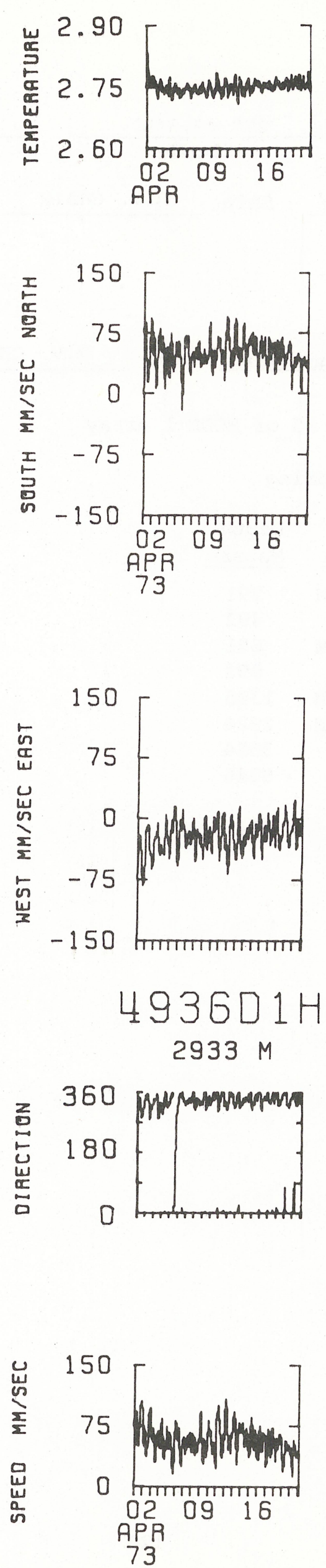
Mooring No. 494

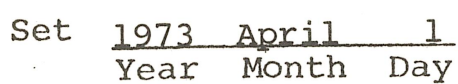

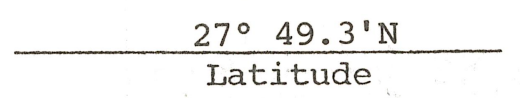

$\frac{70^{\circ} 39.8^{\prime} \mathrm{W}}{\text { Longitude }}$

Set by J. Gifford - R. Heinmiller

Ship R.V. CHAIN

Cruise 112 Leg 2

Retrieved $\frac{1973 \text { June } 29}{\text { Year Month Day }}$

Retrieved by J. Gifford - R. Heinmiller Ship R.V. CHAIN Cruise 112 Leg 6

Purpose of Mooring: Mooring \#5 of MODE 1 array

Mooring Type: Subsurface mooring

$\begin{array}{ccclccc}\text { Key } & \begin{array}{c}\text { Data } \\ \text { Number }\end{array} & \begin{array}{c}\text { Instrument } \\ \text { Number }\end{array} & & \text { Type } & \begin{array}{c}\text { Depth } \\ \text { Meters }\end{array} & \text { Comments } \\ * & 4941 & \text { V-0127 } & & \text { VACM } & 391 & \\ \# & 4942 & \text { \#33 } & & \text { T/P } & 492 & \text { M.I.T. } \\ & 4943 & \text { V-0157 } & \text { VACM } & 691 & \text { I.O.S. } \\ \# & 4944 & \text { \#51 } & \text { T/P } & 893 & \text { M.I.T. } \\ + & 4945 & \text { V-0118 } & \text { VACM } & 1395 & \\ + & 4946 & \text { V-0133 } & \text { VACM } & 2924 & \text { M.I.T. } \\ \# & 4947 & \text { \#24 } & \text { T/P } & 3954 & \text { U.R.I. } \\ & 4948 & \text { M-280 } & 850 & 5346 & \end{array}$

COMMENTS ON MOORING: 


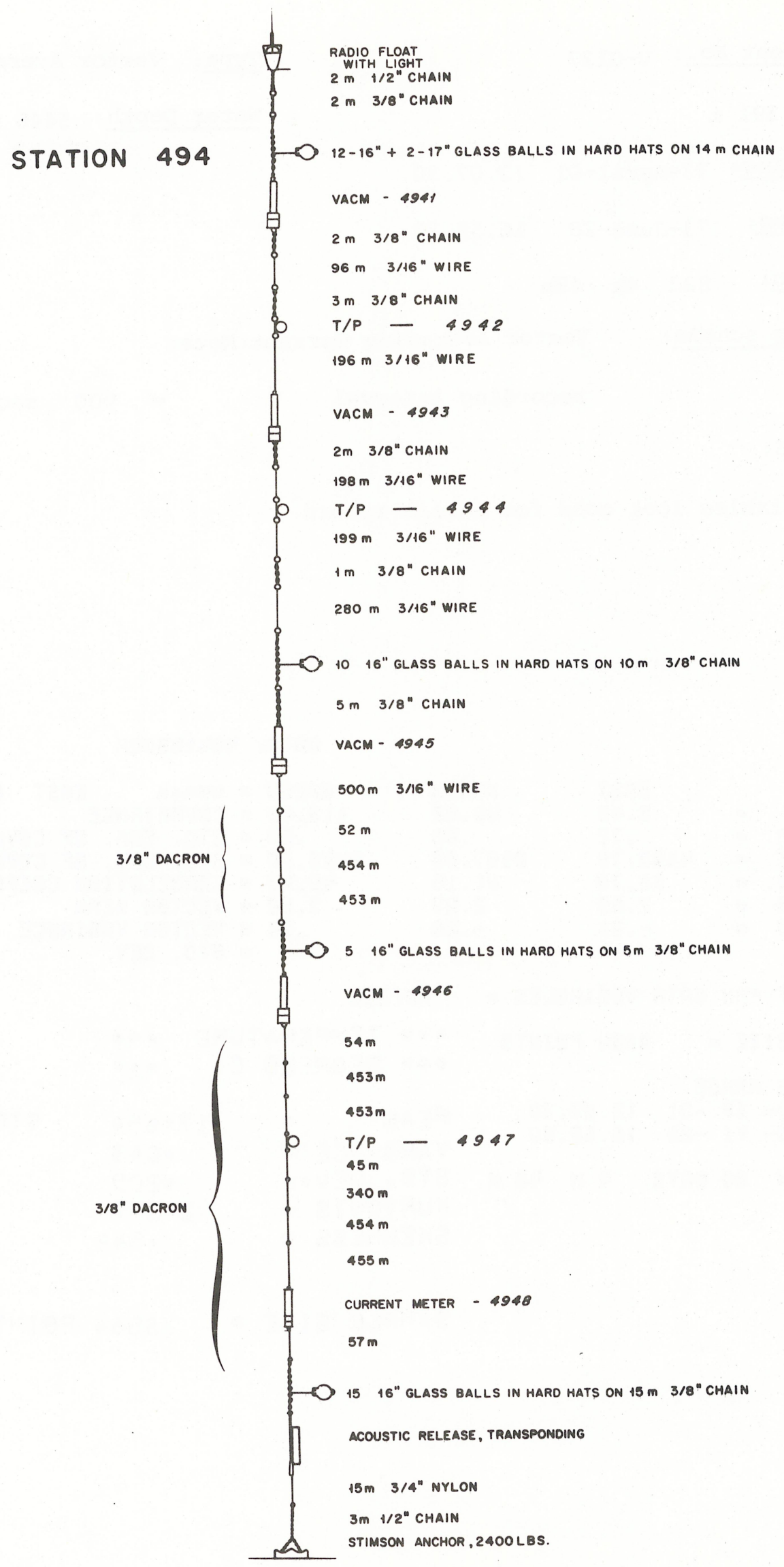


DATA NUMBER 4941

Instrument No.: $\mathrm{V}-0127$

Depth: $391 \mathrm{~m}$

Start time: 73-April-01 12.07.30.

Stop time: 73 -June-28 16.52 .30 .

Duration: $\quad 88 \mathrm{~d} \quad 4 \mathrm{~h} 45 \mathrm{~m}$

Sampling scheme: Vector Averaging Current Meter

$$
\text { recording interval }=900 \text { seconds }
$$

COMMENTS :

All variables look good for entire record
Type: Vector Averaging Current Meter Water Depth: $5446 \mathrm{~m}$
STATS

$\begin{array}{lrr} & \text { EAST } & \text { NORTH } \\ \text { MEAN } & 8.43 & 48.92 \\ \text { STO. ERR. } ~ & .72 & .98 \\ \text { VARIANCE }= & 4448.79 & 8307.65 \\ \text { STD. DEV. } & 66.70 & 81.15 \\ \text { KURTOSIS } & 2.63 & 2.29 \\ \text { SKENNESS }= & -.21 & -.29\end{array}$

DATAP $49420900 A$

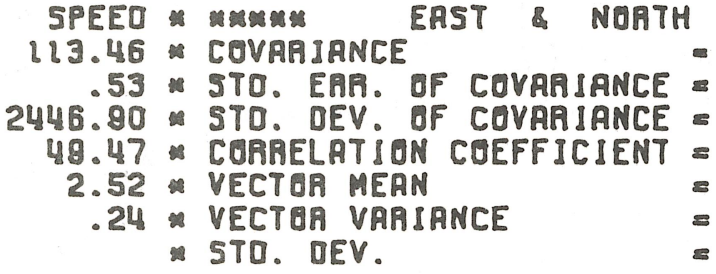

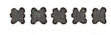

324.17

70.79

6551.10

.053

50.63

6378.22

79.86

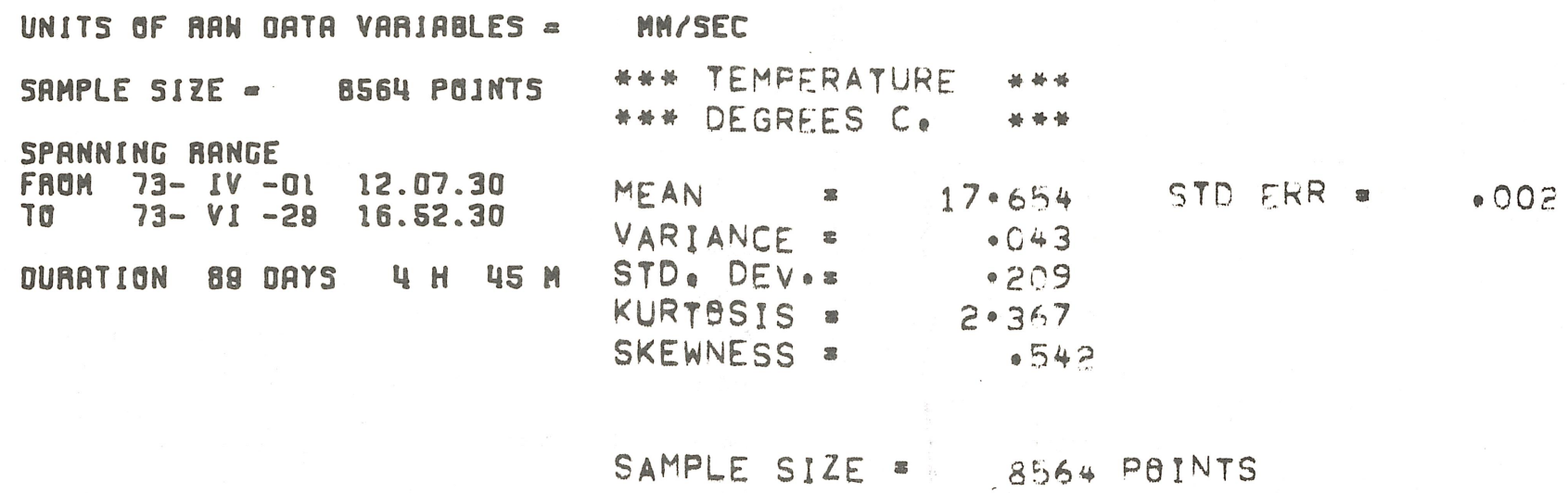



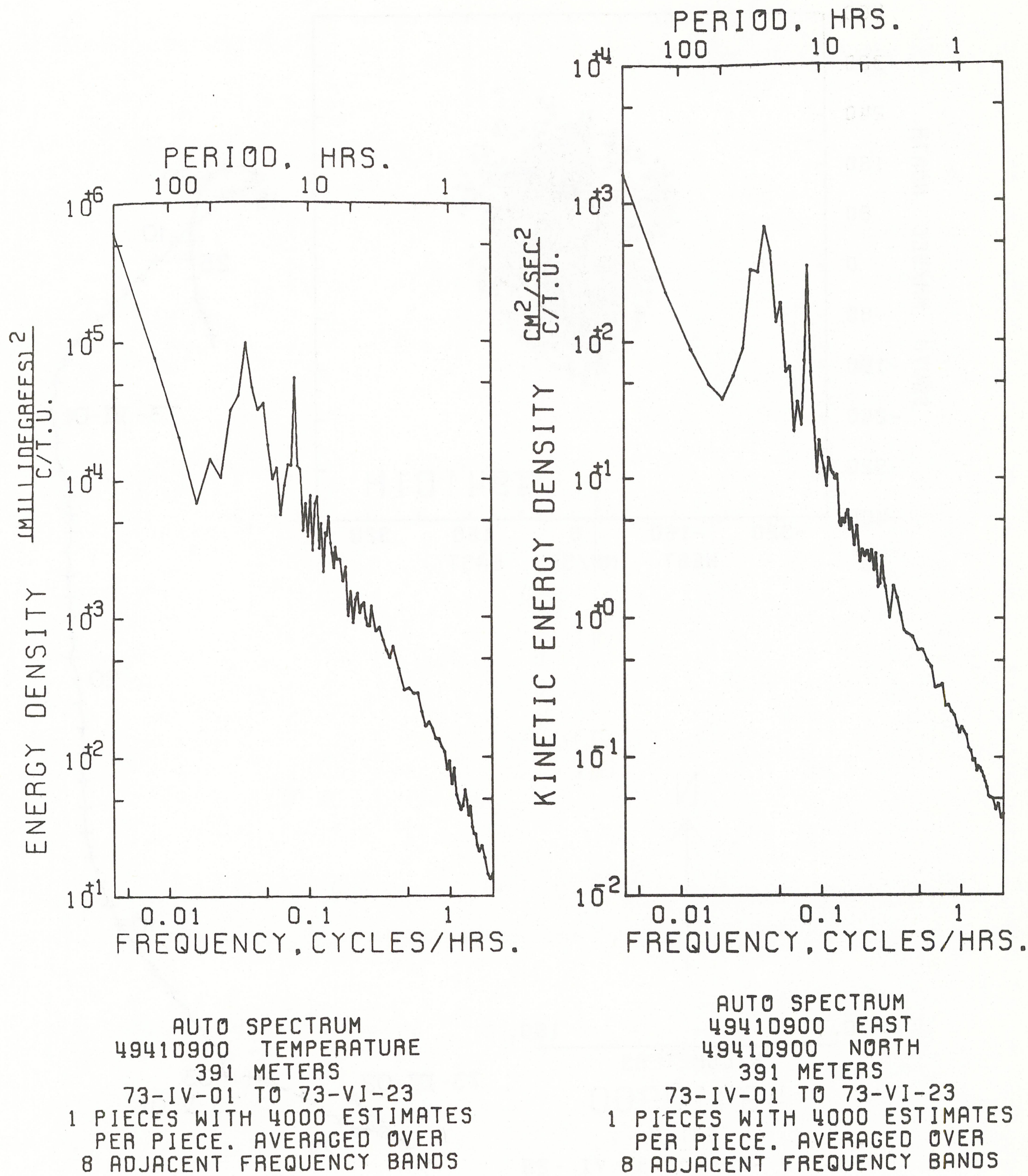

AUTO SPECTRUM 49410900 EAST 49410900 NORTH 391 METERS 73-IV-01 TO 73-VI-23

1 PIECES WITH 4000 ESTIMATES PER PIECE. AVERAGED OVER 8 ADJACENT FREQUENCY BANDS 

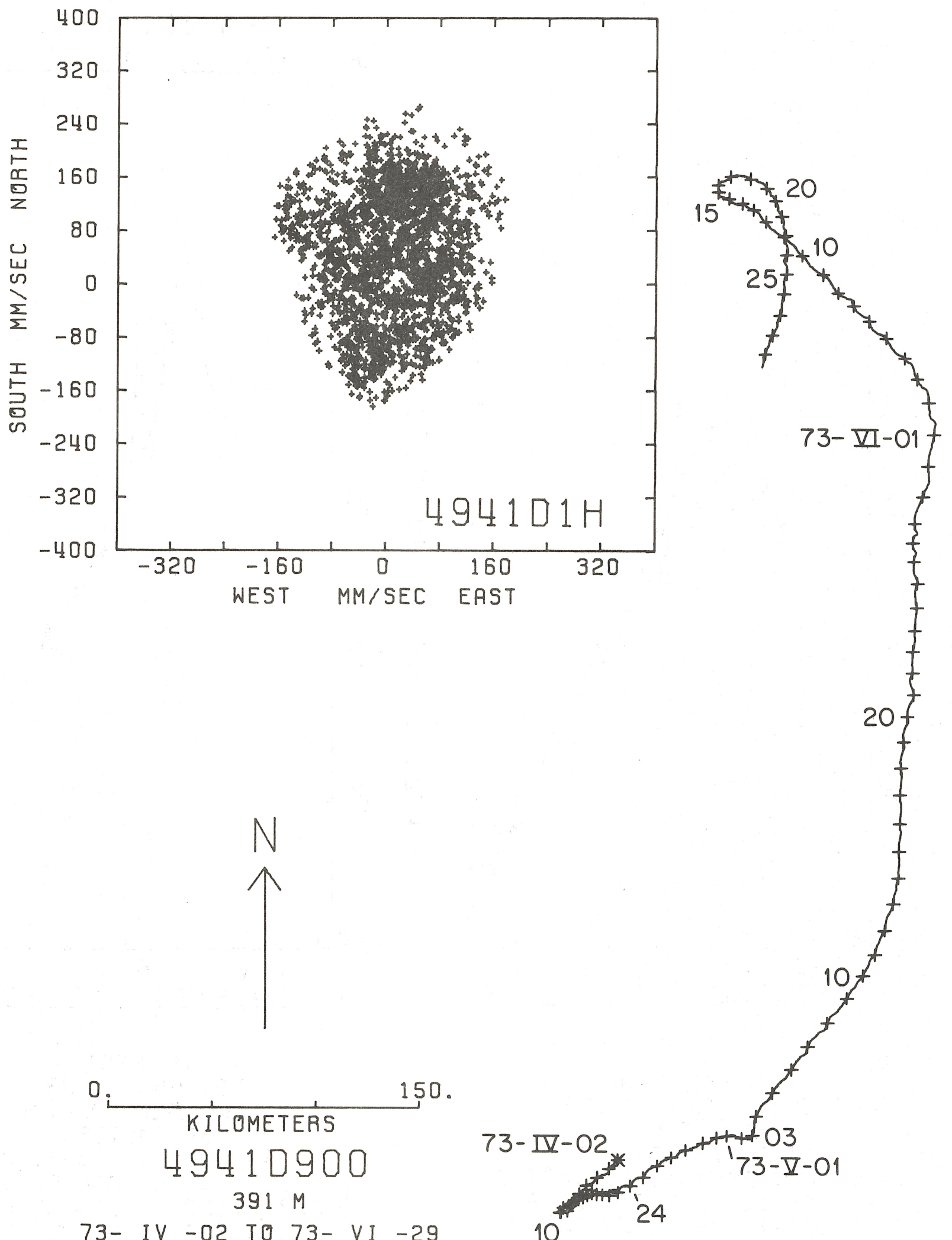

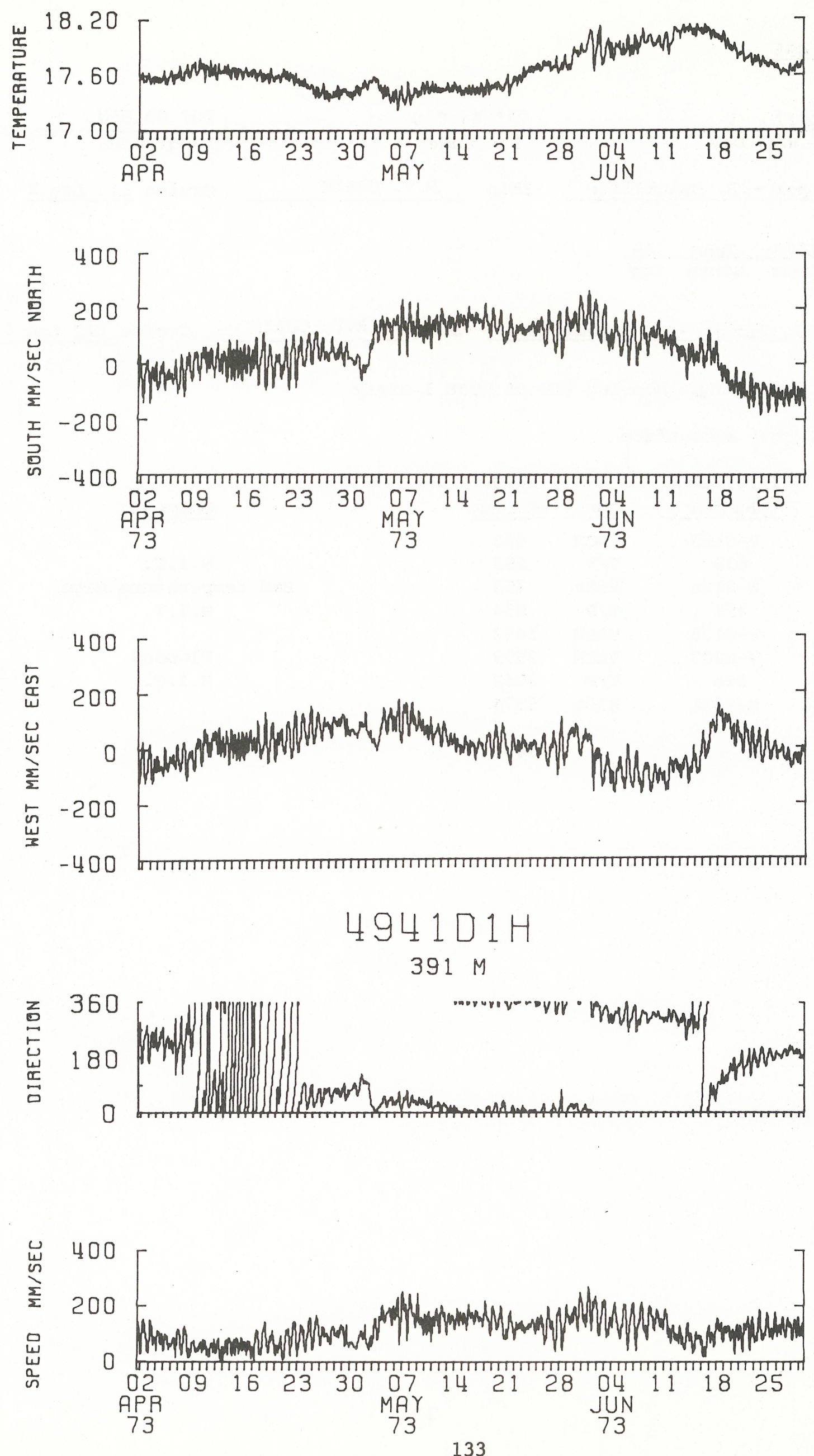
Mooring No. 495

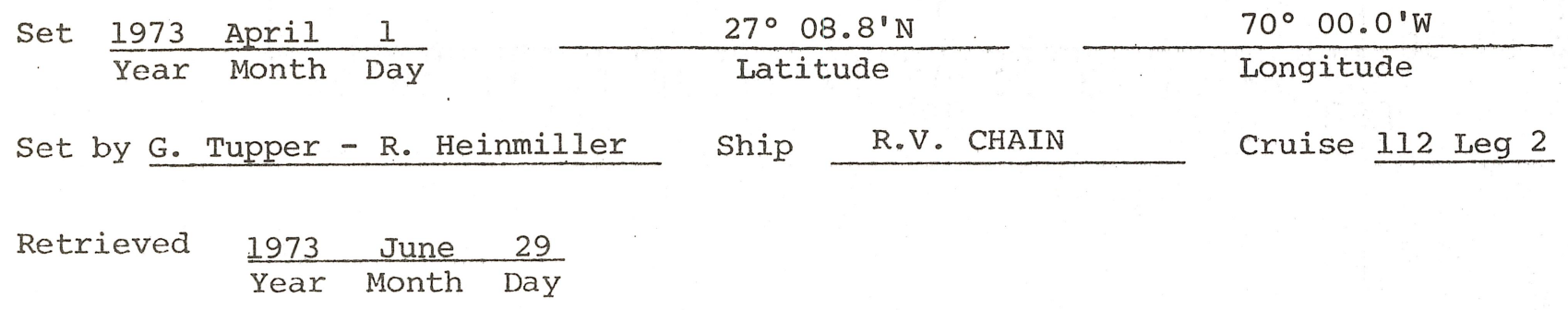

Retrieved by G. Tupper - R. Heinmiller Ship R.V. CHAIN Cruise 112 Leg 6

Purpose of Mooring: Mooring \#10 of MODE 1 array

Mooring Type: Subsurface

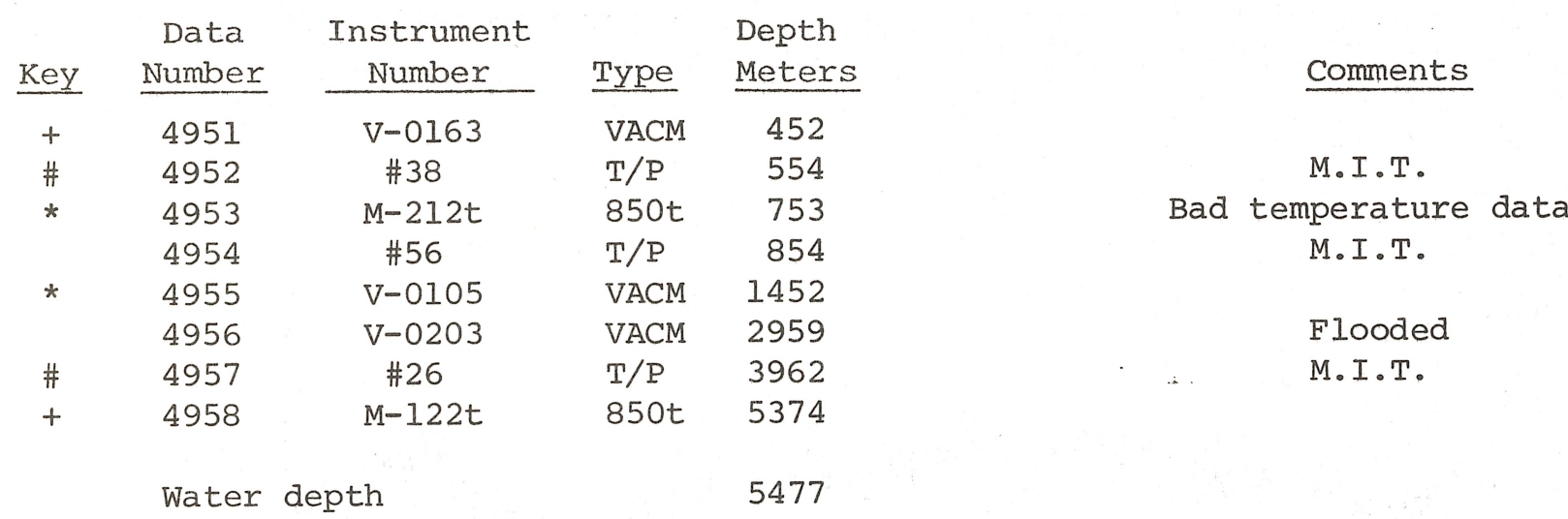

COMMENTS ON MOORING: 


\section{STATION 495}

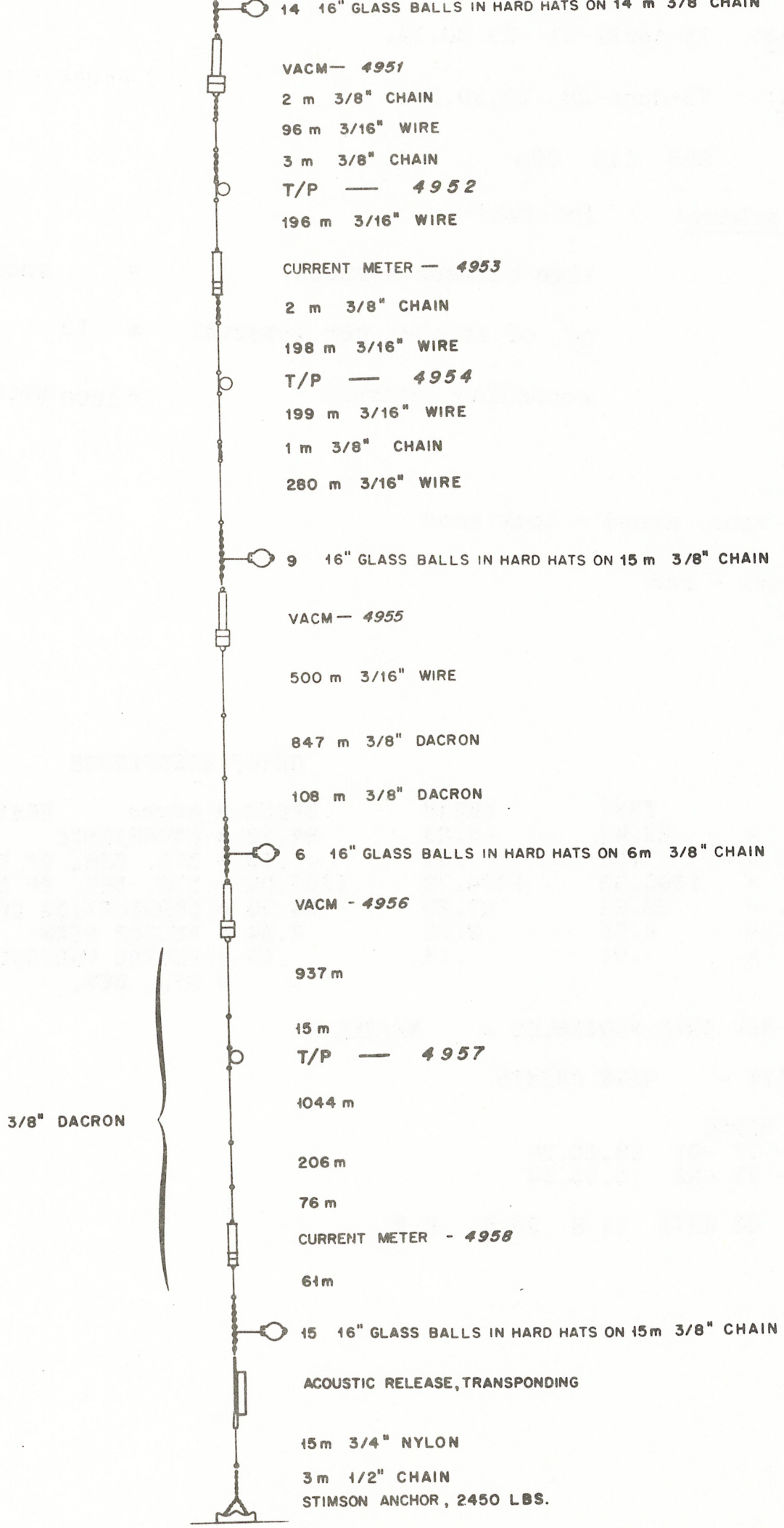


DATA NUMBER 4953

Instrument No.: $\mathrm{M}-212 \mathrm{t}$

Depth: $753 \mathrm{~m}$

Start time: 73-April-01 23.00.34.

Stop time: 73 -June-28 10.30.34.

Duration: $\quad 88 \mathrm{~d}$ 11h $30 \mathrm{~m}$

Sampling scheme: Interval

$$
\begin{array}{ll}
\text { time between strobes } & =13 \\
\text { no. of strobes per interval } & =13 \\
\text { recording interval } & =1800 \text { seconds }
\end{array}
$$

\section{COMMENTS :}

Compass, vane, speed - look good

Temperature - bad

STATS

$\begin{array}{lrr} & \text { EAST } & \text { NORTH } \\ \text { MEAN } & -83.47 & -5.23 \\ \text { STD. ERR. } & .57 & .63 \\ \text { VARIANCE }= & 1366.39 & 1704.72 \\ \text { STD. DEV }= & 96.96 & 41.29 \\ \text { KURTOSIS }= & 2.98 & 2.95 \\ \text { SKENNESS }= & .01 & .14\end{array}$

UNITS OF RAW OATA VARIABLES - MMPEC

SAMPLE SIZE = 4248 PUINTS

SPANNING RANGE

FROM 73-IV -01 23.00.34

TO 73-VI -28 10.30.34

DURATION 88 DAYS II H 30 M O
Type: Magnetic Tape Recording Current Meter Water depth: $5477 \mathrm{~m}$ 

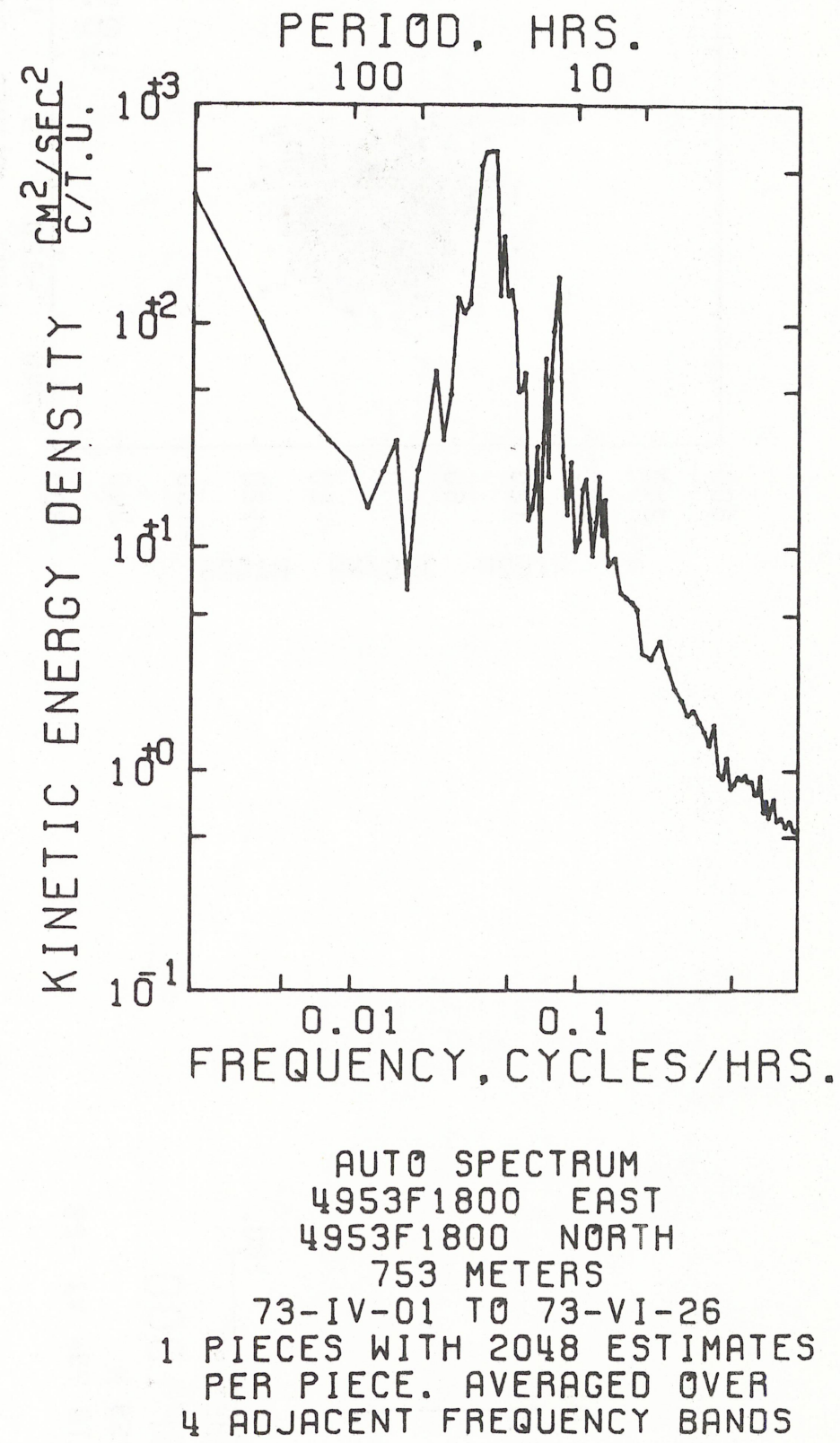

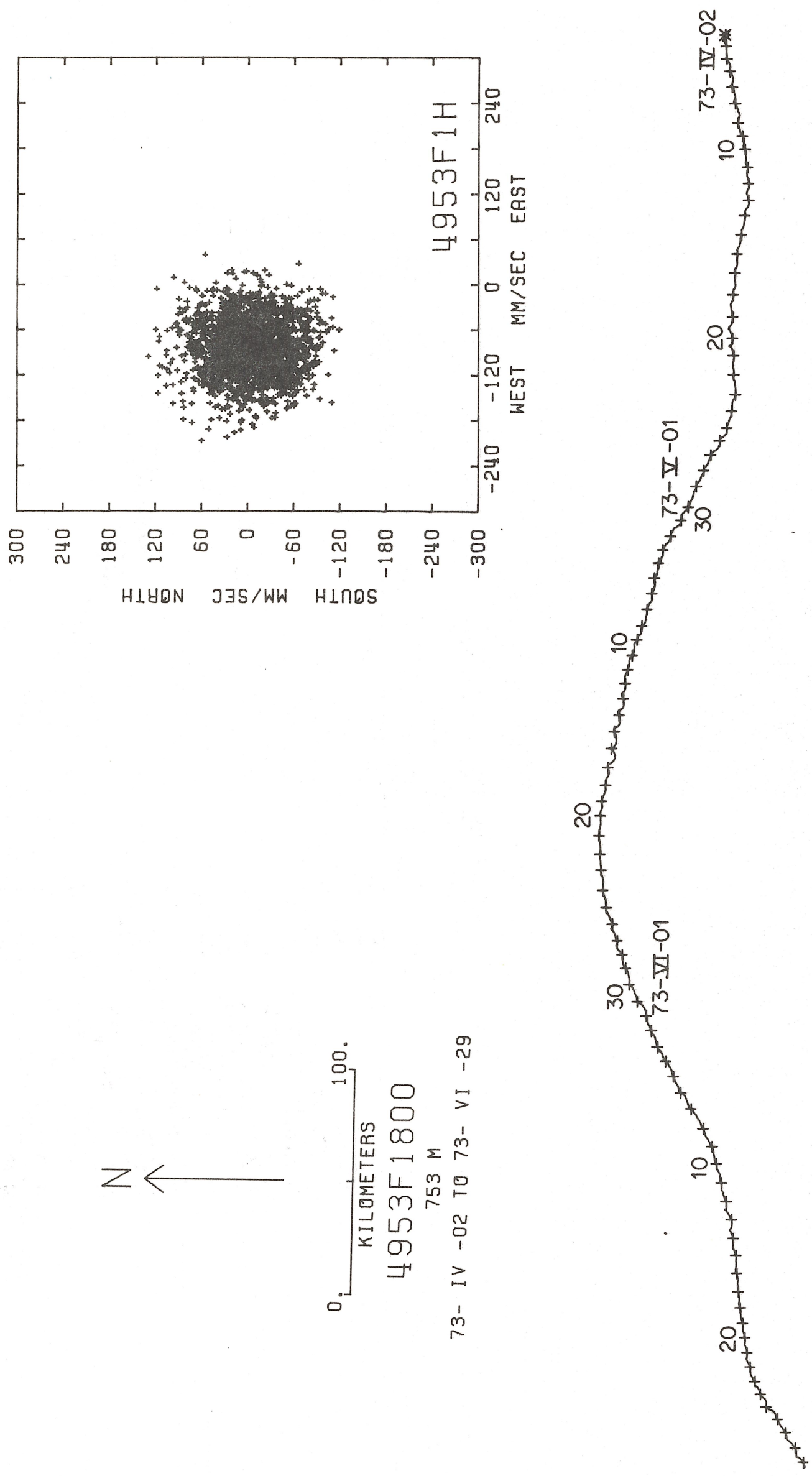

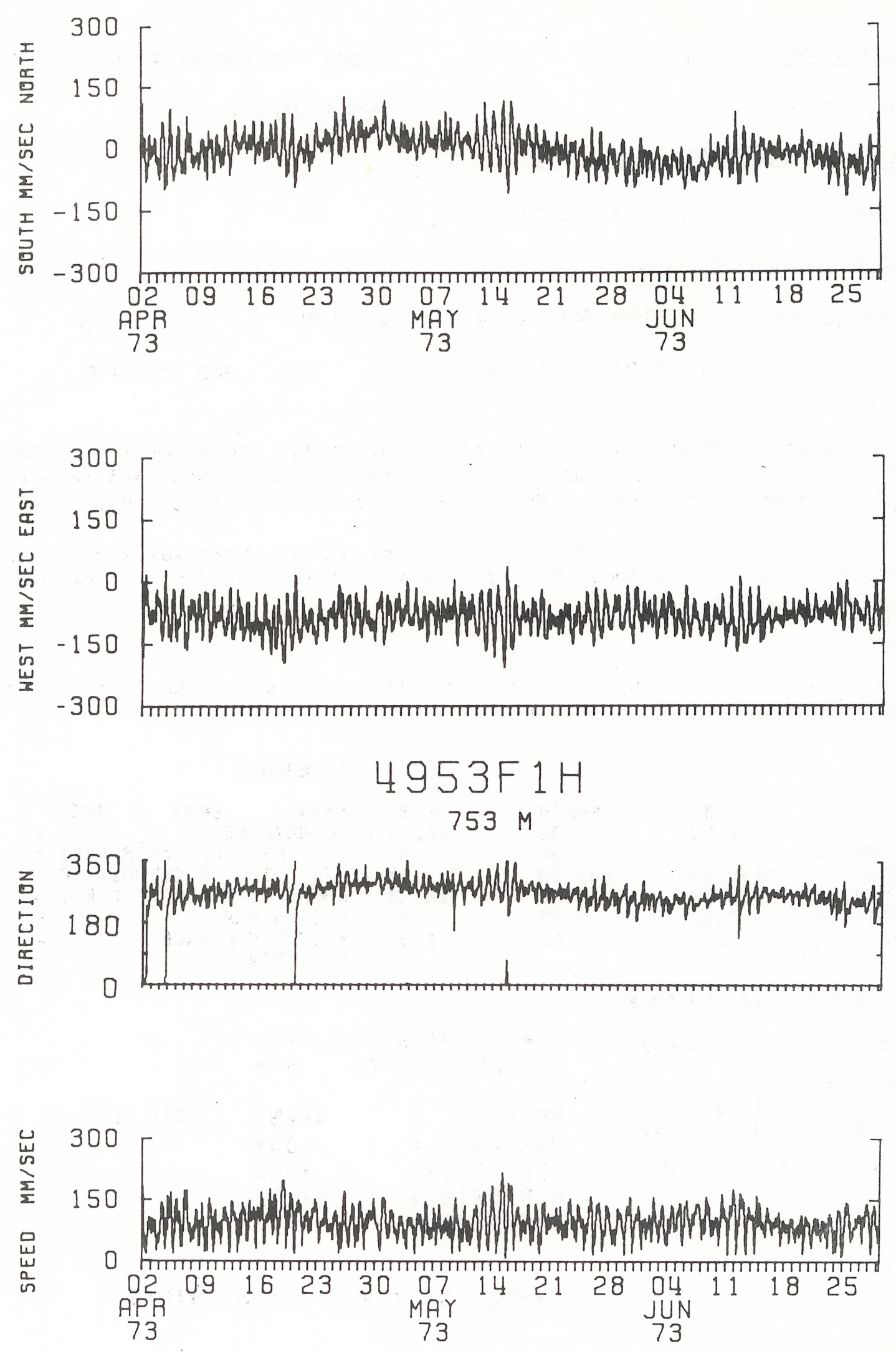
DATA NUMBER 4955

Instrument No.: $\mathrm{V}-0105$

Depth: $1452 \mathrm{~m}$

Start time: 73-April-01 21.07.30.

Stop time: 73-June-14 23.52.30.

Duration: $\quad 74 \mathrm{~d} \quad 2 \mathrm{~h} \quad 45 \mathrm{~m}$

Sampling scheme: Vector Averaging Current Meter

$$
\text { recording interval }=900 \text { seconds }
$$

COMMENTS :

Internal vector computer did not function correctly. To recover data East and North components were computed from instantaneous compass and vane values and accumulated rotor counts. Speed amplitude may be a little high.

Compass and vane data were not stored on tape unless there was a rotor count during the recording period. Temperature values were stored on tape regardless of rotor value.

Current data looks good until June 15th after which some data is lost due to speed being at threshold (0 rotor count).

STATS

oatar $4955 E 900 A$

$\begin{array}{lrr} & \text { EAST } & \text { NORTH } \\ \text { MEAN } & -33.65 & 8.38 \\ \text { STD. ERR. }= & .40 & .34 \\ \text { YARIANCE }= & 1139.69 & 806.38 \\ \text { STD. DEV. }= & 33.76 & 28.40 \\ \text { KURTESIS }= & 3.24 & 2.93 \\ \text { SREWNESS }= & -.41 & -.23\end{array}$

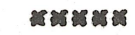

229.75

27.62

1486.49

.125

34.68

973.04

$3 L .19$

UNITS GF RAW DATA VARIRBLES = MMSEC

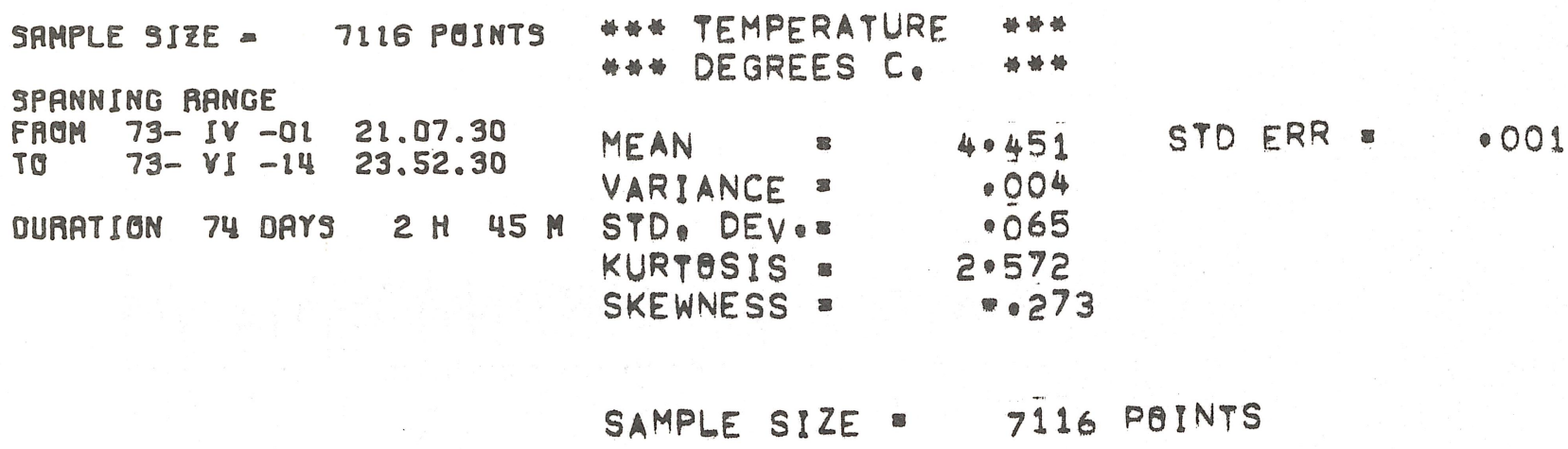



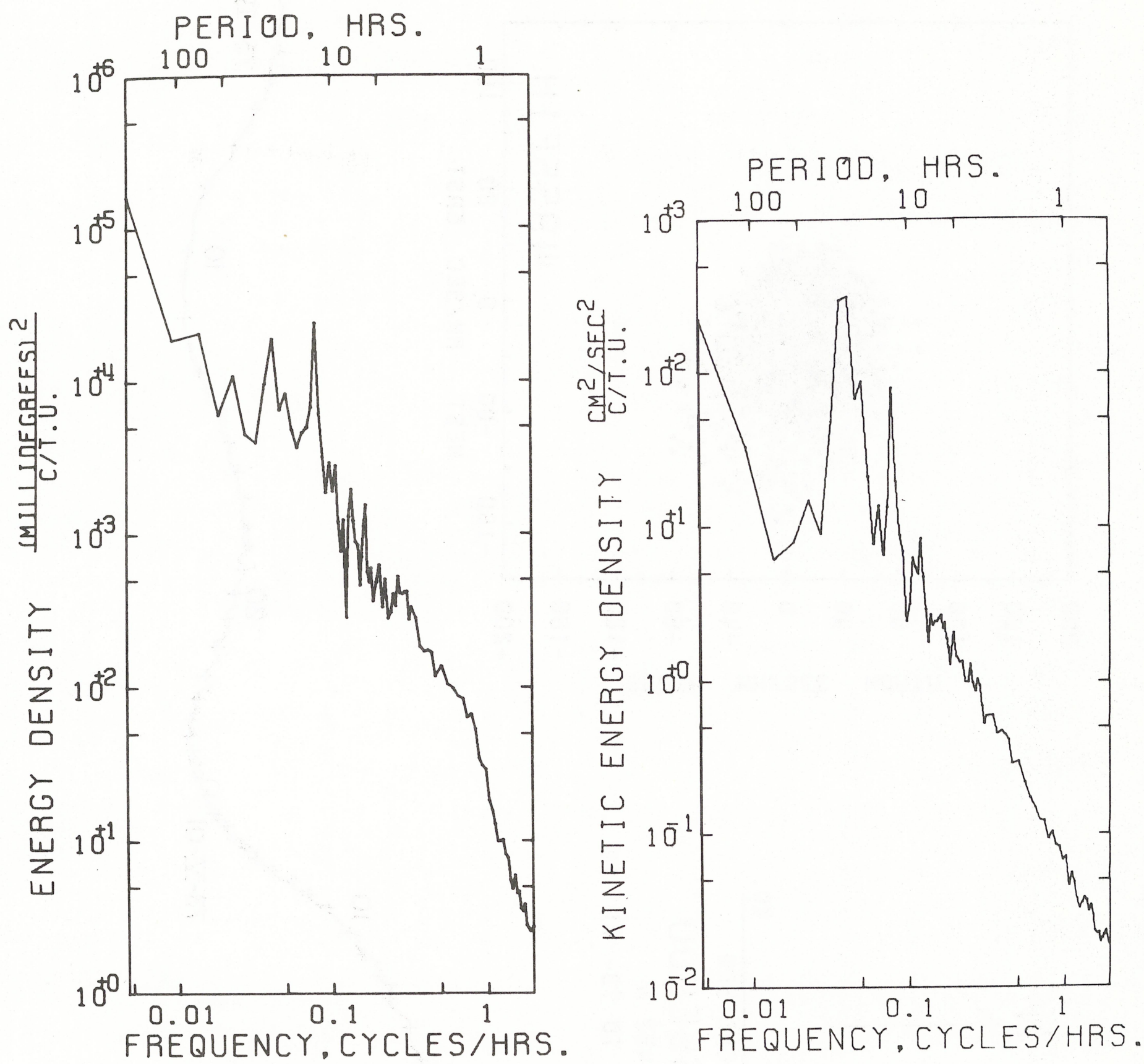

AUTO SPECTRUM 4955E900 TEMPERATURE 1452 METERS 73-IV-01 TO 73-VI-11

1 PIECES WITH 3456 ESTIMATES PER PIECE. AVERAGED OVER 8 ADJACENT FREQUENCY BANDS 


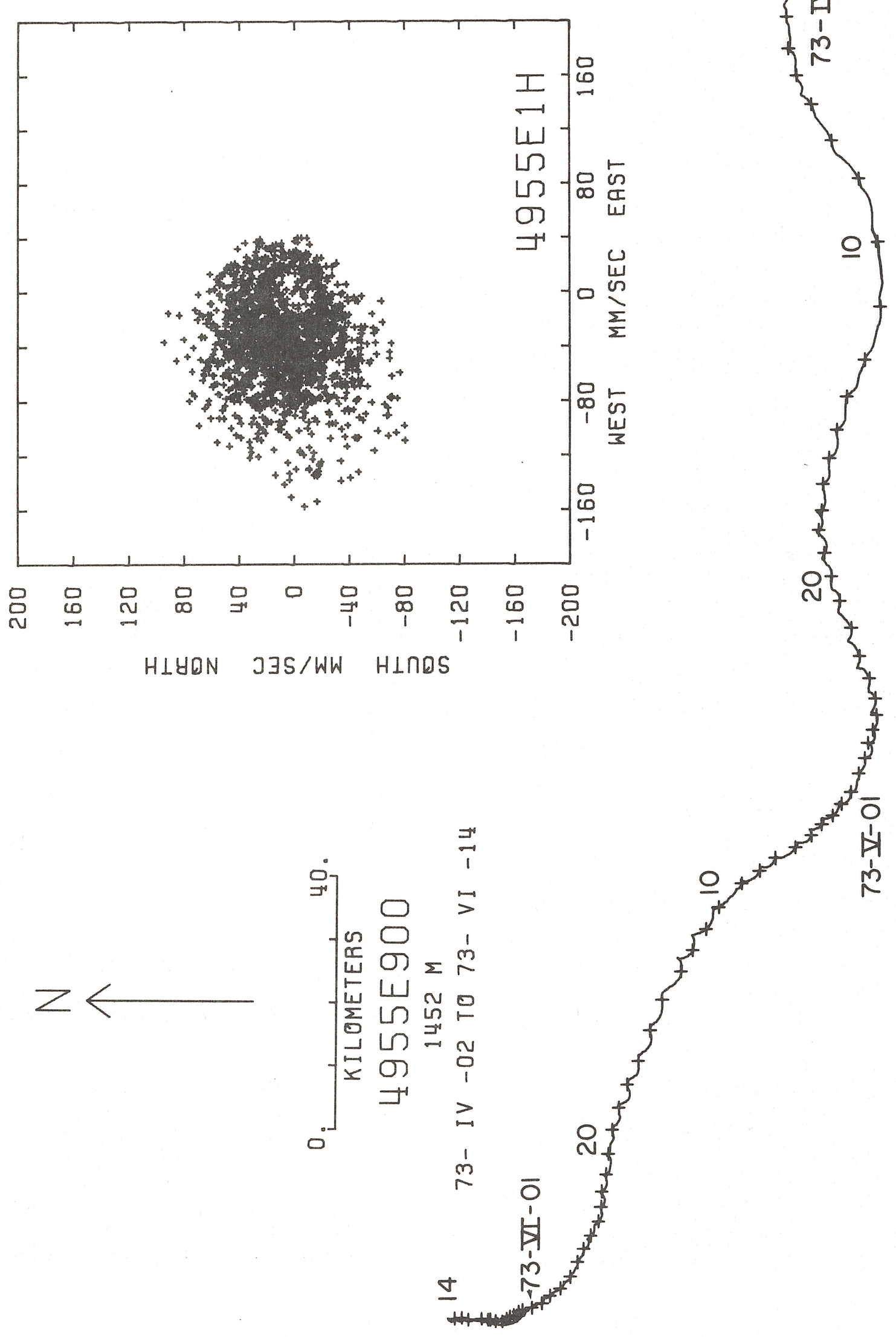



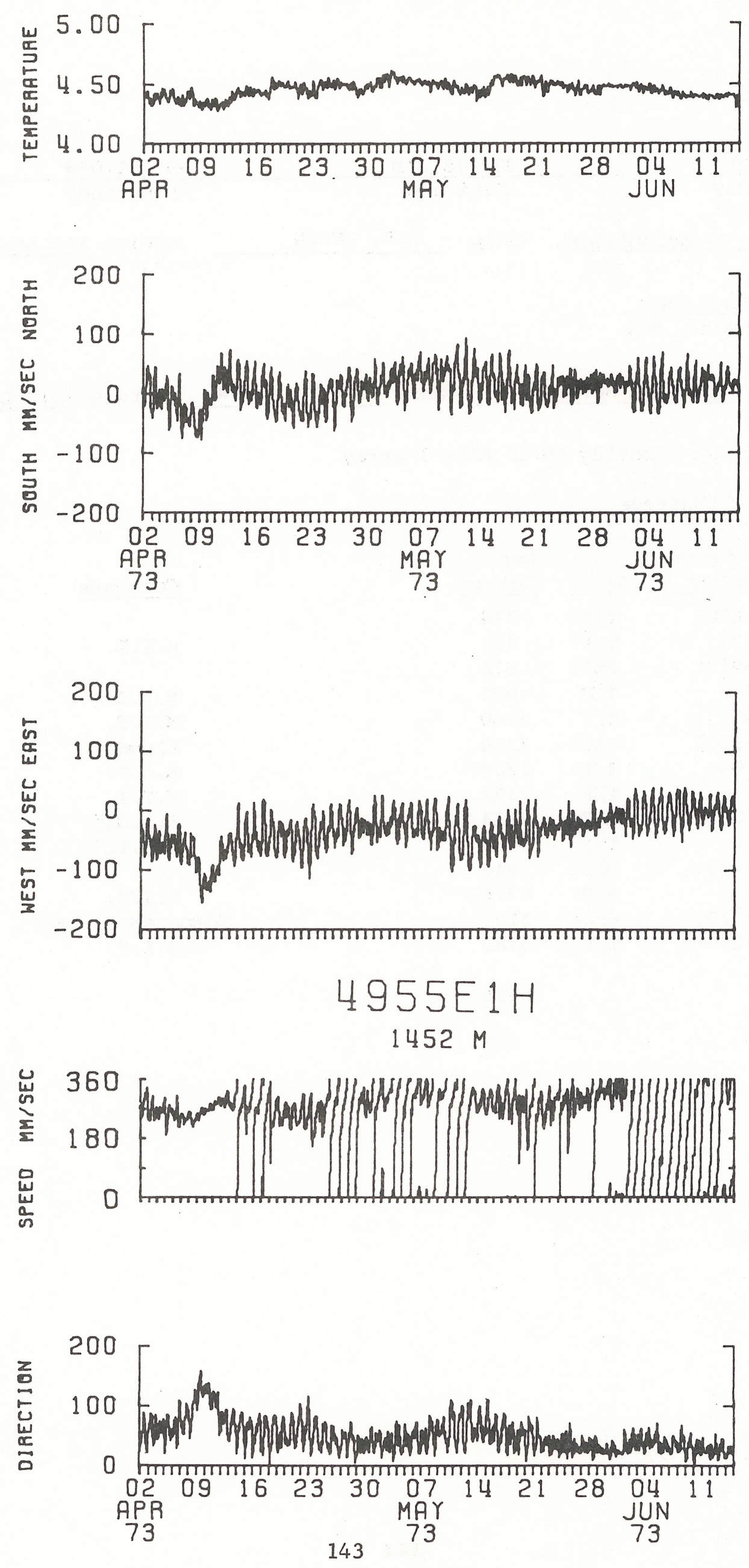
Mooring No. 497

Set $\frac{1973 \text { April } 2}{\text { Year Month Day }}$

$27^{\circ} 18.0^{\circ} \mathrm{N}$

$69^{\circ} 01.0^{\circ} \mathrm{W}$

Latitude

Longitude

Set by G. Tupper - R. Heinmiller

Ship R.V. CHAIN

Cruise 112 Leg 2

Retrieved

$\frac{1973 \text { June } 28}{\text { Yeax Month Day }}$

Retrieved by J. Gifford - R. Heinmiller

Ship

R. V. CHAIN

Cruise 112 Leg 6

Purpose of Mooring: Mooring \#9 of MODE 1 array

Mooring Type: Subsurface

\begin{tabular}{|c|c|c|c|c|c|}
\hline Key & $\begin{array}{c}\text { Data } \\
\text { Number }\end{array}$ & $\begin{array}{c}\text { Instrument } \\
\text { Number }\end{array}$ & Type & $\begin{array}{l}\text { Depth } \\
\text { Meters }\end{array}$ & Comments \\
\hline * & 4971 & $\mathrm{~V}-0120$ & VACM & 374 & \\
\hline \# & 4972 & \#37 & $\mathrm{T} / \mathrm{P}$ & 478 & M.I.T. \\
\hline+ & 4973 & $M-213 t$ & $850 t$ & 676 & \\
\hline \# & 4974 & \#55 & $\mathrm{T} / \mathrm{P}$ & 880 & M.I.T. \\
\hline \multirow[t]{3}{*}{$\#$} & 4975 & \#59 & $\mathrm{T} / \mathrm{P}$ & 1080 & M.I.T. \\
\hline & 4976 & $V-0202$ & VACM & 1380 & Flooded \\
\hline & 4977 & \#06 & $\mathrm{T} / \mathrm{P}$ & 1889 & M.I.T. \\
\hline \multirow[t]{2}{*}{ \# } & 4978 & $\# 18$ & $\mathrm{~T} / \mathrm{P}$ & 2392 & $\mathrm{M} . \mathrm{I} . \mathrm{T}$. \\
\hline & 4979 & $V-0196$ & VACM & 2913 & Flooded \\
\hline$\#$ & 497,10 & \#08 & $\mathrm{T} / \mathrm{P}$ & 3433 & M.I.T. \\
\hline * & 497,11 & $M-206$ & $850 t$ & 3940 & \\
\hline$\#$ & 497,12 & \#31 & $\mathrm{T} / \mathrm{P}$ & 4346 & M.I.T. \\
\hline * & 497,13 & $M-129 t$ & $850 t$ & 5182 & Temperature ba \\
\hline$\#$ & 497,14 & \#09 & $\mathrm{T} / \mathrm{P}$ & 5185 & M.I.T. \\
\hline
\end{tabular}

COMMENTS ON MOORING: 


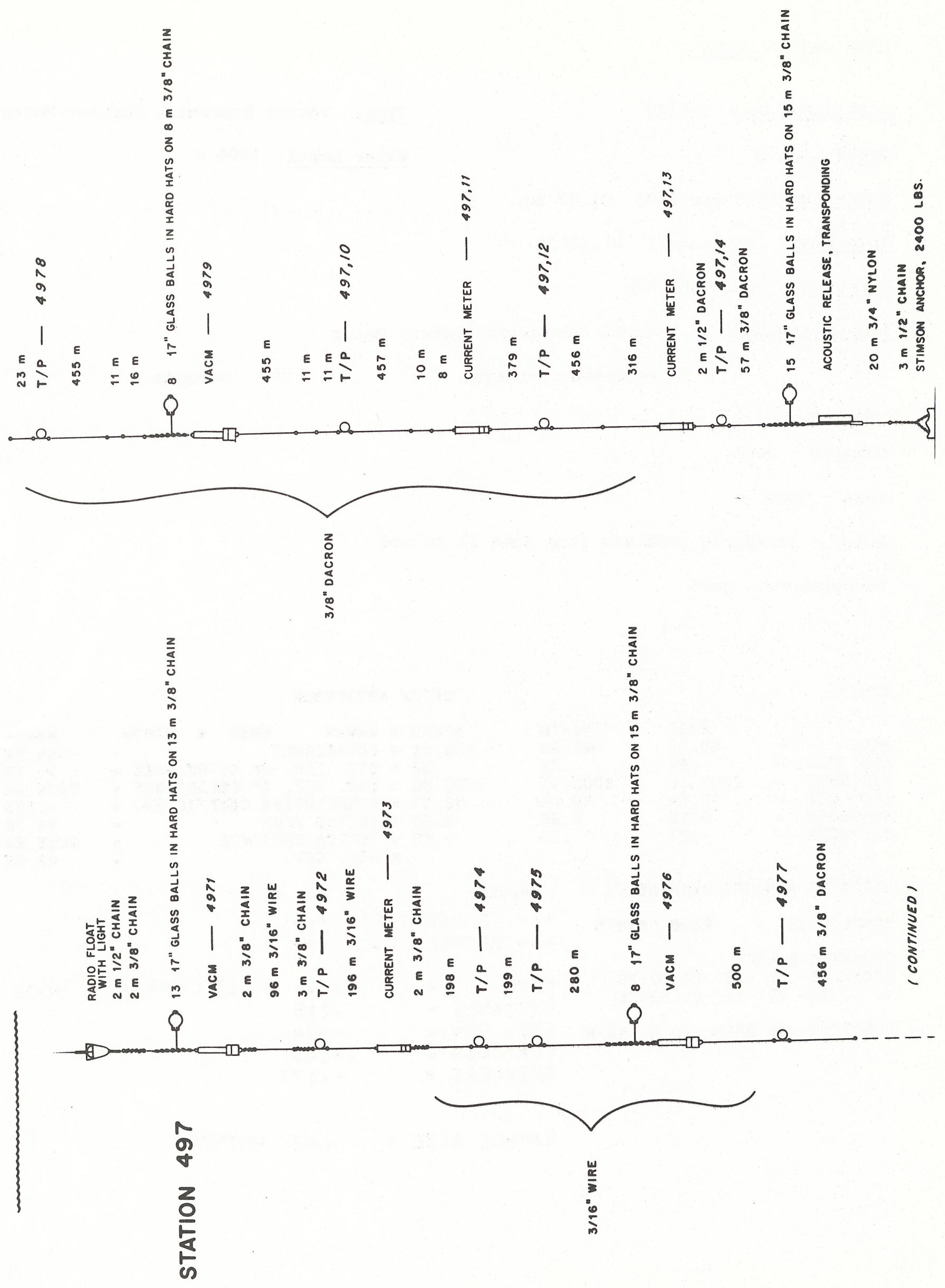


DATA NUMBER 4971

Instrument No.: $\mathrm{V}-0120$

Depth: $374 \mathrm{~m}$
Type: Vector Averaging Current Meter Water Depth: $5296 \mathrm{~m}$

Start time: 73-April-03 01.07.30.

Stop time: 73-June-11 01.52 .30 .

Duration: $\quad 68 \mathrm{~d}$ Oh $45 \mathrm{~m}$

Sampling scheme: Vector Averaging Current Meter recording interval $=900$ seconds

COMMENTS:

Compass - good

Vane - good

Rotor - threshold problems from June 11 to end

Temperature - good

รTคTร

\begin{tabular}{|c|c|c|c|}
\hline & & $\begin{array}{r}\text { EAST } \\
79\end{array}$ & NOลTH \\
\hline 3T0. Enศ. & $\approx$ & $\begin{array}{r}.64 \\
.64\end{array}$ & 5 \\
\hline YAR I ANCE & $\approx$ & 2700.10 & 2203.7 \\
\hline $\begin{array}{l}\text { STD. DEV. } \\
\text { KURTOSIS }\end{array}$ & $\approx$ & $\begin{array}{r}52.04 \\
2.25\end{array}$ & \\
\hline KEWNESS & $\approx$ & -.07 & \\
\hline
\end{tabular}

DATAR 497IC9OOA

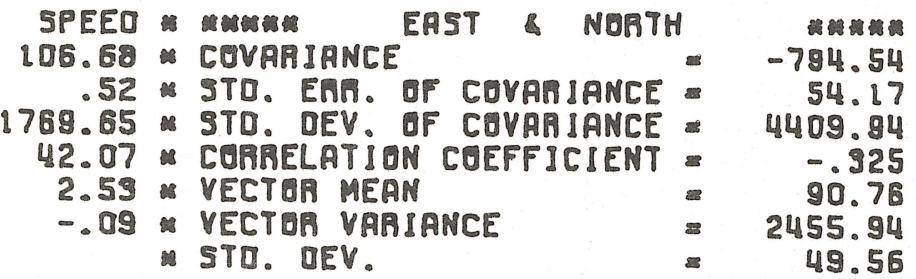

UNITS OF APW DATA YARIABLES - MM/SEC

SAMPLE SIZE - 6628 PUINTS

SPANKING RANGE

F月OH 73- IV -03 01.07.30

T0 73-VI- 11 01.52.30

DURATION 69 DAYS O H 45 M

MEAN

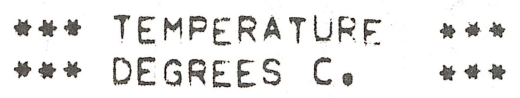

VARIANCE :

$17 \cdot 449$

- 15

STD. DEV :

KURTOSIS:

SKEWNESS :

- 124

$2 \cdot 149$

$-.177$

SAMPLE SIZE GGE' DOINTS 

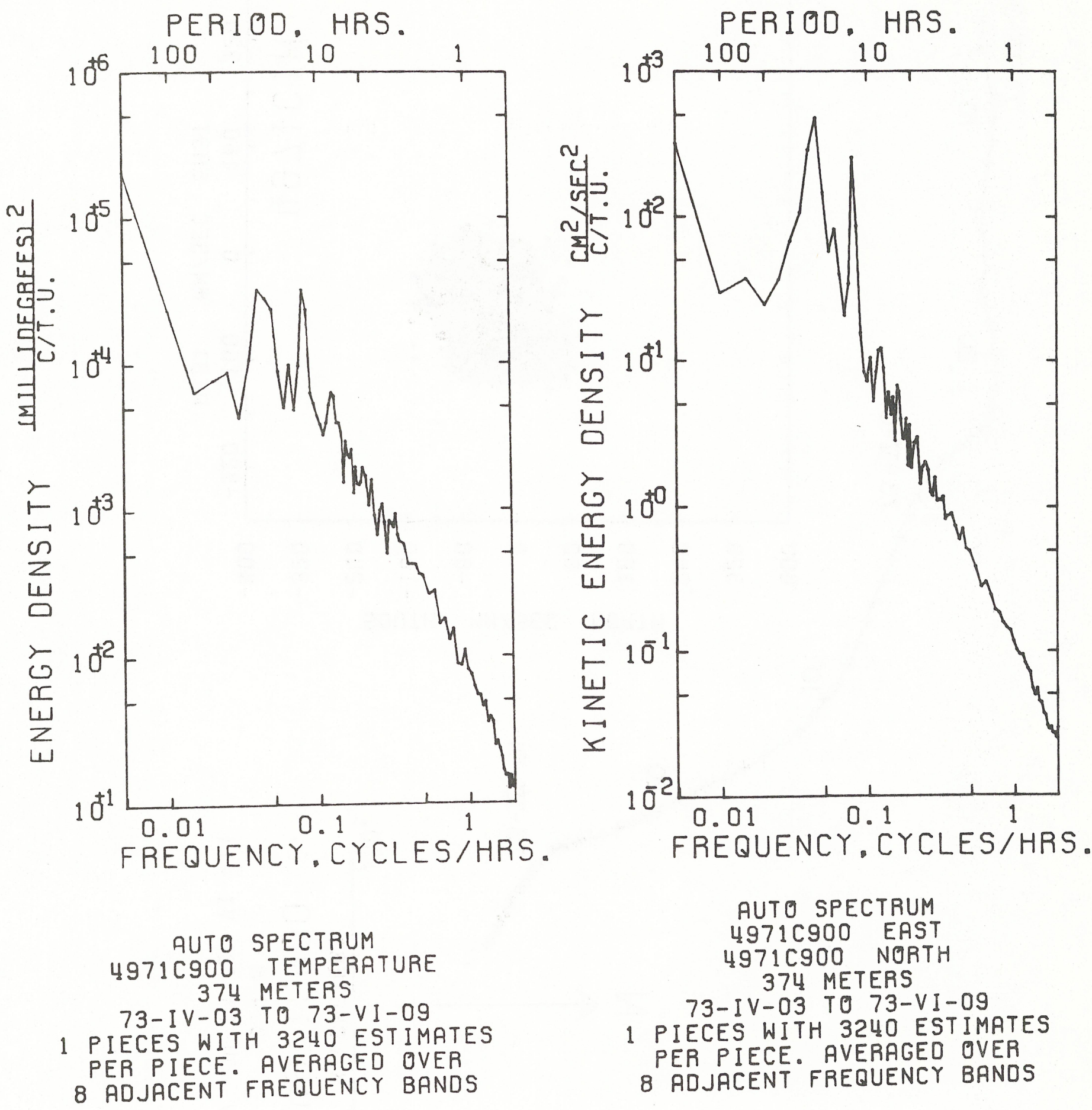

$$
\text { 73-IV-03 TO 73-VI-09 }
$$

1 PIECES WITH 3240 ESTIMATES PER PIECE. AVERAGED OVER 8 ADJACENT FREQUENCY BANDS 


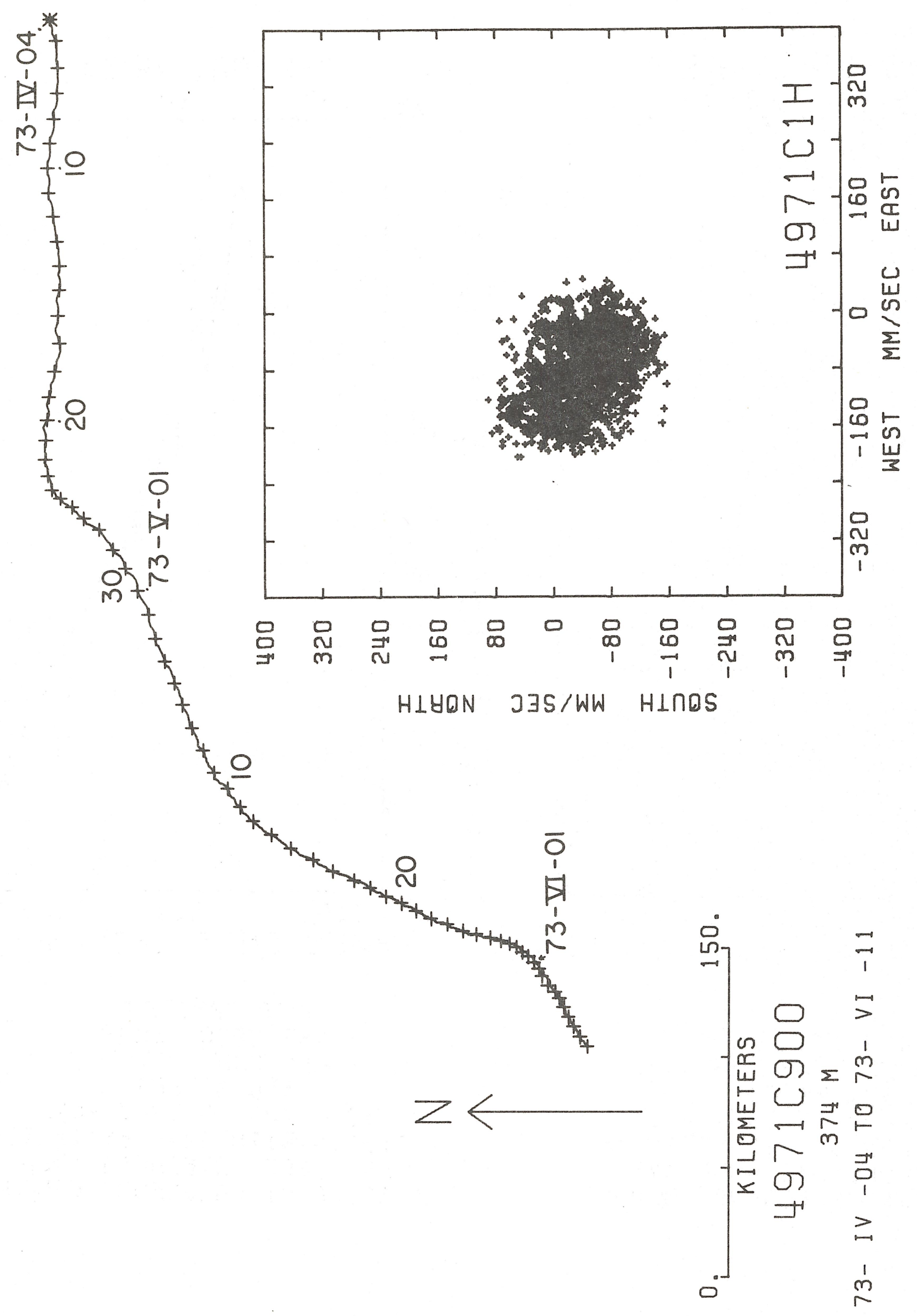



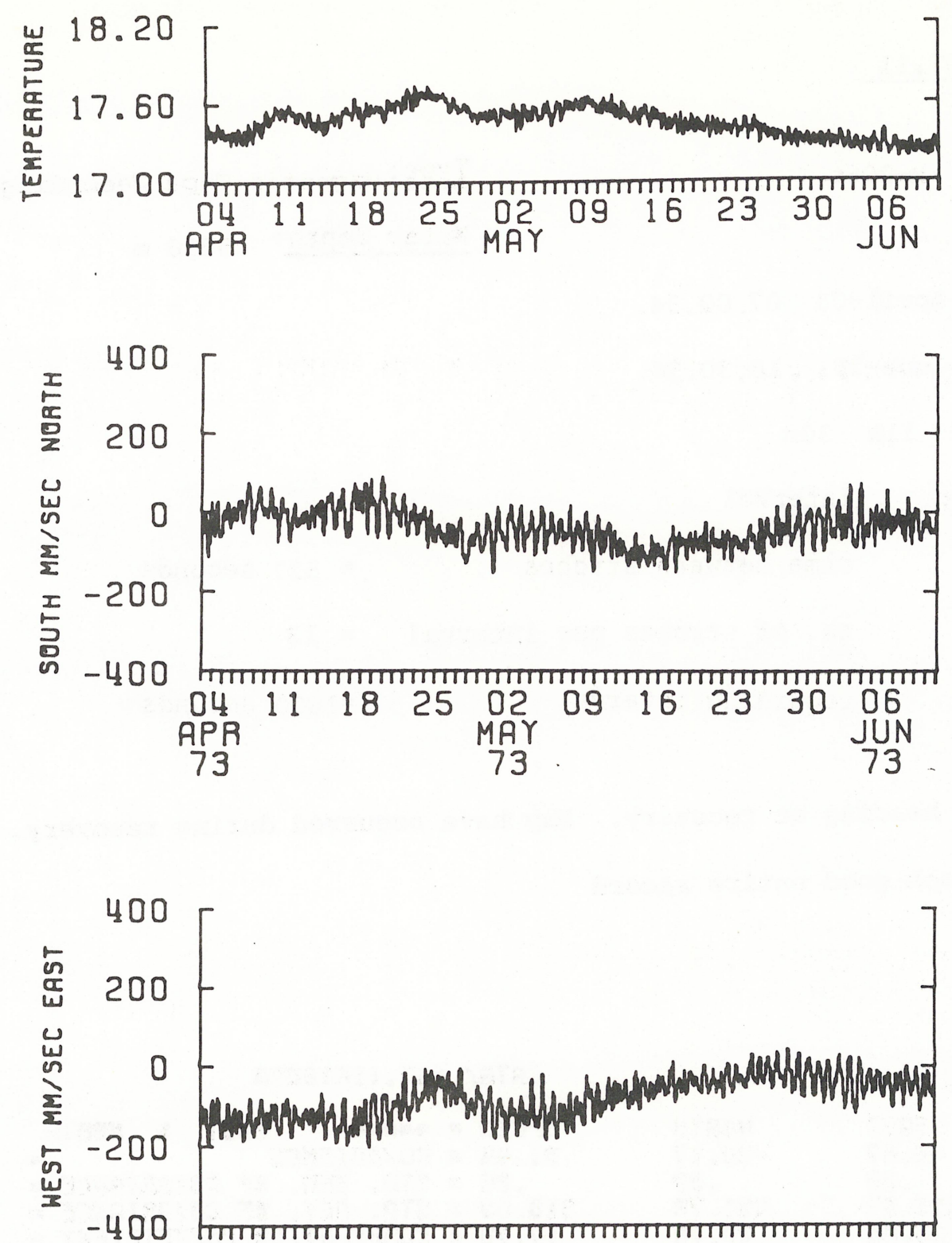

$$
\begin{gathered}
4971 \mathrm{C} 1 \mathrm{H} \\
374 \mathrm{M}
\end{gathered}
$$
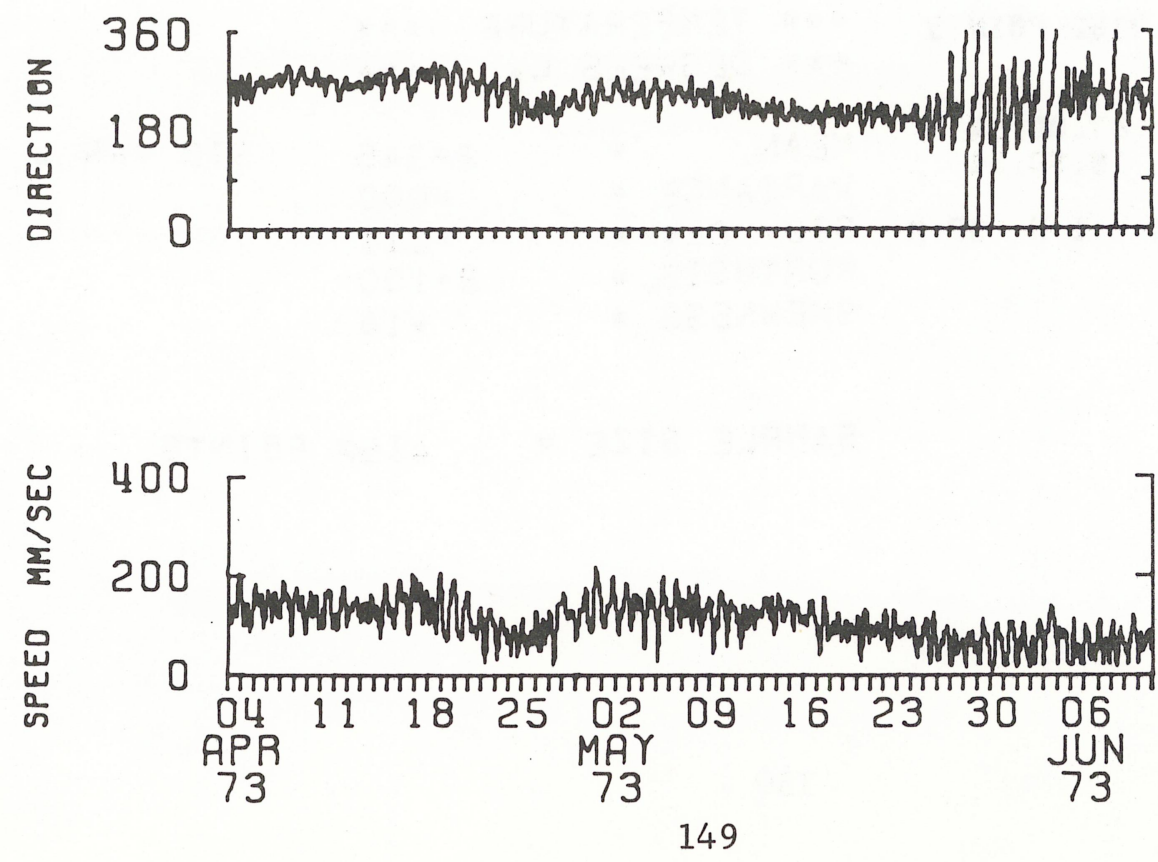
DATA NUMBER 497,11

Instrument No: : $M-206 t$

Depth: $3940 \mathrm{~m}$

Start time: 73-April-03 07.00.34.

Stop time: 73 -June-28 18.30.34.

Duration: $86 \mathrm{~d}$ 11h $30 \mathrm{~m}$

Sampling scheme: Interval
Type: Magnetic Tape Recording Current Meter Water depth: $5296 \mathrm{~m}$

$$
\begin{array}{ll}
\text { time between strobes } & =527 \text { seconds } \\
\text { no. of strobes per interval } & =13 \\
\text { recording interval } & =1800 \text { seconds }
\end{array}
$$

COMMENTS:

\begin{tabular}{|c|c|c|c|}
\hline & & EAST & MONTH \\
\hline 3. E⿱⺈. & $m$ & & 0.17 \\
\hline I ANCE & $\approx$ & 495.67 & 491.76 \\
\hline DEV. & a & 20.83 & 20.70 \\
\hline 0915 & $=$ & 3.15 & 4.05 \\
\hline WNESS & ๓ & -.32 & .46 \\
\hline
\end{tabular}

Vane out of top bearing at recovery. May have occurred during recovery.

All variables look good entire record

STATS
DATAR 497. เ 1K1800A

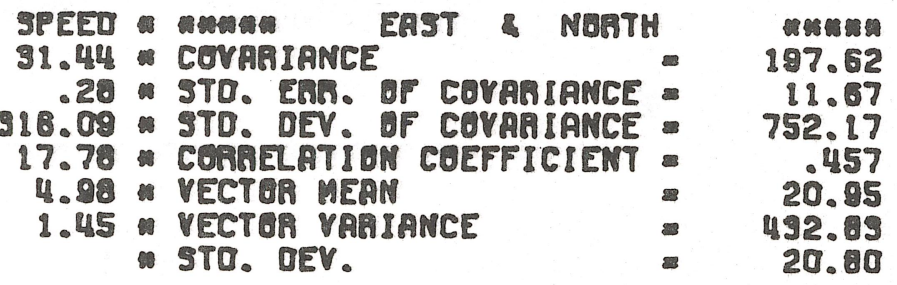

UNITS OF RAM DATA VARIARLES - MMPSEC

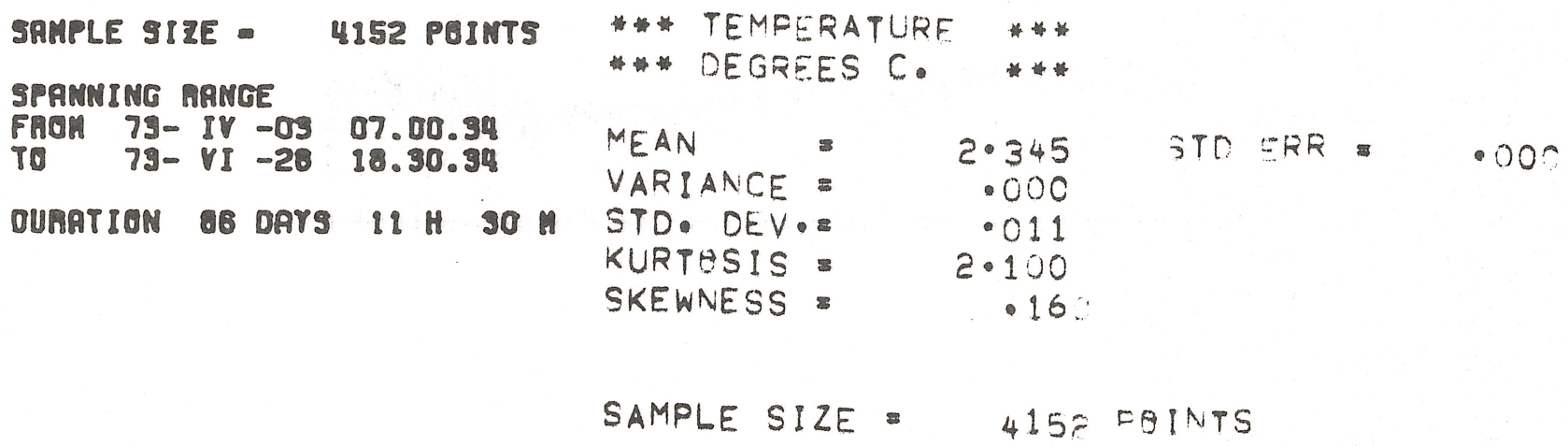




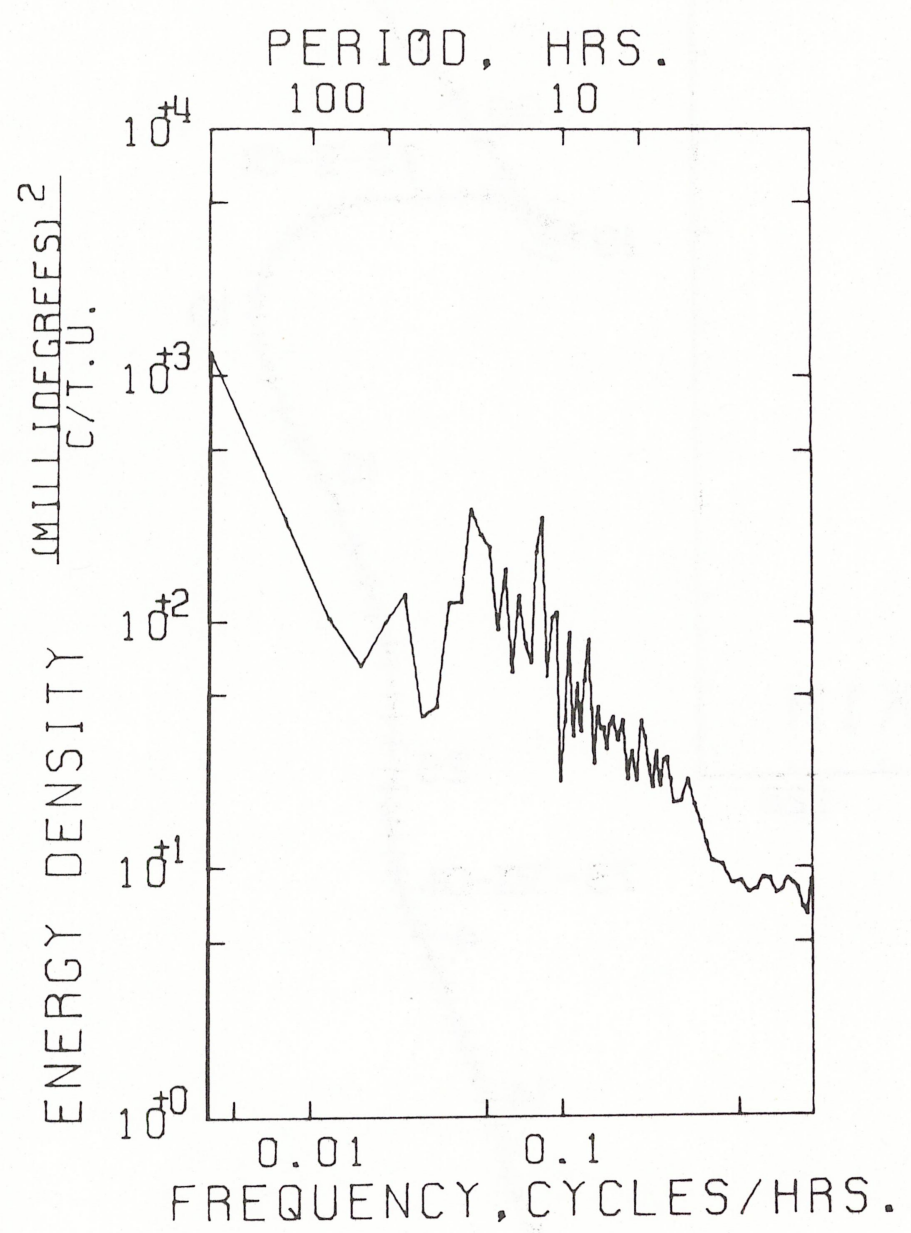

AUTO SPECTRUM

$497.11 \mathrm{~K} 1800$ TEMPERATURE

3940 METERS

73-IV-03 TO 73-VI-27

1 PIECES WITH 2048 ESTIMATES

PER PIECE. AVERAGED OVER

8 ADJACENT FREQUENCY BANDS
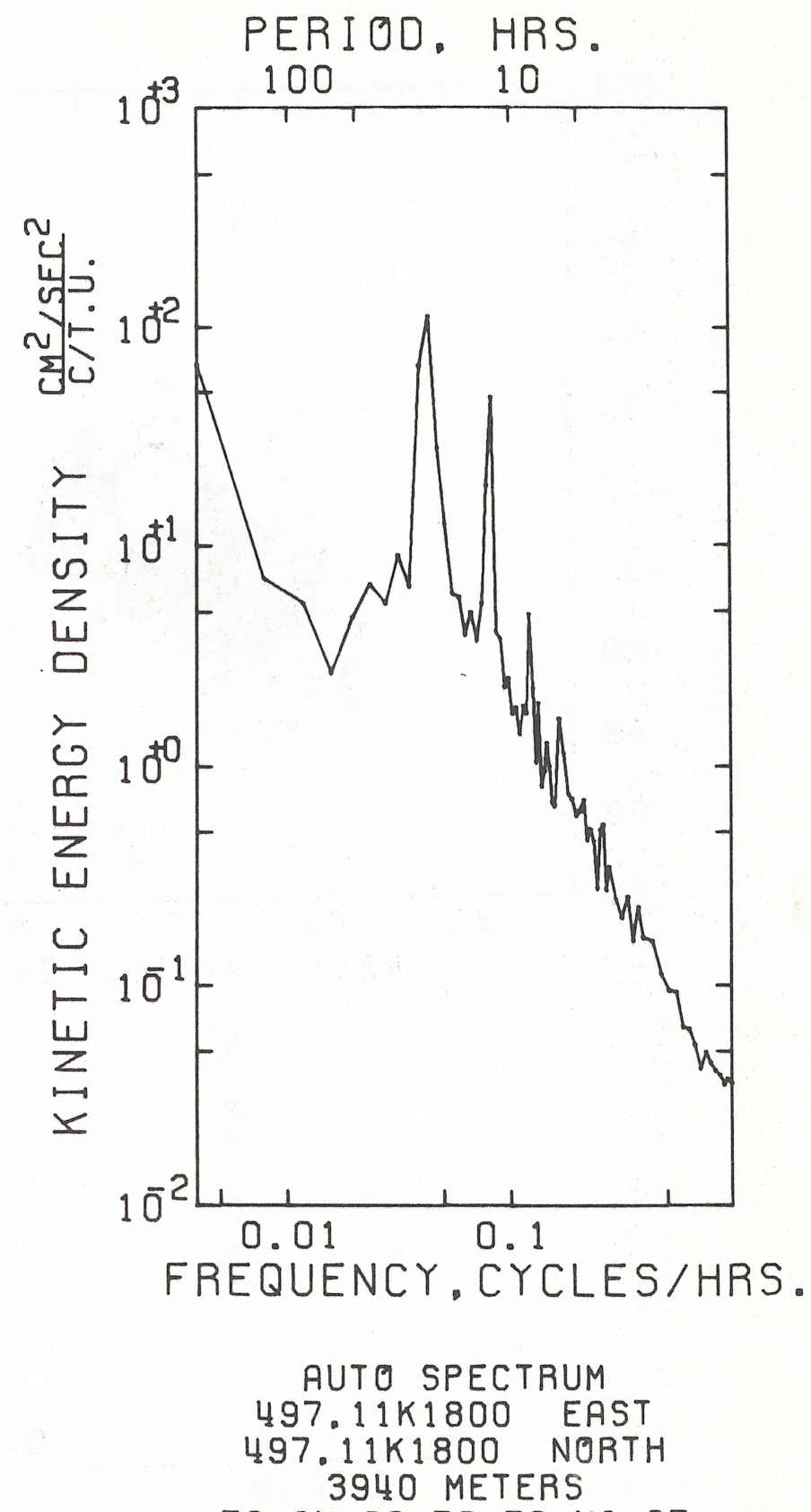

$73-I V-03$ TO $73-V I-27$

1 PIECES WITH 2048 ESTIMATES

PER PIECE. AVERAGED OVER

8 ADJACENT FREQUENCY BANDS 


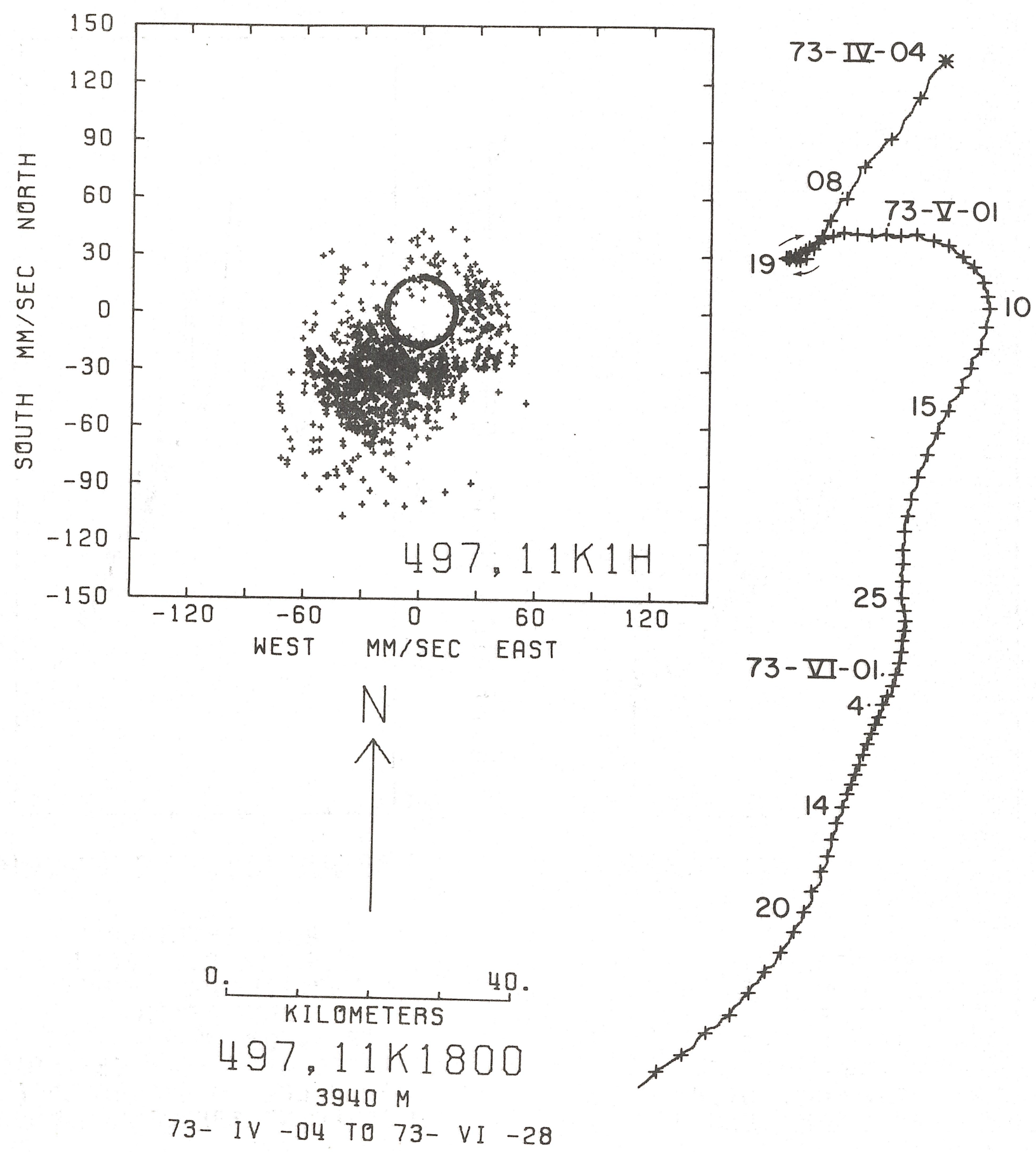



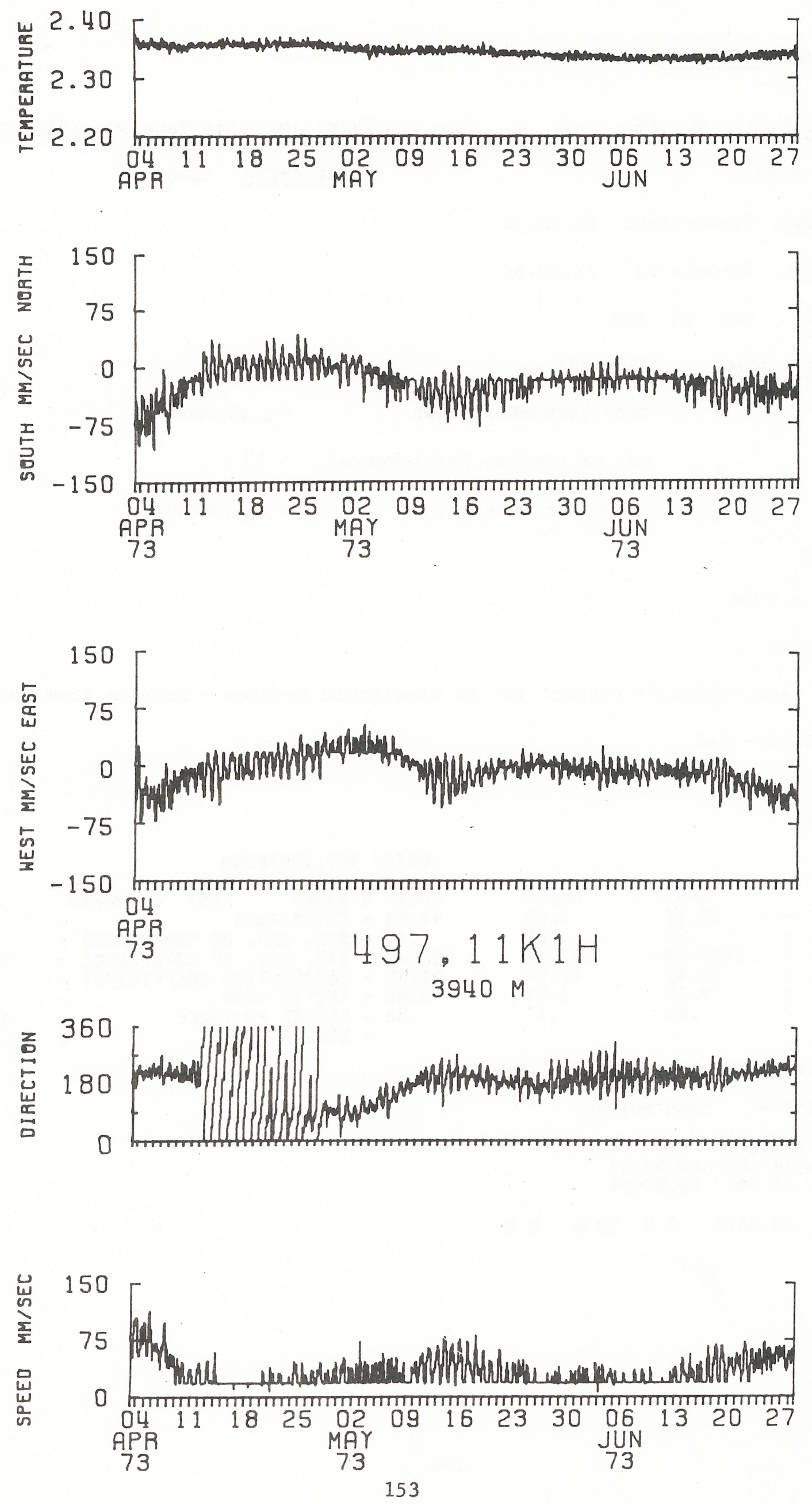
DATA NUMBER 497,13

Instrument No.: M-129t

Depth: $5182 \mathrm{~m}$

Start time: 73-April-02 20.00.34.

Stop time: 73-June-21 22.30.34.

Duration: $\quad 80 \mathrm{~d} 2 \mathrm{~h} 30 \mathrm{~m}$

Sampling scheme: Interval

$$
\begin{array}{ll}
\text { time between strobes } & =5.27 \text { seconds } \\
\text { no. of strobes per interval } & =13 \\
\text { recording interval } & =1800 \text { seconds }
\end{array}
$$

COMMENTS:

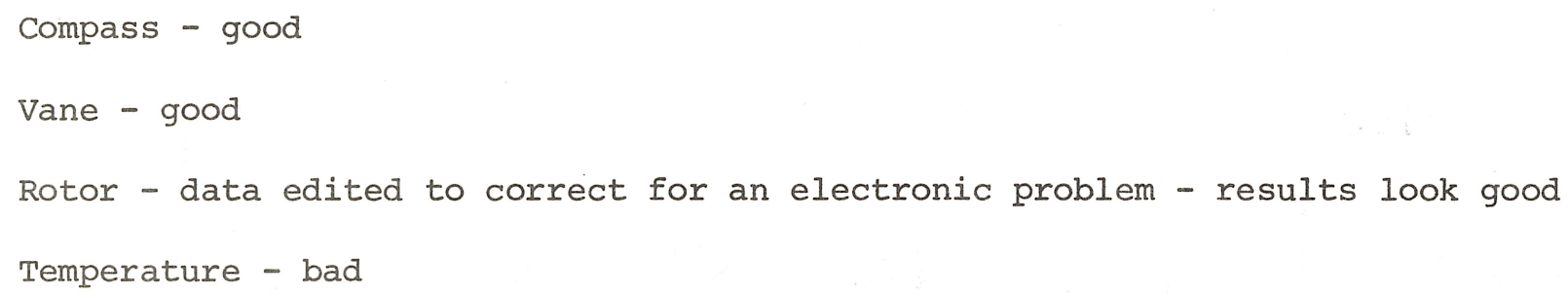

STATS

$$
\text { DATA/ 497.13618009 }
$$

$\begin{array}{lrr} & \text { EAST } & \text { NORTH } \\ \text { MEAN } & 26.16 & 3.82 \\ \text { STD. ENR. }= & .56 & .20 \\ \text { VARIANCE }= & 1209.29 & 307.82 \\ \text { STD. DEV. }= & 34.89 & 17.54 \\ \text { KUATESIS }= & 3.20 & 9.44 \\ \text { SKEWMESS }= & -.36 & .17\end{array}$

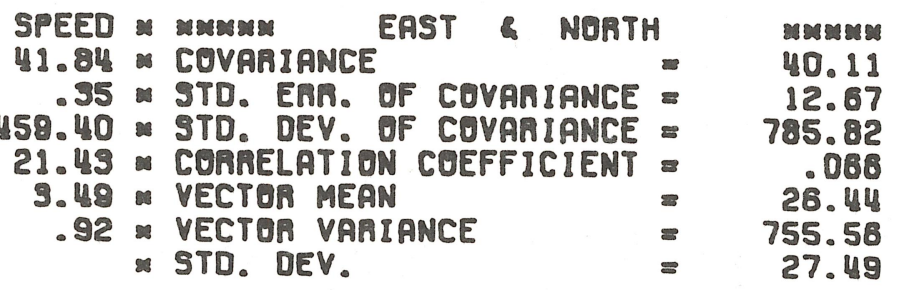

UNITS OF AAW DATA VARIABLES $=$ MM/SEC

SAMPLE SIZE $=3846$ POINTS

SPANNING RANGE

FROM 73- IV -02 20.00.34

TO $73-V I-21 \quad 22.30 .34$

DURATION 80 DAYS $2 \mathrm{H} 30 \mathrm{M}$ O $\mathrm{S}$ 

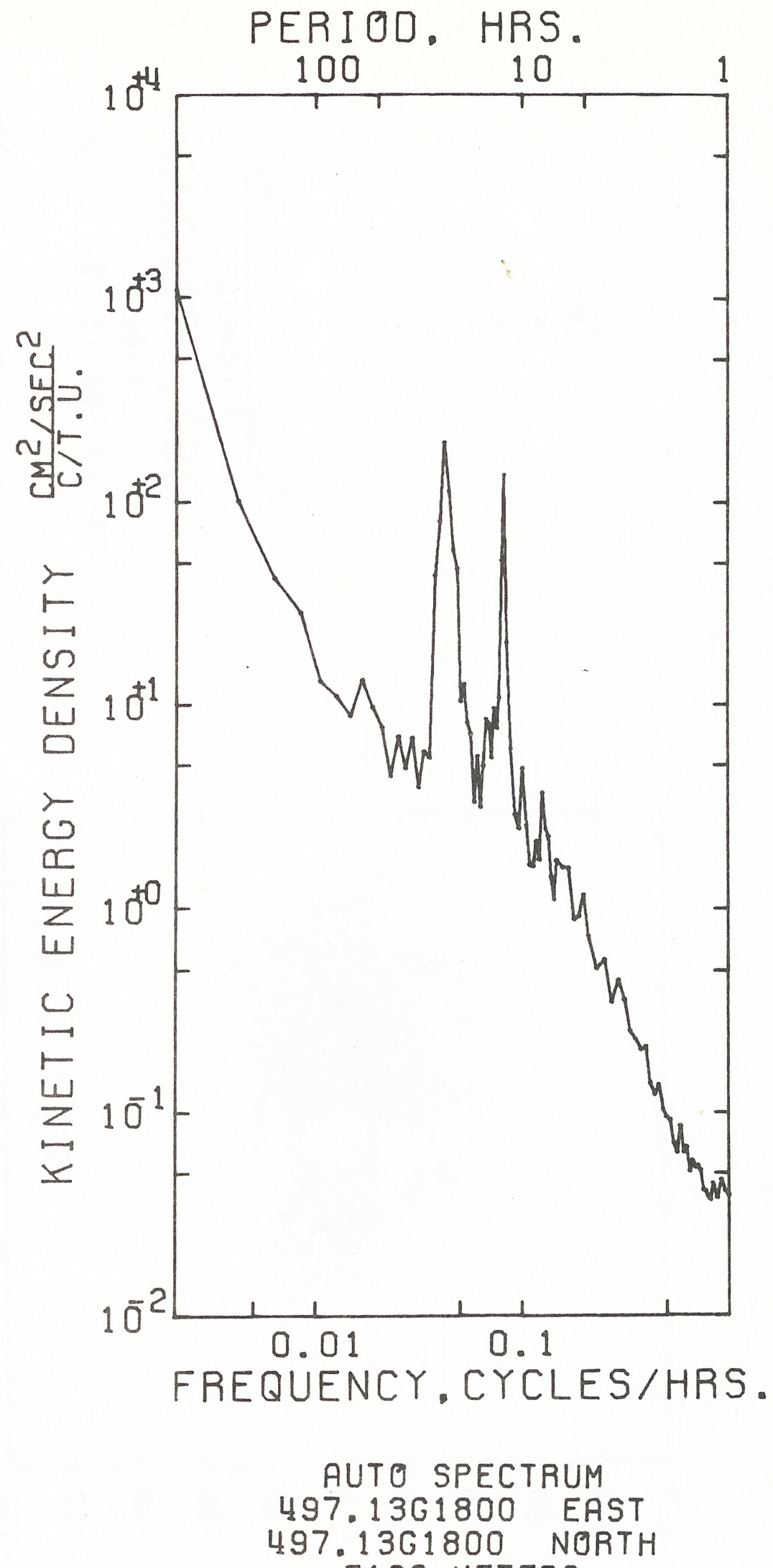
5182 METERS

73-IV-02 TO 73-VI-21

1 PIECES WITH 1920 ESTIMATES PER PIECE. AVERAGED OVER 4 ADJACENT FREQUENCY BANDS 


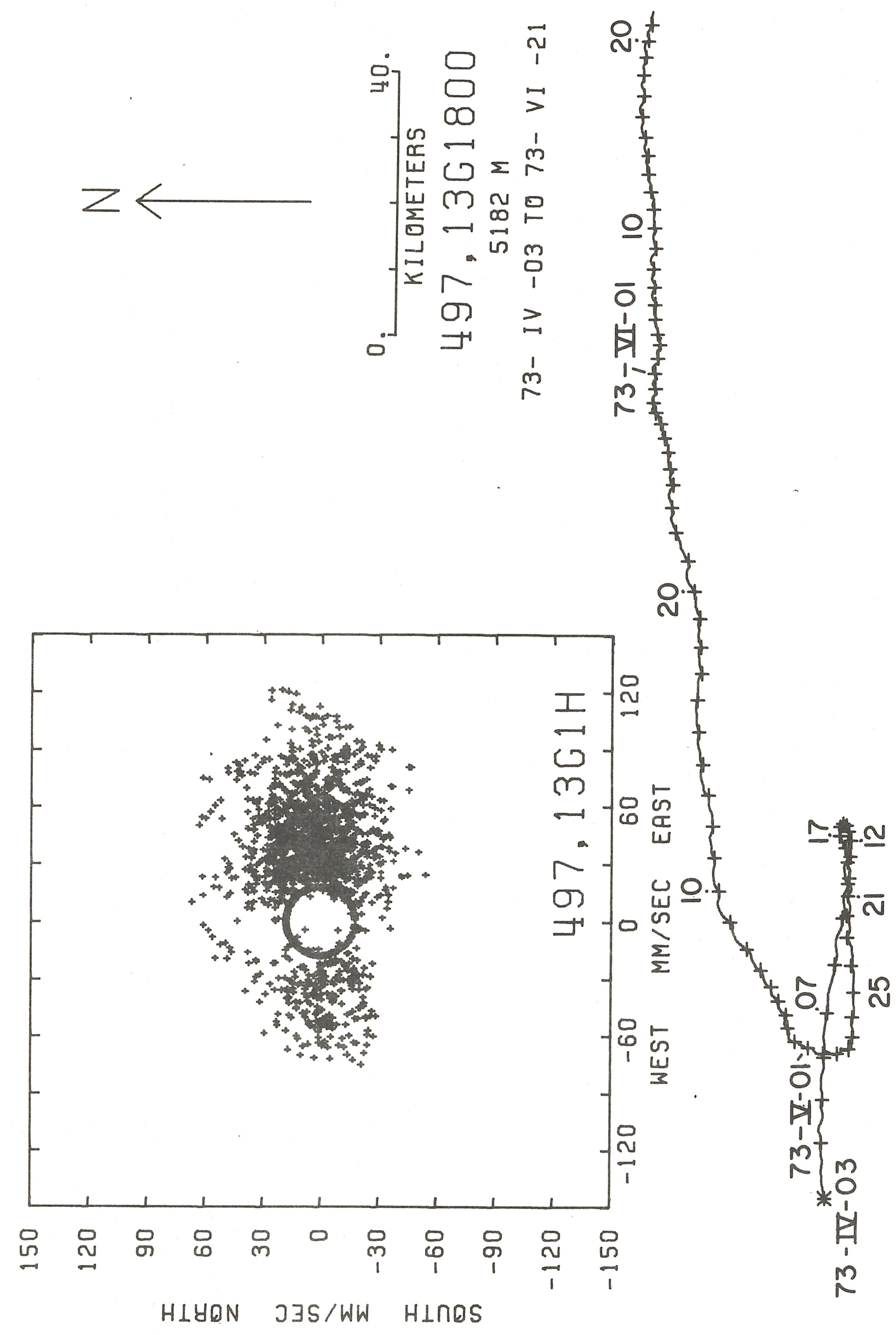



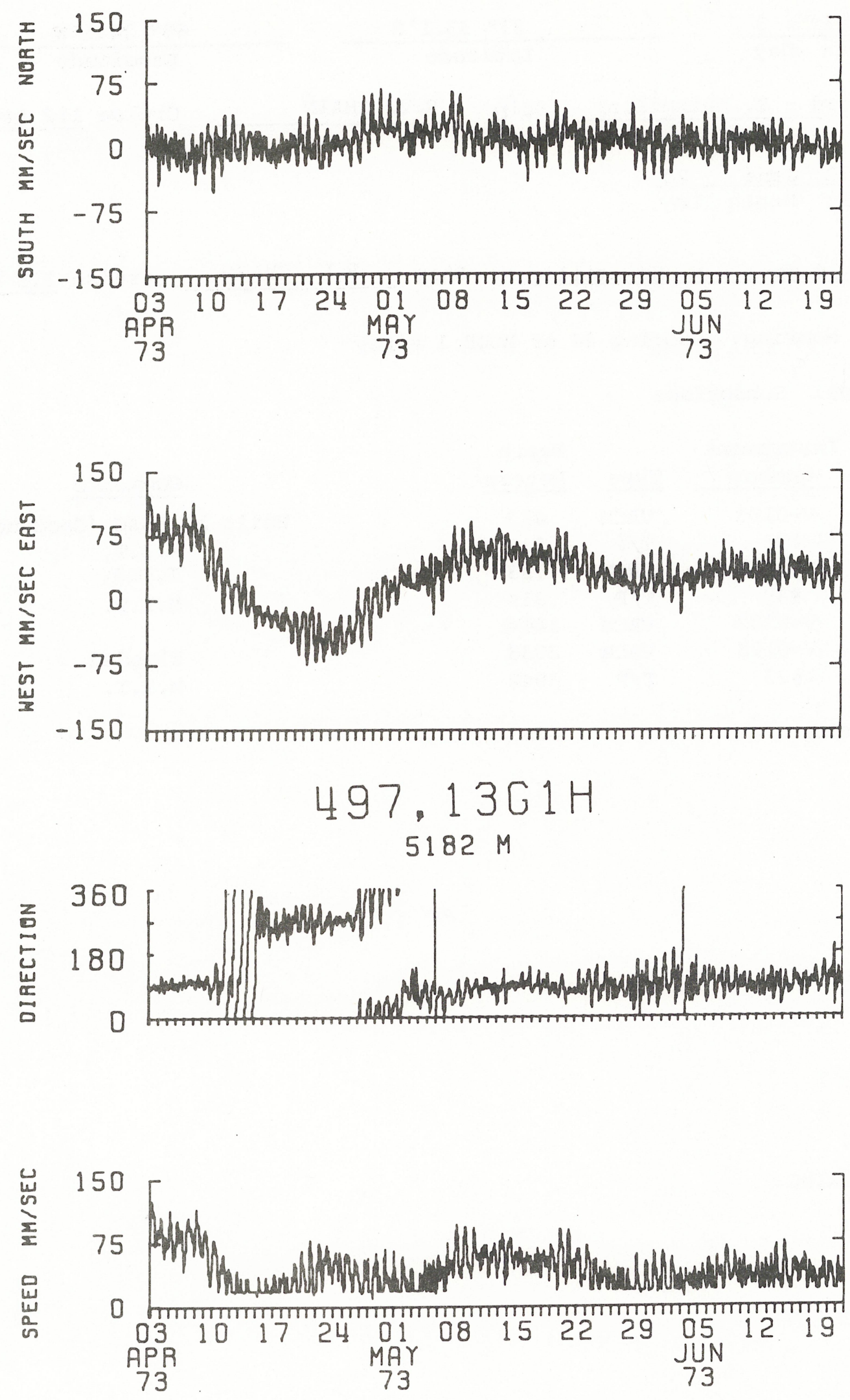
Mooring No. 498

Set $\frac{1973 \text { April } \quad 3}{\text { Year Month Day }}$
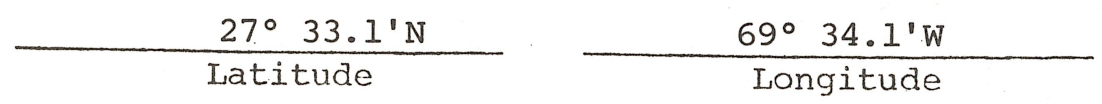

Set by J. Gifford - R. Heinmiller

Ship R.V. CHAIN

Cruise 112 Leg 2

Retrieved $\frac{1973 \text { June }}{\text { Year Month Day }}$

Retrieved by G. Tupper - R. Heinmiller

Ship

R.V. CHAIN

Cruise 112 Leg 6

Purpose of Mooring: Mooring \#4 of MODE 1 array

Mooring Type: Subsurface

\begin{tabular}{|c|c|c|c|c|}
\hline Key & $\begin{array}{c}\text { Data } \\
\text { Number } \\
\end{array}$ & $\begin{array}{c}\text { Instrument } \\
\text { Number } \\
\end{array}$ & Type & $\begin{array}{l}\text { Depth } \\
\text { Meters }\end{array}$ \\
\hline * & 4981 & $V-0103$ & VACM & 413 \\
\hline$\#$ & 4982 & \#36 & $\mathrm{T} / \mathrm{P}$ & 513 \\
\hline * & 4983 & $V-0158$ & VACM & 713 \\
\hline \# & 4984 & $\# 49$ & $\mathrm{~T} / \mathrm{P}$ & 914 \\
\hline+ & 4985 & $V-0202$ & VACM & 1414 \\
\hline & 4986 & $V-0198$ & VACM & 2933 \\
\hline$\#$ & 4987 & $\# 23$ & $\mathrm{~T} / \mathrm{P}$ & 3948 \\
\hline \multicolumn{4}{|c|}{ Water depth } & 5463 \\
\hline
\end{tabular}

Comments

Built by EG\&G (Geodyne)

$M . I . T$.

I.O.S.

M.I.T.

Flooded

M.I.T.

COMMENTS ON MOORING: 


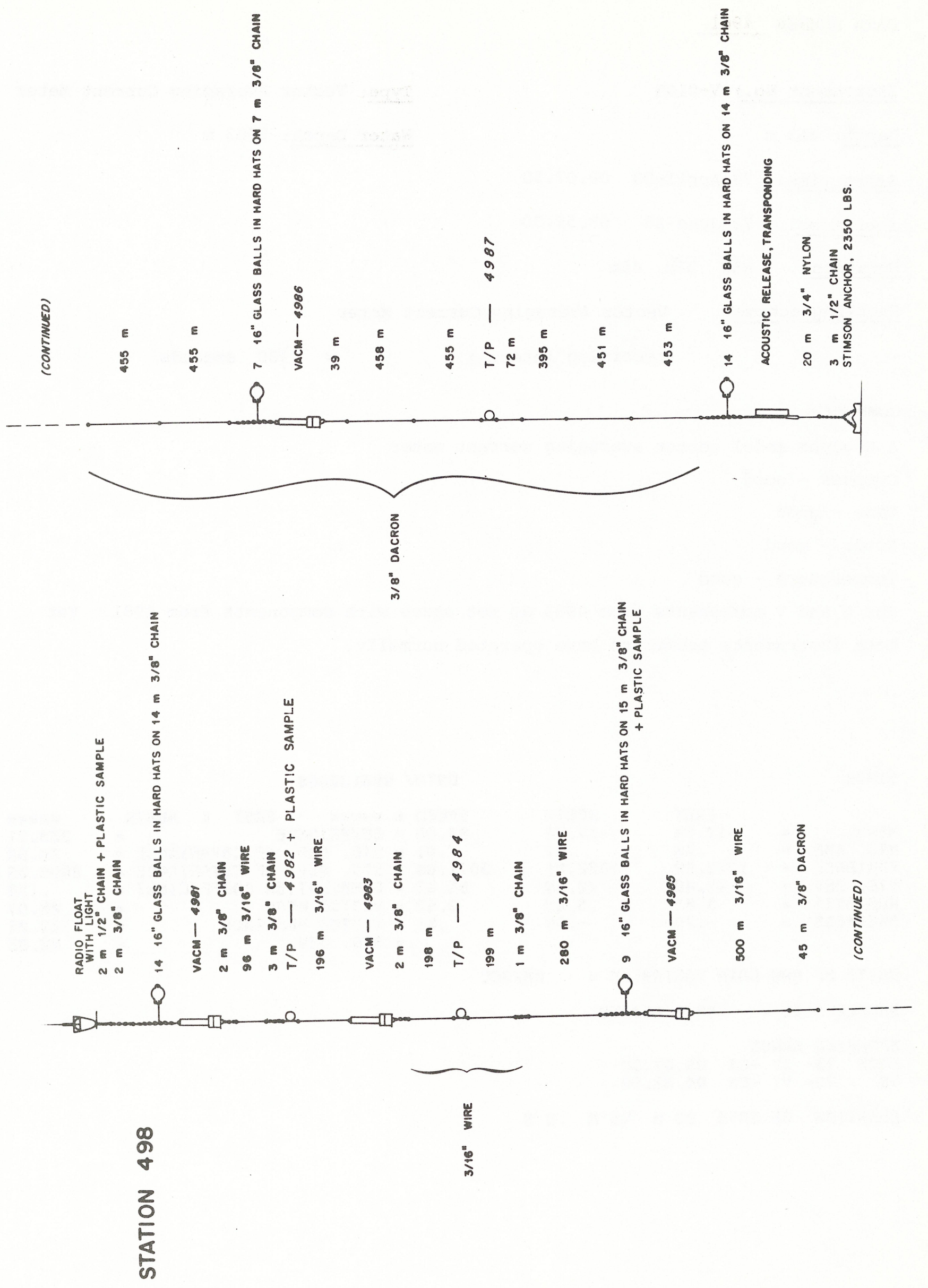


DATA NUMBER 4981

Instrument No.: V-0103

Depth: $413 \mathrm{~m}$
Type: Vector Averaging Current Meter

Water Depth: $5463 \mathrm{~m}$

Start time: 73-April-03 09.07.30

Stop time: 73-June-28 08.52.30

Duration: $\quad 85 \mathrm{~d} \quad 23 \mathrm{~h} 45 \mathrm{~m}$

Sampling scheme: Vector Averaging Current Meter

$$
\text { recording interval }=900 \text { seconds }
$$

COMMENTS:

A Geodyne model vector averaging current meter

Compass - good

Vane - good

Rotor - good

Temperature - good

The $U$ and $V$ components from 4983 do not agree with components from 4981 . Yet both instruments seemed to have operated normally.

STATS

DATA $4981 \mathrm{J9008}$

\begin{tabular}{|c|c|c|c|}
\hline & & EคST & NORTH \\
\hline MEAN & $\approx$ & -17.74 & -17.72 \\
\hline VARIANCE & $\approx$ & 1714.22 & 1022.21 \\
\hline STD. DEV. & $=$ & 41.40 & 42.88 \\
\hline KURTOSIS & $\approx$ & .89 & \\
\hline KEWNESS & $\Xi$ & -.70 & \\
\hline
\end{tabular}

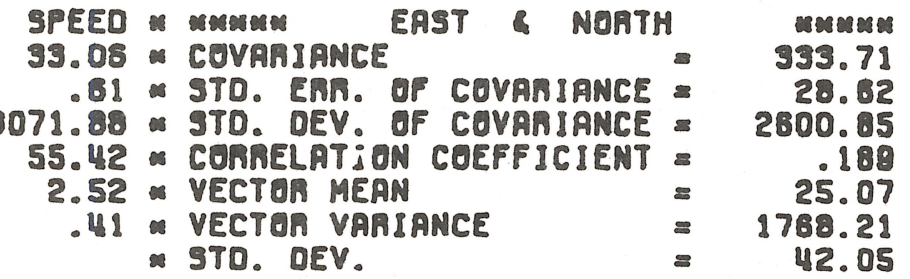

UNITS OF RAH DATA VARIABLES $\cong$ MM/SEC

SAMPLE SIZE = 2256 POINTS

SPANNING RANGE

FROM 73-IV -0309.07 .30$

TO 73-VI -28 08.52.30

DURATION 85 DAYS 23 H 45 M 09 

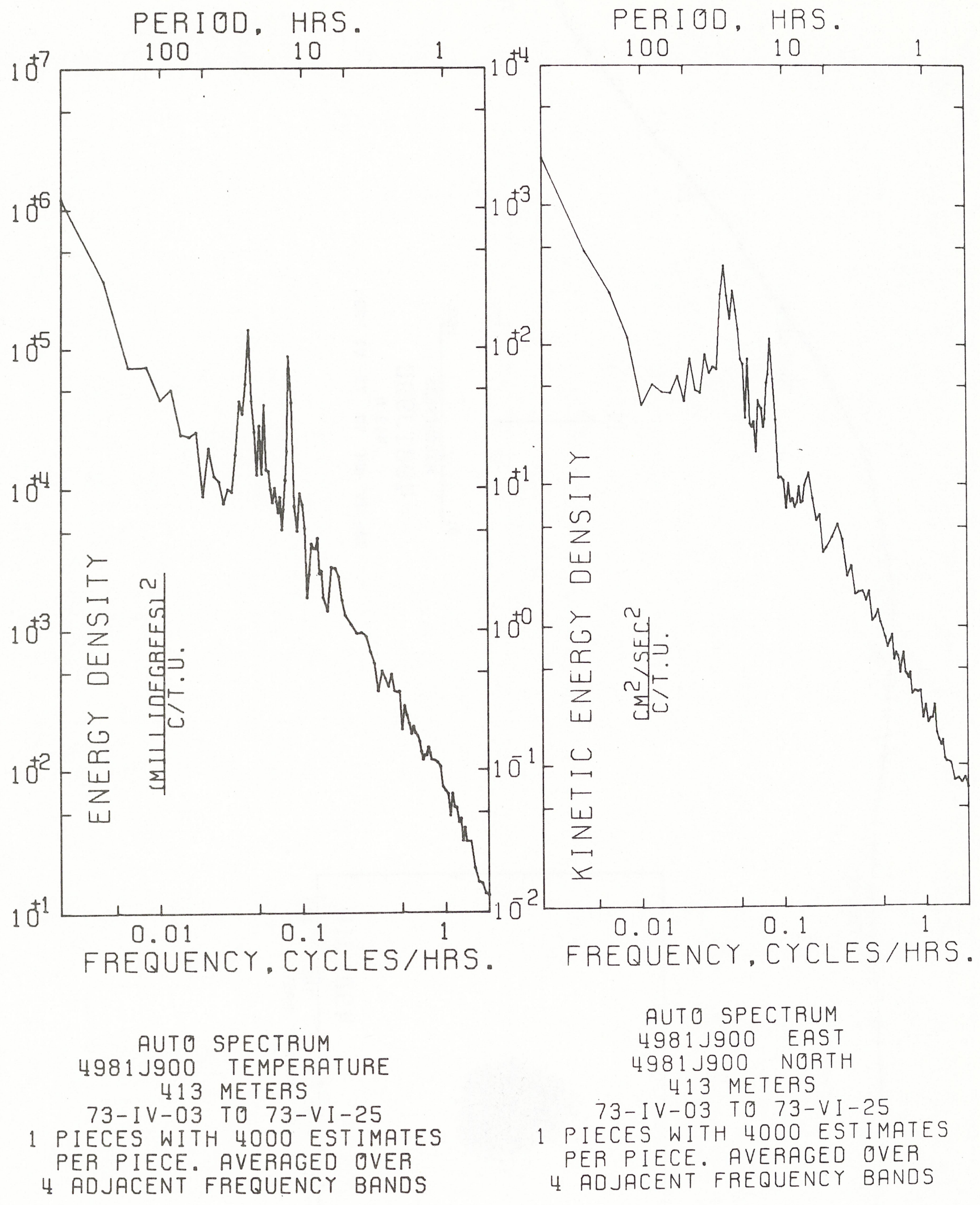

AUTO SPECTRUM $4981 J 900$ EAST $4981 J 900$ NORTH 413 METERS 73-IV-03 TO 73-VI-25 1 PIECES WITH 4000 ESTIMATES PER PIECE. AVERAGED OVER 4 ADJACENT FREQUENCY BANDS 


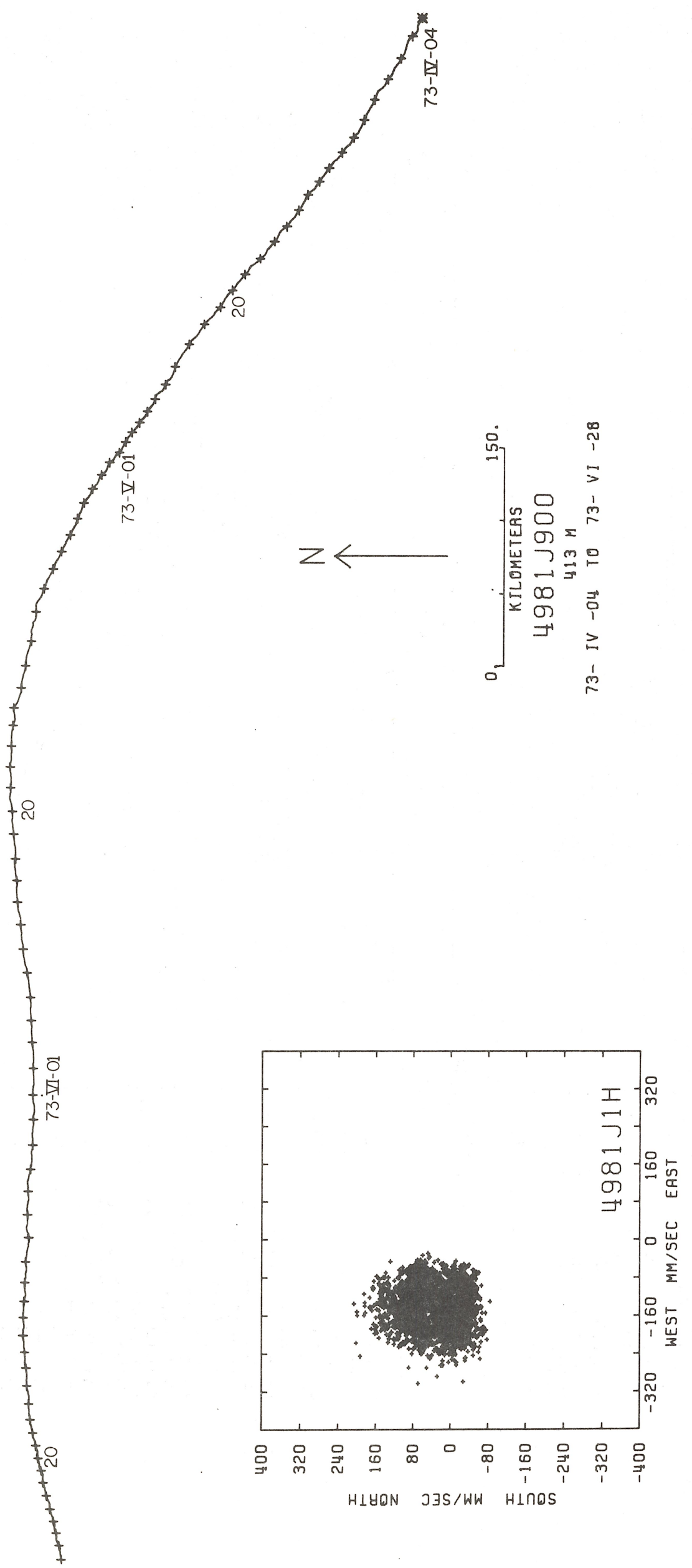



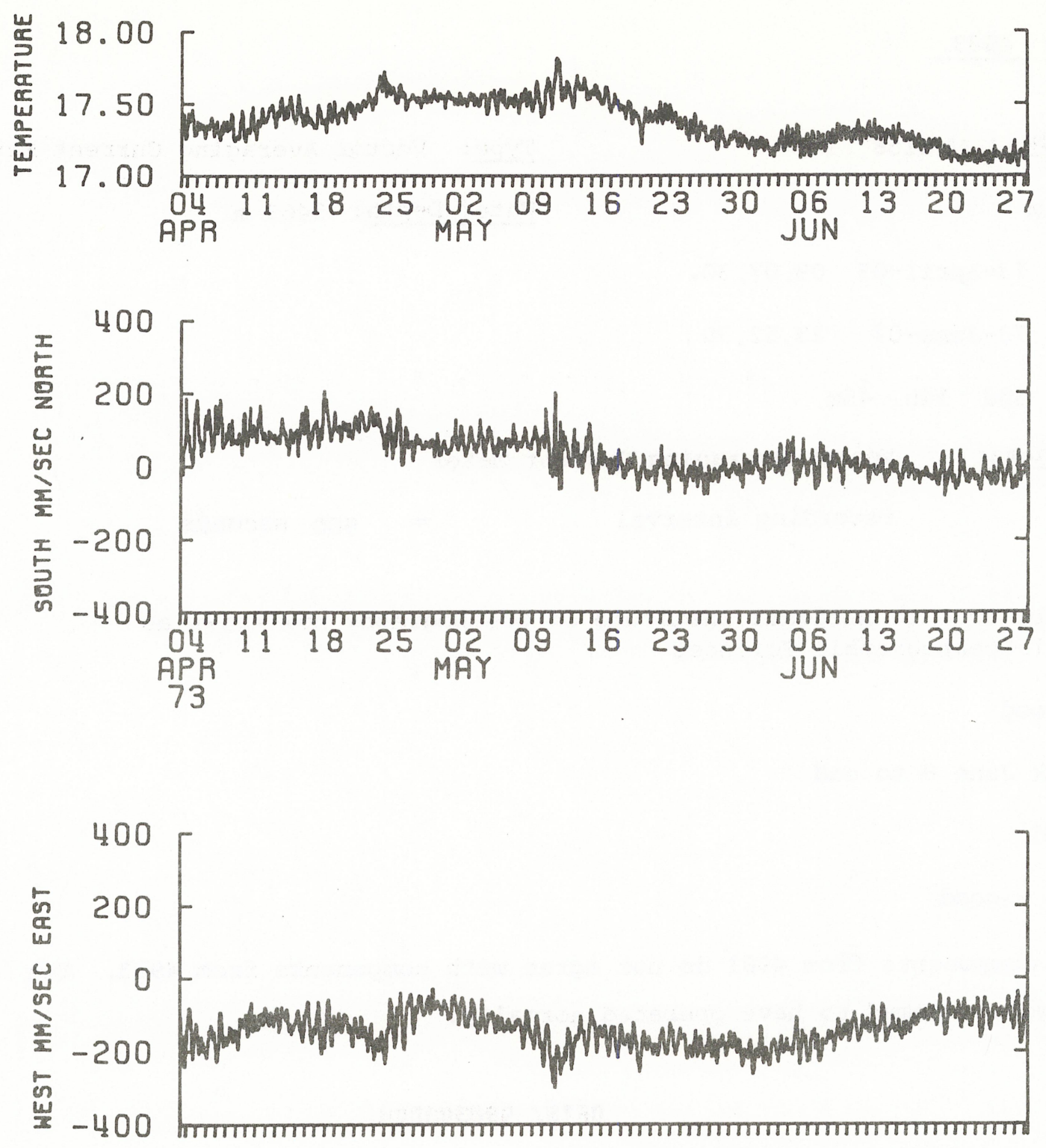

$$
4981 \mathrm{~J} \underset{413}{1 H}
$$
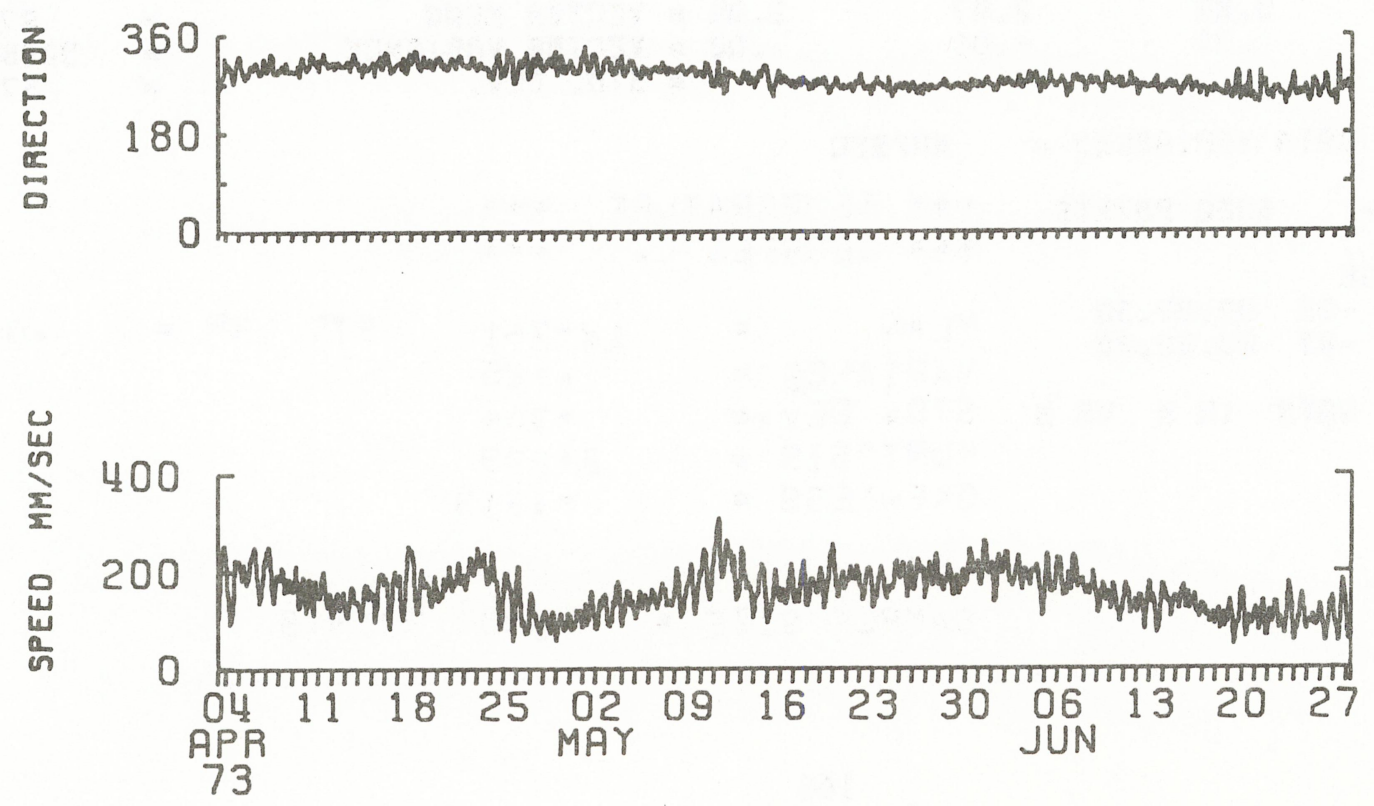
DATA NUMBER 4983

Instrument No.: V-0158

Depth: $713 \mathrm{~m}$
Type: Vector Averaging Current Meter

Water Depth: $5463 \mathrm{~m}$

Start time: 73-April-03 09.07.30.

Stop time: 73-June-07 23.52.30.

Duration: $65 \mathrm{~d}$ 14h $45 \mathrm{~m}$

Sampling scheme: Vector Averaging Current Meter

$$
\text { recording interval }=900 \text { seconds }
$$

COMMENTS :

Instrument belongs to National Institute of Oceanography now known as

Institute of Oceanographic Sciences

Compass - good

Vane - stuck June 8 to end

Rotor - good

Temperature - good

The $U$ and $V$ components from 4981 do not agree with components from $4983 . \quad$ Yet both instruments seemed to have operated normally.

STATS

$\begin{array}{lrr} & \text { EAST } & \text { NOATH } \\ \text { MEAN } & -90.73 & -36.89 \\ \text { STD. EAR. } & .61 & .81 \\ \text { YARIANCE }= & 2970.78 & 4.42 .96 \\ \text { STD. DEV }= & 48.89 & 64.37 \\ \text { KURTESIS }= & 3.84 & 2.47 \\ \text { SKEWNESS }= & .31 & -.01\end{array}$

DATAR 499830900B

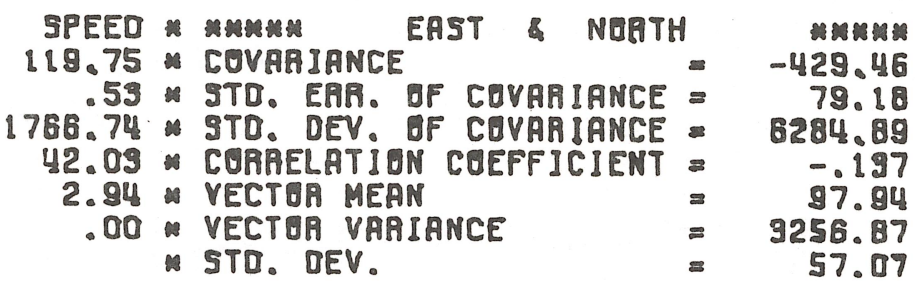

MMPSEC

UNITS OF RAW OATA VARIABLES =

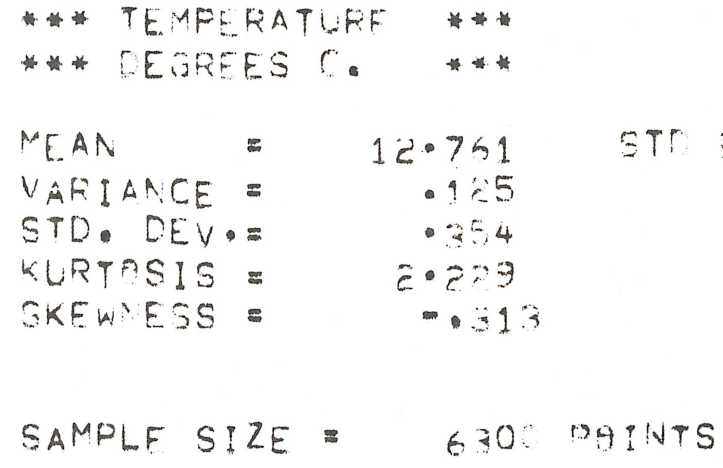



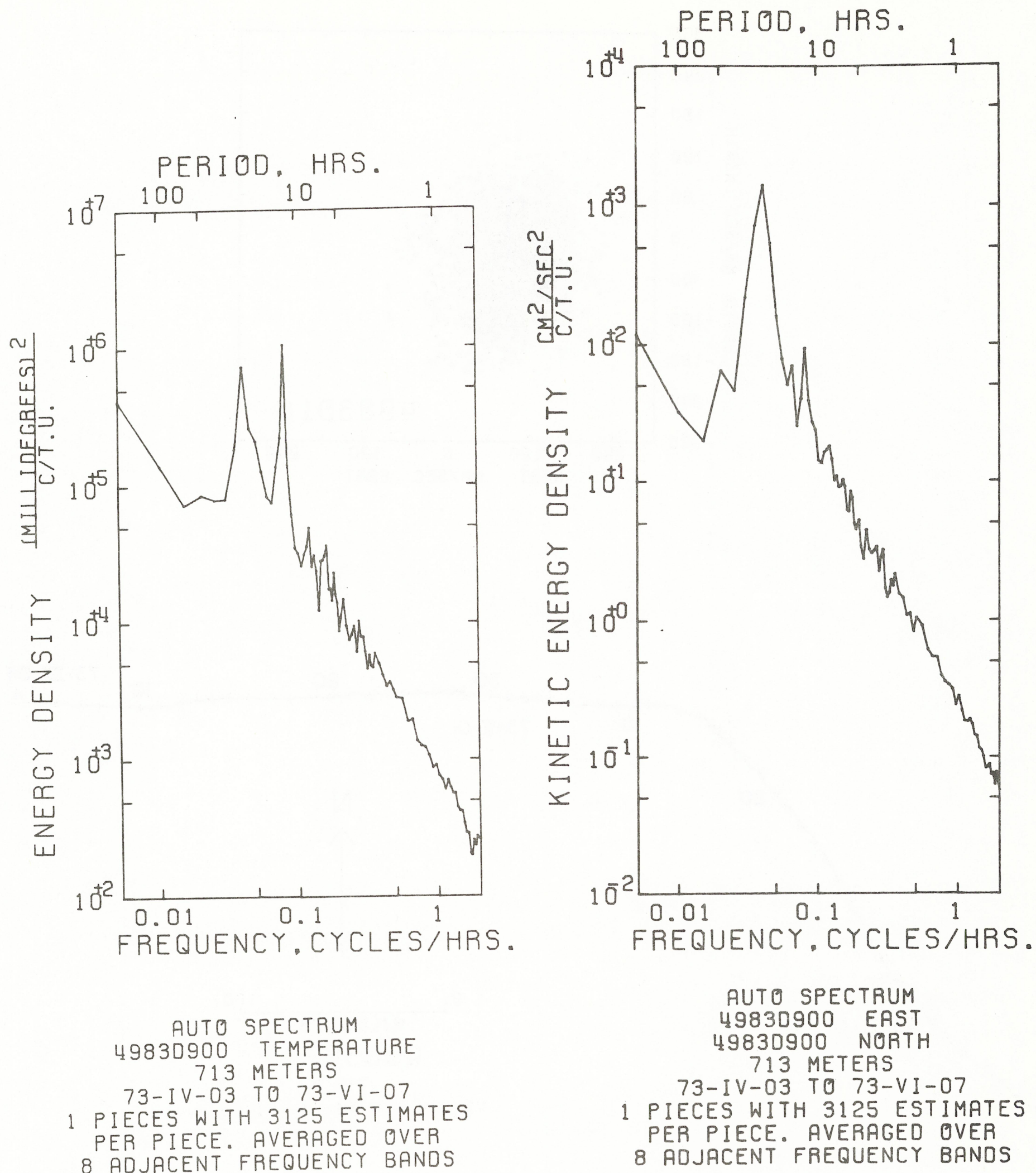

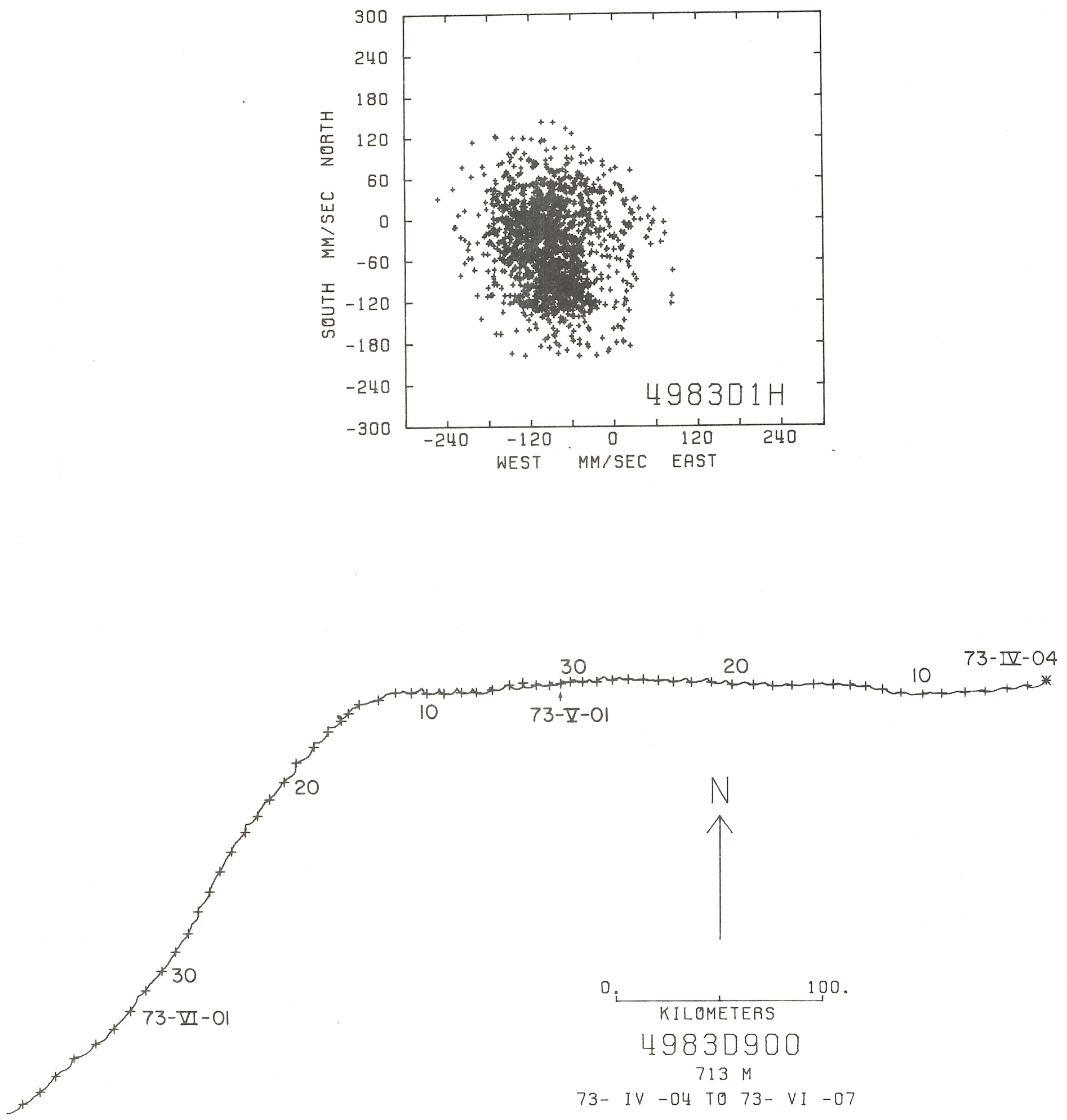

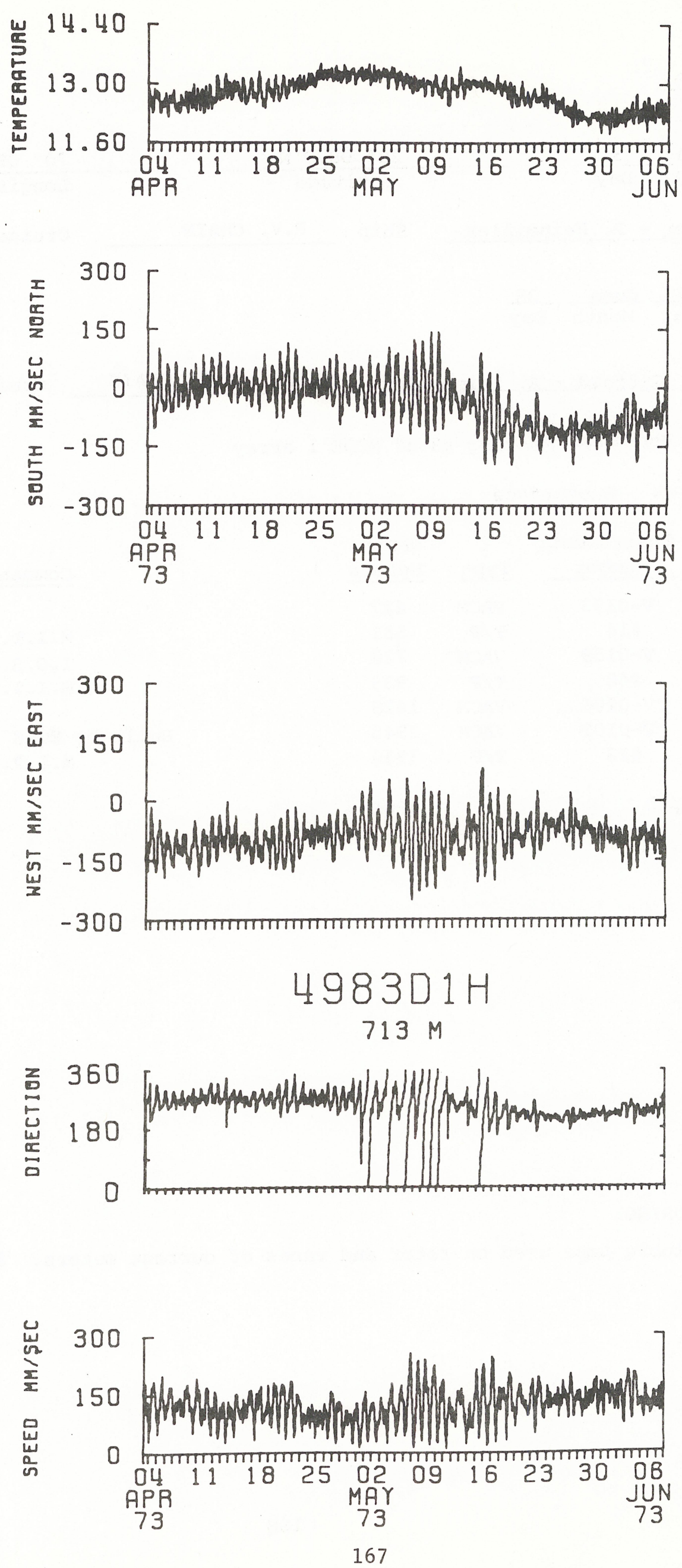
Mooring No. 499

Set $\frac{1973 \text { April } 3}{\text { Year Month Day }} \frac{28^{\circ} 08^{\prime} 9^{\prime} \mathrm{N}}{\text { Latitude }} \frac{70^{\circ} 08^{\prime} 1^{\prime} \mathrm{W}}{\text { Longitude }}$

Set by G. Tupper - R. Heinmiller Ship R.V. CHAIN Cruise 112 Leg 2

Retrieved $\frac{1973}{\text { Year Month Day }} 28$

Retrieved by J. Gifford - R. Heinmiller Ship R.V. CHAIN Cruise 112 Leg 6

Purpose of Mooring: Mooring \#3 of MODE 1 array

Mooring Type: Subsurface

\begin{tabular}{|c|c|c|c|c|c|}
\hline Key & $\begin{array}{c}\text { Data } \\
\text { Number } \\
\end{array}$ & $\begin{array}{c}\text { Instrument } \\
\text { Number } \\
\end{array}$ & Type & $\begin{array}{l}\text { Depth } \\
\text { Meters }\end{array}$ & Comments \\
\hline * & 4991 & $V-0193$ & VACM & 427 & \\
\hline$\#$ & 4992 & \#14 & $\mathrm{T} / \mathrm{P}$ & 531 & $M \cdot I . T$. \\
\hline * & 4993 & $V-0159$ & VACM & 728 & I.O.S. \\
\hline$\#$ & 4994 & $\# 48$ & $\mathrm{~T} / \mathrm{P}$ & 933 & M.I.T. \\
\hline+ & 4995 & $V-0205$ & VACM & 1428 & \\
\hline & 4996 & $V-0102$ & VACM & 2945 & Built by EG\&G (Geodyne) \\
\hline \# & 4997 & $\# 22$ & $\mathrm{~T} / \mathrm{P}$ & 3956 & $\therefore \quad$ M.I.T. \\
\hline \multicolumn{4}{|c|}{ Water depth } & 5461 & \\
\hline
\end{tabular}

COMMENTS ON MOORING:

Water soluble tape used on rotor and vanes of current meters. Shark watched recovery. 


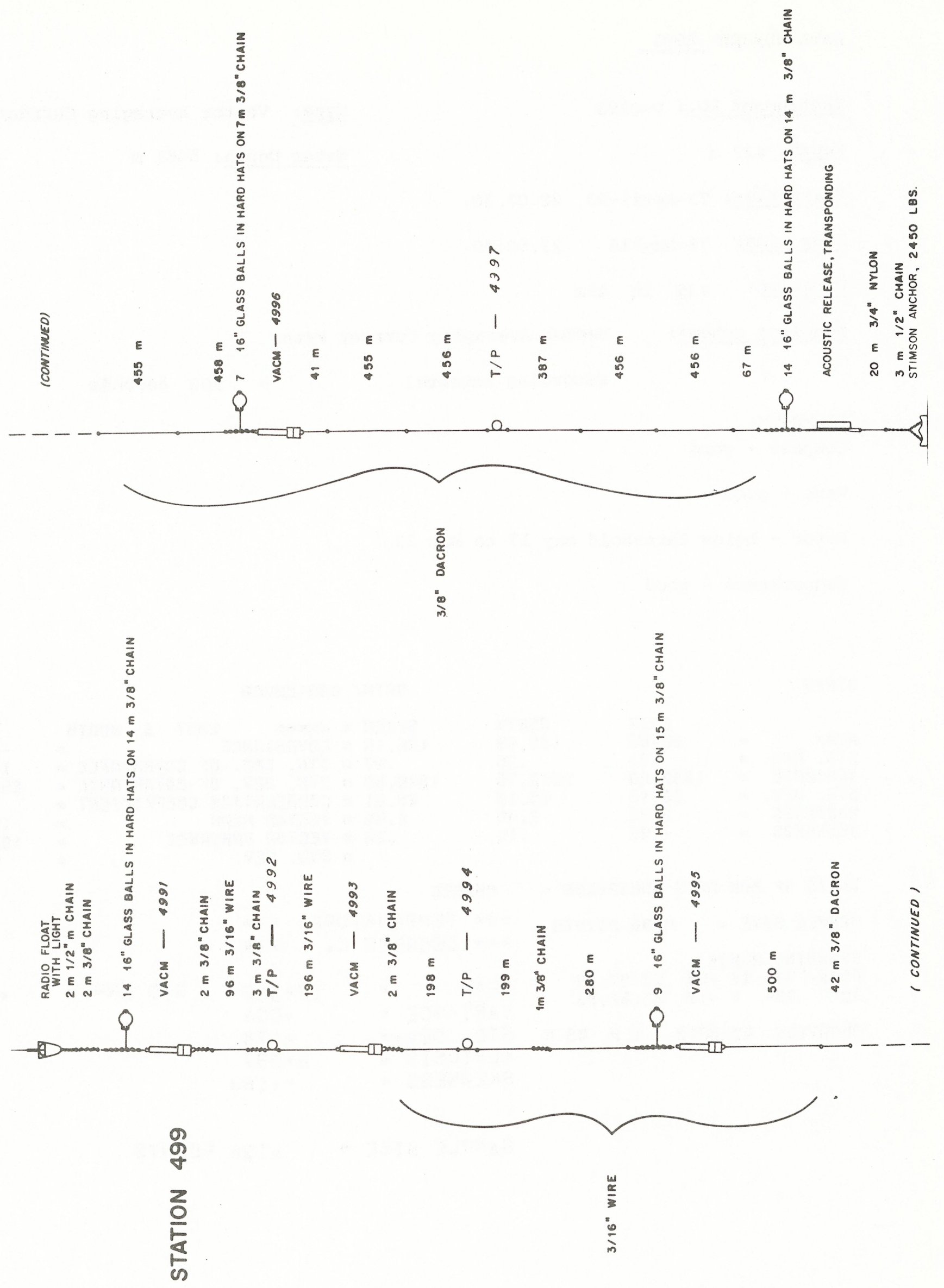


DATA NUMBER 4991

Instrument No.: V-0193

Depth: $427 \mathrm{~m}$

Start time: 73-April-03 22.07.30.

Stop time: 73-May-16 23.52.30.

Duration: $43 \mathrm{~d}$ Ih $45 \mathrm{~m}$

Sampling scheme: Vector Averaging Current Meter recording interval = 900 seconds
Type: Vector Averaging Current Meter

Water Depth: $5461 \mathrm{~m}$

Compass - good

Vane - good

Rotor - below threshold May 17 to May 22

Temperature - good

STATS

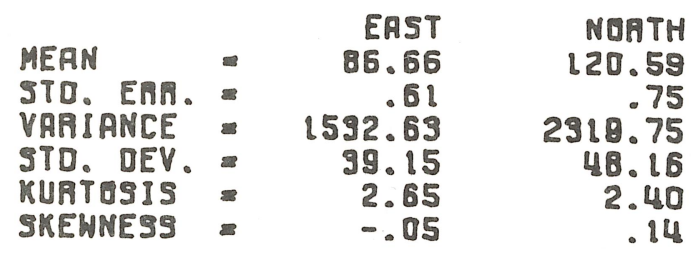

DATAR LLSTESOOA

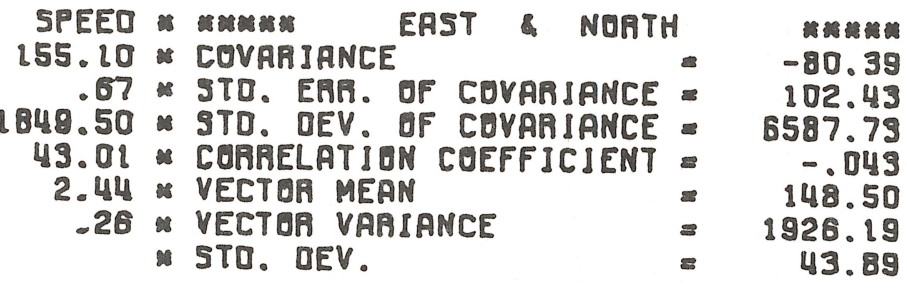

UNITS OF RAW DATA VARIAQLES - MMPEC

SAMPLE SIZE - 4236 POINTS

* TEMPERATURE **

* DEGREES C. * *

SPANNING RANGE

F月OM 73-IV - 03 22.07.30

TO $73-V-16 \quad 23.52 .30$

DURATION 49 DAYS I H 45 M

$\begin{array}{lcc}\text { MEAN } & 17.307 & \text { STD ERR }= \\ \text { VARIANCE }=004 \\ \text { STD. DEV = } & .078 & \\ \text { KURTESIS }= & 2.397 \\ \text { SKEWNESS } & -163 \\ & \\ \text { SAMPLE SIZE } & 4136 \text { POINTS }\end{array}$



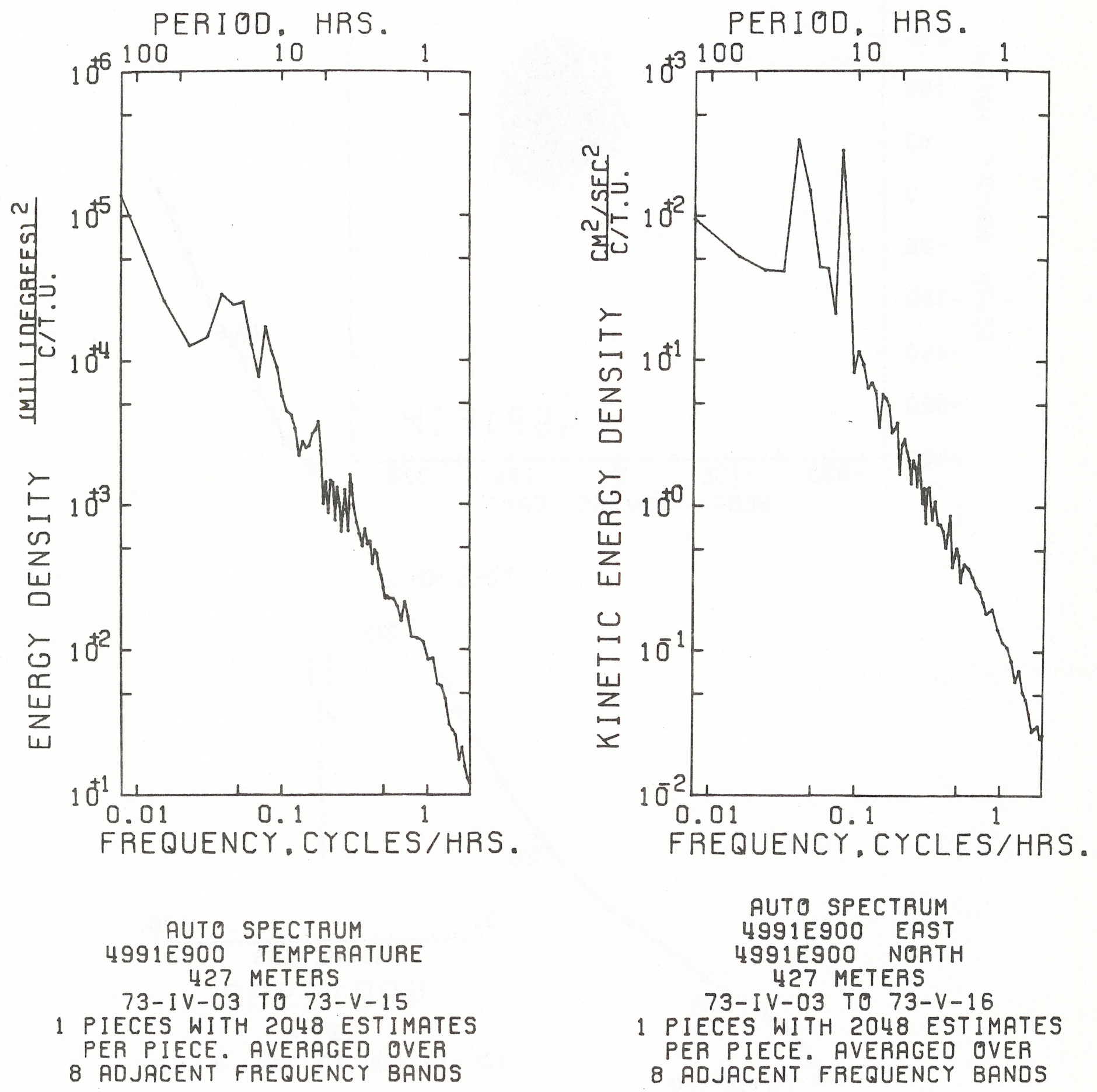


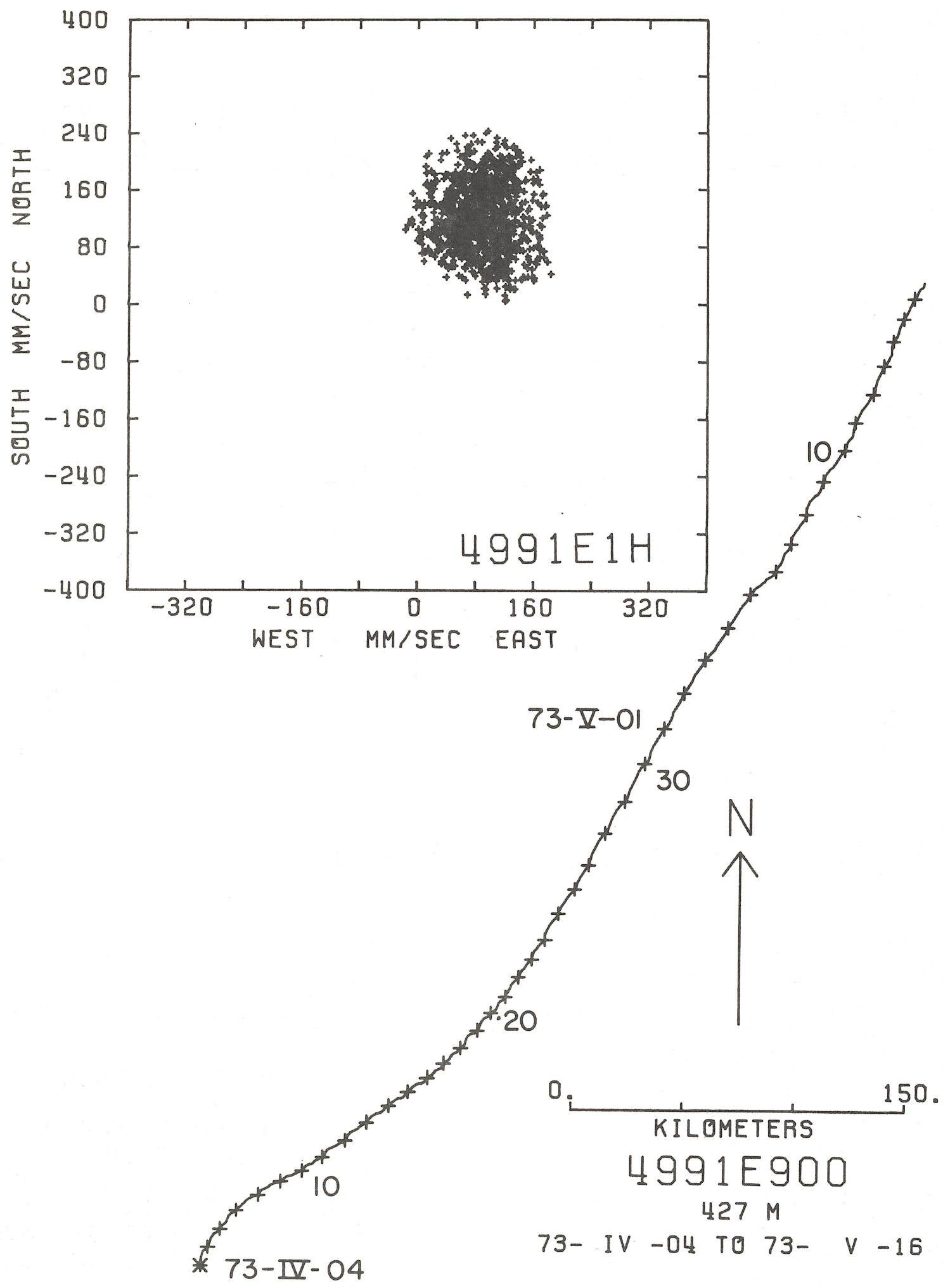



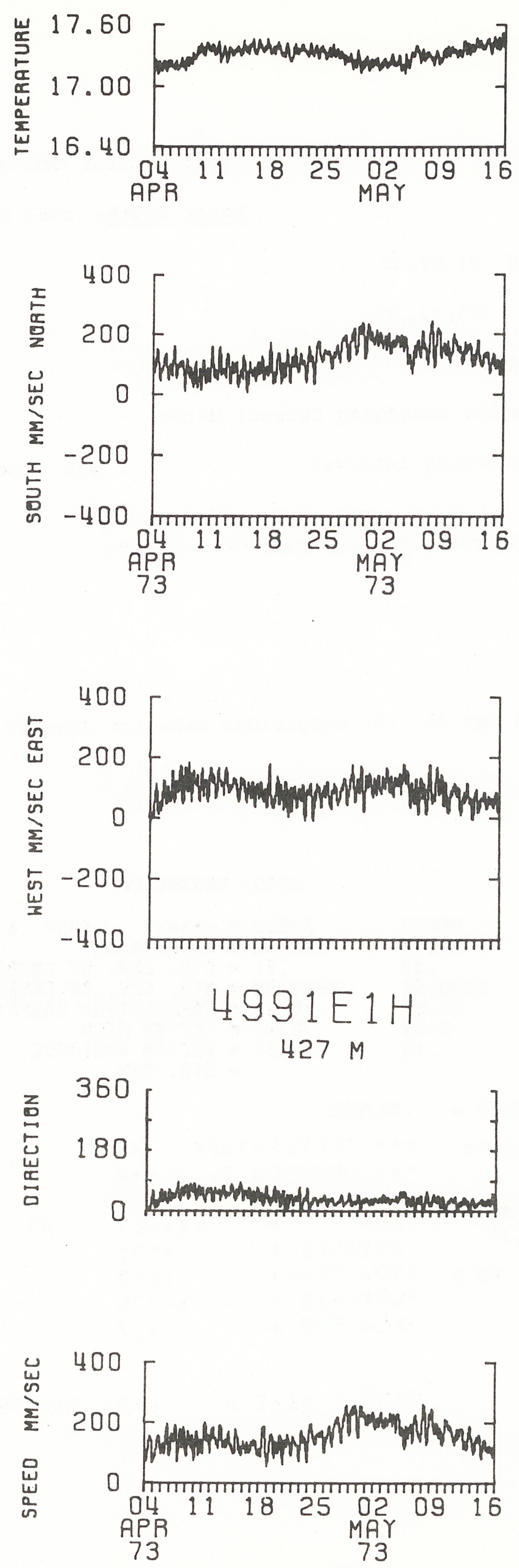
DATA NUMBER 4993

Instrument No.: V-0159

Depth: $728 \mathrm{~m}$

Start time: 73-April-04 01.07.30.

Stop time: $73-$ May-24 23.52.30.

Duration: $\quad 50 \mathrm{~d} 22 \mathrm{~h} \quad 45 \mathrm{~m}$

Sampling scheme: Vector Averaging Current Meter

$$
\text { recording interval }=900 \text { seconds }
$$

COMMENTS :

Instrument owned by Institute of Oceanographic Sciences

Compass - good

Vane - good

Rotor - below threshold May 25, 26, suspicious behavior June 18 to recovery Temperature - good

STATS

DATคЯ 49998900A

\begin{tabular}{|c|c|c|c|}
\hline & & \\
\hline & & EAST & NOBTH \\
\hline STD. EAR. & $=$ & $\begin{array}{r}\text { 53. } \\
.740 \\
.74\end{array}$ & $\begin{array}{r}75.16 \\
.06\end{array}$ \\
\hline VARIANCE & $\cong$ & 2644.78 & 3579.92 \\
\hline $\begin{array}{l}\text { STD. DEV. } \\
\text { KURTOSIS }\end{array}$ & $\begin{array}{l}= \\
=\end{array}$ & 51.43 & $\begin{array}{r}59.03 \\
2.49\end{array}$ \\
\hline NNES & $\approx$ & 00 & \\
\hline
\end{tabular}

UNITS OF RAW DATA VARIABLES = MMPEC

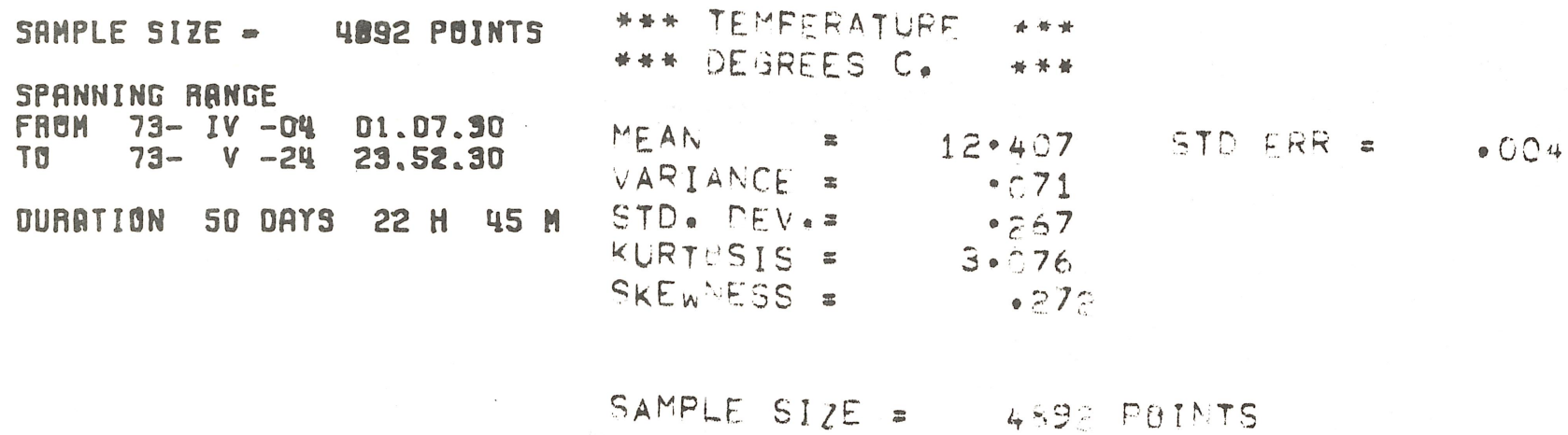




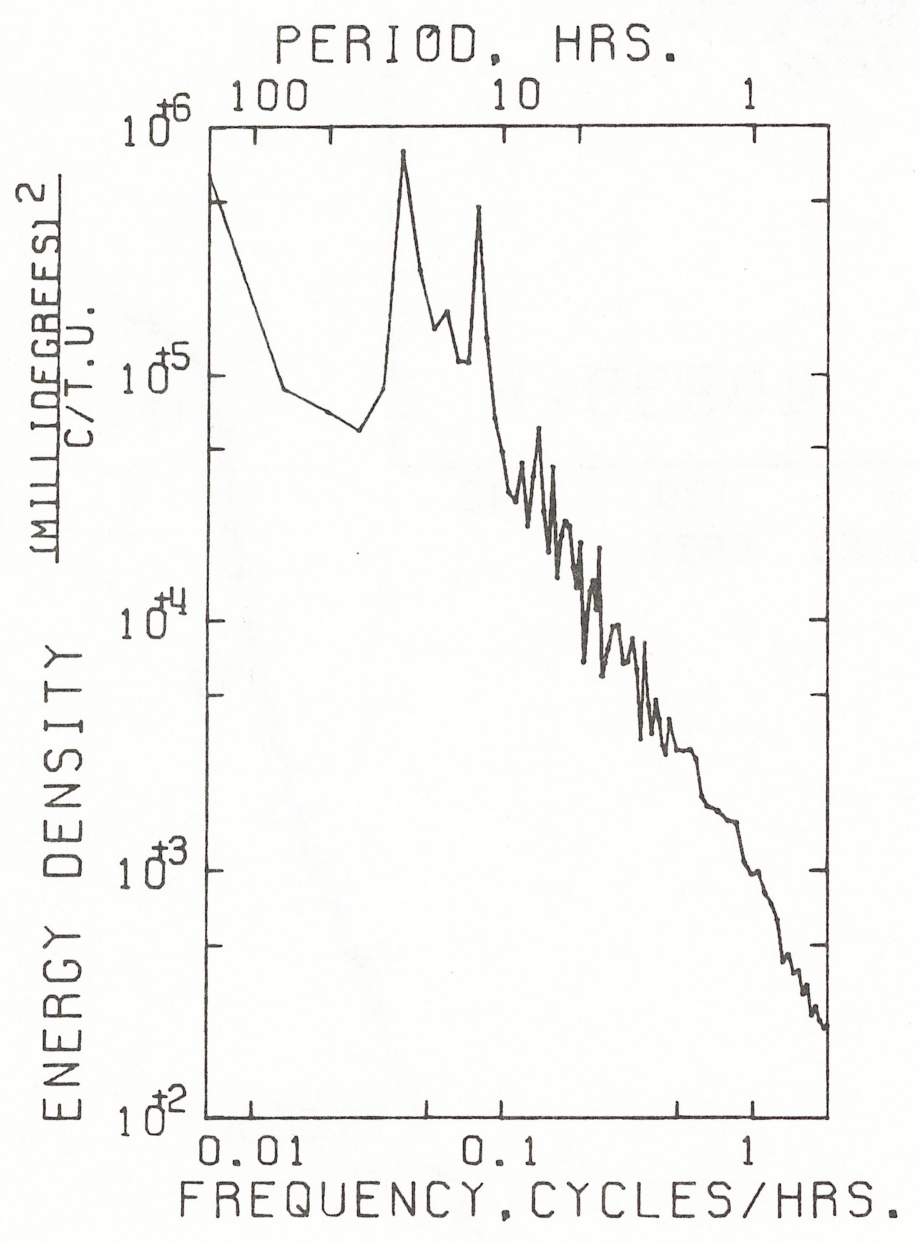

AUTO SPECTRUM
4993B90 TEMPERATURE
728 METERS
$73-I V-04$ TO $73-V-24$
PIECES WITH 2430 ESTIMATES
PER PIECE. AVERAGED OVER
8 ADJACENT FREQUENCY BANDS

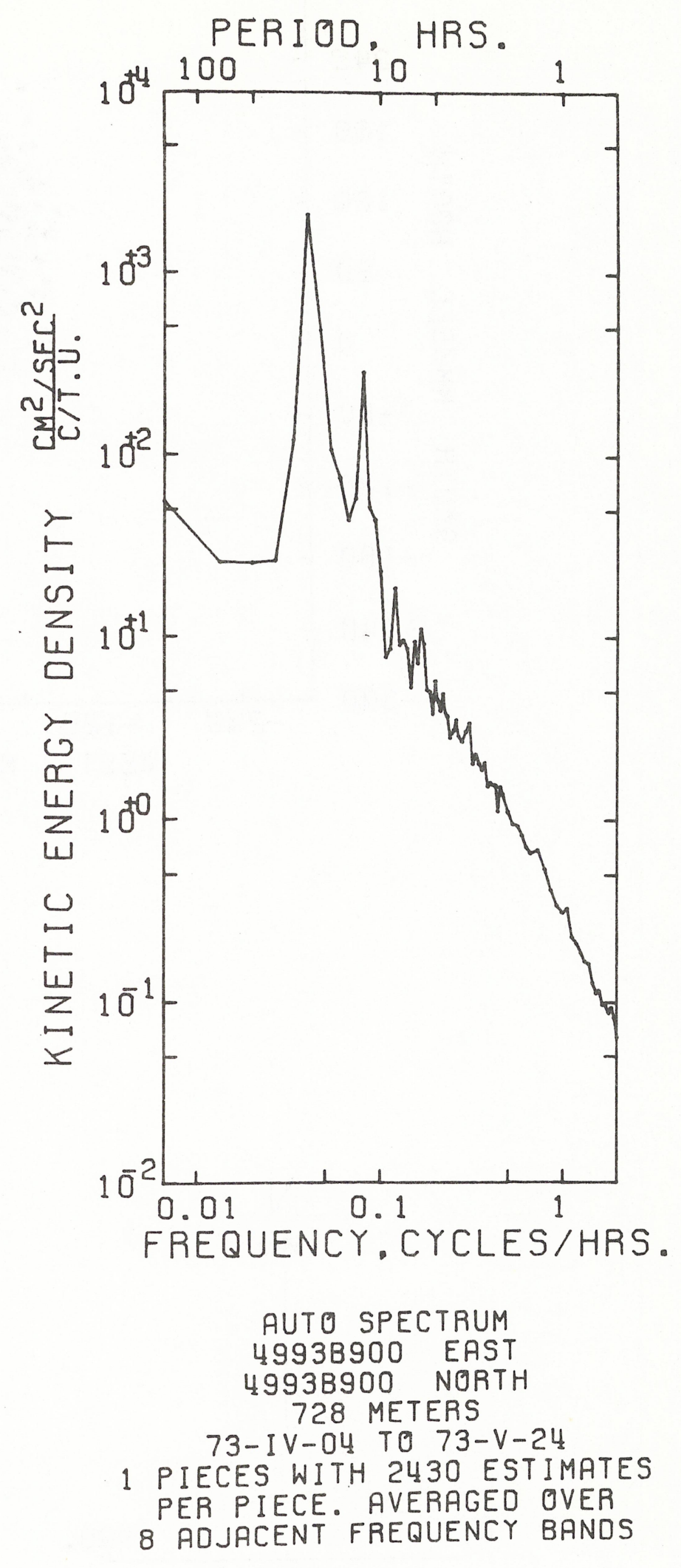




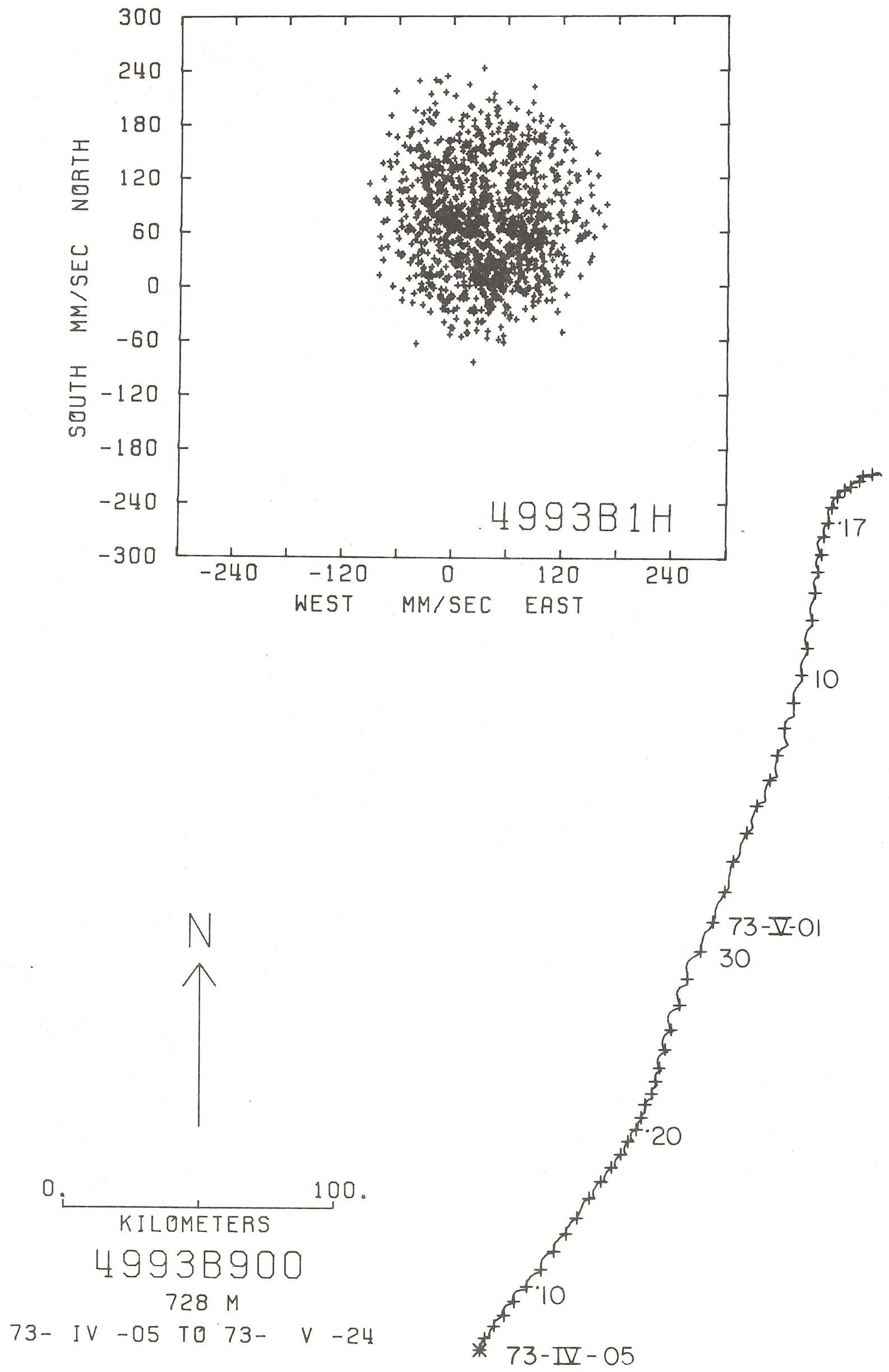



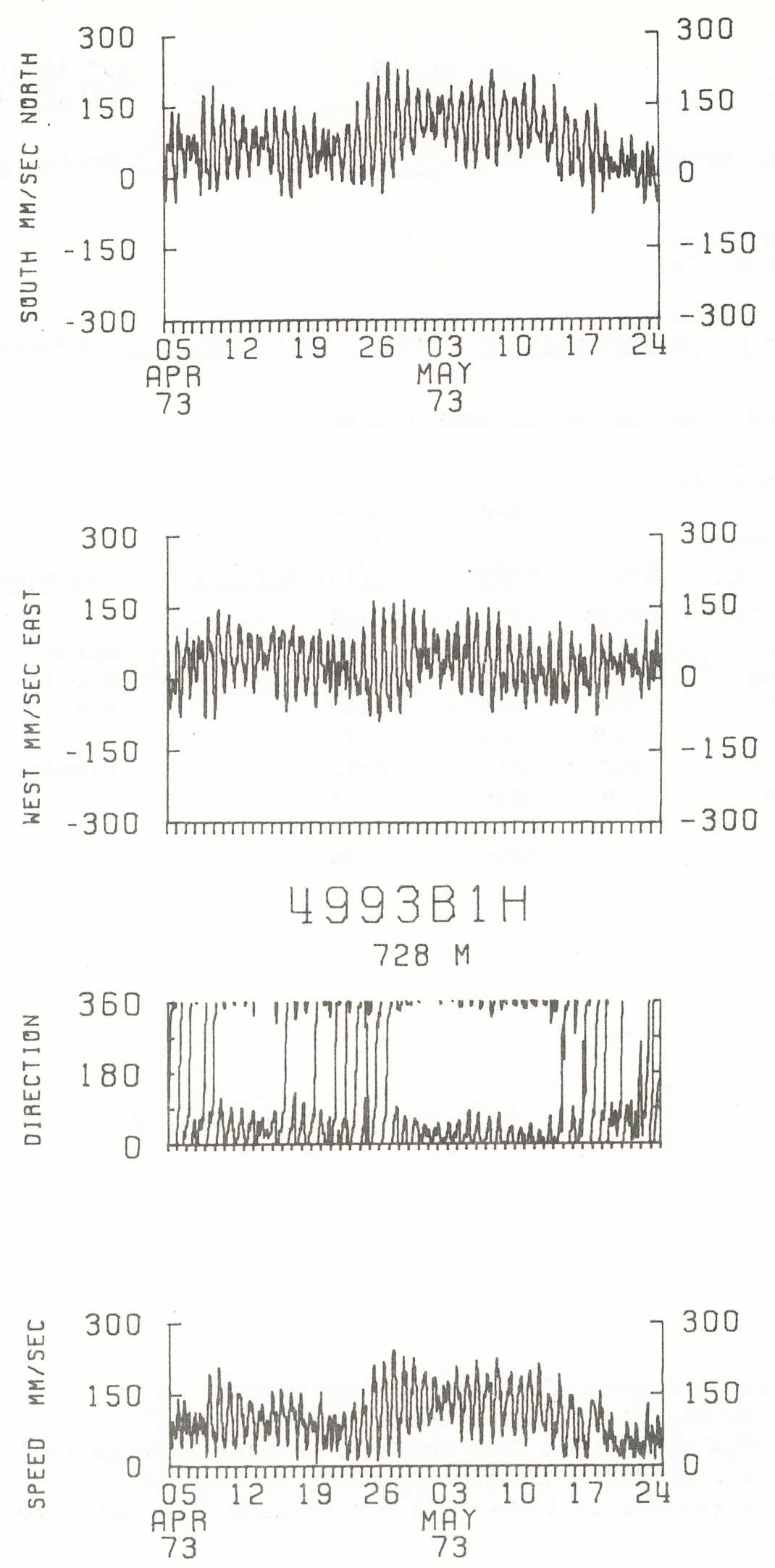
Mooring No. 500

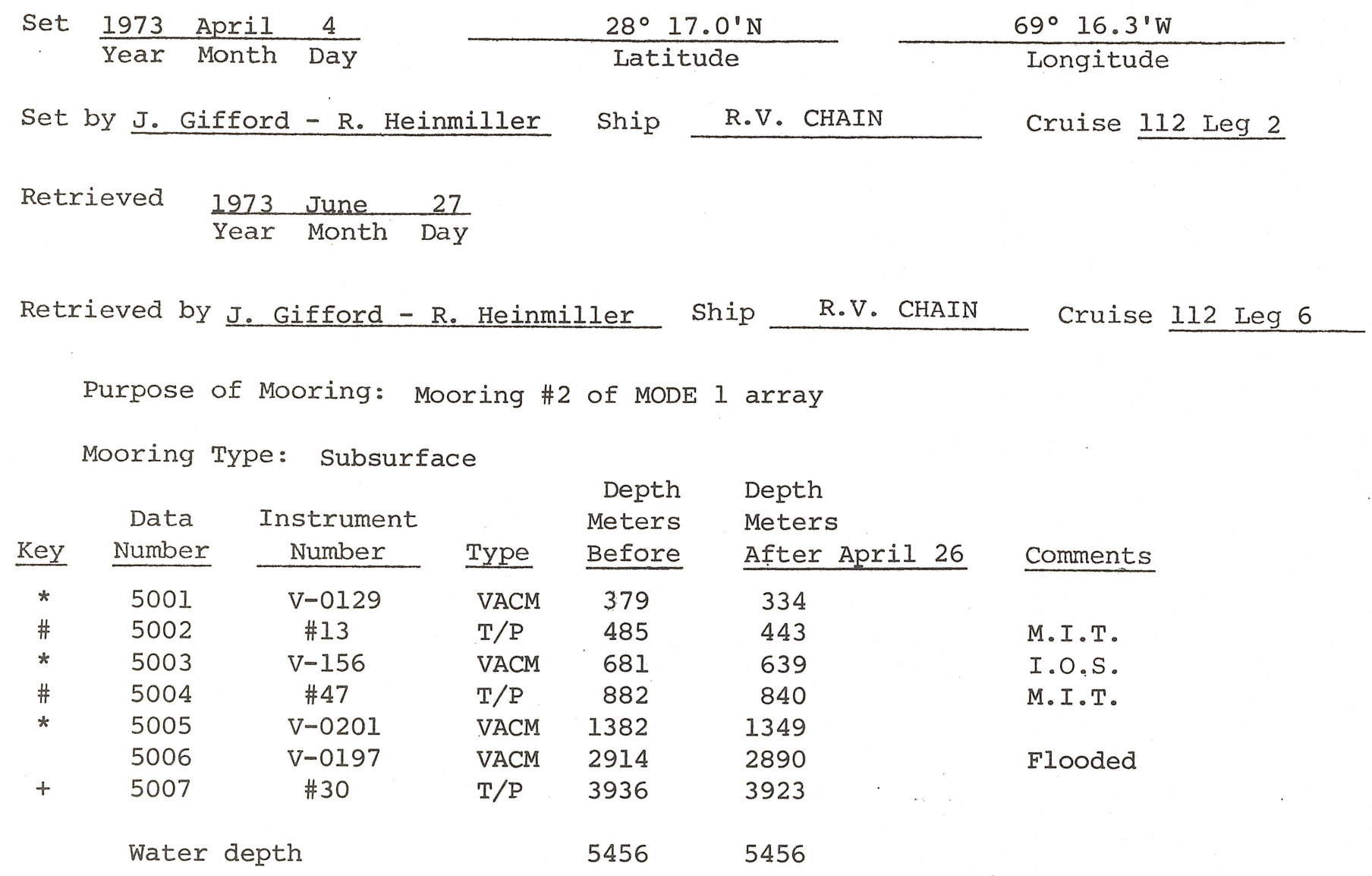

COMMENTS ON MOORING:

April 4, 1973 0434Z Broke bottle of champagne over anchor

April 26, 1973 0900z Mooring fouled by E. Katz' towfish

April 26, 1973 1700z Tow cable free from mooring, mooring left in tact

June 27, $19730608 \mathrm{z}$ Wire at $790 \mathrm{~m}$ damaged, probably by towfish

August 8, 1973 Pressure records indicate that the mooring line was stretched on April 26 


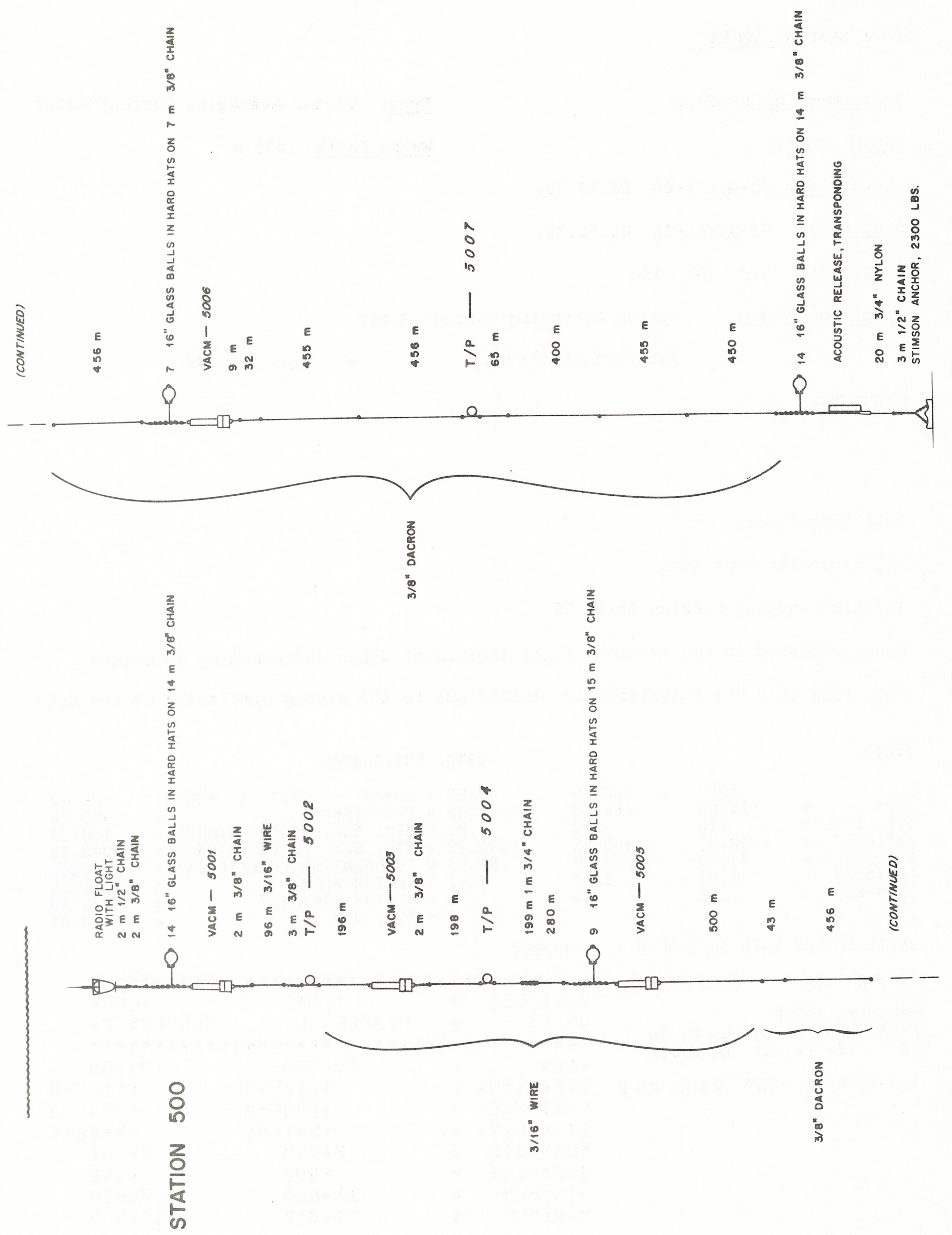


DATA NUMBER 5001A

Instrument No.: V-0129

Depth: $379 \mathrm{~m}$

Start time: 73-April-04 08.07.30.

Stop time: 73-April-26 04.52.30.

Duration: $\quad 21 \mathrm{~d} \quad 20 \mathrm{~h} \quad 45 \mathrm{~m}$

Sampling scheme: Vector Averaging Current Meter

$$
\text { recording interval }=900 \text { seconds }
$$

COMMENTS :

Dual thermistors

All variables look good

Tow fish snagged mooring April 26

Data processed in two sections since instrument depth decreased by 45 meters

Data from only one thermistor is plotted due to the strong similarity of the data

STATS

OATAR 5001909009

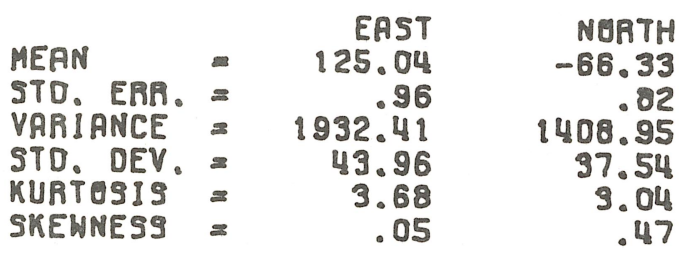

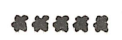
499.45

114.35 5240.13 .303 141.55 1670.68

40.87

UNITS OF RAW DATA VARIABLES $\approx$ MMPSEC

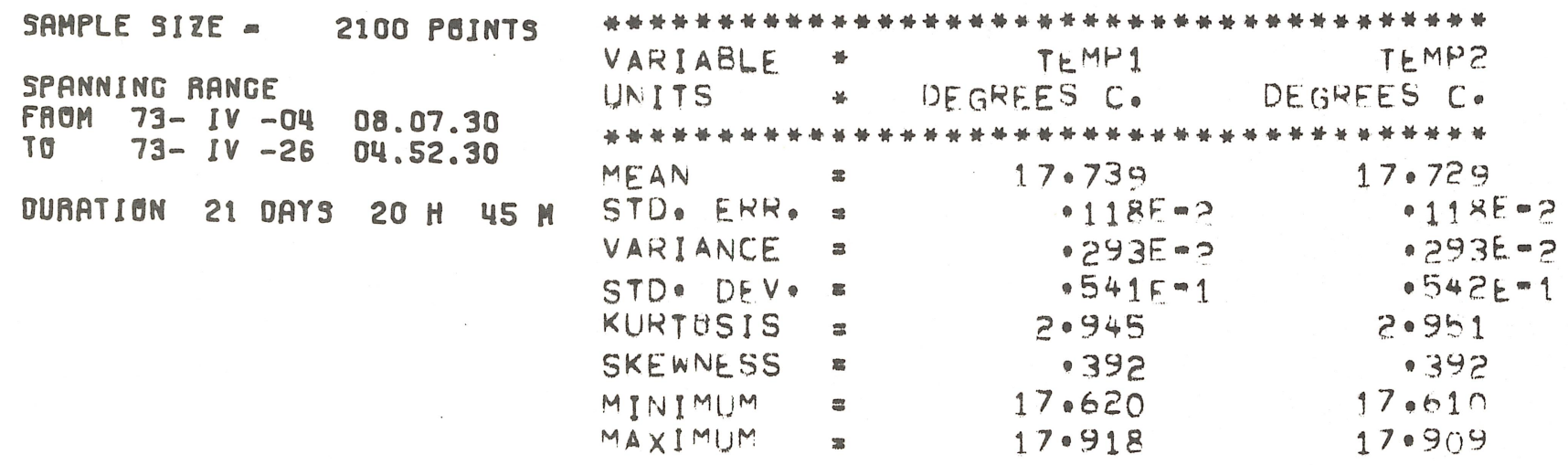




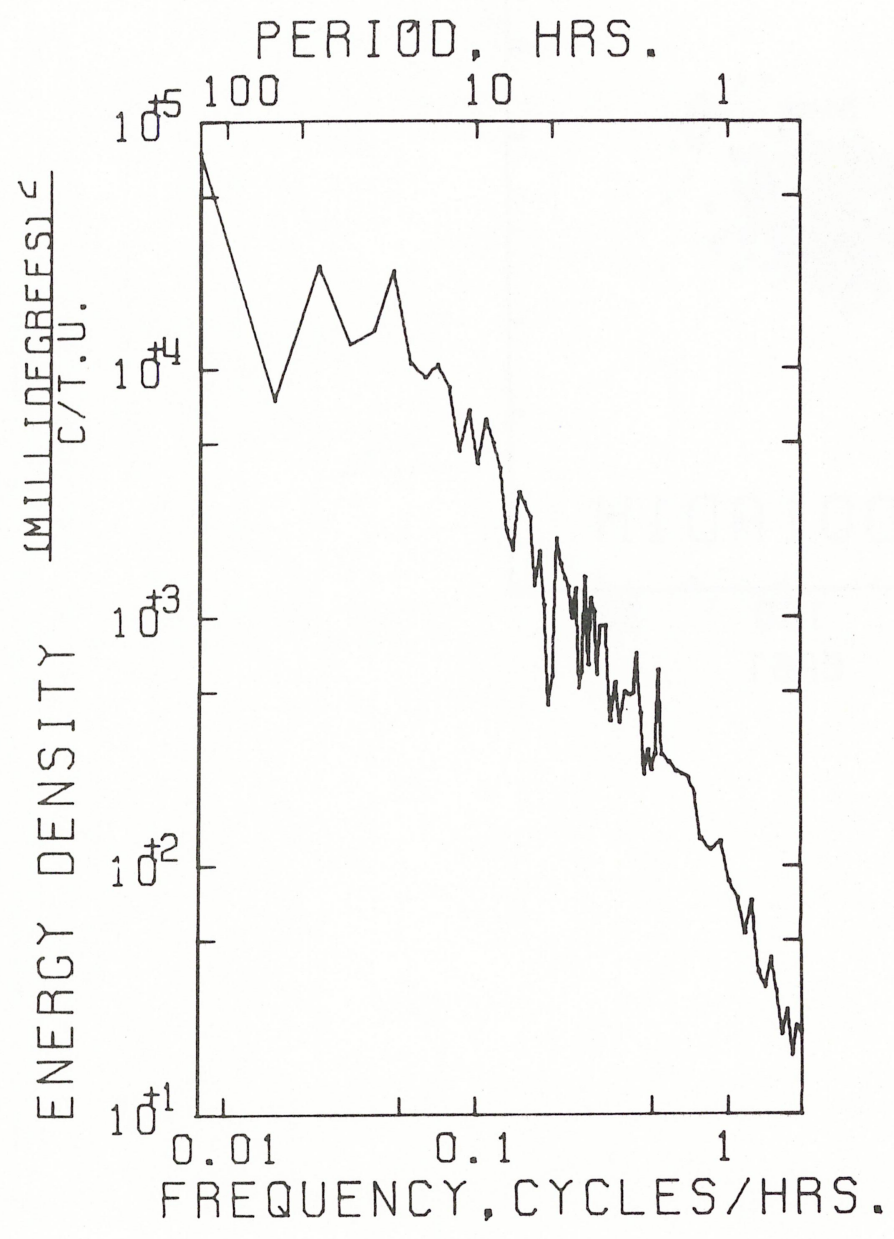

\footnotetext{
AUTO SPECTRUM

5001AD900 TEMPERATURE 379 METERS$$
\text { 73-IV-04 TO 73-IV-25 }
$$

1 PIECES WITH 1024 ESTIMATES

PER PIECE. AVERAGED OVER

4 ADJACENT FREQUENCY BANDS
}

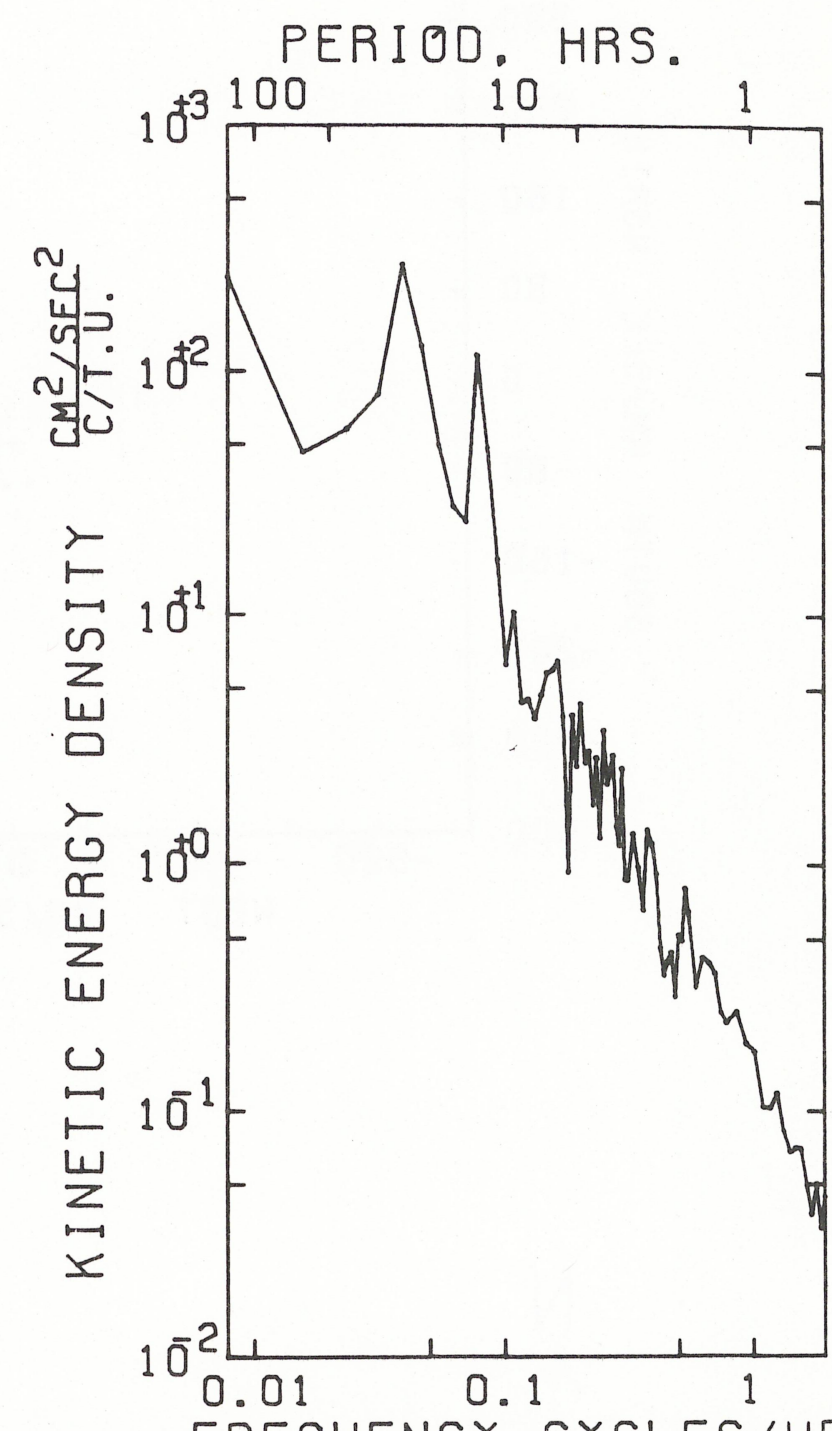

FREQUENCY.CYCLES/HRS.

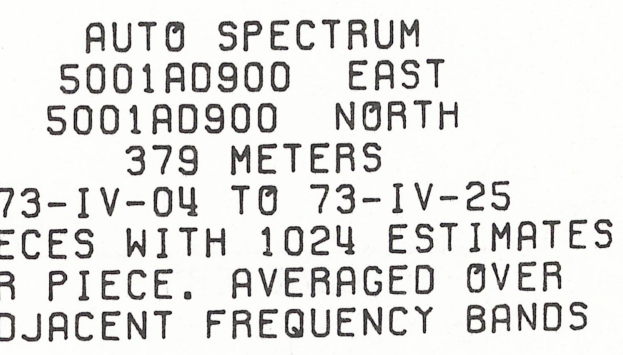



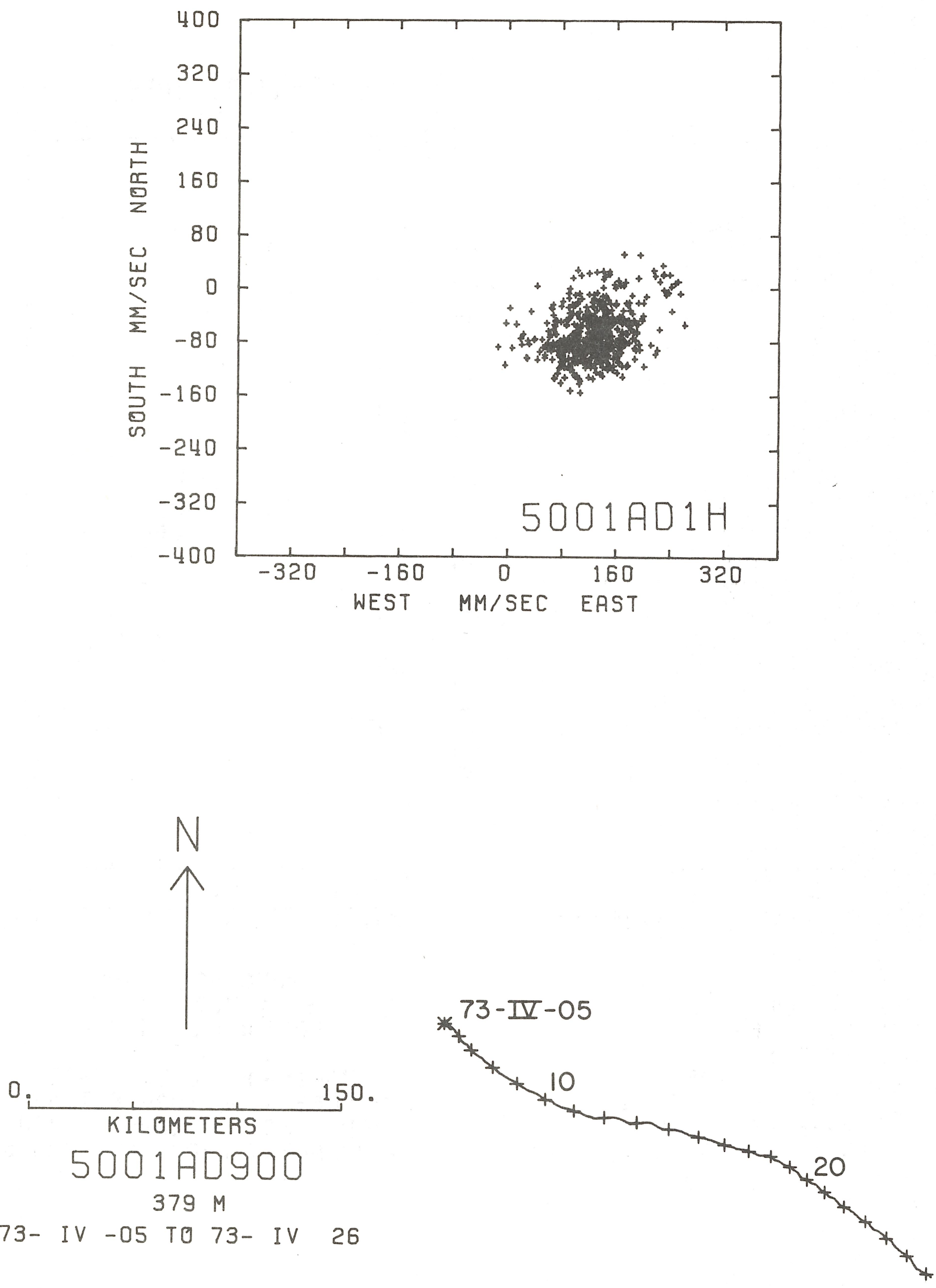

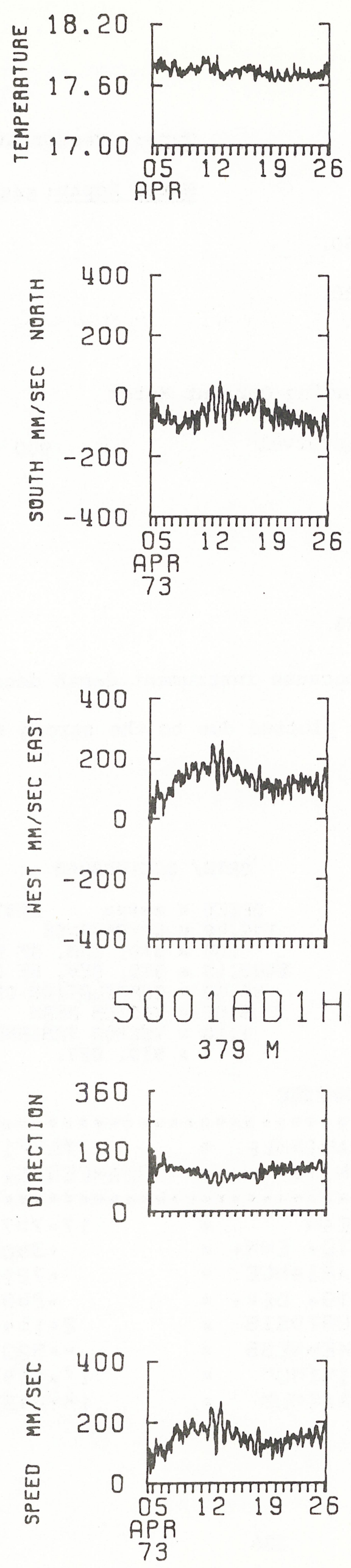
DATA NUMBER 5001B

Instrument No.: V-0129

Depth: $334 \mathrm{~m}$

Start time: 73-April-26 18.07.30.

Stop time: $73-J u n e-27 \quad 02.52 .30$.

Duration: $61 \mathrm{~d}$ 8h $45 \mathrm{~m}$

Sampling scheme: Vector Averaging Current Meter
Type: Vector Averaging Current Meter Water Depth: $5456 \mathrm{~m}$

recording interval $=900$ seconds

COMMENTS :

Dual thermistors

All variables look good

Tow fish snagged mooring April 26

Data processed in two sections because instrument depth decreased by 45 meters Data from only one thermistor is plotted due to the strong similarity of the data

STคTS

$\begin{array}{lrr} & \text { EAST } & \text { NORTH } \\ \text { MEAN = } & 26.61 & -123.05 \\ \text { STD. ERR. = } & .97 & .63 \\ \text { VARIRNCE }= & 5565.04 & 2395.45 \\ \text { STD. DEV. } & 74.60 & 48.39 \\ \text { KURTOSIS }= & 3.84 & 4.19 \\ \text { SKEWNESS }= & .51 & -.59\end{array}$

DATAR 500IBOSOOB

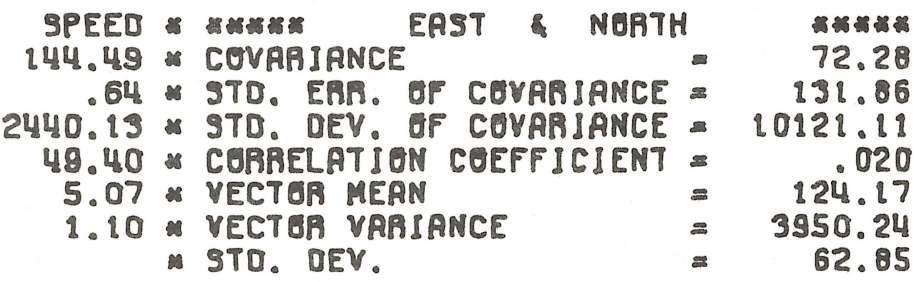

UNITS OF RAW DATA YARIABLES - MM/SEC

SAMPLE SIEE - 5892 PUINTS

SPANNING RANGE

FROM 73- IV - $26 \quad 18.07 .30$

TO 73-VI $-27 \quad 02.52 .30$

DURATION 61 DAYS 8 H 45 M

\begin{tabular}{|c|c|c|c|}
\hline \multirow{2}{*}{$\begin{array}{l}\text { VARIABLE } \\
\text { UNITS }\end{array}$} & \multirow{2}{*}{ H } & $T E M P 1$ & TEMNO \\
\hline & & DEGREES C. & DEGREES C. \\
\hline \multicolumn{4}{|c|}{ 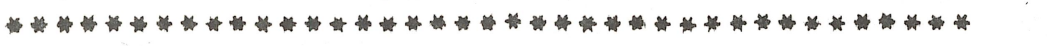 } \\
\hline MEAN & $\approx$ & 17.757 & $17 \cdot 747$ \\
\hline STD. ERK. & 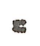 & $350 E \cdot 2$ & - $350 E=2$ \\
\hline VAR\ANCE & $=$ & $.721 E-1$ & $-724 E-1$ \\
\hline STD. DEV. & $=$ & .209 & .269 \\
\hline KURTHSIS & $=$ & $2 \cdot 154$ & $2 \cdot 154$ \\
\hline SKEWNESS & $\Xi$ & .523 & -522 \\
\hline MINIMUM & $\approx$ & 17.154 & 17.143 \\
\hline$M A X I M U M$ & $\approx$ & $13 \cdot 243$ & $18 \cdot 234$ \\
\hline
\end{tabular}



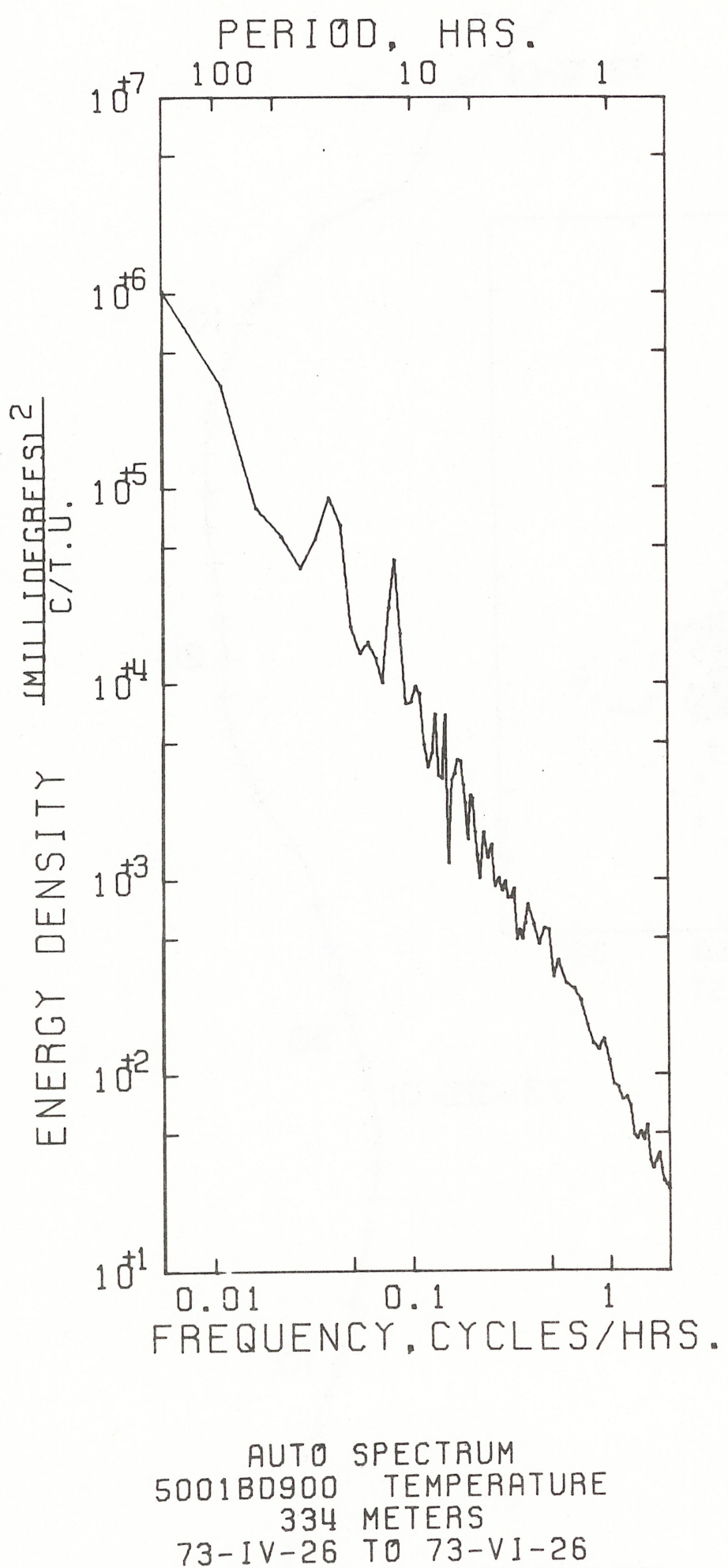

1 PIECES WITH 2916 ESTIMATES PER PIECE. AVERAGED OVER 8 ADJACENT FREQUENCY BANDS

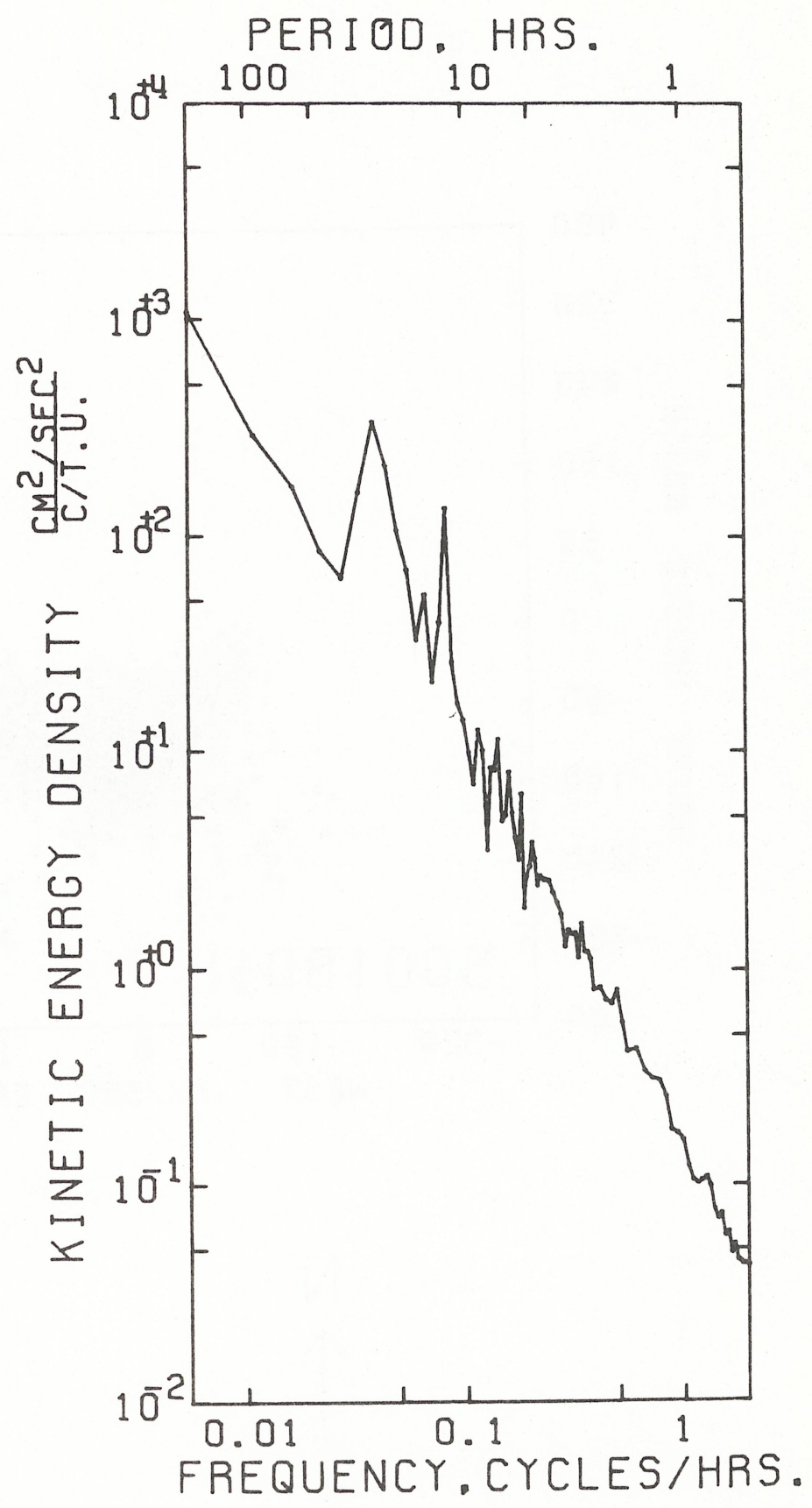

AUTO SPECTRUM $5001 B D 900$ EAST $5001 B 0900$ NORTH 334 METERS 73-IV-26 TO 73-VI-26

1 PIECES WITH 2916 ESTIMATES PER PIECE. AVERAGED OVER 8 ADJACENT FREQUENCY BANDS 


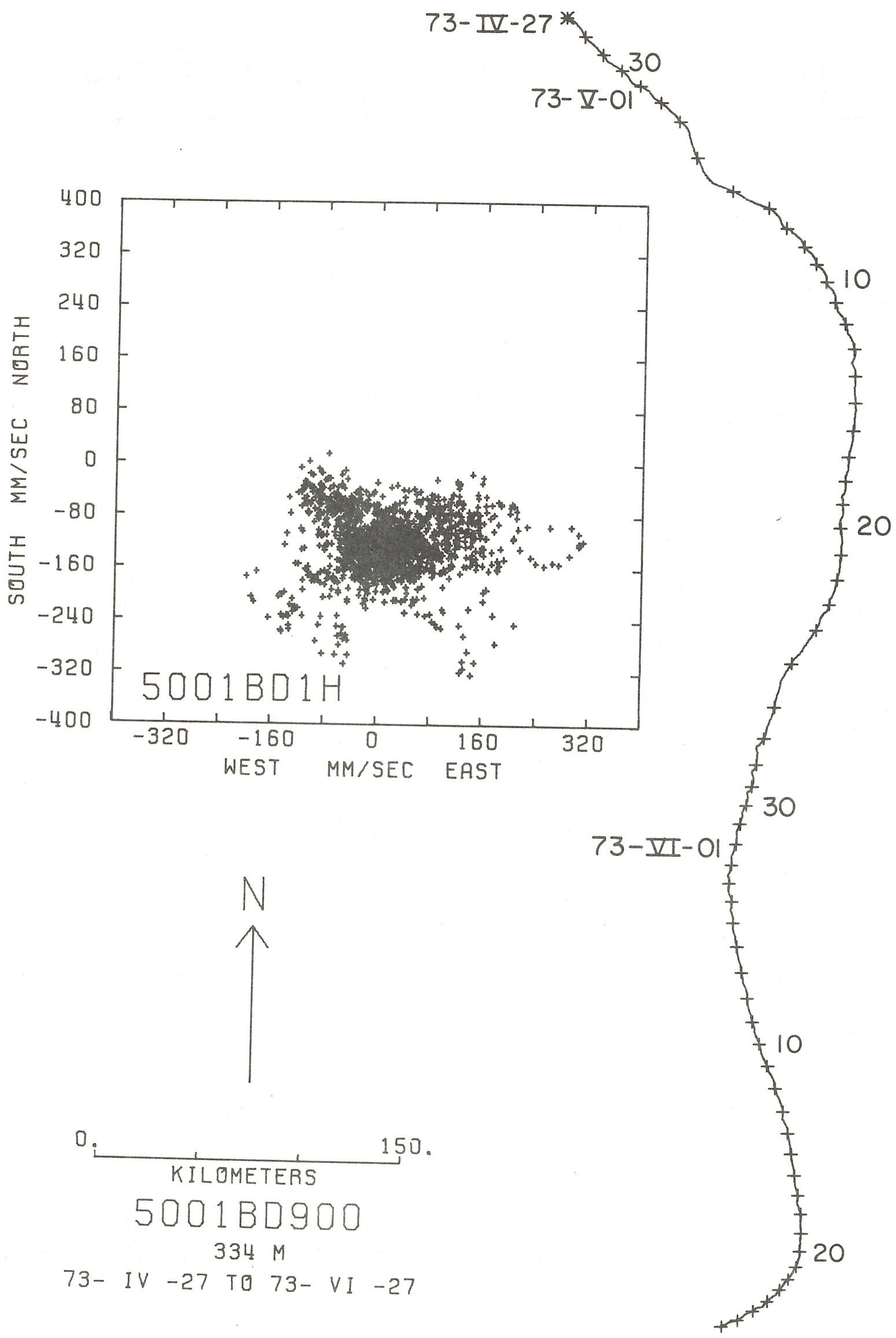



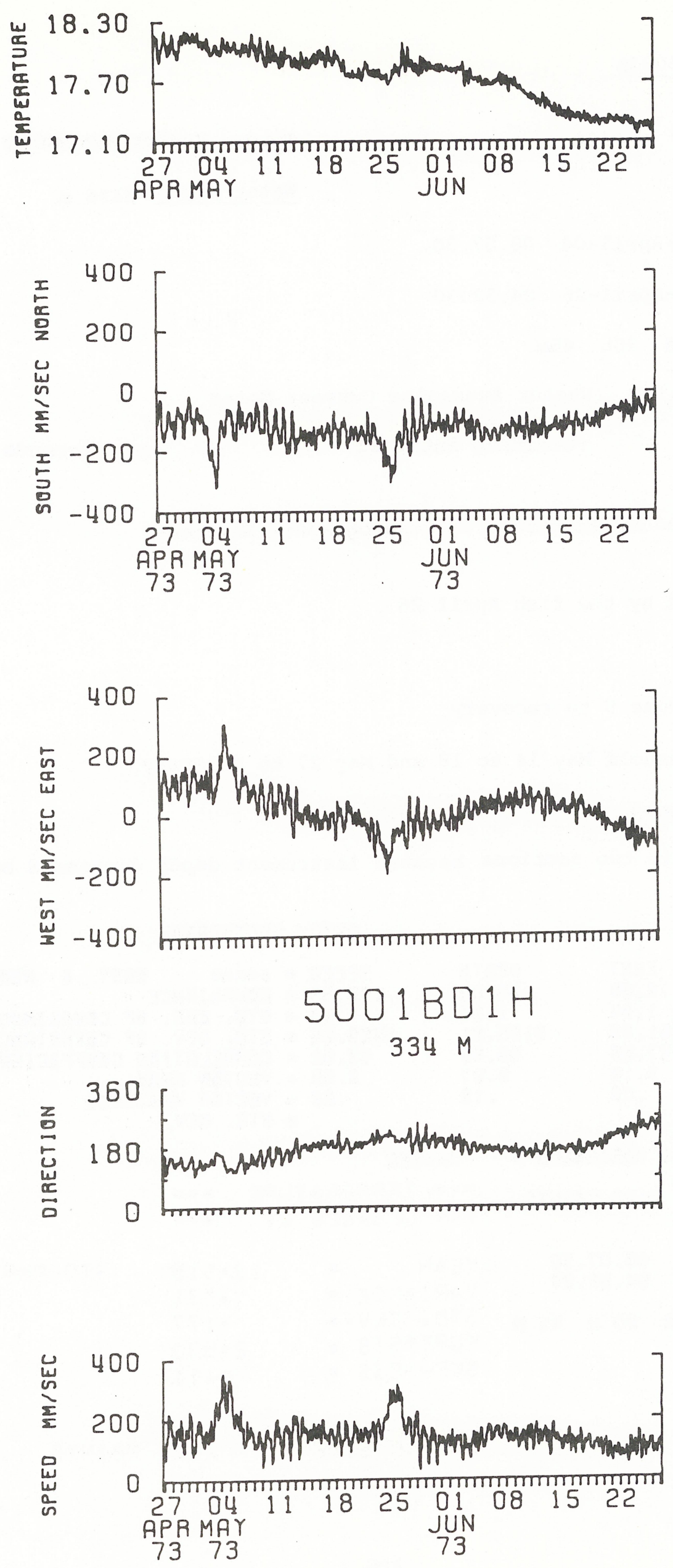
DATA NUMBER 5003A

Instrument No.: $\mathrm{V}-0156$

Depth: $681 \mathrm{~m}$
Type: Vector Averaging Current Meter Water Depth: $5456 \mathrm{~m}$

Start time: 73-April-04 08.07.30.

Stop time: 73-April-26 04.52.30.

Duration: $\quad 2 l d \quad 20 h \quad 45 \mathrm{~m}$

Sampling scheme: Vector Averaging Current Meter

$$
\text { recording interval }=900 \text { seconds }
$$

COMMENTS :

Instrument owned by Institute of Oceanographic Sciences

Mooring snagged by tow fish April 26

Compass - good

Vane - sticky June 9 to recovery

Rotor - At threshold May 14 to 18 and May 27 to recovery

Temperature - good

Data processed in two sections because instrument depth decreased by 42 meters

STATS

$\begin{array}{lrrr} & \text { EAST } & \text { NEATH } \\ \text { MERN } & = & 72.68 & -65.04 \\ \text { STD. EAR. }= & 1.04 & 1.01 \\ \text { VARIANCE }= & 2261.38 & 2155.01 \\ \text { STD. DEV. }= & 47.55 & 48.42 \\ \text { KURTESIS }= & 3.14 & 2.97 \\ \text { SKENMESS } \approx & .06 & .19\end{array}$

Datar 5003AcgoOa

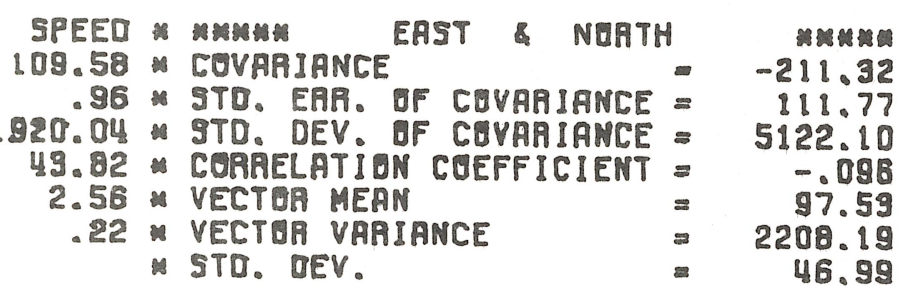

UNITS GF RAW DATA VARIABLES $=$ MMPSEC

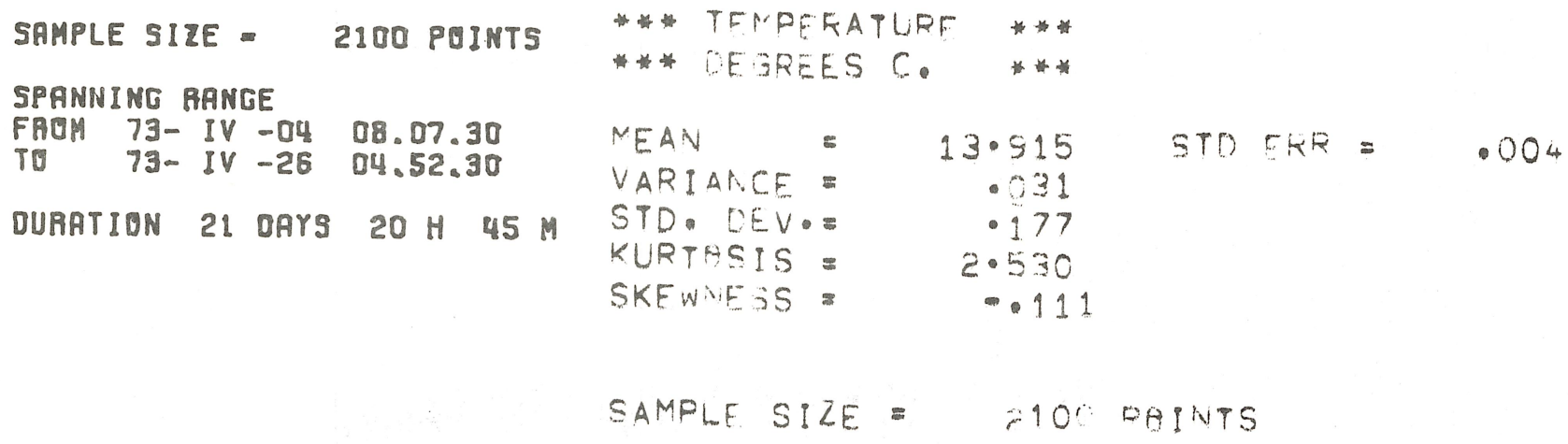



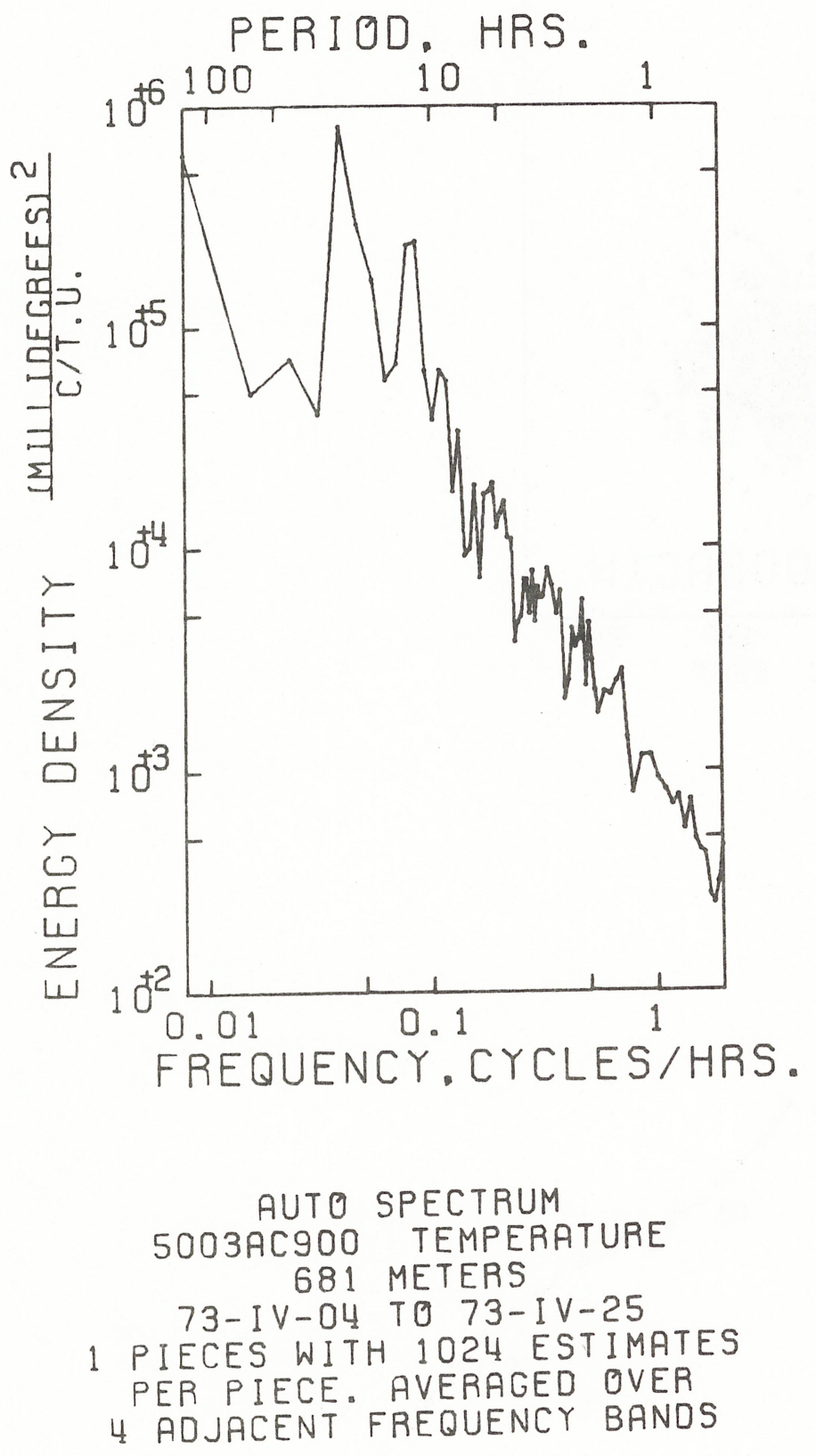

PERIOD, HRS.

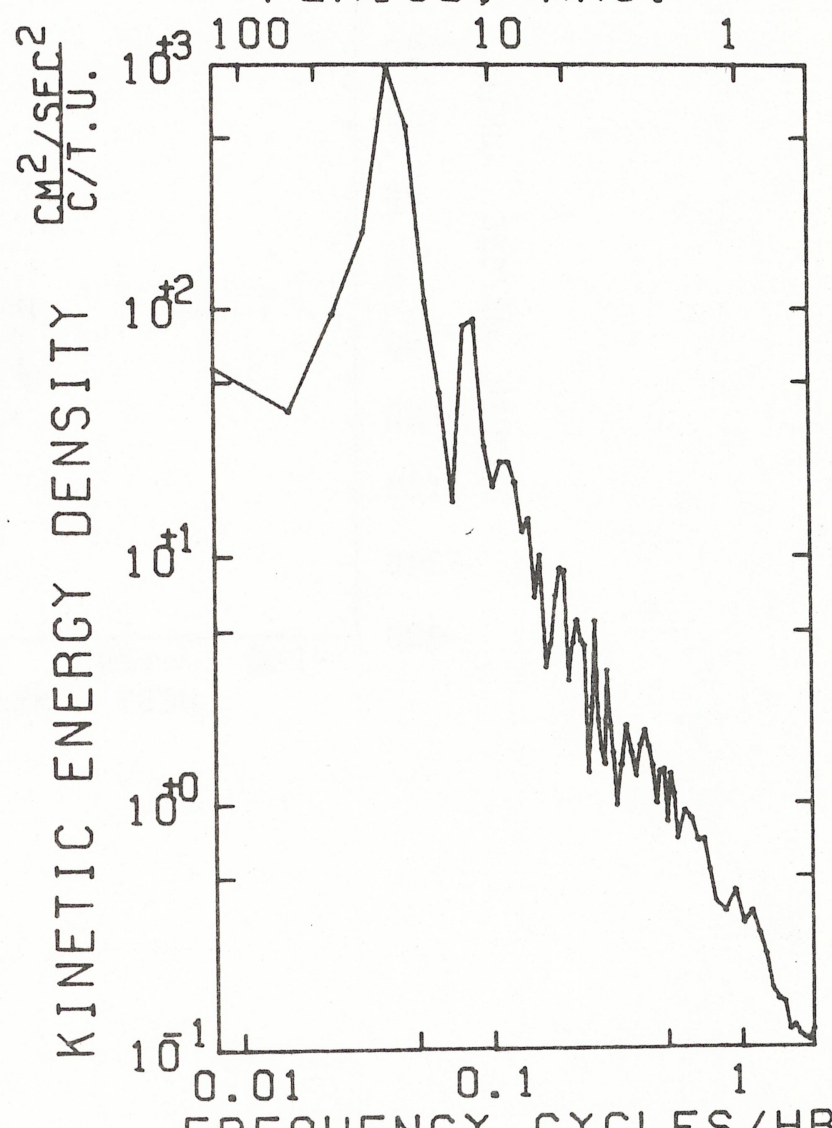

FREQUENCY,CYCLES/HRS.

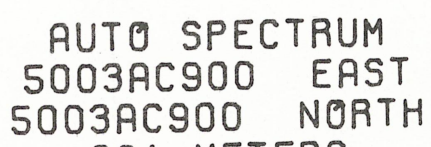
681 METERS

73-IV-04 TO 73-IV-25

1 PIECES WITH 1024 ESTIMATES PER PIECE. AVERAGED OVER 4 ADJACENT FREQUENCY BANDS 

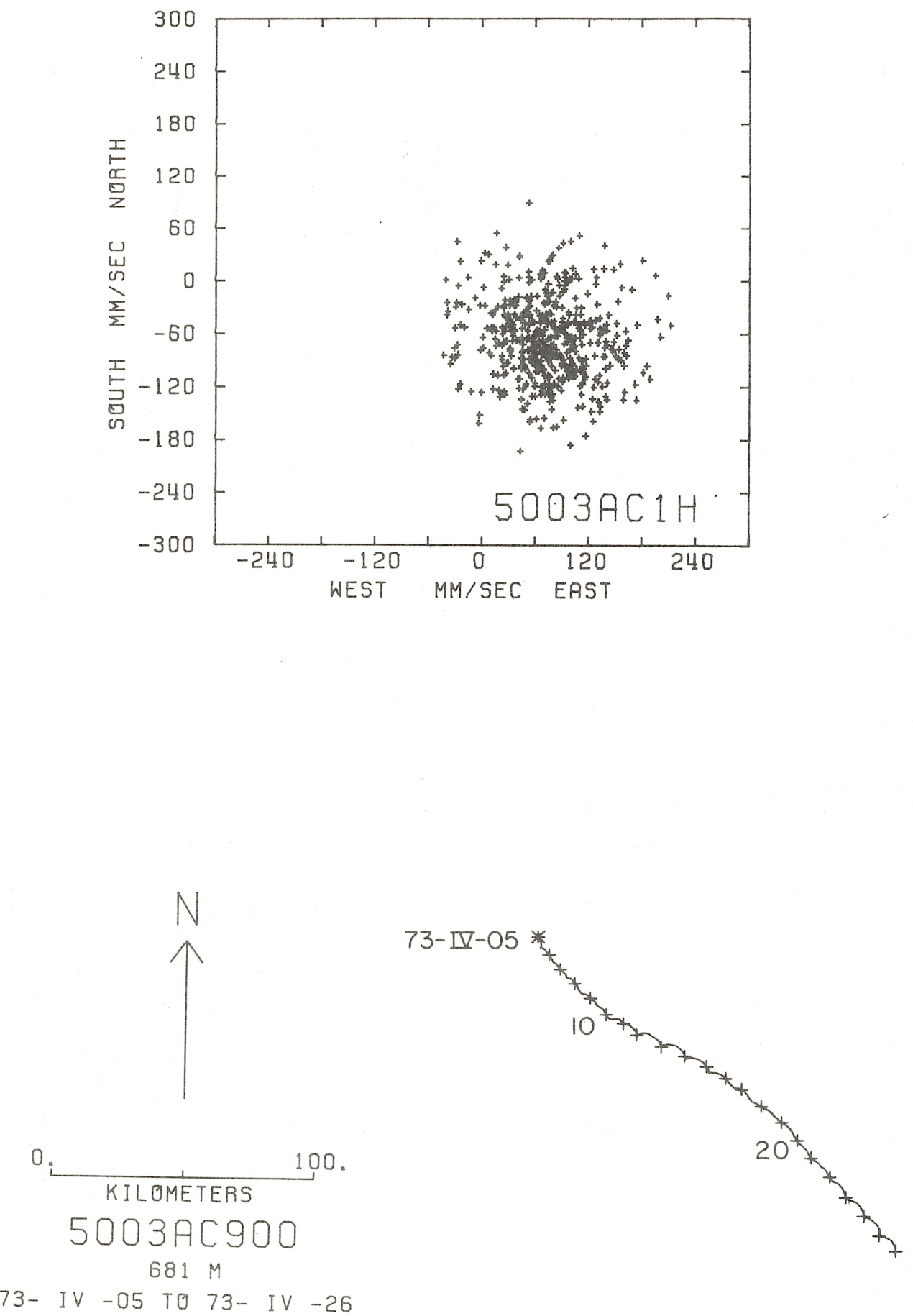

73- IV -05 TO 73- IV - 26 

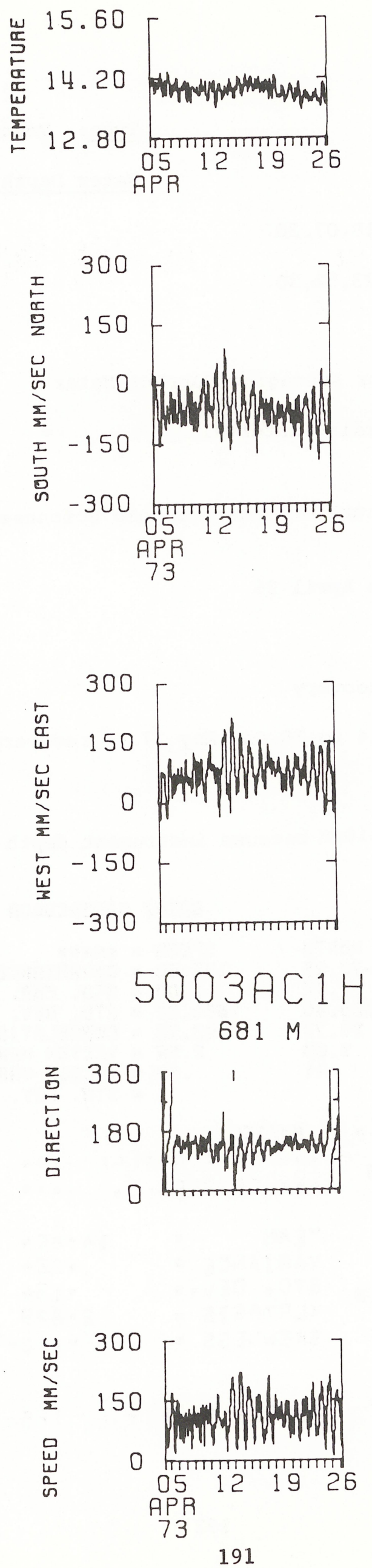
DATA NUMBER $5003 B$

Instrument No.: V-0156

Depth: $639 \mathrm{~m}$
Type: Vector Averaging Current Meter Water Depth: $5456 \mathrm{~m}$

Start time: 73-Apri1-26 18.07.30.

Stop time: 73-May-13 23.52.30.

Duration: $\quad 17 \mathrm{~d}$ 5h $43 \mathrm{~m}$

Sampling scheme: Vector Averaging Current Meter

$$
\text { recording interval }=900 \text { seconds }
$$

COMMENTS:

Instrument owned by Institute of Oceanographic Sciences

Mooring snagged by towfish April 26

Compass - good

Vane - sticky June 9 to recovery

Rotor - at threshold May 14 to 18 and May 27 to recovery

Temperature - good

Data processed in two sections because instrument depth decreased by 42 meters

STATS

DATAR 5003BCgOOA

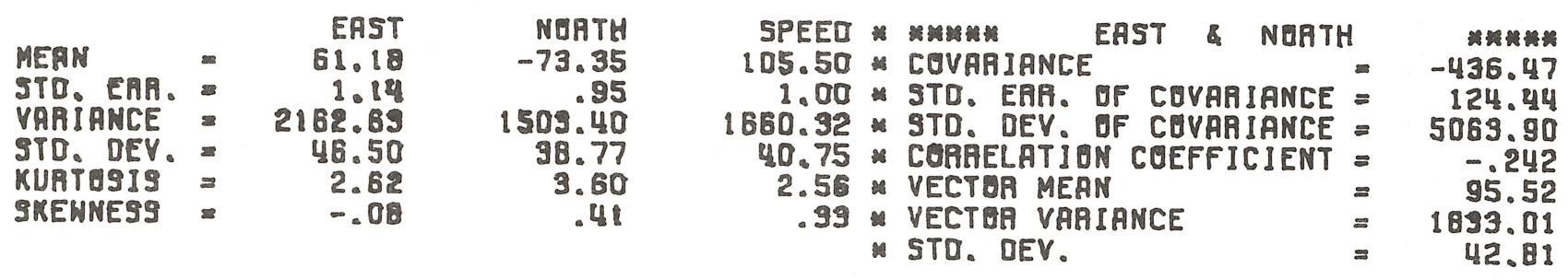

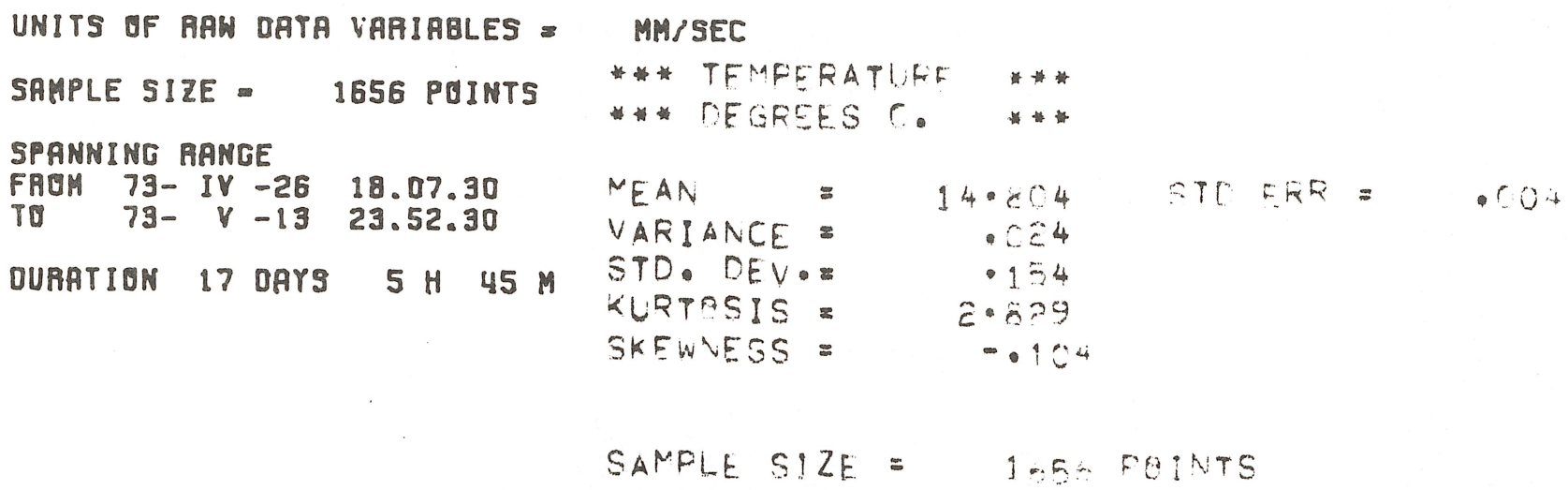



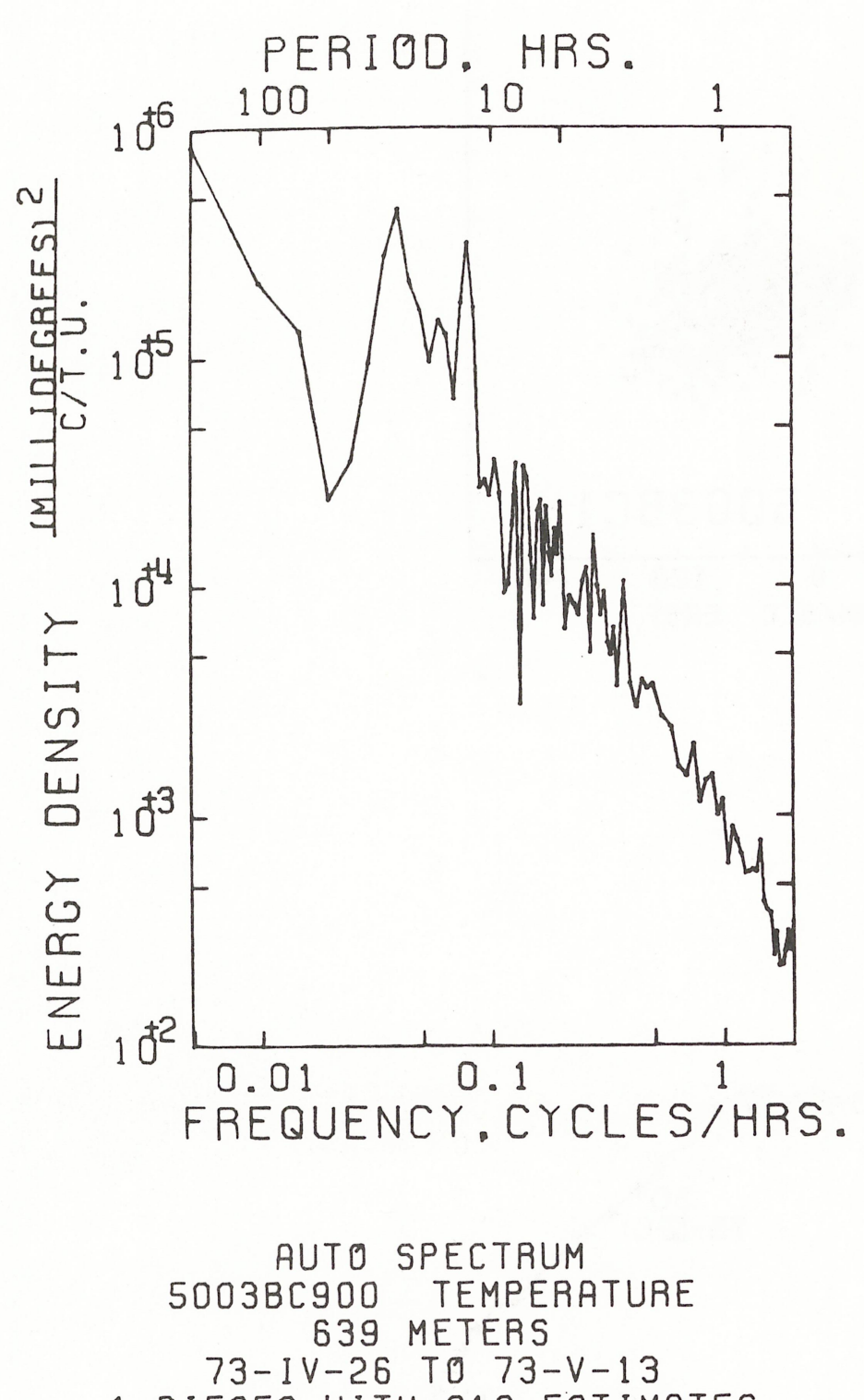

1 PIECES WITH 810 ESTIMATES

PER PIECE. AVERAGED OVER

2 ADJACENT FREQUENCY BANDS
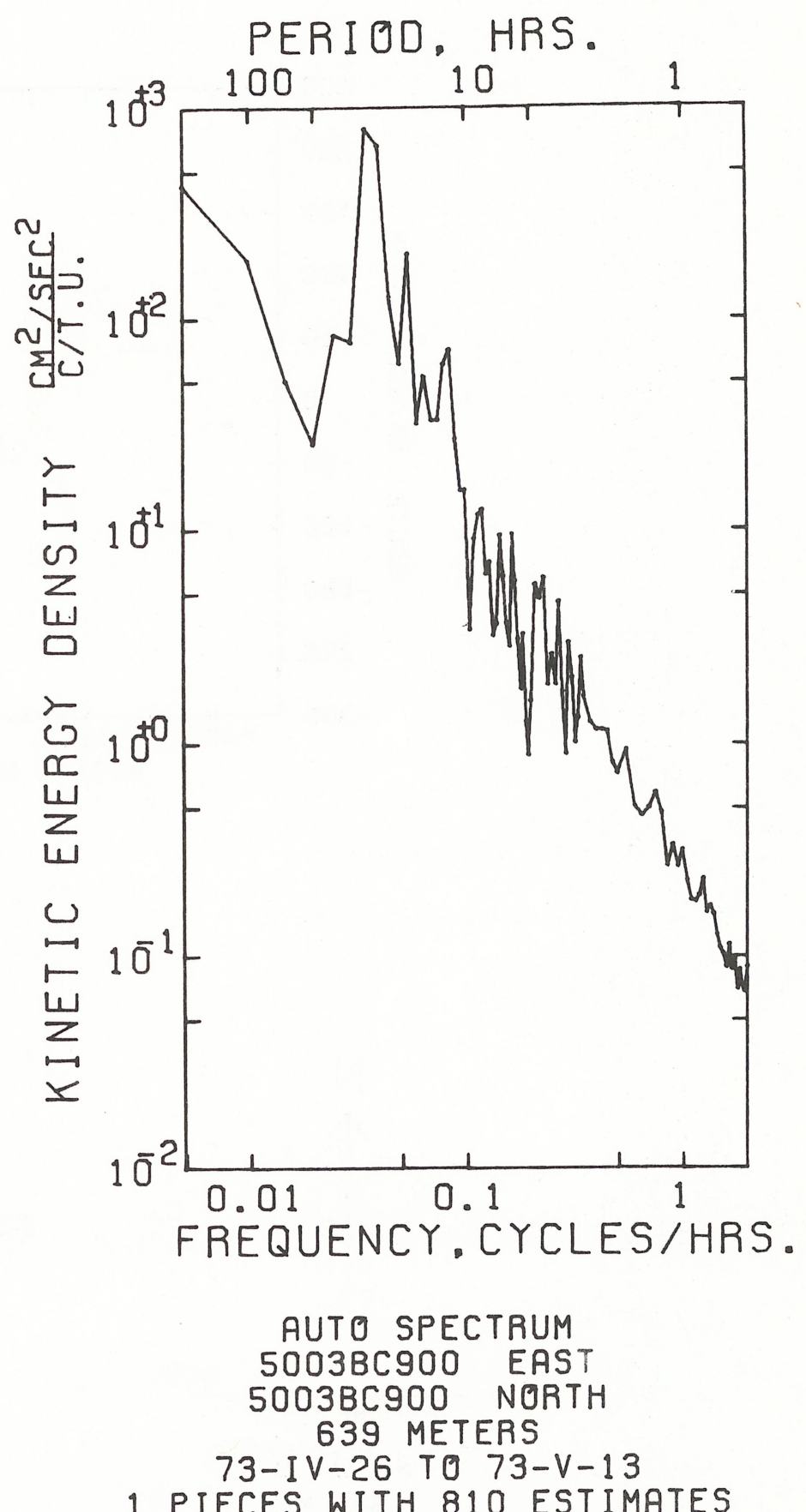

1 PIECES WITH 810 ESTIMATES PER PIECE. AVERAGED OVER 2 ADJACENT FREQUENCY BANOS 


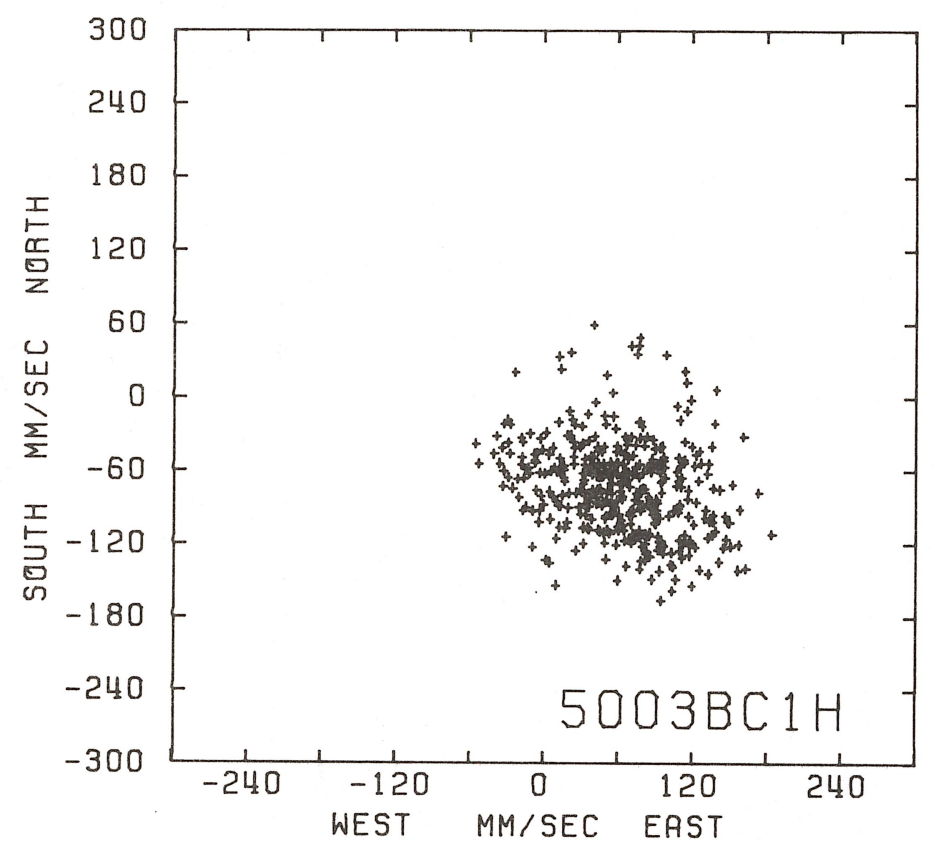

N
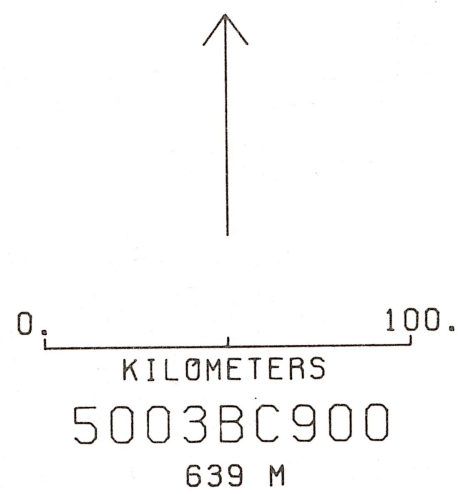

73- IV - 27 TO $73-\quad V-13$

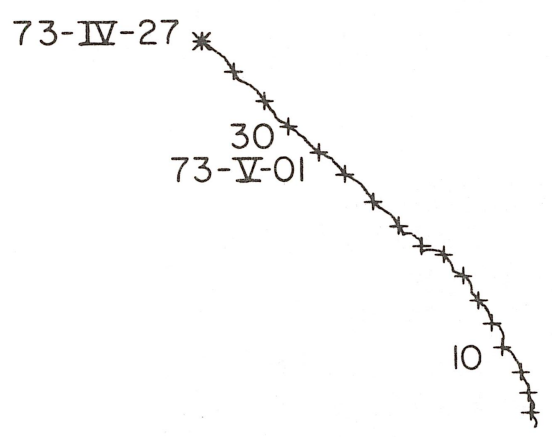



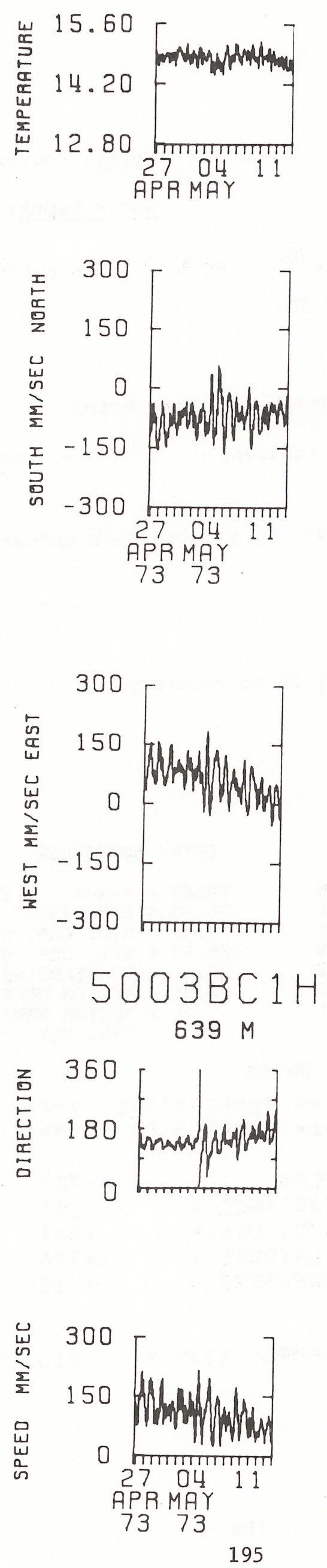
DATA NUMBER 5005

Instrument No.: $\mathrm{V}-0201$

Depth: $1382 \mathrm{~m}$

Start time: 73-April-04 08.07.30.

Stop time: 73-Apri1-26 04.52.30.

Duration: $\quad 21 d$ 20h $45 \mathrm{~m}$

Sampling scheme: Vector Averaging Current Meter recording interval = 900 seconds

COMMENTS:

Mooring snagged by towfish April 26, slight depth change

Compass - good

Vane - stuck May 9 to recovery

Rotor - at threshold from April 26 to recovery

Temperature - good

รTคTร

$\begin{array}{lrrr} & & \text { EAST } & \text { NORTH } \\ \text { MEAN } & -21.75 & -50.10 \\ \text { STD. ERR. }= & .62 & .55 \\ \text { VARIANCE }= & 017.29 & 644.89 \\ \text { STD DEV. }= & 28.59 & 25.39 \\ \text { KURTOSIS }= & 2.37 & 2.56 \\ \text { SKEWNESS } & .16 & -.01\end{array}$

Type: Vector Averaging Current Meter Water Depth: $5456 \mathrm{~m}$ 


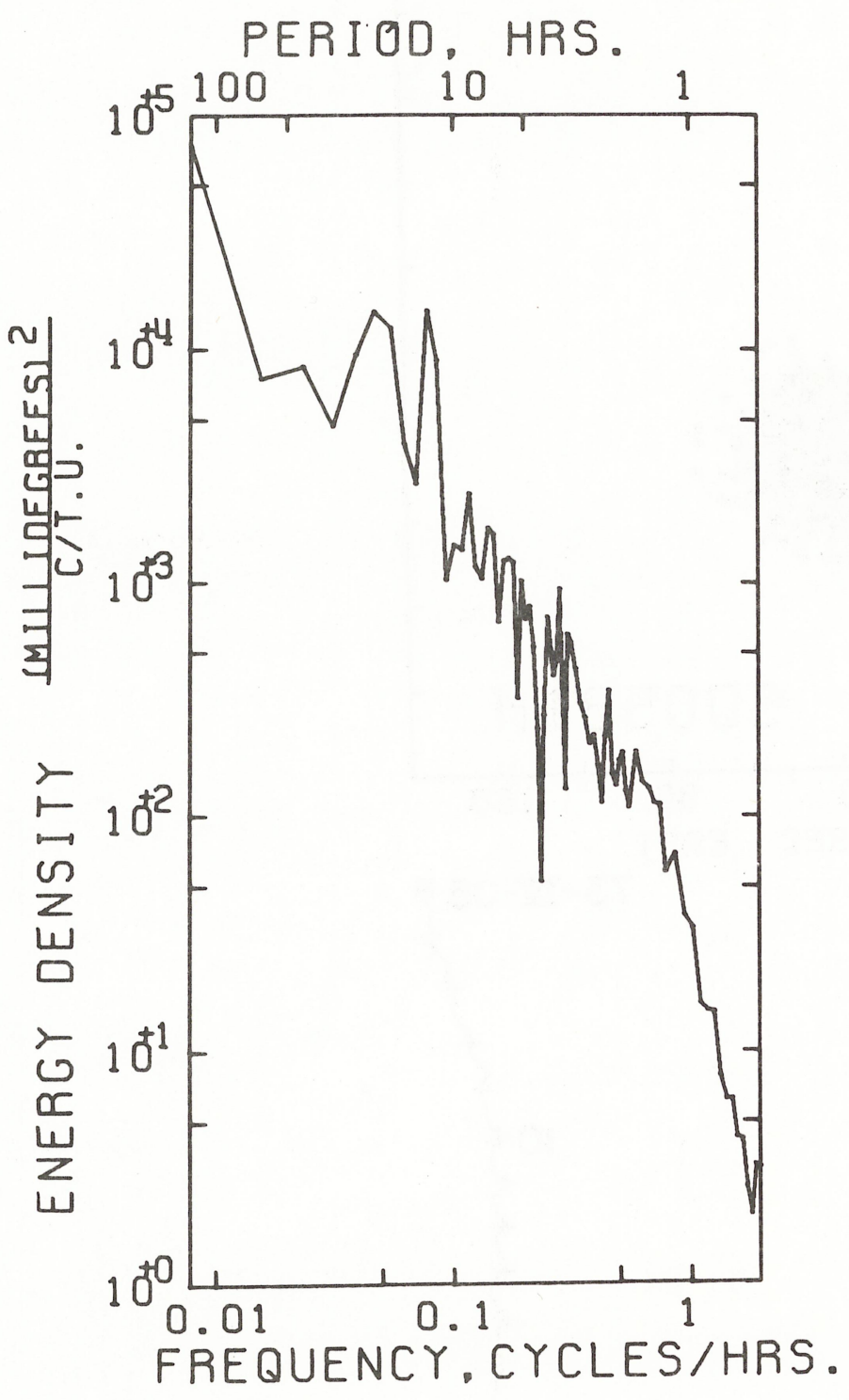

AUTO SPECTRUM

5005B900 TEMPERATURE 1382 METERS 73-IV-04 TO 73-IV-25

1 PIECES WITH 1024 ESTIMATES PER PIECE. AVERAGED OVER 4 ADJACENT FREQUENCY BANDS

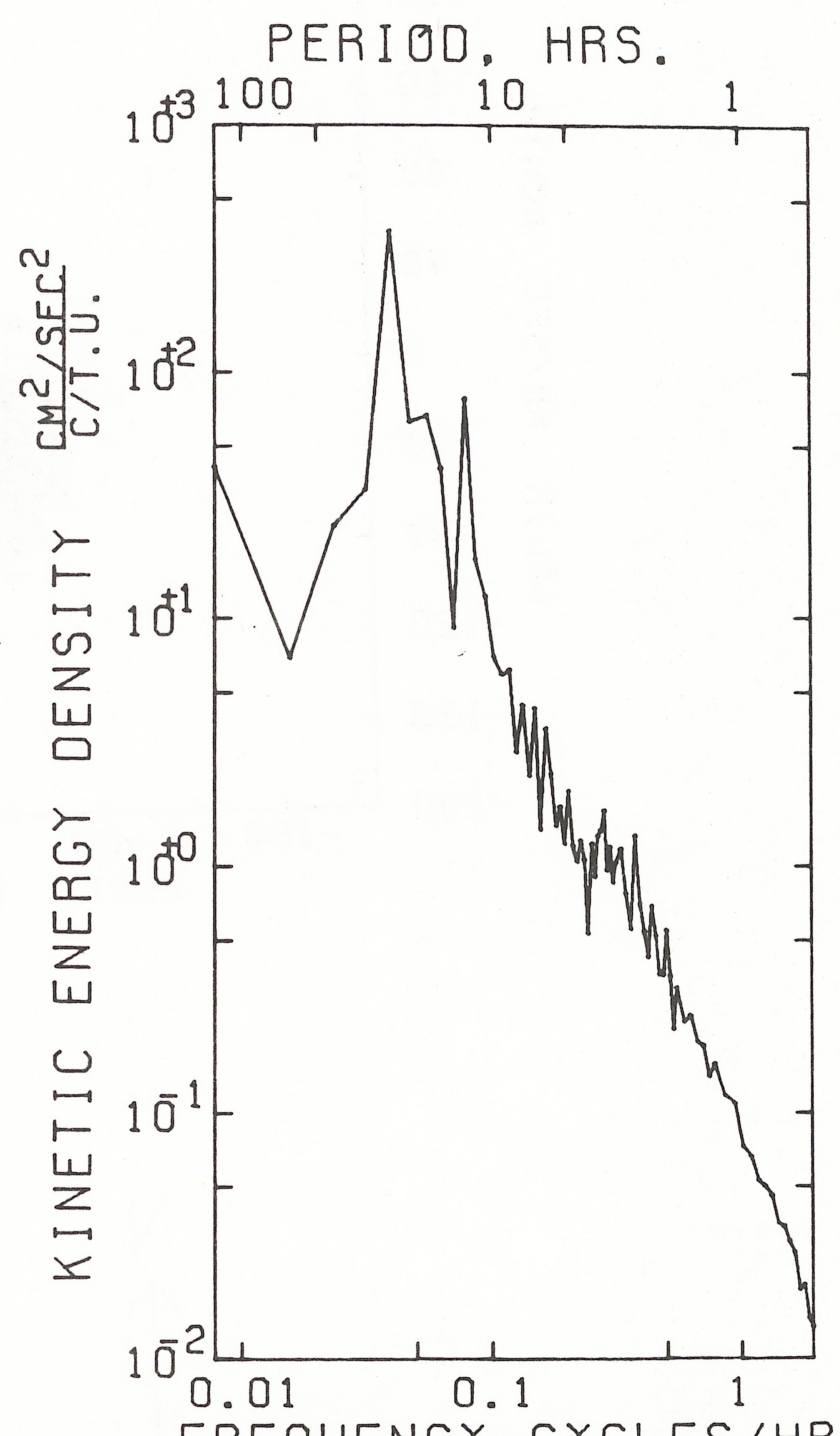

FREQUENCY,CYCLES/HRS.

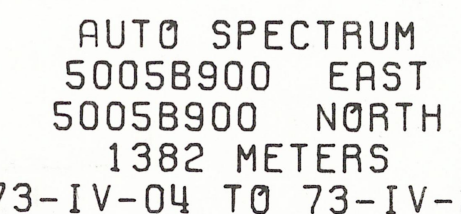

73-IV-04 TO 73-IV-25

1 PIECES WITH 1024 ESTIMATES PER PIECE. AVERAGED OVER 4 ADJACENT FREQUENCY BANOS 

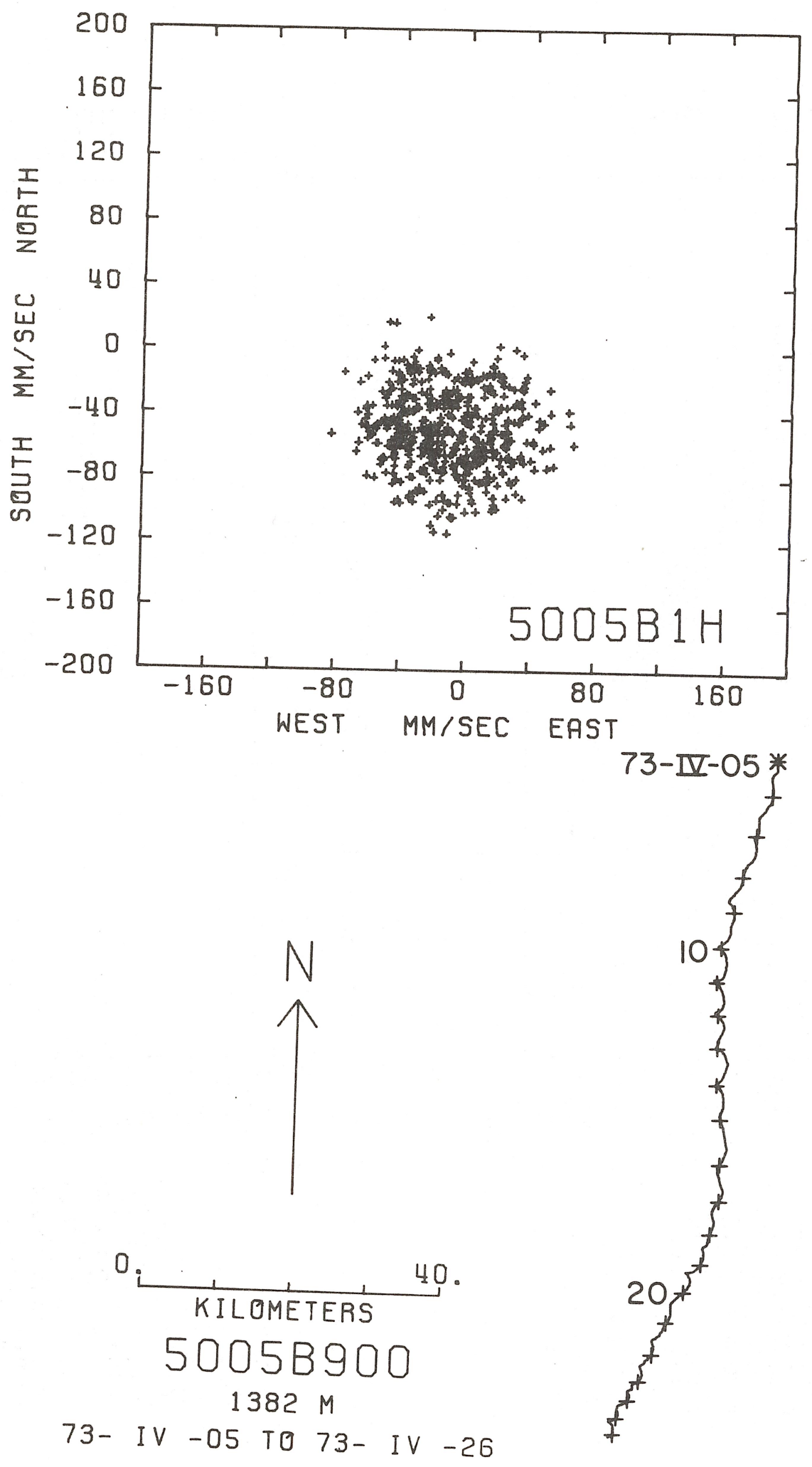

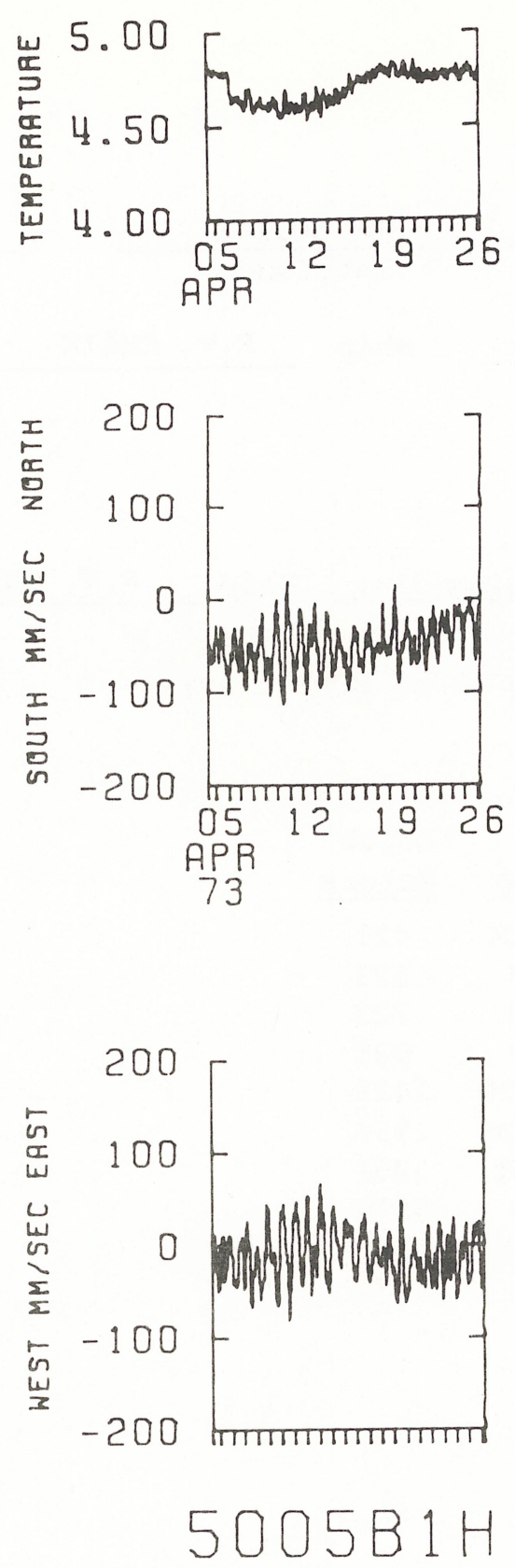
$1382 M$
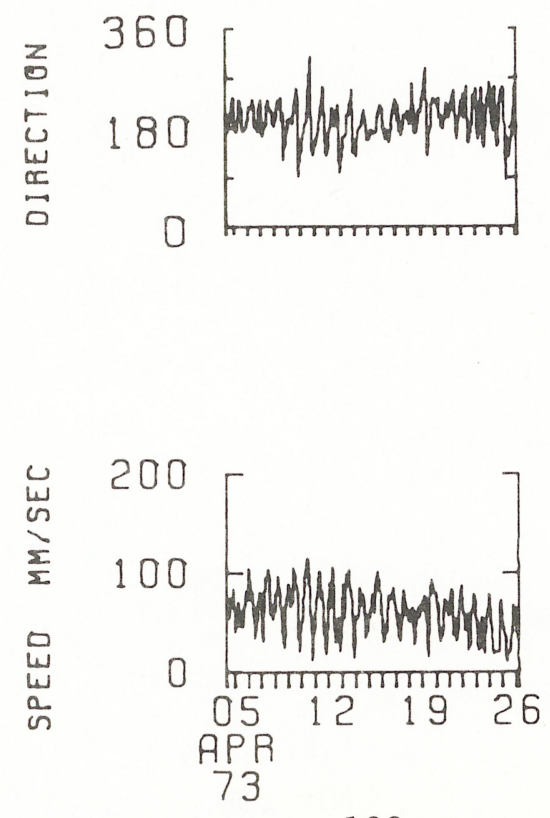


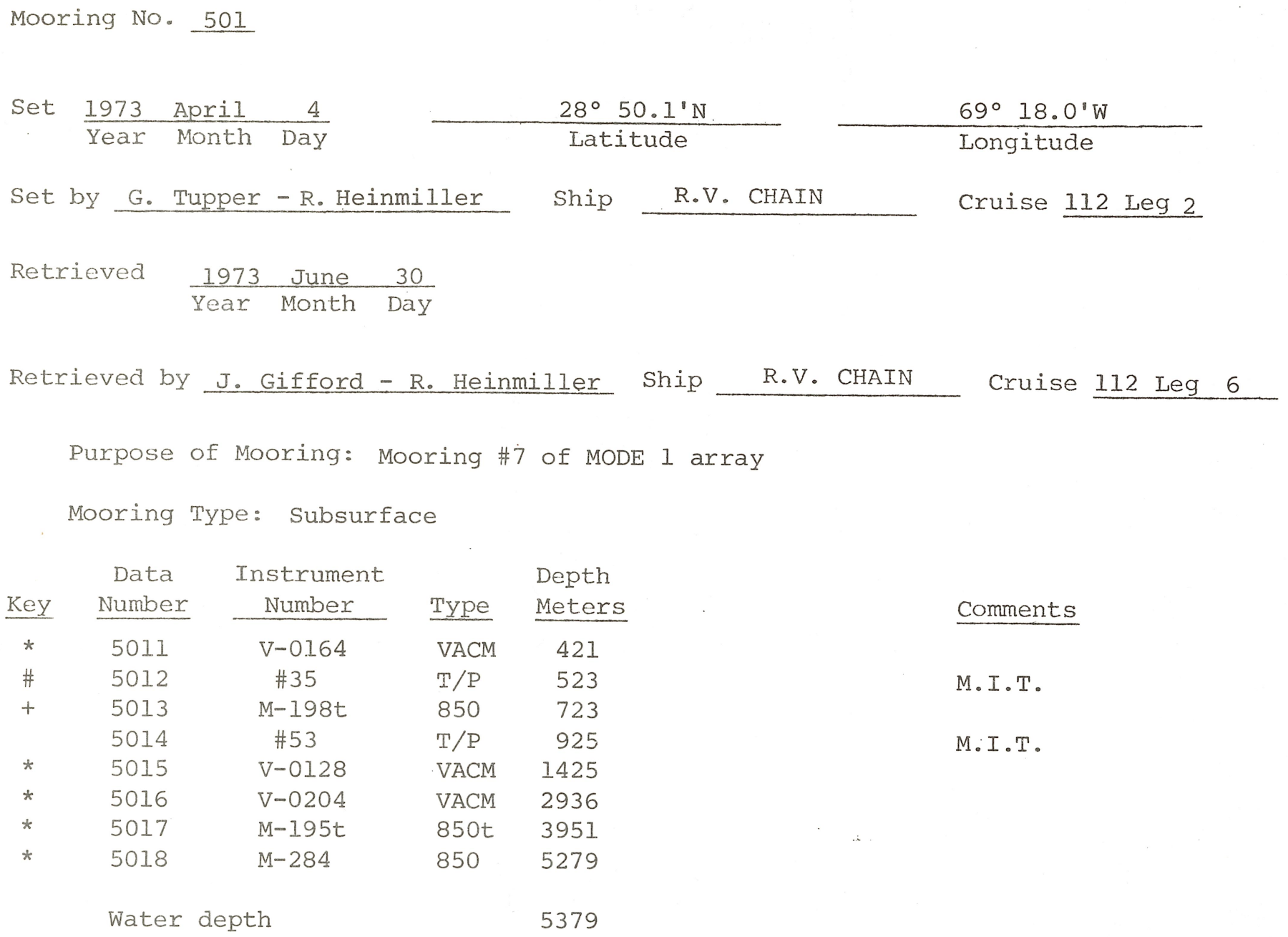

COMMENTS ON MOORING: 


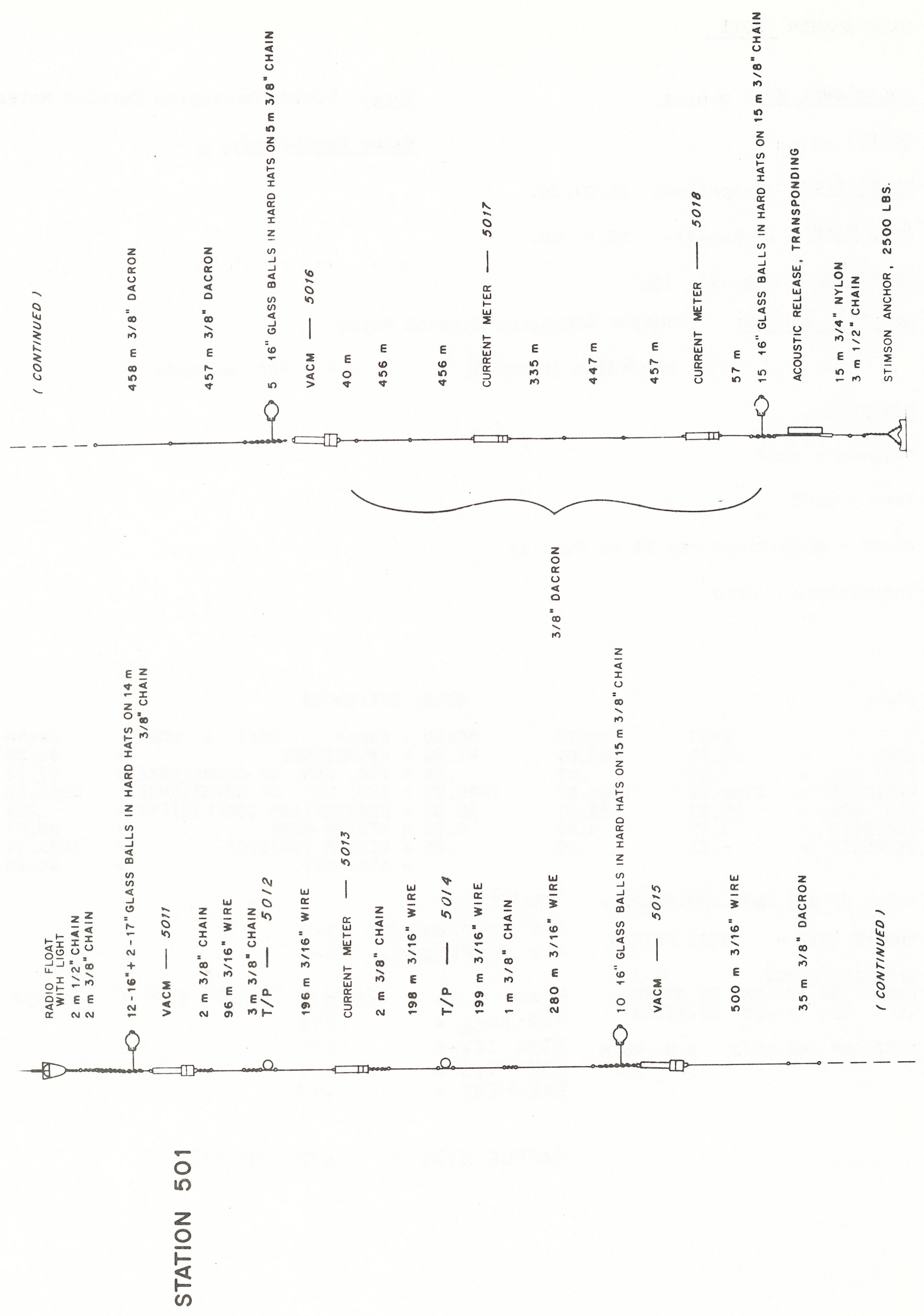


DATA NUMBER 5011

Instrument No.: V-0164

Depth: $421 \mathrm{~m}$

Start time: 73-April-04 20.07.30.

Stop time: 73-May-23 23.52.30.

Duration: $\quad 49 \mathrm{~d} \quad 3 \mathrm{~h} \quad 45 \mathrm{~m}$

Sampling scheme: Vector Averaging Current Meter

recording interval $=900$ seconds

COMMENTS :

Compass - good

Vane - good

Rotor - suspicious May 24 to June 12

Temperature - good

STATS

$\begin{array}{lrr} & \text { EAST } & \text { NORTH } \\ \text { MEAN } & 64.39 & -26.89 \\ \text { STD. ERR. }= & .68 & .48 \\ \text { VARIANCE }= & 2192.36 & 1109.87 \\ \text { STD DEV. }= & 46.82 & 33.31 \\ \text { KURTOSIS }= & 3.09 & 4.89 \\ \text { SKEWNESS } \approx & -.04 & .02\end{array}$

Type: Vector Averaging Current Meter

Watex Depth: $5379 \mathrm{~m}$

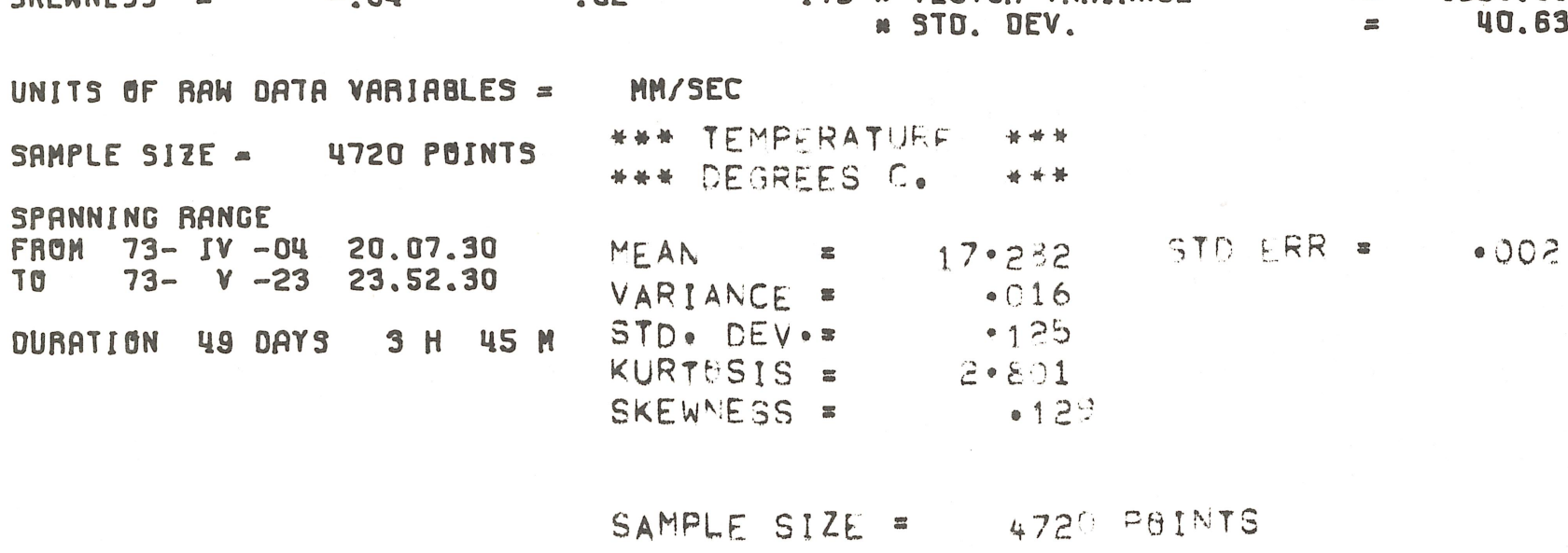



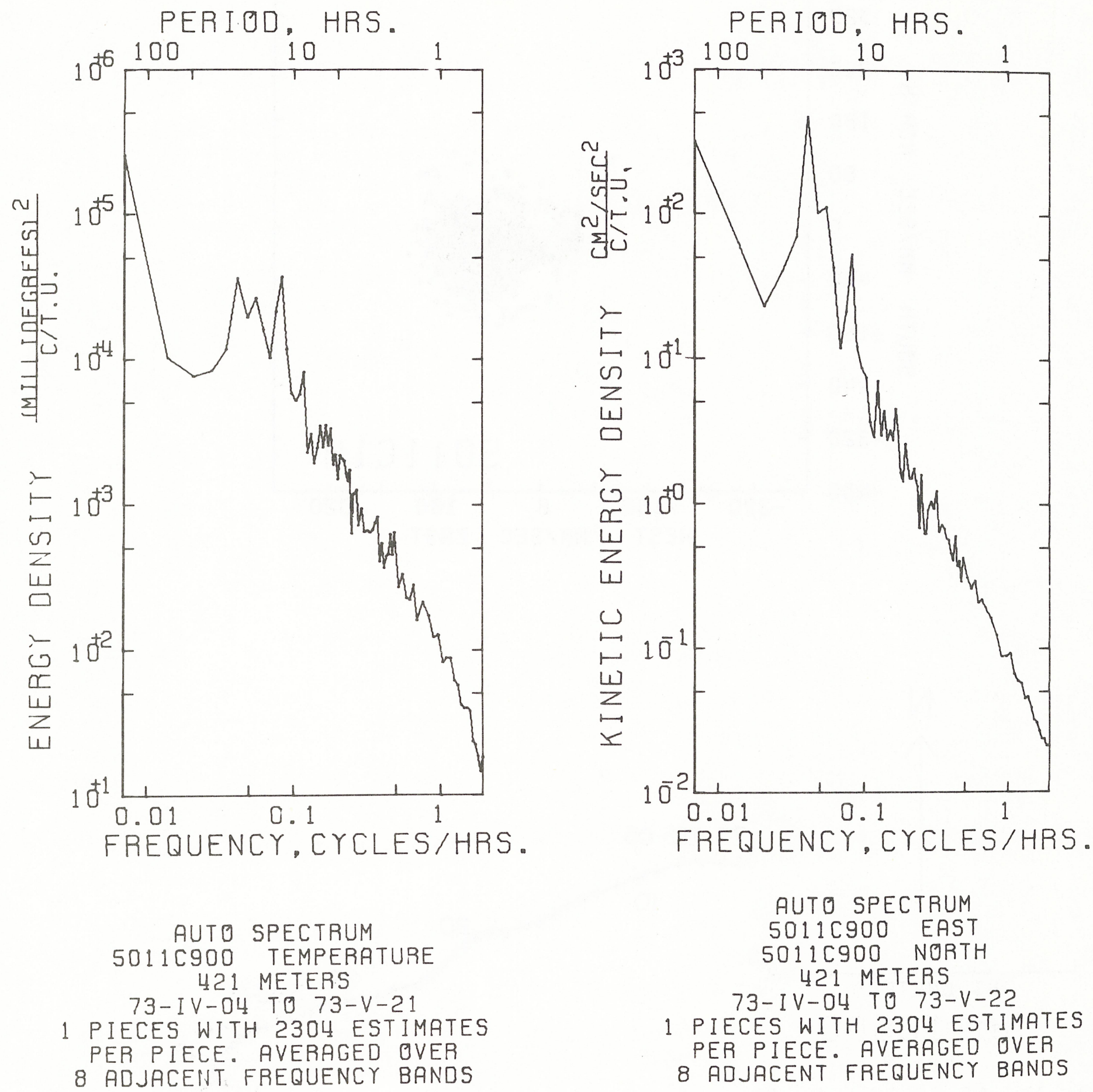

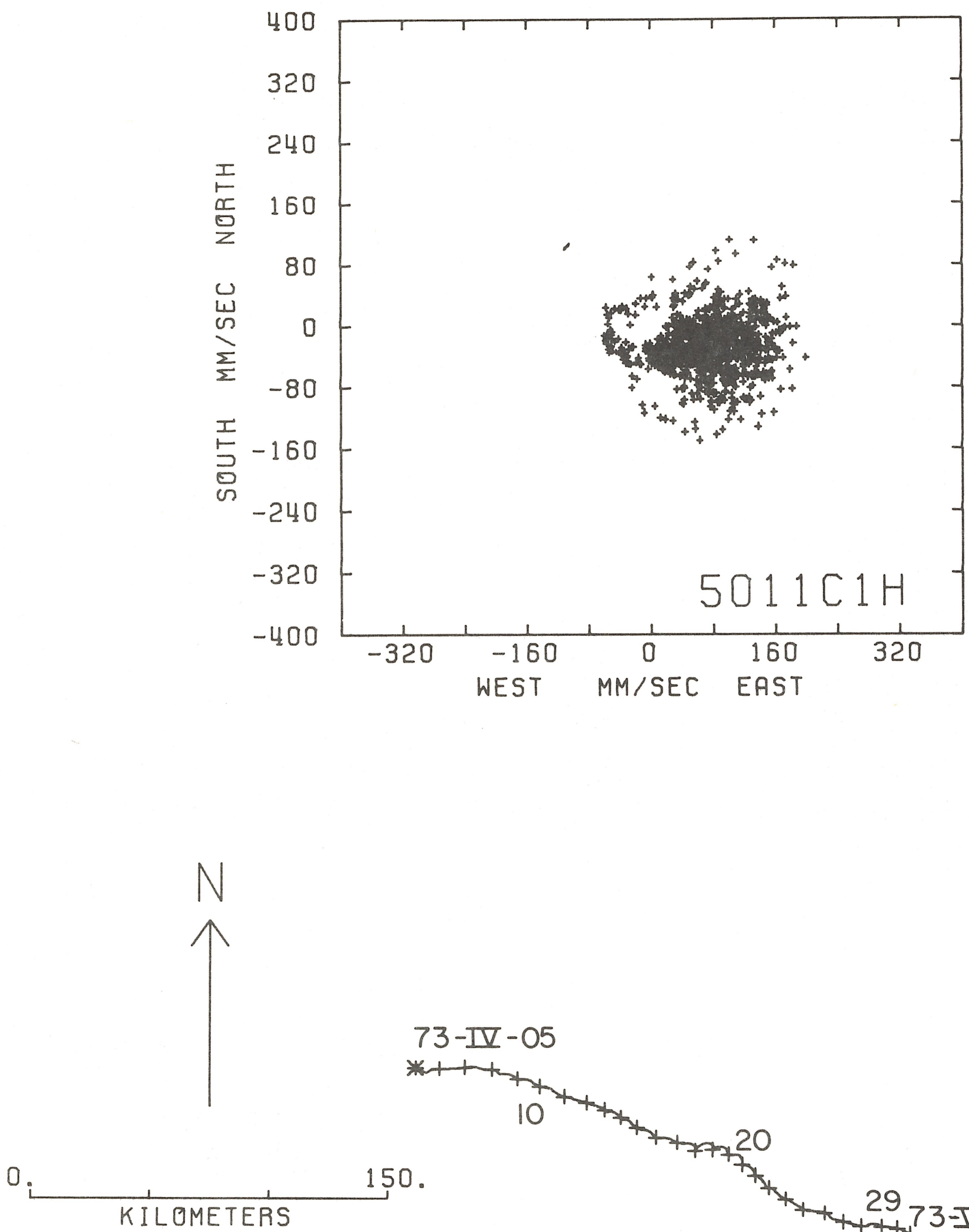

$5011 \mathrm{C} 900$

$421 M$

73- IV -05 TO 73- V - 23

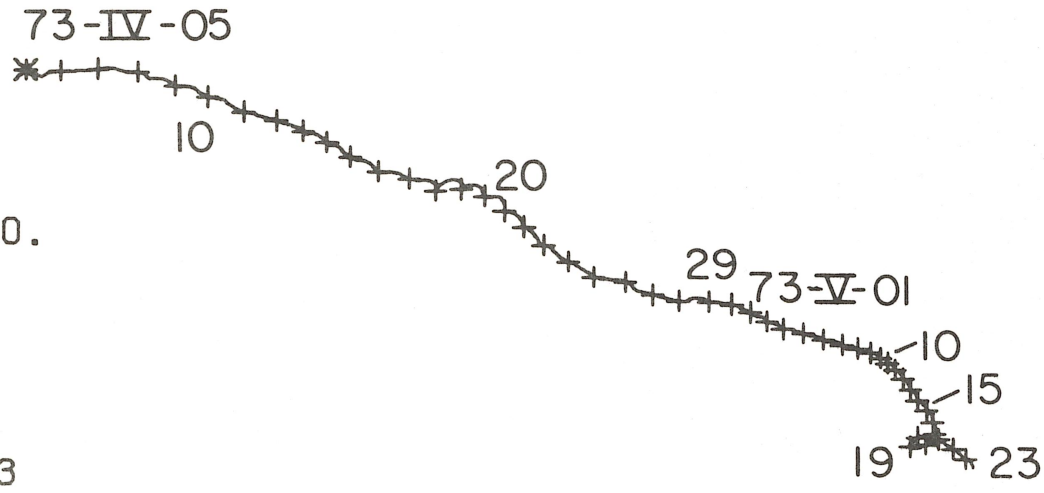



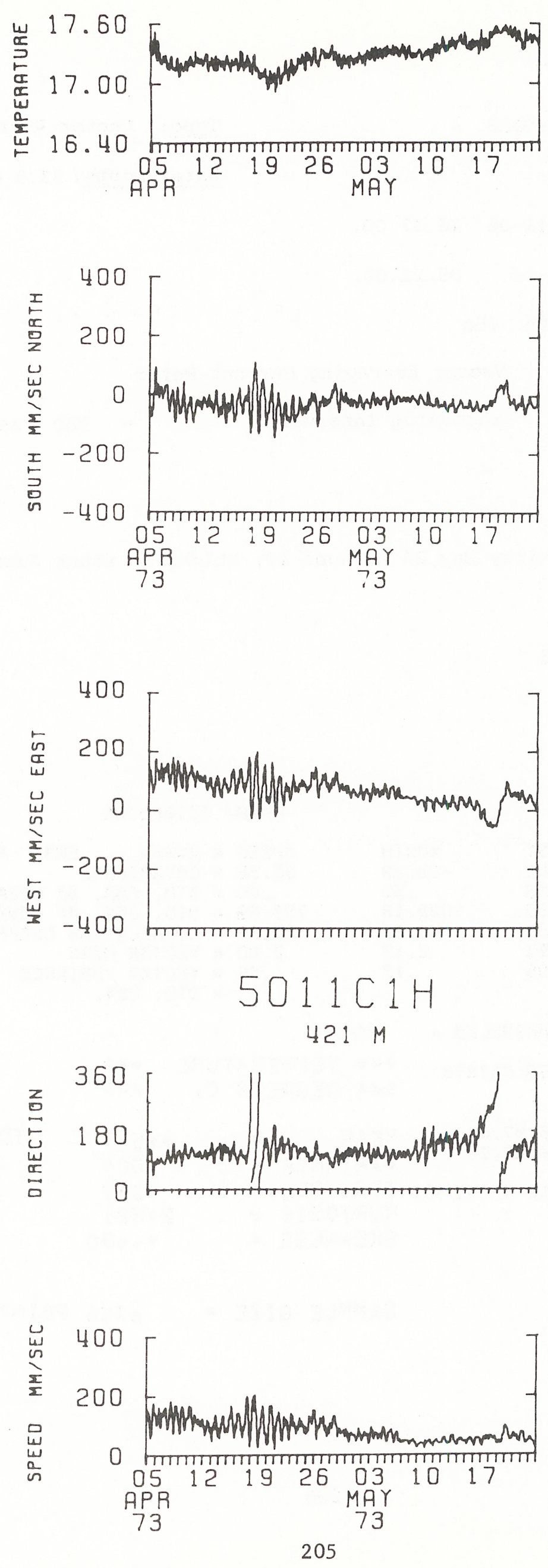
DATA NUMBER 5015

Instrument No.: V-0128

Depth: $1425 \mathrm{~m}$
Type: Vector Averaging Current Meter

Water Depth: $5379 \mathrm{~m}$

Start time: 73-April-04 16.37.00.

Stop time: 73-May-24 05.22.00.

Duration: $49 \mathrm{~d} \quad 12 \mathrm{~h} \quad 45 \mathrm{~m}$

Sampling scheme: Vector Averaging Current Meter

$$
\text { recording interval }=900 \text { seconds }
$$

COMMENTS :

Compass - good

Vane - slightly sticky May 24 to June 10, sticky to stuck June 24 to recovery

Rotor - good

Temperature - good

STATS

$\begin{array}{lrrr} & & \text { EAST } & \text { NOATH } \\ \text { MEAN } & = & -7.02 & -39.28 \\ \text { STD. ERR. }= & .36 & .59 \\ \text { VARIRNCE }= & 601.50 & 2620.18 \\ \text { STD. DEV. }= & 24.53 & 40.35 \\ \text { KURTOSIS }= & 2.94 & 2.12 \\ \text { SKEWNESS }= & .08 & .13\end{array}$

DATAP 5025F900A

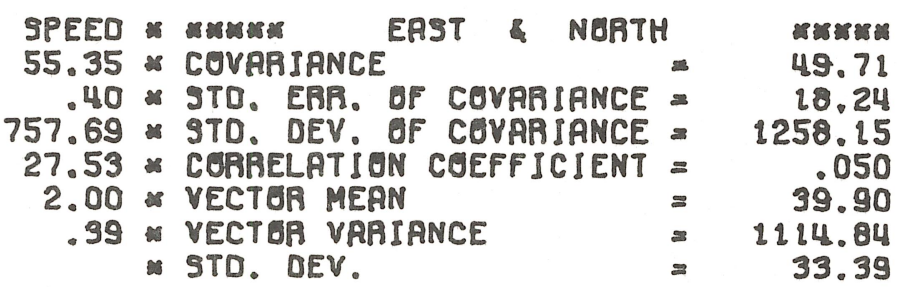

UNJTS GF RAW DATA VARIABLES = MMPEC

SAMPLE SIZE = 4756 POINTS TEMPERATURE * DEGRES C.

SPANNING RANGE

$\begin{array}{llllll}\text { FROM } 73-I V-04 & 16.37 .00 & \text { MEAN } & 4.549 & \text { STD ERR } \\ \text { TO } & 73-V-24 & 05.22 .00 & \text {. }\end{array}$

VARIANCE :

.004

DURATION 49 DAYS $12 \mathrm{H} 45 \mathrm{M}$ STD. DEV. .063

KURTOSIS $\quad 2 \cdot 521$

SKEWNESS $\quad .690$

SAMPLE SIZE - $\quad 4756$ POINTS 

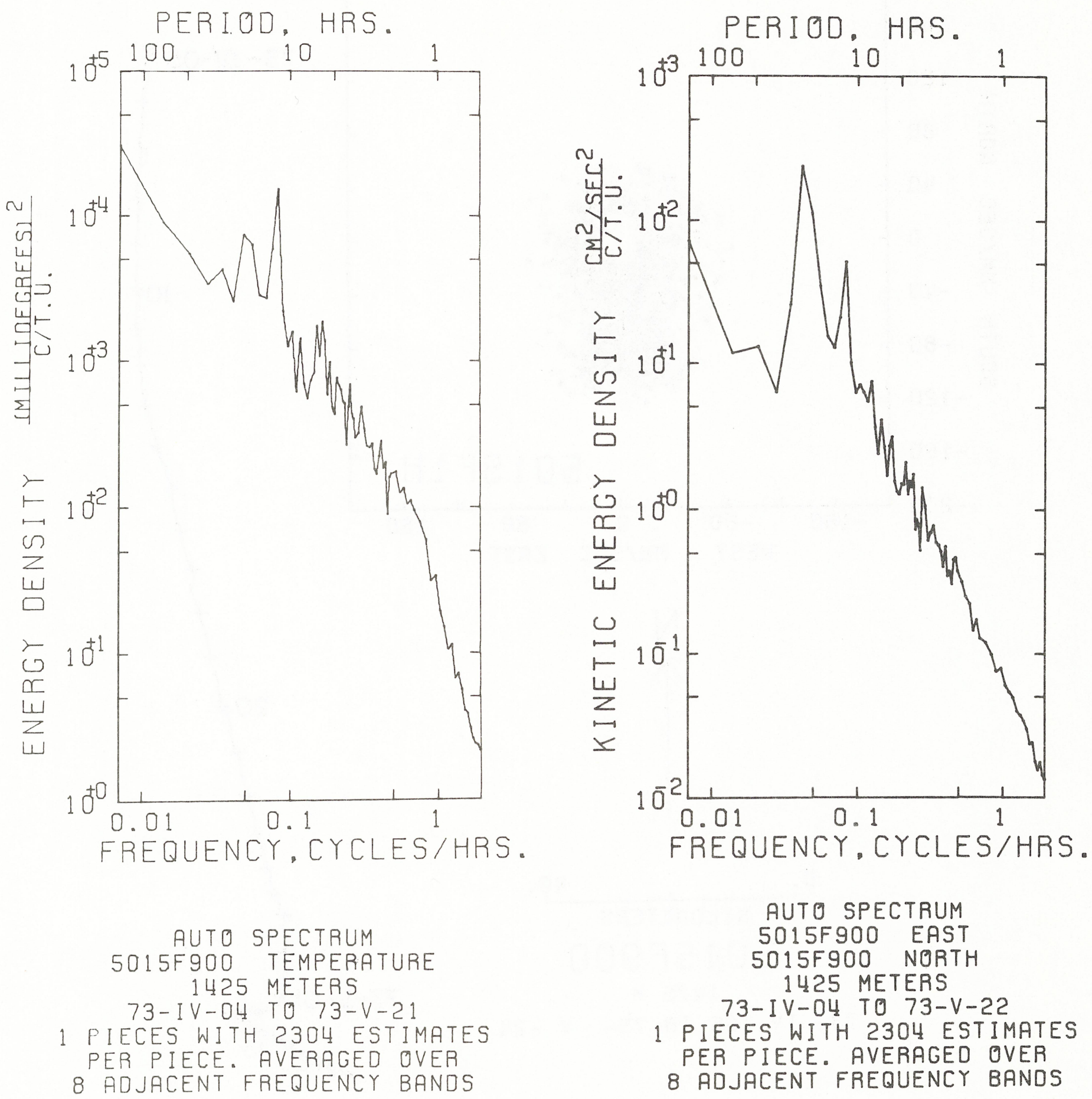


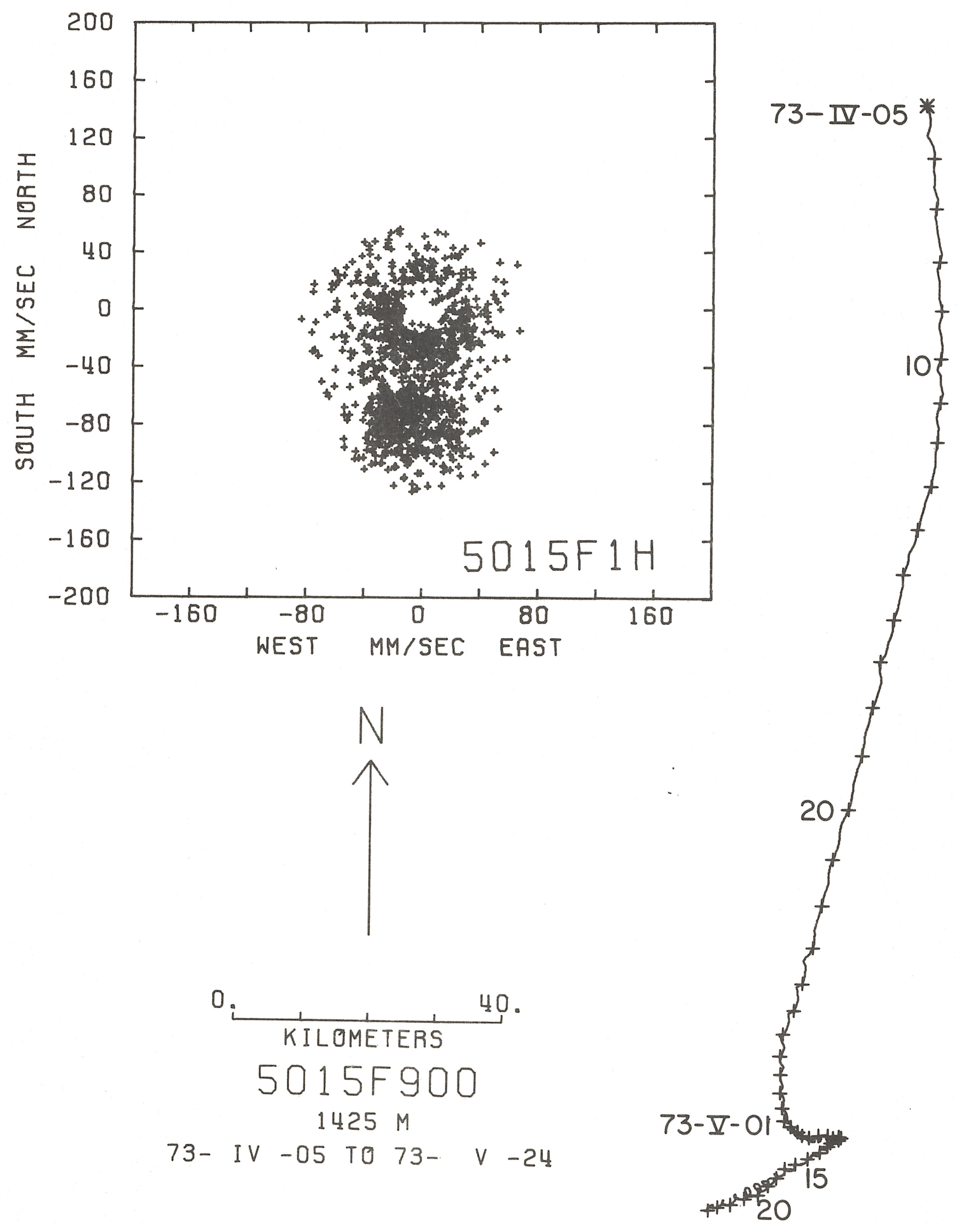



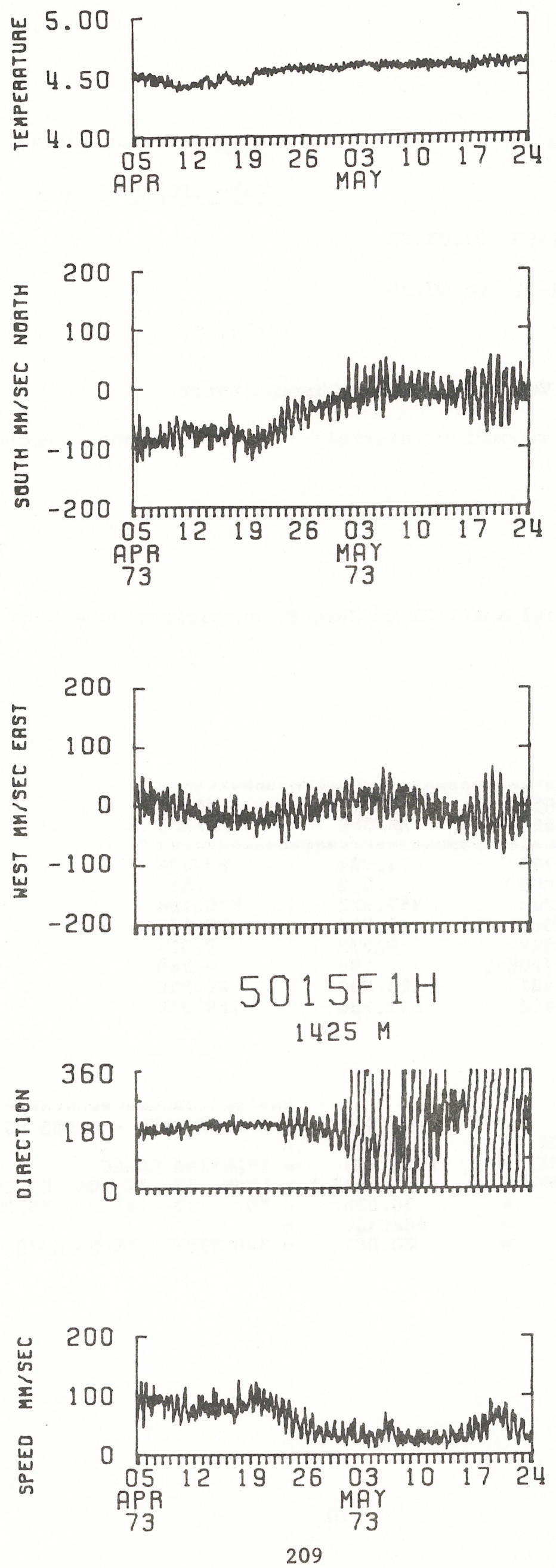
DATA NUMBER 5016

Instrument No.: V-0204

Depth: $2936 \mathrm{~m}$
Type: Vector Averaging Current Meter

Water Depth: $5379 \mathrm{~m}$

Start time: 73-April-05 07.07.30

Stop time: 73-April-21 06.07.30

Duration: $\quad 15 \mathrm{~d} 23 \mathrm{~h}$

Sampling scheme: Vector Averaging Current Meter recording interval $=900$ seconds

COMMENTS :

Compass - good

Vane - good

Rotor - below threshold April 21 to June 9, suspicious June 9 to recovery

Temperature - good

DATA/ 50169900

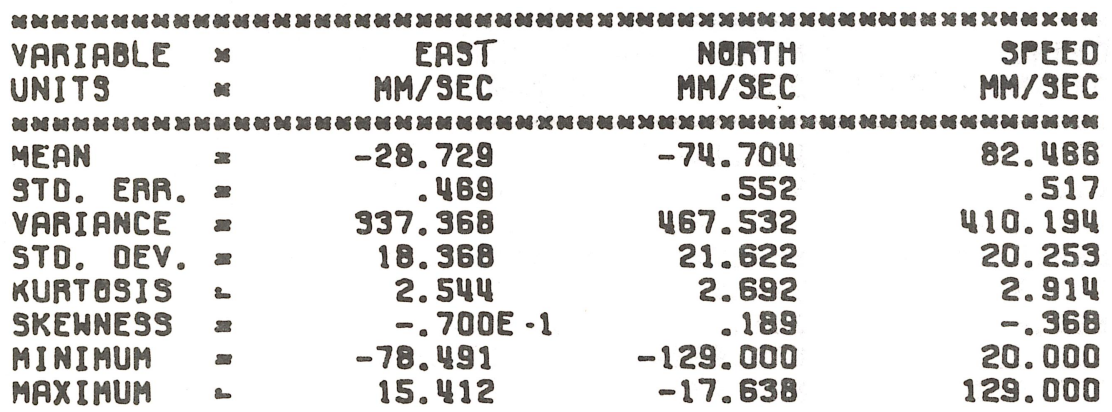

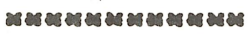

EAST \& NONTH

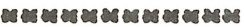

COYARIANCE

STD. EAR. OF COVPRIANCE

STO. DEV. OF COVARIANCE

CORAELATION COEFFICIENT

VECTOR MEPN

VECTOR VARIANCE

IECTOA STO. DEV.

$\begin{array}{cc}= & -34.972 \\ = & 36.342 \\ = & 1422.997 \\ = & -.081 E-1 \\ = & 80.098 \\ = & 402.450 \\ = & 20.061\end{array}$

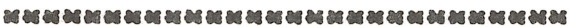

* SAMPLE SIEE = 1533 POINTS

$\varpi$

- SPANNING RANGE

* FREA 73- IV -05 07.07.30

* T0 73-IV -2106.07 .30$

20.061 DURATION 15.96 DAYS 

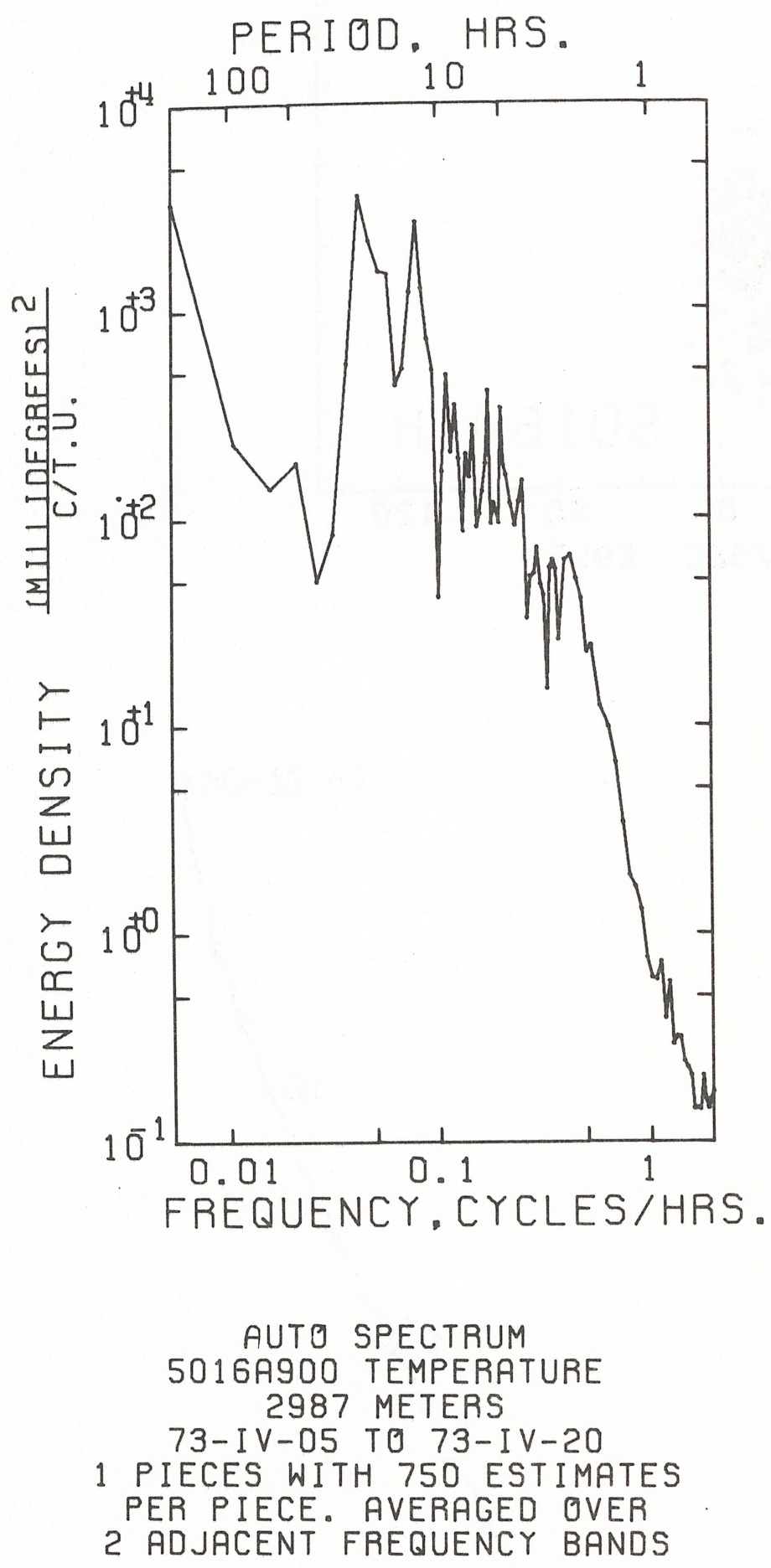
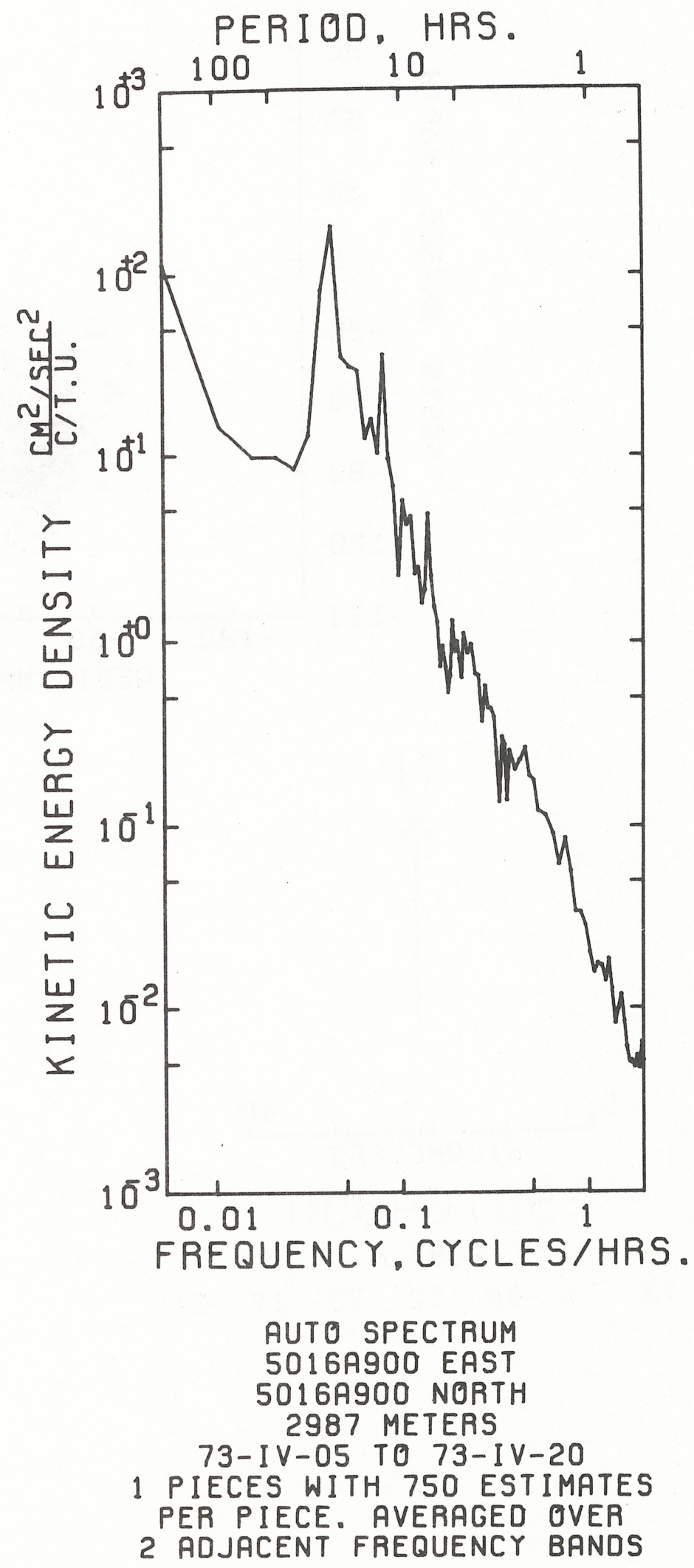

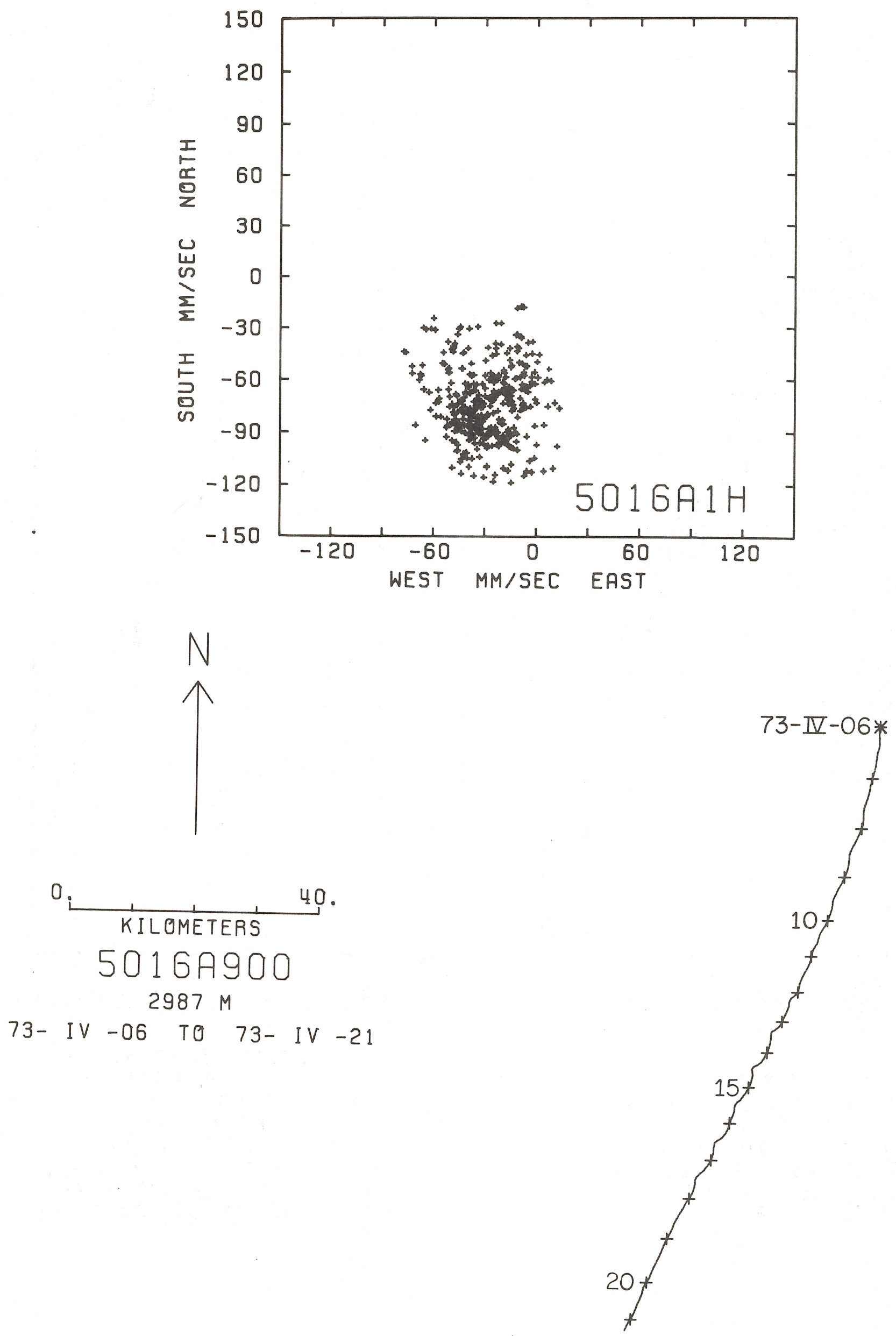

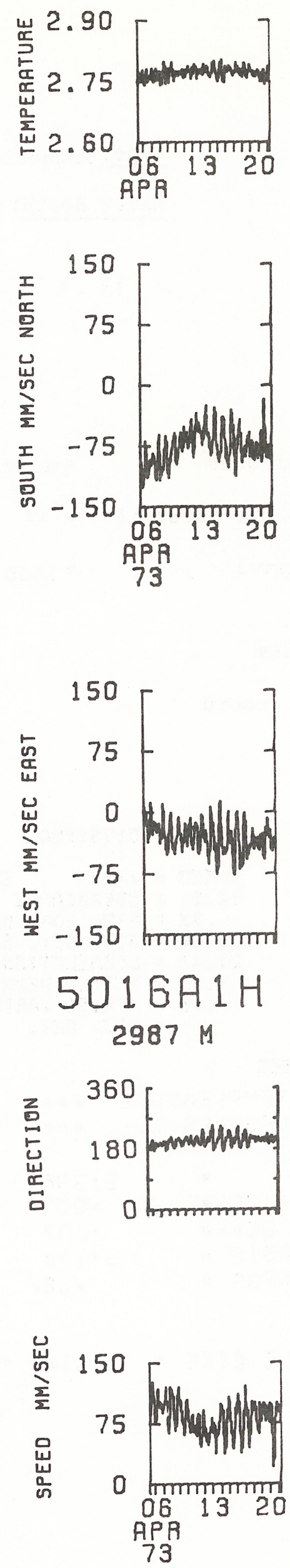
DATA NUMBER 5017

Instrument No. : $M-175 t$

Depth: $3951 \mathrm{~m}$

Start time: 73-April-05 06.00.34.

Stop time: 73 -June-30 12.30.34.

Duration: $86 \mathrm{~d}$ 06h $30 \mathrm{~m}$

Sampling scheme: Interval

$$
\begin{array}{ll}
\text { time between strobes } & =5.27 \text { seconds } \\
\text { no. of strobes per interval } & =13 \\
\text { recording interval } & =1800 \text { seconds }
\end{array}
$$

COMMENTS:

Compass had a correctable bit problem

All variables look good for entire record

\begin{tabular}{|c|c|c|c|}
\hline & & EAST & MOATH \\
\hline MEAN & & -27.45 & \\
\hline $\begin{array}{l}\text { STO. ERT. } \\
\text { YRRIPMCE }\end{array}$ & $=$ & $\begin{array}{r}.30 \\
58\end{array}$ & 57 \\
\hline STO. DEV. & 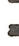 & 10.59 & $\begin{array}{r}1391.6 \\
38.4\end{array}$ \\
\hline RUATOSIS & & 3.18 & 5 \\
\hline REMNESS & & -.29 & \\
\hline
\end{tabular}

รTคTร

DATAR 501751000

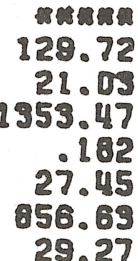

29.27

UNITS OF RAM OATA VAAIABLES - MMPSC

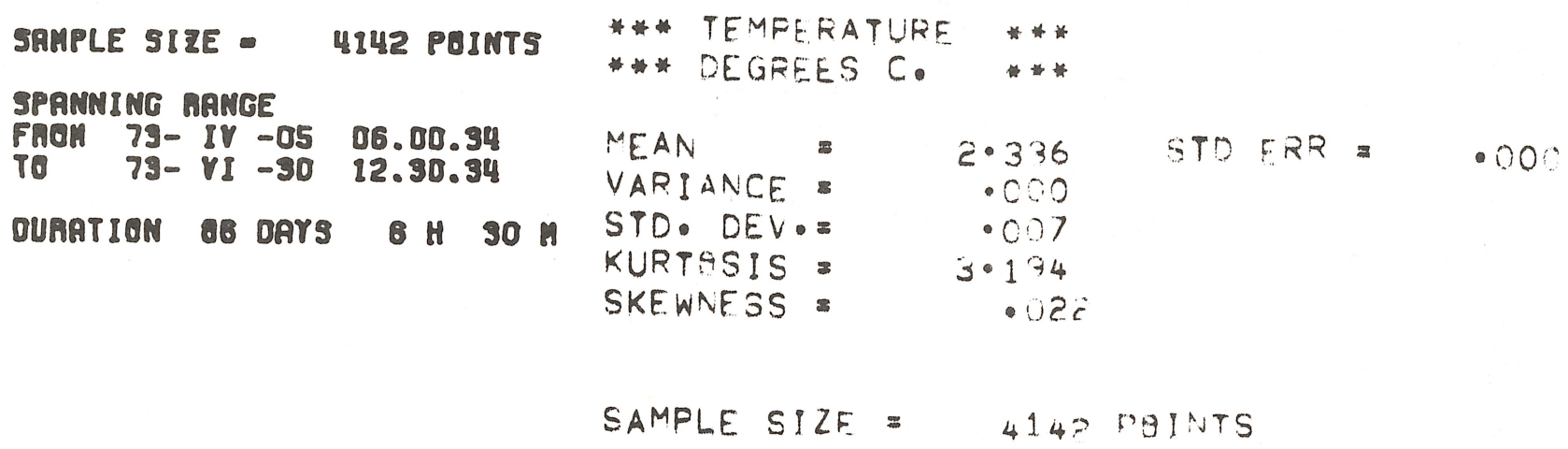




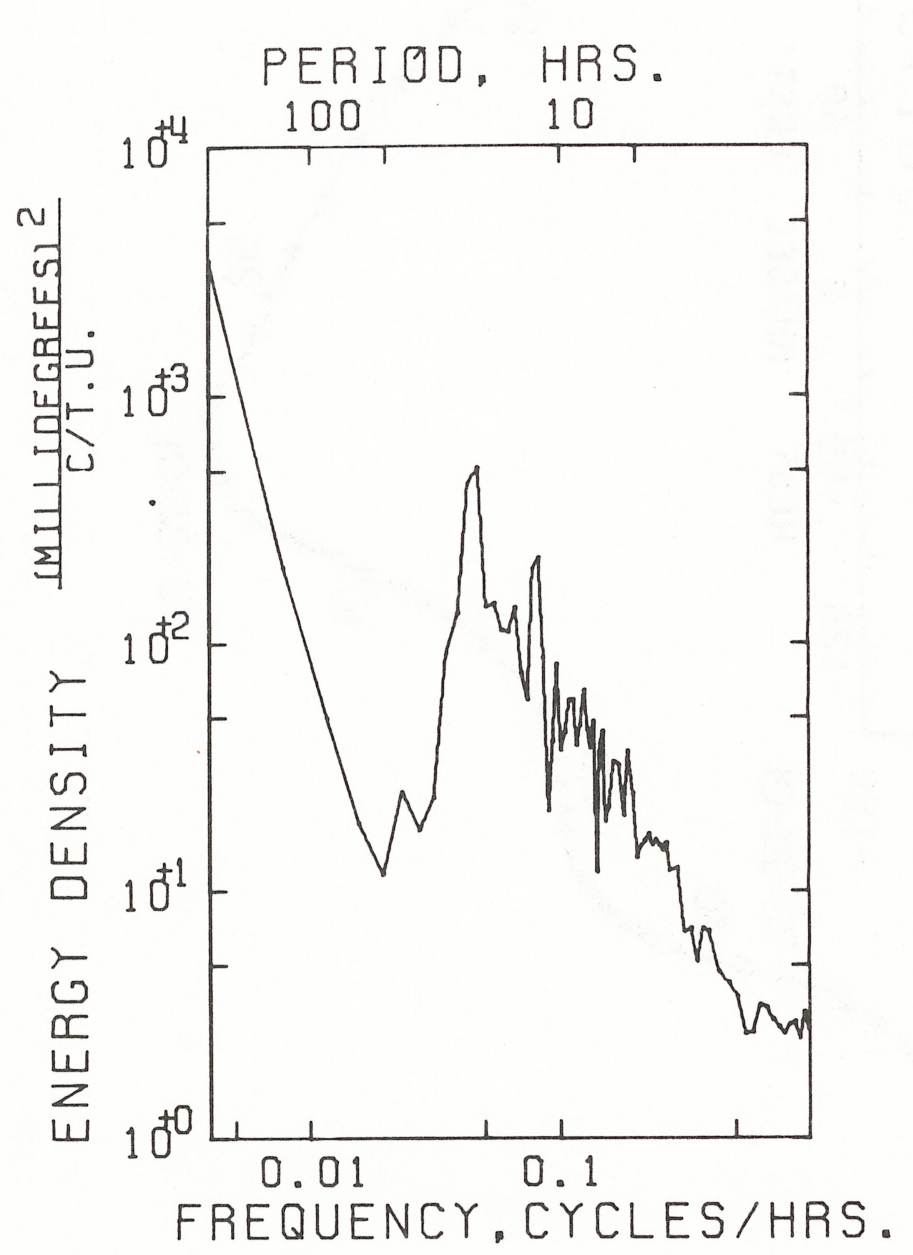

$$
\begin{gathered}
\text { AUTO SPECTRUM } \\
501751800 \text { TEMPERATURE } \\
3951 \text { METERS } \\
\text { 73-IV-05 TO } 73-V I-29 \\
1 \text { PIECES WITH 2048 ESTIMATES } \\
\text { PER PIECE. AVERAGED OVER } \\
\text { 8 ADJACENT FREQUENCY BANDS }
\end{gathered}
$$

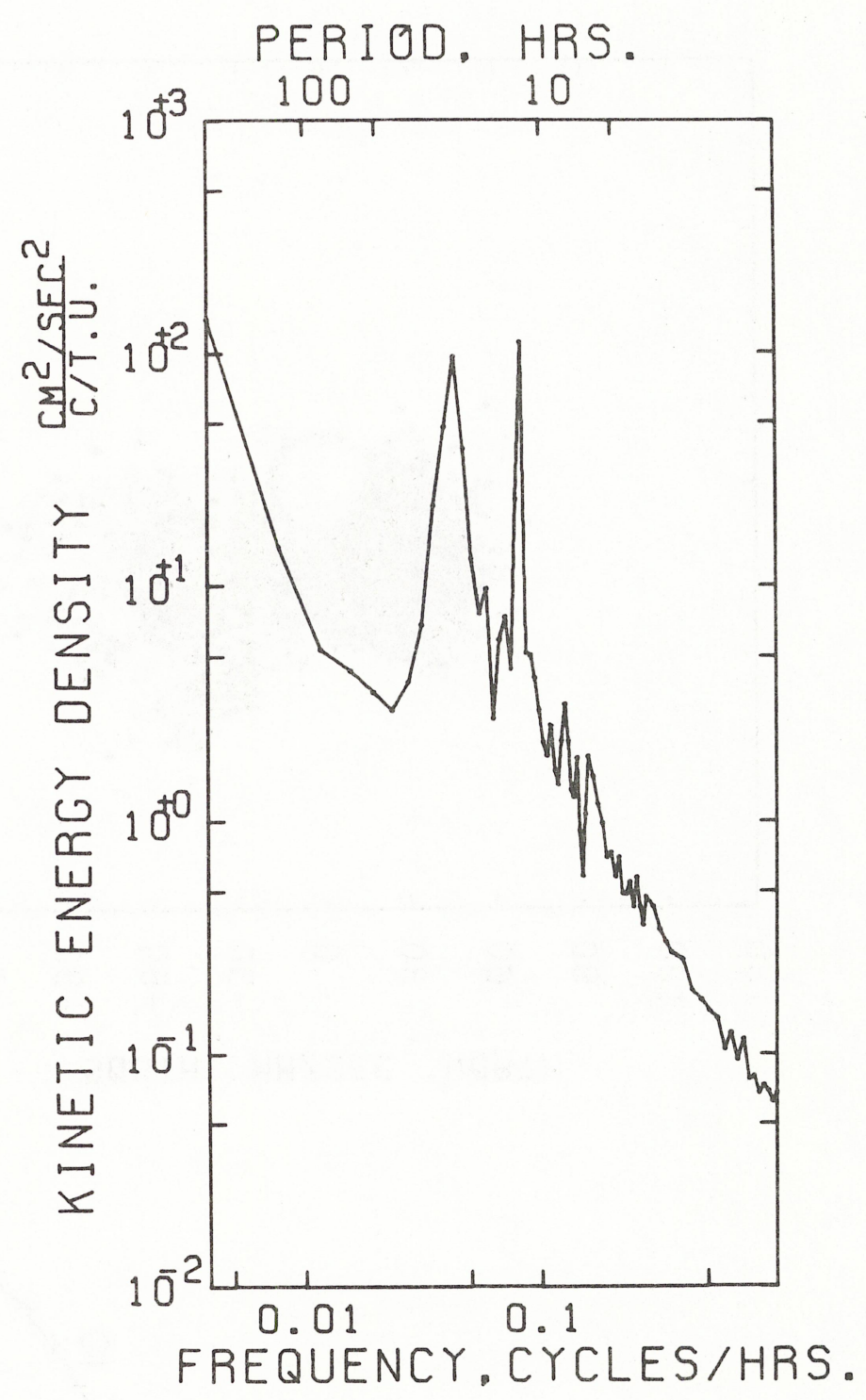

AUTO SPECTRUM 501751800 EAST 501751800 NORTH 3951 METERS 73-IV-05 TO 73-VI-29 1 PIECES WITH 2048 ESTIMATES PER PIECE. AVERAGED OVER 8 ADJACENT FREQUENCY BANDS 


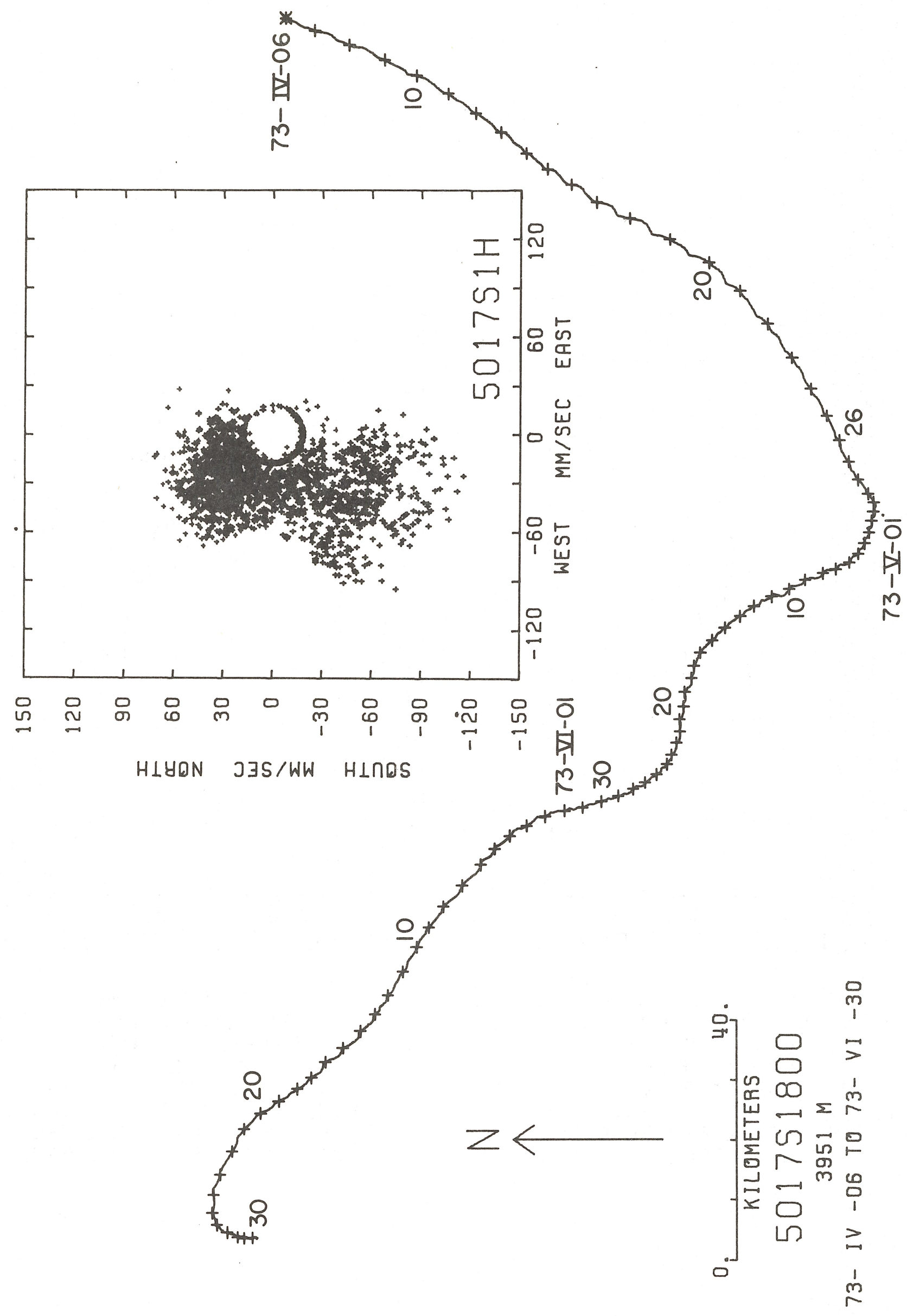



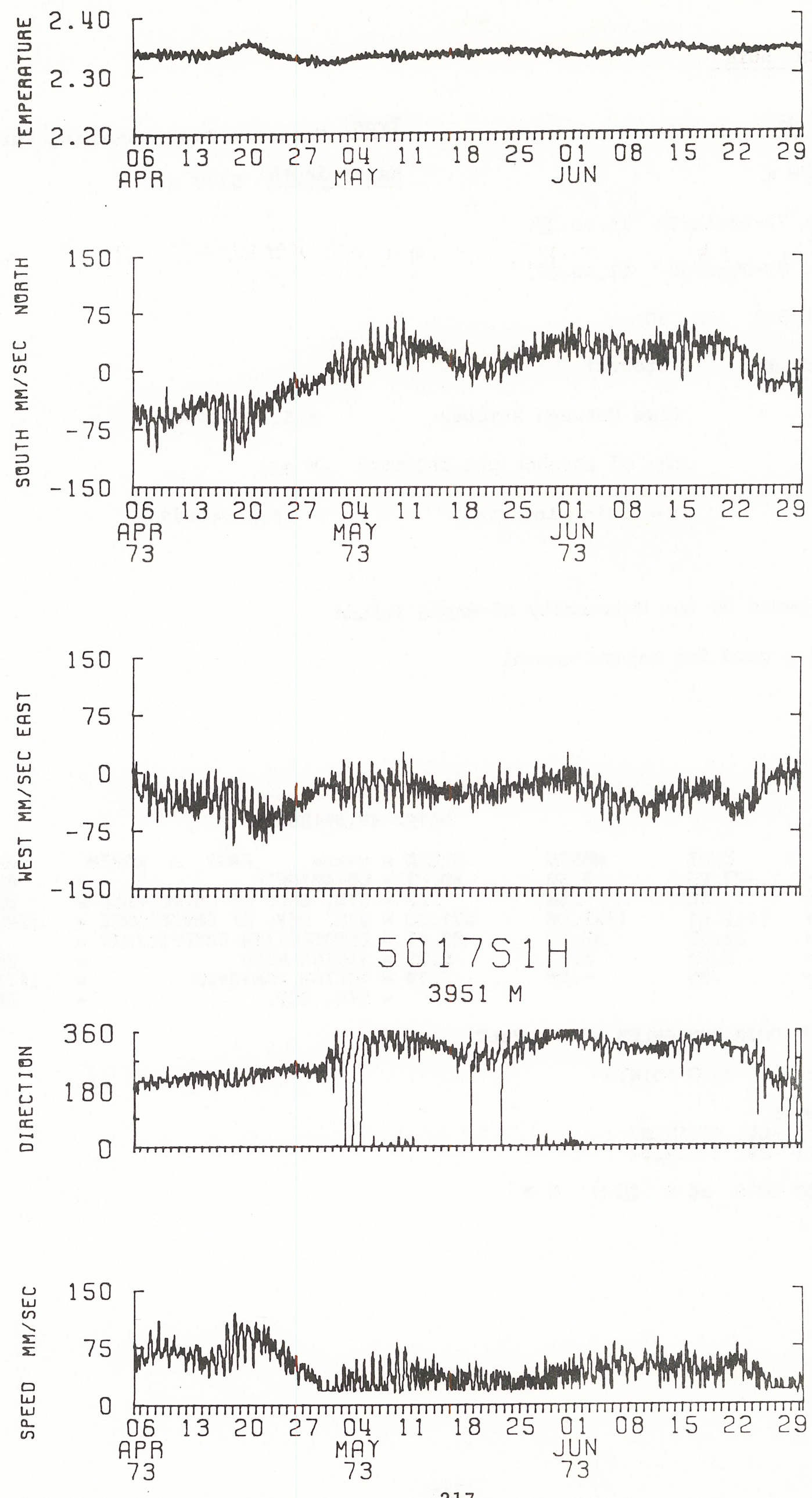
DATA NUMBER 5018

Instrument No.: $\mathrm{M}-284$

Depth: $5279 \mathrm{~m}$

Start time: 73-April-04 15.10.37.

Stop time: $73-J u n e-30 \quad 11.40 .37$.

Duration: $\quad 86 \mathrm{~d} \quad 20 \mathrm{~h} \quad 30 \mathrm{~m}$

Sampling scheme: Interval

$$
\begin{array}{ll}
\text { time between strobes } & \equiv 5.27 \text { seconds } \\
\text { no. of strobes per interval } & =13 \\
\text { recording interval } & =1800 \text { seconds }
\end{array}
$$

COMMENTS :

Instrument owned by the University of Rhode Island

All variables good for entire record

STATS

$$
\text { DATA/ 5018F1800 }
$$

$\begin{array}{lrr} & \text { EAST } & \text { MORTH } \\ \text { MEAN } & -27.65 & 5.59 \\ \text { STD. ENR. }= & .52 & .54 \\ \text { VARIANCE }= & 1111.91 & 1291.06 \\ \text { STD DEV. } & 39.55 & 95.08 \\ \text { KURTOSIS }= & 2.83 & 9.10 \\ \text { SKEHNESS }= & .05 & -.09\end{array}$

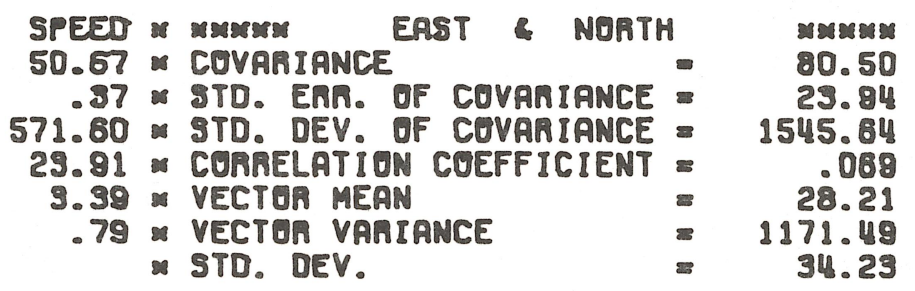

UNITS OF AAH DATA VARIABLES = MM/SEC

SAMPLE SIZE = 4170 POINTS

SPANNING RAMGE

FROM 73- IV -OU 15.10.37

TO 73-VI - 30 11.40.37

DURATION 86 DAYS 20 H 30 M O S 


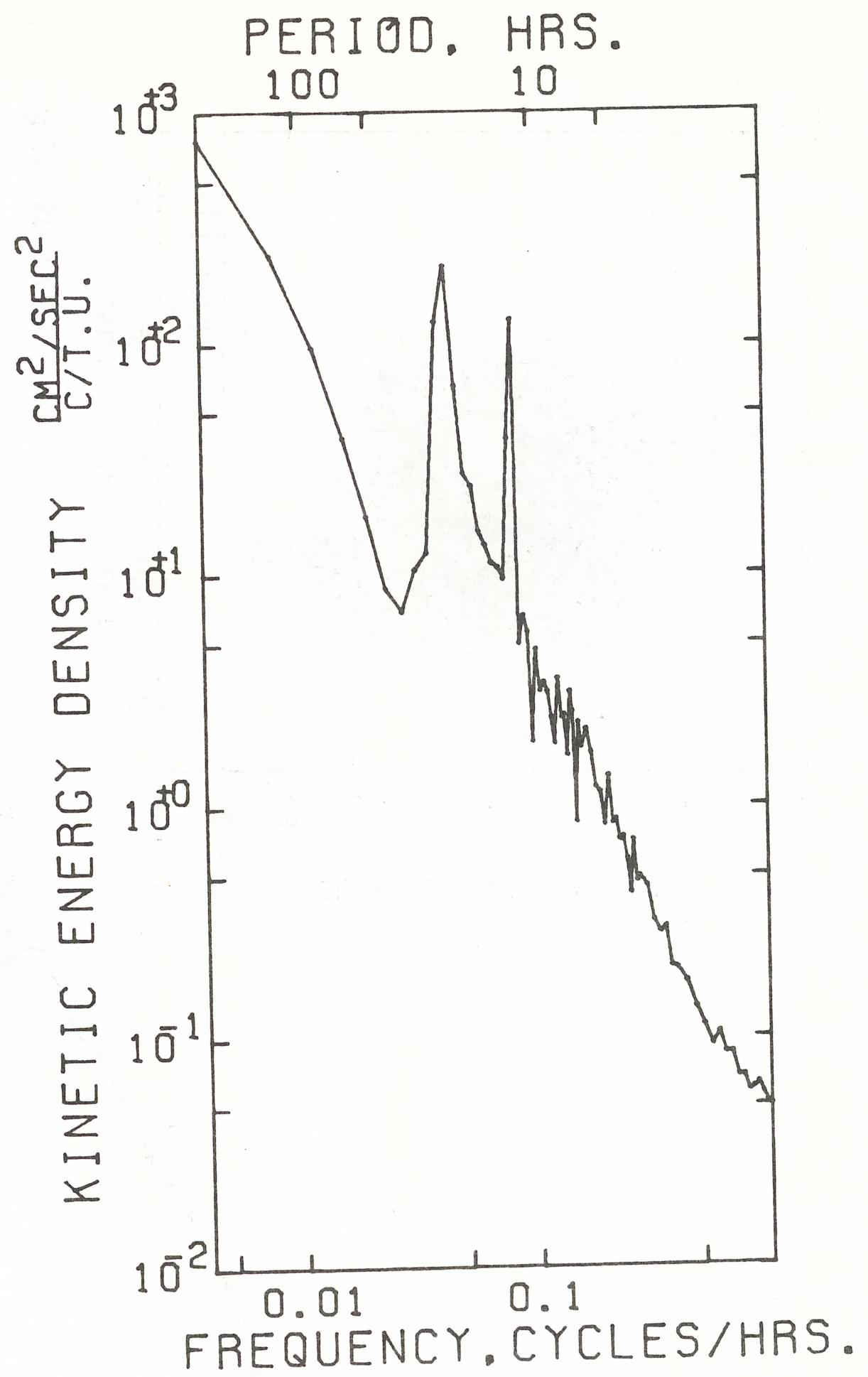

AUTO SPECTRUM

$5018 F 1800$ EAST

5018 F 1800 NORTH

5279 METERS

73-IV-04 TO 73-VI-28

1 PIECES WITH 2048 ESTIMATES PER PIECE. AVERAGED OVER 8 ADJACENT FREQUENCY BANOS 


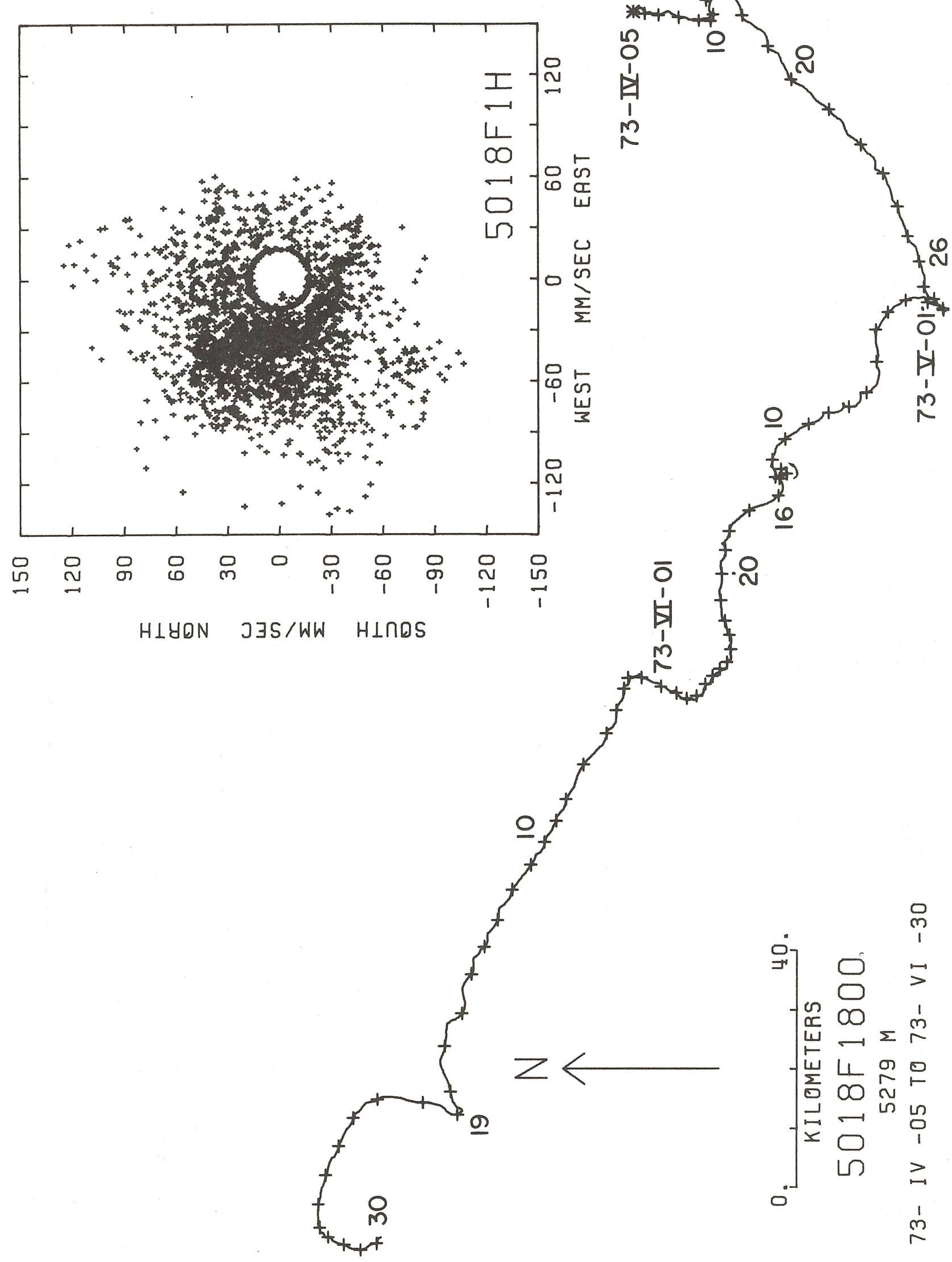



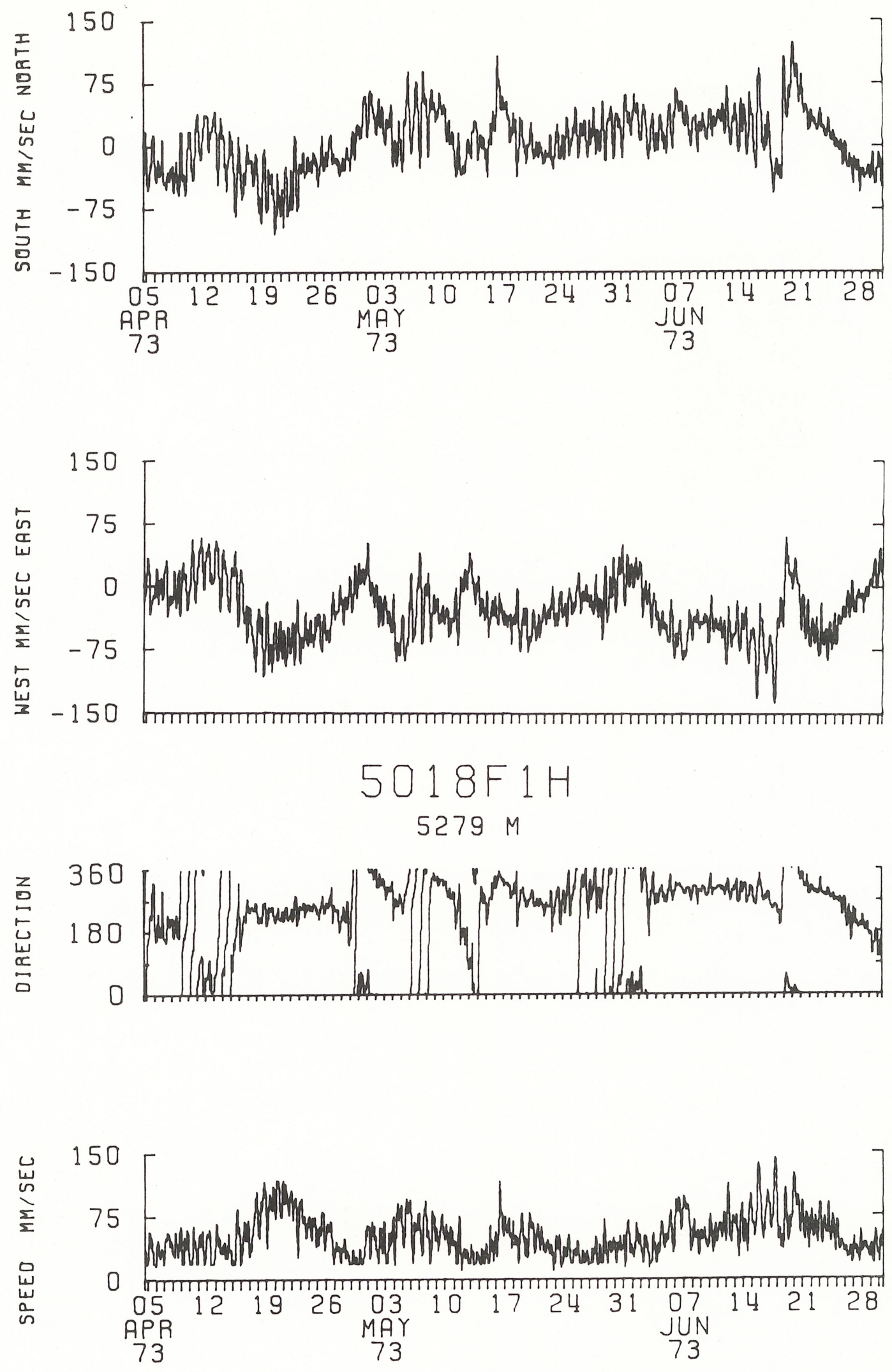

Data Section 2

Page

Temperature and Pressure Plotted by Mooring

224

U, V Vector Components Plotted by Mooring (Stick Plot)

258

U, V Vector Components Plotted by Depth

274 


\section{1}

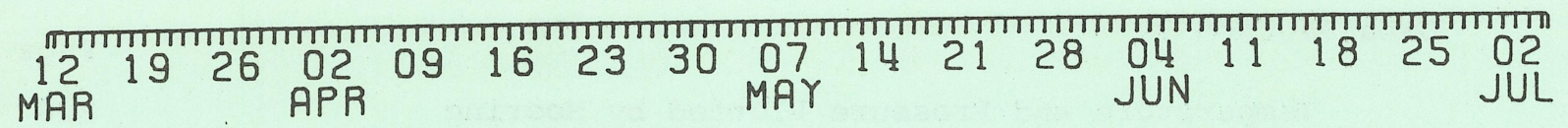

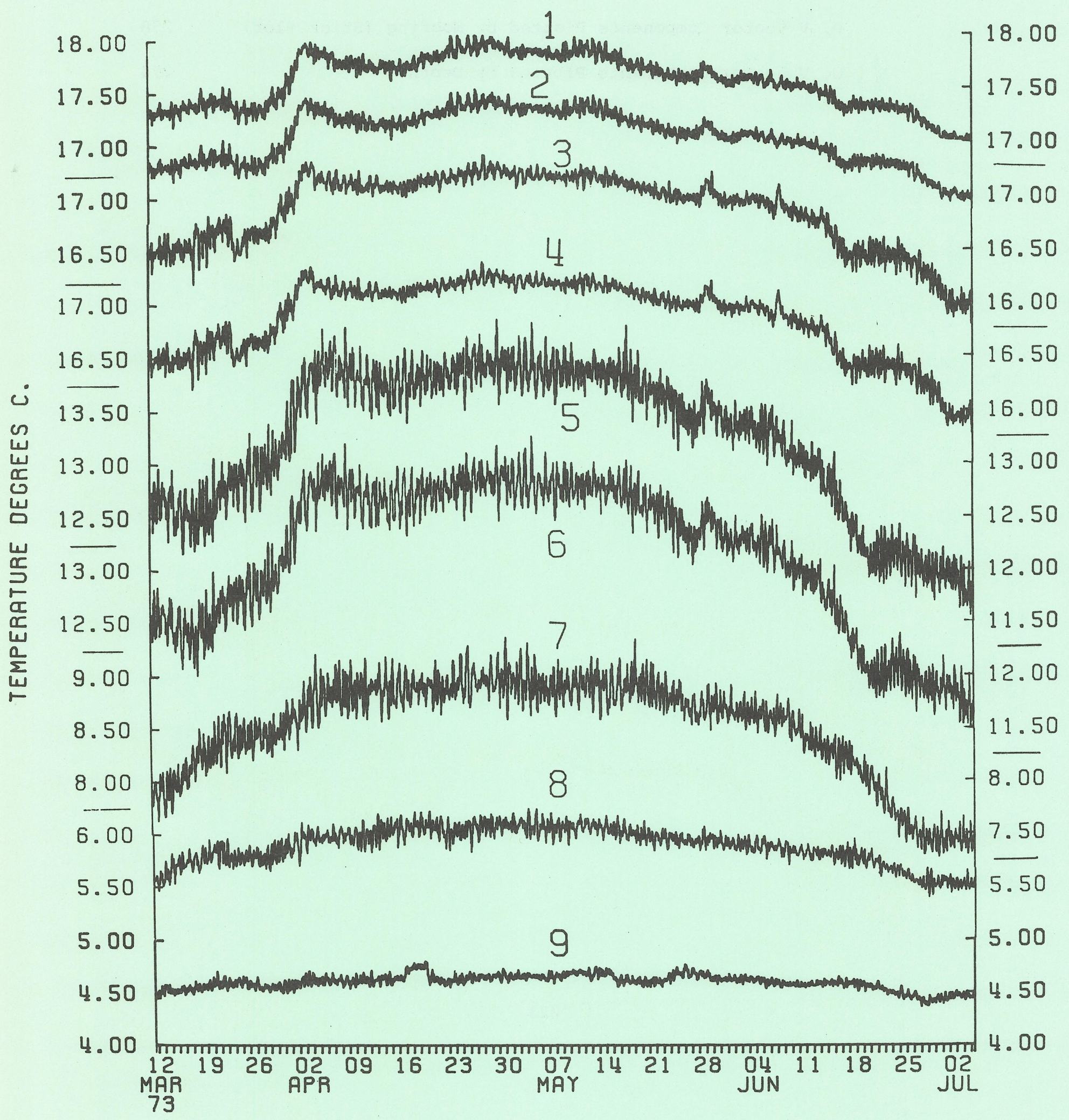




\section{1}

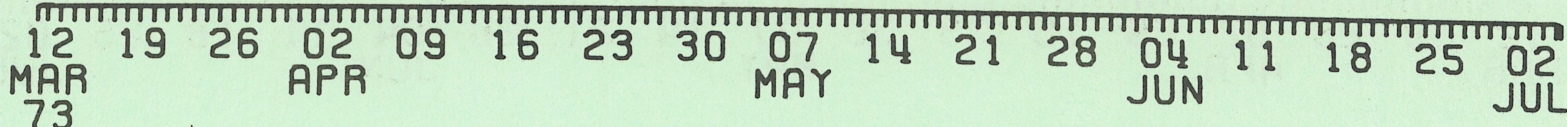

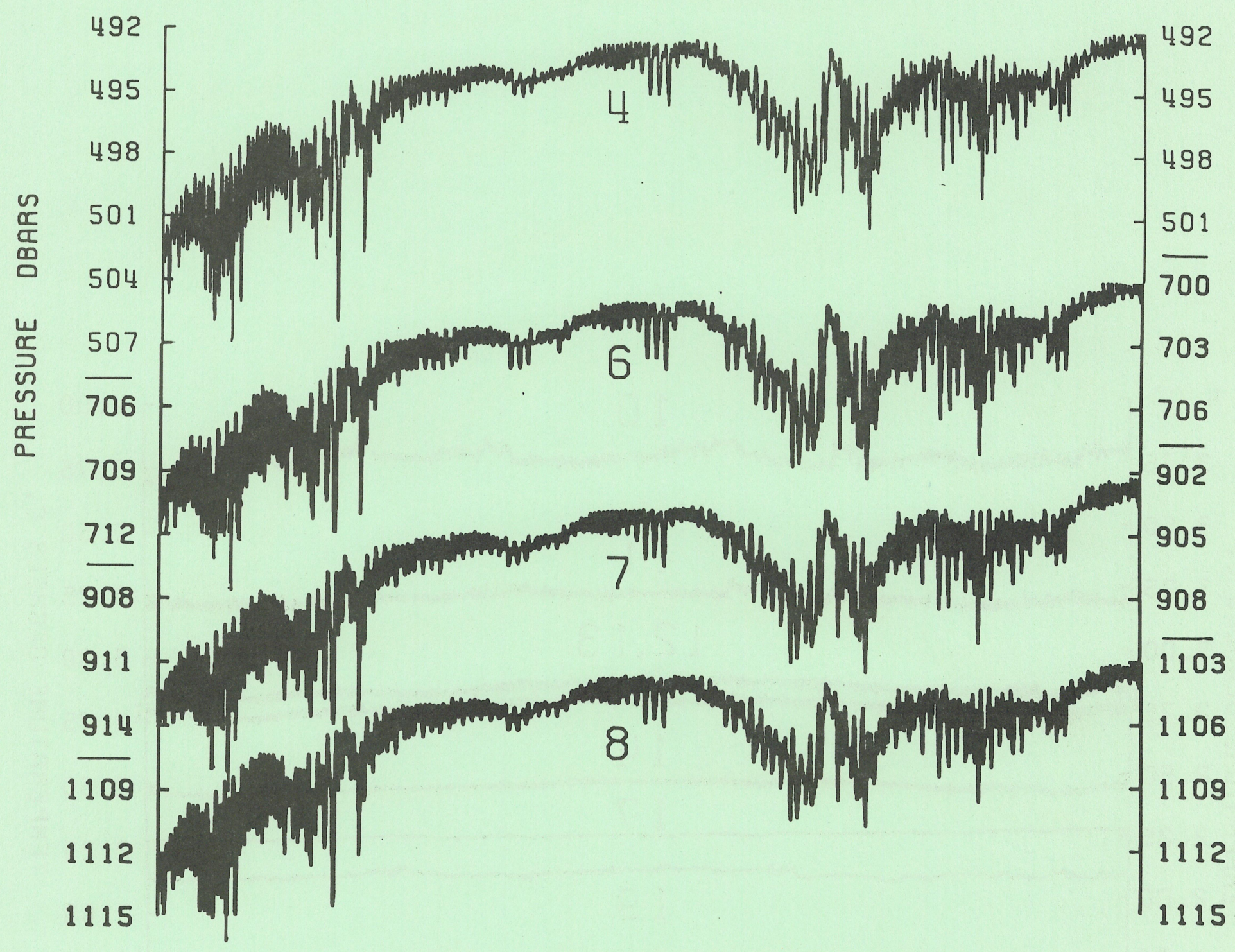

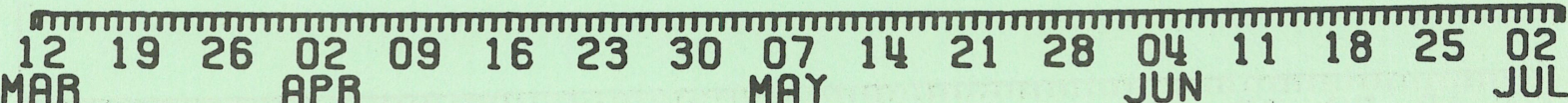




\section{1}

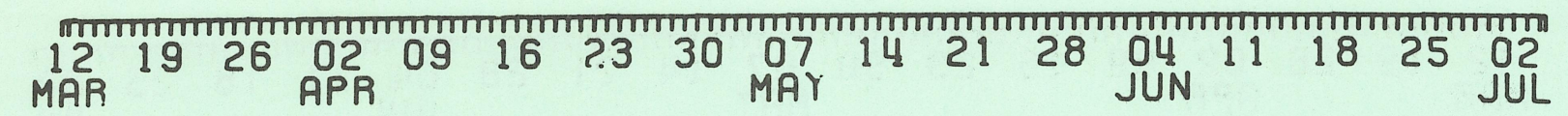

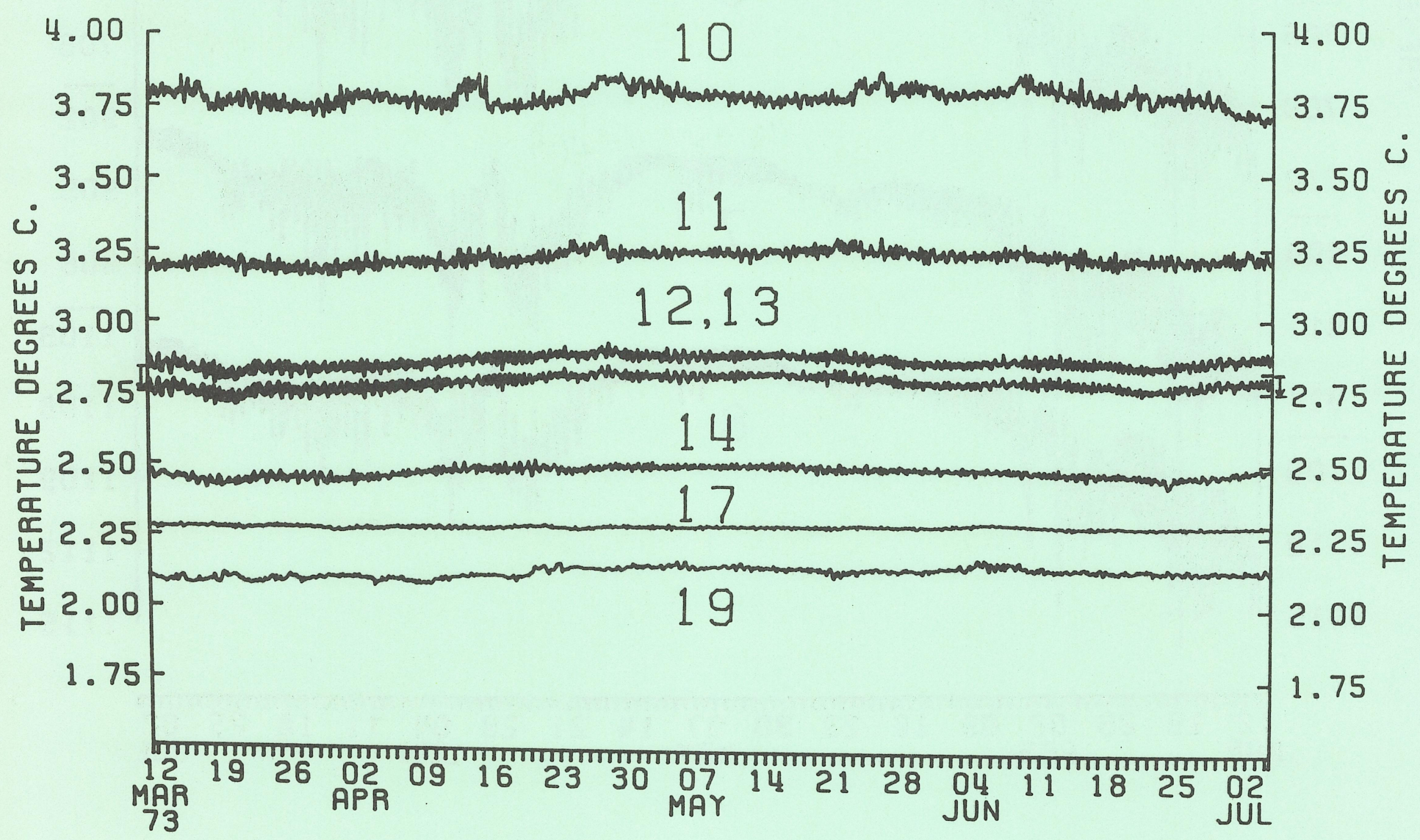




\section{1}

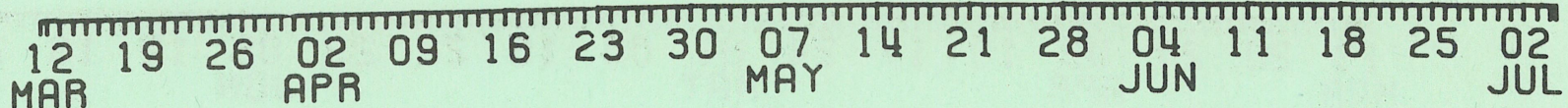
MAR
73

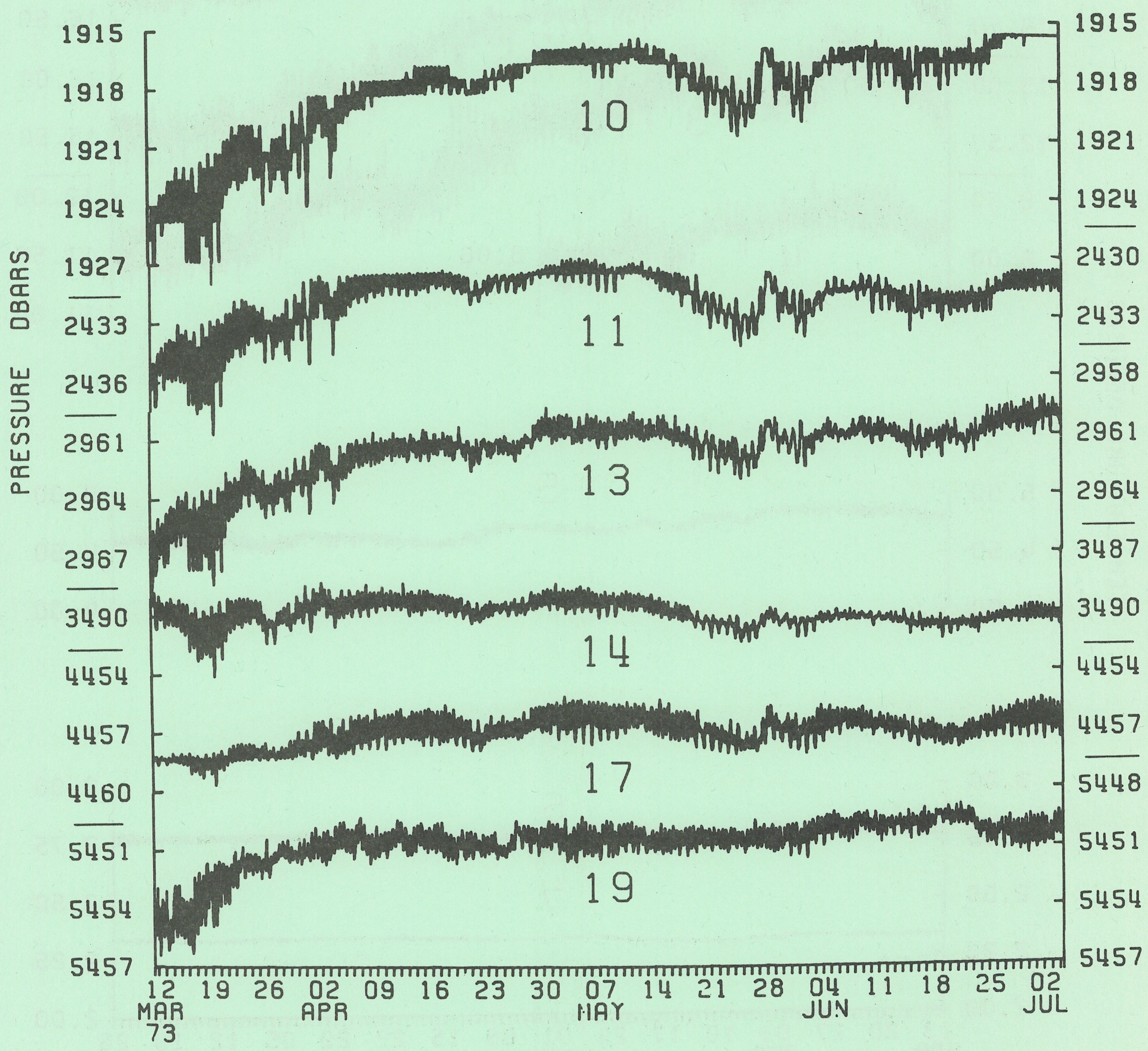




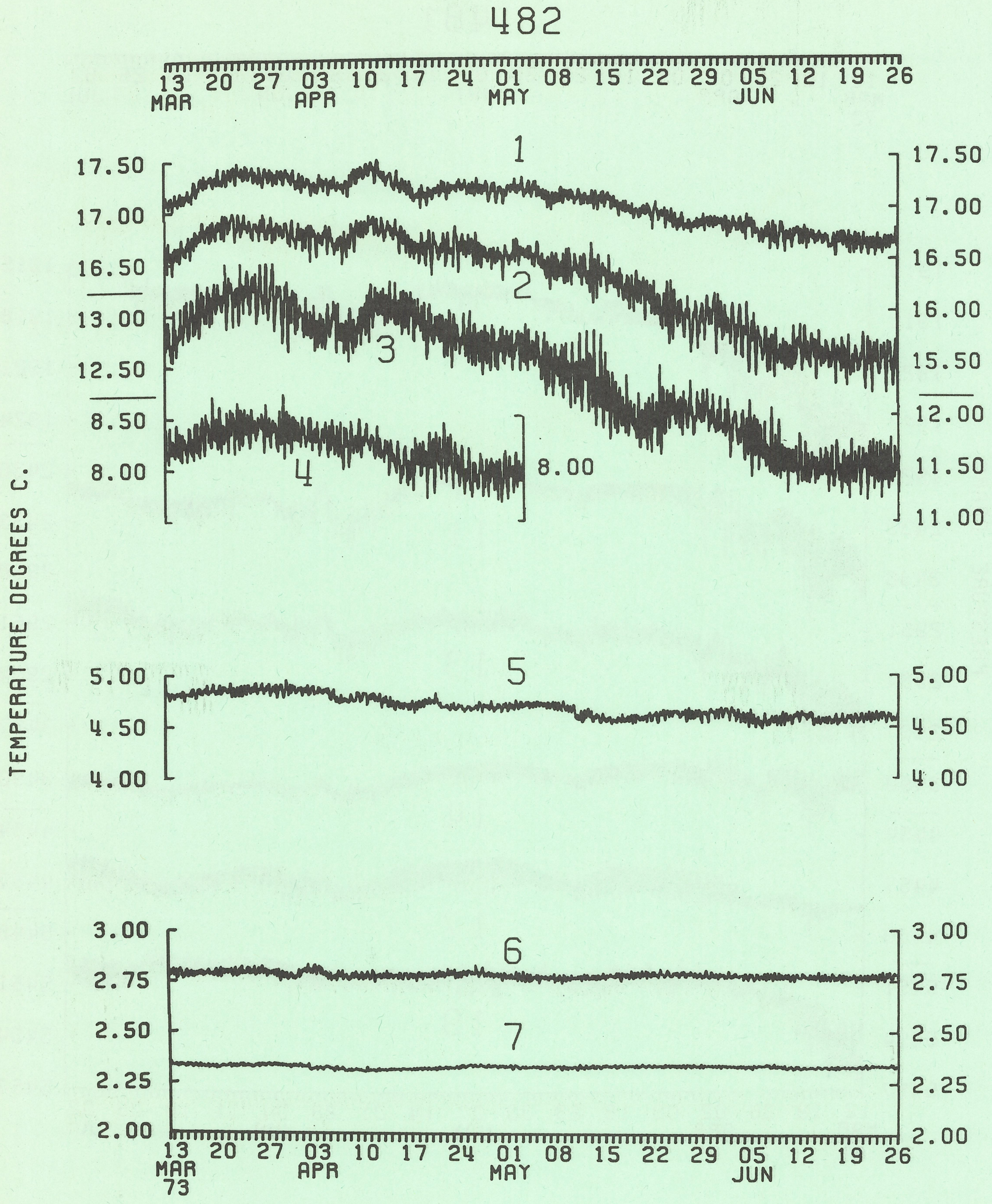




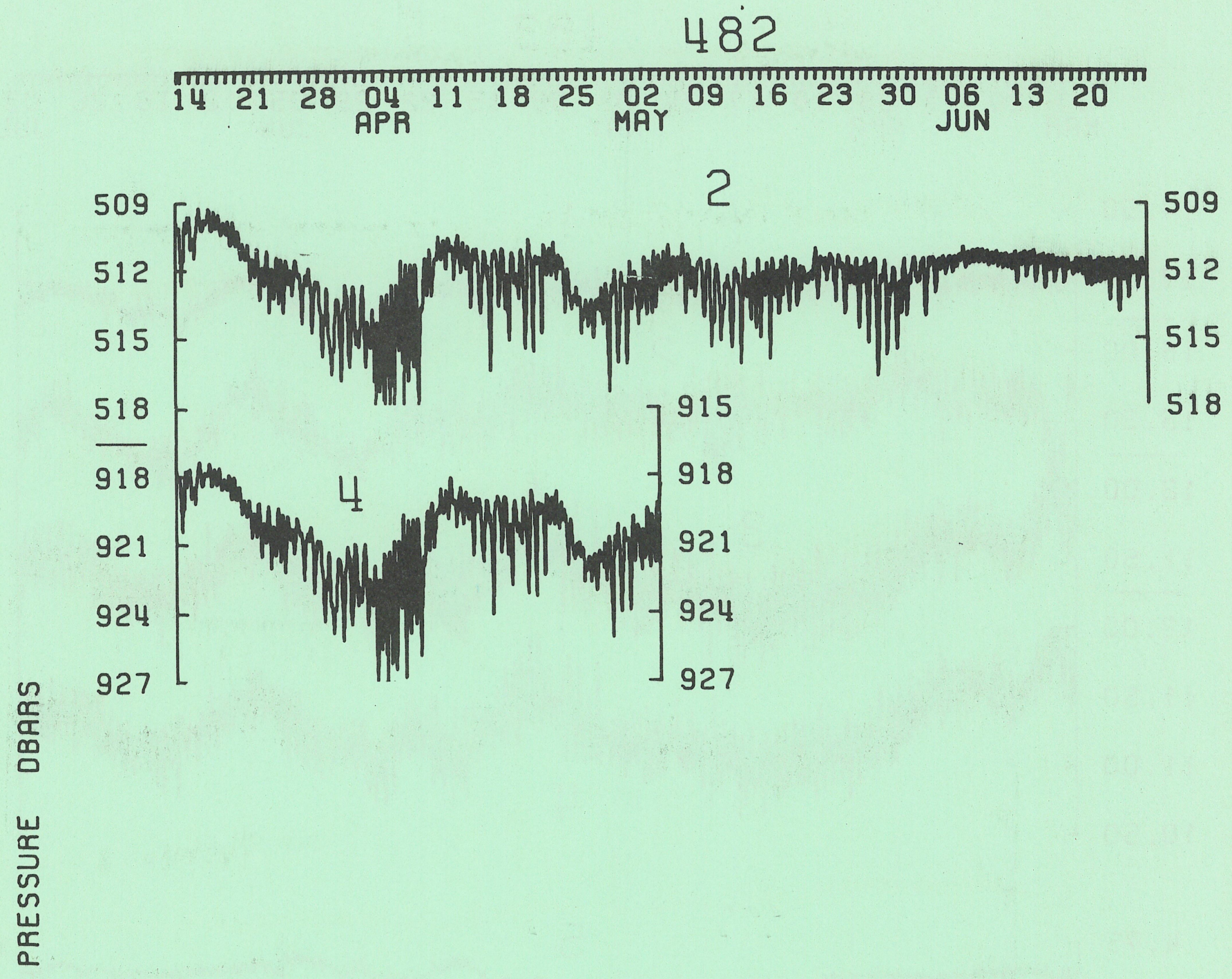

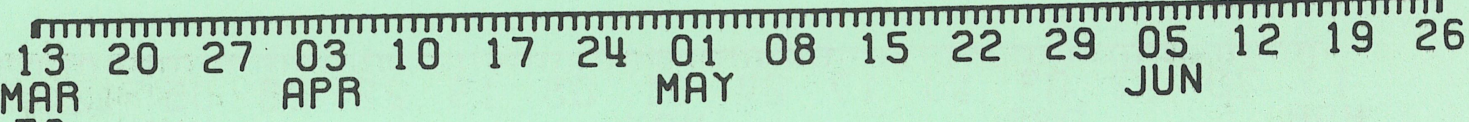
73 


\section{3}

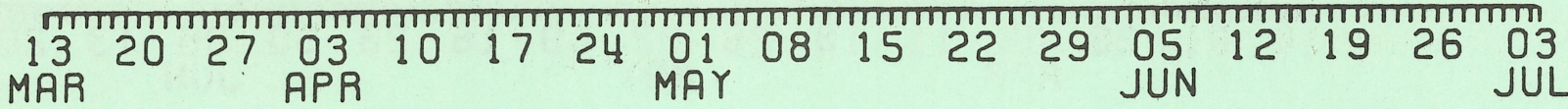

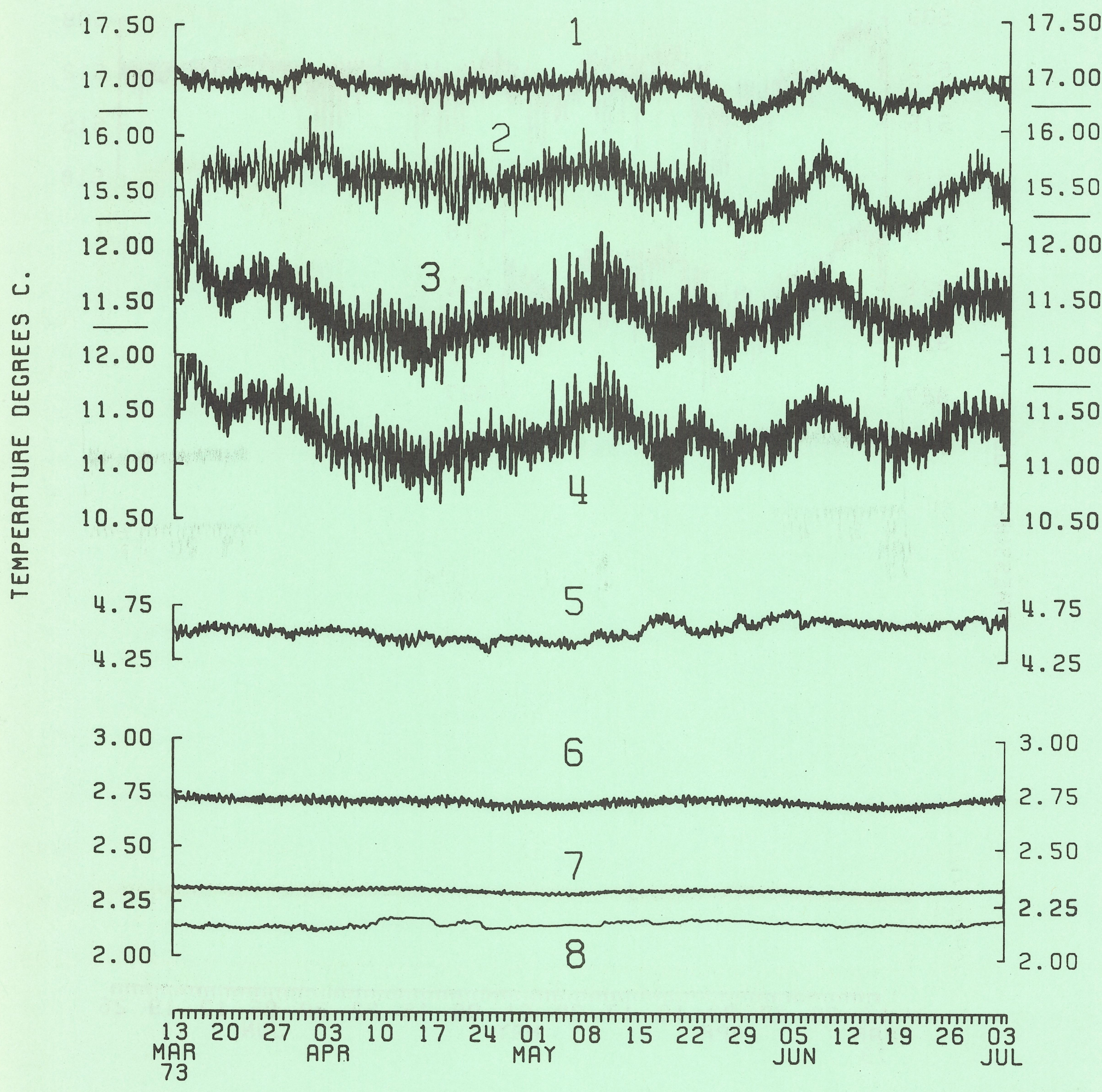




\section{3}

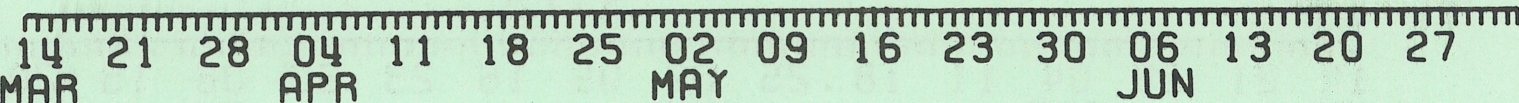
MAR

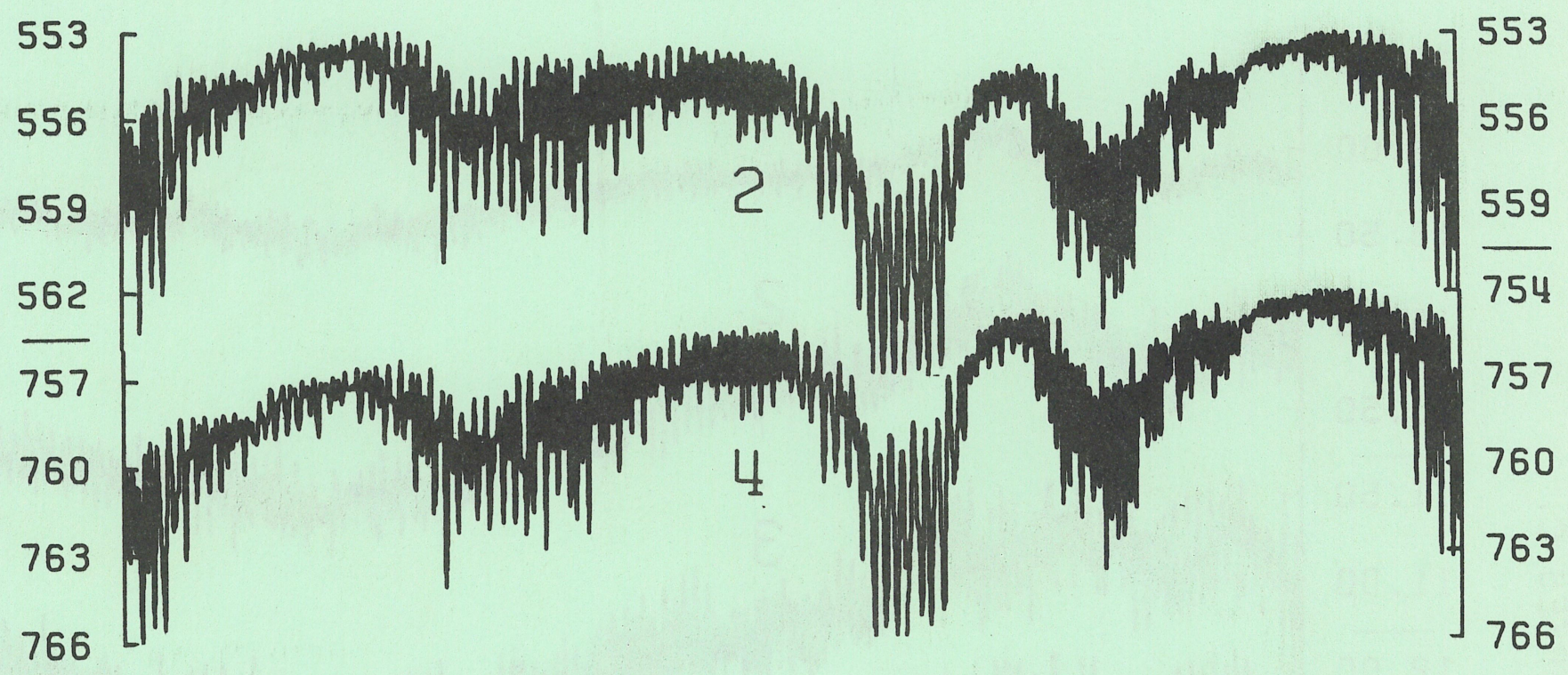

$n$
$\dddot{a}$
$a$
0
0

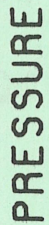

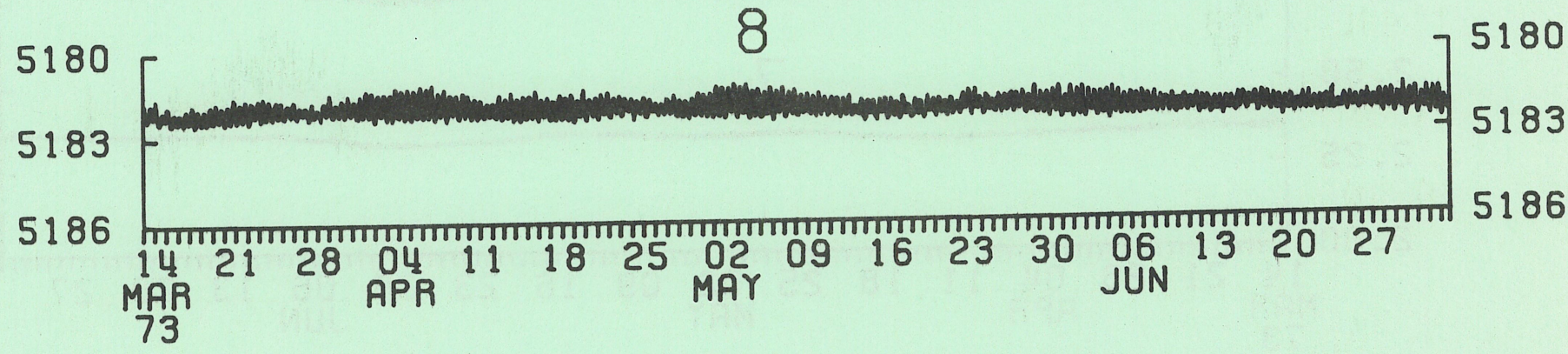




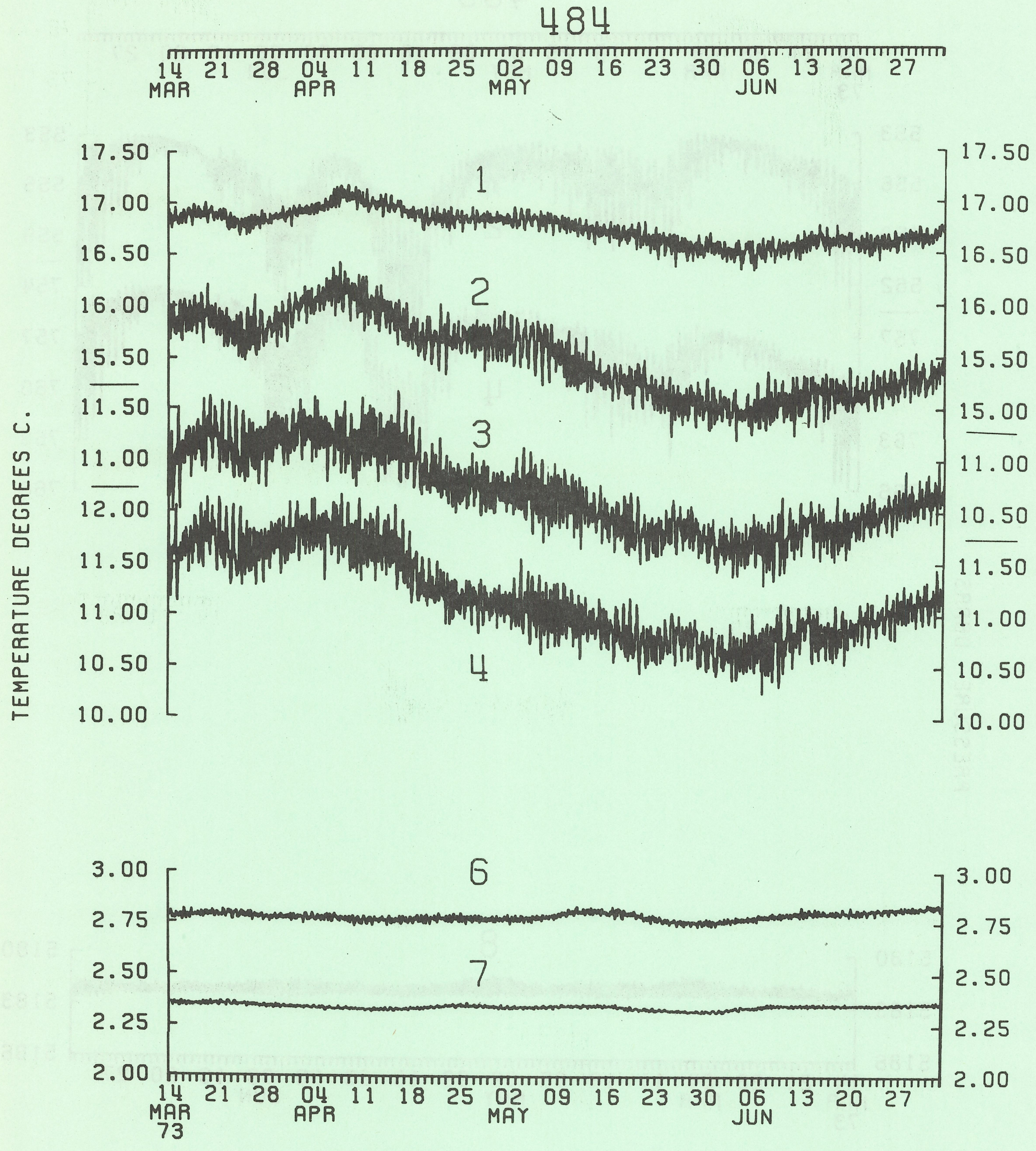




\section{4}

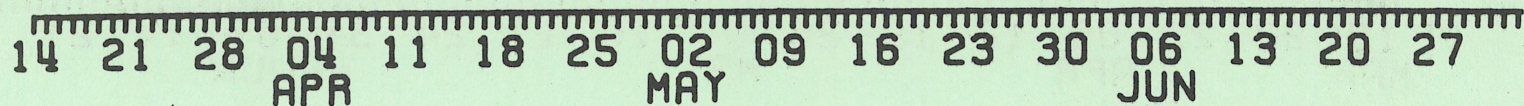

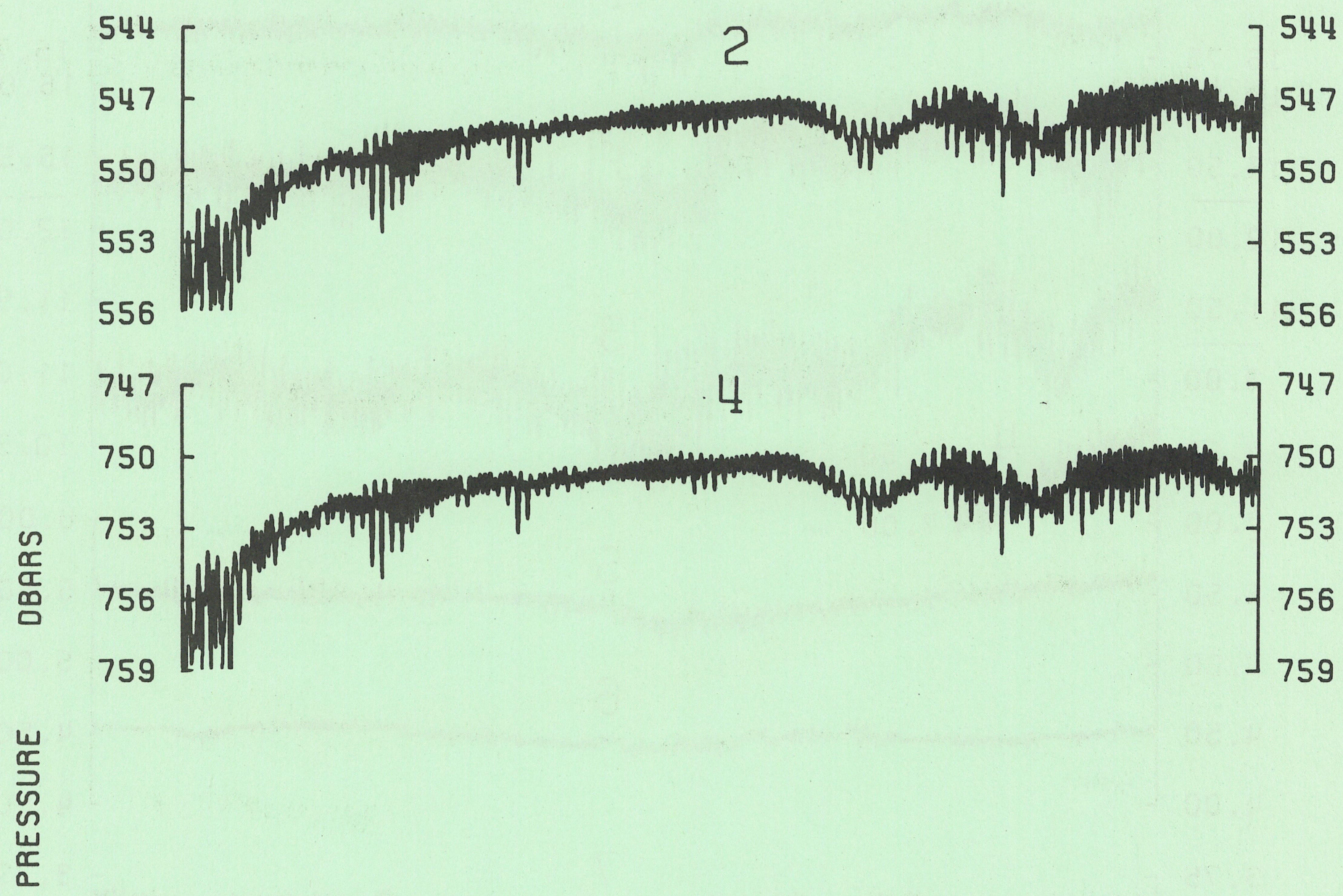

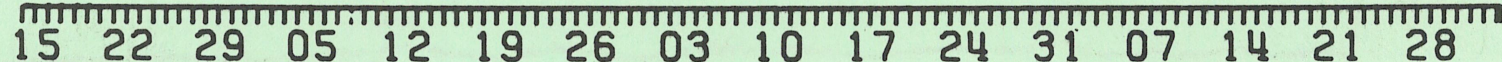
MAR APR MAY JUN 


\section{5}

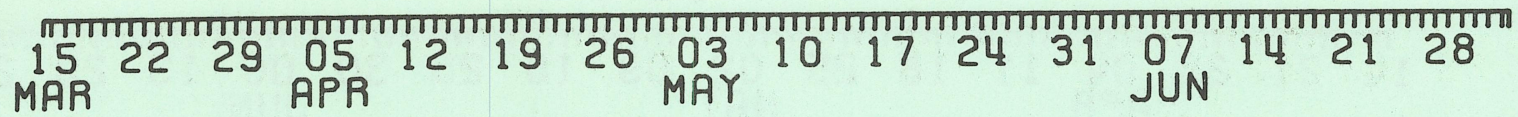

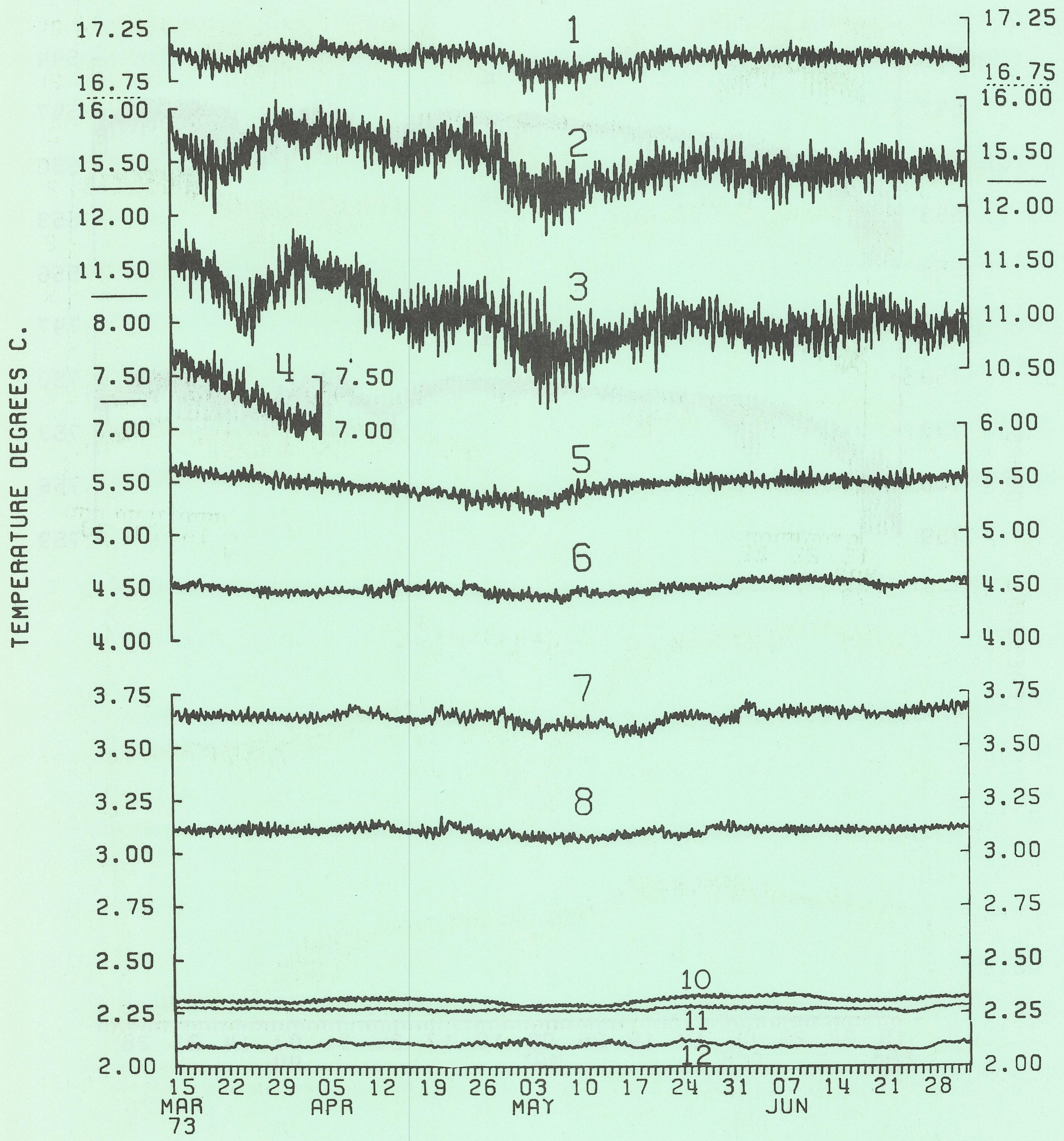




\section{5}

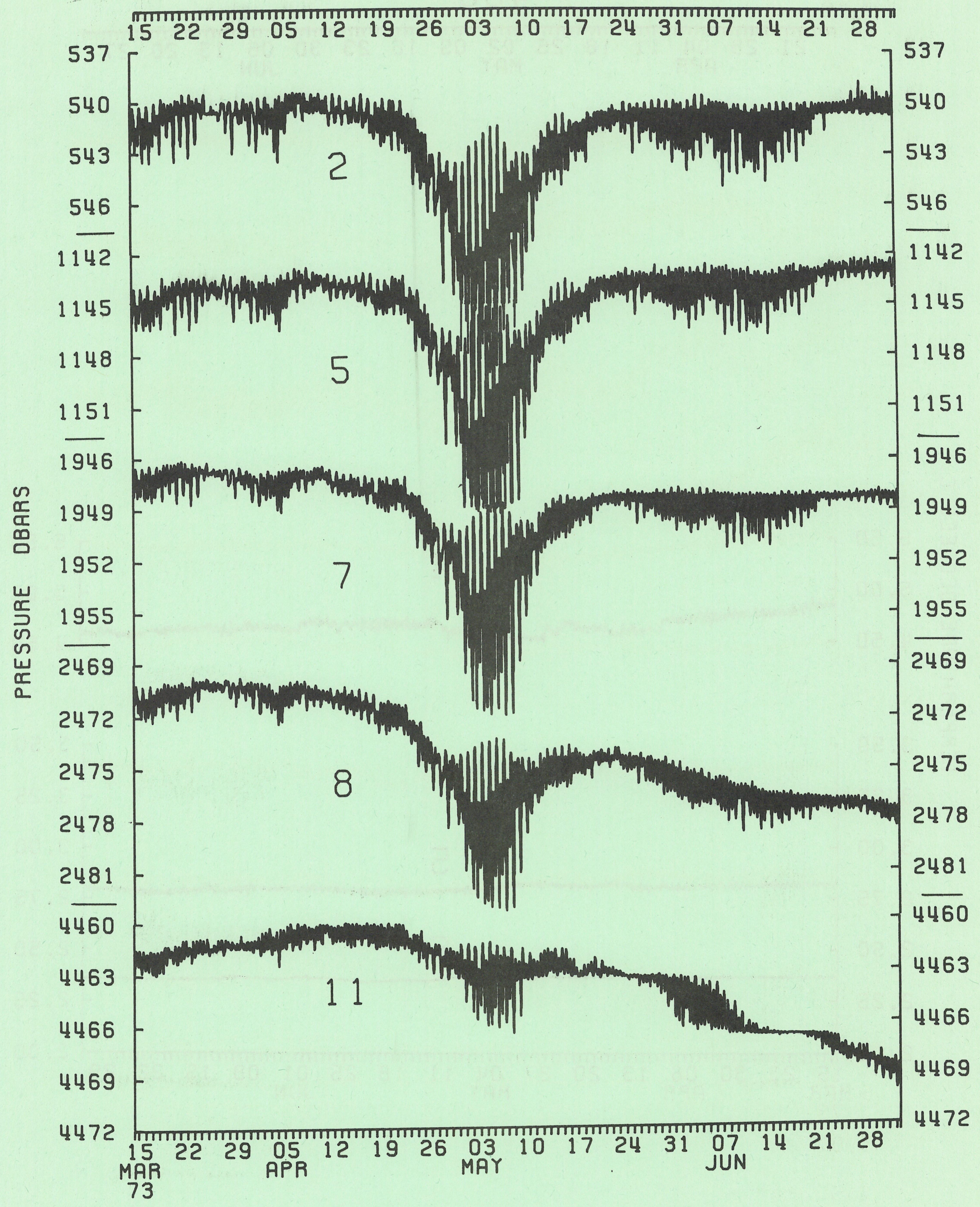




\section{6}

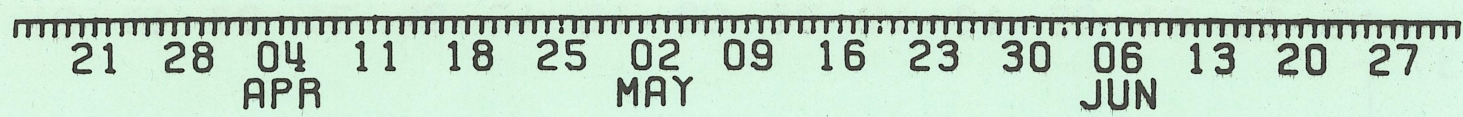

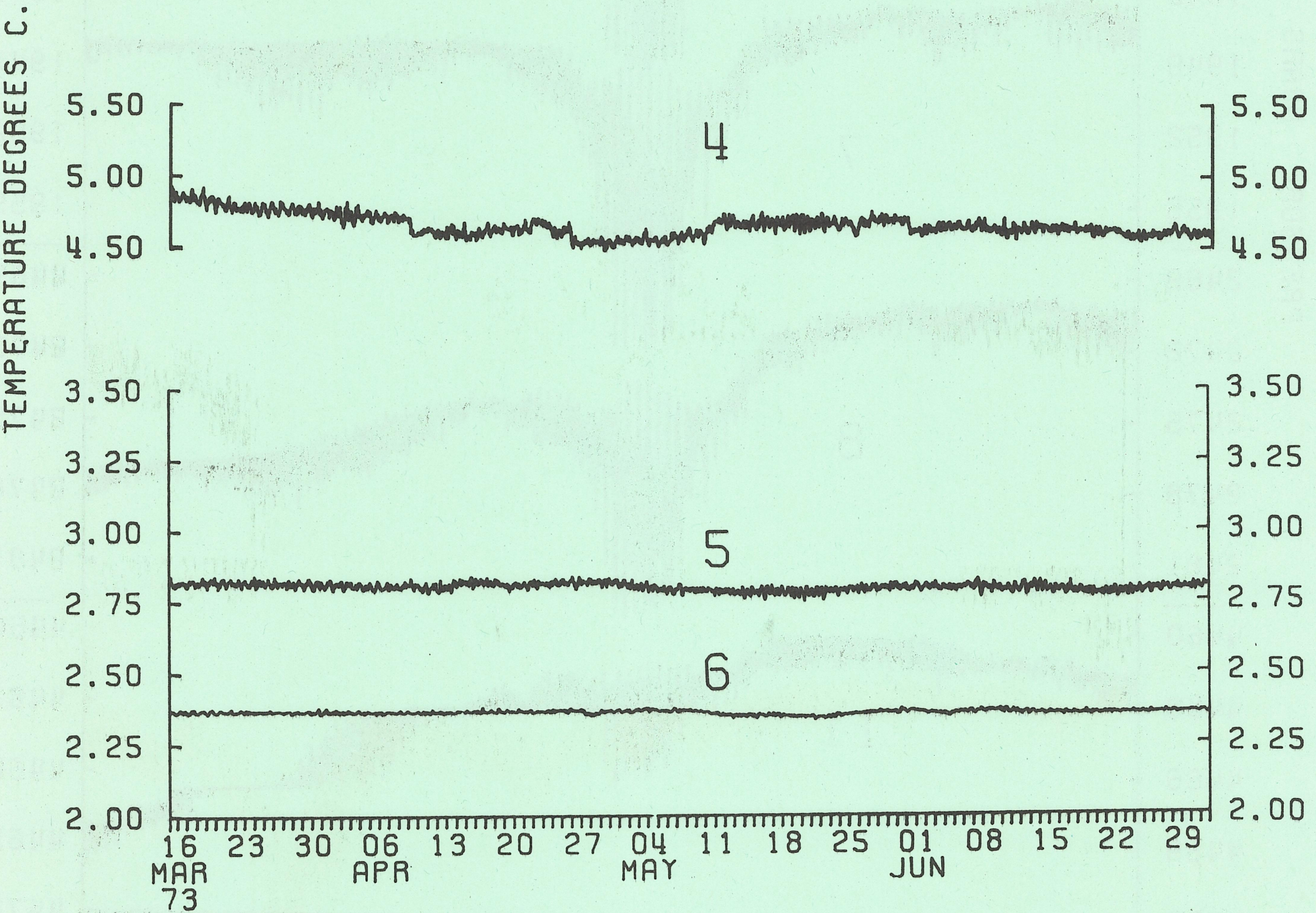




\section{6}

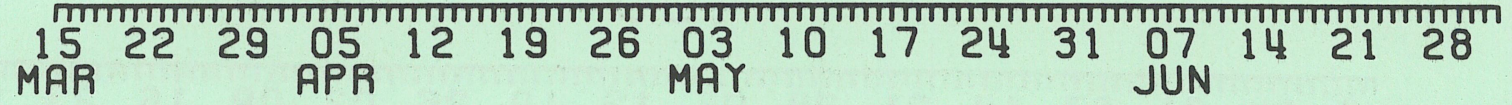

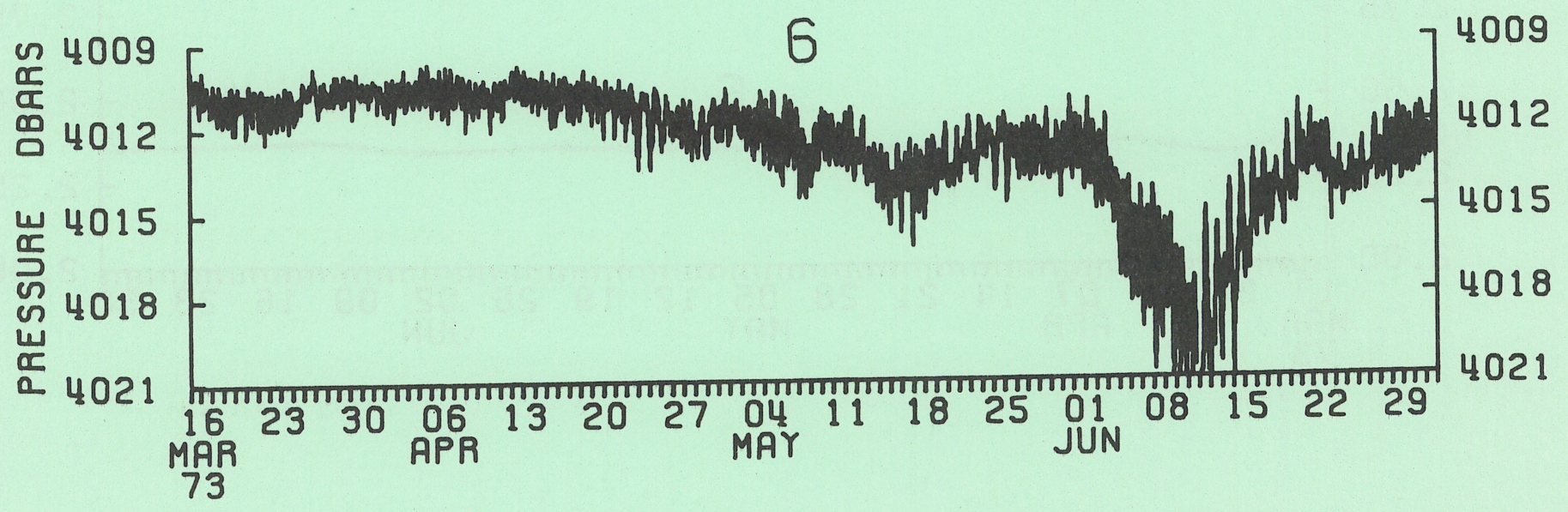




\section{8}

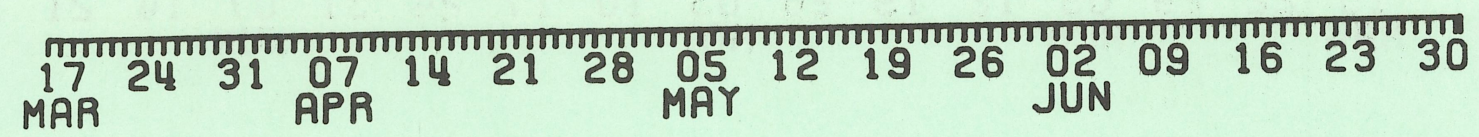

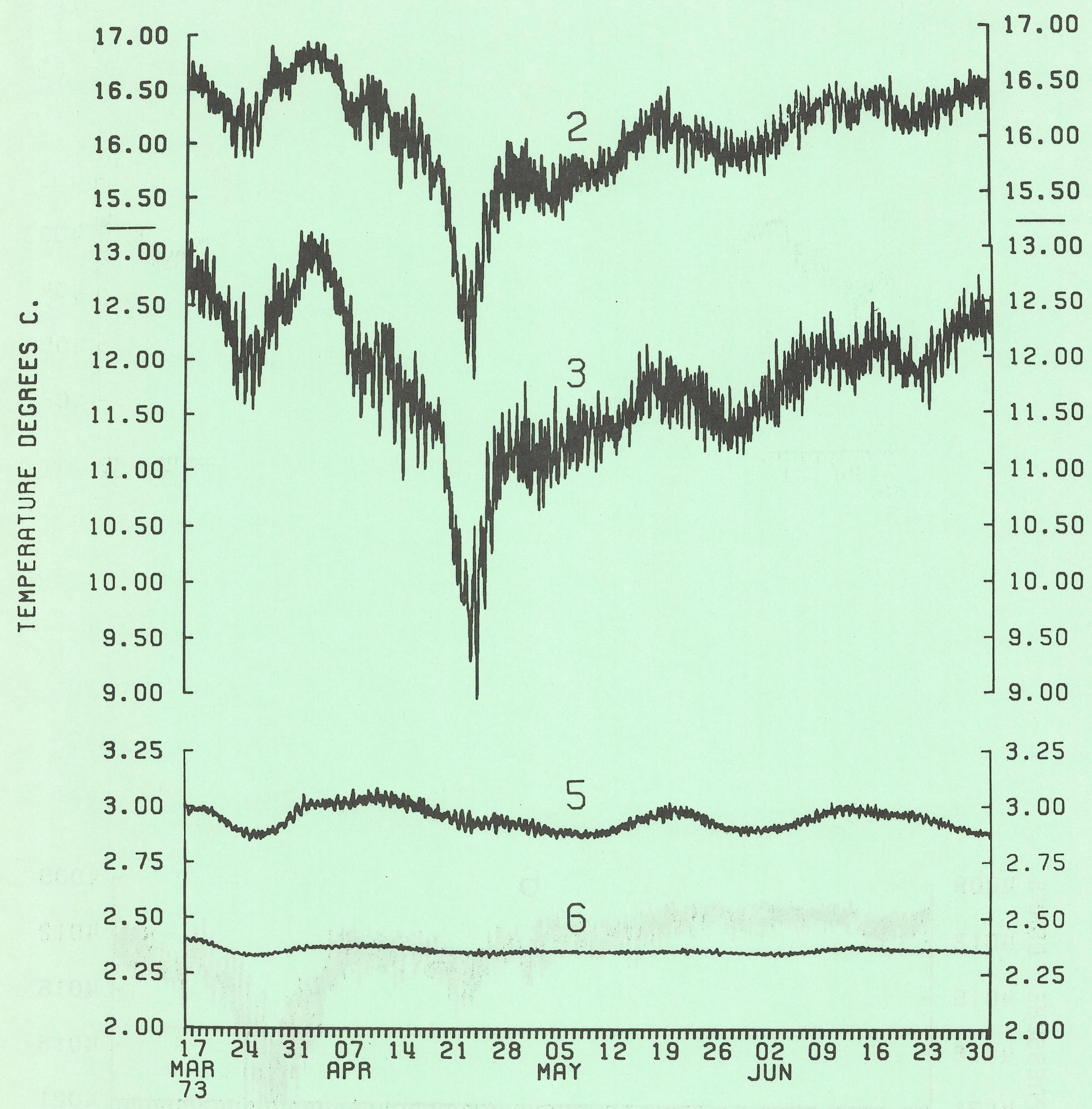




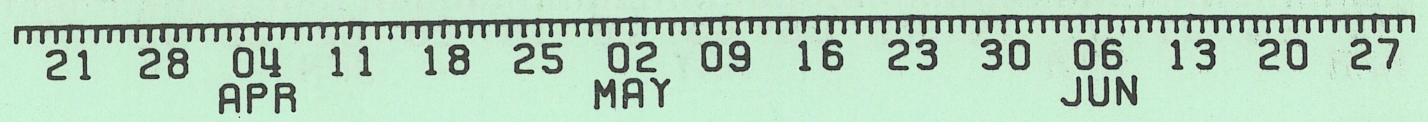
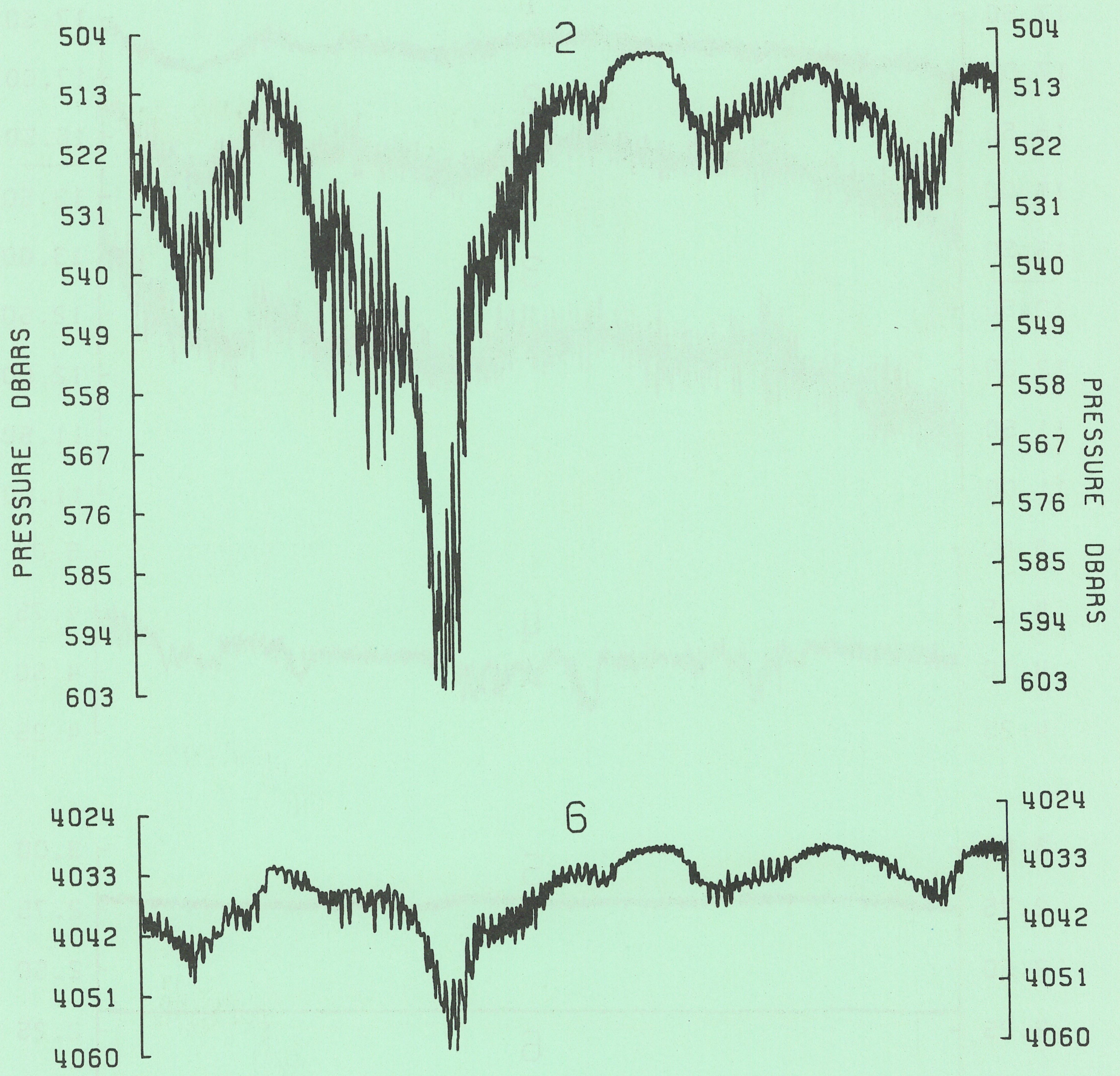

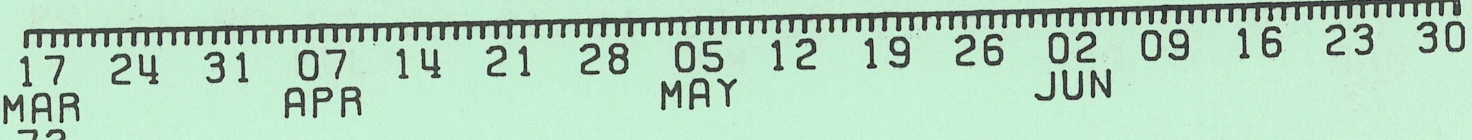

73 


\section{9}

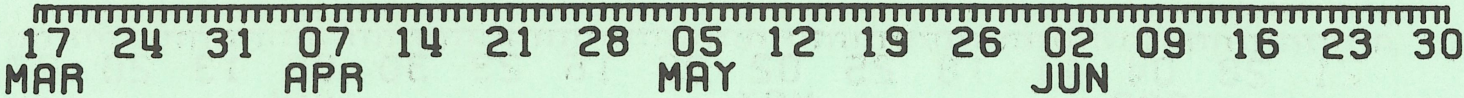
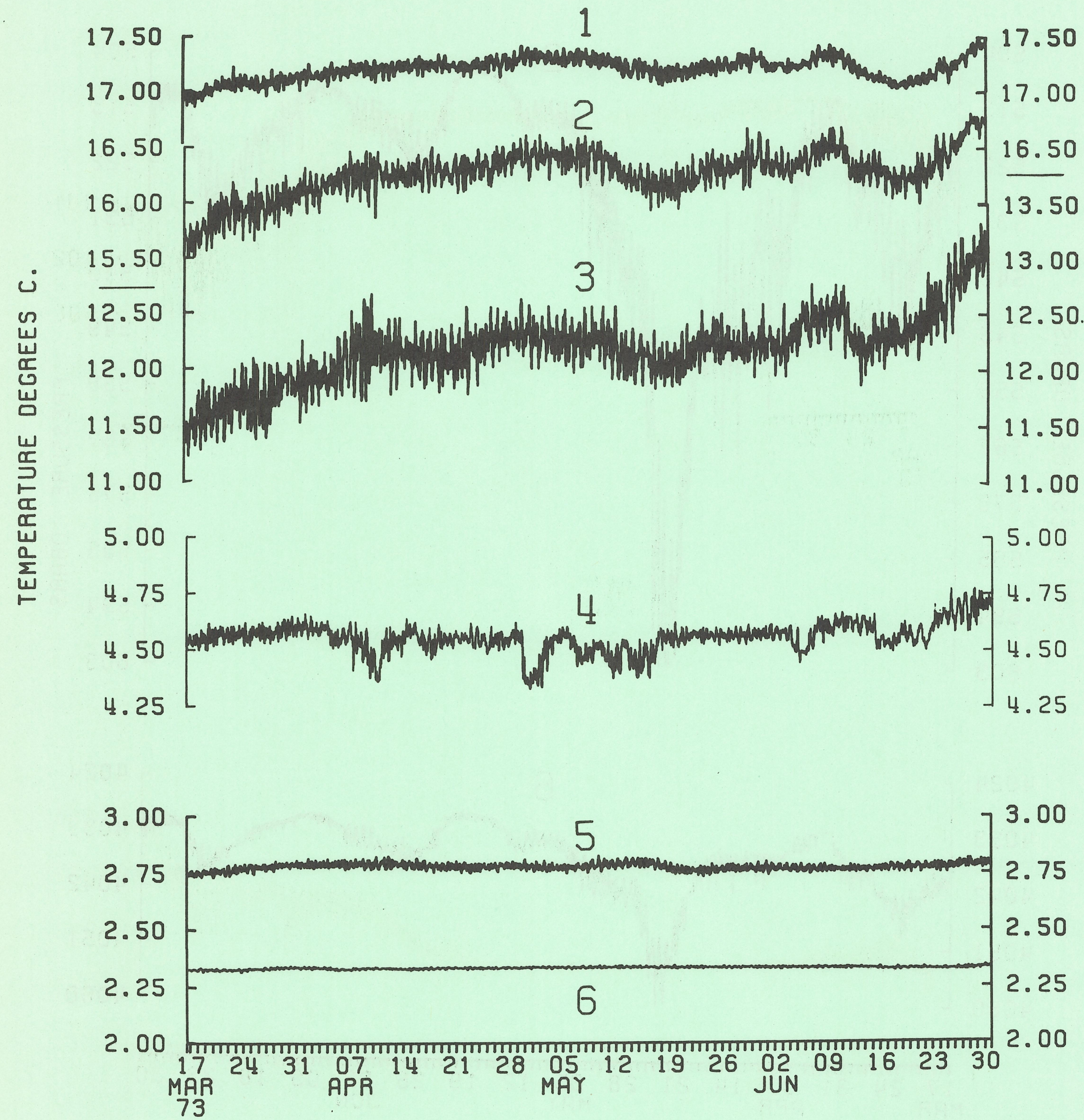


\section{9}

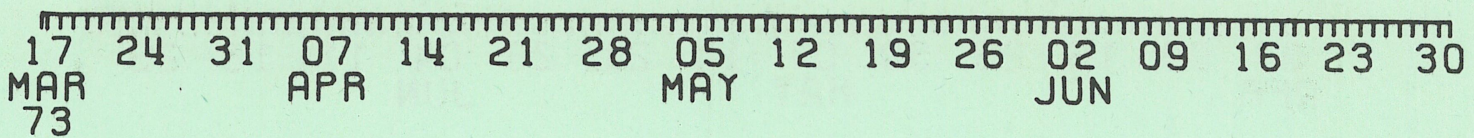

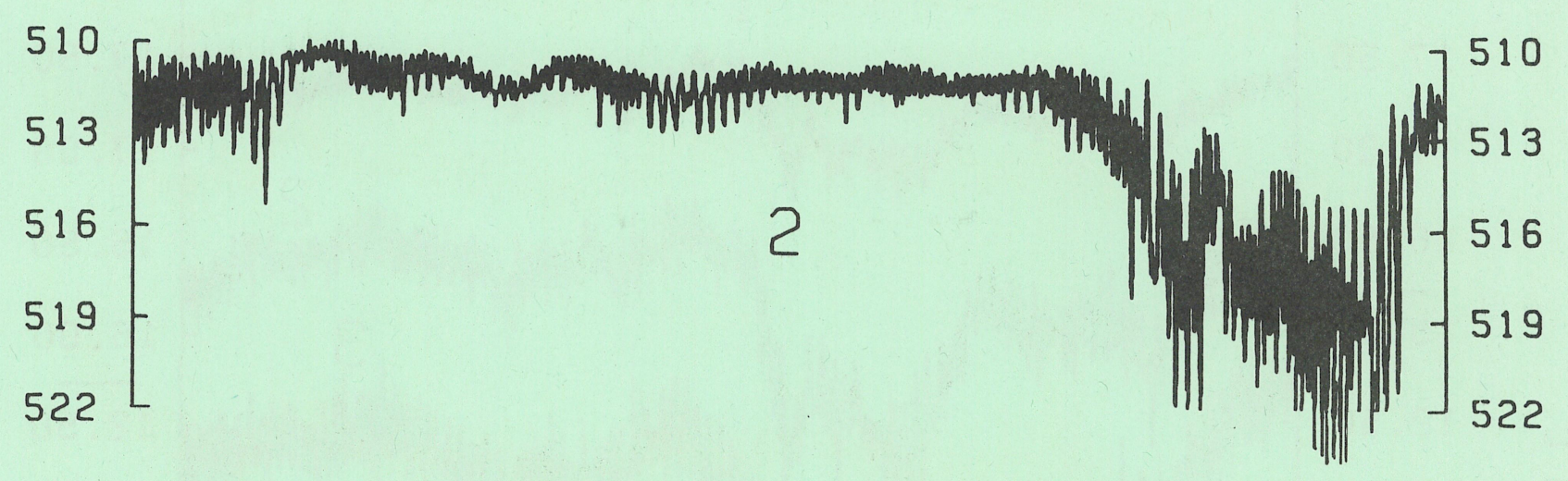

$n$
$\frac{a}{a}$
$\infty$
0

$w$
व
ज
ज
$w$
0
0

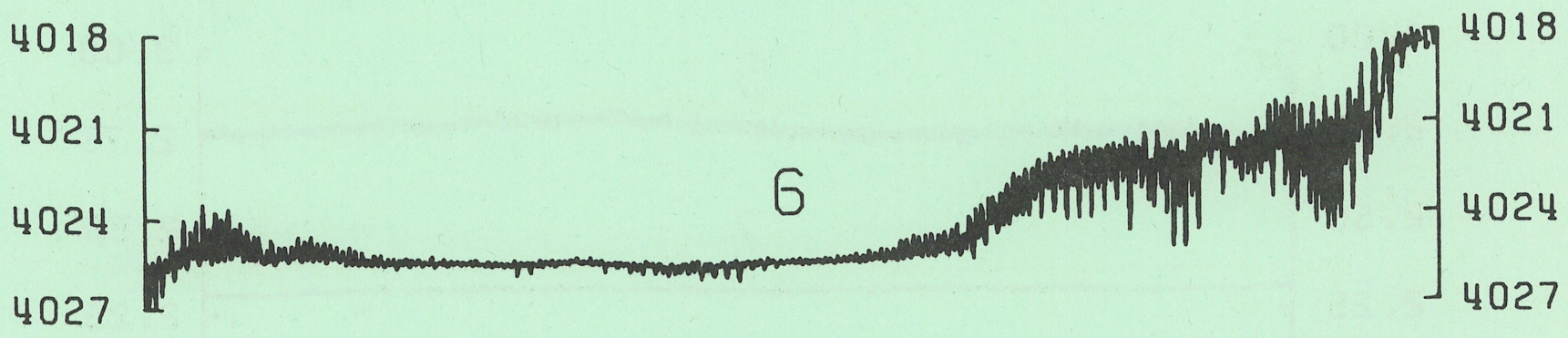

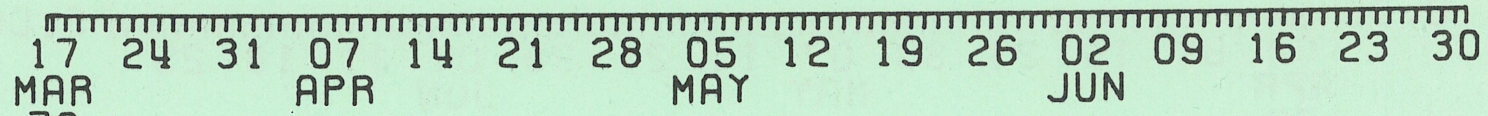
73 


\section{3}

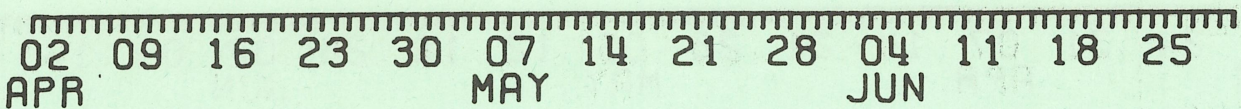

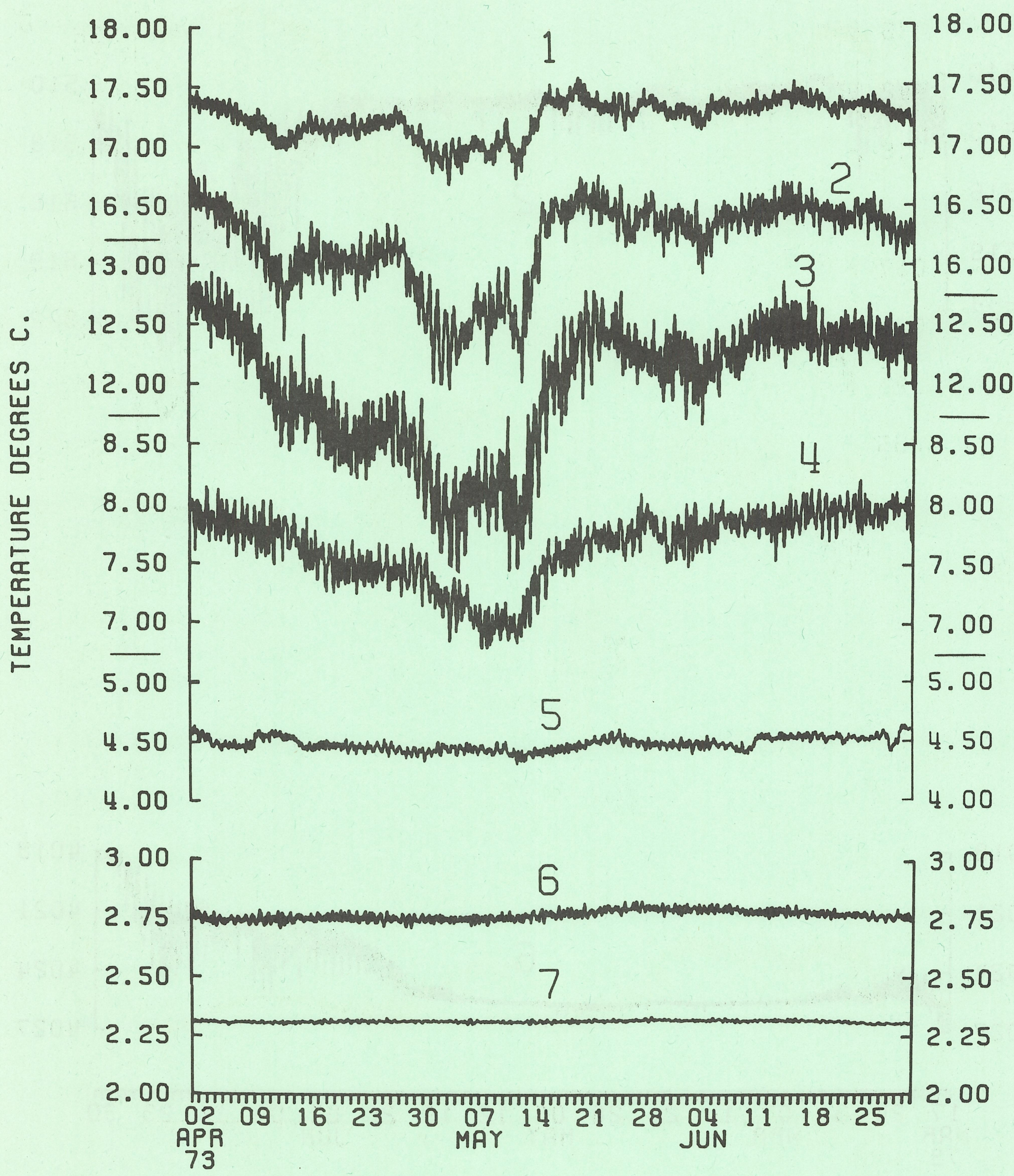




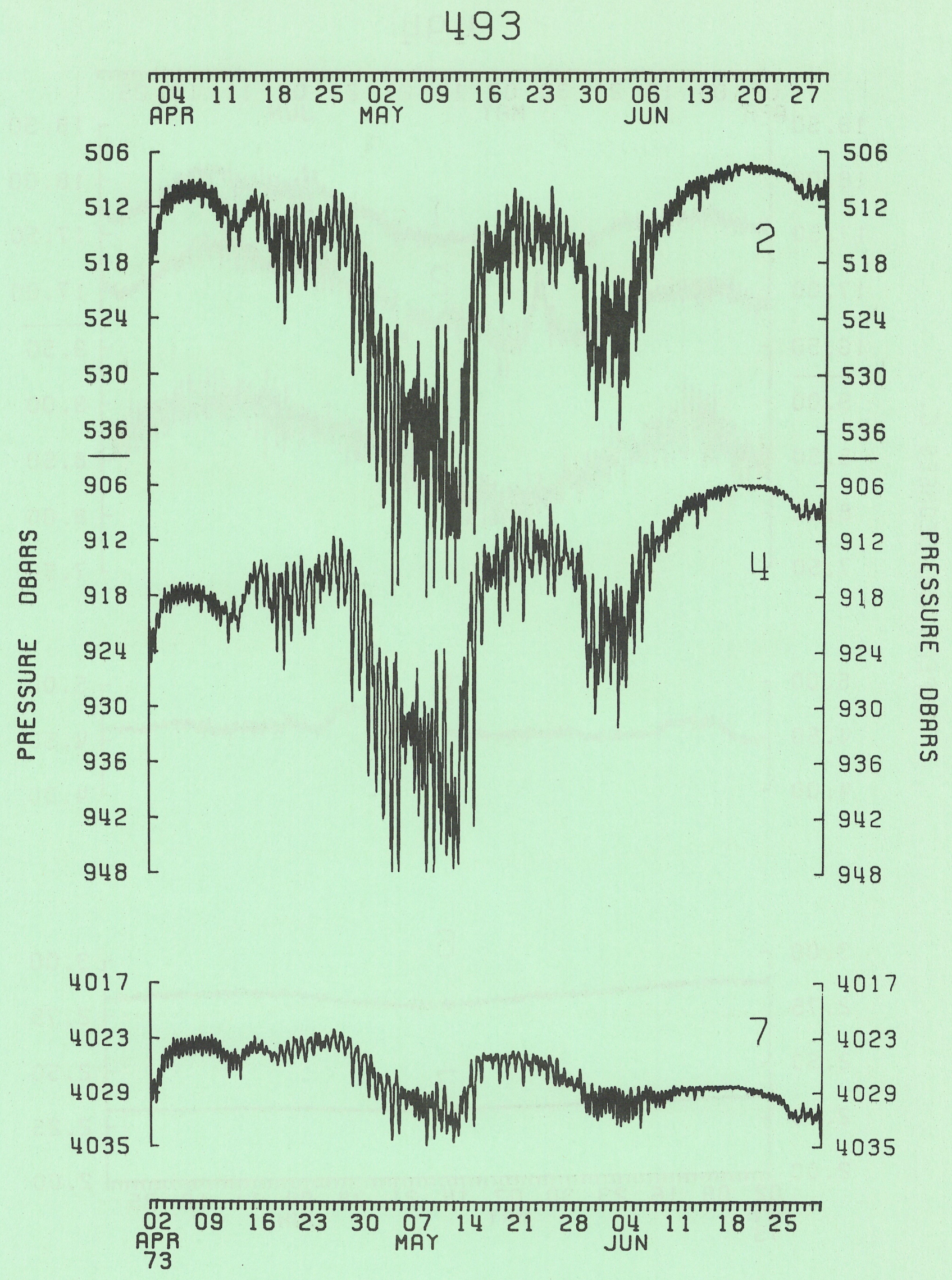



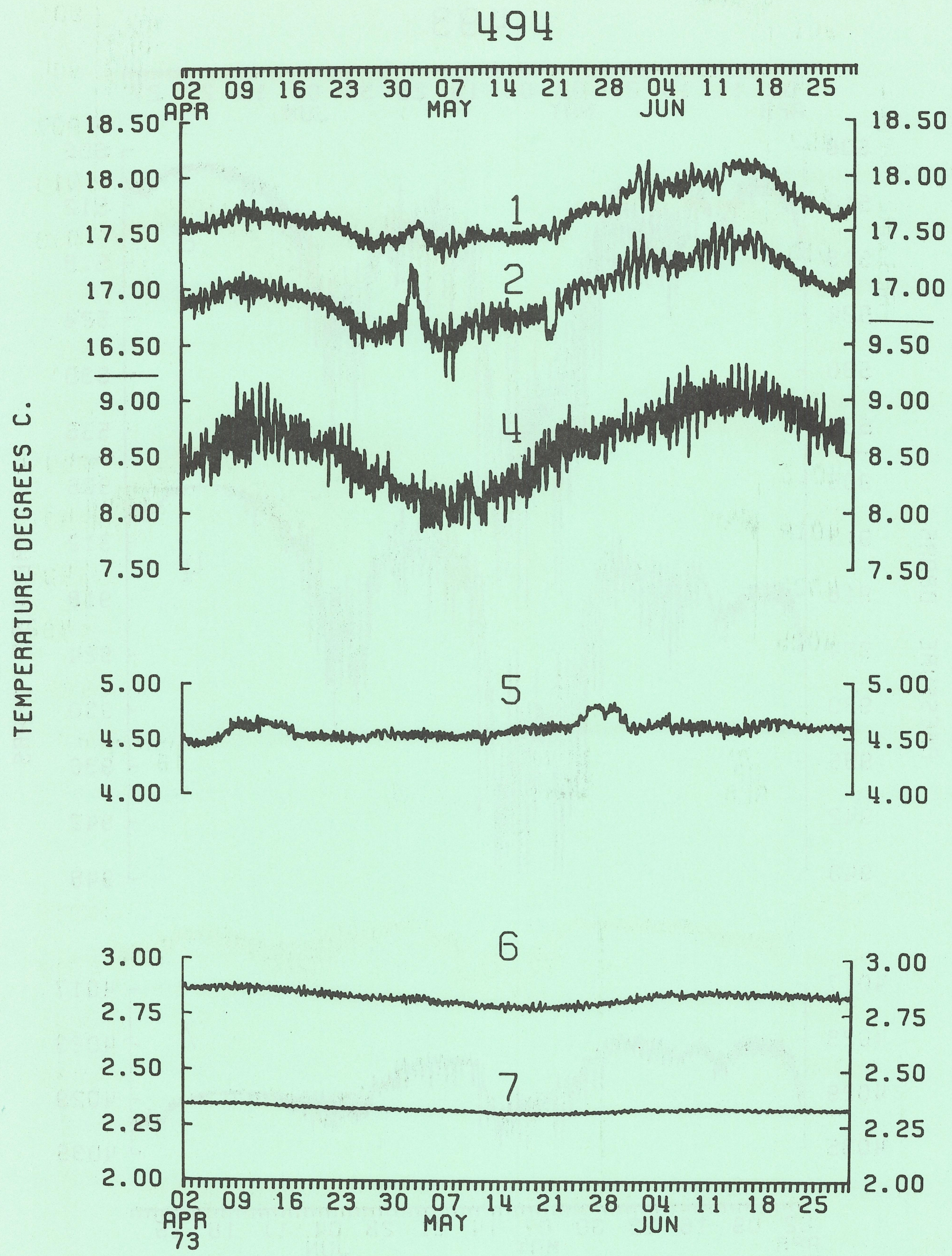


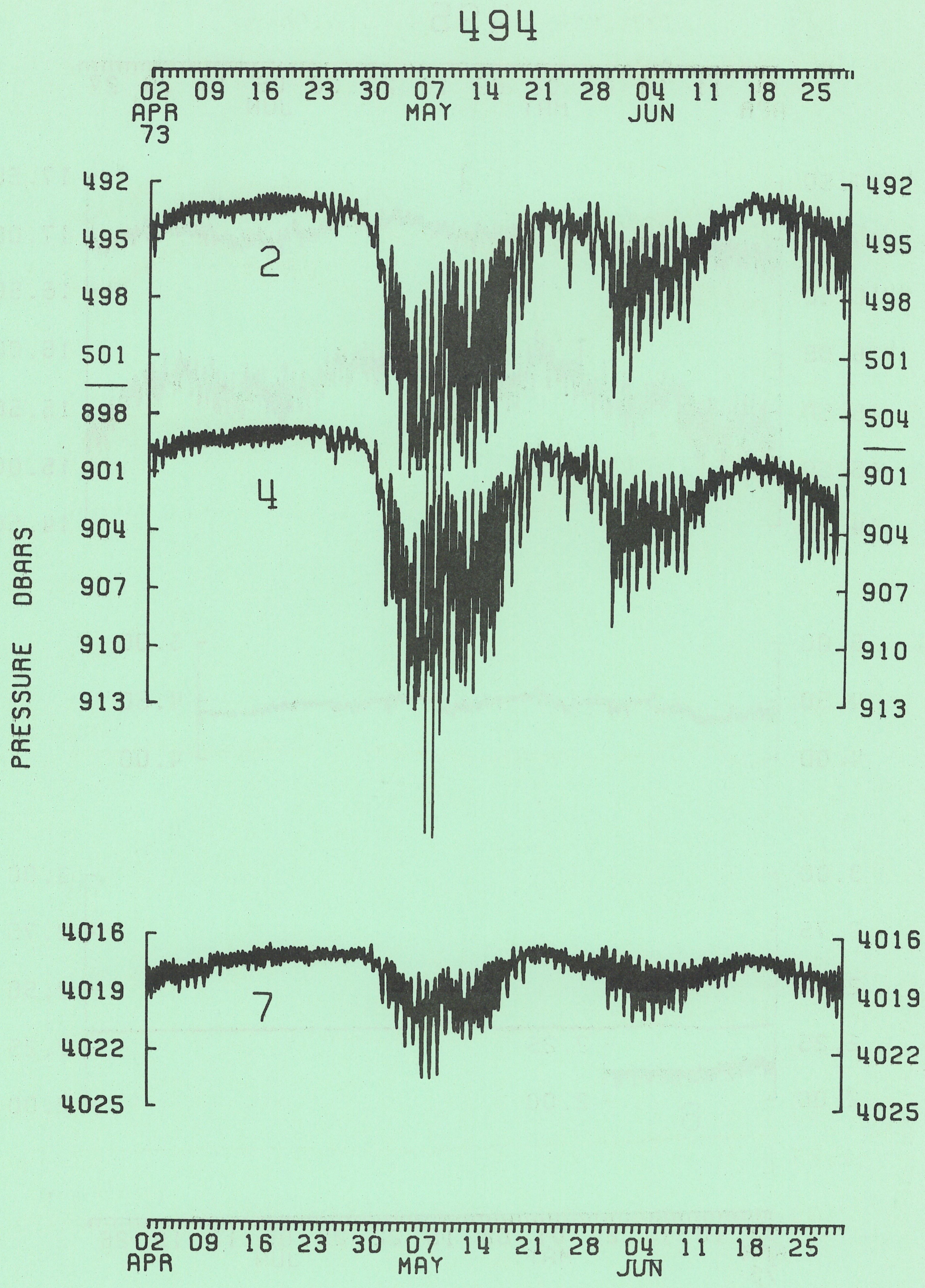




\section{5}

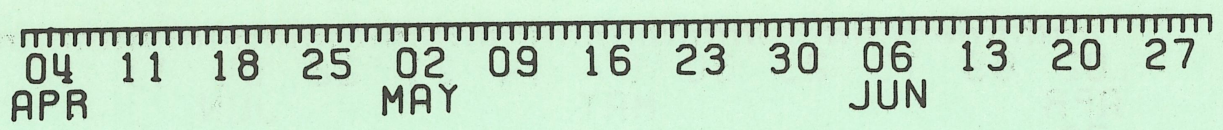
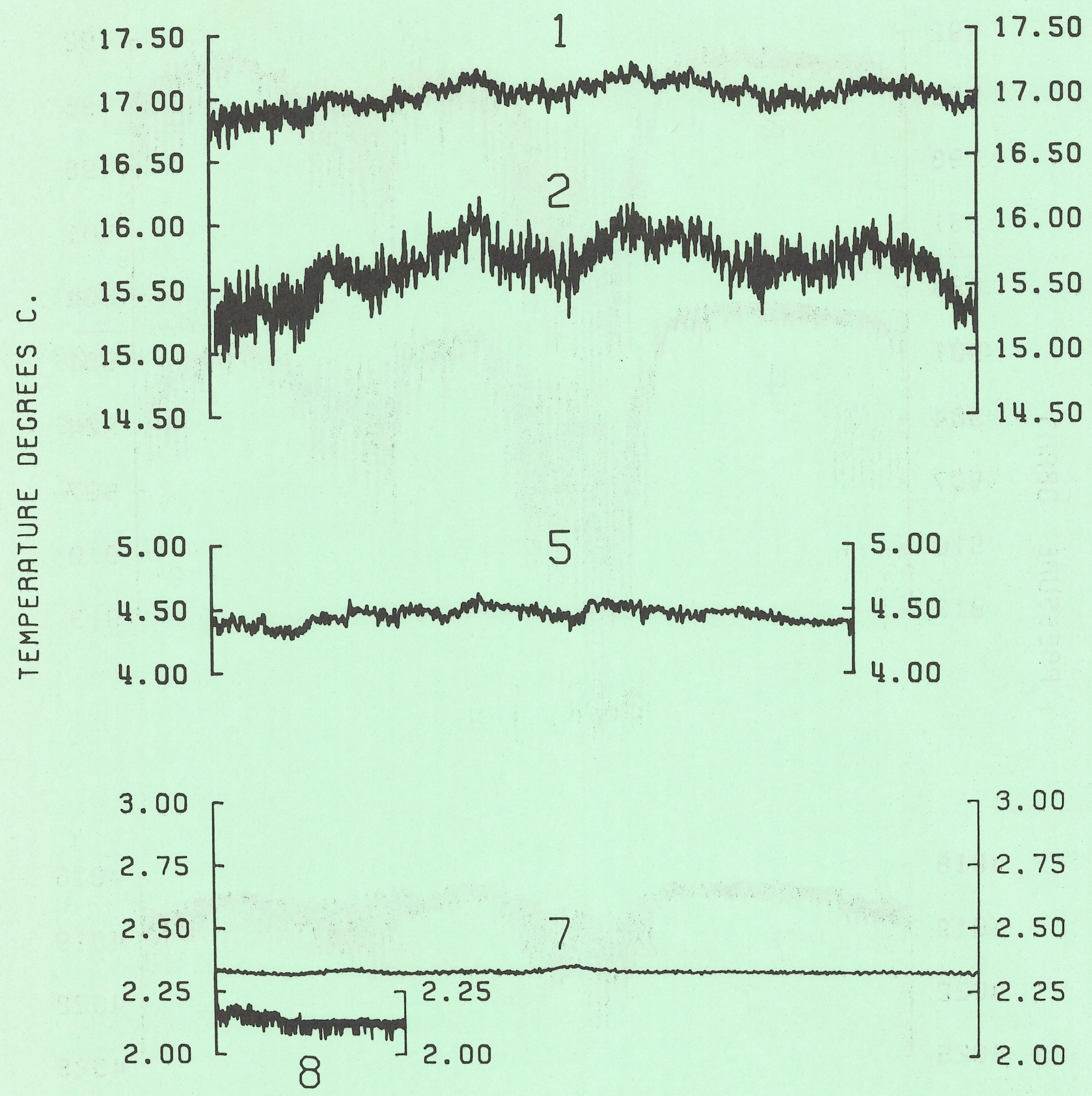

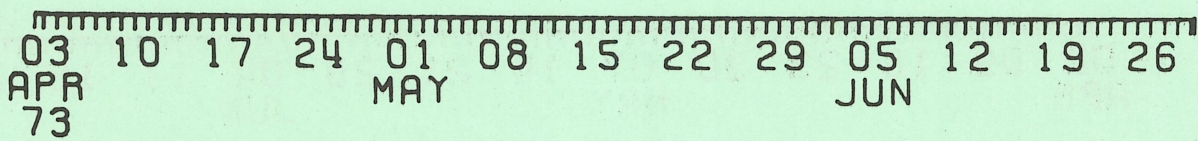




\section{5}

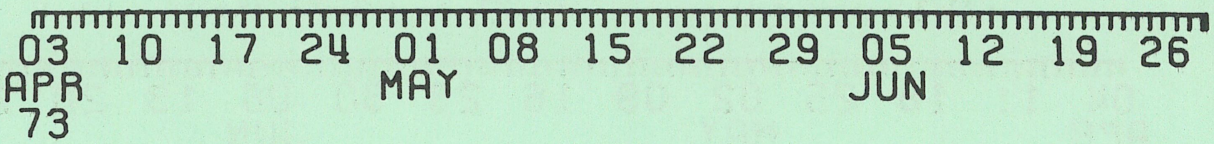

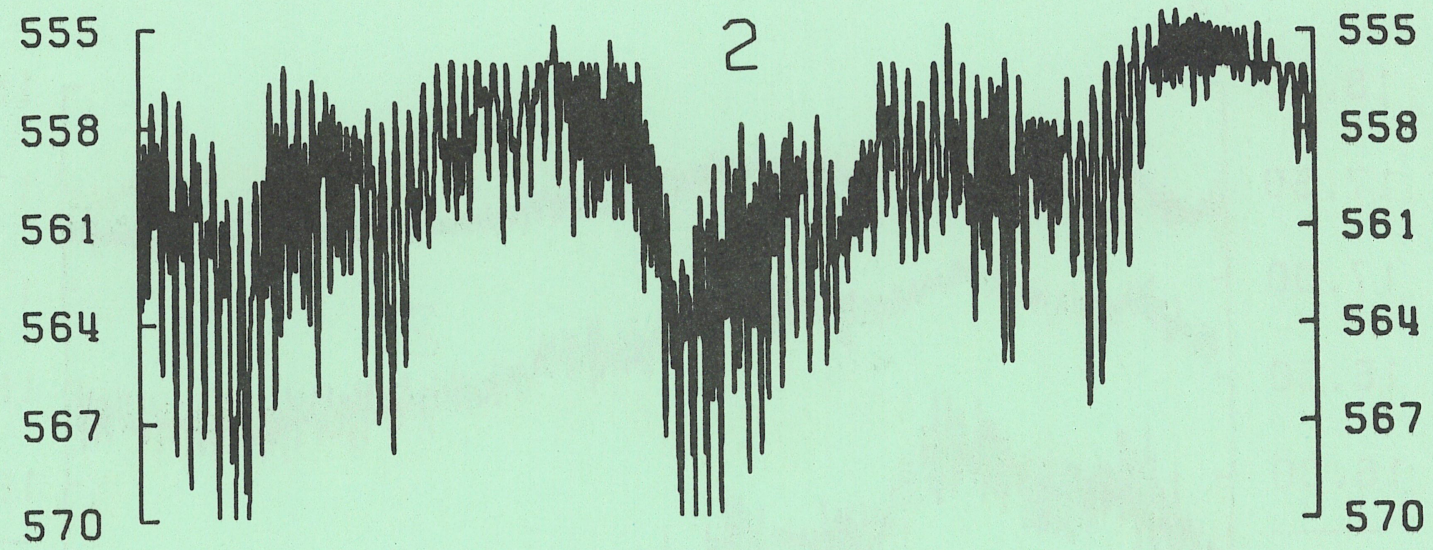

$n$
$\frac{1}{a}$
$\infty$
0

$\omega$
0
0
0
$\omega$
$\frac{1}{a}$
0

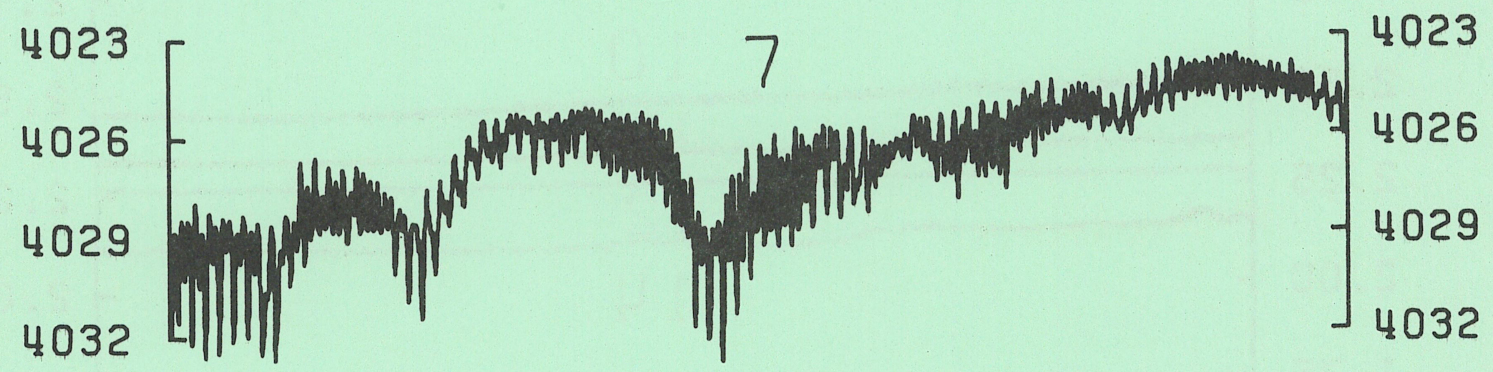

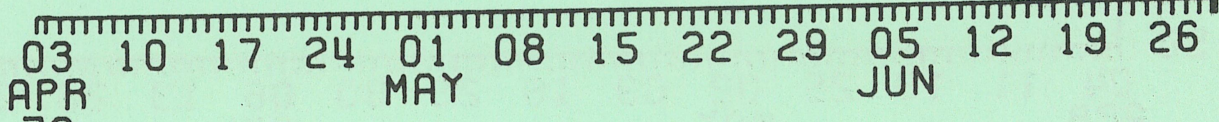
73 


\section{7}

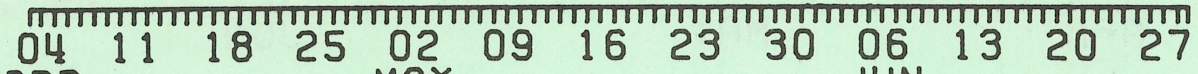
APR

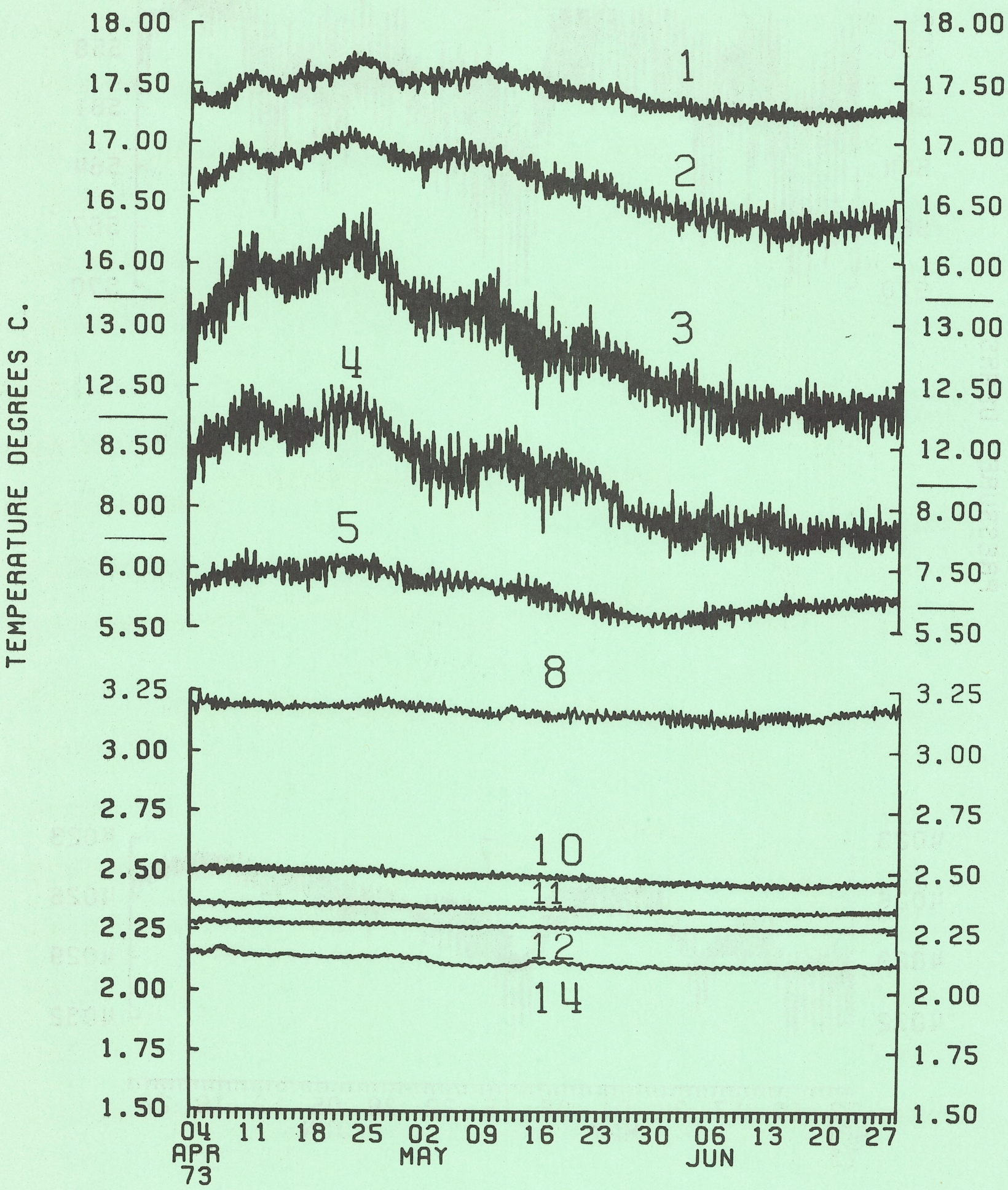




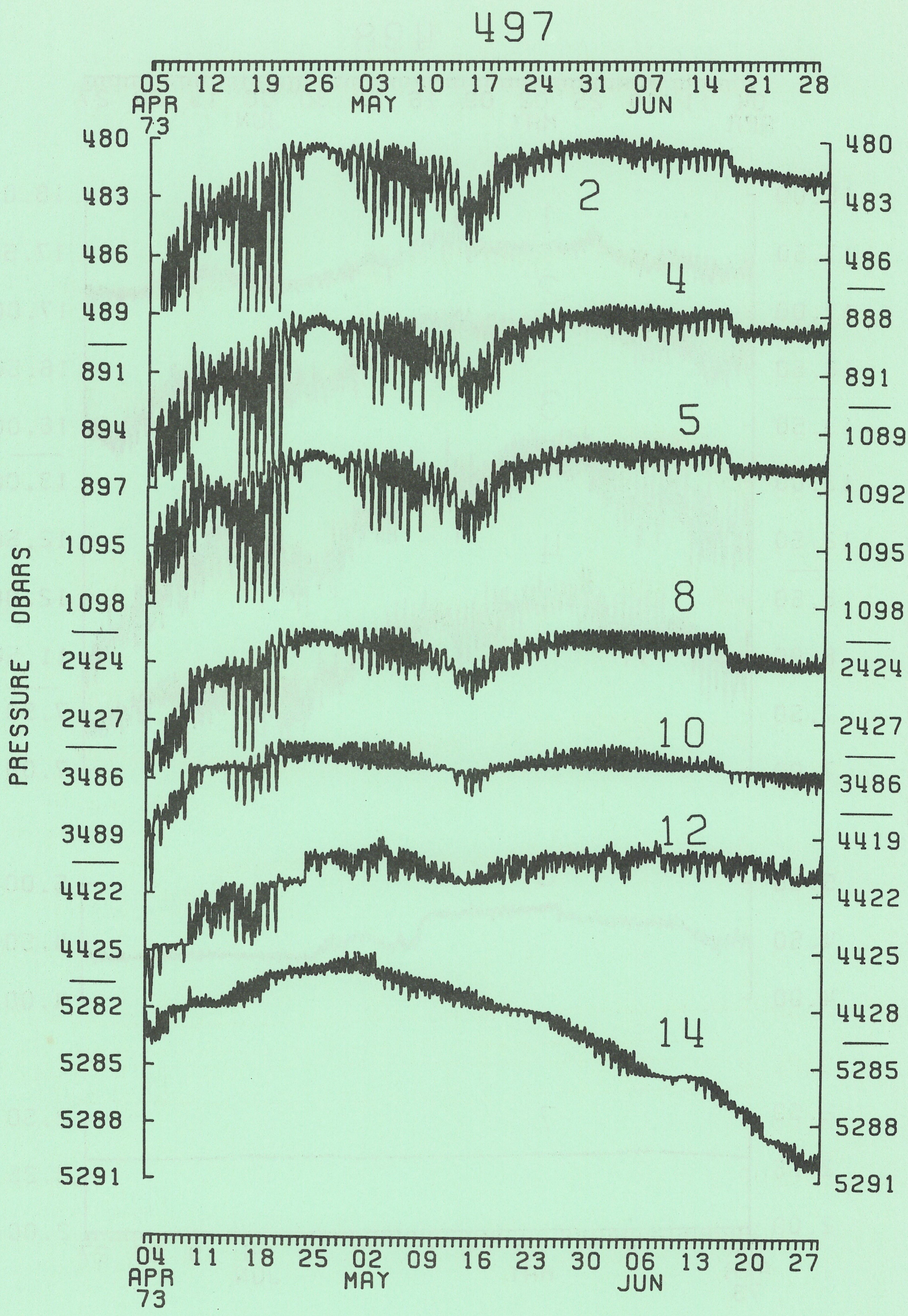




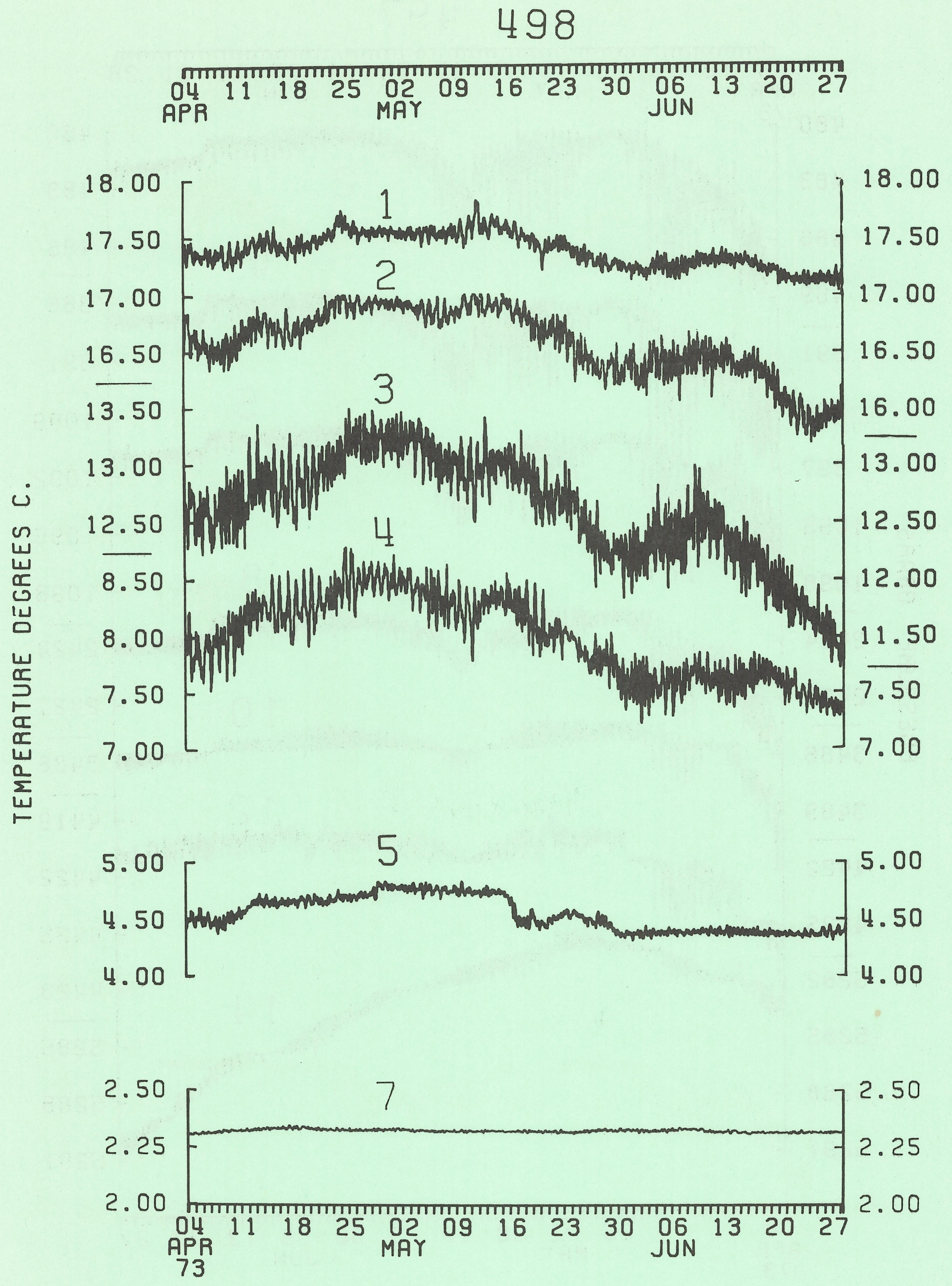




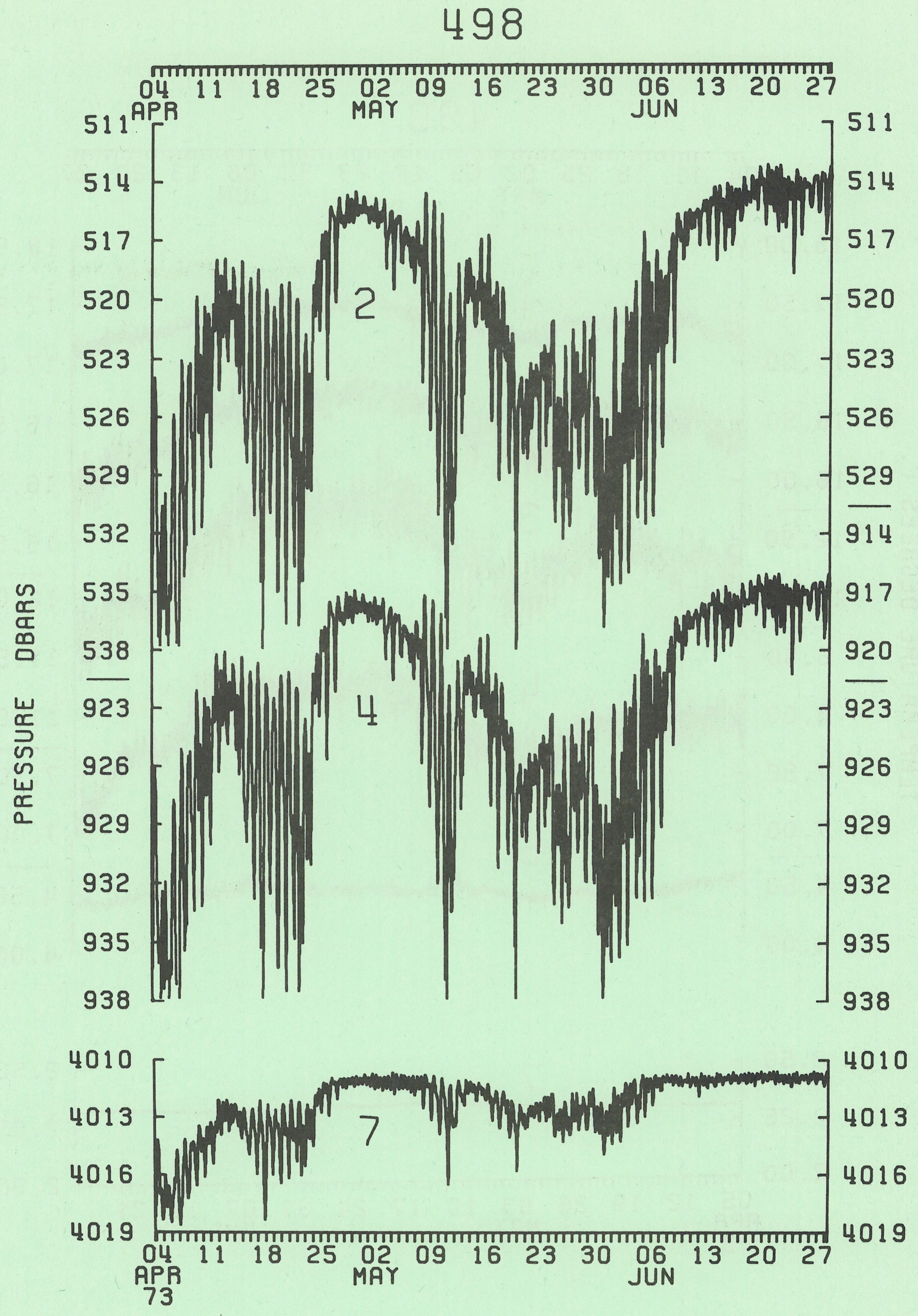




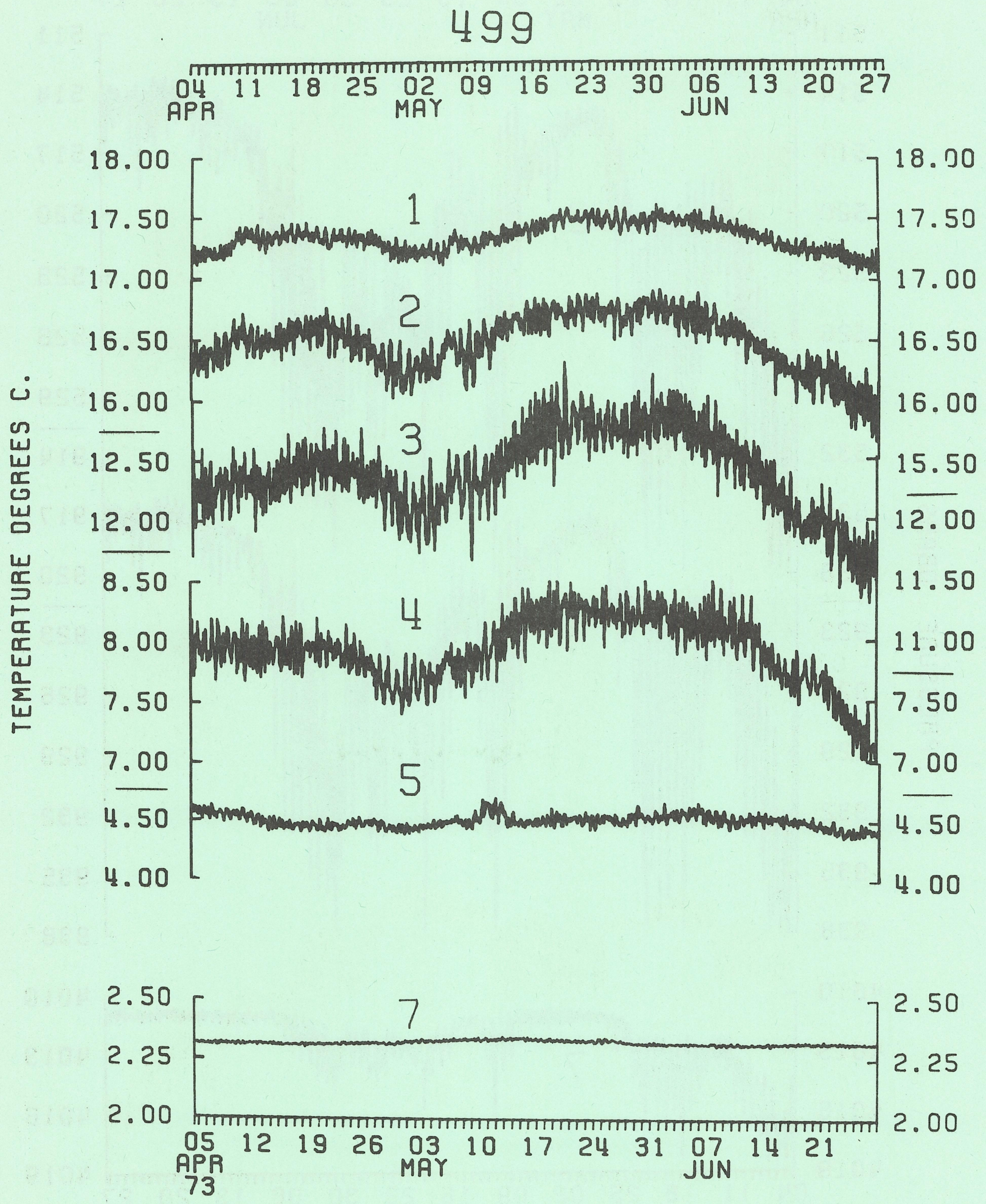




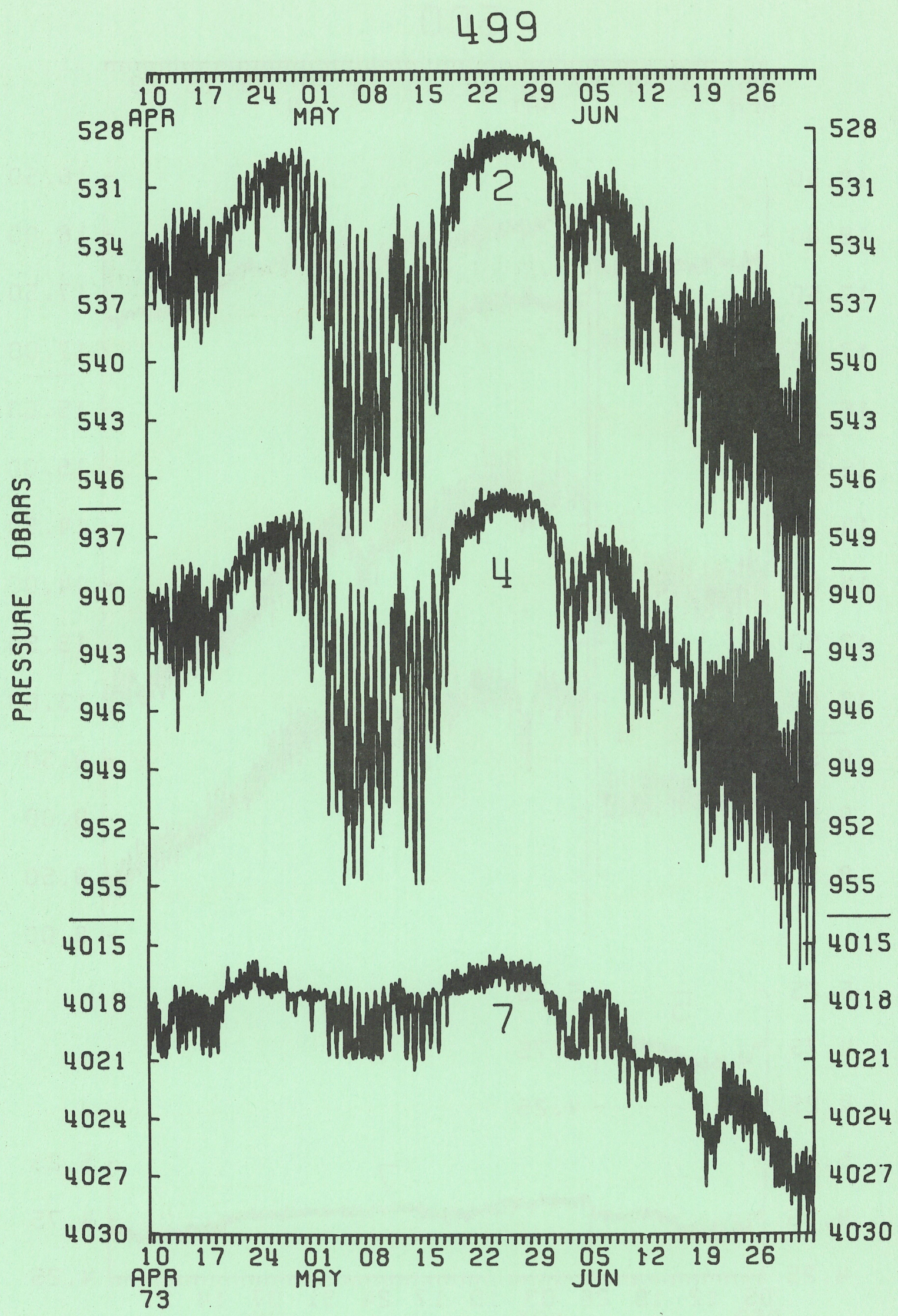


500

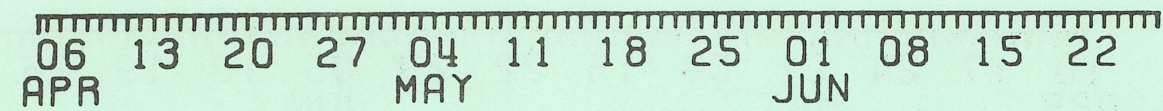
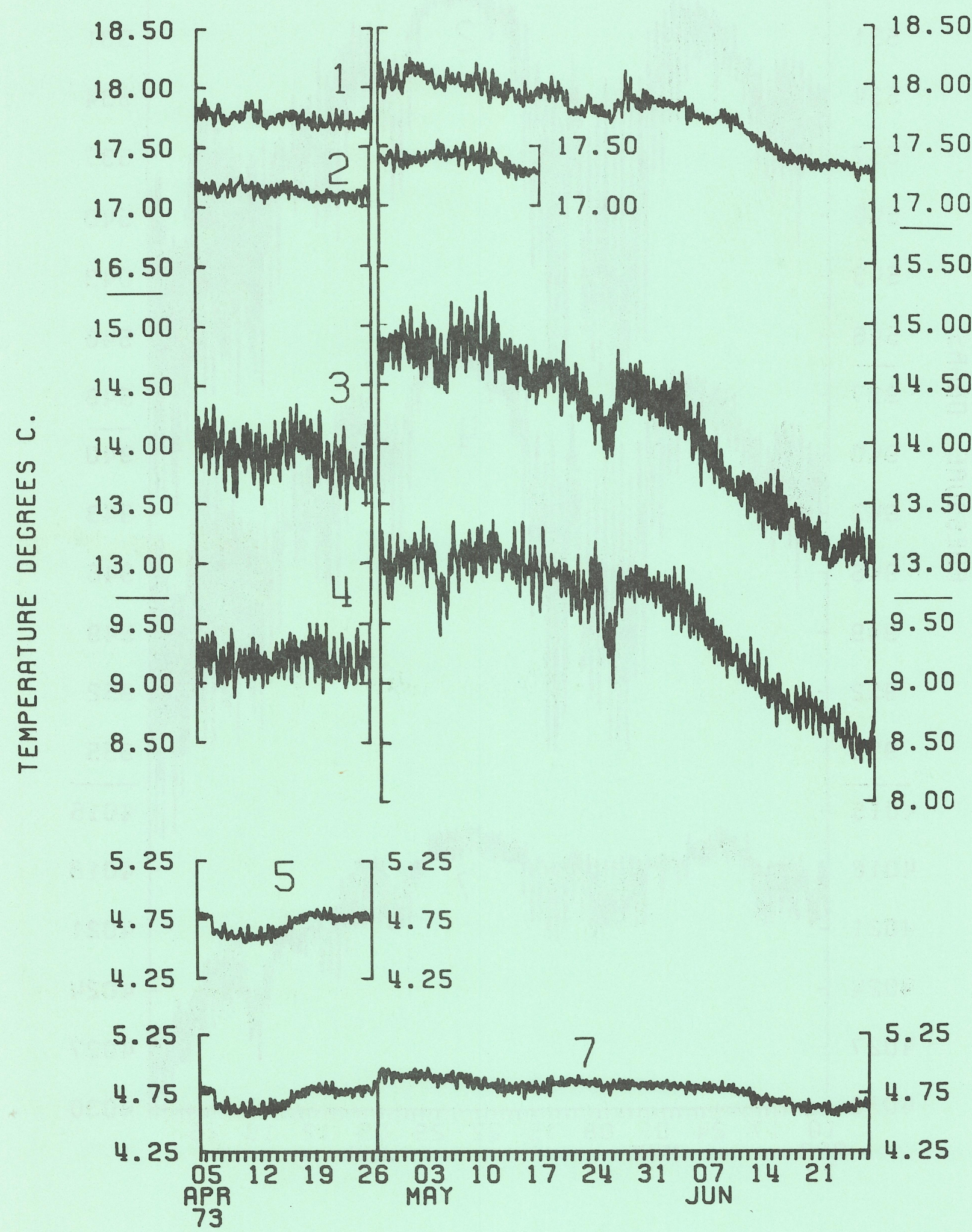


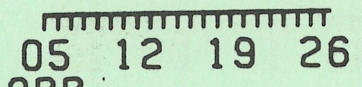
APR

73

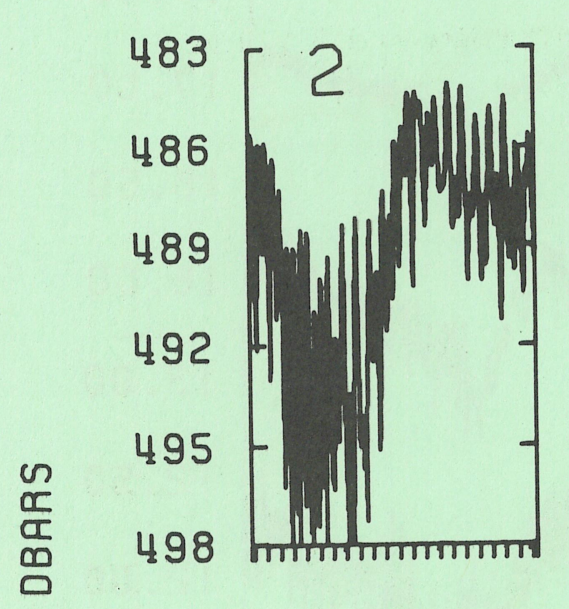

$w$
r
v
v
$w$
$\frac{w}{a}$

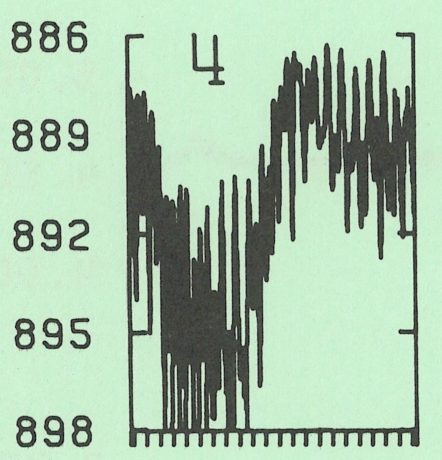

500

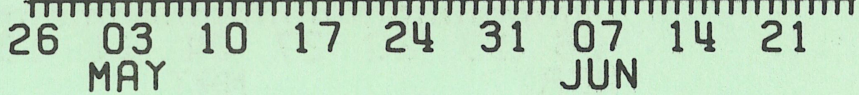

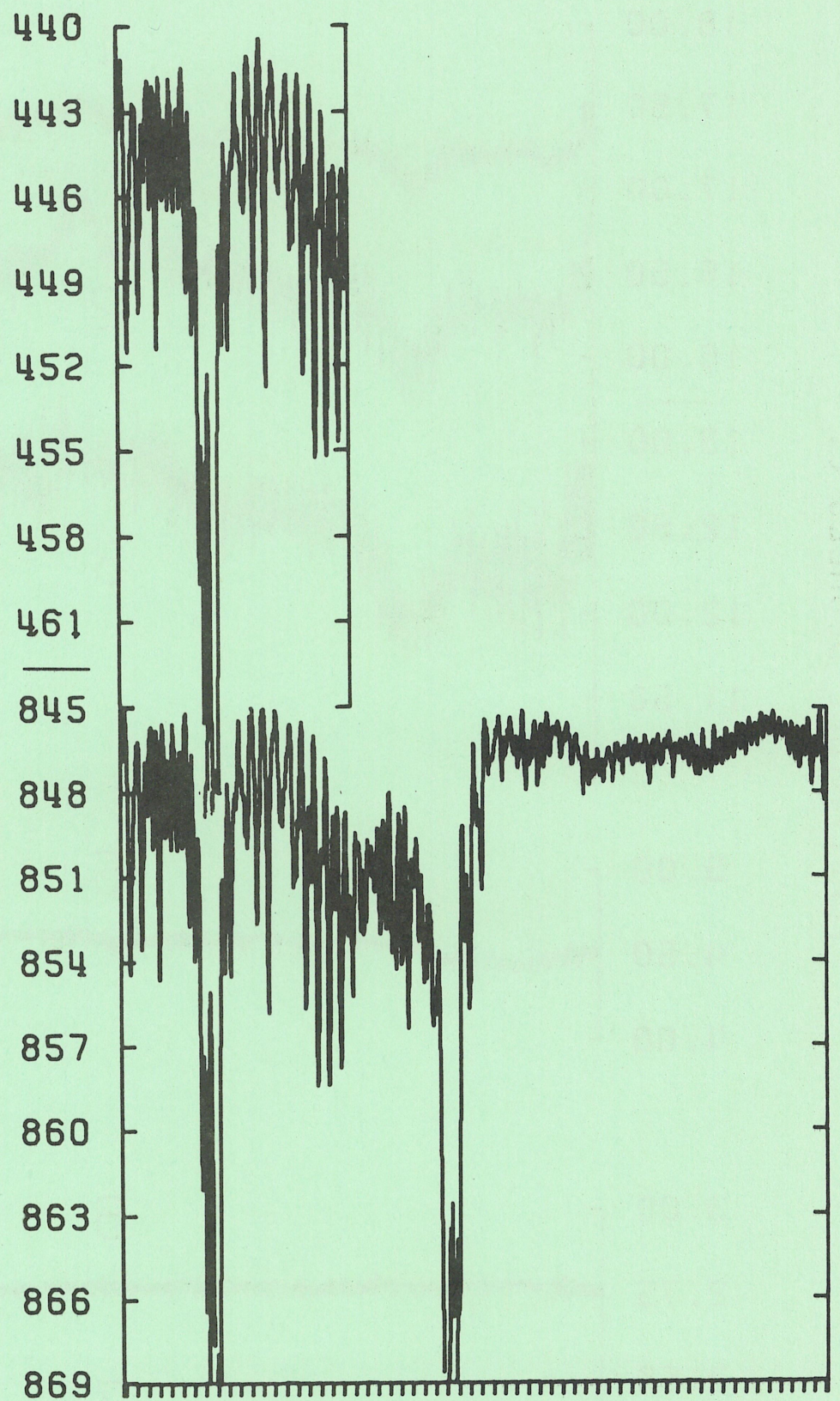

$0512 \quad 1926$

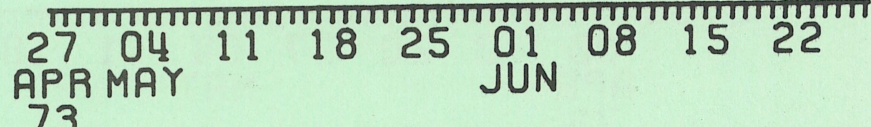




\section{1}

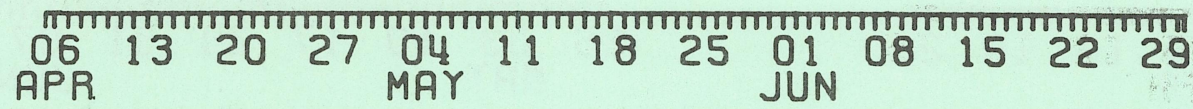
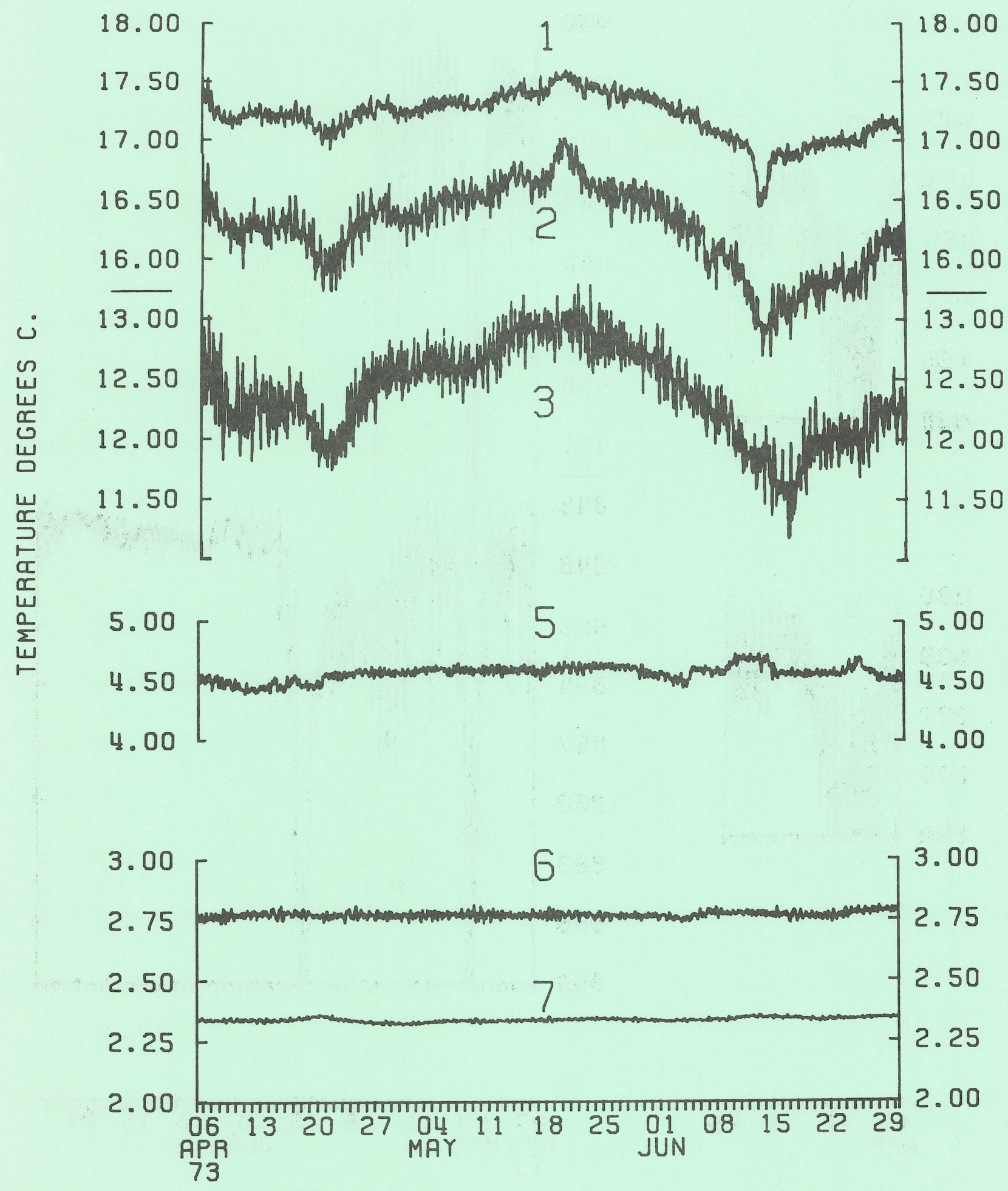


\section{1}

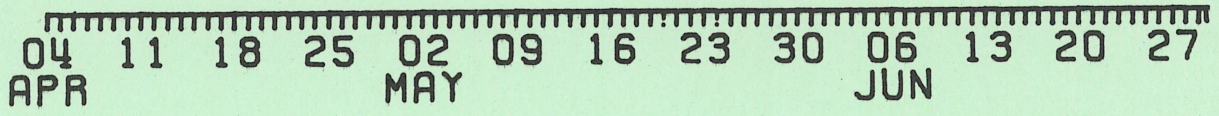

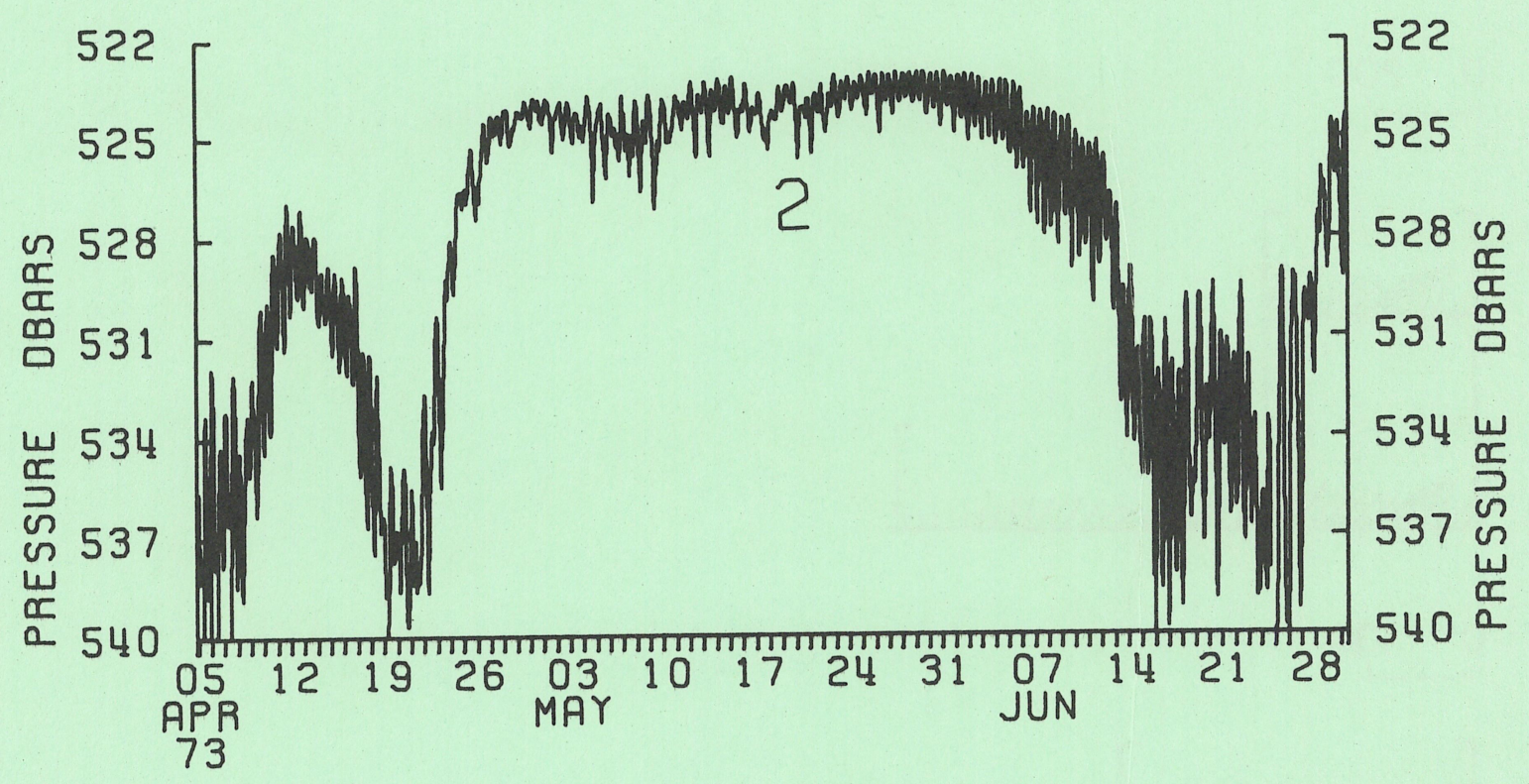




\section{1}

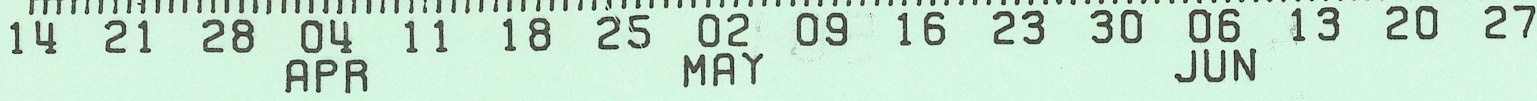

$\stackrel{2}{0} 200$

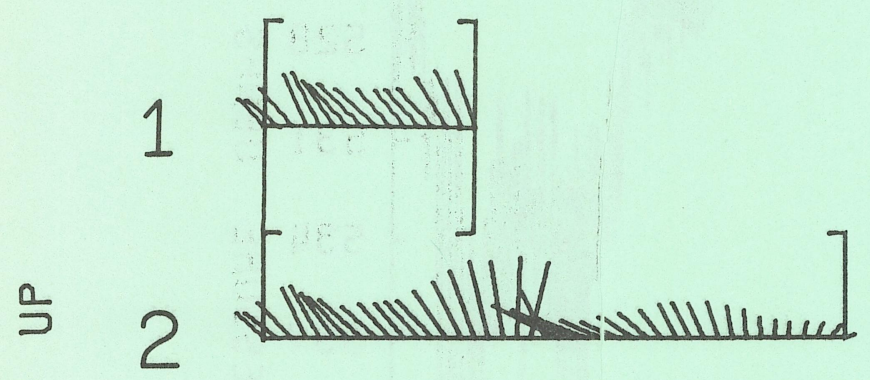

$\stackrel{0}{0 \quad 100}$

r

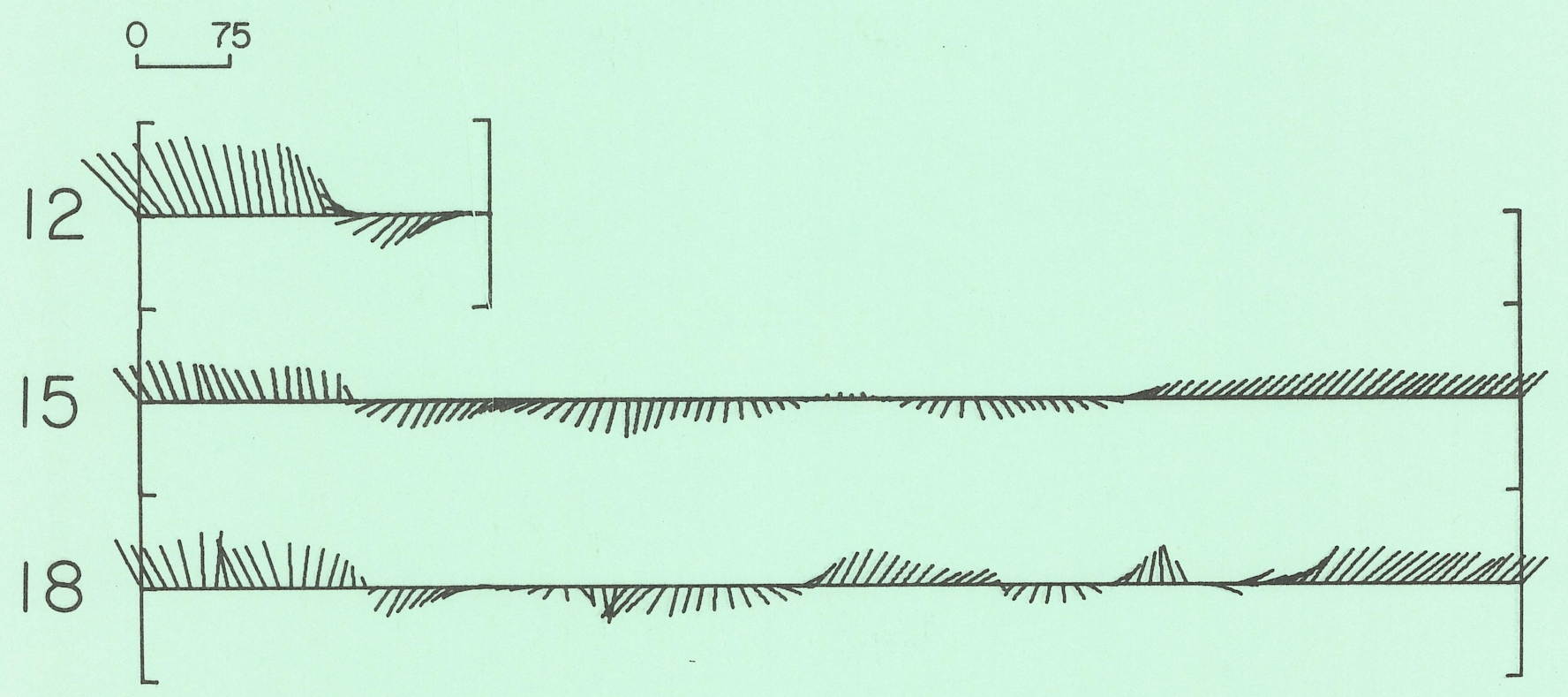

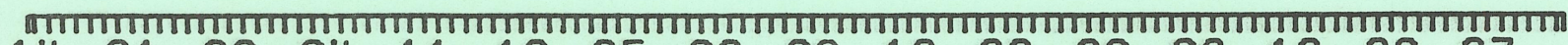

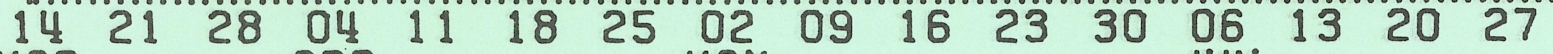
MAR APR MAY JUN

73

73

73

73 


\section{2 \\ 212804 \\ APR}
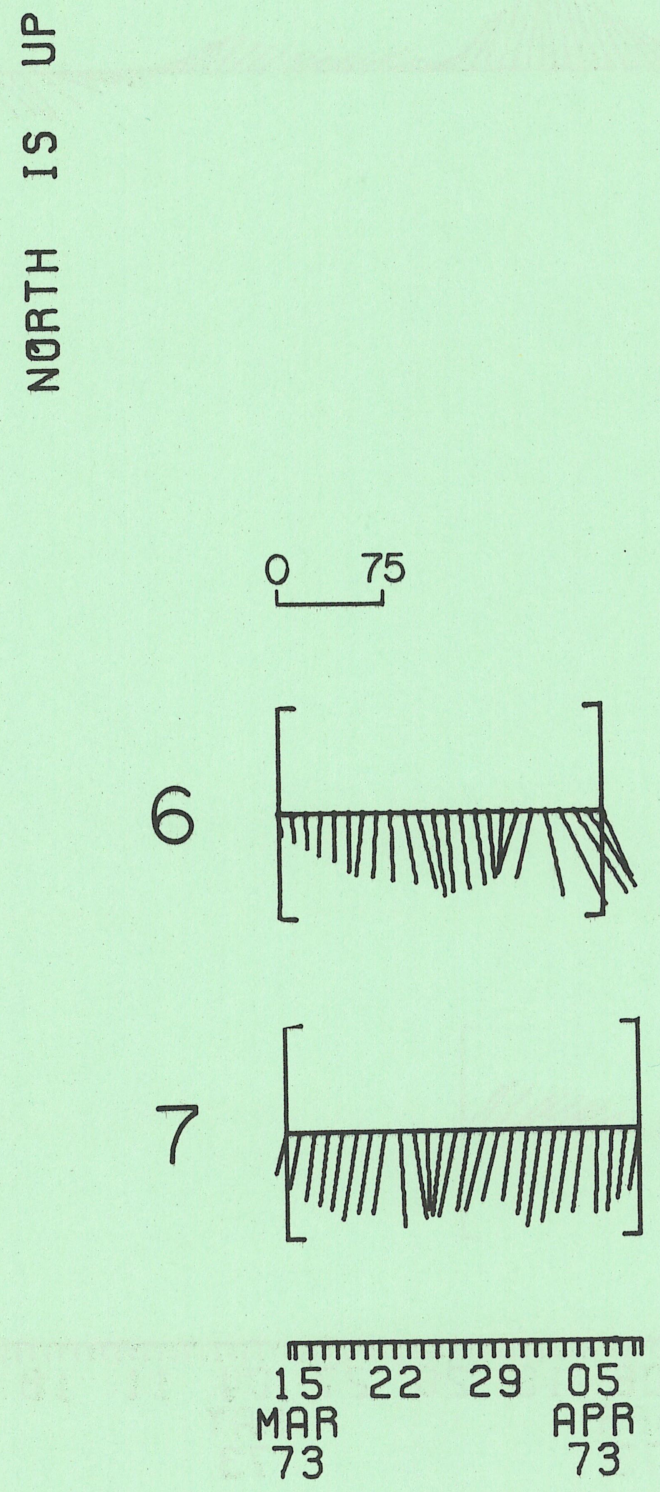


\section{3}

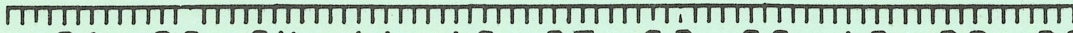

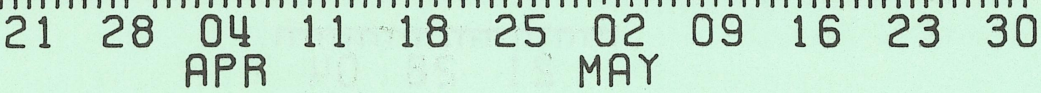

$\stackrel{0}{\longrightarrow} 150$

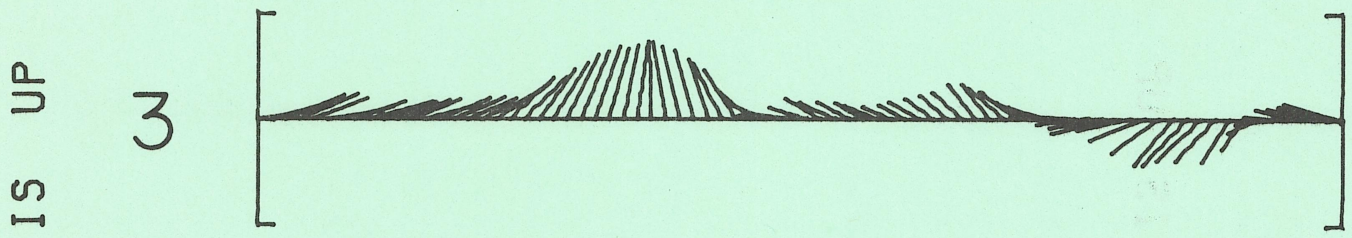

$\frac{I}{\text { I }}$

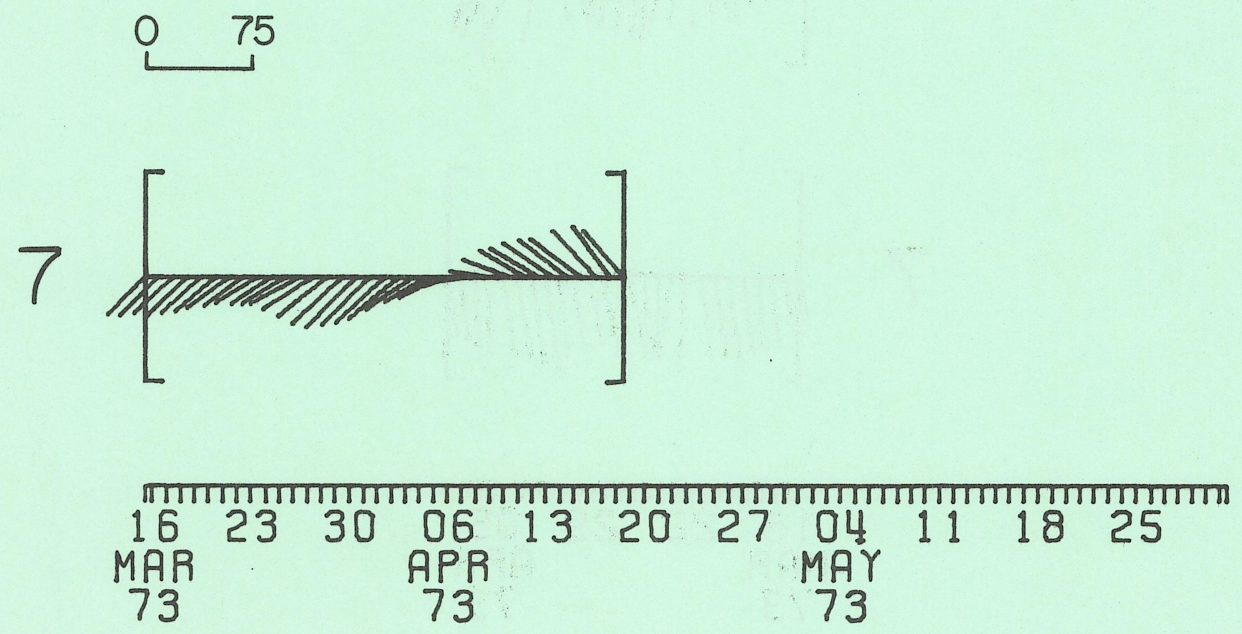




\section{4}

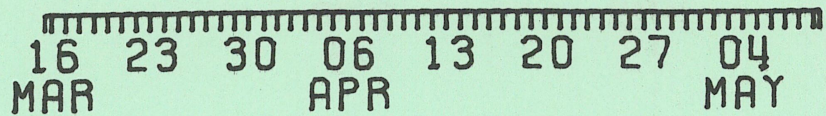

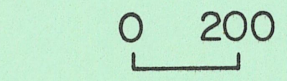

1 [D

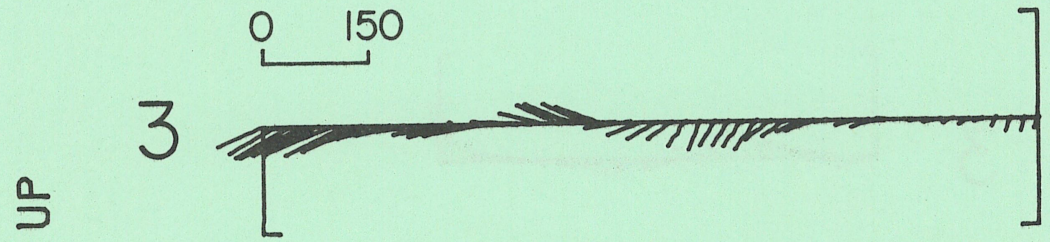

$\stackrel{0}{n}$

$I$
$上$
$\frac{1}{2}$
$Z$

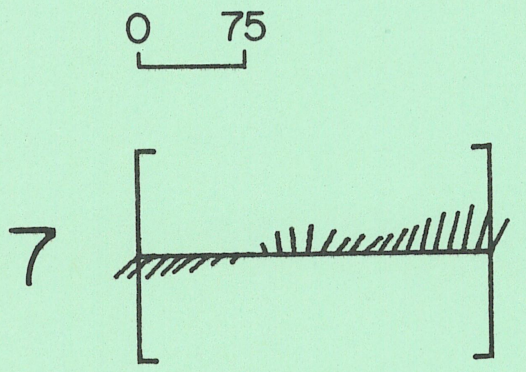

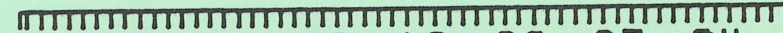
$\begin{array}{llllllll}16 & 23 & 30 & 06 & 13 & 20 & 27 & 04\end{array}$ MAR APR MAY 73

73 


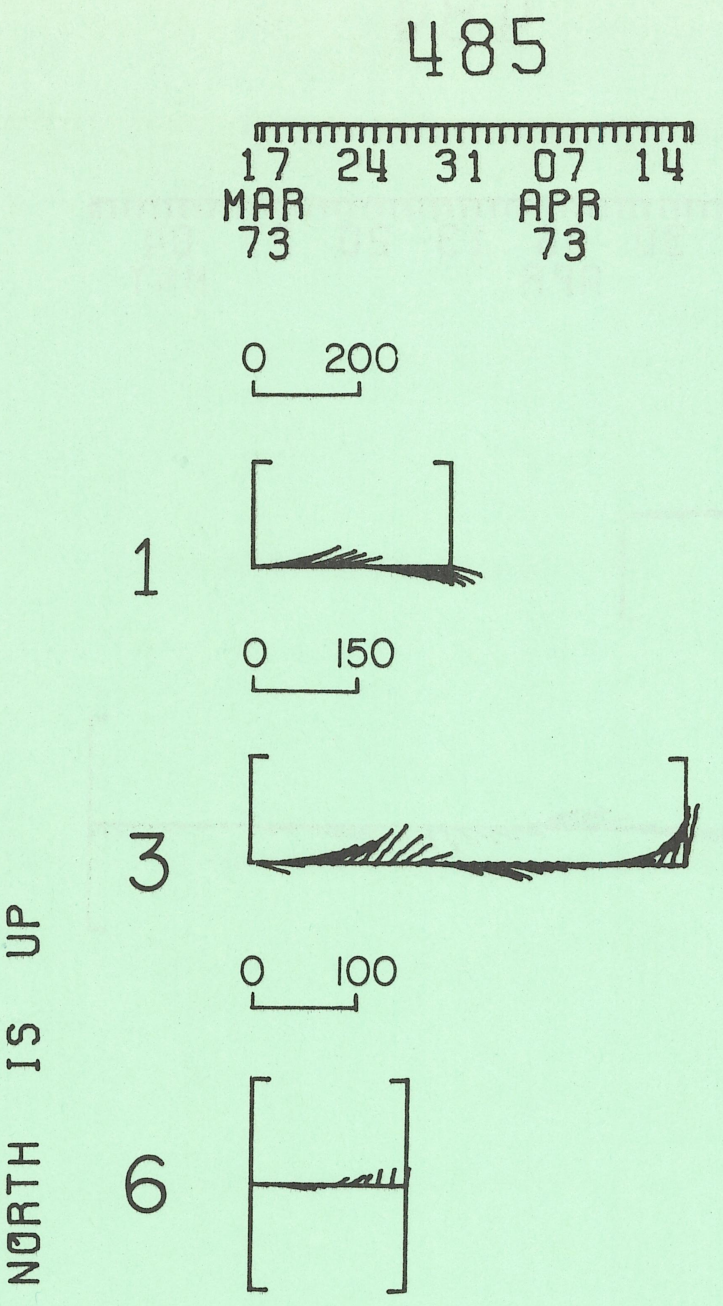

$\begin{array}{lllll}17 & 24 & 31 & 07 & 14\end{array}$

MAR APR

73

73 


\section{6}

लाभागा $\begin{array}{lllllllllllllll}21 & 28 & 04 & 11 & 18 & 25 & 02 & 09 & 16 & 23 & 30 & 06 & 13 & 20 & 27\end{array}$ APR MAY JUN
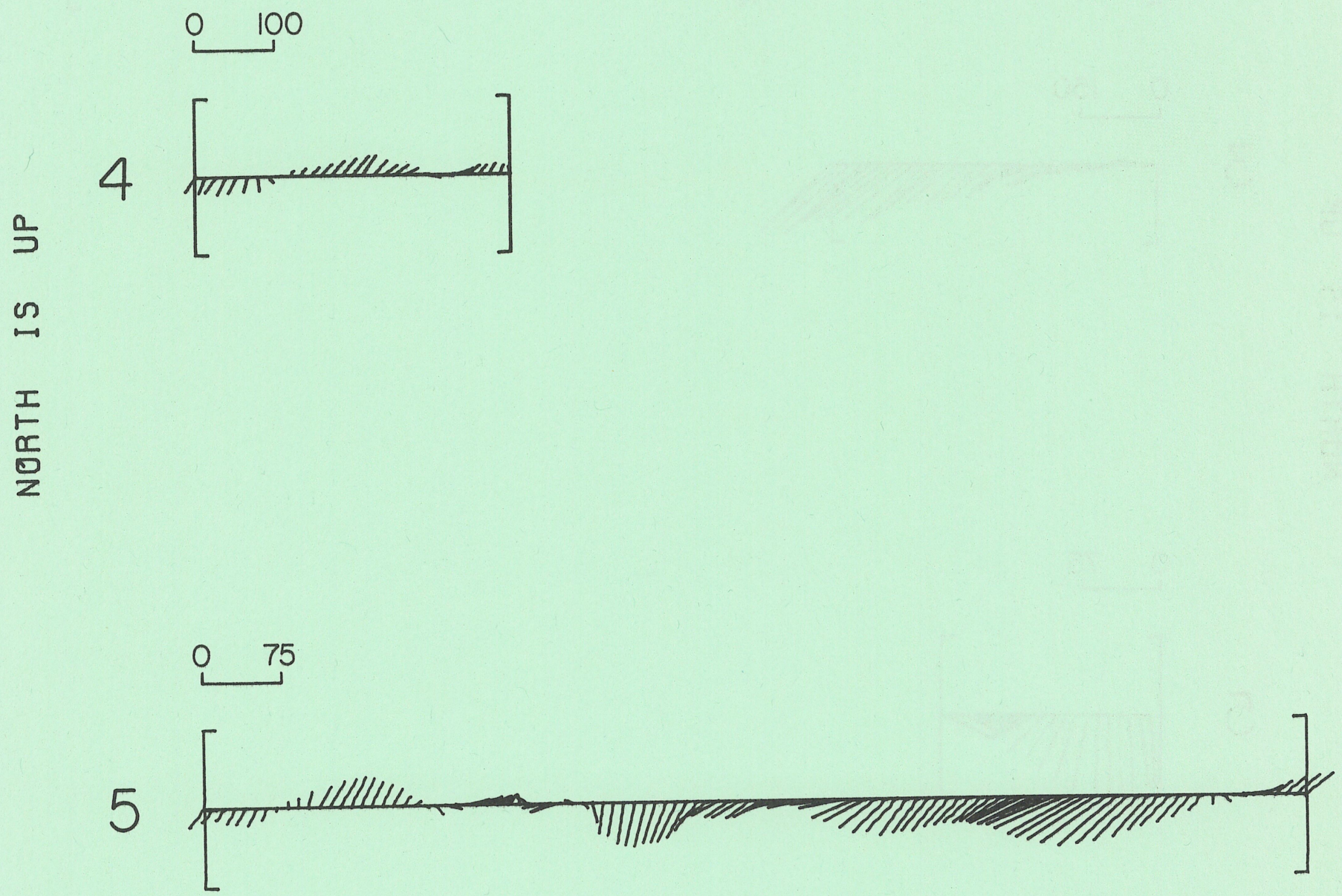

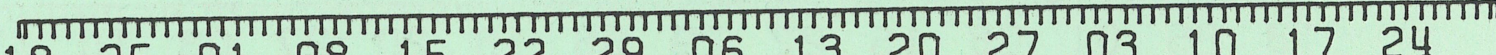

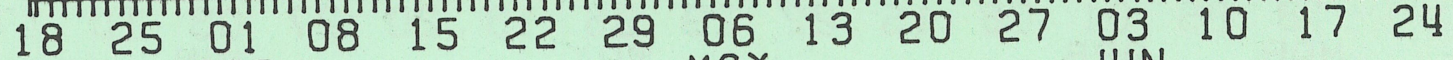
$\begin{array}{cccc}M A B & A P R & \text { MAY } & \text { JUN } \\ 73 & 73 & 73 & 73\end{array}$ 
488

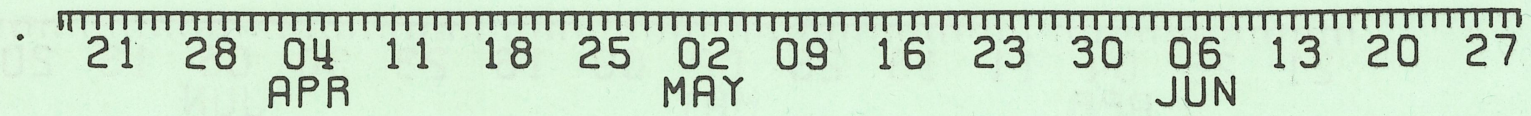

$\underbrace{0 \quad 200}$

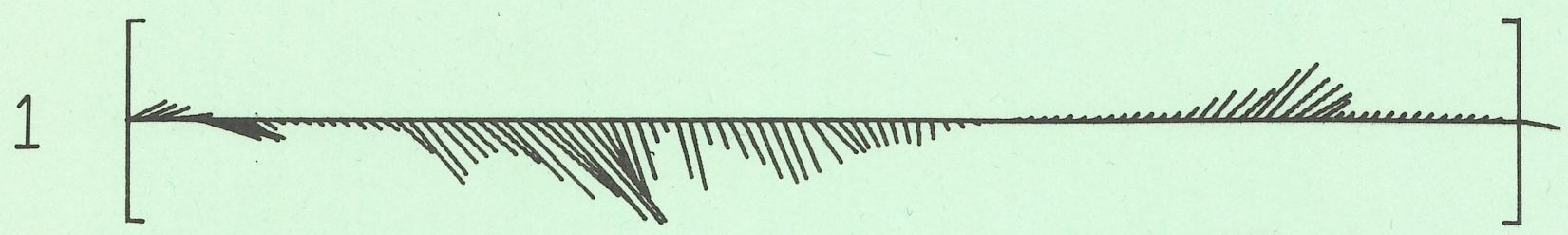

$0 \quad 150$

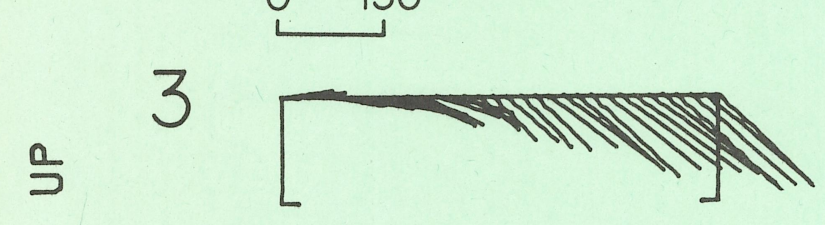

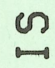

$\frac{I}{E}$

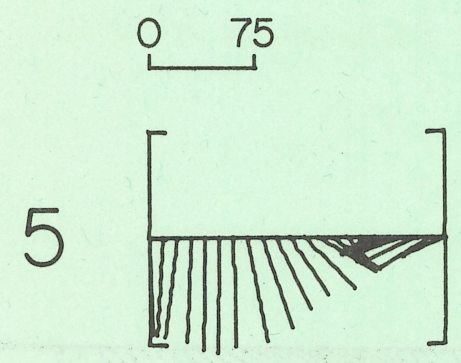

$\begin{array}{lllllllllllllll}18 & 25 & 01 & 08 & 15 & 22 & 29 & 06 & 13 & 20 & 27 & 03 & 10 & 17 & 24\end{array}$ MAR 73 


\section{9}

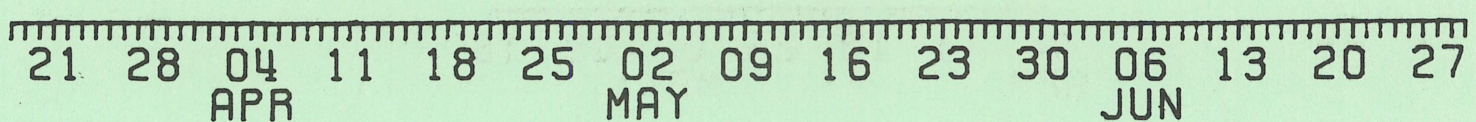
APR MAY JUN

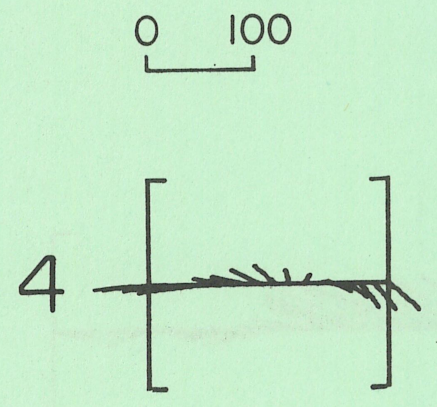

今 $\quad \underbrace{7} \quad 75$

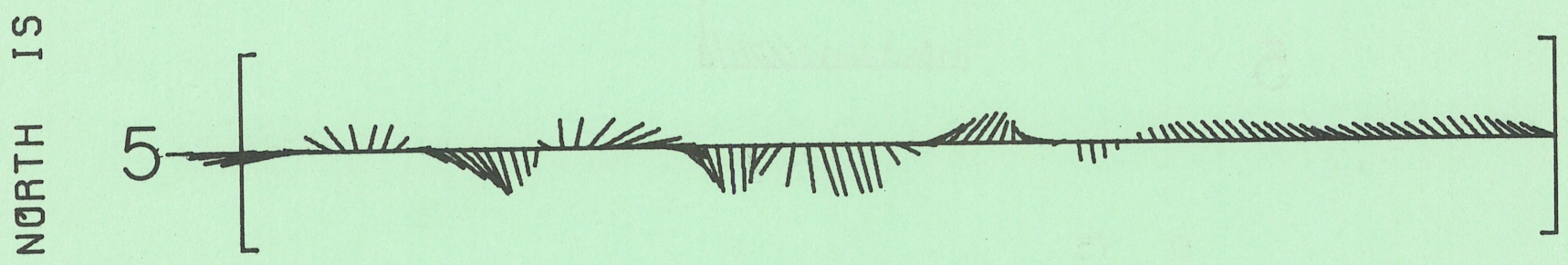

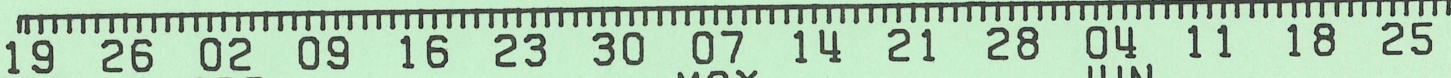
MAR APR MAY JUN 73 


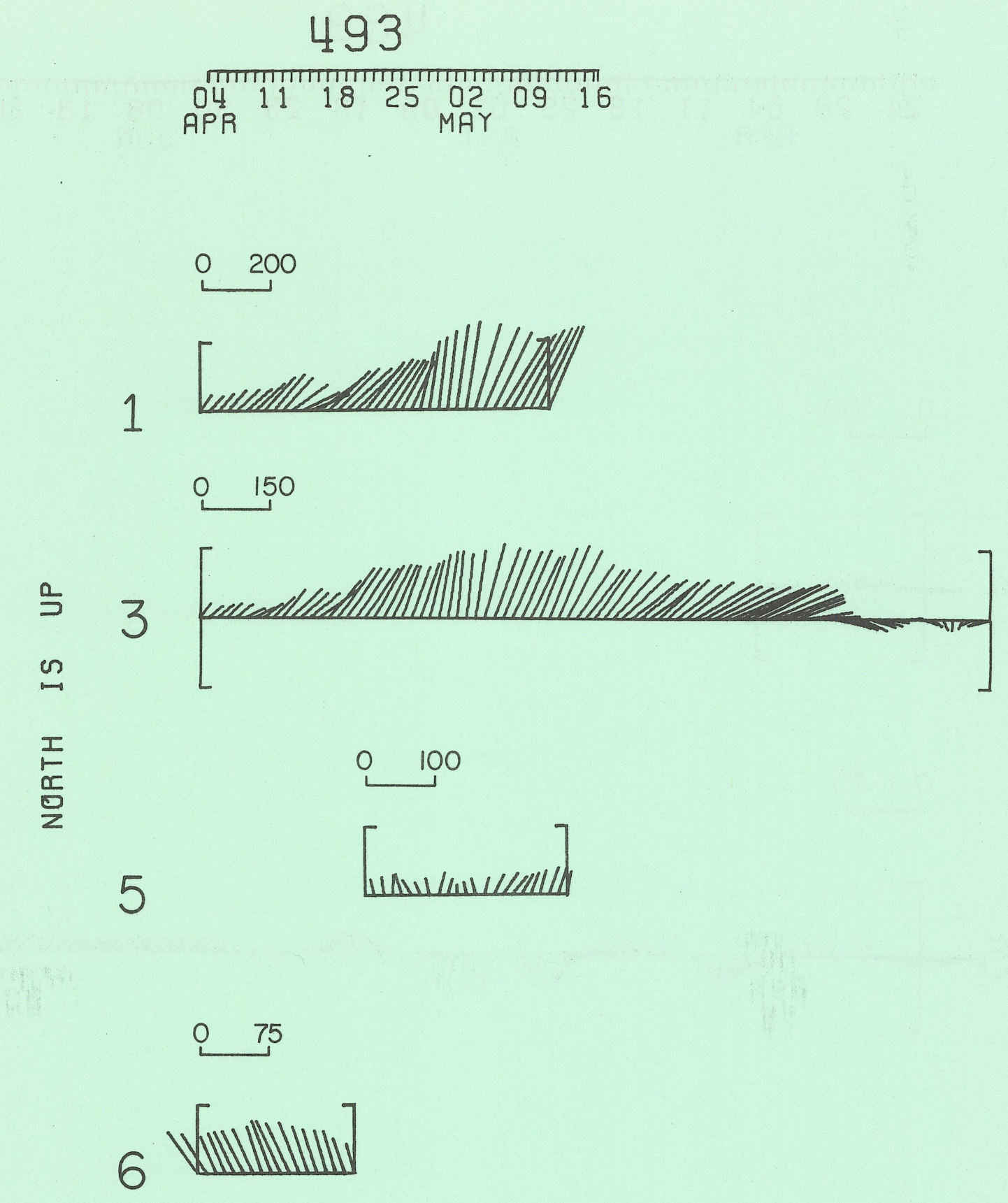

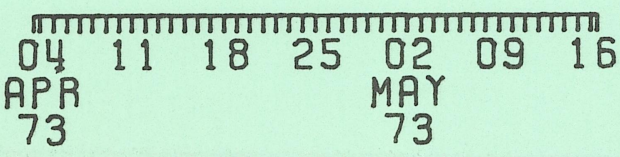




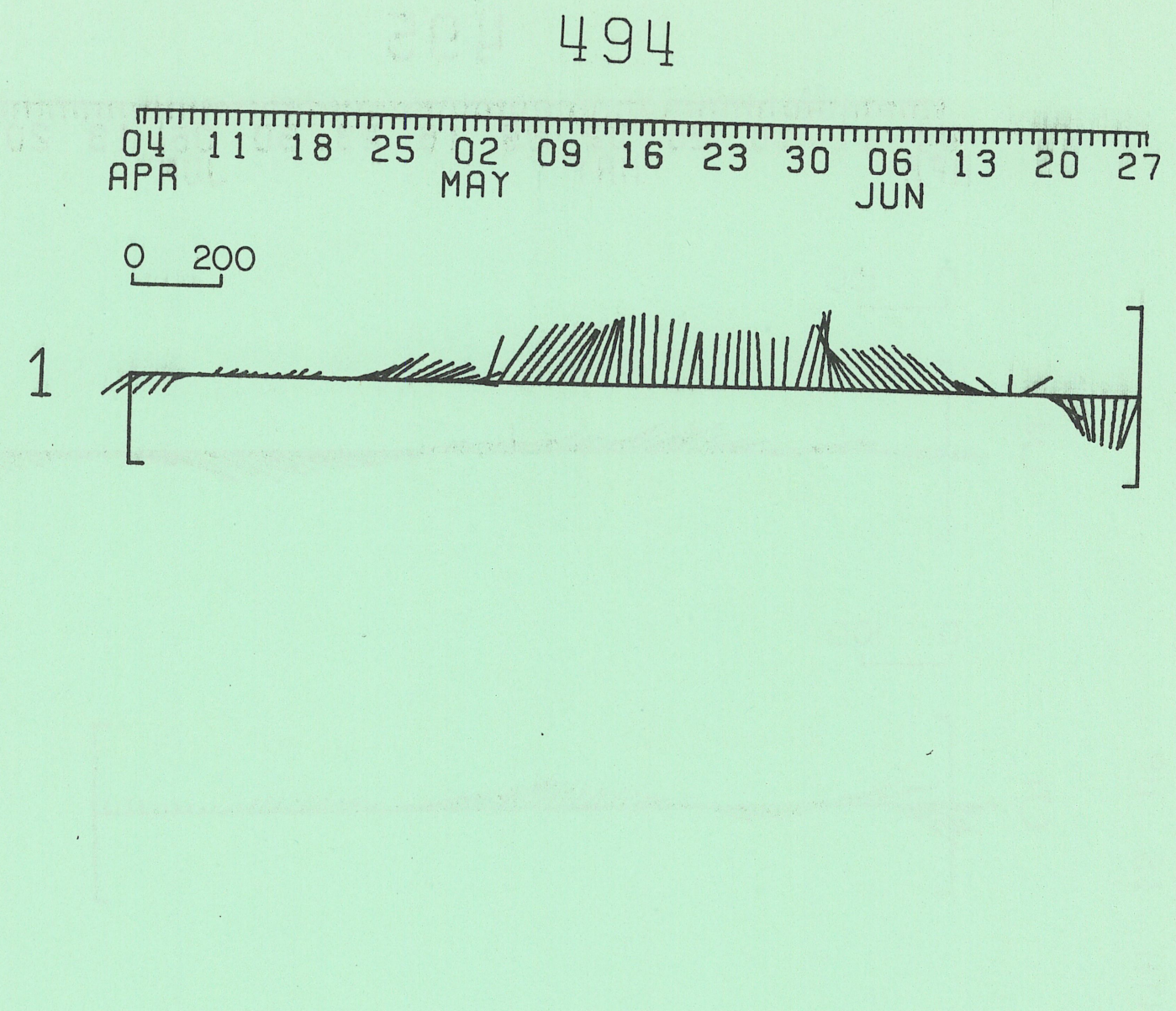

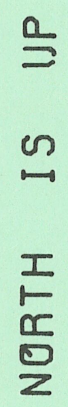

$04111825 \quad 02 \quad 09161030$

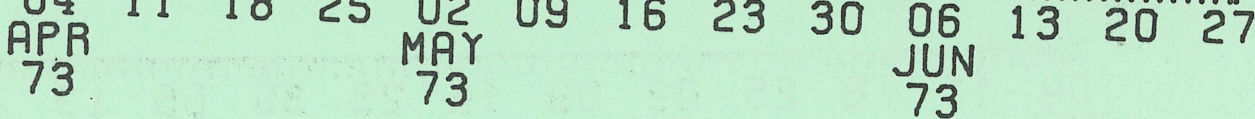




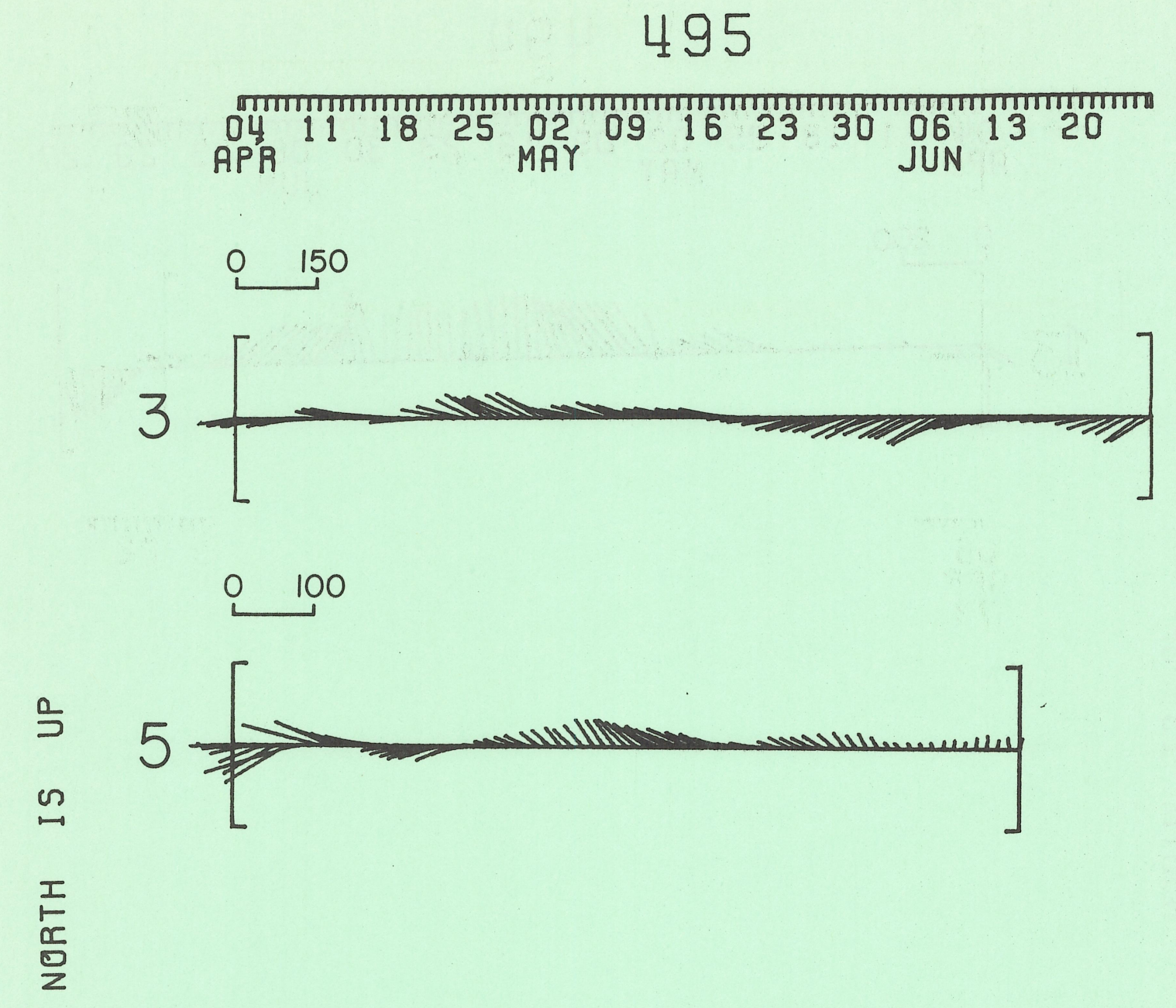

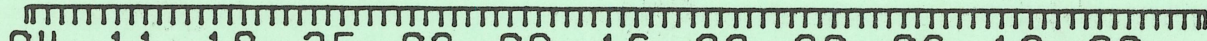
$\begin{array}{ccc}\text { APR } & \text { MAY } & \text { JUN } \\ 73 & 73 & 73\end{array}$ 


\section{7}

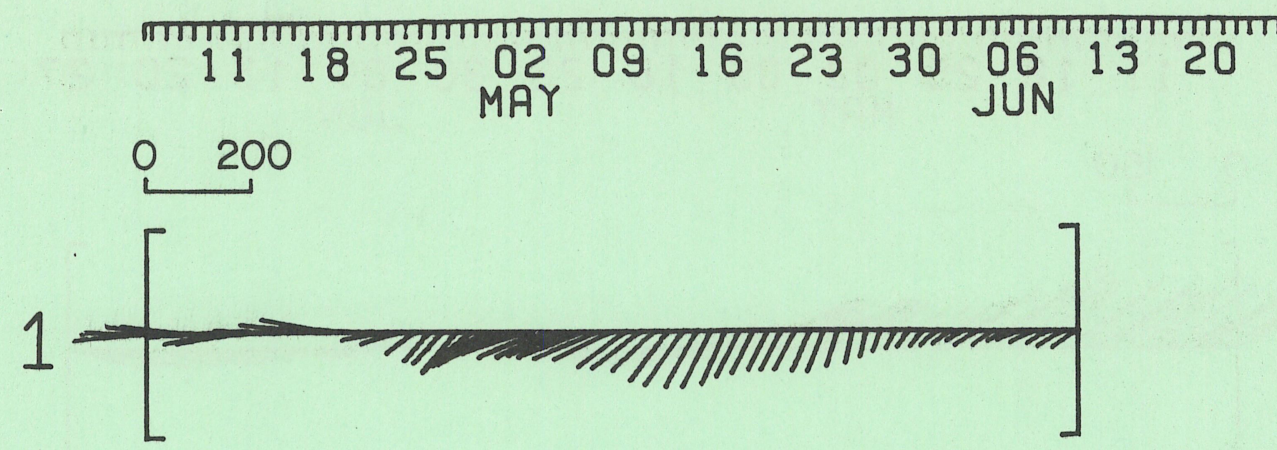

0
0
0
1
0
0
0
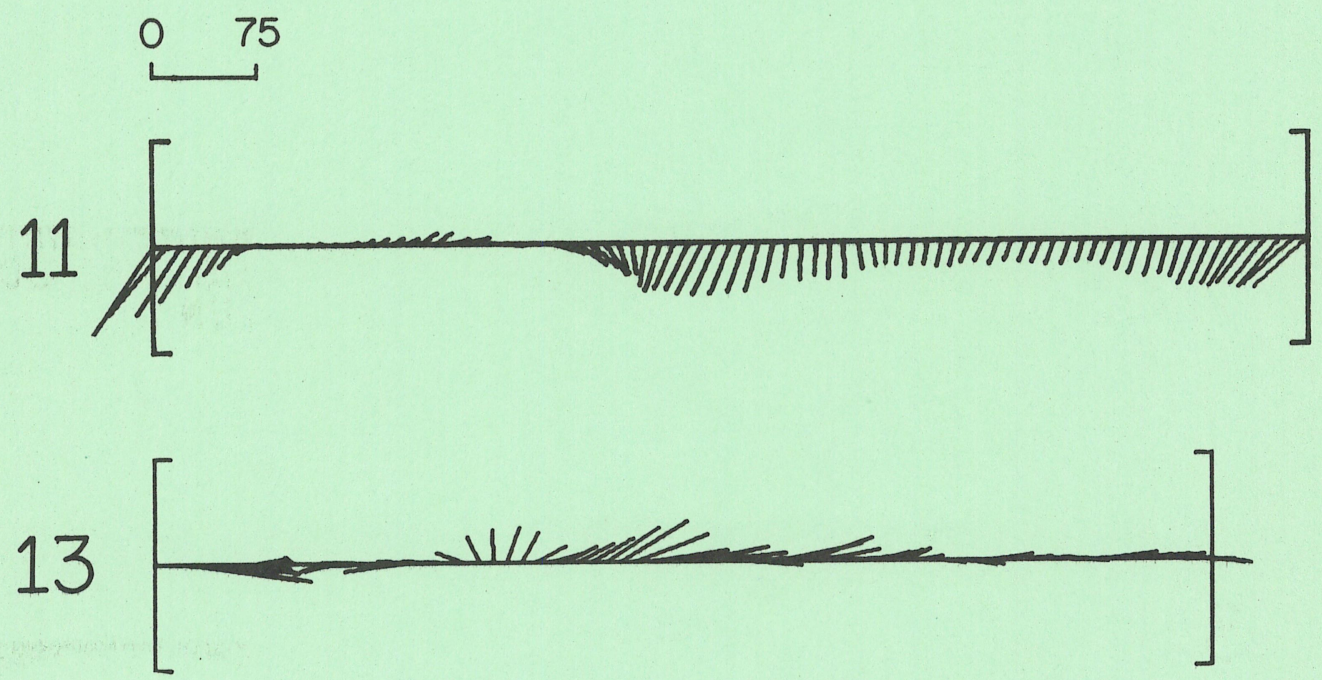

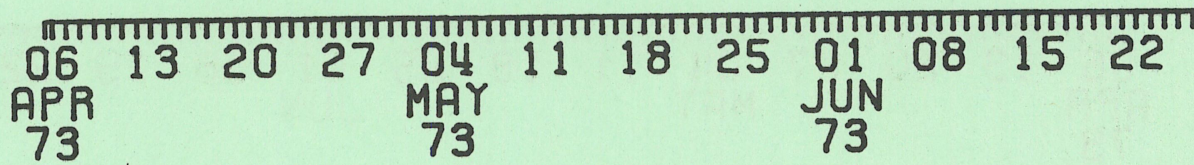




\section{8}
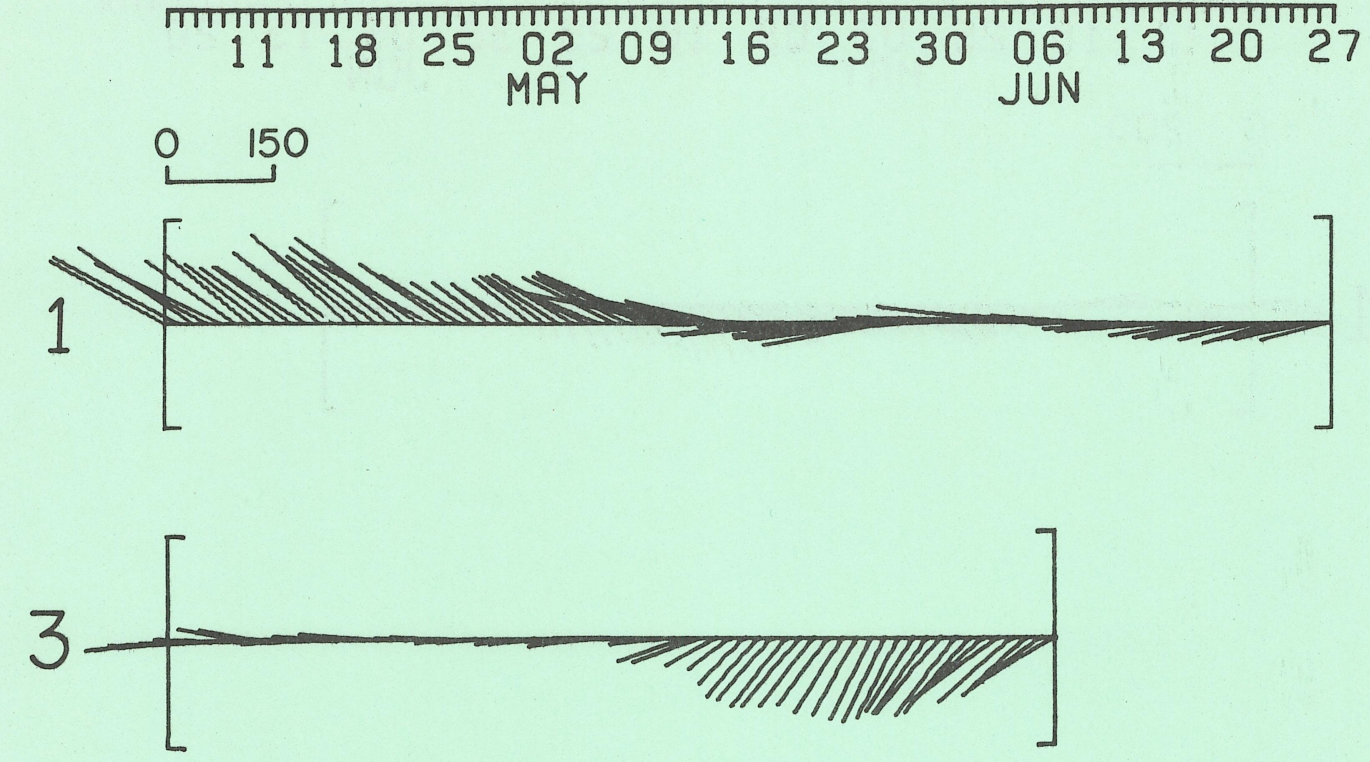

\section{气 \\ $\stackrel{n}{\rightarrow}$ \\ $I$
$\frac{\pi}{0}$
$\frac{0}{2}$}

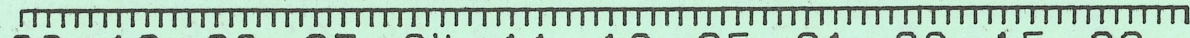
$\begin{array}{llllllllllll}06 & 13 & 20 & 27 & 04 & 011 & 18 & 25 & \text { OIN } & 08 & 15 & 22 \\ \text { APR } & & & \text { MAY } & & & & \text { JUN } & & & \end{array}$ APR
73 MAY 


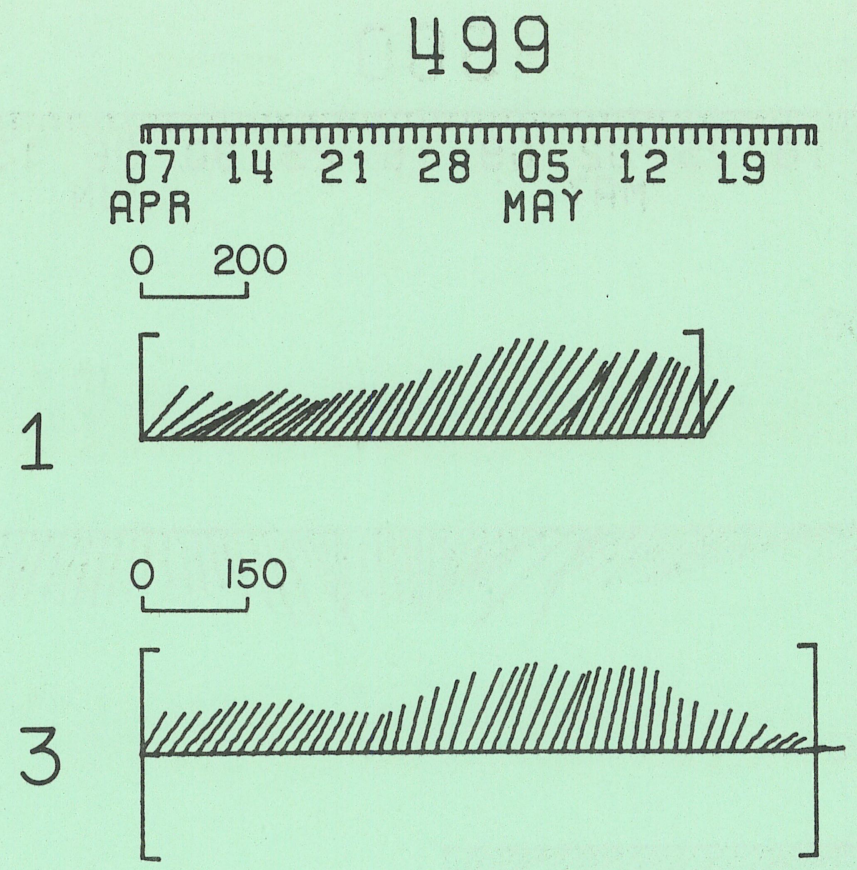

옥

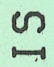

$\frac{I}{5}$

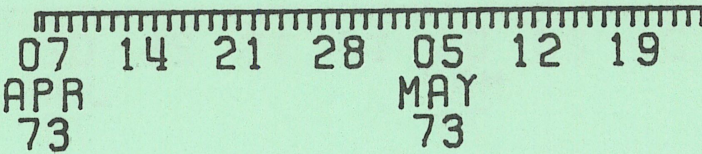




\section{0}

ला1ा

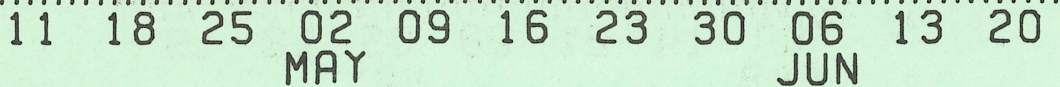

0200

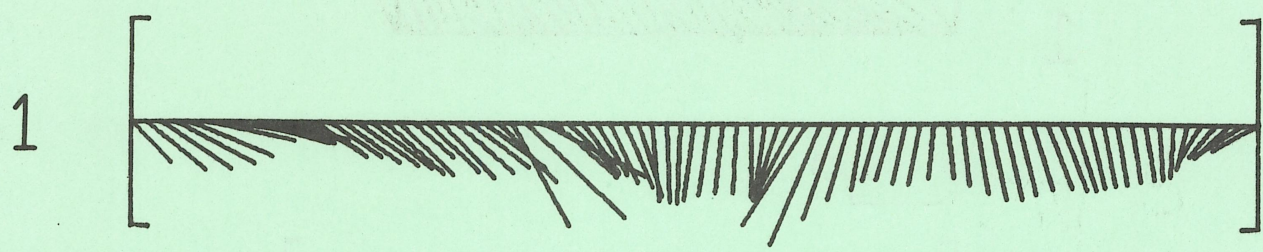

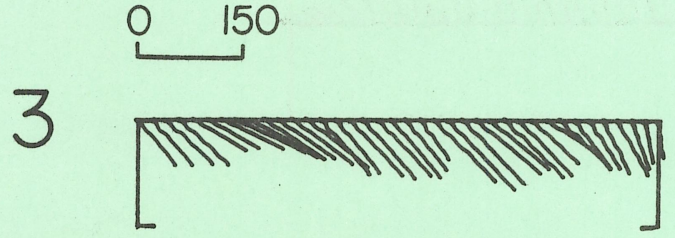

号

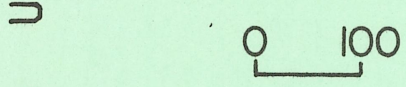

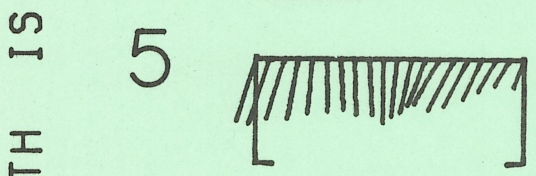

而

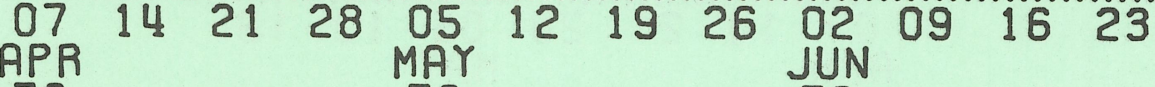

$\begin{array}{lll}73 & 73 & 73\end{array}$ 

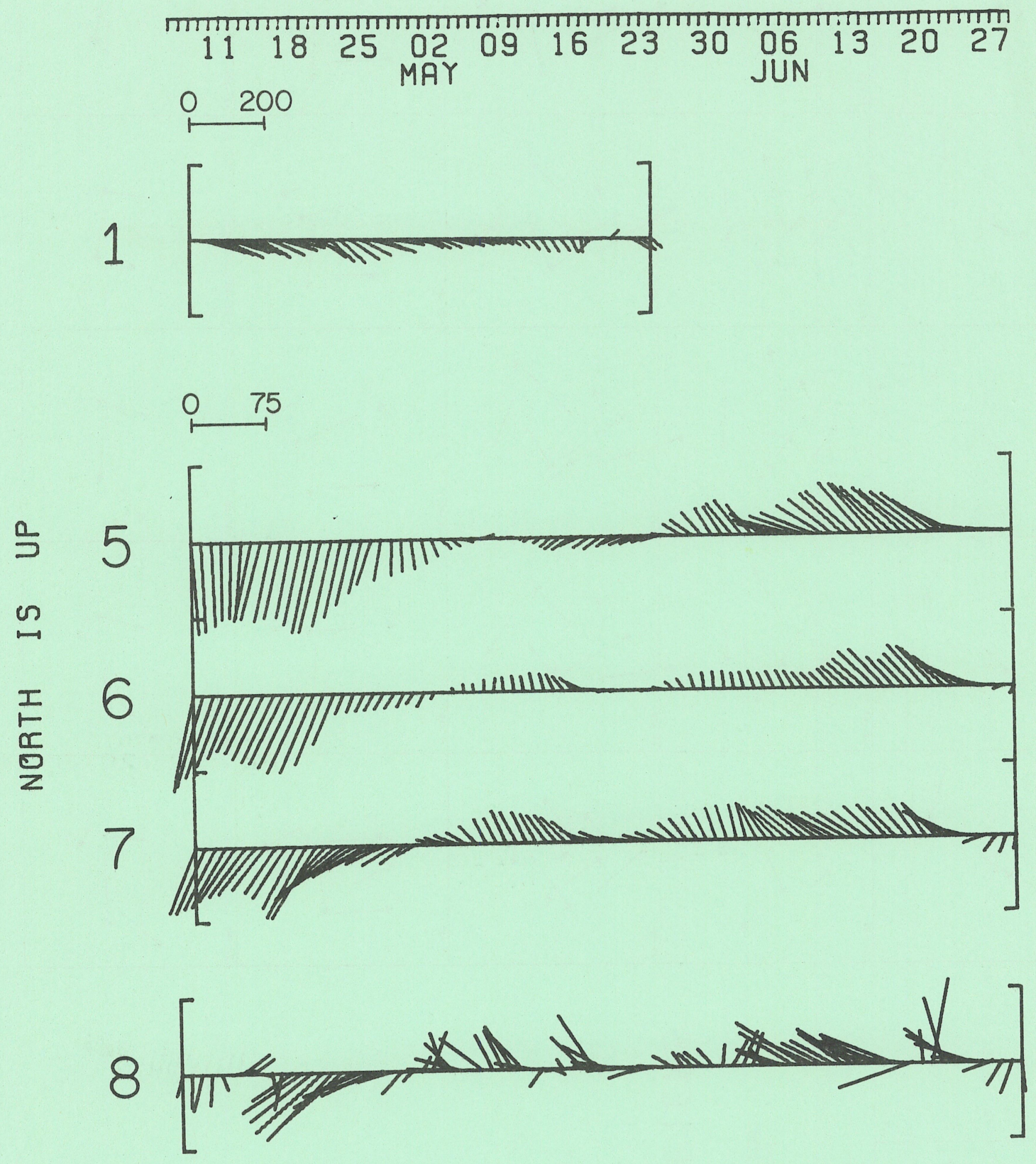

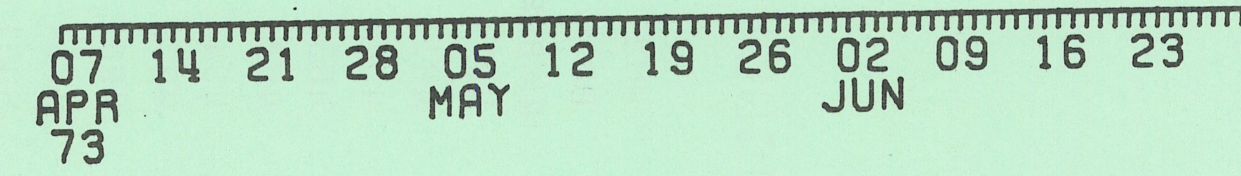



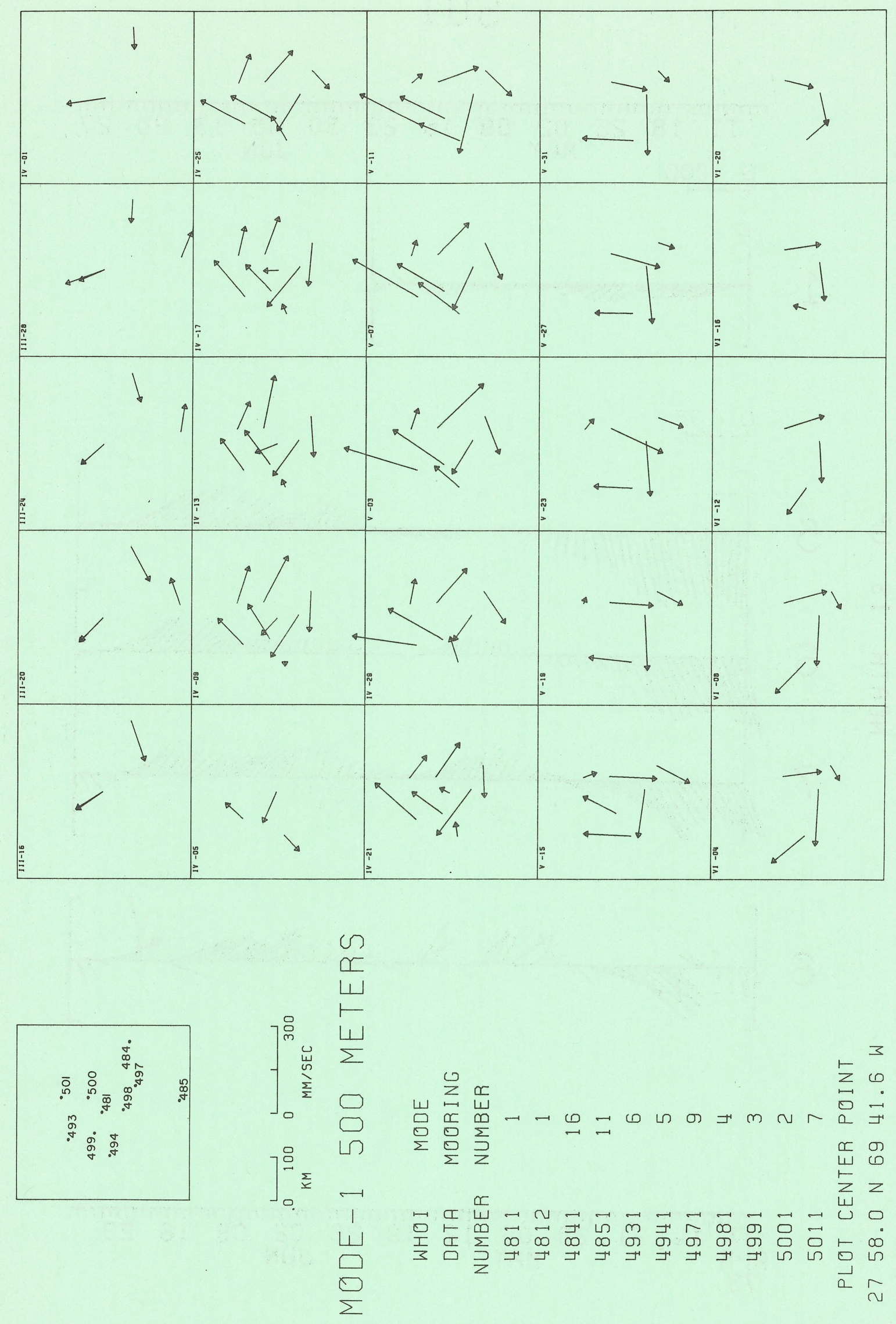

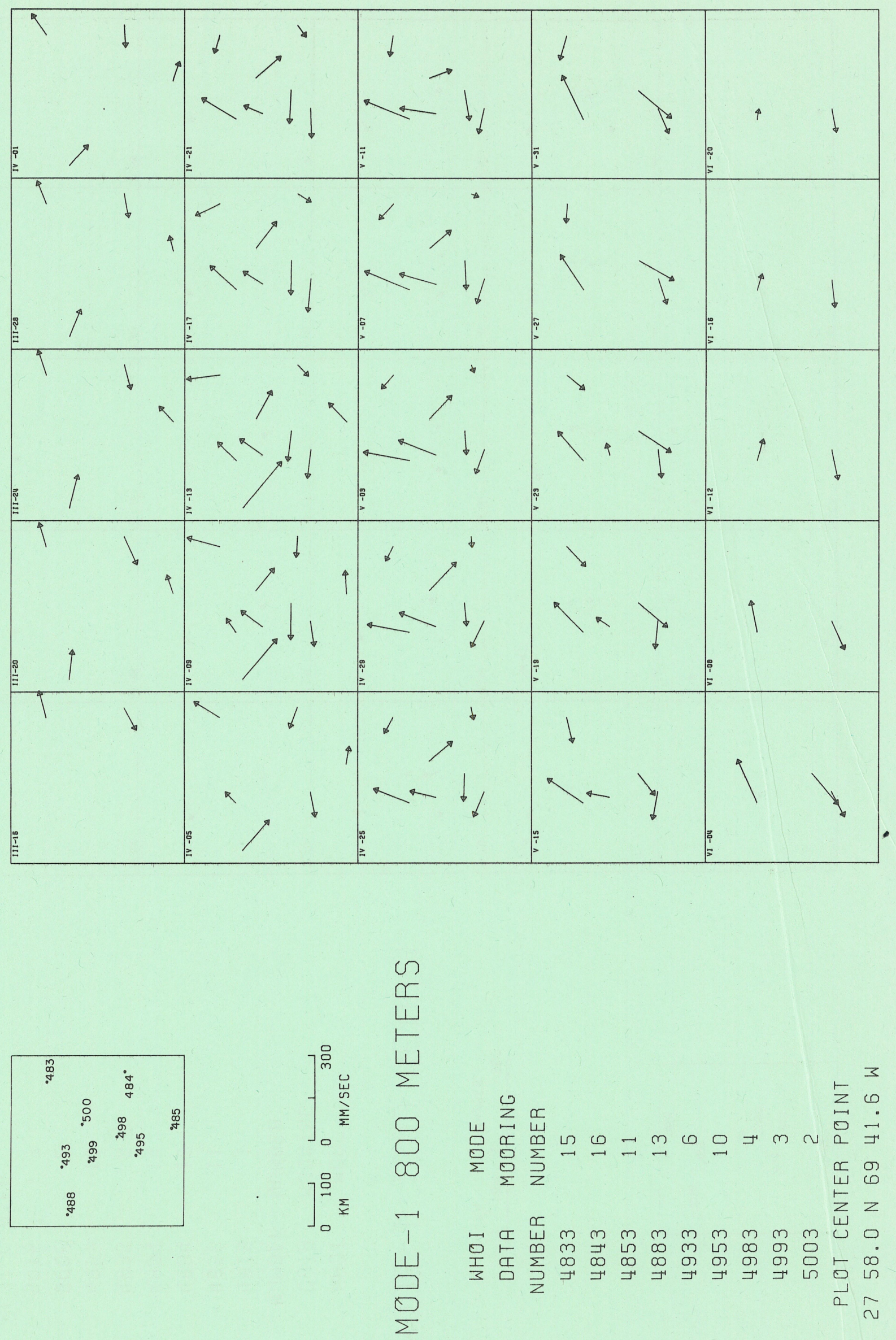

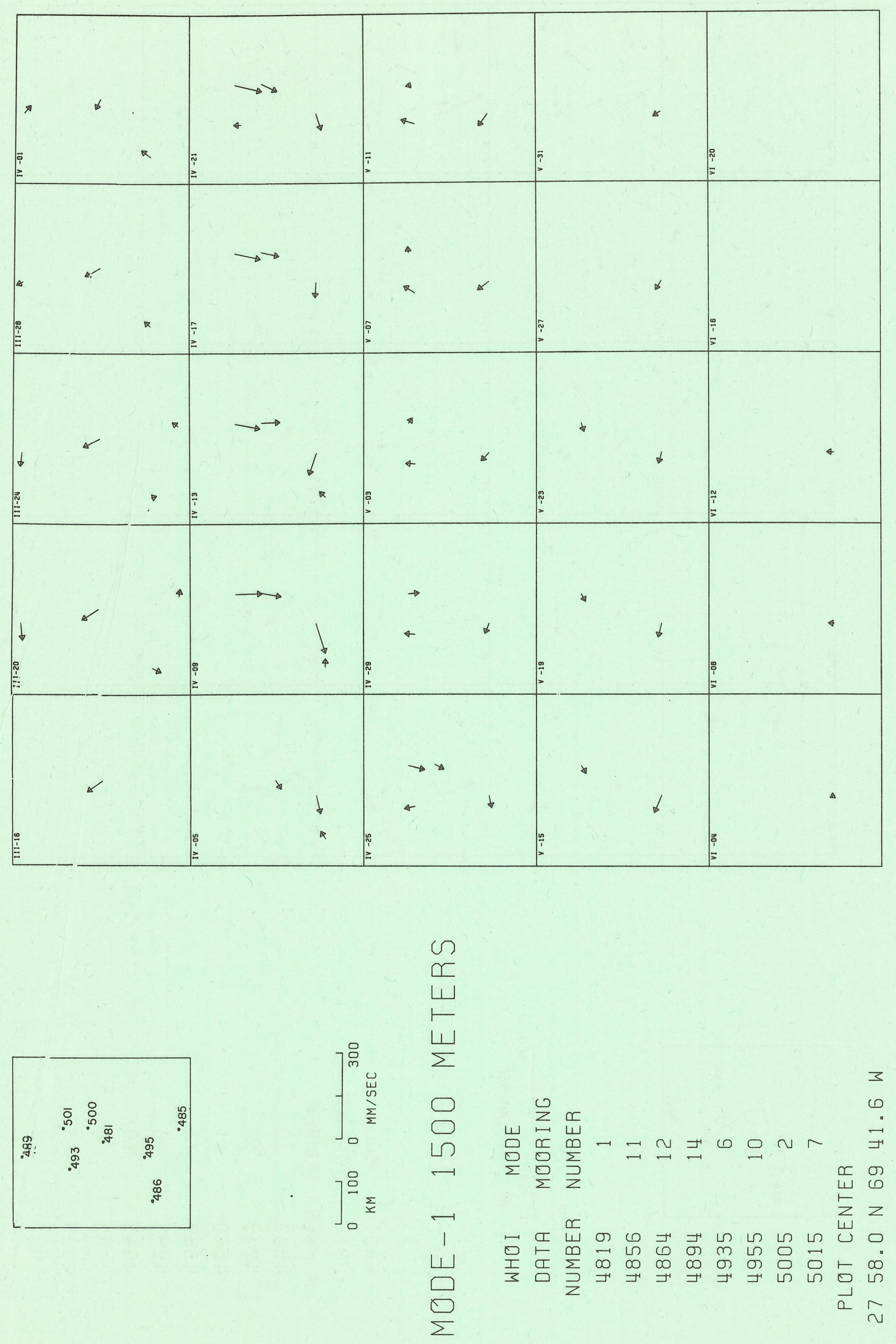

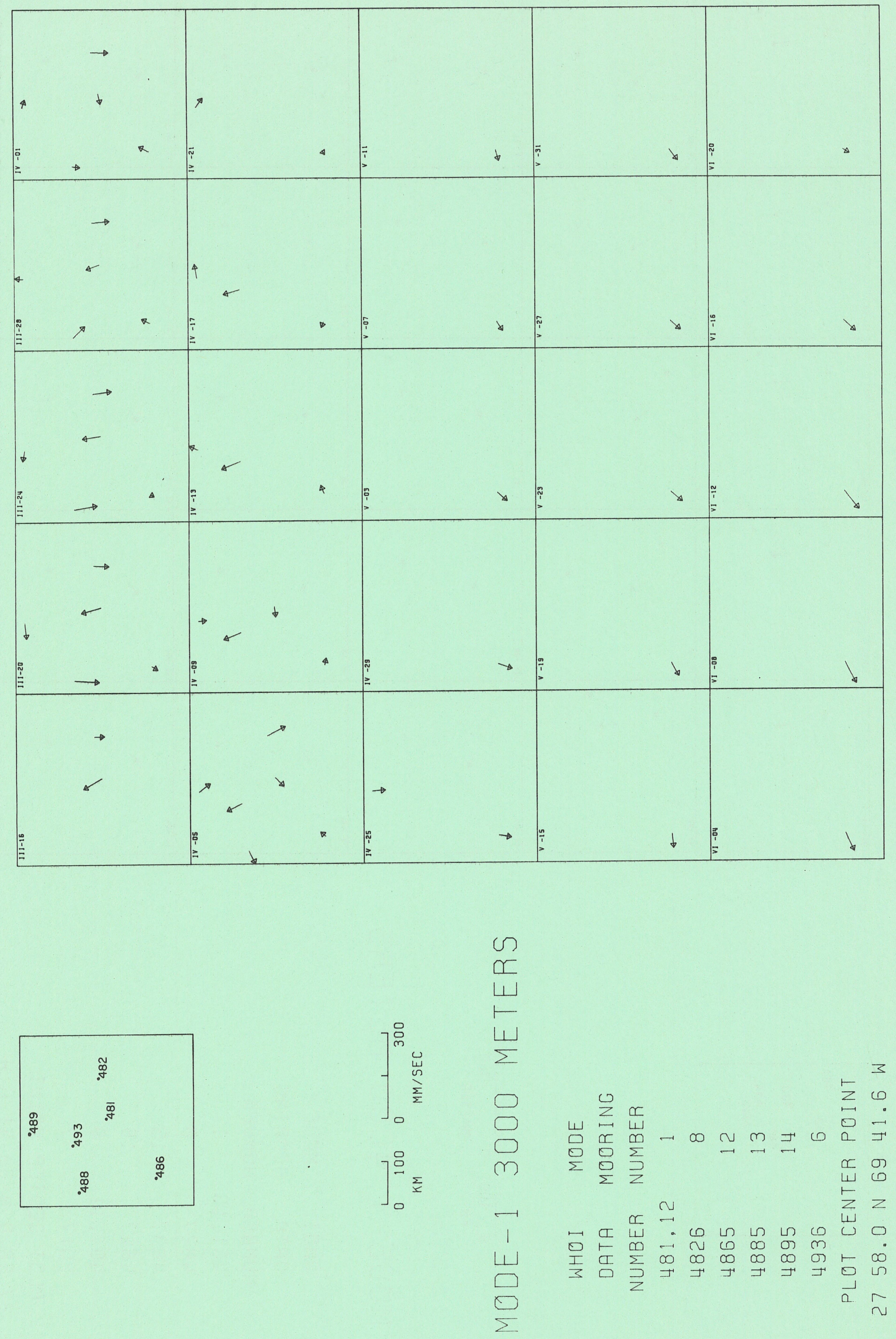

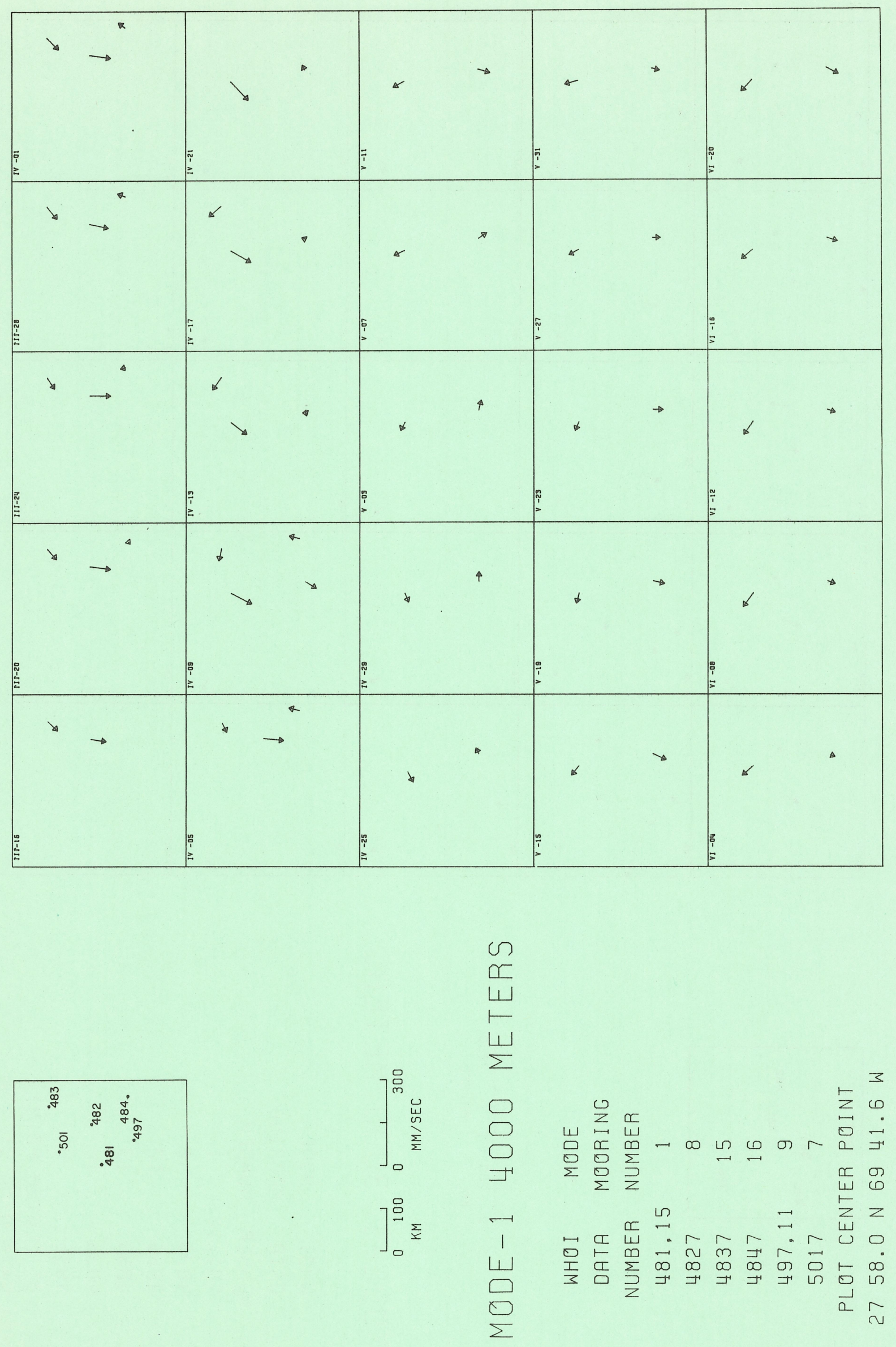

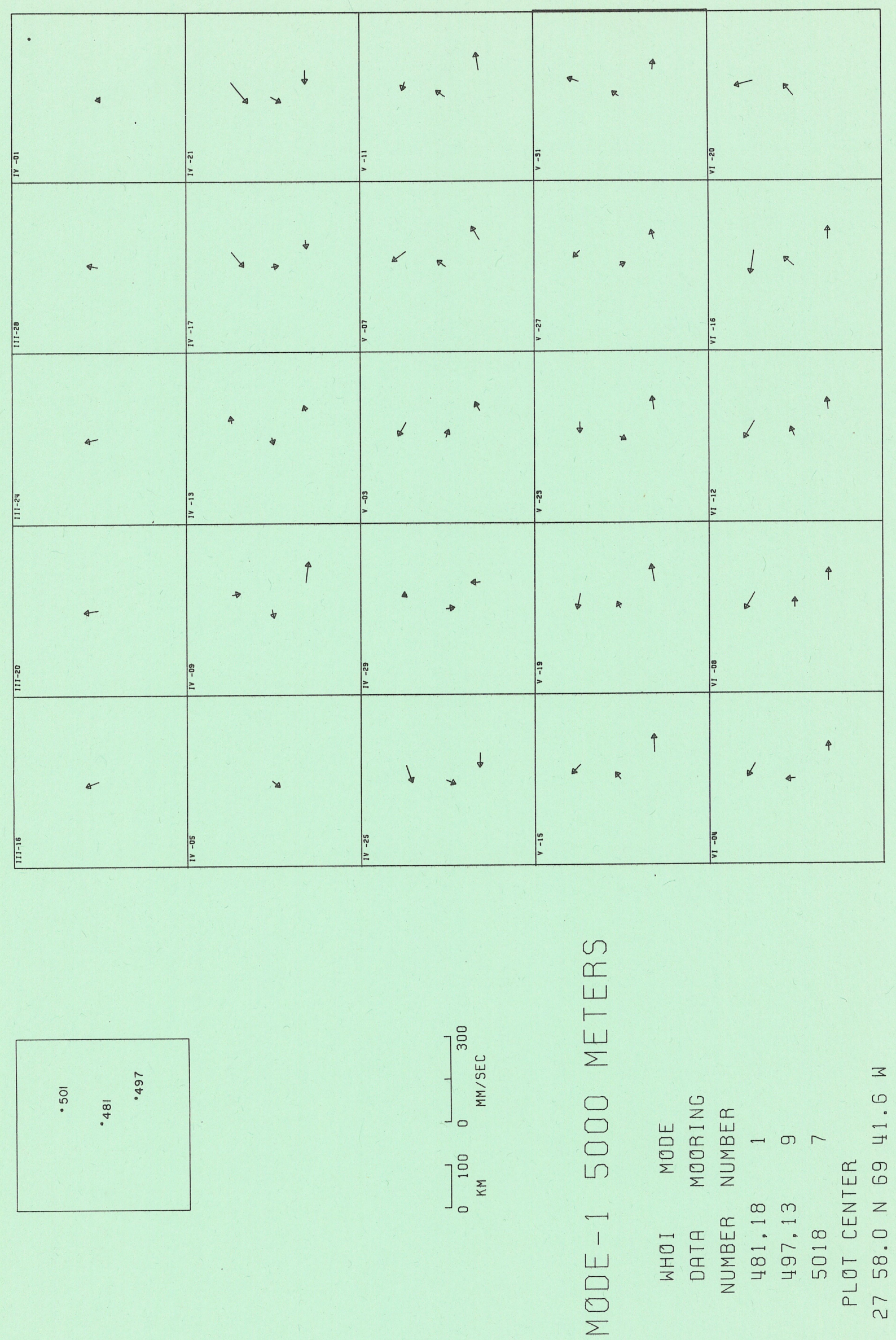

279 



\section{Appendixes}

Appendix I Project's Principal Investigators and Institutions

Page

Appendix II MODE Contribution Numbers

282

Appendix III MODE Hot Line News Index

285

293 


\section{APPENDIX I}

\section{Projects, Principal investigators and Institutions}

MOORED CURRENT METER ARRAYS

16 moorings with 48 current

meters each

5 moorings with 4 current meters each

8 moorings with 1 or 2 current

meters each

\section{BOTTOM MOUNTED INSTRUMENTS}

2 IGPP capsules, 1 month lifetime, 1 IGPP capsule, 1 year lifetime

(temperature, current, pressure

bottom kilometer)

6 inverted echo sounders

3 electric field recorders, and 3

bottom mounted magnetometers

5 bottom pressure recorders

(fused silica bourdon type)

FLOAT TRACKING

20 longrange SOFAR type

floats using MILS listening

stations

36 intermediate range acoustic

floats tracked by shipborne

hydrophones

Hydrophone arrays for

locating SOFAR floats
N. Fofonoff, W. Schmitz and

F. Webster

J. Swallow

J. Knauss

W. Sturges

W. Munk,

F. Snodgrass and

W. Brown

H. T. Rossby

C. Cox, V. Vacquier, Scripps Institution of

J. Filloux and

R. Parker

Oceanography, Univ. of

Calif., San Diego

D. J. Baker, Jr. Harvard University

Woods Hole Oceanographic Institution and Yale

University

J. Swallow

National Institute of Oceanography, England

R. Walden

H. Bertaux

Woods Hole Oceanographic Institution 
DENSITY MEASUREMENTS

Shipboard STD and CTD casts

MOORED THERMAL ARRAY

60 temperature-pressure recorders

(on W.H.O.I. moorings)

\section{TOWED INSTRUMENTS}

STD tows to map

isopycnal surfaces

FREE FALL INSTRUMENTS

Velocity profilers acoustically

tracked by using bottom mounted

transponders

Electric field free falling

probe and bottom recorders

Displacement type current probe

Airborne expendable (2000)

NUMERICAL MODELING AND

THEORETICAL STUDIES

Synoptic maps for MODE-I

Interactions between short internal gravity waves and larger scale motions in the

ocean

MODE array design as an

inverse problem

Theory and computer experiments on oceanic eddies and waves

Analytic and numerical studies

of mesoscale motions
D. Hansen

J. Crease

A. Leetmaa

R. Scarlet

C. Wunsch

E. Katz

R. Nowak

T. Pochapsky

T. Sanford

W. S. Richardson

F. Bretherton

K. Hasselmann

M. Hendershott,

R. Davis and

W. Munk

P. Rhines

A. R. Robinson
Atlantic Oceanographic and Meteorological Laboratory

National Institute of Oceanography, England Atlantic Oceanographic and Meteorological Laboratory Massachusetts Institute of Technology

Massachusetts Institute of Technology and Draper Laboratory

Woods Hole Oceanographic Institution

Columbia University

Woods Hole Oceanographic Institution

Nova University

The Johns Hopkins University

University of Hamburg
Scripps Institution of Oceanography

Woods Hole Oceanographic Institution

Harvard University 

A theoretical-numerical study of
P. Welander
University of Gothenburg
geostrophic eddy motions in the
oceans

\section{ADMINISTRATIVE \\ Funds for travel, executive of ficer, meetings, etc.}

H. Stommel and

D. Moore (on

Massachusetts Institute of leave from Nova

University)

\section{Additional Associated Projects}

5 Filloux-type bottom

H. Mofjeld

Atlantic Oceanographic and
Meteorological Laboratory

mounted tide gauges and

1 Hewlett Packard pressure

gauge

Monitoring earth's magnetic

J. Larsen

field at island stations

Bottom mounted vertical

electric field measurements

R. Harvey

Bottom mounted magnetometers

R. Von Herzen

\section{Ships}

R/V CHAIN Woods Hole Oceanographic Institution, Woods Hole, Mass.

R/V EASTWARD Duke University Marine Laboratory, Beaufort, N.C.

R/V TRIDENT University of Rhode Island, Narragansett, Rhode Island

RRS DISCOVERY National Institute of Oceanography, Wormley, England

R/V RESEARCHER Atlantic Oceanographic and Meteorological Laboratory, Miami, Florida

R/V HUNT MODE charter from TRACOR/Marine Acoustical Services,

Ft. Lauderdale, Florida 


\section{APPENDIX II}

\section{MODE CONTRIBUTIONS}

No.

1. Scarlet, R. I., 1973

STD's in MODE -- A Grab-Bag of Calibration Problems, Proceedings of the Second STD Conference (Supplement), Plessey, Environmental Systems, San Diego CA, January 1973.

2. Wunsch, C., and J. Dahlen, 1974

A Moored Temperature and Pressure Recorder, Deep-Sea Research, 21

(2) , 145-154 .

3. Katz, E., 1973

Profile of an Isopycnal Surface in the Main Thermocline of the Sargasso Sea, Journal of Physical Oceanography, 3 (4), 448-457.

4. Dantzler, L., Jr., 1974

Dynamic Salinity Calibration of Continuous Salinity/Temperature/ Depth Data, Deep-Sea Research, 21 (8), 675-682.

5. Gould, J., W. Schmitz, and C. Wunsch, 1974

Preliminary Field Results for a Mid-Ocean Dynamics Experiment (MODE-O), Deep-Sea Research, 21 (11), 911-932.

6. Baker, D. J., R. B. Wearn, Jr., and W. Hill, 1973

Pressure and Temperature Measurements at the Bottom of the Sargasso Sea, Nature 245 (141), 25-26.

7. Robinson, A. R., and J. McWilliams, 1974

The Baroclinic Instability of the Open Ocean, Journal of Physical Oceanography, 4 (3), 281-294.

8. McWilliams, J., 1973

Forced Transient Flow and Small-Scale Topography, Geophysical Fluid Dynamics, 6 (1), 49-79.

9. MCWilliams, J., and A. R. Robinson, 1974

A Wave Analysis of the Polygon Array in the Tropical Atlantic, DeepSea Research, 21 (5), 359-368.

10. Bryden, H. L., 1974 Geostrophic Comparisons Using MODE-I Moored Current Meter and Temperature Measurements, Nature 251, 409-410.

11. Swallow, J., B. S. McCartney, and N. W. Millard, 1974

The Minimode Float Tracking System, Deep-Sea Research, 21 (7), 573-595.

12. Scarlet, R. I., 1975

A Data Processing Method for STD Profiles, Deep-Sea Research, 22 (7), 509-515. 


\section{APPENDIX II (cont.)}

MODE CONTRIBUTIONS

No.

13. Katz, E. J., 1975

Tow Spectra from MODE, Journal of Geophysical Research, 80 (9), 1163-1167.

14. Snodgrass, F., W. Brown, and W. Munk, 1975

MODE: IGPP Measurements of Bottom Pressure and Temperature, Journal of Physical Oceanography, 5 (1), 63-74.

15. Brown, W., W. Munk, F. Snodgrass, H. Mofjeld, and B. Zetler, 1975

MODE Bottom Experiment, Journal of Physical Oceanography, 5 (1), 75-85.

16. Zetler, B., W. Munk, H. Mofjeld, W. Brown, and F. Dormer, 1975 MODE Tides, Journal of Physical Oceanography, 5, (1), 430-441.

17. Sanford, T. B., 1974 Observations of Strong Current Shears in the Deep Ocean and Some Implications on Sound Rays, Journal of the Acoustic Society of America, 56 (4), 1118-1121.

18. Zenk, W., and E. J. Katz, 1975

On the Stationarity of Temperature Spectra at High Horizontal Wave Numbers, Journal of Geophysical Research, 80 (27), 3885-3891.

19. Leaman, K. D., and T. B. Sanford, 1975

Vertical Energy Propagation of Inertial Waves: A Vector Spectral Analysis of Velocity Prcfiles, Journal of Geophysical Research, 80 (15), 1975-1978.

20. Withdrawn.

21. Bruce, J. G., and E. Firing, 1974 Temperature Measurements in the Upper $10 \mathrm{~m}$ with Modified Expendable Bathythermograph Probes, Journal of Geophysical Research, 79 (27), 4110-4111.

22. Scarlet, R. I., 1975

STDs in MODE: A Grab Bag of Calibration Problems: The Results, Proceedings of the Third STD Conference (Supplement), Plessey Environmental Systems, San Diego CA.

23. Bretherton, F. P., and M. Karweit, 1975

Midocean Mesoscale Modelling, Proceedings of Symposium on Numerical Models of Ocean Circulation, ISBN 0-309-02225-8, National Academy of Sciences.

24. Frankignoul, C., 1974

Observed Anisotropy of Spectral Characteristics of Internal Waves Induced by Low Frequency Current, Journal of Physical Oceanography, 4 (4), 625-634. 
APPENDIX II (cont.)

MODE CONTRIBUTIONS

25. Müller, P., 1974

On the Interaction Between Short Internal Waves and Larger Scale Motions

in the Ocean, Hamburger Geophysikalische Einzelschriften 23, 1-107.

26. Olbers, D., 1974

On the Energy Balance of Small-Scale Internal Waves in the Deep-Sea, Hamburger Geophysikalische Einzelschriften 24.

27. Müller, P., and D. J. Olbers, 1975

On the Dynamics of Internal Waves in the Deep Ocean, Journal of

Geophysical Research, 80 (27), 3848-3860.

28. Hayes, S. P., T. M. Joyce, and R. C. Millard, Jr., 1975

Measurements of Vertical Fine Structure in the Sargasso Sea, Journal of Geophysical Research (Internal Wave Issue), 80, (3), 314-319.

29. Dexter, S., J. Milliman, and W. Schmitz, 1975

Mineral Deposition in Current Meter Bearings, Deep-Sea Research, 22 (10), 703-706.

30. Bennett, D., and J. H. Filloux, 1975

Magnetotelluric Deep Electrical Sounding and Resistivity, Reviews of Geophysics and Space Physics, 13 (3), 197-239.

31. Hayes, S. P., 1975

Preliminary Measurements of the Time Lagged Coherence of Vertical

Temperature Profiles, Journal of Geophysical Research, 80 (3), 307-311.

32. Hayes, S. P., 1975

The Temperature and Salinity Fine Structure of the Mediterranean Water in the Western Atlantic, Deep-Sea Research, 22. (1), 1-11.

33. Sanford, T. B., 1975

Observations of the Vertical structure of Internal Waves, Journal of Geophysical Research, 80 (27), 3861-3871.

34. Robinson, A. R., 1975

The Variability of Ocean Currents, Reviews of Geophysics and Space

Physics, 13 (3), 598-601.

35. MCWilliams, J., 1976

Large Scale Inhomogeneities and Mesoscale Ocean Waves: A Single Stable Wave Field, Journal of Marine Research, 34 (3), 423-455.

36. McWilliams, J., and G. Flierl, 1976

Optimal Quasi-geostrophic Wave Analyses of MODE Array Data, Deep-Sea Research, 23. (4), 285-300.

37. Milliman, J., 1975

Dissolution of Aragonite, Mg-Calcite and Calcite in the North Atlantic, Geology, 3, 461-462. 
APPENDIX II (cont.)

MODE CONTRIBUTIONS

No.

38. Bretherton, F. P., R. E. Davis, and C. B. Fandry, 1976

A Technique for Objective Analysis and Design of Oceanographic Experiments Applied to MODE-73, Deep-Sea Research, 23 (7), 559-582.

39. Rhines, P., 1975

Waves and Turbulence on a Beta-Plane, Journal of Fluid Mechanics,

69, Part 3, 417-443.

40. Freeland, H., P. Rhines, and H. T. Rossby, 1975

Statistical Observations of the Trajectories of Neutrally Buoyant Floats in the North Atlantic, Journal of Marine Research, 33, Part 3, 383-404.

41. Rossby, H. T., A. Voorhis, and D. Webb, 1975

A Quasi-Lagrangian Study of Mid-Ocean Variability Using Long Range SOFAR Floats, Journal of Marine Research, 33, Part 3, 355-382.

42. Bretherton, F., 1975

Recent Developments in Dynamical Oceanography, Quarterly Journal of the Royal Meteorological Society, 101, 705-721.

43. Hogg, N., 1976

On Spatially Growing Baroclinic Waves in the Ocean, Journal of Fluid Mechanics (in press).

44. Huppert, H., and K. Bryan, 1976

Topographically Generated Eddies, Deep-Sea Research, 23 (8), 655-679.

45. Frankignoul, C., 1976

Observed Interaction Between Oceanic Internal Waves and Mesoscale

Eddies, Deep-Sea Research, 23 (9), 805-820.

46. Olbers, D., 1976

Nonlinear Energy Transfer and the Energy Balance of the Internal Wave

Field in the Deep Ocean, Journal of Fluid Mechanics, 74, Part 2, 375-399.

47. MCWilliams, J., 1975

On the Large Scale Circulation of the Ocean: A Discussion for the Unfamiliar, Proceedings from Symposium on the Prediction of Sound Scattering in the Oceans from Physical/Chemical/Biological Information, Asilomar, California, Plenum Press, Nov. 10-14.

48. McWilliams, J.,

Maps from the Mid-Ocean Dynamics Experiment, I., Geostrophic Streamfunction, Journal of Physical Oceanography (submitted).

49. McWilliams, J.,

Maps from the Mid-Ocean Dynamics Experiment, II., Potential Vorticity and its conservation, Journal of Physical Oceanography (submitted). 
APPENDIX II (cont.)

MODE CONTRIBUTIONS

No.

50. Hirsch, J. E., and W. L. Briggs,

Sparse Matrix Problems in a Finite Element Open Ocean Model, Sparse Matrix Computations, J. R. Burch and D. J. Rose, editors, Academic Press, NY (to appear).

51. Rhines, P., The Dynamics of Unsteady Currents, Volume Six of The Sea, John Wiley and Sons, New York (in press).

52. Bryden, H. , Horizontal Advection of Temperature for Low Frequency Motions, Deep-Sea Research (submitted).

53. Dantzler, L., 1976

Geostrophic Variations in Intensity of the North Atlantic and North Pacific Oceanic Eddy Fields, Deep-Sea Research, 23 (9), 783-794.

54. Wunsch, C., 1976

Geographical Variability of the Internal Wave Field: A Search

for Sources and Sinks, Journal of Physical Oceanography, 6 (4), 471-485.

55. Milliman, J.,

Dissolution of Calcium Carbonate in the Sargasso Sea, Fate of Fossil $\mathrm{CO}_{2}$

in the Oceans, Studies in Oceanography, Plenum Press (In press).

56. Leaman, K.,

Observations on the Vertical Polarization and Energy Flux of NearInertial Waves, Journal of Physical Oceanography (submitted).

57. Bush, P., Bathymetry of the MODE-I Region, Deep-Sea Research (submitted).

58. Pochapsky, T. E., 1976

Vertical Structure of Currents and Deep Temperatures in the Western Sargasso Sea, Journal of Physical Oceanography, 6 (1), 45-56.

59. McWilliams, J., 1976

Mapping the Weather in the Sea, Oceanus, 19 (3), 77-81.

60. Bryden, H. ,

Geostrophic Comparisons from Moored Measurements of Current and Temperature During MODE, Deep-Sea Research (submitted).

61. Schmitz, W. J., Jr., 1976

Eddy Kinetic Energy in the Deep Western North Atlantic, Journal of Geophysical Research, 81 (27), 4981-2. 
No.

62. Schmitz, W. J., Jr., 1976

Observation of a New Abyssal Current, Geophysical Research Letters 3 ( $(7), 373-374$.

63. Freeland, H., and J. Gould, Objective Analysis of Mesoscale Ocean Circulation Features, Deep-Sea Research (submitted).

64. Leetmaa, A., A Study of MODE Dynamics, Deep-Sea Research (submitted).

65. Voorhis, A. D., E. H. Schroeder, and A. Leetma, The Influence of the Deep Mesoscale Eddies on Sea Surface Temperature in the North Atlantic Subtropical Convergence, Journal of Physical Oceanography (submitted).

66. Schmitz, W. J., Jr., On the Deep General Circulation in the Western North Atlantic, Journal of Marine Research (submitted).

67. Owens, W. B., and F. P. Bretherton, A Numerical Study of Mid-Ocean Mesoscale Eddies, Deep-Sea Research (submitted).

68. Joyce, T. M., Large-Scale Variations in Small-Scale Temperature/Salinity Fine Structure in the Main Thermocline of the Northwest Atlantic, Deep-Sea Research (submitted).

69. Bryden, H., and N. Fofonoff, Horizontal Divergence and Vorticity Estimates from Velocity and Temperature Measurements in the MODE region, Journal of Physical Oceanography (submitted).

70. Hendry, R., Observations of the Semidiurnal Internal Tide in the Western North Atlantic Ocean, Philosophical Transactions of the Royal Society of London, Series A (in press).

71. Leetmaa, A., Observations of Large Scale Depth Perturbations of the Main Thermocline, Journal of Physical Oceanography (submitted).

72. Flierl, G., and J. McWilliams, On the sampling Requirements for Measuring Moments of Eddy Variability, Journal of Marine Research (submitted).

73. MCWilliams, J., A Note on a Consistent Quasigeostrophic Model in a Multiply-Connected Domain with Both Buoyancy and Mechanical Forcing and Diffusion, Dynamics of Atmosphere and Oceans (submitted). 
APPENDIX II (cont.)

MODE CONTRIBUTIONS

\section{Technical Reports}

1. Walden, R. G., H. O. Berteaux, and F. Striffler, 1973

The Design, Logistics, and Installation of a SOFAR Float Tracking

Station at Grand Turk Island, B.W.I.,W.H.O.I. Ref. 73-73 (unpublished manuscript).

2. MCWilliams, J. C., and A. Robinson, 1973

On the Greater MODE Region Linearized Wave Properties, Reports in Meteorology and Oceanography, No. 7, Division of Engineering and Applied Physics, Harvard University.

3. Swallow, J. C., 1973

R.R.S. Discovery Cruise 53 (April-June, 1973), N.I.O. Cruise Report 58.

4. Voorhis, A., and D. Webb, 1973

Data Summary and Review of Shipboard SOFAR Float Program, from September, 1972 through July, 1973, W.H.O.I. Ref. 73-74 (unpublished manuscript).

5. Sanford, T., R. Drever, and J. H. Dunlap, 1974

The Design and Performance of a Free-Fall Electro-Magnetic Velocity Profiler (EMVP) July, 1974, W.H.O.I. Ref. 74-46 (unpublished manuscript).

6. Voorhis, A. D., and R. Benoit, 1974

MODE SOFAR Float in situ Data Summary, W.H.O.I. Ref. 74-37 (unpublished manuscript).

7. MCCullough, J. R., 1974

In search of moored current sensors, Reprinted from Marine Technology Society 10th Annual Conference Proceedings, September 1974.

8. Bryden, H. , 1975

Momentum, mass, heat, and vorticity balances from oceanic measurements of current and temperature, Ph.D. Thesis, W.H.O.I.-M.I.T. Joint Program.

9. Pochapsky, T. E., and F. D. Malone, 1975

Profiles of transport, current, and temperature in the MODE Areas of the North Atlantic, July 1975, Technical Report No. 2 CU-2-75, Office of Naval Research, Technical Report No. 1 CU-1-75, National Science Foundation.

10. Karweit, M., 1975

Some visual interpretations of MODE - a 17-minute color computer film interpretation of MODE-I data with subtitles. The Johns Hopkins University.

11. MCWilliams, J., and B. Owens, 1976

Estimation of spatial covariance from the Mid-Ocean Dynamics

Experiment, National Center for Atmospheric Research, T/N-115 - STR, February 1976 


\section{Technical Reports}

12. Heinmiller, R. H., 1975

Mooring operations of the Woods Hole Buoy Group for the Mid-Ocean Dynamics Experiment, W.H.O.I. Ref. 75-42 (unpublished manuscript).

13. MODE-I Theoretical Panel, 1972

Dynamics and the design of MODE-I, A Report to the MODE-I Scientific Council.

14. MODE-I Scientific Council, 1973

MODE-I: The Program and Plan.

15. MODE-I Data Analysis Committee (compiled by K. Daly), 1974

MODE-I Data Inventory.

16. MODE-I Dynamics Group, 1975

Dynamics and the Analysis of MODE-I, A Report to the MODE-I Scientific Council.

17. MODE-I Intercomparison Group, 1974

Instrument Description and Intercomparison Report, A Report to the MODE-I Scientific Council.

18. MODE-I Synoptic Atlas Group, 1974

MODE-I Draft Synoptic Atlas, A Report to the MODE-I Scientific Council.

19. Walen, R., 1976

Shore based receivers used in the POLYMODE Program, W.H.O.I. Ref. 76-72 (unpublished manuscript).

20. Drever, R., and T. Sanford, 1976

A velocity profiler based on acoustic Doppler principles, W.H.O.I. Ref. 76-96 (unpublished manuscript).

21. Chausse, D., and S. Tarbell, A compilation of moored current data and associated ocean observations, Volume XII, 1973 MODE, W.H.O.I.Ref. (submitted). 


\section{MODE HOT LINE NEWS NOS. $1-85$ TABLE OF CONTENTS}

No. 1 (March 17, 1972)

Anonymous: Purpose of MODE Hot Line News

Schmitz, Sturges: First results from Array-1 current measurements

No. 2 (March 31, 1972)

Bretherton: A numerical model of meso-scale ocean

Moller: Now survey of MODE region bottom topography Rhines: MODE monster

No. 3 (April 14, 1972)

Anonymous: MODE Scientific Council Meeting

Fuglister: First results from a non-existent current

meter array

Gould, Sturges: Velocity vectors from Array-1

No. 4 (April 28, 1972)

Katz: Results of tows along isopycnal surfaces

McCullough: A temperature time series

Wunsch: Array-1 wavenumber spectrum

No. 5 (May 12, 1972)

Fofonoff: Brunt-Väisälä periods in the MODE region

Millard: CTD microprofiler temperature measurements

No. 6 (May 26, 1972)

Churgin: Temperature profiles in the upper layer

Moller: Navigation in the MODE region

Rossby, Sanford: Vertical profiles of horizontal

motions

No. 7 (June 9, 1972)

Scarlet: Dynamic height maps

Scarlet: Temperature-salinity microstructure

Volkmann: Temperature profile contrasts

No. 8 (June 23, 1972)

Baker, Hill, Wearn: A test of the fused-quartz pressure

Fofonoff: MODE-O cruise schedule

Savonious Rotor: What's Going Around

Schmitz, Gould: Summary of velocities from Array-1

No. 9 (July 7, 1972)

Anonymous: Committee Structure of MODE

Anonymous: Schedule for formulating MODE-I final plan Beckerle, LaCasce: Horizontal sound-speed patterns in the main thermocline

Fofonoff: Tentative MODE-I ship schedule

Gould: A vertical-velocity spectrum

Savonius Rotor: What's Going Around

No. 10 (July 21, 1972)

Davis: Array-1, velocity correlations

Fandry, Bretherton: Correlation functions and

synoptic maps from Array-1 data

Gould: Current measurements from surface and subsurface moorings

No. 11 (August 4, 1972)

Anonymous: Indices for MODE Hot Line News Nos. 1-11

Gould: Current measurements from Array-2

Savonius Rotor: What's Going Around

Wunsch: Coherence between current meter records on mooring 406

Wunsch: Wavenumber spectrum from Array-1

No. 12 (August 18, 1972)

Fomin: Vertical structure of mesoscale currents

from the POLYGON array
Gould: Horizontal velocity spectra from Array- 2 Koshlyakov, Grachev: Mesoscale currents from the POLYGON array

Richman: Vertical modal structure of currents

Riser, Wunsch: Periodograms of Array-1 current meter and temperature records

Savonius Rotor: What's Going Around
No. 13 (September 1, 1972)

Munk: Early prejudices on MODE units

Savonius Rotor: What's Going Around

Stommel: Vertical modal structure of long-period

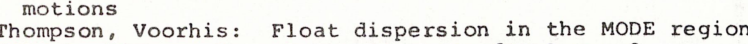
Wunsch, Riser: Wavenumber spectrum for Array-2

No. 14 (September 15, 1972)

Anonymous: MODE meeting

Gould: The SCOR intercomparison experiment

Katz: Repeated horizontal profile of a density surface

Katz: Vertical temperature gradients in the main thermocline

Scarlet: Shear measurements: Hydro stations vs. current meters

Webster: Comments on the variability of ocean current spectra

No. 15 (September 29, 1972)

Anonymous: Where will MODE be meeting?

Baker: R/V Eastward cruise, $18-30$ August 1972 motion investigation based on data from the POLYGON array

McWilliams, Robinson: Free-wave geography of the greater MODE region

Millard: Vertical temperature spectra in the MODE region

Savonius Rotor: What's Going Around

No. 16 (October 13, 1972)

Chhabra: Mooring dynamics experiment

Gould: SCOR mocrings--some preliminary results

Rhines: Scales, patterns, and spectra

No. 17 (October 27,1972 )

Dantzler: Stability of small-scale density patterns McWilliams: Bathymetry of the greater MODE region Savonius Rotor: What's Going Around

No. 18 (November 10, 1972)

Brown: Deep-sea pressure-temperature measurements in the Pacific

Millard, Joyce, Garrett: Further comments on vertical temperature spectra in the MODE region

Po topsky. Malone: MODE region bottom topography.

Rossby: SOFAR float tracks

Rcarlet: Temperature profile for the MODE region

No. 19 (November 24, 1972)

Anonymous: MODE Scientific Council Meeting

Fofonoff: MODE-I ship schedule

Katz: Spatial description of a temperature surface

Sanford: Vertical profiles of velocity

Swallow: MINIMODE trial results

No. 20 (December 8, 1972)

Hansen: Near-surface drogue buoy trajectories

Hayes: Isotherm depth changes in the MODE region

Rossby: SOFAR float tracks, 28 Sept. -14 Nov.

Savonius Rotor: What's Going Around

Walden, Berteaux: Hydrophone array off Turks Island

No. 21 (December 22, 1972)

Anonymous: Indices for MODE Hot Line News Nos. 1-20 Gould: Further data from the SCOR intercomparison Savonius Rotor: What's Going Around Stommel, Volkmann: MODE topography revisited Sturges: $10^{\circ}$ isotherm topography Sullivan: Bottom photographs in the MODE region Volkmann: A MODE seamount

No. 22 (January 5, 1973)

Baker: R/V Eastward cruise, 20 November-3 December

Gould: Velocity vectors from Array-3

Rossby: SOFAR float tracks, 19 Nov.-4 Dec. 


\section{MODE HOT LINE NEWS NOS. 1-85 \\ TABLE OF CONTENTS (CONTINUED)}

\section{No. 23 (January 19, 1973)}

Bretherton, Fandry: Data analysis competition Fofonoff: Revised MODE-I ship schedule

Richardson, Yager: Airdrop current measurements in

Wunsch: preliminary results from the MODE temperaturepressure recorders

No. 24 (February 2, 1973)

Bretherton, Fandry: Solution for mapping competition Chekotillo, Schmitz: Notes on the properties of mesoscale motions from Array-3

Hasselmann: The VACM: a parametric amplifier?

Katz: Isotropy of tow spectra

Moore: MODE contribution numbers

Savonius Rotor: What's Going Around

No. 25 (February 16, 1973)

Bretherton, Fandry: Objective analysis of MODE-

type simulated data

Fofonoff: MODE-I ship schedule

No. 26 (March 2, 1973)

Rhines: Eddies and waves

Rossby: SOFAR float tracks

Walden, Berteaux: Vertical excursions of an instrument on a surface mooring

No. 27 (March 16, 1973)

MODE Scientific Council: Scientific program and detailed plan of the field program for MODE-I

No. 28 (March 30, 1973)

Appel1, Kalvaitis, Woodward: Dynamic testing of current meters by NOIC

Baker, Wearn, Hill: Bottom pressure and temperature measurements in the MODE area

Hasselmann, Müller, Olbers: Comments on micro-

Heinmiller: First MODE leg of $R / V$ Chain

No. 29 (April 13, 1973)

Heinmiller: Second MODE leg of $R / V$ Chain

Kalvaitis: Further comments on dynamic testing of current meters by NOIC

Millard: Homogeneous bottom layer in the MODE area Rossby: Four-month SOFAR float tracks for Floats 4

and 12

Scarlet: First MODE leg of R/V Trident

No. 30 (April 27, 1973)

Hogg: Steady flow past isolated topography

Joyce: Contamination of horizontal velocities

by internal waves

Richman: Brunt-Väisälä frequency for the MODE area

No. 31 (May 11, 1973)

Anonymous: Indices for MODE Hot Line News Nos. 1-30

Filloux: First MODE leg of R/V llunt

Hess, Nowak, Katz: The towfish



olbers: Energy transfer in an internal wave field

Swallow: First MODE leg of RRS Discovery

No. 32 (May 25, 1973)

Monget: Time-space pattern classification of current meter array

üller: Interaction between internal waves and larger-scale motions

Saunders: Flow tracing by isotherm tracking
No. 33 (June 8, 1973)

Gould, Cherriman: Mooring work for RRS Discovery Howe: Surface currents derived from ship's navigational system

Sturges: Second MODE leg of $R / V$ Hunt

Swallow: Second MODE leg of RRS Discovery

Wunsch: Fourth MODE leg of $R / V$ chain

No. 34 (June 22, 1973)

Briscoe: The Internal Wave Experiment (IWEX) Brown: Third MODE leg of $R / V$ Hunt

Leetmaa: MODE legs I, II, and III of $R / V$ Researcher

Müller: Correlations between internal waves and

mean currents

Pochapsky, Malone: Current profiles

Scarlet: "Second MODE leg of $R / V$ Trident

No. 35 (July 6, 1973)

Bryan: A model study of abyssal currents in the Array -2 area

Dahlen: MODE temperature-pressure recorde

Hansen: Fourth MODE leg of R/V Researcher

Moore: MODE leg III-1/2 of $\mathrm{R} / \mathrm{V}$ Hunt

Robinson: Fifth MODE leg of $R / V$ Chain
Stommel: Fourth MODE leg of $R / V$ Hunt

No. 36 (July 20, 1973)

Baker: Second MODE leg of R/V Eastward

Heinmiller: Sixth MODE leg of R/V Chain

Mofjeld: Fifth MODE leg of $R / V$ Researcher

First MODE leg of R/V Eastward

No. 37 (August 3, 1973)

Brown, Munk, Snodgrass, Zetler: Bottom pressures in the MODE region

crépon: Complex correlation and characteristic

curves for MODE-O data

Hasselmann: Wave influences on current measurements

No. 38 (August 24, 1973)

Brown: Bottom temperature and pressure measurements in the MODE region

Brown: Fifth MODE leg of $R / V$ Hunt

Daly: Survey of MODE-I field program results

MODE Office: MODE contribution numbers $1-5$

No. 39 (September 14, 1973)

Davis: MODE-I temperature field statistics

Rossby: Trajectories of the MODE I-1/2 float experiment for July and Augus

Welander: The "frozen field"-model versus the vorticity equation

No. 40 (September 28, 1973)

Briscoe, Hayes: Current measurements from surface

Pollard, Halpern: and subsurface moorings

No. 41 (October 12, 1973)

Anonymous: Indices for MODE Hot Line News Nos: 1-40

Katz: Horizontal profiles of the $12^{\circ} \mathrm{C}$ isotherm during MODE-I

Rossby: Inverted Echo Sounder--a description and some results from MODE-I

No. 42 (October 26, 1973)

Anonymous: MODE Scientific Council Meeting

Flierl, McWilliams, Robinson, Harrison: Subjective mapping and quasigeostrophic wave interpretation of MODE-I real-time density data

Rossby, Taylor: Trajectories of the MODE-I-1/2

float experiment for September

Addendum: MODE-I moored array and bottom-mounted ins trumentation 


\section{MODE HOT LINE NEWS NOS. $1-85$ \\ TABLE OF CONTENTS (CONTINUED)}

No. 43 (November 9, 1973)

Bruce: potential temperature/salinity sections across the western North Atlantic

Bruce: Surface temperature measurements with modified XBT probes

Fofonoff: Potential temperature/salinity curves from MODE-I CTD stations

Millard, Bryden: Spatially-averaged MODE-I CTD stations

No. 44 (November 23, 1973)

Boicourt: Current measurements from a subsurface mooring and a rigid tower

Flierl, McWilliams, Robinson, Harrison: Subjective mapping and quasigeostrophic wave interpretation of MODE-I real-time density data--a clarification Kalvaitis: Current measurements from surface and subsurface moorings

Notes from the Editor

No. 45 (December 7, 1973)

Haycs: Temperature and salinity fine structure due to Mcditerranean water

Hunkins: Subsurface eddies in the Arctic Ocean zenk: Could the Mediterranean double maxima have influenced the MODE-I CTD stations?

Notes from the Editor

No. 46 (Decembcr 21, 1973)

Gould: MINIMODE current meter results

Joyce: MODE-I temperature measurements

Notes from the Editor

No. 47 (January 4, 1974)

Briscoe: Near surface experiment at MODE site scarlet: Progress report from the MODE-I density profile experiment

profile experiment
Schmitz: MODE session at American Geophysical

Union meeting

Union meeting

No. 48 (January 18, 1974)

Bush: Preliminary ship track and bathymetric data

for the MODE area

for the MOFAR float trajectories for october

Schmitz, Payne: Status of MODE-I moored temperature

Schmitz, Payne:

Swallow: Velocity differences between closely spaced floats

No. 49 (February

Gi11: Mid-ocean eddies in Ocean Weather Ship records Rossby: Westward intensification of the eddy field

Notes from the Editor

No. 50 (February 15, 1974)

Bryden: Geostrophic calculations from MODE-I moored

temperature data
wunsch, Hogg, Richman: Results from temperaturepressure recorders during MODE-I

No. 51 (March 1, 1974)

Moller: Acoustic navigation during IWEX

Rossby: SOFAR float trajectories for November,

December, and January

No. 52 (March 15, 1974)

Dantzler: Main-thermocline depth fluctuations in the central North Atlantic

Millard: CTD averages from IWEX

Mamburo: Vertical temperature coherence from MODE-I tows Notes from the Editor
No. 53 (March 29, 1974)

Freeland, Rossby, Rhines: Westward intensification of the MODE eddy field

Joyce: IWEX temperature measurements

Simmons: POLYMODE planning

Zenk: Vertical displacement spectra at high wavenumbers from MODE-I tows

No. 54 (April 12, 1974)

Dean: Threshold test of current meters

Frankignoul: Energy correspondences in the internal wave field

Pochapsky, Malone: Current profiles and hodographs

№. 55 (April 26, 1974)

Holland, Lin: Generation of MODE-like eddics and their interaction with the mean circulation

Saunders: Intercomparison of near-surface current

measurements

No. 56 (May 10, 1974)

Bryan: Can baroclinically unstable eddies be detected by hydrographic stations?

Dantzler: The western boundary current system as a source for the mesoscale baroclinic eddy field

Rhines: B-plane mean flow: a simple theory

Notes from the Editor

No. 57 (May 24, 1974)

McWilliams: MODE mean currents and eddy surface intensification

Robinson, Mintz, Haney, Han, Takano: Meso-scale eddies in a model gyre: a numerical experiment with steady forcing and simplified geometry

Scarlet: (a) Densities without salinities (b) Waves vs. eddies: a "dynamic signature" in the density data? (c) Modal dynamic height maps

No. 58 (June 7, 1974)

Dantzler: Structure functions of dynamic thickness anomaly across the thermocline

Katz: High-resolution contour maps of the $12^{\circ}$ isotherm

Leaman: Current profiles from MODE-I electromagnetic data

Rossby: SOFAR float trajectories for April-December, 1973

Rossby, Dow: SOFAR flnat trajectories for March and Aprii, 1974

No. 59 (June 21, 1974)

Briscoe: Exponential fits to the MODE area Väisälä protile

Gill: Theoretical studies on mid-ocean eddies: Part I MODE Officc: MODE contribution and technical report nos

No. 60 (July 5,1974 )

Gill: Theoretical studies on mid-ocean eddics: II Millard: Bottom layer observations from MODE and IWEX Scarlet: Correlation functions from the MODE-I density data

No. 61 (July 19, 1974)

Schmitz, Luyten, Sturges: The role of eddies in the general circulation of the deep western North

zenk: Tenperature variability below the mixed layer in the MODE-I area

Index for MODE Hot Line News nos. 1-60

No. 62 (August 2, 1974)

Frankignoul: Intcraction between internal waves and low-frequency currents

Mofjeld: Bottom pressure fluctuations in MODE-I Panicker: Effect of cable material on surface mooring dynamics 


\section{MODE HOT LINE NEWS NOS. 1-85 TABLE OF CONTENTS (CONTINUED)}

No. 63 (August 16, 1974)

Freeland: Spatial dependence of variance in the MODE area Koshlyakov: Phase velocity of mesoscale baroclinic eddies along the $56^{\circ} \mathrm{W}$ meridian (for the POLYMODE project) Richman: Vertical correlation and horizontal coherence

of moored temperature

No. 64 (August 30,1974 )

Fedorov: Temperature inversions of intrusive origin in the ocean

Rossby: Float tracks for May and June, 1974

Sturges: Extended pattern recognition array

No. 65 (September 13, 1974)

Heinmiller: Bottom topography at POLYMODE-O sites Heinmiller: Report on $R / V$ Chain cruise 116

Holloway and Hendershott: The effect of bottom relief on barotropic eddy fields

Huppert and Freeland: MODE bathymetry

No. 66 (September 27, 1974)

Scarlet: Correlation maps for the MODE-I density data

sturges: Time scales of deep currents during MODE-I

No. 67 (October 25, 1974)

Dorson: Experimental float no. 4

MODE Office: MODE contribution numbers and technical

reports

MODE Office: POLYMODE ships of opportunity

Moore: Empirical orthogonal functions--a non-statistical

view

No. 68 (December 6, 1974)

Gonella, Lamy: The effect of platform on an intercomparison of Aanderaa current meters

Saunders: More intercomparisons of near-surface current measurements

No. 69 (January 17, 1975)

Clay: The all-wire mooring

Richman: Temperature records from MODE-I moorings 1 and 8 Schmitz, Heinmiller: Notes on mooring and current meter performance

No. 70 (February 14, 1975)

Bush: Bathymetry of the MODE-I region

Rossby, Evans: An XBT section

No. 71 (March 14, 1975)

Larichev, Reznik: Nonlinear Rossby waves as a model of mesoscale eddies

Riser: Temperature events at MODE-I site moorings 1 and 8 Index for MODE Hot Line News nos. 1-70

Notes from the Editor

No. 72 (April 11, 1975)

Frankignoul: Some statistical features of high-

frequency motions in MODE-I

killworth and Anderson: Meaningless modes?

No. 73 (May 9, 1975)

Killworth and Anderson: Some thoughts on eddy generation Mirabel, Vasilenko: Velocity spectra from 15 days to 2 hours at a hydrophysical polygon in the tropical Atlantic

No. 74 (June 6, 1975)

Gould, Swallow: First field phase of the I.O.S. topographic experiment

McCartney: Big Babies in the northern Sargasso sea
№. 75 (July 4, 1975)

Frankignoul: Modulation of the internal wave field by mesoscale eddies

Gould: A comparison of measured surface currents and geostrophic shear

Tranter and Webb: Rossby-wave simulation of SOFAR float. tracks

No. 76 (July 18, 1975)

Owens, Bretherton: A numerical simulation of the MODE-I statistics

Rossby, Dow, Freeland: Float tracks for May-December, 1974

No. 77 (August 1, 1975)

Huppert: Cold water over Brontosaurus Bump

Wunsch: Dynamical and kinematical tests with coherent arrays

No. 78 (August 15, 1975)

Killworth, Anderson: Horizontal and vertical fitting of MODE-type data

Williams: Some ocean-Jupiter connections

No. 79 (September 5, 1975) Albuisson, Monget: The structure function as a criterion
for data quality assessment of oceanographic time-serie Cheney, Richardson: Distribution of Gulf Stream rings in the northwestern Sargasso Sea

MODE Office: MODE contribution numbers

No. 80 (September 26,1975 )

Lai, Richardson: Distribution and movement of cyclonic Gulf Stream rings from historical data (1970-1973)

Rhines: Moscow Diary (13 June-2 July, 1975)

Thompson: Dynamical tests with single current meters

No. 81 (October 10, 1975)

Robinson: Summary of theoretical and numerical modelling of low-frequency mesoscale processes

Indices for MODE Hot Line News Nos. 1-80

No. 82 (October 17, 1975)

Gould, Hendry, Huppert, Webb: I.O.S. topographic cruise leg 2

Rooth: Eddy fluctuations refelected in tracer fields

No. 83 (November 7, 1975)

Cheney, Gemmil1, Shank, Richardson, Webb: Ring

tracking experiment

Strong, McClain: Satellite applications to oceanography by NOAA

No. 84 (November 28,1975 )

Noble: XBT sections from $R / V$ Chain 127

Seaver: Two XBT sectios in the North Atlantic Volkmann: Some XBT sections in the western North Atlantic

Wilson, Dugan: Thermal structure south of the Kuroshio Extension

No. 85 (December 19, 1975)

Flierl: Quasi-Eulerian quantities derived from float measurements and true Eulerian quantities

Grachev: Statistical structure and velocity mapping at the North Atlantic hydrophysical Polygon Yamagata: Rossby wave propagation in a lateral 


\section{MODE Hot Line News Nos. 1-85 CONTRIBUTOR INDEX} Anonymous: $1,3,9,11,14,15$
$19,21,31,41,51,61,71,81$ Albuisson: 79

Anderson: $72,73,78$

Baker: $8,15,22,28,36$

Beckerle: 9

Berteaux: 20,26

Bishev: 15

Boicourt: 44

Bretherton: 2, 10, 23, 24, 25, $7 \epsilon$

Briscoe: $34,40,47,59$

Brown: $18,34,37,38$

Bruce: 43

Bryan: 35,56

Bryden: 43,50

Bush: 48,70

Chekotillo: 15, 24

Cheney: 79,83

Cherriman: 33

Chhabra: 16

churgin: 6

clay: 69

Crépon: 37

Dahlen: 35

Daly: 38

Dantzler: 17, 52, 56, 58

Davis: 10,39

Dean: 54

Dorson: 67

Dow: 68,76

Dugan: 84

Fandry: $10,23,24,25$

Fedorov: 64

Flierl: $42,44,85$

Fofonoff: $5,8,9,19,23$ 25,43

Fomin: 12

Frankignoul: $54,62,72,75$

Freeland: $53,63,65,76$

Fuglister: 3

Garrett: 18

Gemmill: 83

Gill: 49, 59, 60

Gonella: 68

Gould: $3,8,9,10,11,12,14$ $16,21,22,33,46,74,75$

$$
82
$$

Grachev: 12,85

Halpern: 40

Han: 57

Haney: 57

Hansen: 20,35

Harrison: 42,44
Hasselmann: $24,28,37$

Hayes: $20,40,45$

Heinmiller: $28,29,36,65$ 69

Hendershott: 65

Hendry: 82

Hess: 31

Hill: 8,28

Hogg: 30,50

Holl and : 55

Holloway: 65

Howe: 33

Hunkins: 45

Huppert: $65,77,82$

Joyce: $18,30,46,53$

Kalvaitis: $28,29,44$

Katz: $4,14,19,24,31$ 41,58

Killworth: $72,73,78$

Koshlyakov: 12, 63

Lacasce: 9

Lai: 80

Lamy: 68

Larichev: 71

Leaman : 58

Leetmaa: 34

Lin: 55

Luyten: 61

Malone: $18,34,36$

McCartney: 74

MCClain: 83

McCullough: 4

McWilliams: $15,17,42$

$$
44,57
$$

Millard: $5,15,18,29,43$

Mintz: 57

Mirabel: 73

MODE office: $38,59,67,79$

MODE Scientific Council: 27

Mofjeld: 36,62

Mollex: 2, 6, 51

Monget: 32,79

Moore: $24,35,67$

Müller: $28,32,34$

Munk: 13,37

Noble: 84

NOTES from the Editor: 44, 45 $46,49,52,56,71$

Nowak: 31

Olbers: 28,31

Owens: 76

Panicker: 62

Pochapsky: 18, 34, 36, 54

Pollard: 40

Payne: 48
Reznik: 71

Rhines: 2, 16, 26, 53, 56, 80

Richardson, P.: 79, 80,83

Richardson, W.: 23

Richman: $12,30,50,63,69$

Riser: $12,13,71$

Robinson: $15,35,42,44,57$ 81

Rossby: $6,18,20,22,26$

$29,39,41,42,48,49,51$

$53,55,58,64,70,76$

Sambuco: 52

Sanford: 6,19

Saunders: $32,55,68$

Savonius Rotor: $8,9,11,12$

$13,15,17,20,21,24$

Scarlet: $7,14,18,29,34,47$ $57,60,66$

Schmitz: $1,8,24,47,48,61$ 69

Seaver: 84

Shank: 83

Simmons: 53

Snodgrass: 37

Stommel: $13,21,35$

Strong: 83

Sturges: $1,3,21,33,61$ 64,66

Sullivan: 21

Swallow: 19, 31, 33, 48, 74

Takano: 57

Taylox: 42

Thompson: 13, 80

Tranter: 75

Vasilenko: 73

Volkmann: 7, 21, 84

Voorhis: 13

Walden: 20,26

Wearn: 8,28

Webb, D. C.: 83

Webb, D. J.: 75, 82

Webster: 14

Welander: 39

Whitehead: 56

Williams: 78

Wilson: 84

Woodward: 28

Worthington: 47

Wunsch: $4,11,12,13,23$

$33,40,77$

Yager: 23

Yamagata: 85

zenk: $45,53,61$

zetler: 37 



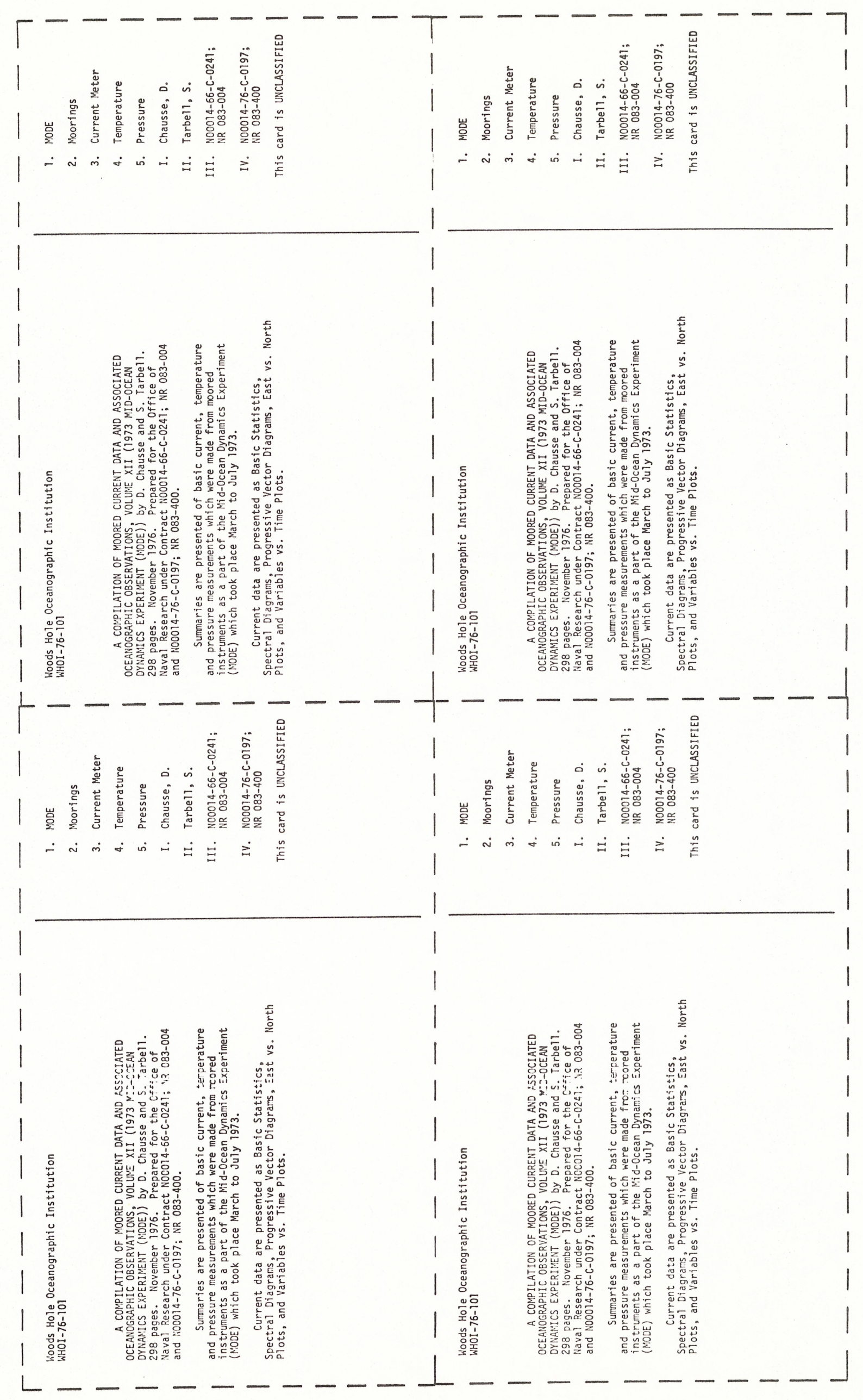



MANDATORY DISTRIBUTION LIST

FOR UNCLASSIFIED TECHNICAL REPORTS, REPRINTS, \& FINAL REPORTS

PUBLISHED BY OCEANOGRAPHIC CONTRACTORS

OF THE OCEAN SCIENCE AND TECHNOLOGY DIVISION

OF THE OFFICE OF NAVAL RESEARCH

(REVISED OCT 。 1975)

Director of Defense Research and Engineering

Office of the Secretary of Defense Washington, DC 20301

ATTN: Office Assistant Director (Research)

Office of Naval Research

Arlington, VA 22217

3 ATTN: (Code 480)

1 ATTN: (Code 460)

1 ATTN: (Code 102-OS)

6 ATTN: (Code 102IP)

1 ATTN: (Code 200)

1 LCDR David Cacchione, (USN)

ONR Representative

Woods Hole Oceanographic Inst.

Woods Hole, MA 02543

1 Office of Naval Research

Branch Office

495 Summer Street

Boston, MA 02210

Director

Naval Research Laboratory

Washington, DC 20375

6 ATTN: Library, Code 2620

1 National Oceanographic Data Center

National Oceanic \& Atmospheric

Administration

Rockvilie, MD 20852
12 Defense Documentation

Center

Cameron Station

Alexandria, VA 22314

\section{Commande $\mathrm{r}$}

Naval Oceanographic

Office

Washington, DC 20390

1 ATTN: Code 1640

1 ATTN: Code 70 



\begin{tabular}{|c|c|c|}
\hline \multicolumn{2}{|r|}{ REPORT DOCUMENTATION PAGE } & $\begin{array}{l}\text { READ INSTRUCTIONS } \\
\text { BEFORE COMPLETING FORM }\end{array}$ \\
\hline & \begin{tabular}{l|l|} 
REPORT NUMAER & 2. GOVT ACCESSION NO. \\
WHOI-76-101 & \\
\end{tabular} & 3. RFCIPIENT'S CATALOG NUMEEA \\
\hline \multirow{2}{*}{\multicolumn{2}{|c|}{$\begin{array}{l}\text { A COMPILATION OF MOORED CURRENT DATA AND } \\
\text { ASSOCIATED OCEANOGRAPHIC OBSERVATIONS, VOLUME } \\
\text { XII ( } 1973 \text { MID-OCEAN DYNAMICS EXPERIMENT (MODE)) }\end{array}$}} & $\begin{array}{l}\text { 5. TYPE OF REPORT \& PERIOD COVEREO } \\
\text { Technical }\end{array}$ \\
\hline & & 6. PERFORMING ORG. REPORT NUMBER \\
\hline \multicolumn{2}{|r|}{$\begin{array}{l}\text { AUTHOR(s) } \\
\text { D. Chausse and S. Tarbell }\end{array}$} & $\begin{array}{l}\text { CONTRACT OR GRANT NUMEER(O) } \\
\text { N00014-66-C-0247; } \\
\text { N00014-76-C-0197; }\end{array}$ \\
\hline \multicolumn{2}{|r|}{$\begin{array}{l}\text { PERFORMING ORGANIZATION NAME AND ADDRESS } \\
\text { Woods Hole Oceanographic Institution } \\
\text { Woods Hole, MA } 02543\end{array}$} & $\begin{array}{l}\text { 10. PROGRAM ELEMENT. PROJECT, TASK } \\
\text { AREAQ WORK UNIT NUMEERS } \\
\text { NR } 083-004 \\
\text { NR } 083-400\end{array}$ \\
\hline \multirow{2}{*}{\multicolumn{2}{|c|}{$\begin{array}{l}\text { CONTROLLING OFFICE NAME AND ADORESS } \\
\text { Office of Naval Research } \\
\text { Code } 480\end{array}$}} & $\begin{array}{l}\text { 12. REPORT DATE } \\
\text { November } 1976\end{array}$ \\
\hline & & $\begin{array}{l}\text { 13. NUMBER OF PAGES } \\
298\end{array}$ \\
\hline \multirow{2}{*}{\multicolumn{2}{|c|}{ T4 MONITORING AGENCY NAME \& ADDRESS(If difforent from Confrolling Oflice) }} & $\begin{array}{l}\text { 15. SECURITY CLASS. (ol this POPOPE) } \\
\text { Unclassified }\end{array}$ \\
\hline & & $\begin{array}{l}\text { 15. DECLASSIFICATION/DOWNGRADING } \\
\text { SCHEDULE }\end{array}$ \\
\hline \multicolumn{3}{|c|}{$\begin{array}{l}\text { 16. DISTRIBUTION STATEMENT (ol thl ROPOPt) } \\
\text { Approved for public release; distribution unlimited. }\end{array}$} \\
\hline \multicolumn{3}{|c|}{ 17. DISTRIBUTION STATEMENT (of the abetpect ontered in Block 20, "l difforent from Ropopt) } \\
\hline \multicolumn{3}{|c|}{18 SUPPLEMENTARY NOTES } \\
\hline \multicolumn{3}{|c|}{ 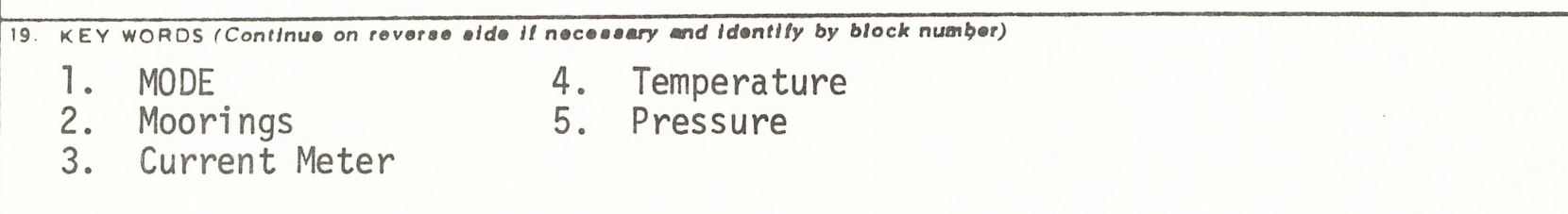 } \\
\hline \multirow{2}{*}{\multicolumn{3}{|c|}{$\begin{array}{l}\text { 20. ABSTRACT (Continue on peverse ald } 18 \text { nocoseary end idontlfy by block number) } \\
\text { Summaries are presented of basic current, temperature and pressure } \\
\text { measurements which were made from moored instruments as a part of the Mid- } \\
\text { Ocean Dynamics Experiment (MODE) which took place March to July 1973. } \\
\text { Current data are presented as Basic Statistics, Spectral Diagrams, Pro- } \\
\text { gressive Vector Diagrams, East vs. North Plots, and Variables vs. Time Plots. }\end{array}$}} \\
\hline & & \\
\hline & $\begin{array}{ll}\text { FORM } \\
\text { I JAN } 73073 & \text { EDITION OF I NOV BS IS OESOLETE } \\
\text { S/N } 0102-014 \cdot 6601\end{array}$ & SIFIED $\quad 11 / 76$ \\
\hline
\end{tabular}




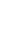

